Coastal Ocean Data System

\title{
Two- and Three-Dimensional Laboratory Studies of Wave Breaking, Dissipation, Setup, and Runup on Reefs
}

Ernest R. Smith, Tyler J. Hesser, and Jane McKee Smith

September 2012

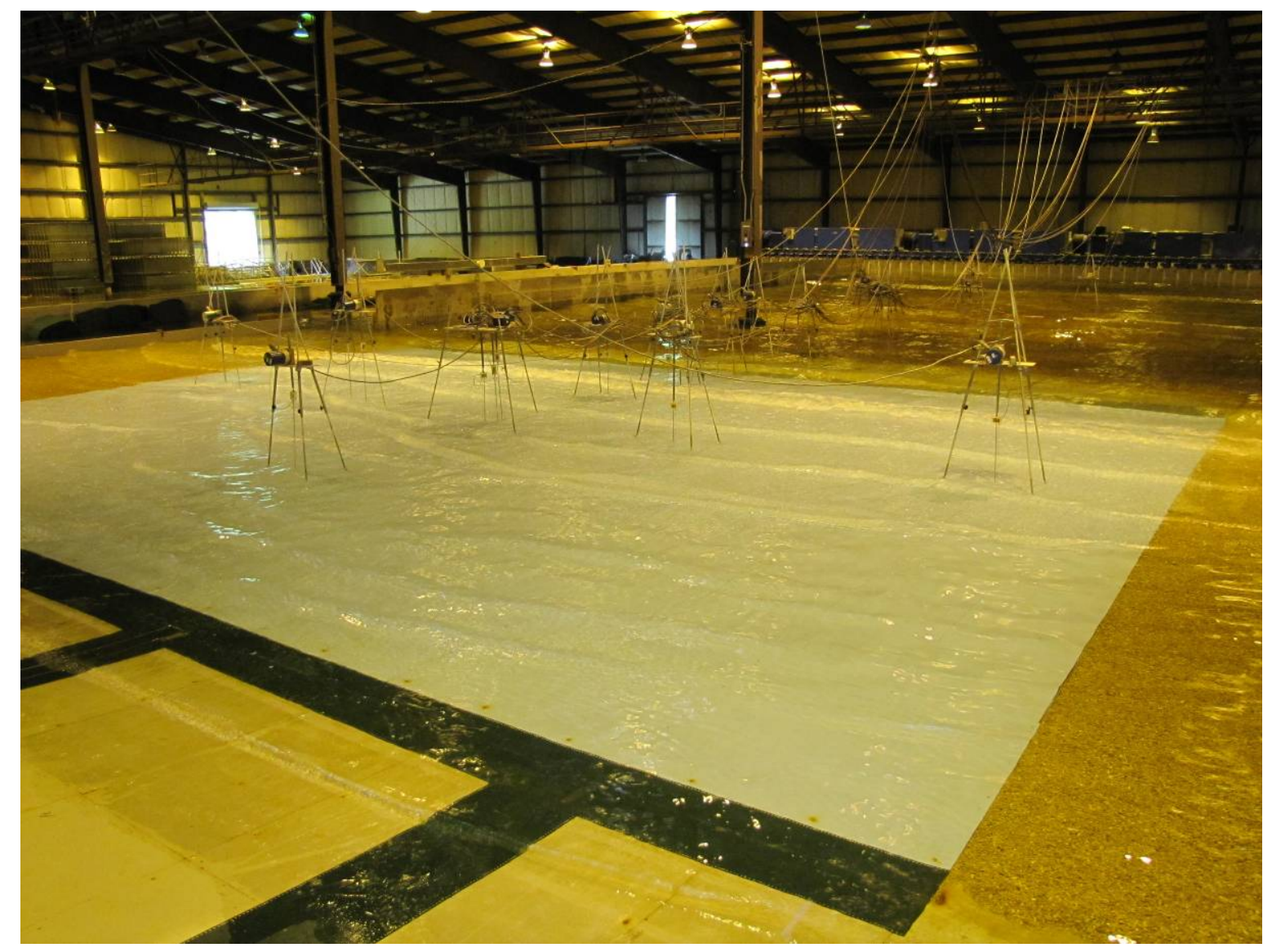




\section{Two- and Three-Dimensional Laboratory Studies of Wave Breaking, Dissipation, Setup, and Runup on Reefs}

Ernest R. Smith, Tyler J. Hesser, and Jane McKee Smith

Coastal and Hydraulics Laboratory

U.S. Army Engineer Research and Development Center

3909 Halls Ferry Road

Vicksburg, MS 39180-6199

Final report

Approved for public release; distribution is unlimited.

Prepared for U.S. Army Corps of Engineers

441 G Street, NW

Washington, DC 20314-1000

Monitored by Coastal and Hydraulics Laboratory

U.S. Army Engineer Research and Development Center

3909 Halls Ferry Road, Vicksburg, MS 39180-6199 


\section{Abstract}

Physical model studies were performed at a 1-to-50 model to prototype scale in a three-dimensional (3-D) basin and a two-dimensional (2-D) flume to measure wave transformation, setup, and runup on a generalized reef to improve understanding of the processes. Incident wave and water level conditions encompassed a range of combinations representative of the geographical locations where reefs are present.

Results from the 3-D experiment indicated higher frequency wave energy dissipated across the reef, whereas low frequency wave energy remained nearly constant. Three-dimensional patterns were observed on the reef, which was attributed to non-symmetrical bathymetry, wave-wave interactions, wave refraction, and reflection. Additional tests with an angled channel cut into the reef showed net offshore flow in the channel and near the reef centerline, where a net alongshore velocity also was measured. Wave heights were similar to the non-channel tests, but longer-period waves with higher incident heights generally showed greater heights in the shoaling region.

A cross-section of the 3-D reef was duplicated and installed in a wave tank. The 2-D experiments were performed for similar wave and water level conditions as in the three-dimensional tests. Reef slopes of 1:2.5 and 1:5 were examined. Transformation results were similar to the 3 -D tests except that a strong return flow was observed which increased breaker height. Reef roughness was varied for both the 2-D and 3-D tests, but had little effect on the waves, indicating that a rougher surface would be necessary to reduce wave height.

DISCLAIMER: The contents of this report are not to be used for advertising, publication, or promotional purposes. Citation of trade names does not constitute an official endorsement or approval of the use of such commercial products. All product names and trademarks cited are the property of their respective owners. The findings of this report are not to be construed as an official Department of the Army position unless so designated by other authorized documents. 


\section{Contents}

Abstract................................................................................................................................... ii

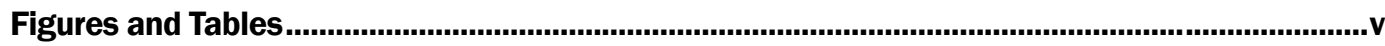

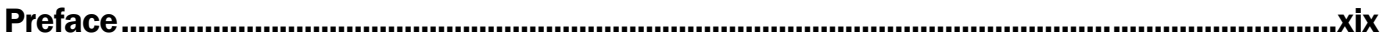

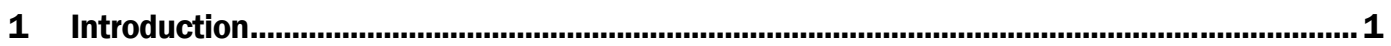

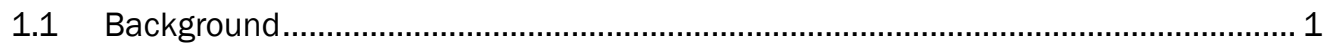

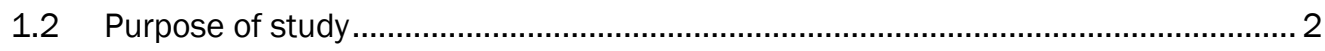

2 Three-Dimensional Physical Model Experiments ................................................................. 4

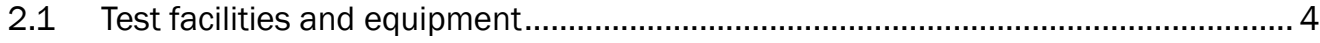

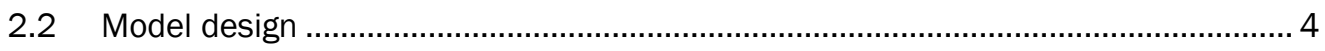

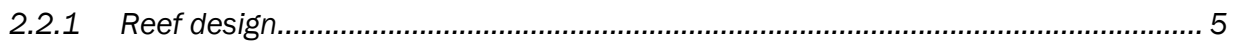

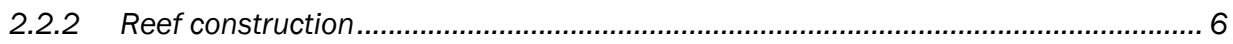

2.2.3 Reef roughness ................................................................................................. 10

2.3 Wave and water level conditions ............................................................... 10

2.4 Test procedures ............................................................................................. 14

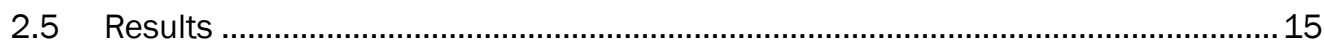

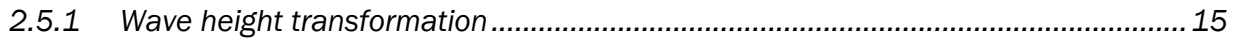

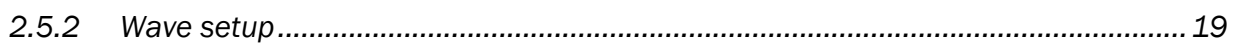

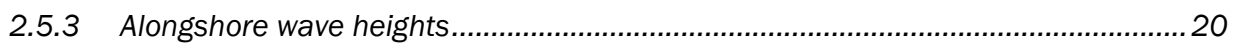

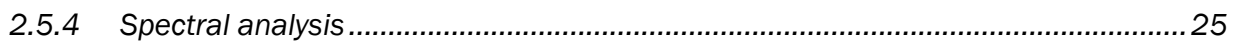

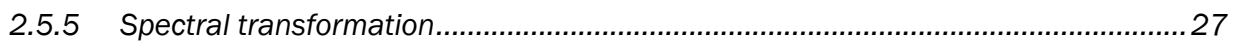

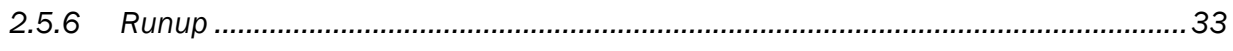

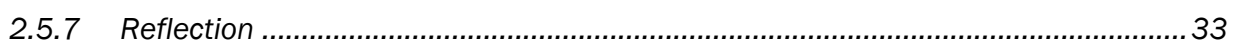

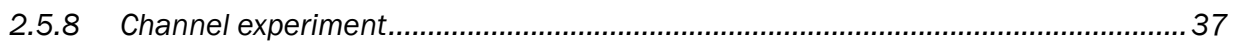

3 Two-Dimensional Physical Model Experiments ......................................................................49

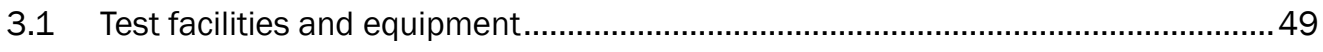

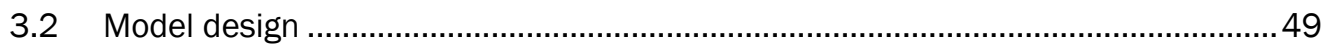

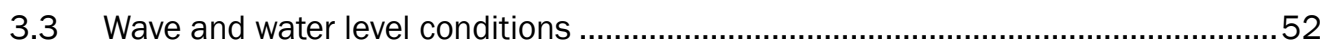

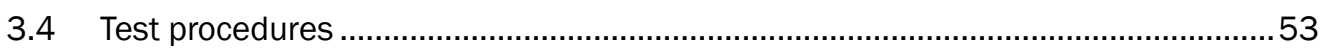

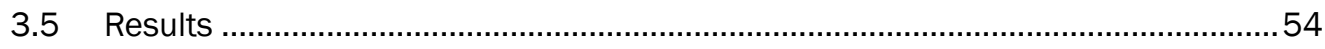

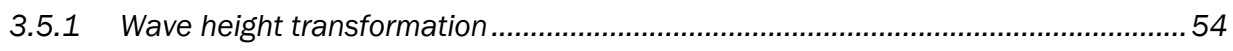

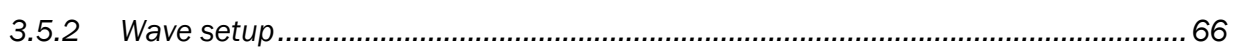

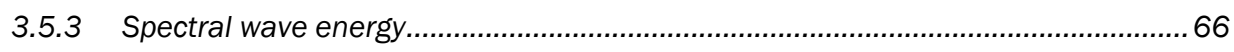

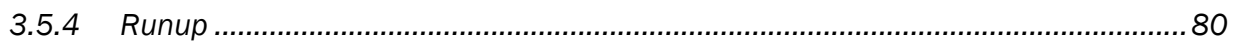

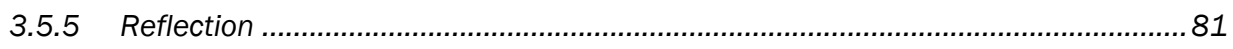

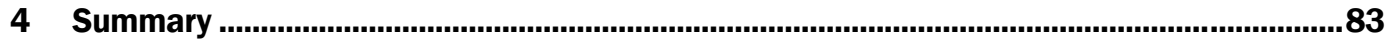

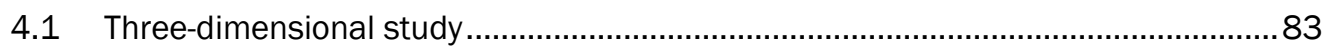

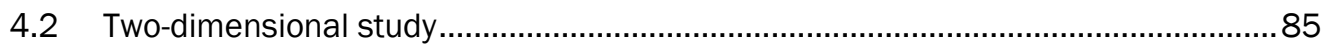




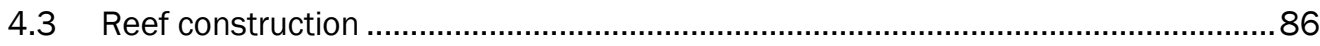

References...................................................................................................................................8

Appendix A: Wave transformation figures from the three-dimensional experiments ................89

Appendix B: Alongshore wave height figures from the three-dimensional experiments......... 110

Appendix C: Channel wave-height and current figures from the three-dimensional

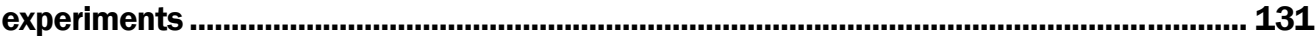

Appendix D: Wave transformation figures from UF 2-D experiments..................................... 167

Appendix E: Wave transformation figures from the two-dimensional experiments ................ 211

Report Documentation Page 


\section{Figures and Tables}

\section{Figures}

Figure 1. Reef design bathymetry.

Figure 2. Bathymetry applied onto a 0.61-m square acrylic panel. .................................................. 7

Figure 3. Installation of reef panels on compound slope..................................................................... 8

Figure 4. Elevation differences between measured and design bathymetry................................... 9

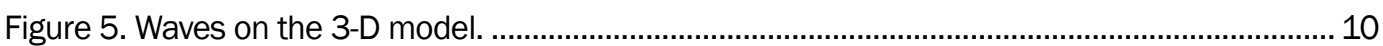

Figure 6. Grain size distribution of roughening agent.................................................................. 11

Figure 7. Three-dimensional wave basin and gauge location. ........................................................ 13

Figure 8. Wave transformation for 1-sec, 0.1-m waves at low water................................................. 16

Figure 9. Wave transformation for 2.8-sec, 0.14-m waves at low water. ...................................... 16

Figure 10. Wave transformation for $1-\mathrm{sec}, 0.1-\mathrm{m}$ waves at mid water............................................... 17

Figure 11. Wave transformation for 2.8-sec, 0.13-m waves at mid water....................................... 17

Figure 12. Wave transformation for 1-sec, 0.1-m waves at high water............................................. 18

Figure 13. Wave transformation for 2.8-sec, 0.15-m waves at high water. ....................................... 18

Figure 14. Highest mean water level recorded for each wave condition.........................................20

Figure 15. Overhead photograph of 2.8-sec, 0.1-m waves at low water........................................... 21

Figure 16. Alongshore wave heights for 1.0-sec, 0.1-m waves at low water. ................................... 22

Figure 17. Alongshore wave heights for 2.8-sec, 0.14-m waves at low water..................................... 22

Figure 18. Alongshore wave heights for 1.0-sec, 0.1-m waves at mid water................................... 23

Figure 19. Alongshore wave heights for 2.8-sec, 0.13-m waves at mid water.................................. 23

Figure 20. Alongshore wave heights for 1.0-sec, 0.1-m waves at high water.................................. 24

Figure 21. Alongshore wave heights for 2.8-sec, 0.15-m waves at high water. ................................ 24

Figure 22. Time series of 2.8-sec, 0.1-m waves at low water measured at Gauge 3........................25

Figure 23. Full energy density spectrum for 2.8-sec, $0.1 \mathrm{~m}$ waves at low water measured at Gauge 3.

Figure 24. Time series of 2.8-sec, 0.1-m waves at low water measured at Gauge 3 after low frequency cut off was applied: black line is total time series, red line is low-frequency time series. 28

Figure 25. Energy density spectrum transformation of 2.3-sec, 0.13-m waves at low water........... 29

Figure 26. Energy density spectrum transformation of 2.3-sec, 0.14-m waves at mid water.

Figure 27. Energy density spectrum transformation of 2.3-sec, 0.16-m waves at high water.

Figure 28. Low frequency energy density spectrum for 2.3-sec, 0.13-m waves at low water.

Figure 29. Low frequency energy density spectrum for 2.3-sec, 0.14-m waves at mid water. 
Figure 30. Low frequency energy density spectrum for 2.3-sec, 0.16-m waves at high water.

Figure 31. Two-percent runup as a function of wave power grouped by water level and surface roughness.

Figure 32. Two-percent runup as a function of wave power grouped by alongshore location...............34

Figure 33. Reflection coefficient as a function of surf similarity parameter. …................................36

Figure 34. Location of channel and ADVs during channel experiment........................................... 37

Figure 35. Wave transformation for 1-sec, 0.1-m waves at mid water.................................................39

Figure 36. Wave transformation for 2.8-sec, 0.12-m waves at mid water........................................39

Figure 37. Wave transformation for 1-sec, 0.1-m waves at high water. .......................................... 40

Figure 38. Wave transformation for 2.3-sec, 0.16-m waves at high water........................................ 40

Figure 39. Current rose patterns for 1-sec, 0.1-m waves at mid water........................................... 42

Figure 40. Current rose patterns for 2.8-sec, 0.12-m waves at mid water...................................... 43

Figure 41. Current rose patterns for 1-sec, 0.1-m waves at high water. .......................................... 44

Figure 42. Current rose patterns for 2.3-sec, 0.16-m waves at high water. .................................... 45

Figure 43. Alongshore wave heights for 1-sec, 0.1-m waves at mid water....................................... 46

Figure 44. Alongshore wave heights for 2.8-sec, 0.12-m waves at mid water. ................................. 47

Figure 45. Alongshore wave heights for 1-sec, 0.1-m waves at high water. ..................................... 47

Figure 46. Alongshore wave heights for 2.3-sec, 0.16-m waves at high water. ................................ 48

Figure 47. Area of bathymetry installed in 2-D flume....................................................................... 50

Figure 48. Sketch of 2-D wave flume and reef........................................................................... 51

Figure 49. Wave transformation for 1-sec, $0.1 \mathrm{~m}$ waves at low water with a 1:25 reef

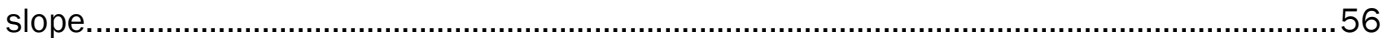

Figure 50. Wave transformation for 2.8-sec, $0.15 \mathrm{~m}$ waves at low water with a 1:25

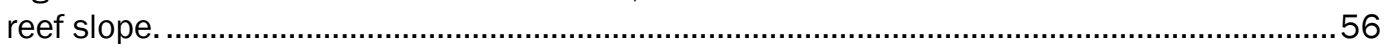

Figure 51. Wave transformation for 1-sec, $0.1 \mathrm{~m}$ waves at mid water with a 1:25 reef

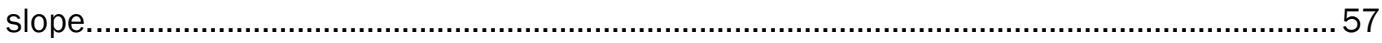

Figure 52. Wave transformation for 2.8-sec, $0.15 \mathrm{~m}$ waves at mid water with a 1:25 reef slope.

Figure 53. Wave transformation for 1-sec, $0.11 \mathrm{~m}$ waves at high water with a 1:25 reef slope.

Figure 54. Wave transformation for 2.8-sec, $0.16 \mathrm{~m}$ waves at high water with a 1:25 reef slope.

Figure 55. Wave transformation for 1-sec, $0.1 \mathrm{~m}$ waves at low water with a 1:5 reef slope.

Figure 56. Wave transformation for 2.8-sec, $0.15 \mathrm{~m}$ waves at low water with a $1: 5$ reef slope.

Figure 57. Wave transformation for $1-\mathrm{sec}, 0.1 \mathrm{~m}$ waves at mid water with a 1:5 reef slope.

Figure 58. Wave transformation for 2.8-sec, $0.15 \mathrm{~m}$ waves at mid water with a 1:5 reef slope. 60

Figure 59. Wave transformation for 1-sec, $0.1 \mathrm{~m}$ waves at high water with a 1:5 reef slope. 
Figure 60 . Wave transformation for $2.8-\mathrm{sec}, 0.16 \mathrm{~m}$ waves at high water with a 1:5 reef slope.

Figure 61. Wave transformation comparison between reef slopes for 1-sec, $0.1 \mathrm{~m}$ waves at low water with rough reef surface.

Figure 62. Wave transformation comparison between reef slopes for 2.8-sec, $0.15 \mathrm{~m}$ waves at low water with rough reef surface.

Figure 63. Wave transformation comparison between reef slopes for 1-sec, $0.1 \mathrm{~m}$ waves at mid water with rough reef surface.

Figure 64. Wave transformation comparison between reef slopes for 2.8-sec, $0.15 \mathrm{~m}$ waves at mid water with rough reef surface.

Figure 65. Wave transformation comparison between reef slopes for 1-sec, $0.1 \mathrm{~m}$ waves at high water with rough reef surface.

Figure 66. Wave transformation comparison between reef slopes for 2.8-sec, $0.16 \mathrm{~m}$ waves at high water with rough reef surface.

Figure 67. Highest mean water level recorded for each wave condition.

Figure 68. Energy density spectrum transformation of 1.8-sec, 0.11-m waves at low water with a 1:2.5 reef slope and smooth reef surface.

Figure 69. Energy density spectrum transformation of 1.8-sec, 0.11-m waves at mid water with a 1:2.5 reef slope and smooth reef surface.

Figure 70. Energy density spectrum transformation of 1.8-sec, 0.12-m waves at high water with a 1:2.5 reef slope and smooth reef surface.

Figure 71. Energy density spectrum transformation of 1.8-sec, 0.12-m waves at low water with a 1:5 reef slope and smooth reef surface.

Figure 72. Energy density spectrum transformation of 1.8-sec, 0.12-m waves at mid water with a 1:5 reef slope and smooth reef surface.

Figure 73. Energy density spectrum transformation of 1.8-sec, 0.12-m waves at high water with a 1:5 reef slope and smooth reef surface.

Figure 75. Low frequency energy density spectrum for 1.8-sec, 0.11-m waves at mid water with a 1:2.5 reef slope and smooth reef surface.

Figure 76. Low frequency energy density spectrum for 1.8-sec, 0.12-m waves at high water with a 1:2.5 reef slope and smooth reef surface.

Figure 77. Low frequency energy density spectrum for 1.8-sec, 0.12-m waves at low water with a 1:5 reef slope and smooth reef surface.

Figure 78. Low frequency energy density spectrum for 1.8-sec, 0.12-m waves at mid water with a 1:5 reef slope and smooth reef surface.

Figure 79. Low frequency energy density spectrum for 1.8-sec, 0.12-m waves at high water with a 1:5 reef slope and smooth reef surface.

Figure 80. Two-percent runup as a function of wave power......................................................... 80

Figure 81. Reflection coefficient as a function of surf similarity parameter..................................... 81

Figure A1. Wave transformation for 1-sec, 0.08-m waves at low water............................................. 89

Figure A2. Wave transformation for 1-sec, 0.1-m waves at low water...............................................90

Figure A3. Wave transformation for 1.4-sec, 0.08-m waves at low water........................................90

Figure A4. Wave transformation for 1.4-sec, 0.11-m waves at low water......................................... 91

Figure A5. Wave transformation for 1.8-sec, 0.11-m waves at low water.......................................... 91

Figure A6. Wave transformation for 1.8-sec, 0.14-m waves at low water.........................................92 
Figure A7. Wave transformation for 1.8-sec, 0.15-m waves at low water. ......................................92

Figure A8. Wave transformation for 2.3-sec, 0.11-m waves at low water.......................................93

Figure A9. Wave transformation for 2.3-sec, 0.14-m waves at low water........................................93

Figure A10. Wave transformation for 2.3-sec, 0.15-m waves at low water........................................94

Figure A11. Wave transformation for 2.8-sec, 0.1-m waves at low water........................................94

Figure A12. Wave transformation for 2.8-sec, 0.13-m waves at low water. .....................................95

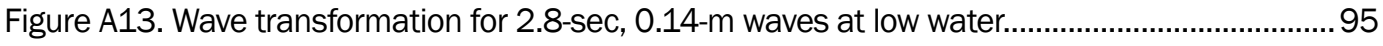

Figure A14. Wave transformation for 1-sec, 0.08-m waves at mid water......................................96

Figure A15. Wave transformation for 1-sec, 0.1-m waves at mid water. ...........................................96

Figure A16. Wave transformation for 1.4-sec, 0.07-m waves at mid water...................................... 97

Figure A17. Wave transformation for 1.4-sec, 0.12-m waves at mid water. ..................................... 97

Figure A18. Wave transformation for 1.4-sec, 0.14-m waves at mid water.......................................98

Figure A19. Wave transformation for 1.8-sec, 0.09-m waves at mid water. ……….........................98

Figure A20. Wave transformation for 1.8-sec, 0.16-m waves at mid water.......................................99

Figure A21. Wave transformation for 2.3-sec, 0.11-m waves at mid water.......................................99

Figure A22. Wave transformation for 2.3-sec, 0.15-m waves at mid water....................................100

Figure A23. Wave transformation for 2.3-sec, 0.16-m waves at mid water....................................100

Figure A24. Wave transformation for 2.8-sec, 0.09-m waves at mid water..................................... 101

Figure A25. Wave transformation for 2.8-sec, 0.13-m waves at mid water................................... 101

Figure A26. Wave transformation for 1-sec, 0.08-m waves at high water.......................................102

Figure A27. Wave transformation for 1-sec, 0.1-m waves at high water. .......................................102

Figure A28. Wave transformation for 1.4-sec, 0.09-m waves at high water..................................103

Figure A29. Wave transformation for 1.4-sec, 0.13-m waves at high water...................................103

Figure A30. Wave transformation for 1.4-sec, 0.14-m waves at high water...................................104

Figure A31. Wave transformation for 1.4-sec, 0.16-m waves at high water...................................104

Figure A32. Wave transformation for 1.8-sec, 0.1-m waves at high water. ...................................105

Figure A33. Wave transformation for 1.8-sec, 0.16-m waves at high water...................................105

Figure A34. Wave transformation for 1.8-sec, 0.17-m waves at high water....................................106

Figure A35. Wave transformation for 2.3-sec, 0.09-m waves at high water....................................106

Figure A36. Wave transformation for 2.3-sec, 0.15-m waves at high water.................................... 107

Figure A37. Wave transformation for 2.3-sec, 0.17-m waves at high water..................................... 107

Figure A38. Wave transformation for 2.8-sec, 0.09-m waves at high water....................................108

Figure A39. Wave transformation for 2.8-sec, 0.15-m waves at high water...................................108

Figure A40. Wave transformation for 2.8-sec, 0.17-m waves at high water.................................109

Figure B1. Alongshore wave heights for 1.0-sec, 0.08-m waves at low water...............................110

Figure B2. Alongshore wave heights for 1.0-sec, 0.1-m waves at low water..................................111

Figure B3. Alongshore wave heights for 1.4-sec, 0.08-m waves at low water...............................111

Figure B4. Alongshore wave heights for 1.4-sec, 0.11-m waves at low water..............................112

Figure B5. Alongshore wave heights for 1.8-sec, 0.11-m waves at low water...............................112

Figure B6. Alongshore wave heights for 1.8-sec, 0.14-m waves at low water..............................113 
Figure B7. Alongshore wave heights for 1.8-sec, 0.15-m waves at low water...............................113

Figure B8. Alongshore wave heights for 2.3-sec, 0.11-m waves at low water..............................114

Figure B9. Alongshore wave heights for 2.3-sec, 0.14-m waves at low water...............................114

Figure B10. Alongshore wave heights for 2.3-sec, 0.15-m waves at low water. ...........................115

Figure B11. Alongshore wave heights for 2.8-sec, 0.1-m waves at low water...............................115

Figure B12. Alongshore wave heights for 2.8-sec, 0.13-m waves at low water. ............................116

Figure B13. Alongshore wave heights for 2.8-sec, 0.14-m waves at low water. .............................116

Figure B14. Alongshore wave heights for 1.0-sec, 0.08-m waves at mid water............................. 117

Figure B15. Alongshore wave heights for 1.0-sec, 0.1-m waves at mid water............................... 117

Figure B16. Alongshore wave heights for 1.4-sec, 0.07-m waves at mid water.............................118

Figure B17. Alongshore wave heights for 1.4-sec, 0.12-m waves at mid water.............................118

Figure B18. Alongshore wave heights for 1.4-sec, 0.14-m waves at mid water.............................119

Figure B19. Alongshore wave heights for 1.8-sec, 0.09-m waves at mid water............................119

Figure B20. Alongshore wave heights for 1.8-sec, 0.16-m waves at mid water.............................120

Figure B21. Alongshore wave heights for 2.3-sec, 0.11-m waves at mid water............................120

Figure B22. Alongshore wave heights for 2.3-sec, 0.15-m waves at mid water.............................121

Figure B23. Alongshore wave heights for 2.3-sec, 0.16-m waves at mid water.............................121

Figure B24. Alongshore wave heights for 2.8-sec, 0.09-m waves at mid water............................122

Figure B25. Alongshore wave heights for 2.8-sec, 0.13-m waves at mid water.............................122

Figure B26. Alongshore wave heights for 1.0-sec, 0.08-m waves at high water............................123

Figure B27. Alongshore wave heights for 1.0-sec, 0.1-m waves at high water. ............................123

Figure B28. Alongshore wave heights for 1.4-sec, 0.09-m waves at high water............................124

Figure B29. Alongshore wave heights for 1.4-sec, 0.13-m waves at high water.............................124

Figure B30. Alongshore wave heights for 1.4-sec, 0.14-m waves at high water............................125

Figure B31. Alongshore wave heights for 1.4-sec, 0.16-m waves at high water............................125

Figure B32. Alongshore wave heights for 1.8-sec, 0.1-m waves at high water. .............................126

Figure B33. Alongshore wave heights for 1.8-sec, 0.16-m waves at high water............................126

Figure B34. Alongshore wave heights for 1.8-sec, 0.17-m waves at high water.............................127

Figure B35. Alongshore wave heights for 2.3-sec, 0.09-m waves at high water............................127

Figure B36. Alongshore wave heights for 2.3-sec, 0.15-m waves at high water............................128

Figure B37. Alongshore wave heights for 2.3-sec, 0.17-m waves at high water............................128

Figure B38. Alongshore wave heights for 2.8-sec, 0.09-m waves at high water............................129

Figure B39. Alongshore wave heights for 2.8-sec, 0.15-m waves at high water..............................129

Figure B40. Alongshore wave heights for 2.8-sec, 0.17-m waves at high water...........................130

Figure $\mathrm{C} 1$. Wave transformation for 1-sec, 0.09-m waves at mid water......................................132

Figure C2. Wave transformation for 1.4-sec, 0.13-m waves at mid water.....................................132

Figure C3. Wave transformation for 1.8-sec, 0.15-m waves at mid water....................................133

Figure C4. Wave transformation for 2.3-sec, 0.15-m waves at mid water....................................133

Figure C5. Wave transformation for 2.8-sec, 0.12-m waves at mid water....................................134

Figure C6. Wave transformation for 1-sec, 0.08-m waves at high water......................................134 
Figure C7. Wave transformation for 1-sec, 0.1-m waves at high water........................................135

Figure C8. Wave transformation for 1.4-sec, 0.09-m waves at high water..................................135

Figure C9. Wave transformation for 1.4-sec, 0.13-m waves at high water....................................136

Figure C10. Wave transformation for 1.4-sec, 0.15-m waves at high water. ................................136

Figure C11. Wave transformation for 1.8-sec, 0.1-m waves at high water...................................137

Figure C12. Wave transformation for 1.8-sec, 0.15-m waves at high water. ................................137

Figure C13. Wave transformation for 1.8-sec, 0.17-m waves at high water. ................................138

Figure C14. Wave transformation for 2.3-sec, 0.09-m waves at high water. ................................138

Figure C15. Wave transformation for 2.3-sec, 0.16-m waves at high water. ................................139

Figure C16. Wave transformation for 2.8-sec, 0.09-m waves at high water. ................................139

Figure C17. Wave transformation for 2.8-sec, 0.14-m waves at high water. ................................140

Figure C18. Current rose patterns for 1-sec, 0.09-m waves at mid water..................................... 141

Figure C19. Current rose patterns for 1.4-sec, 0.13-m waves at mid water..................................142

Figure C20. Current rose patterns for 1.8-sec, 0.15-m waves at mid water...................................143

Figure C21. Current rose patterns for 2.3-sec, 0.15-m waves at mid water...................................144

Figure C22. Current rose patterns for 2.8-sec, 0.12-m waves at mid water...................................145

Figure C23. Current rose patterns for 1-sec, 0.08-m waves at high water...................................146

Figure C24. Current rose patterns for 1-sec, 0.1-m waves at high water.................................... 147

Figure C25. Current rose patterns for 1.4-sec, 0.09-m waves at high water.................................148

Figure C26. Current rose patterns for 1.4-sec, 0.13-m waves at high water..................................149

Figure C27. Current rose patterns for 1.4-sec, 0.15-m waves at high water.................................150

Figure C28. Current rose patterns for 1.8-sec, 0.1-m waves at high water.................................. 151

Figure C29. Current rose patterns for 1.8-sec, 0.15-m waves at high water.................................152

Figure C30. Current rose patterns for 1.8-sec, 0.17-m waves at high water.................................153

Figure C31. Current rose patterns for 2.3-sec, 0.09-m waves at high water..................................154

Figure C32. Current rose patterns for 2.3-sec, 0.16-m waves at high water.................................155

Figure C33. Current rose patterns for 2.8-sec, 0.09-m waves at high water..................................156

Figure C34. Current rose patterns for 2.8-sec, 0.14-m waves at high water................................... 157

Figure C35. Alongshore wave heights for 1-sec, 0.09-m waves at mid water................................158

Figure C36. Alongshore wave heights for 1-sec, 0.13-m waves at mid water. ...............................158

Figure C37. Alongshore wave heights for 1.84-sec, 0.15-m waves at mid water. ..........................159

Figure C38. Alongshore wave heights for 2.3-sec, 0.15-m waves at mid water.............................159

Figure C39. Alongshore wave heights for 2.8-sec, 0.12-m waves at mid water.............................160

Figure C40. Alongshore wave heights for 1-sec, 0.08-m waves at high water. .............................160

Figure C41. Alongshore wave heights for 1-sec, 0.1-m waves at high water................................. 161

Figure C42. Alongshore wave heights for 1.4-sec, 0.09-m waves at high water............................. 161

Figure C43. Alongshore wave heights for 1.4-sec, 0.13-m waves at high water............................162

Figure C44. Alongshore wave heights for 1.4-sec, 0.15-m waves at high water............................162

Figure C45. Alongshore wave heights for 1.8-sec, 0.1-m waves at high water. .............................163

Figure C46. Alongshore wave heights for 1.8-sec, 0.15-m waves at high water............................163 
Figure C47. Alongshore wave heights for 1.8-sec, 0.17-m waves at high water............................164

Figure C48. Alongshore wave heights for 2.3-sec, 0.09-m waves at high water............................164

Figure C49. Alongshore wave heights for 2.3-sec, 0.16-m waves at high water............................165

Figure C50. Alongshore wave heights for 2.8-sec, 0.09-m waves at high water............................165

Figure C51. Alongshore wave heights for 2.8-sec, 0.14-m waves at high water............................166

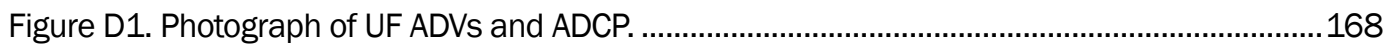

Figure D2. Wave transformation for 1-sec, $0.09 \mathrm{~m}$ waves at low water with a 1:25

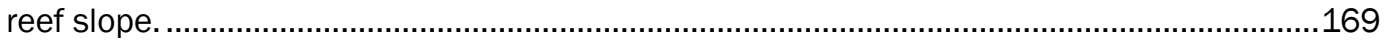

Figure D3. Wave transformation for 1-sec, $0.09 \mathrm{~m}$ waves at low water with a 1:25 reef slope.

Figure D4. Wave transformation for 1.4-sec, $0.09 \mathrm{~m}$ waves at low water with a 1:25 reef slope.

Figure D5. Wave transformation for 1.4-sec, $0.12 \mathrm{~m}$ waves at low water with a 1:25 reef slope.

Figure D6. Wave transformation for 1.8-sec, $0.11 \mathrm{~m}$ waves at low water with a 1:25 reef slope.

Figure D7. Wave transformation for 1.8-sec, $0.12 \mathrm{~m}$ waves at low water with a 1:25 reef slope.

Figure D8. Wave transformation for 1.8-sec, $0.14 \mathrm{~m}$ waves at low water with a 1:25 reef slope.

Figure D9. Wave transformation for 2.3-sec, $0.11 \mathrm{~m}$ waves at low water with a 1:25 reef slope.

Figure D10. Wave transformation for 2.3-sec, $0.12 \mathrm{~m}$ waves at low water with a 1:25 reef slope.

Figure D11. Wave transformation for 2.3-sec, $0.14 \mathrm{~m}$ waves at low water with a 1:25 reef slope.

Figure D12. Wave transformation for 2.8-sec, $0.1 \mathrm{~m}$ waves at low water with a 1:25 reef slope.

Figure D13. Wave transformation for 2.8-sec, $0.12 \mathrm{~m}$ waves at low water with a 1:25 reef slope.

Figure D14. Wave transformation for 2.8-sec, $0.15 \mathrm{~m}$ waves at low water with a 1:25 reef slope.

Figure D15. Wave transformation for 1-sec, $0.08 \mathrm{~m}$ waves at mid water with a 1:25 reef slope.

Figure D16. Wave transformation for 1-sec, $0.1 \mathrm{~m}$ waves at mid water with a 1:25 reef slope.

Figure D17. Wave transformation for 1.4-sec, $0.08 \mathrm{~m}$ waves at mid water with a 1:25 reef slope.

Figure D18. Wave transformation for 1-sec, $0.08 \mathrm{~m}$ waves at mid water with a 1:25 reef slope.

Figure D19. Wave transformation for 1.4-sec, $0.13 \mathrm{~m}$ waves at mid water with a 1:25 reef slope.

Figure D20. Wave transformation for 1.8-sec, $0.09 \mathrm{~m}$ waves at mid water with a 1:25 reef slope. 
Figure D21. Wave transformation for 1.8-sec, $0.12 \mathrm{~m}$ waves at mid water with a 1:25 reef slope.

Figure D22. Wave transformation for 1.8-sec, $0.15 \mathrm{~m}$ waves at mid water with a 1:25 reef slope.

Figure D23. Wave transformation for 2.3-sec, $0.09 \mathrm{~m}$ waves at mid water with a 1:25 reef slope.

Figure D24. Wave transformation for 2.3-sec, $0.12 \mathrm{~m}$ waves at mid water with a 1:25 reef slope.

Figure D25. Wave transformation for 2.3-sec, $0.15 \mathrm{~m}$ waves at mid water with a 1:25 reef slope.

Figure D26. Wave transformation for 2.8-sec, $0.08 \mathrm{~m}$ waves at mid water with a 1:25 reef slope.

Figure D27. Wave transformation for 2.8-sec, $0.12 \mathrm{~m}$ waves at mid water with a 1:25 reef slope.

Figure D28. Wave transformation for 2.8-sec, $0.13 \mathrm{~m}$ waves at mid water with a 1:25 reef slope.

Figure D29. Wave transformation for 2.8-sec, $0.15 \mathrm{~m}$ waves at mid water with a 1:25 reef slope.

Figure D30. Wave transformation for 1-sec, $0.08 \mathrm{~m}$ waves at high water with a 1:25 reef slope.

Figure D31. Wave transformation for 1-sec, $0.1 \mathrm{~m}$ waves at high water with a 1:25

reef slope.

Figure D32. Wave transformation for 1.41-sec, $0.09 \mathrm{~m}$ waves at high water with a 1:25 reef slope.

Figure D33. Wave transformation for 1.4-sec, $0.12 \mathrm{~m}$ waves at high water with a 1:25 reef slope.

Figure D34. Wave transformation for 1.4-sec, $0.15 \mathrm{~m}$ waves at high water with a 1:25 reef slope.

Figure D35. Wave transformation for 1.8-sec, $0.09 \mathrm{~m}$ waves at high water with a 1:25 reef slope.

Figure D36. Wave transformation for 1.8-sec, $0.15 \mathrm{~m}$ waves at high water with a 1:25 reef slope.

Figure D37 Wave transformation for 1.8-sec, $0.16 \mathrm{~m}$ waves at high water with a 1:25 reef slope...

Figure D38. Wave transformation for 2.3-sec, $0.09 \mathrm{~m}$ waves at high water with a 1:25 reef slope.

Figure D39. Wave transformation for 2.3-sec, $0.12 \mathrm{~m}$ waves at high water with a 1:25 reef slope.

Figure D40. Wave transformation for 2.3-sec, $0.16 \mathrm{~m}$ waves at high water with a 1:25 reef slope.

Figure D41. Wave transformation for 2.8-sec, $0.09 \mathrm{~m}$ waves at high water with a 1:25 reef slope.

Figure D42. Wave transformation for 2.8-sec, $0.12 \mathrm{~m}$ waves at high water with a 1:25 reef slope.

Figure D43. Wave transformation for 2.8-sec, $0.17 \mathrm{~m}$ waves at high water with a 1:25 reef slope. 
Figure D44. Wave transformation for 1-sec, 0.09-m waves at low water with a 1:5 reef slope.

Figure D45. Wave transformation for 1-sec, 0.1-m waves at low water with a 1:5 reef slope.

Figure D46. Wave transformation for 1.4-sec, 0.09-m waves at low water with a 1:5 reef slope.

Figure D47. Wave transformation for 1.4-sec, 0.12-m waves at low water with a 1:5 reef slope.

Figure D48. Wave transformation for 1.8-sec, 0.11-m waves at low water with a 1:5 reef slope.

Figure D49. Wave transformation for 1.8-sec, 0.13-m waves at low water with a 1:5 reef slope.

Figure D50. Wave transformation for 1.8-sec, 0.15-m waves at low water with a 1:5 reef slope.

Figure D51. Wave transformation for 2.3-sec, 0.11-m waves at low water with a 1:5 reef slope.

Figure D52. Wave transformation for 2.3-sec, 0.12-m waves at low water with a 1:5 reef slope.

Figure D53. Wave transformation for 2.3-sec, 0.14-m waves at low water with a 1:5 reef slope.

Figure D54. Wave transformation for 2.8-sec, 0.10-m waves at low water with a 1:5 reef slope.

Figure D55. Wave transformation for 2.83-sec, 0.12-m waves at low water with a 1:5 reef slope.

Figure D56. Wave transformation for 2.83-sec, 0.15-m waves at low water with a 1:5 reef slope.......

Figure D57. Wave transformation for 1-sec, 0.08-m waves at mid water with a 1:5 reef slope.

Figure D58. Wave transformation for 1-sec, 0.10-m waves at mid water with a 1:5 reef slope.

Figure D59. Wave transformation for 1.4-sec, 0.08-m waves at mid water with a 1:5 reef slope.

Figure D60. Wave transformation for 1.4-sec, 0.13-m waves at mid water with a 1:5 reef slope...

Figure D61. Wave transformation for 1.4-sec, 0.14-m waves at mid water with a 1:5 reef slope.

Figure D62. Wave transformation for 1.8-sec, 0.09-m waves at mid water with a 1:5 reef slope.

Figure D63. Wave transformation for 1.8-sec, 0.12-m waves at mid water with a 1:5 reef slope.

Figure D64. Wave transformation for 1.8-sec, 0.15-m waves at mid water with a 1:5 reef slope.

Figure D65. Wave transformation for 2.3-sec, 0.09-m waves at mid water with a 1:5 reef slope.

Figure D66. Wave transformation for 2.3-sec, 0.12-m waves at mid water with a 1:5 reef slope. 
Figure D67. Wave transformation for 2.3-sec, 0.15-m waves at mid water with a 1:5 reef slope.

Figure D68. Wave transformation for 2.8-sec, 0.08-m waves at mid water with a 1:5 reef slope.

Figure D69. Wave transformation for 2.8-sec, 0.12-m waves at mid water with a 1:5 reef slope.

Figure D70. Wave transformation for 2.8-sec, 0.14-m waves at mid water with a 1:5 reef slope.

Figure D71. Wave transformation for 2.8-sec, 0.16-m waves at mid water with a 1:5 reef slope.

Figure D72. Wave transformation for 1-sec, 0.08-m waves at high water with a 1:5 reef slope.

Figure D73. Wave transformation for 1-sec, 0.1-m waves at high water with a 1:5 reef slope.

Figure D74. Wave transformation for 1.4-sec, 0.1-m waves at high water with a 1:5 reef slope.

Figure D75. Wave transformation for 1.4-sec, 0.13-m waves at high water with a 1:5 reef slope. .205

Figure D76. Wave transformation for 1.4-sec, 0.15-m waves at high water with a 1:5 reef slope.

Figure D77. Wave transformation for 1.8-sec, 0.09-m waves at high water with a 1:5 reef slope. .206

Figure D78. Wave transformation for 1.8-sec, 0.16-m waves at high water with a 1:5 reef slope.

Figure D79. Wave transformation for 1.8-sec, 0.17-m waves at high water with a 1:5 reef slope.......

Figure D80. Wave transformation for 2.3-sec, 0.09-m waves at high water with a 1:5 reef slope.

Figure D81. Wave transformation for 2.3-sec, 0.13-m waves at high water with a 1:5 reef slope......

Figure D82. Wave transformation for 2.3-sec, 0.17-m waves at high water with a 1:5 reef slope.

Figure D83. Wave transformation for 2.8-sec, 0.09-m waves at high water with a 1:5 reef slope.

Figure D84. Wave transformation for 2.8-sec, 0.13-m waves at high water with a 1:5 reef slope.

Figure D85. Wave transformation for 2.8-sec, 0.17-m waves at high water with a 1:5 reef slope.

Figure E1. Wave transformation for 1-sec, 0.08-m waves at low water with a 1:25 reef slope.

Figure E2. Wave transformation for 1-sec, 0.1-m waves at low water with a 1:25 reef slope.

Figure E3. Wave transformation for 1.4-sec, 0.08-m waves at low water with a 1:25 reef slope.

Figure E4. Wave transformation for 1.4-sec, 0.11-m waves at low water with a 1:25 reef slope. 
Figure E5. Wave transformation for 1.4-sec, 0.13-m waves at low water with a 1:25 reef slope.

Figure E6. Wave transformation for 1.8-sec, 0.1-m waves at low water with a 1:25 reef slope.

Figure E7. Wave transformation for 1.8-sec, 0.11-m waves at low water with a 1:25 reef slope.

Figure E8. Wave transformation for 1.8-sec, 0.14-m waves at low water with a 1:25 reef slope.

Figure E9. Wave transformation for 2.3-sec, 0.1-m waves at low water with a 1:25 reef slope.

Figure E10. Wave transformation for 2.3-sec, 0.11-m waves at low water with a 1:25 reef slope.

Figure E11. Wave transformation for 2.3-sec, 0.14-m waves at low water with a 1:25 reef slope.

Figure E12. Wave transformation for 2.8-sec, 0.1-m waves at low water with a 1:25 reef slope.

Figure E13. Wave transformation for 2.8-sec, 0.11-m waves at low water with a 1:25 reef slope.

Figure E14. Wave transformation for 2.8-sec, 0.15-m waves at low water with a 1:25 reef slope.

Figure E15. Wave transformation for 1-sec, 0.08-m waves at mid water with a 1:25 reef slope.

Figure E16. Wave transformation for 1-sec, 0.1-m waves at mid water with a 1:25 reef slope.

Figure E17. Wave transformation for 1.4-sec, 0.07-m waves at mid water with a 1:25 reef slope.

Figure E18. Wave transformation for 1.4-sec, 0.11-m waves at mid water with a 1:25 reef slope.

Figure E19. Wave transformation for 1.4-sec, 0.13-m waves at mid water with a 1:25 reef slope.

Figure E20. Wave transformation for 1.8-sec, 0.08-m waves at mid water with a 1:25 reef slope.

Figure E21. Wave transformation for 1.8-sec, 0.11-m waves at mid water with a 1:25 reef slope.

Figure E22. Wave transformation for 1.8-sec, 0.14-m waves at mid water with a 1:25 reef slope.

Figure E23. Wave transformation for 2.3-sec, 0.09-m waves at mid water with a 1:25 reef slope.

Figure E24. Wave transformation for 2.3-sec, 0.12-m waves at mid water with a 1:25 reef slope.

Figure E25. Wave transformation for 2.3-sec, 0.15-m waves at mid water with a 1:25 reef slope.

Figure E26 Wave transformation for 2.8-sec, 0.08-m waves at mid water with a 1:25 reef slope.

Figure E27. Wave transformation for 2.8-sec, 0.12-m waves at mid water with a 1:25 reef slope. 
Figure E28. Wave transformation for 2.8-sec, 0.14-m waves at mid water with a 1:25 reef slope.

Figure E29. Wave transformation for 2.8-sec, 0.15-m waves at mid water with a 1:25 reef slope.

Figure E30. Wave transformation for 1-sec, 0.08-m waves at high water with a 1:25 reef slope.

Figure E31. Wave transformation for 1-sec, 0.11-m waves at high water with a 1:25 reef slope.

Figure E32. Wave transformation for 1.4-sec, 0.09-m waves at high water with a 1:25 reef slope.

Figure E33. Wave transformation for 1.4-sec, 0.13-m waves at high water with a 1:25 reef slope.

Figure E34. Wave transformation for 1.4-sec, 0.14-m waves at high water with a 1:25 reef slope.

Figure E35 Wave transformation for 1.8-sec, 0.09-m waves at high water with a 1:25 reef slope.

Figure E36. Wave transformation for 1.8-sec, 0.12-m waves at high water with a 1:25 reef slope.

Figure E37. Wave transformation for 1.8-sec, 0.15-m waves at high water with a 1:25 reef slope.

Figure E38. Wave transformation for 2.3-sec, 0.1-m waves at high water with a 1:25 reef slope. .230

Figure E39. Wave transformation for 2.3-sec, 0.13-m waves at high water with a 1:25 reef slope.

Figure E40. Wave transformation for 2.3-sec, 0.16-m waves at high water with a 1:25 reef slope.

Figure E41. Wave transformation for 2.8-sec, 0.09-m waves at high water with a 1:25 reef slope.

Figure E42. Wave transformation for 2.8-sec, 0.13-m waves at high water with a 1:25 reef slope.

Figure E43. Wave transformation for 2.8-sec, 0.16-m waves at high water with a 1:25 reef slope.

Figure E44. Wave transformation for 1-sec, 0.08-m waves at low water with a 1:5 reef slope.

Figure E45. Wave transformation for 1-sec, 0.1-m waves at low water with a 1:5 reef slope.

Figure E46. Wave transformation for 1.4-sec, 0.08-m waves at low water with a 1:5 reef slope.

Figure E47. Wave transformation for 1.4-sec, 0.11-m waves at low water with a 1:5 reef slope.

Figure E48. Wave transformation for 1.4-sec, 0.13-m waves at low water with a 1:5 reef slope.

Figure E49. Wave transformation for 1.8-sec, 0.1-m waves at low water with a 1:5 reef slope.

Figure E50. Wave transformation for 1.8-sec, 0.11-m waves at low water with a 1:5 reef slope. 
Figure E51. Wave transformation for 1.8-sec, 0.14-m waves at low water with a 1:5 reef slope.

Figure E52. Wave transformation for 2.3-sec, 0.1-m waves at low water with a 1:5 reef slope.

Figure E53. Wave transformation for 2.3-sec, 0.11-m waves at low water with a 1:5 reef slope.

Figure E54. Wave transformation for 2.3-sec, 0.14-m waves at low water with a 1:5 reef slope.

Figure E55. Wave transformation for 2.8-sec, 0.09-m waves at low water with a 1:5 reef slope.

Figure E56. Wave transformation for 2.8-sec, 0.11-m waves at low water with a 1:5 reef slope.

Figure E57. Wave transformation for 2.8-sec, 0.15-m waves at low water with a 1:5 reef slope.

Figure E58. Wave transformation for 1-sec, 0.08-m waves at mid water with a 1:5 reef slope.

Figure E59. Wave transformation for 1-sec, 0.1-m waves at mid water with a 1:5 reef slope.

Figure E60. Wave transformation for 1.4-sec, 0.07-m waves at mid water with a 1:5 reef slope.

Figure E61. Wave transformation for 1.4-sec, 0.11-m waves at mid water with a 1:5 reef slope.

Figure E62. Wave transformation for 1.4-sec, 0.13-m waves at mid water with a 1:5 reef slope.

Figure E63. Wave transformation for 1.8-sec, 0.08-m waves at mid water with a 1:5 reef slope.

Figure E64. Wave transformation for 1.8-sec, 0.11-m waves at mid water with a 1:5 reef slope.

Figure E65. Wave transformation for 1.8-sec, 0.14-m waves at mid water with a 1:5 reef slope.

Figure E66. Wave transformation for 2.3-sec, 0.09-m waves at mid water with a 1:5 reef slope.

Figure E67. Wave transformation for 2.3-sec, 0.12-m waves at mid water with a 1:5 reef slope.

Figure E68. Wave transformation for 2.3-sec, 0.15-m waves at mid water with a 1:5 reef slope.

Figure E69. Wave transformation for 2.8-sec, 0.08-m waves at mid water with a 1:5 reef slope.

Figure E70. Wave transformation for 2.8-sec, 0.12-m waves at mid water with a 1:5 reef slope.

Figure E71. Wave transformation for 2.8-sec, 0.14-m waves at mid water with a 1:5 reef slope.

Figure E72. Wave transformation for 2.8-sec, 0.15-m waves at mid water with a 1:5 reef slope.

Figure E73. Wave transformation for 1-sec, 0.08-m waves at high water with a 1:5 reef slope. 
Figure E74. Wave transformation for 1-sec, 0.1-m waves at high water with a 1:5 reef slope.

Figure E75. Wave transformation for 1.4-sec, 0.09-m waves at high water with a 1:5 reef slope.

Figure E76. Wave transformation for 1.4-sec, 0.12-m waves at high water with a 1:5 reef slope.

Figure E77. Wave transformation for 1.4-sec, 0.14-m waves at high water with a 1:5 reef slope.

Figure E78. Wave transformation for 1.8-sec, 0.09-m waves at high water with a 1:5 reef slope. 250

Figure E79. Wave transformation for 1.8-sec, 0.12-m waves at high water with a 1:5 reef slope.

Figure E80. Wave transformation for 1.8-sec, 0.15-m waves at high water with a 1:5 reef slope.

Figure E81. Wave transformation for 2.3-sec, 0.1-m waves at high water with a 1:5 reef slope.

Figure E82. Wave transformation for 2.3-sec, 0.13-m waves at high water with a 1:5 reef slope.

Figure E83. Wave transformation for 2.3-sec, 0.16-m waves at high water with a 1:5 reef slope.

Figure E84. Wave transformation for 2.8-sec, 0.09-m waves at high water with a 1:5 reef slope.

Figure E85. Wave transformation for 2.8-sec, 0.12-m waves at high water with a 1:5 reef slope.

Figure E86. Wave transformation for 2.8-sec, 0.16-m waves at high water with a 1:5 reef slope.

\section{Tables}

Table 1. Model-prototype scale relations (1:50 scale)...................................................................... 5

Table 2. SWIMS 3-D reef design wave conditions in prototype, $\mathrm{m}$ (Model, $\mathrm{m}$ )..................................12

Table 3. SWIMS 3-D reef wave gauge locations......................................................................... 12

Table 4. SWIMS 3-D reef ADV locations (see Figure 7 for coordinate system)....................................38

Table 5. SWIMS 2-D reef design wave conditions in prototype, $\mathrm{m}$ (Model, $\mathrm{m}$ )...................................52

Table 6. SWIMS 2-D reef wave gauge locations................................................................................53

Table D1. ERDC - UF 2-D reef design wave conditions in prototype, $m$ (Model, m). .........................167 


\section{Preface}

This study was conducted under the Coastal Ocean Data System Program's Surge and Wave Island Modeling Studies (SWIMS) Project. The Program Manager was William Birkemeier and the technical lead was Dr. Jane McKee Smith.

This report was prepared by Drs. Ernest R. Smith, Tyler J. Hesser, and Jane McKee Smith, Coastal Processes Branch (CEERD-HF-C), of the Flood and Storm Protection Division (CEERD-HF), Coastal and Hydraulic Laboratory (CHL), U.S. Army Engineer Research and Development Center (ERDC). Work was performed under the general administrative supervision of Dr. Ty Wamsley, Chief, CEERD-HF-C; Bruce Ebersole, former Chief, CEERD-HF; Jose E. Sanchez, Deputy Director, (CHL) and Dr. William D. Martin, Director (CHL). William Curtis was the Technical Director for the Flood and Coastal Storm Damage Reduction. Experiments were conducted by Drs. Smith and Hesser, and Hugh F. Acuff formerly of Harbors, Entrances, and Structures Branch (CEERD-HN-H). Tim Nisley and David Daily, of the ERDC Information Technology Laboratory provided instrumentation support. Dr. Michael J. Briggs (CEERD-HN-H) provided valuable review of the draft version of the report.

At the time of publication, COL Kevin D. Wilson was Commander and Executive Director of ERDC. Dr. Jeffery P. Holland was Director. 


\section{Introduction}

\subsection{Background}

Island coasts and populations are extremely vulnerable to tropical storms. Waves propagating on top of storm surge from tropical cyclones (typhoons and hurricanes) can penetrate inland from the coast, and the resulting inundation and wave runup can cause significant damage.

Methodologies for analyzing tropical cyclone waves were developed for mainland coasts, which generally consist of gently sloping beaches. Islands differ from mainland coasts in that they are adjacent to deep water, exposed to very large storm waves, and most include fringing coral reefs. Waves undergo significant transformation as they shoal and break on fringing reefs and then run up the shore, flooding coastal areas. Storm waves and storm surge are closely linked. Large waves increase water levels due to wave setup (wave setup can account for half of storm surge in island environments) and increased water levels allow waves to break further inland, which further increases runup and flooding on island shorelines. The interaction between surge and waves is nonlinear and complicates reliable estimates of the maximum inundation caused by tropical cyclone waves, which is important for establishing flood risk and managing emergency response.

The topography of reefs in the Pacific and Atlantic Oceans and Caribbean Sea are non-uniform, which results in non-uniform bottom roughness, and the size, profile, and morphology of the reefs can vary significantly. Research studies have shown that the physical structure of reef geometry determines the characteristics of wave transformation and resulting wave breaking and attenuation over reefs (Hardy et al. 1991; Gourlay 1994, 1996a, 1996b; Massel and Gourlay 2000). These site- and reef geometryspecific data may not be used for other reef geometries. Also, waves impinging on complex and unique reef topography are influenced by a variety of hydrodynamic processes including the turbulence generated by waves and flow over the specific structure of a given reef.

Numerical modeling of waves over reefs remains a difficult coastal engineering challenge because of the nonlinear interaction between waves and water levels, and because it is not easy to characterize the topography of reefs. Earlier attempts to model waves over reefs have relied on simplified 
models, limiting the success of numerical wave models applied over long reef reaches with complex morphology. Additionally, the current design guidance for estimating wave setup and wave runup for island flooding is based on empirical formulae derived from laboratory experiments for planar beaches and sloping structures (Headquarters, U.S. Army Corps of Engineers 2002). It is generally recognized that nonlinear wave-wave interactions lead to strong low-frequency (infragravity) oscillations of the water level close to the shoreline. Wave runup estimates associated with the low-frequency oscillations over reef-type topographies can be significantly higher than those for planar beaches (Nwogu and Demirbilek 2006, 2010). For example, runup during Hurricane Iniki on the island of Kauai in Hawaii was 6 to $8 \mathrm{~m}$ in some locations. In summary, coastal inundation calculation methodologies for island coasts have not received attention commensurate with the importance and complexity of the processes.

\subsection{Purpose of study}

The U.S. Army Corps of Engineers (USACE) initiated the Surge and Wave Island Modeling Studies (SWIMS) program in 2005 to develop improved methodologies for predicting coastal flooding associated with typhoons and hurricanes in the U.S. Pacific and Caribbean islands. A comprehensive understanding of the physics of wave inundation over reef systems is required to develop reliable predictive models of inundation for emergency planning and for calculation of flooding. The objectives of SWIMS are to:

- Acquire coastal hydrodynamic and meteorological data under tropical cyclone and high-wind conditions in island environments.

- Use these data to improve understanding of the physics of coastal waves and water levels in these environments.

- Incorporate this understanding to improve analytic and computerbased models for application to tropical cyclone hazard mitigation studies.

- Contribute these results to improved emergency management tools and procedures for islands.

As part of SWIMS, three-dimensional (3-D) and two-dimensional (2-D) physical model studies were performed to measure wave transformation, setup, runup, and reflection on steep bathymetry and fringing reefs to improve the understanding of the processes. In conjunction with field measurements collected under a sister program (Pacific Islands 
Land-Ocean Typhoon Experiment), the physical model data are being used to validate numerical models and modeling systems.

The 3-D model included a generalized reef surface overlaid on an embayed surface. A total of 94 tests were performed on the 3-D reef, which included 16 tests conducted with a channel cut into the reef bathymetry. A 0.61-mwide section of the 3-D reef was reproduced and installed in a wave tank for the 2-D experiments. Prior to conducting the SWIMS 2-D experiments, a set of data was collected in collaboration with the University of Florida (UF), which included measurements of currents on the reef slope in addition to wave, water surface, and runup measurements. A total of 172 tests was performed in the SWIMS 2-D experiment that included two slopes fronting the reef. Additionally, tests were performed in both the 3-D and 2-D studies with smooth and roughened reef surfaces.

Model wave conditions were selected to encompass a range of wave period and height combinations representative of the geographical locations where reefs are present: the Pacific and Atlantic Oceans, and the Caribbean Sea. The test conditions also included waves at multiple water levels because wave breaking and transformation is controlled in part by the water depth.

The design, construction, and results from the 3-D and 2-D experiments are discussed in Chapters 2 and 3, respectively. A summary of the study is given in Chapter 4. Appendix A includes wave transformation figures from the 3-D experiments and Appendix B contains figures of wave heights measured in the longshore direction of the 3-D experiment. Figures produced from the 3-D experiment with the channel installed are found in Appendix C. Wave transformation figures from the 2-D UF experiments are in Appendix D, and Appendix E contains wave transformation figures from the SWIMS 2-D experiment. 


\section{Three-Dimensional Physical Model Experiments}

This chapter describes the design and construction of the three-dimensional reef experiments. The purpose of these experiments was to obtain wave, setup and runup data over an idealized reef, which included an irregular surface and an embayment.

\subsection{Test facilities and equipment}

Tests were conducted in a 51.8-m-long, 29.0-m-wide, and 1.5-m-deep wave basin. The basin contained a $14.8-\mathrm{m}$-long by $22.0-\mathrm{m}$-wide compound-slope steel platform on which reef bathymetry was installed. The platform was configured into a compound slope that included reef slopes of 1:4 (0.29-m long) and 1:13 (2.43-m long), a 7.3-m flat section, and a 1:10 foreshore slope (4.8-m long).

Waves were produced by the Directional Spectral Wave Generator (DSWG). The DSWG is 27.4-m long and consists of 60 paddles, divided into four modules. Each paddle is $0.46-\mathrm{m}$ wide by $1-\mathrm{m}$ high and driven at the paddle joints by an electric motor in piston mode. Displacement of the DSWG wave paddles was controlled by a command signal transmitted to the board. Irregular wave command signals to drive the board were generated to simulate a TMA shallow-water unidirectional spectrum (Bouws et al. 1985), with a gamma value of $3 \cdot 3$.

The DSWG has a stroke of $0.36 \mathrm{~m}$, and angles between paddles can be continuously varied using the "snake principle" to produce waves at angles approaching 85 deg from normal. However, only shore-normal waves, i.e., perpendicular to the DSWG, were generated for the present study. Additionally, Module 1 was not operated due to technical difficulties. Modules 2 through 4 provided sufficient coverage of the reef model to accomplish the experiment.

\subsection{Model design}

Model experiments were conducted at a geometrically-undistorted linear scale of 1:50, model to prototype. Scale was based on the capabilities of the wave generator to produce required wave heights at modeled water depths. 
Time relations were scaled according to Froude Model Law (Stevens et al. 1942) and model-prototype relations were defined in terms of length $l$ and time $t$ shown in Table 1.

Table 1. Model-prototype scale relations (1:50 scale)

\begin{tabular}{|l|l|l|}
\hline Characteristic & Dimension & $\begin{array}{l}\text { Scale Relations } \\
\text { Model:Prototype }\end{array}$ \\
\hline Length & $I$ & $I_{r}=1: 50$ \\
\hline Area & $I^{2}$ & $a_{r}=1: 2500$ \\
\hline Volume & $I^{3}$ & $v_{r}=1: 125,000$ \\
\hline Time & $11 / 2$ & $t_{r}=1: 7.07$ \\
\hline
\end{tabular}

\subsubsection{Reef design}

The goal of the physical model experiments was to provide the numerical modeling effort detailed wave transformation, setup, and runup observations on a generalized reef topography/bathymetry. The physical model was designed and constructed to include idealized reef topography to provide roughness, and an embayment to provide three-dimensional wave transformation.

Two approaches were considered in the design of the irregular reef surface. The first approach considered a range of random depths generated over a grid. However, it was important that the idealized reef surface represented bathymetry that reproduced realistic hydrodynamic conditions, and this approach, though feasible, would require judgment as to what constituted a "realistic" idealized reef. The second approach, which was adopted, was more straightforward and consisted of modifying bathymetry of an existing reef. LIDAR data were available for southeast Guam. To idealize the Guam bathymetry, features specific to Guam were removed from the data. To remove the site-specific features, the bathymetry was interpolated to a uniform grid with $0.5-\mathrm{m}$ prototype spacing. The mean slope was determined by a polynomial solution along each cross-shore grid line, and deviations from the mean slope were saved as idealized reef bathymetry. The result was a horizontal irregular surface having a standard deviation of approximately $1 \mathrm{~m}$. The reef bathymetry was then overlaid onto an embayed surface, which was 375-m long (prototype), and centered at the offshore portion of the reef with a shoreward extent of $250 \mathrm{~m}$. The contours of the resulting idealized bathymetry at model scale are shown in Figure 1. 


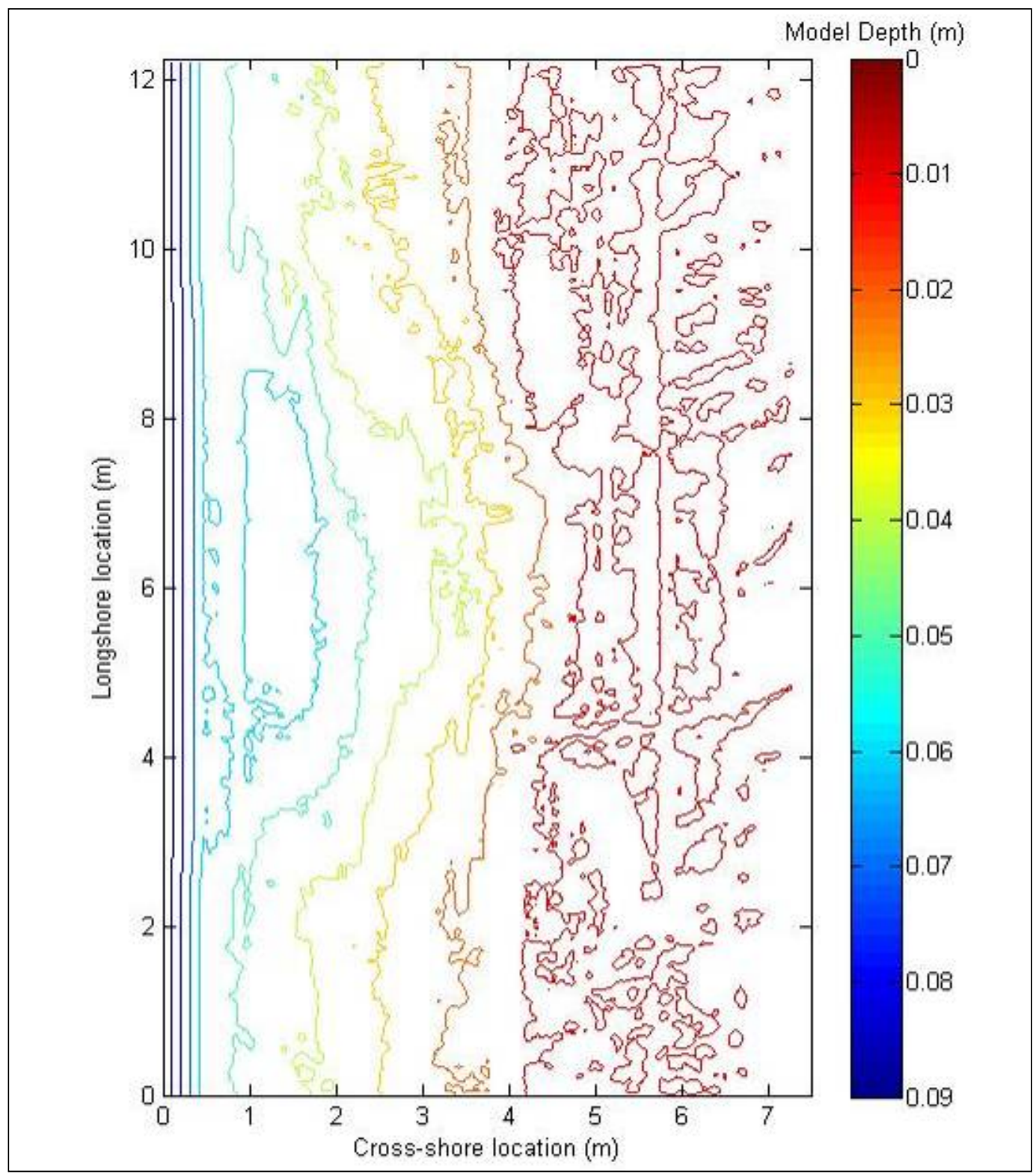

Figure 1. Reef design bathymetry.

\subsubsection{Reef construction}

A durable material was required for the model reef to allow installation of numerous wave gauges and the ability to accommodate personnel to access the gauges. Three materials were considered for reef construction: concrete, high-density pressed foam, and acrylic glass. Traditionally, three-dimensional bathymetric models have been constructed of concrete. However, it was not feasible to achieve the desired bathymetric resolution with concrete construction. Whether or not pressed foam could support personnel and maintain the desired bathymetry was uncertain; therefore, the reef was constructed of acrylic glass. 
The idealized reef bathymetry was reduced to model scale, which yielded a horizontal model spatial resolution of $0.01 \mathrm{~m}$. A programmable automated table router cut the reef surface from the bathymetric xyz coordinates onto o.61-m wide, 2.44-m long, 0.05-m thick acrylic glass sheets. After the surface was cut, the sheets were reduced to $0.61-\mathrm{m}$ square panels, which improved installation efficiency over the heavier rectangular sheets. An example of the reef surface resolution on one of the square panels is shown in Figure 2.

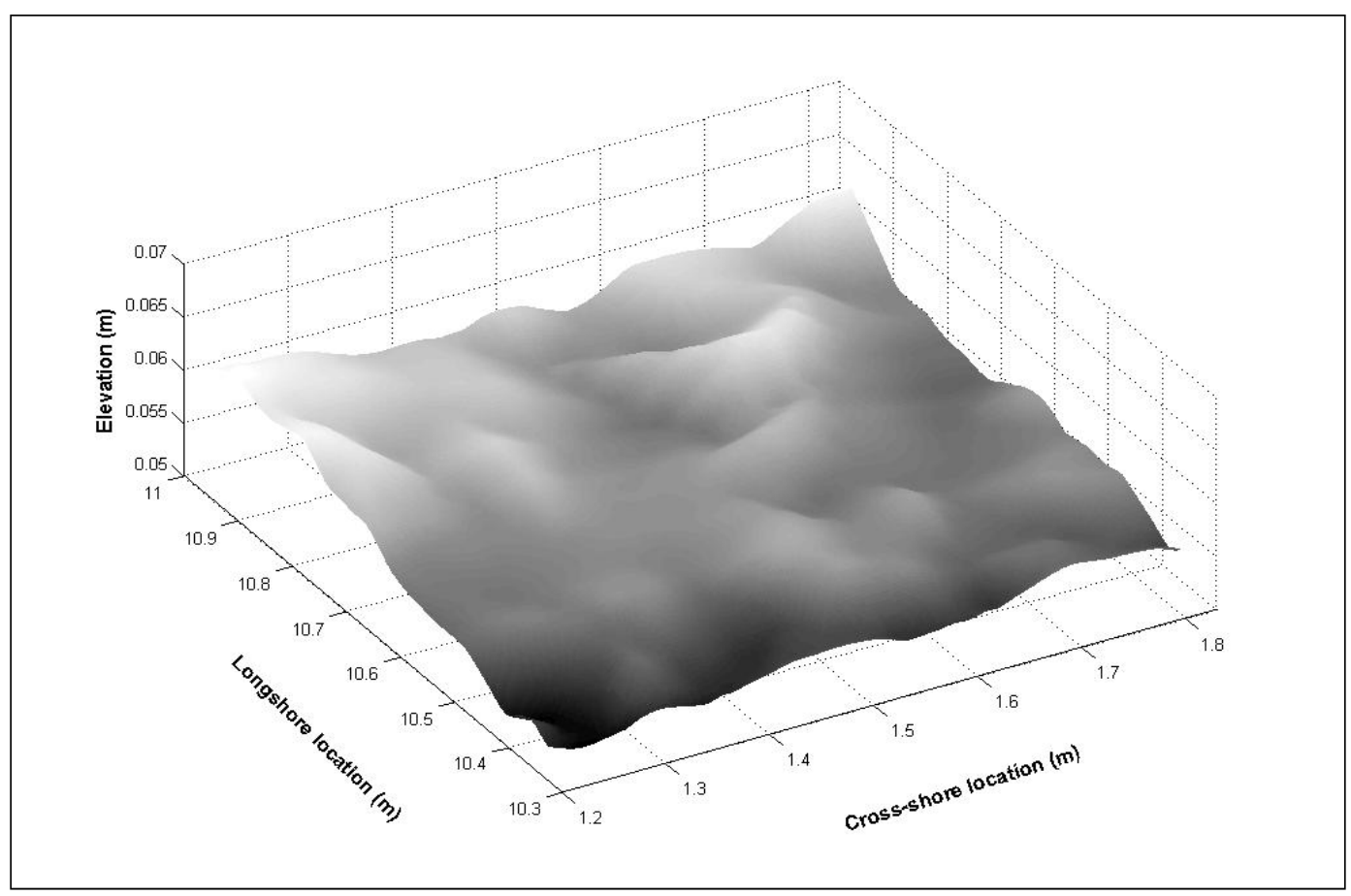

Figure 2. Bathymetry applied onto a 0.61-m square acrylic panel.

The reef panels were installed on the horizontal portion of the compound slope platform (Figure 3). Legs were attached to the corners of each panel and were adjusted to the design elevation. The priority of installing the panels was to maintain a uniform surface between sections. Some sections were adjusted to maintain smooth transitions between adjacent acrylic sections. This procedure resulted in a continuous reef surface, although actual elevations deviated slightly from the design. After a section was adjusted to a satisfactory elevation, the legs were attached to the steel platform with a marine urethane adhesive/sealant.

A post-construction survey of the reef was performed with a 3-D-scanning laser to determine constructed elevations. Figure 4 shows differences between measured and design elevations. The majority of the reef is within 


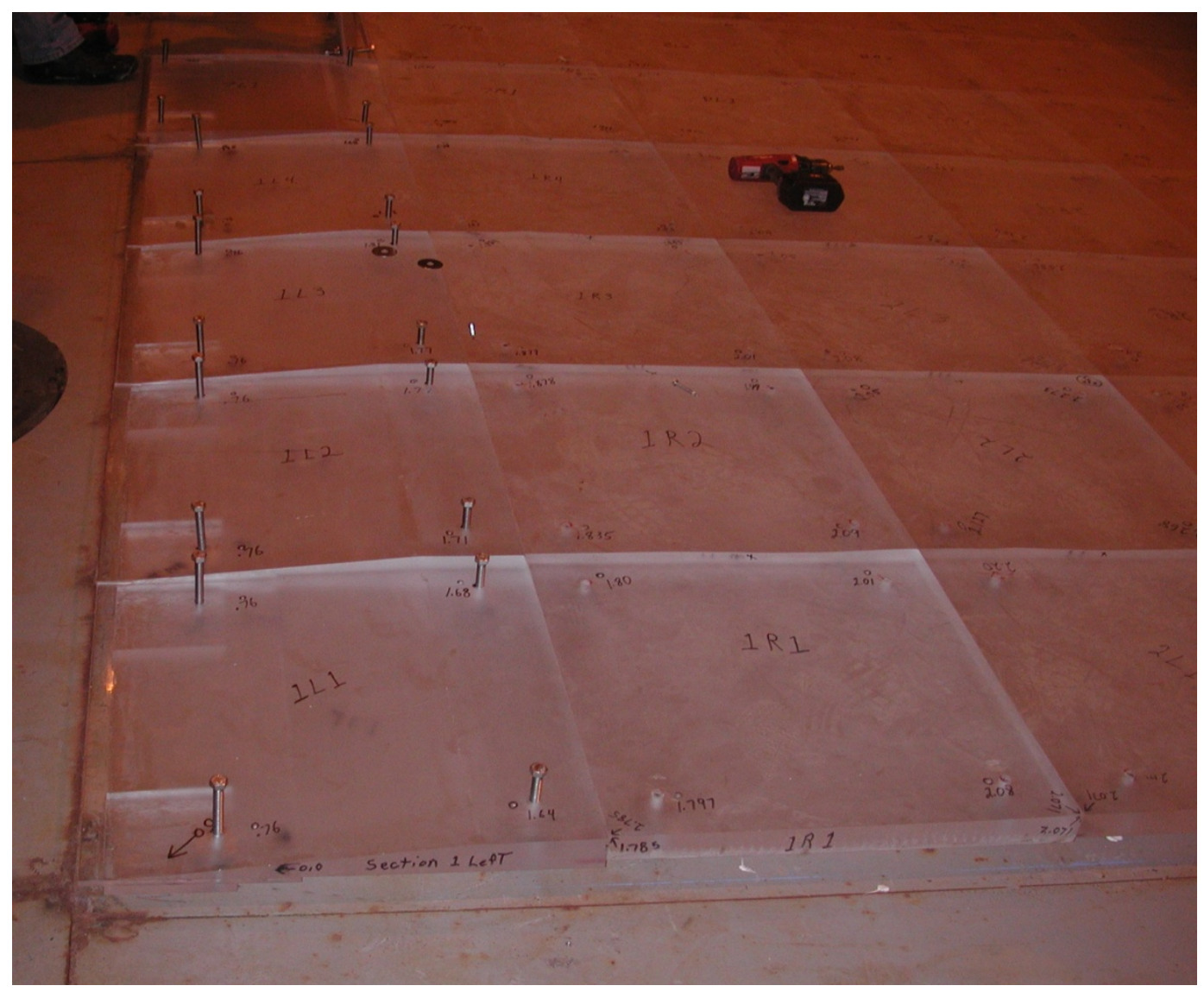

Figure 3. Installation of reef panels on compound slope.

$0.004 \mathrm{~m}$ of the design elevation. The figure indicates elevation differences as high as $0.04 \mathrm{~m}$ along the offshore reef boundary where the acrylic is very thin and nearly transparent. The scanning laser resolves elevations from captured images, and we believe that the underlying surface was captured along the offshore boundary rather than the reef surface. Elevations were approximately $0.01 \mathrm{~m}$ higher at many points between longshore locations of 8.0 and $12.2 \mathrm{~m}$. These anomalies were a result of non-uniformity of the underlying steel platform where the acrylic pieces were adjusted to maintain a flush surface. The result is that depths are slightly shallower in this region.

The reef height was approximately $0.15 \mathrm{~m}$ above the steel platform on the landward side of the reef, and plywood was installed as a transition between the platform and reef in the cross shore. Additionally, the steel platform was $4.9 \mathrm{~m}$ longer than the reef on each side in the longshore direction. Gravel of size $d_{50} \approx 6.4 \mathrm{~mm}$ was placed on each end of the acrylic reef to provide consistent depth and prevent refraction at the side boundary. A concrete layer was added to stabilize the gravel in the breaking zone. 


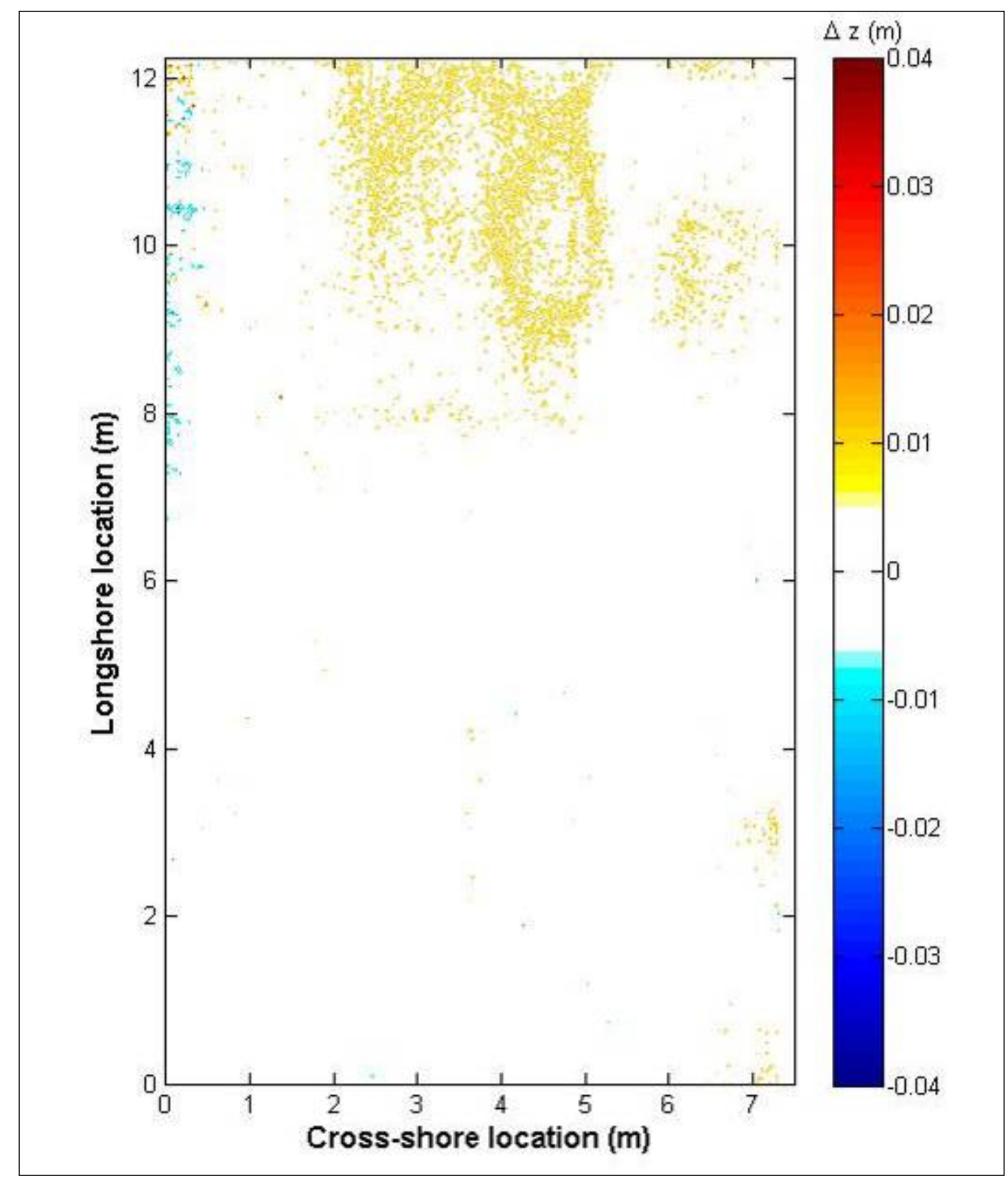

Figure 4. Elevation differences between measured and design bathymetry.

Figure 5 shows the completed reef during an experiment with wave gauges on the reef and the DSWG in the background. Gravel bordering the reef sides is shown as brown. Insulated tape, shown as dark stripes in the foreground of the figure, prevented runup gauges from short circuiting with the underlying steel foreshore slope. The tape also was placed alongshore to provide a seal at the transition between the reef and foreshore slope 


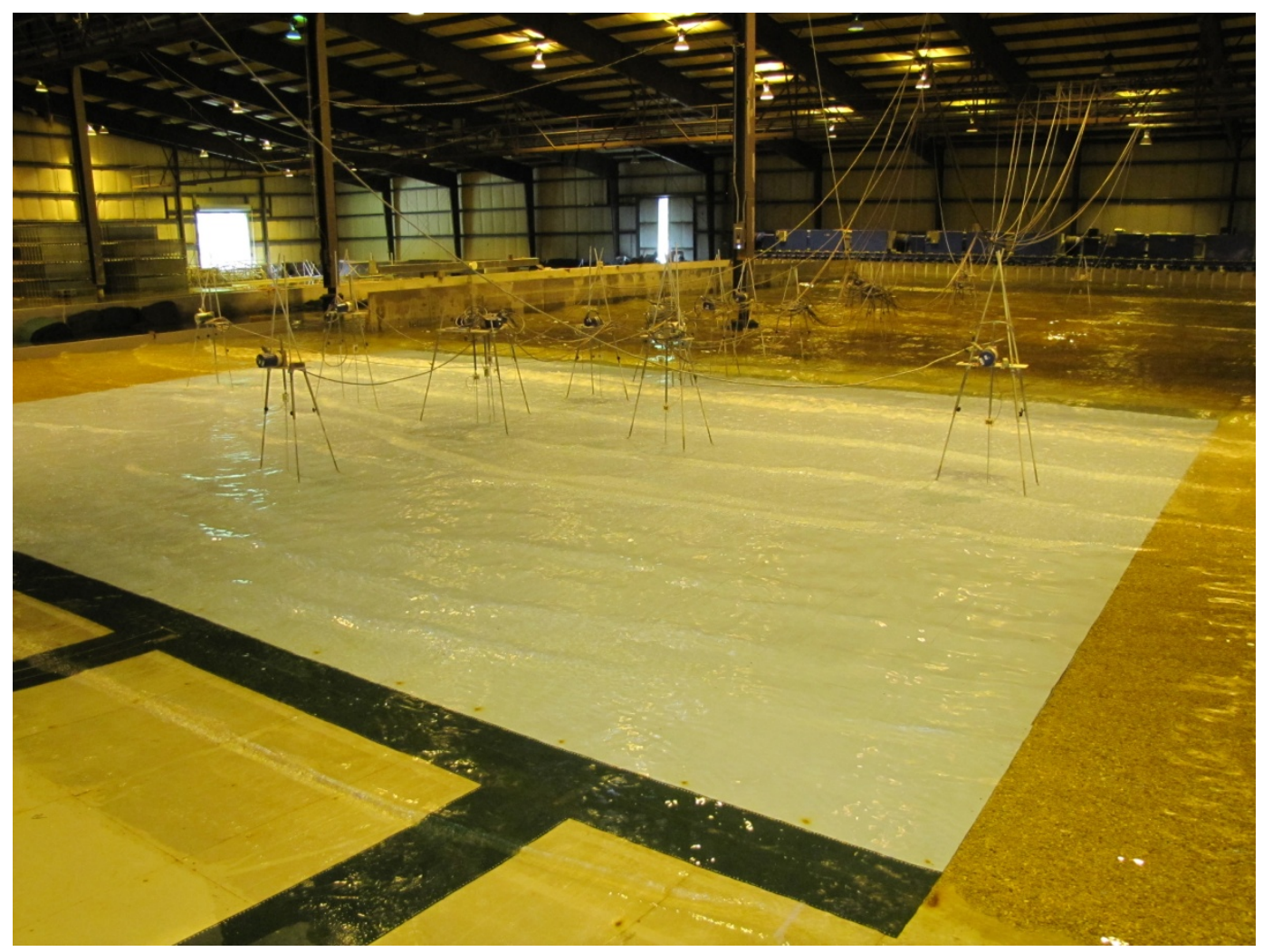

Figure 5. Waves on the 3-D model.

\subsubsection{Reef roughness}

One of the complexities of modeling waves on reefs is specifying the roughness coefficient in the numerical model. Tests were performed for two roughnesses. Roughness for the initial tests consisted solely of the surface bathymetry molded into the acrylic. Although the bathymetry was irregular, the surface was smooth. Following experiments with the "smooth" surface, a roughening agent was added to paint and applied to the acrylic. The roughening agent was commercially obtained and had a $d_{50}$ of $0.33 \mathrm{~mm}$ (Figure 6).

\subsection{Wave and water level conditions}

Islands with fringing reefs are present in the Pacific and Atlantic Oceans and the Caribbean Sea, each characterized by different wave conditions.

Fetch lengths and depths of the Pacific generally produce higher waves with longer periods, whereas storm conditions in the Atlantic and Caribbean Sea have shorter periods and lower wave heights due to shorter fetch and shallower depths. Consequently, the model design wave conditions were selected to encompass a range of wave period and height combinations 


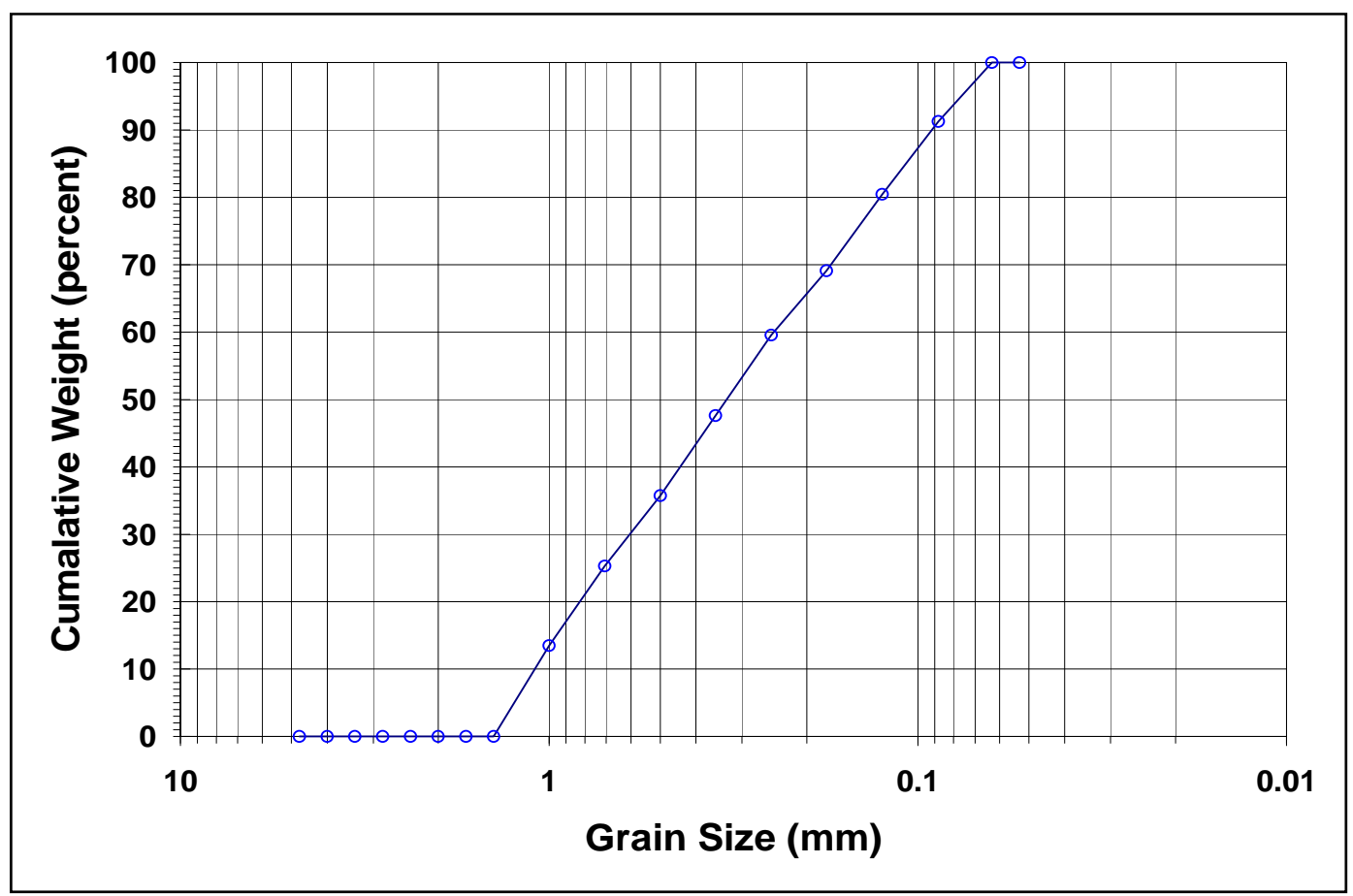

Figure 6. Grain size distribution of roughening agent.

representative of the geographical locations where reefs are present. Wave breaking and transformation is controlled in part by the water depth; therefore, test conditions included waves at multiple water levels. A total of forty wave and water level conditions were generated for the study and are given in Table 2 in prototype units with the corresponding model units in parenthesis. Table 2 provides peak wave period, $T_{p}$, incident zero-moment wave height, $H_{i}$, and still water depth, $h$, measured relative to the basin floor. However, all results in this report are presented in model units. Water surface elevations were collected with 19 single wire capacitance-type gauges distributed in both across and along the shore.

Five continuous wire capacitance gauges were placed on the foreshore slope to measure runup. It was necessary to convert measurements from the runup gauges from distance along the 1:10 slope to vertical elevations to obtain runup. The wave and runup gauges all sampled water surface elevations at $20 \mathrm{~Hz}$. Eleven of the wave gauges were positioned along the centerline of the reef to measure waves as they transform from deeper water, through breaking, and across the reef. Five gauges were placed in the longshore direction to capture three-dimensional effects of waves propagating across the reef. The coordinates of the gauge locations are listed in Table 3 where the origin is at the wave generator in line with the left edge of the acrylic reef looking offshore (Figure 7). Figure 7 shows the basin and locations of the reef and the wave gauges. 
Table 2. SWIMS 3-D reef design wave conditions in prototype, $m$ (Model, $m$ ).

\begin{tabular}{|l|l|l|l|}
\hline \multirow{2}{*}{$\begin{array}{l}T_{p} \\
\text { sec }\end{array}$} & $\begin{array}{l}h=20.9 \mathrm{~m}(0.418 \mathrm{~m}) \\
\mathrm{m}(\mathrm{m})\end{array}$ & $\begin{array}{l}h=22.1 \mathrm{~m}(0.442 \mathrm{~m}) \\
\mathrm{m}(\mathrm{m})\end{array}$ & $\begin{array}{l}h=24.6 \mathrm{~m}(0.493 \mathrm{~m}) \\
\mathrm{m}(\mathrm{m})\end{array}$ \\
\hline $7(1.0)$ & $4.1(0.08)$ & $4.2(0.08)$ & $4.0(0.08)$ \\
\hline $7(1.0)$ & $4.8(0.10)$ & $5.0(0.10)$ & $5.1(0.10)$ \\
\hline $10(1.4)$ & - & - & $4.7(0.09)$ \\
\hline $10(1.4)$ & $4.1(0.08)$ & $3.5(0.07)$ & $6.4(0.13)$ \\
\hline $10(1.4)$ & $5.6(0.11)$ & $5.9(0.12)$ & $7.3(0.15)$ \\
\hline $10(1.4)$ & - & $7.0(0.14)$ & $7.9(0.16)$ \\
\hline $13(1.8)$ & $5.7(0.11)$ & $4.6(0.09)$ & $4.8(0.10)$ \\
\hline $13(1.8)$ & $6.9(0.14)$ & $8.0(0.16)$ & $7.8(0.16)$ \\
\hline $13(1.8)$ & $7.7(0.15)$ & - & $8.5(0.17)$ \\
\hline $16(2.3)$ & $5.5(0.11)$ & $5.6(0.11)$ & $4.7(0.09)$ \\
\hline $16(2.3)$ & $6.9(0.14)$ & $7.4(0.15)$ & $7.6(0.15)$ \\
\hline $16(2.3)$ & $7.3(0.15)$ & $7.9(0.16)$ & $8.7(0.17)$ \\
\hline $20(2.8)$ & $5.1(0.10)$ & $4.3(0.09)$ & $4.4(0.09)$ \\
\hline $20(2.8)$ & $6.7(0.13)$ & $6.3(0.13)$ & $7.5(0.15)$ \\
\hline $20(2.8)$ & $6.9(0.14)$ & - & $8.5(0.17)$ \\
\hline
\end{tabular}

Values in parenthesis refer to values in model seconds and meters

Table 3. SWIMS 3-D reef wave gauge locations.

\begin{tabular}{|l|l|l|}
\hline Gauge & $x(\mathrm{~m})$ & $y(\mathrm{~m})$ \\
\hline 1 & 14.02 & 4.73 \\
\hline 2 & 14.02 & 7.93 \\
\hline 3 & 18.50 & 6.10 \\
\hline 4 & 22.98 & 6.10 \\
\hline 5 & 23.32 & 6.10 \\
\hline 6 & 23.93 & 6.10 \\
\hline 7 & 26.79 & 6.10 \\
\hline 8 & 28.19 & 6.10 \\
\hline 9 & 29.23 & 6.10 \\
\hline 10 & 30.45 & 6.10 \\
\hline 11 & 31.61 & 6.10 \\
\hline 12 & 33.40 & 1.32 \\
\hline
\end{tabular}




\begin{tabular}{|l|l|l|}
\hline Gauge & $x(\mathrm{~m})$ & $y(\mathrm{~m})$ \\
\hline 13 & 33.40 & 4.06 \\
\hline 14 & 33.40 & 6.10 \\
\hline 15 & 33.40 & 8.13 \\
\hline 16 & 33.40 & 10.94 \\
\hline 17 & 33.58 & 6.03 \\
\hline 18 & 33.58 & 6.16 \\
\hline 19 & 35.91 & 6.10 \\
\hline 20 & 38.44 & 1.23 \\
\hline 21 & 38.44 & 3.67 \\
\hline 22 & 38.44 & 6.10 \\
\hline 23 & 38.44 & 8.55 \\
\hline 24 & 38.44 & 10.98 \\
\hline
\end{tabular}

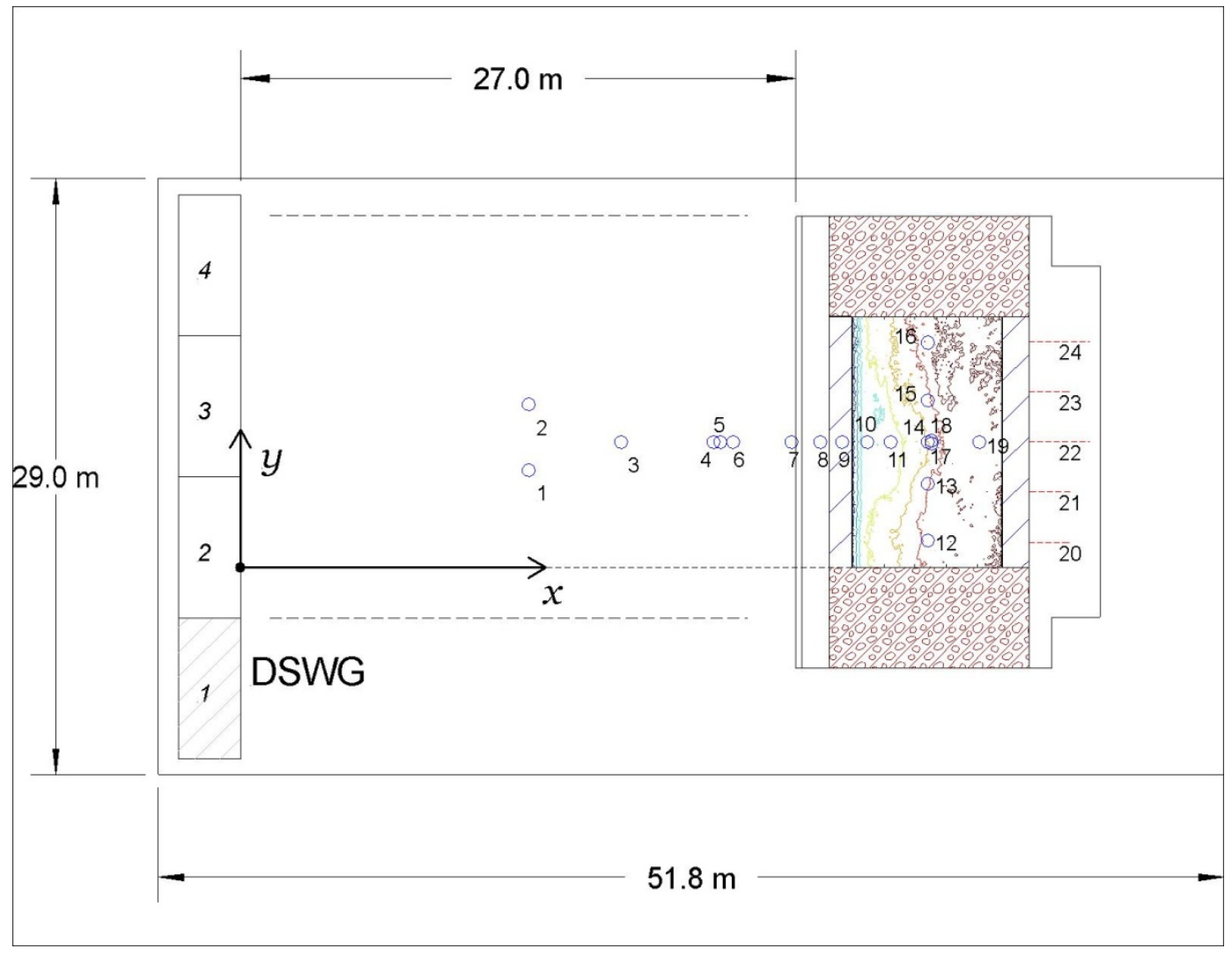

Figure 7. Three-dimensional wave basin and gauge location.

Data analysis performed on wave data from individual gauges included (a) mean up-crossing analysis to obtain significant $H_{s}$, maximum and average wave heights, significant and average wave periods, and mean water levels 
at each gauge, and (b) spectral analysis to calculate zero-moment wave height, $H_{m o}$, and peak wave period, $T_{p}$. Three gauges (Gauges 4 to 6 in Figure 7) were positioned on the flat portion of the basin floor in front of the generator in an array that allowed calculation of incident and reflected wave heights using the method of Goda and Suzuki (1976). Operations performed on the wave gauge array were unidirectional spectral density incident/ reflection analysis to determine incident $T_{p}$, incident $H_{m o}$, and reflection coefficient, $K_{r}$.

\subsection{Test procedures}

Wave gauges were calibrated daily before performing experiments. For the mid- and low-water levels, some gauges that were positioned on the reef had insufficient depth to perform a calibration. Under these circumstances, the last calibration obtained at the high-water level was applied for these gauges. At a minimum, all gauges were calibrated once per week, which required raising the water level to high water, performing wave gauge calibration, and then lowering the water to the desired level to perform experiments. For days in which the gauges weren't calibrated, the calibration was verified by generating a previously tested wave condition and the results compared to the previous run.

Prior to each run of a test case, the water level of the basin was checked and adjusted as needed. Water level could be adjusted to within $0.03 \mathrm{~cm}$ of the target value. The desired wave condition file was selected on the control computer and the waves were started. Each wave time series was 20-min duration and waves ramped down and ceased at the end of the time series. Data collection was synchronized with the start of the wave generator and ended at the conclusion of the wave time series. As a result, the initial portion of the time series included still water and waves produced by the wavemaker ramping. Therefore, this data segment was not included in analysis.

A video camera mounted above the model was used to document each experiment. The camera gave an overhead oblique view of tests. Video of the experiment was recorded digitally and was started manually immediately following initiation of waves. The camera was set to record for 20 minutes and ended at approximately the same time as the wave action. The experiments were also documented through photos and video taken from the model shoreline and from the side of the model. 


\subsection{Results}

Data were collected for a total of 78 runs, which include 40 runs on the smooth reef surface and 38 on the roughened surface. Wave runs at the 0.492-m water level were not completed for $0.17-\mathrm{m}$ high waves at 2.3-sec and 2.8-sec periods on the roughened surface. Several attempts were made to collect data for these conditions; however, portions of the acrylic reef bathymetry became dislodged when subjected to the waves. A discussion of this issue is found in Chapter 4.

This section includes results from the wave and runup gauges, including presentation of wave transformation, alongshore wave heights, and analysis of spectral transformation, wave setup, runup and reflection.

\subsubsection{Wave height transformation}

Figures of wave height, $H_{m o}$, and mean water level (setup), $\eta$, measured along the centerline of the model are plotted with distance from the wave generator in Appendix A. Data collected for both the smooth and rough surfaces are shown for each wave and water level condition. The initial water level and reef bathymetry also are presented as a reference, but both are reduced by a factor of 10 for the plot.

The initial level for the low-water experiments was below the elevations of several wave gauges and the runup gauges, i.e., the gauges were out of the water at the onset of the test. Mean water levels recorded for these gauges were adjusted by adding the elevation difference between the particular gauge and the initial water elevation.

The highest waves generated for 1.0- and 2.8-sec periods at each water level for both reef surfaces are shown in Figures 8 to 13. The figures show a common trend of relatively uniform wave heights in the flat portion of the basin, shoaling as the wave encounters the reef slope, and a sharp decrease in height shoreward of breaking and as the wave propagates across the flat reef surface. Several of the plots also indicate a slight decrease in height immediately offshore of the breakpoint, particularly for the longer wave periods.

As expected through linear wave theory, shoaling is greater for the longer wave periods, and wave breaking and dissipation offshore of the reef are reduced with higher water levels. Wave heights over the reef are similar for 


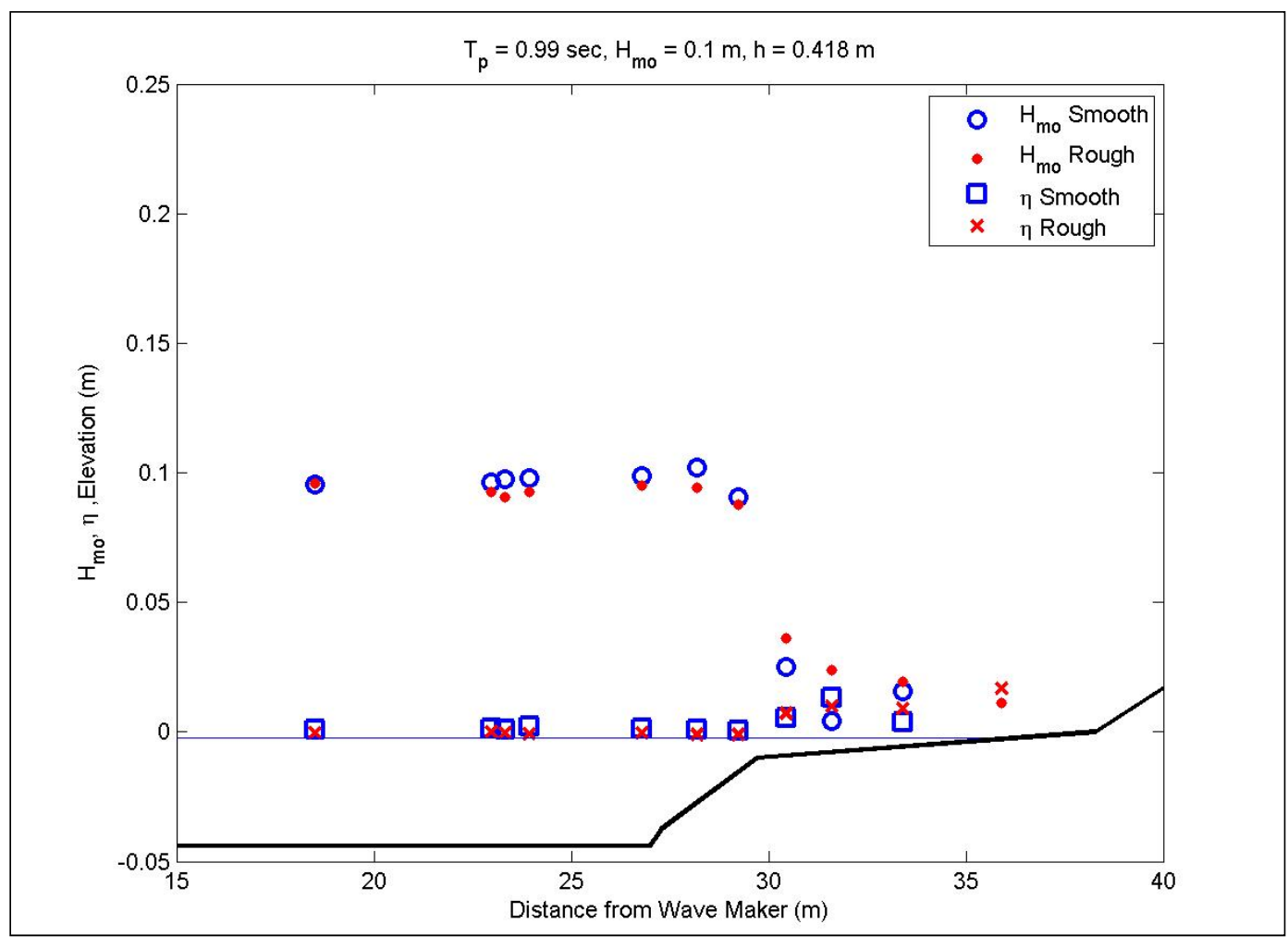

Figure 8. Wave transformation for 1-sec, 0.1-m waves at low water.

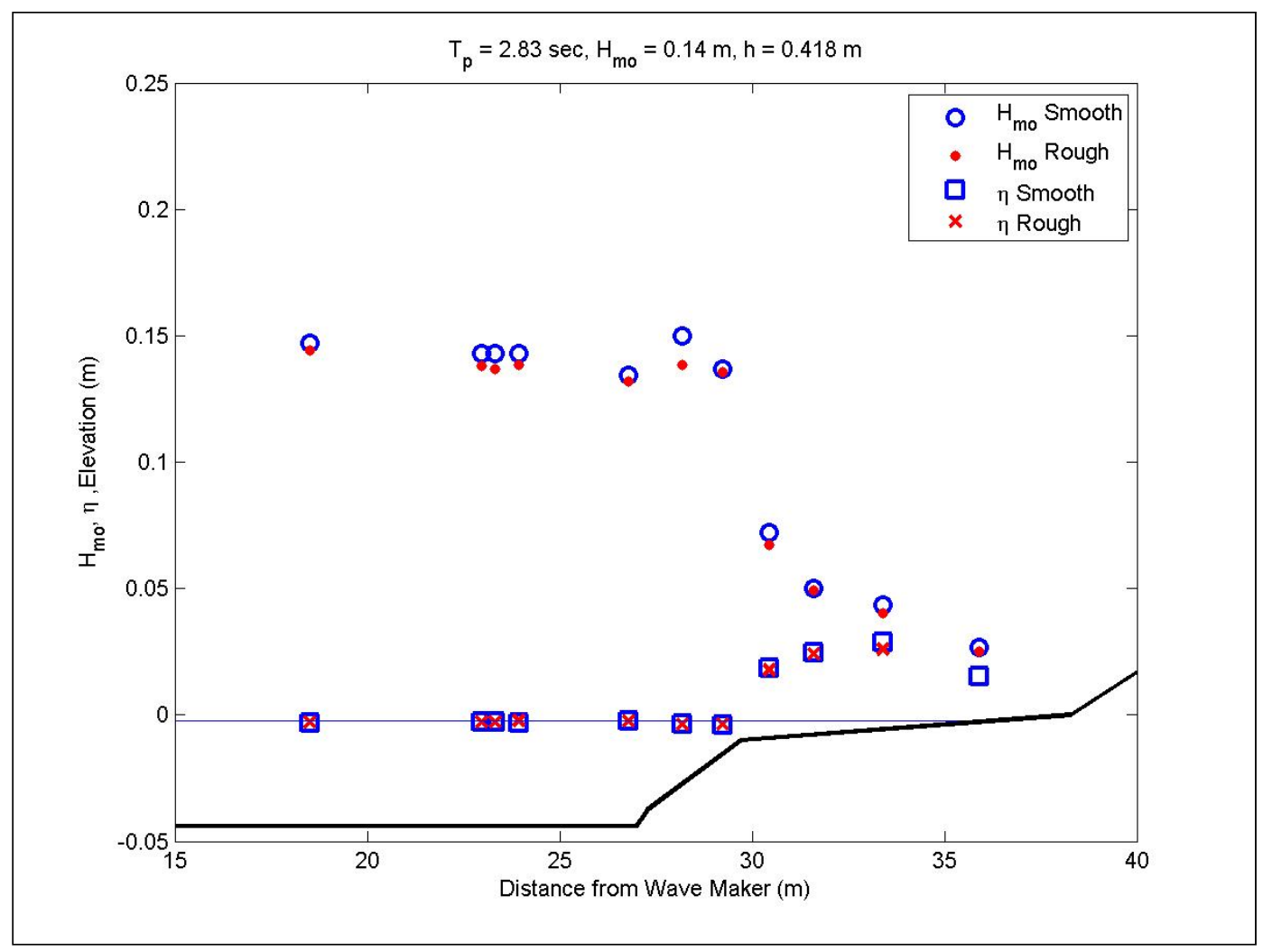

Figure 9. Wave transformation for 2.8-sec, 0.14-m waves at low water. 


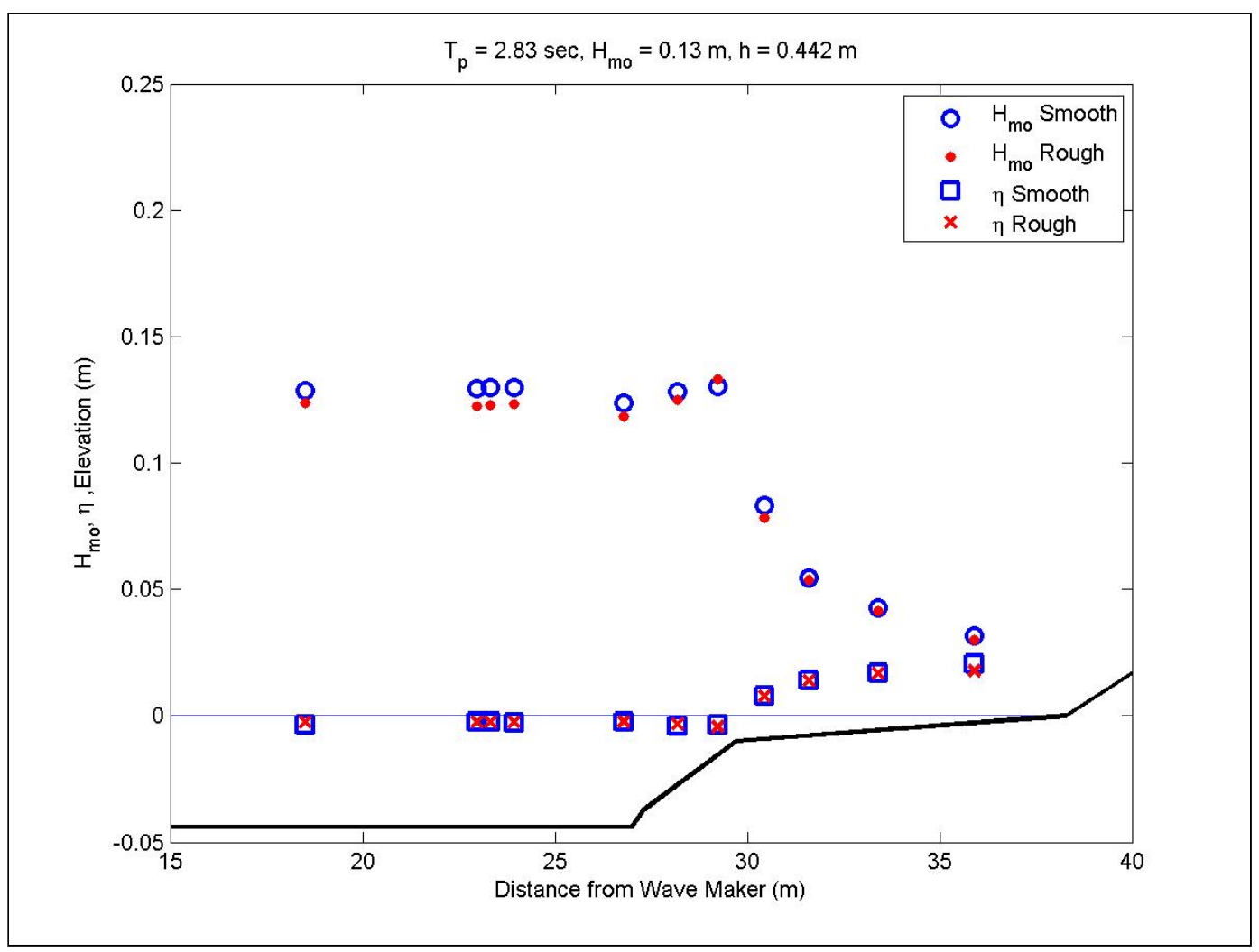

Figure 10. Wave transformation for 1-sec, 0.1-m waves at mid water.

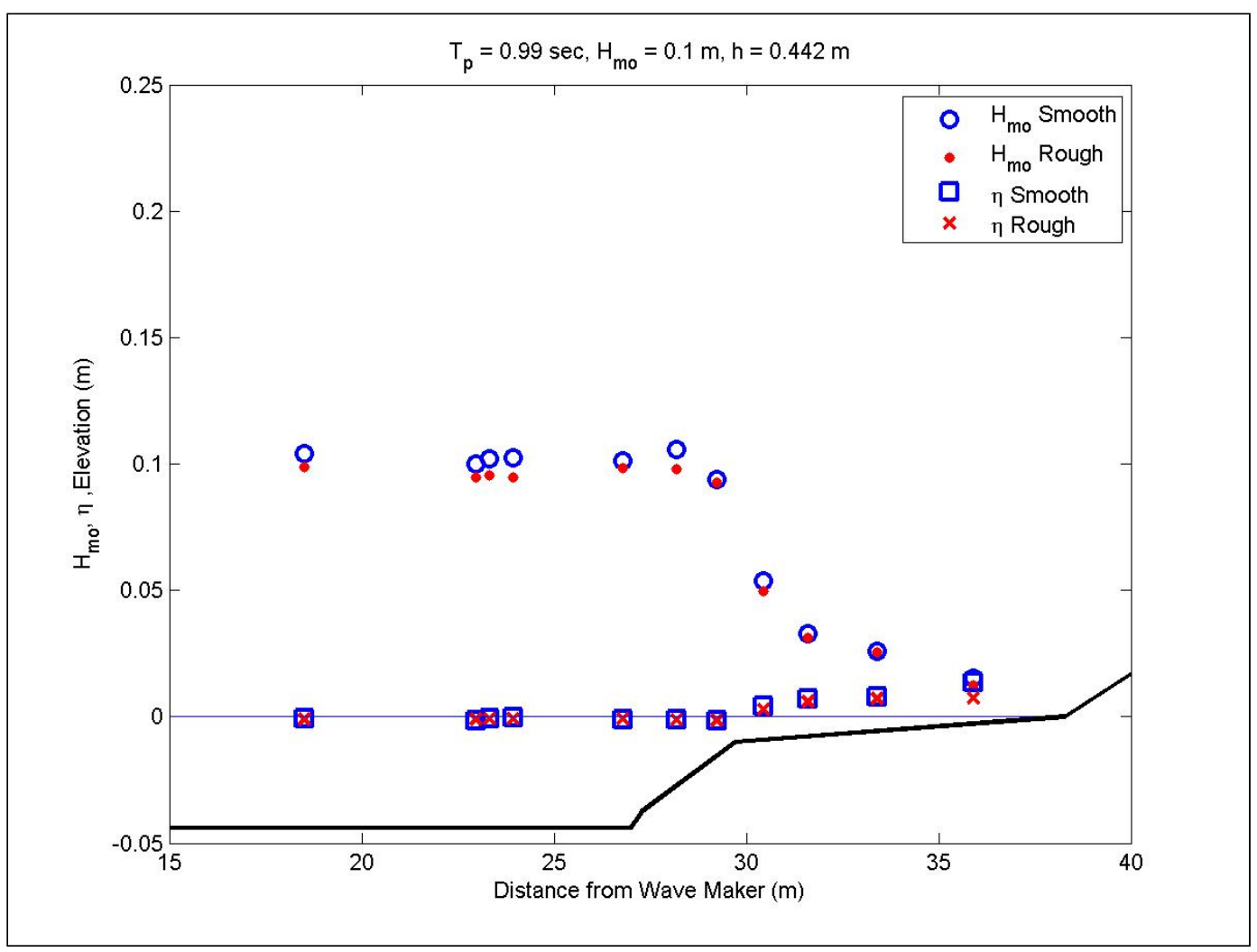

Figure 11. Wave transformation for $2.8-\mathrm{sec}, 0.13-\mathrm{m}$ waves at mid water. 


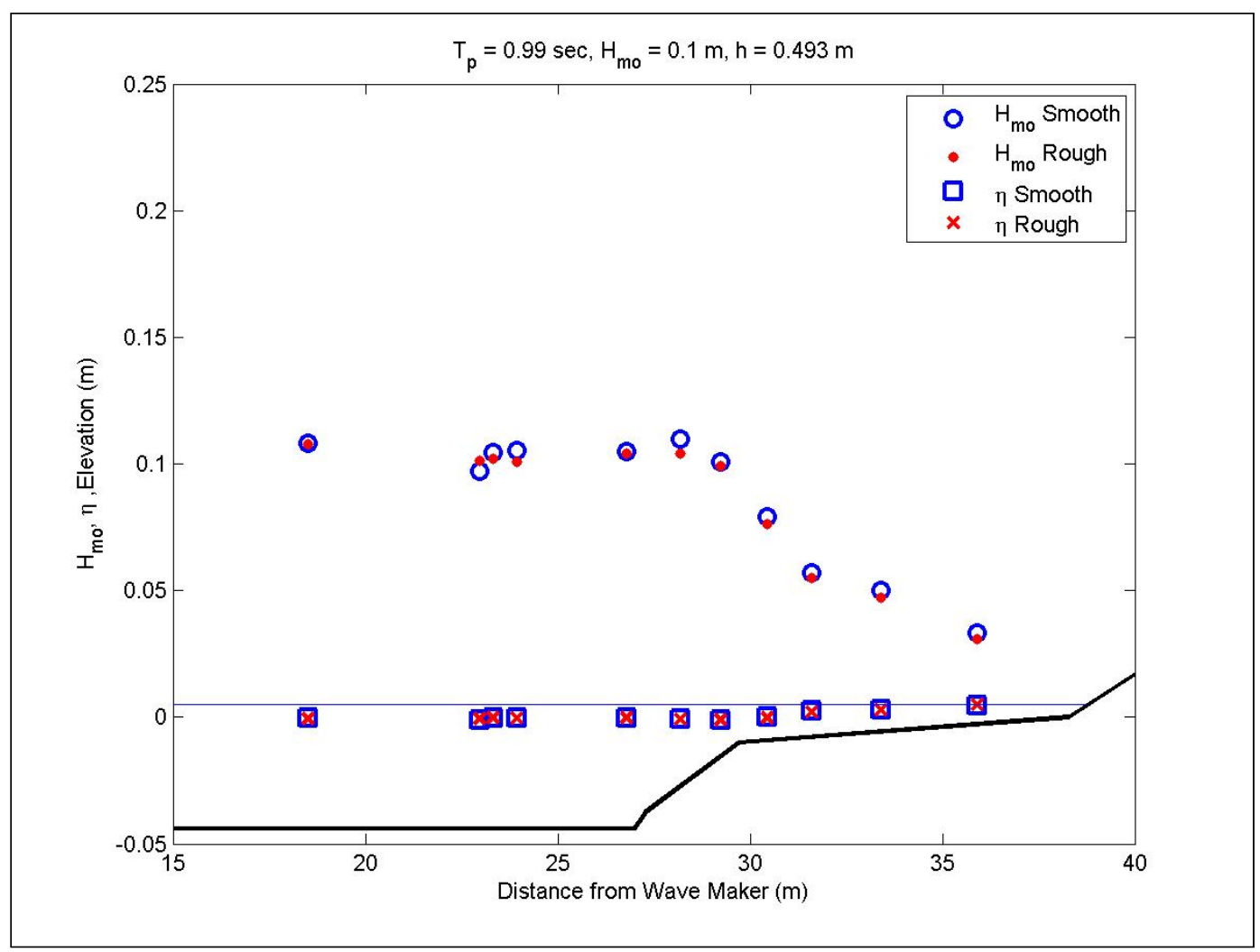

Figure 12. Wave transformation for 1-sec, 0.1-m waves at high water.

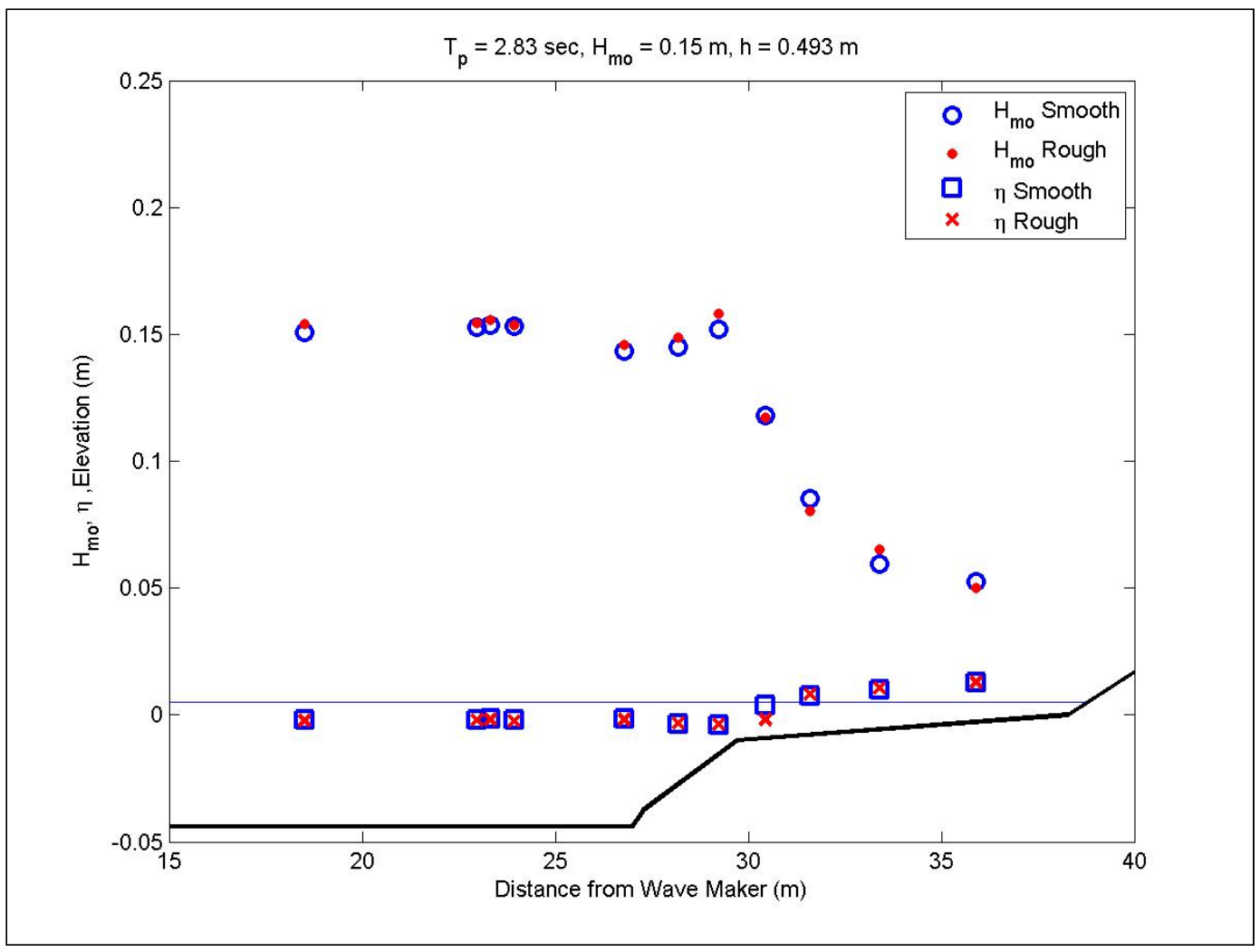

Figure 13. Wave transformation for $2.8-\mathrm{sec}, 0.15-\mathrm{m}$ waves at high water. 
the two reef roughness. This indicates that energy is saturated for both roughness conditions and that the difference between the surface roughnesses is negligible. It shouldn't be implied that roughness doesn't affect wave height, only that the surface roughness applied for the experiment didn't significantly affect wave heights on the reef.

\subsubsection{Wave setup}

Wave setup measured for the two reef roughnesses was also similar. Both conditions show a decrease in water level, setdown, as the wave approaches the break point and increasing setup over the reef, which is greater for the longer period and for the lower water levels. Setup continues to increase over the reef as the wave propagates into shallower water for the mid- and high-water levels. Figure 8 also indicates increasing setup with shallower depth for the shorter-period wave condition with the rough surface. However, following an increase of setup shoreward of breaking, setup decreases for 1-sec waves with the smooth reef surface. Although, it should be noted that no data were available for the most shoreward gauge (Gauge 19). Figure 9 shows a sharp increase in setup immediately shoreward of breaking, shoreward of which setup decreases at Gauge 19 for the longerperiod wave on the smooth surface (no data were available at Gauge 19 with the rough surface).

In previous laboratory tests by Gourlay (1996a) and Demirbilek et al. (2007), setup decreased with increasing water depth on reefs. The highest setup recorded for each test is plotted as a function of deepwater wave power, $P$, in Figure 14 (gauges at elevations above the initial water level were excluded). Wave power is defined as:

$$
P=H_{0}^{2} T_{p}
$$

in which $H_{o}$ is $H_{m o}$ in deep water, which was calculated from the incident wave height obtained from reflection analysis. With the exception to a few tests with low water, the highest setup occurred with the lowest water levels. The figure shows that setup follows the trend from the previously cited laboratory experiments of Seelig (1983) and Gourlay (1996b) who also found that setup increased with wave power. 


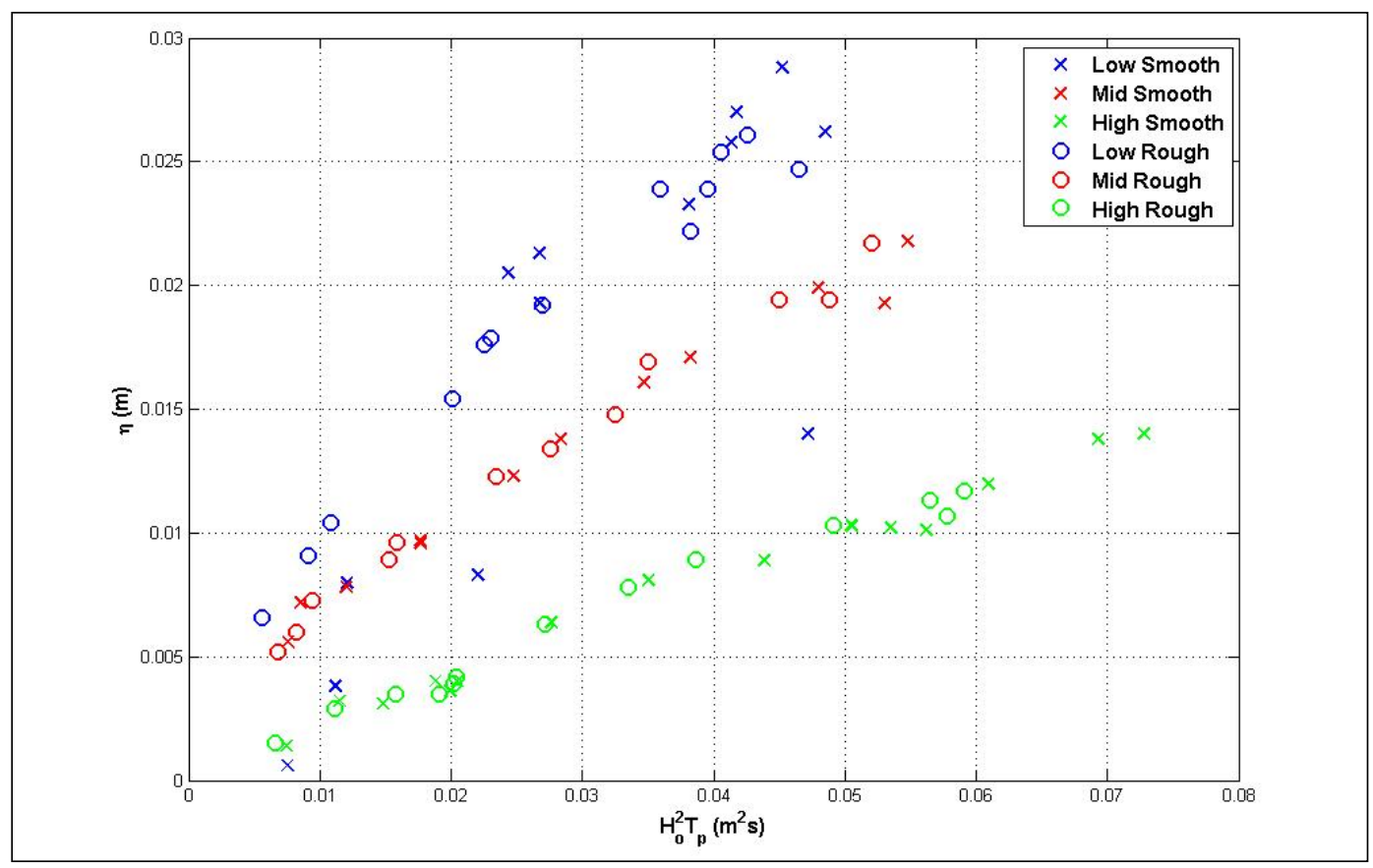

Figure 14. Highest mean water level recorded for each wave condition.

\subsubsection{Alongshore wave heights}

Figure 15 shows a photograph taken from above the model during an experiment at the low water level with 2.8-sec, o.1-m waves approaching the reef from the bottom of the photo. The photograph was taken immediately after a wave broke on the reef and shows that the crest of the broken wave is not uniform. The broken wave crest has propagated farther onshore on the left of the photo than the right. It is believed that the non-uniform breaker line is due to return flow on the right side of the reef and reflection from the foreshore slope.

The non-uniform breaker line results in waves breaking at an angle to shore normal. Also shown are wave crests that refract toward the reef sides from the center of the model, which interact with other directional incident and reflected waves; all of which is measured by the alongshore gauges shown in the center of the reef.

Appendix B includes figures of $H_{m o}$ and $\eta$ measured at the alongshore reef gauges (Gauges 12 through 16) for both smooth and rough surface conditions. For reference, the figures include the initial water levels and measured bathymetry elevations along the gauge transect. 


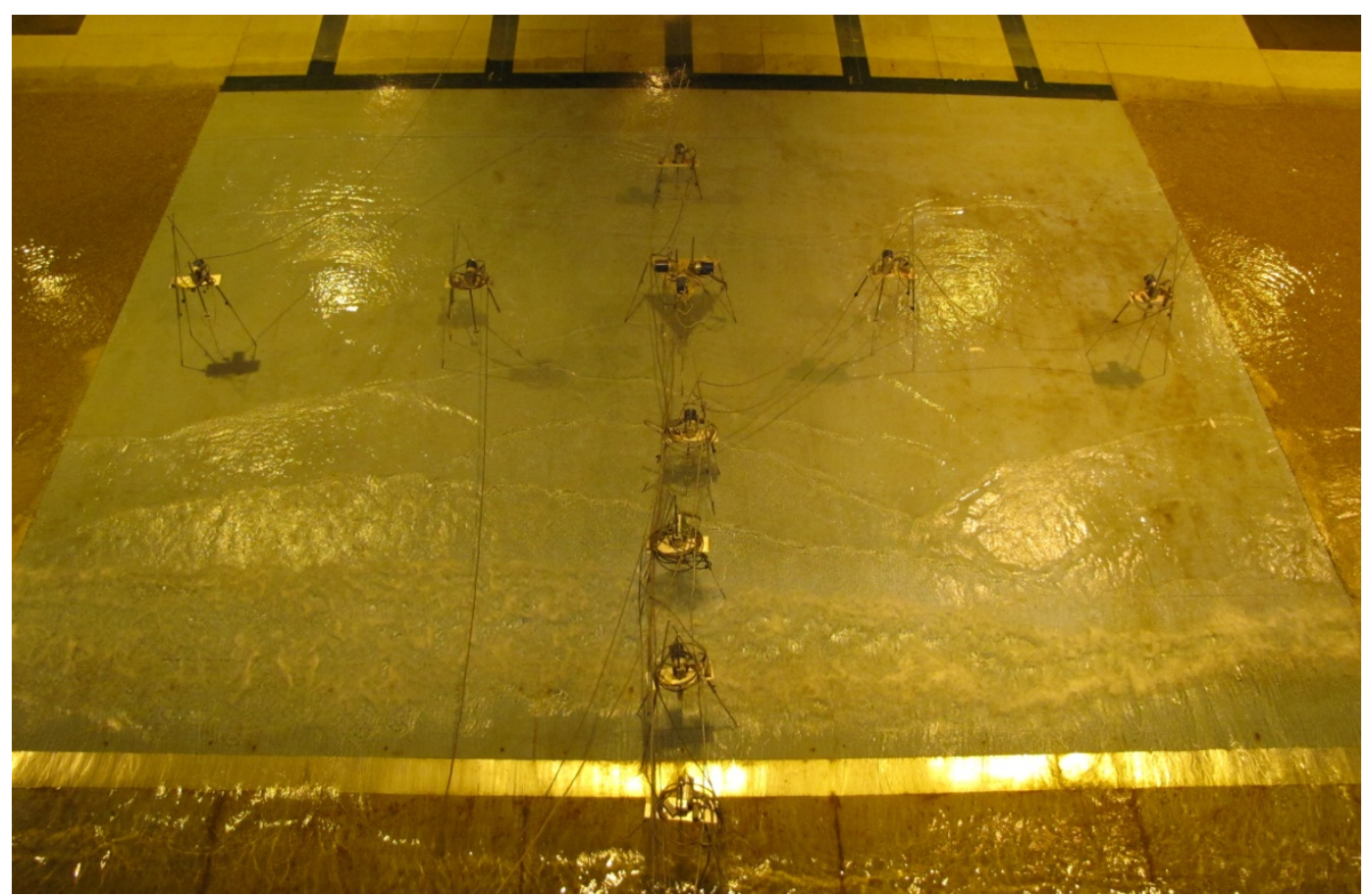

Figure 15. Overhead photograph of 2.8-sec, 0.1-m waves at low water.

Figures 16 to 21 show alongshore heights and setup for the highest waves generated for 1.0- and 2.8-sec periods at each water level for both surfaces. It should be noted that the initial water level at the low water level was lower than the gauges on the sides of the reef (Gauges 12 and 16), i.e., the gauges were not in the water at the start of the experiment. Wave heights were generally highest at Gauge 14, the center gauge, where water depth is greatest and lowest at Gauges 12 and 16, the side gauges, as expected in a two-dimensional regime. However, Figure 20 illustrates the threedimensionality of the waves over the reef, which is neither uniform nor symmetrical in the alongshore direction.

The figure shows an asymmetrical distribution with the highest wave measurement at Gauge 13, one gauge to the left of center in the figure. The gauge measurements in the figures show total wave height and setup and as a result, refraction, reflection and the wave-wave interactions are all included in the measurement.

Differences in wave height and setup are minor between the smooth and rough surfaces. The greatest differences between surface roughnesses occur at the low water level. An observation during the low-water experiments is that the drawdown of waves consistently extended seaward of the alongshore gauge locations. 


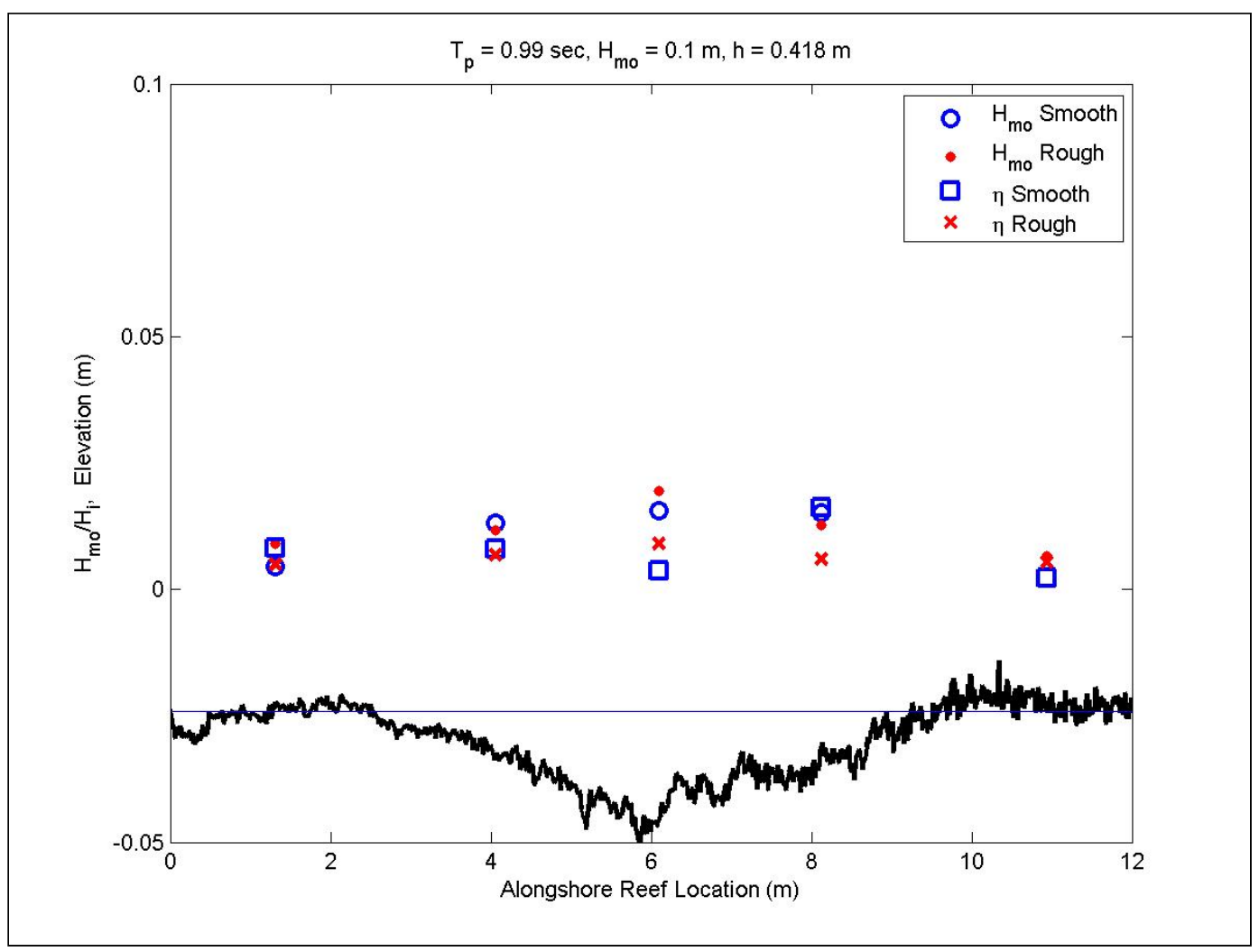

Figure 16. Alongshore wave heights for 1.0-sec, 0.1-m waves at low water.

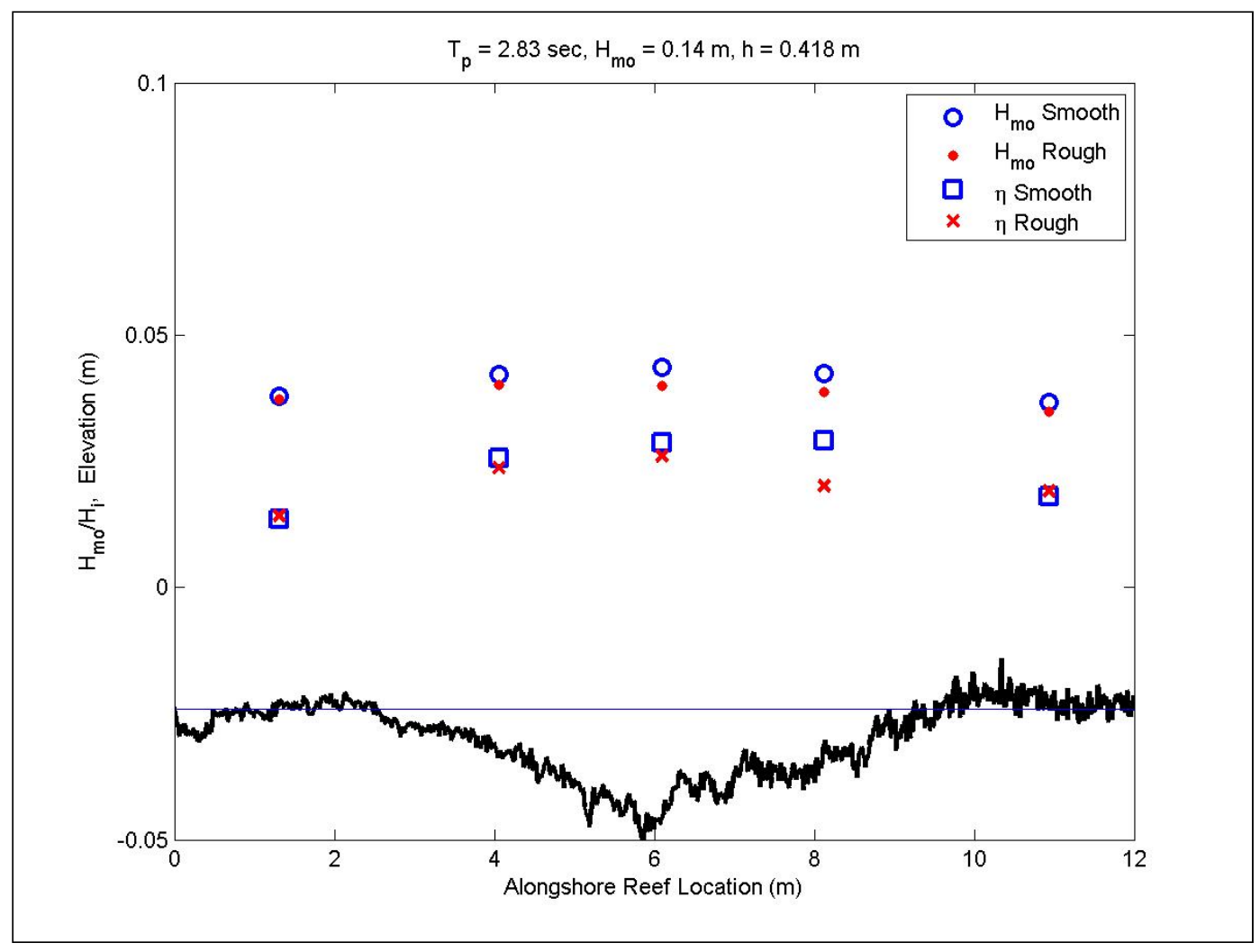

Figure 17. Alongshore wave heights for $2.8-\mathrm{sec}, 0.14-\mathrm{m}$ waves at low water. 


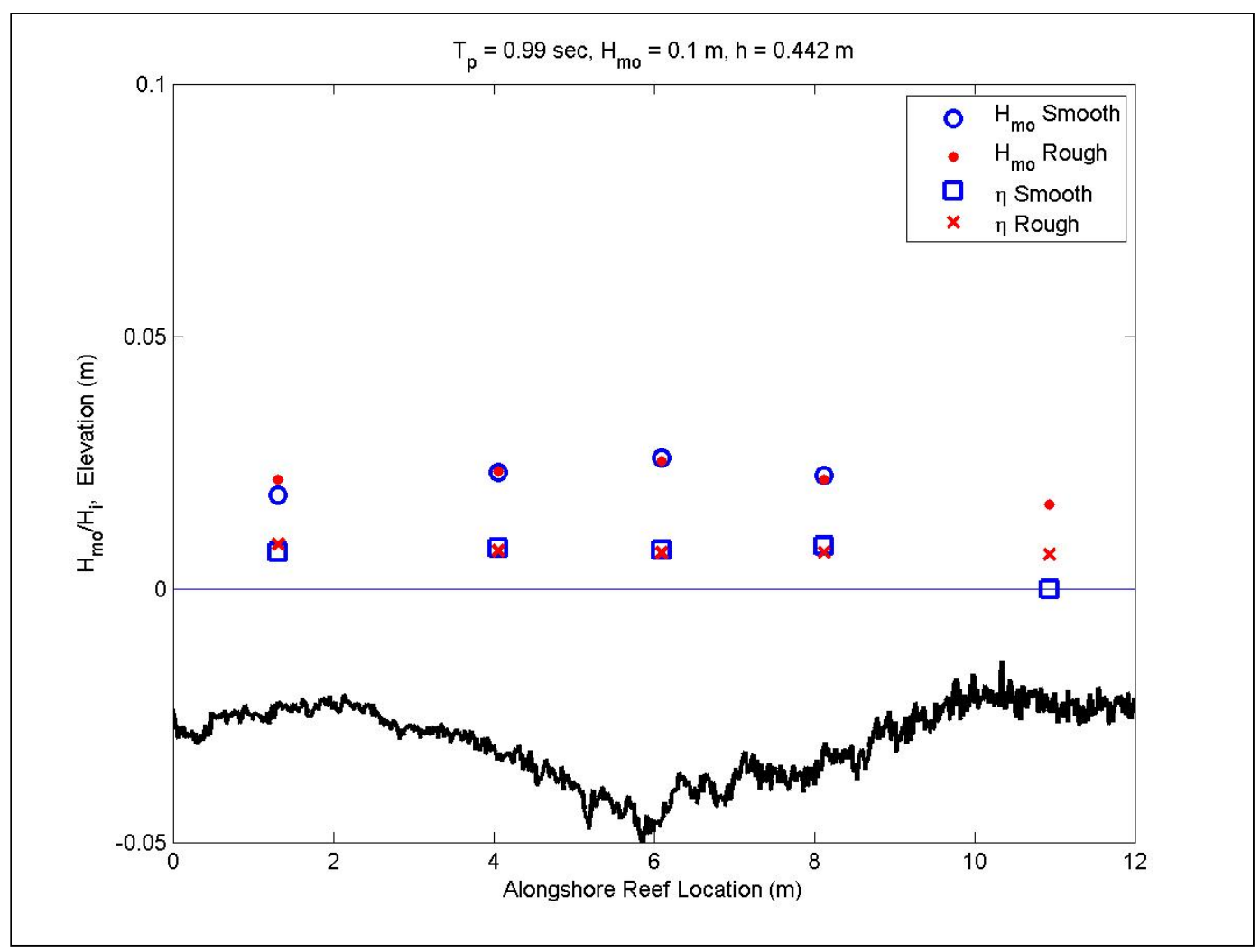

Figure 18. Alongshore wave heights for 1.0-sec, 0.1-m waves at mid water.

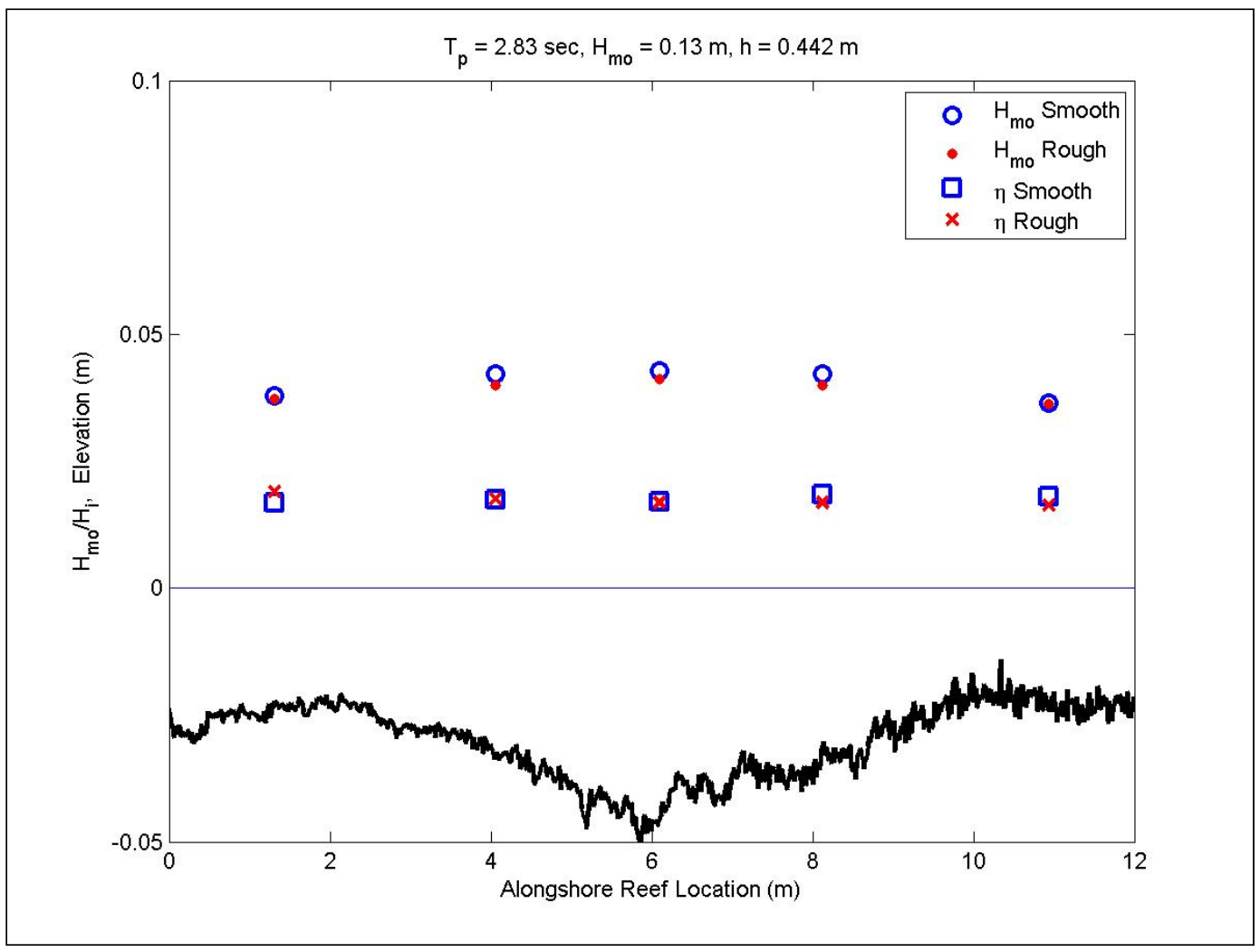

Figure 19. Alongshore wave heights for $2.8-\mathrm{sec}, 0.13-\mathrm{m}$ waves at mid water. 


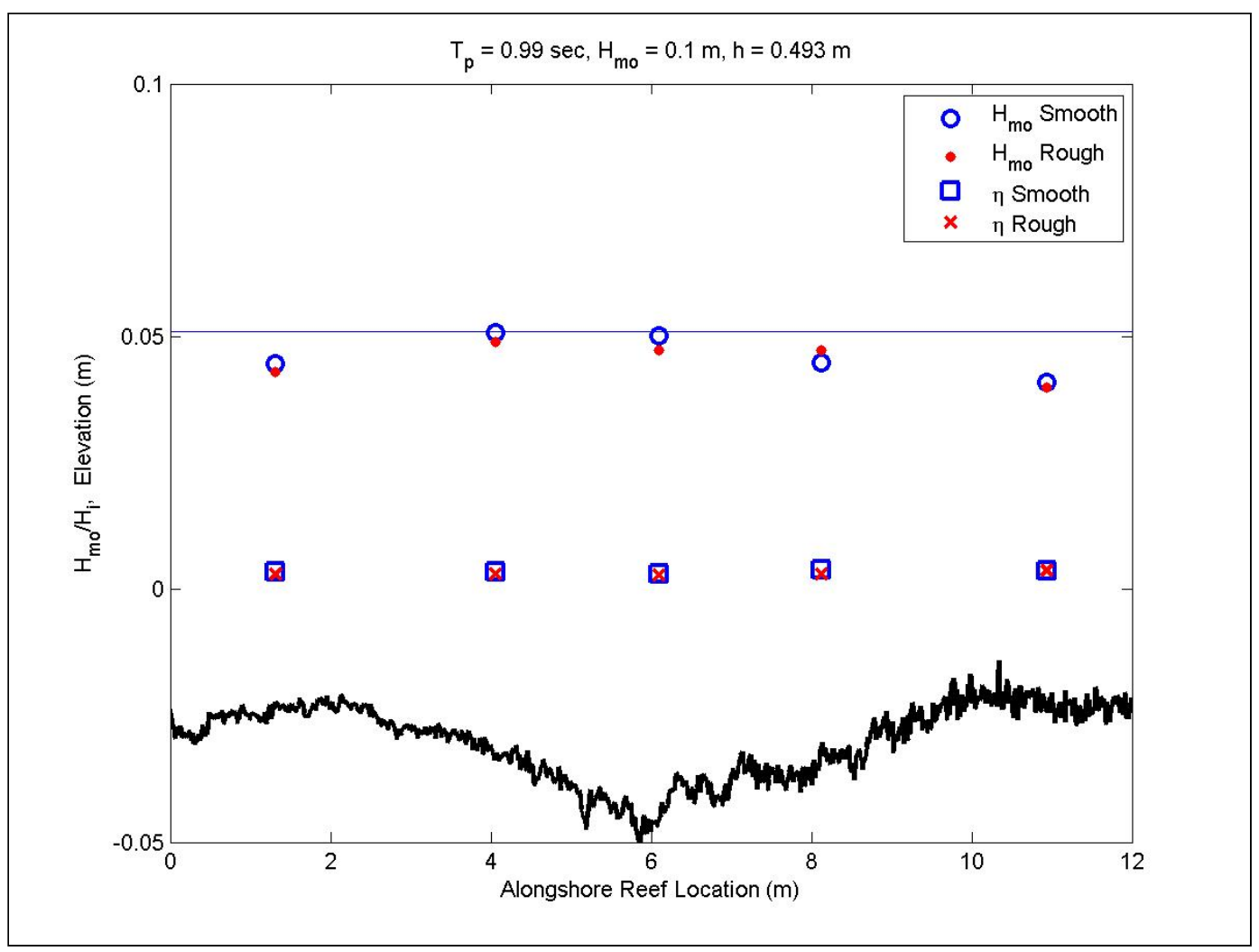

Figure 20. Alongshore wave heights for 1.0-sec, 0.1-m waves at high water.

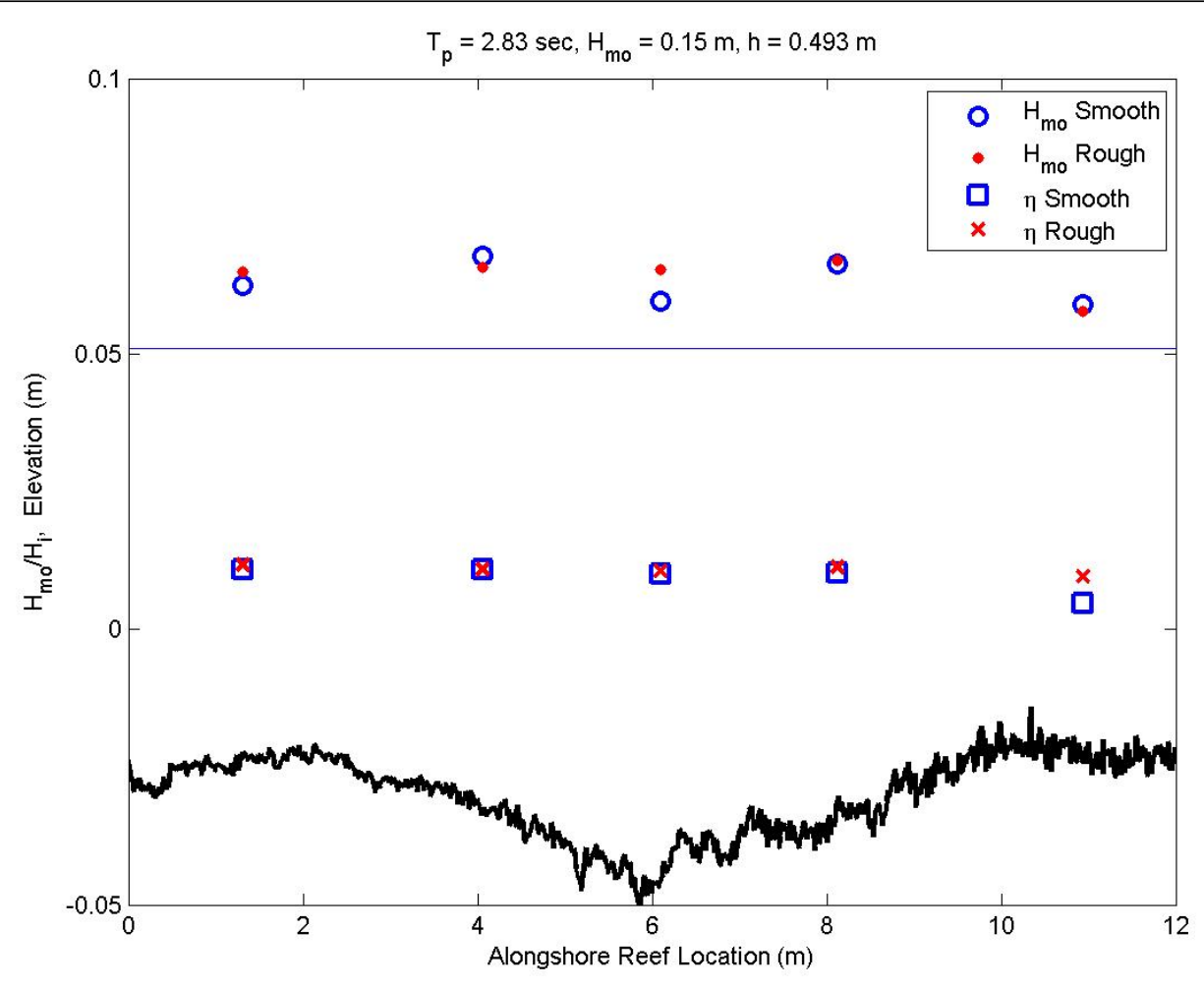

Figure 21. Alongshore wave heights for $2.8-\mathrm{sec}, 0.15-\mathrm{m}$ waves at high water. 


\subsubsection{Spectral analysis}

All laboratory runs were recorded for $20 \mathrm{~min}$ or $1200 \mathrm{sec}$. The time series of water surface elevations at Gauge 3 is shown in Figure 22 for a 2.8-sec, o.10-m wave at low water. For consistency, all analysis was performed for measured data between 30 seconds and 20 minutes.

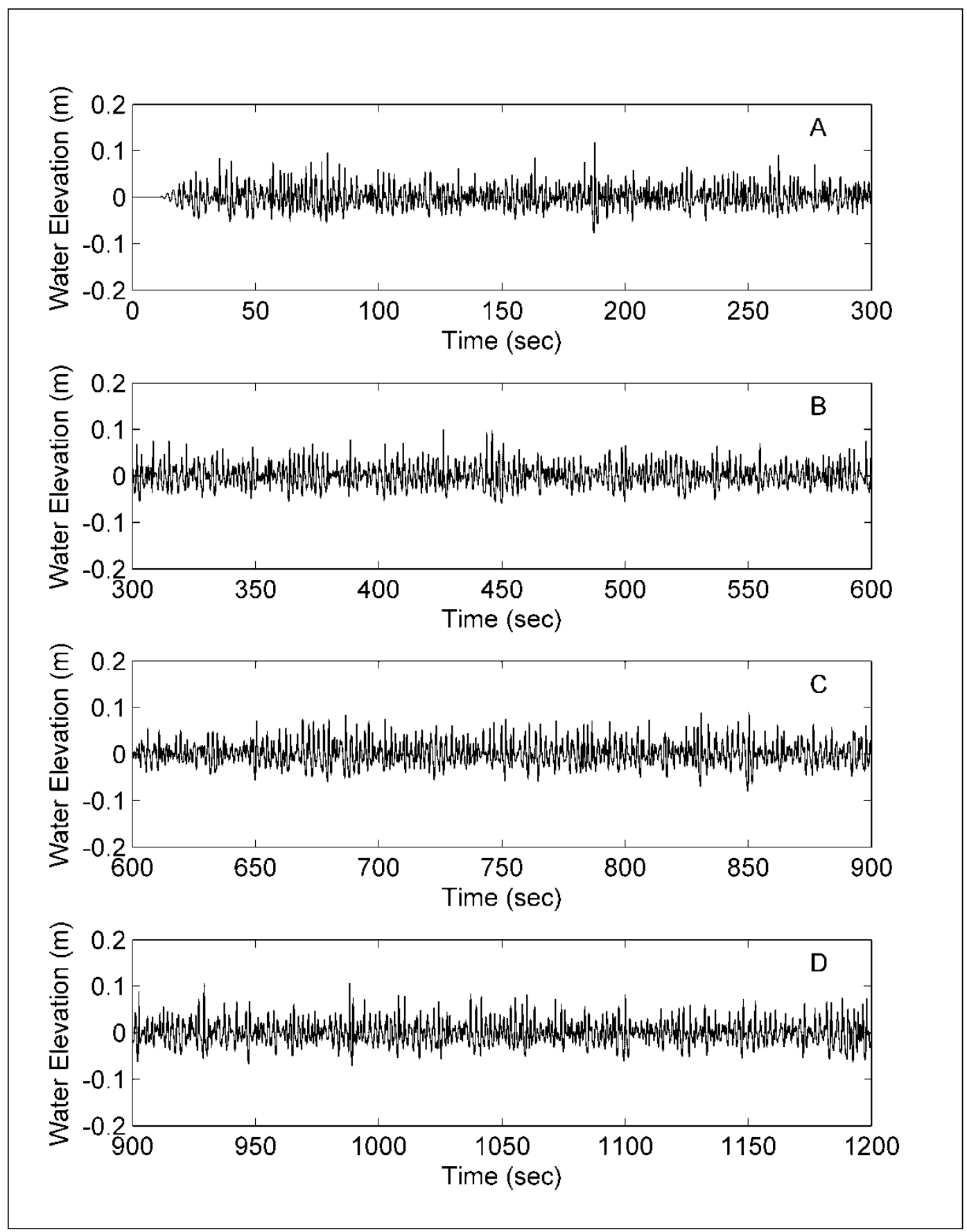

Figure 22. Time series of 2.8-sec, 0.1-m waves at low water measured at Gauge 3. 
During spectral analysis the mean was removed from the data. A Welch method for spectral analysis was performed and the resulting energy density spectrum is shown (Figure 23 (top panel)). The Welch method uses a Hamming window with 50 percent overlap. The data set was separated into 4096 point sections for the analysis. The Welch method calculates the spectrum for each section and then averages the spectra from all sections to produce the final spectrum.
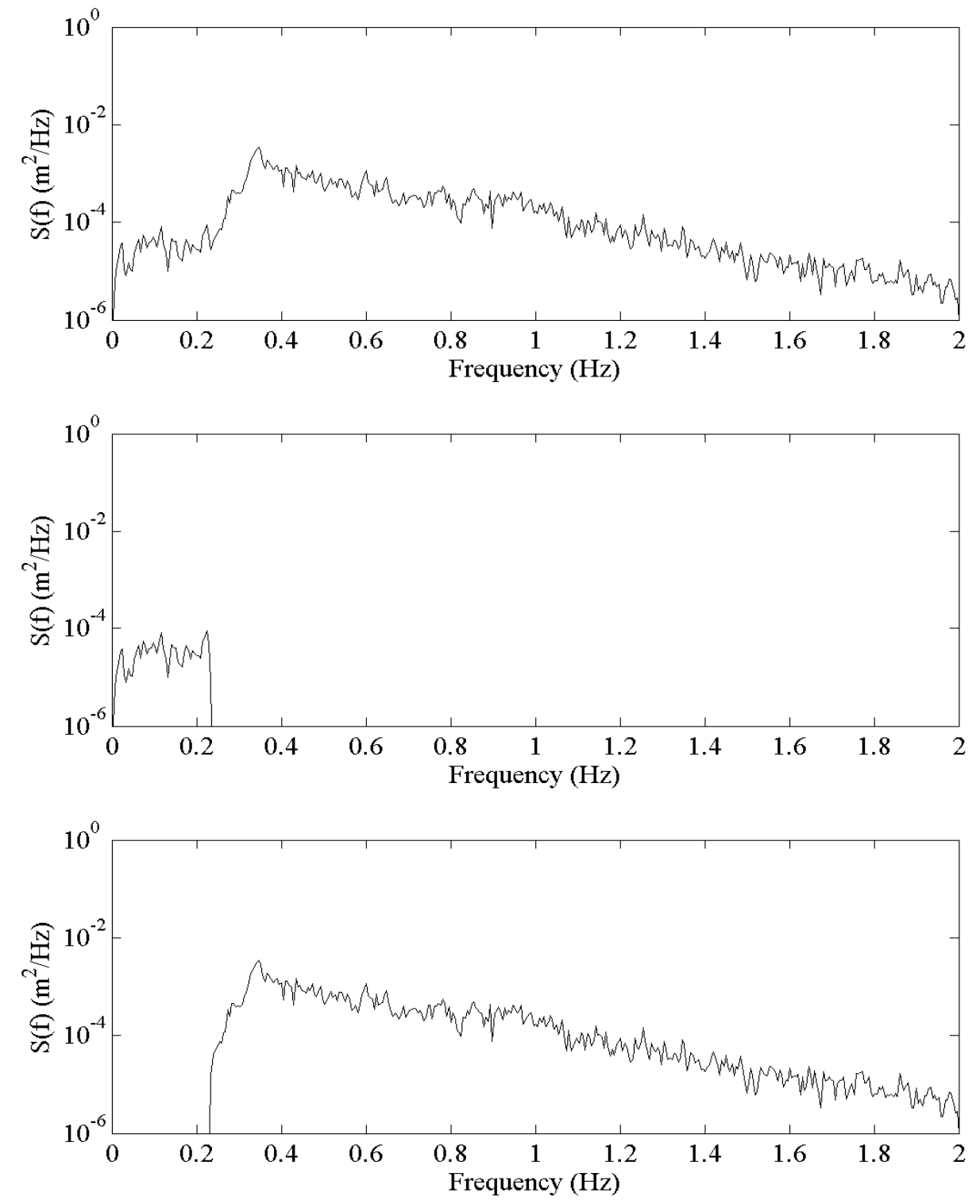

Figure 23. Full energy density spectrum for $2.8-\mathrm{sec}, 0.1 \mathrm{~m}$ waves at low water measured at Gauge 3. 
A filter was used to remove all energy greater than $2.0 \mathrm{~Hz}$ to eliminate very high oscillations from the data. A second filter separated the low and high frequency oscillations. The applied cut off for the low-frequency oscillations was $f_{p} / 1.5$, where $f_{p}$ is the peak frequency. This cutoff was selected based on observations of the full energy density spectrum. In Figure 23, the full energy density spectrum is shown in the top panel, along with the lowfrequency (middle panel) and the high-frequency (bottom panel) spectra. The separated low-frequency and high-frequency oscillations for the analyzed portion of the time series are shown in Figure 24 after the lowfrequency cut off was applied to the raw data. Low frequencies (red line) are generally associated with reflection, seiching or infragravity waves, and high frequencies (black line) are associated with the incident wave signal and nonlinear harmonics. Wave parameters such as $H_{m o}$, and $T_{p}$ were derived from analysis of the full spectrum.

\subsubsection{Spectral transformation}

The transformation of the wave spectrum across the bathymetric profile is shown in Figures 25 to 27. Energy density, $S(f)$, is shown at gauges along the centerline of the basin progressing from offshore to the reef. Energy densities are shown at Gauges 5, 8, 9, 10, 11, and 14 in the analysis.

Gauge 5 was located in the offshore region of the basin, Gauges 8 and 9 were on the offshore reef slope, and Gauges 10, 11, and 14 were located on the reef top. Note the scale change between the top and bottom plots in each figure.

The change in energy density at the peak frequency between Gauges 5 and 9 was similar at all three water levels. The energy peak remained at the same frequency, but the energy at the peak decreased as the waves approached the top of the reef slope. Dissipation of energy from offshore to the reef slope was caused by a combination of friction and wave breaking. Inshore of breaking, usually between Gauges 9 and 10, wave attenuation is observed by the rapid decrease of energy density at the peak frequency. In the low-water tests (Figure 25), the energy at the peak frequency was completely diminished at Gauge 11, due to the very shallow water depth at Gauge 11. The additional depth at Gauge 11 for the mid- and high-water tests allowed wave energy at the peak frequency to reach Gauge 11. A small amount energy remained in the peak frequency at Gauge 14 for the mid- (Figure 26) and high-water (Figure 27) tests. At all water levels, the majority of the energy shifted to the low frequencies at Gauge 14. 

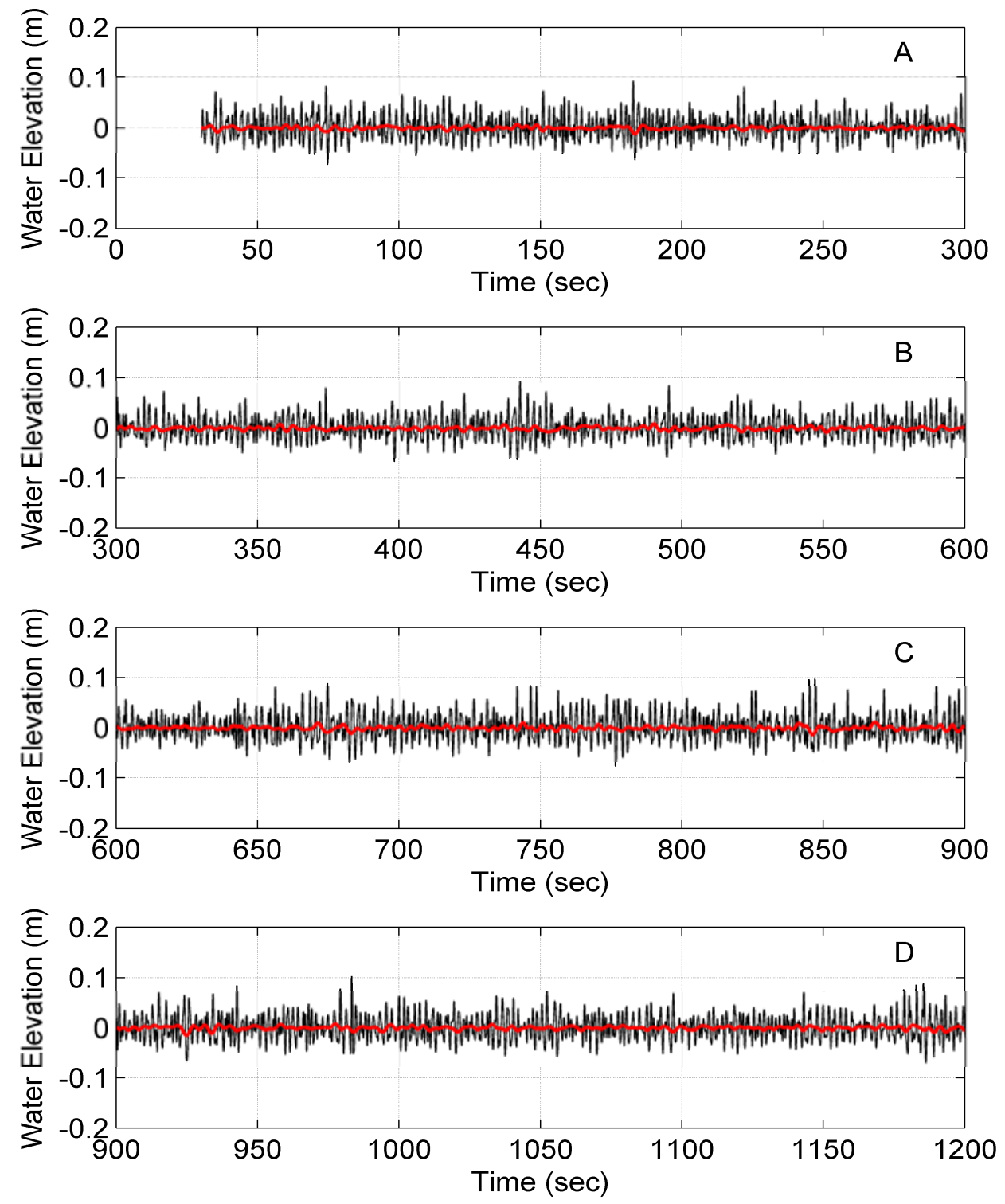

Figure 24. Time series of 2.8-sec, 0.1-m waves at low water measured at Gauge 3 after low frequency cut off was applied: black line is total time series, red line is low-frequency time series. 


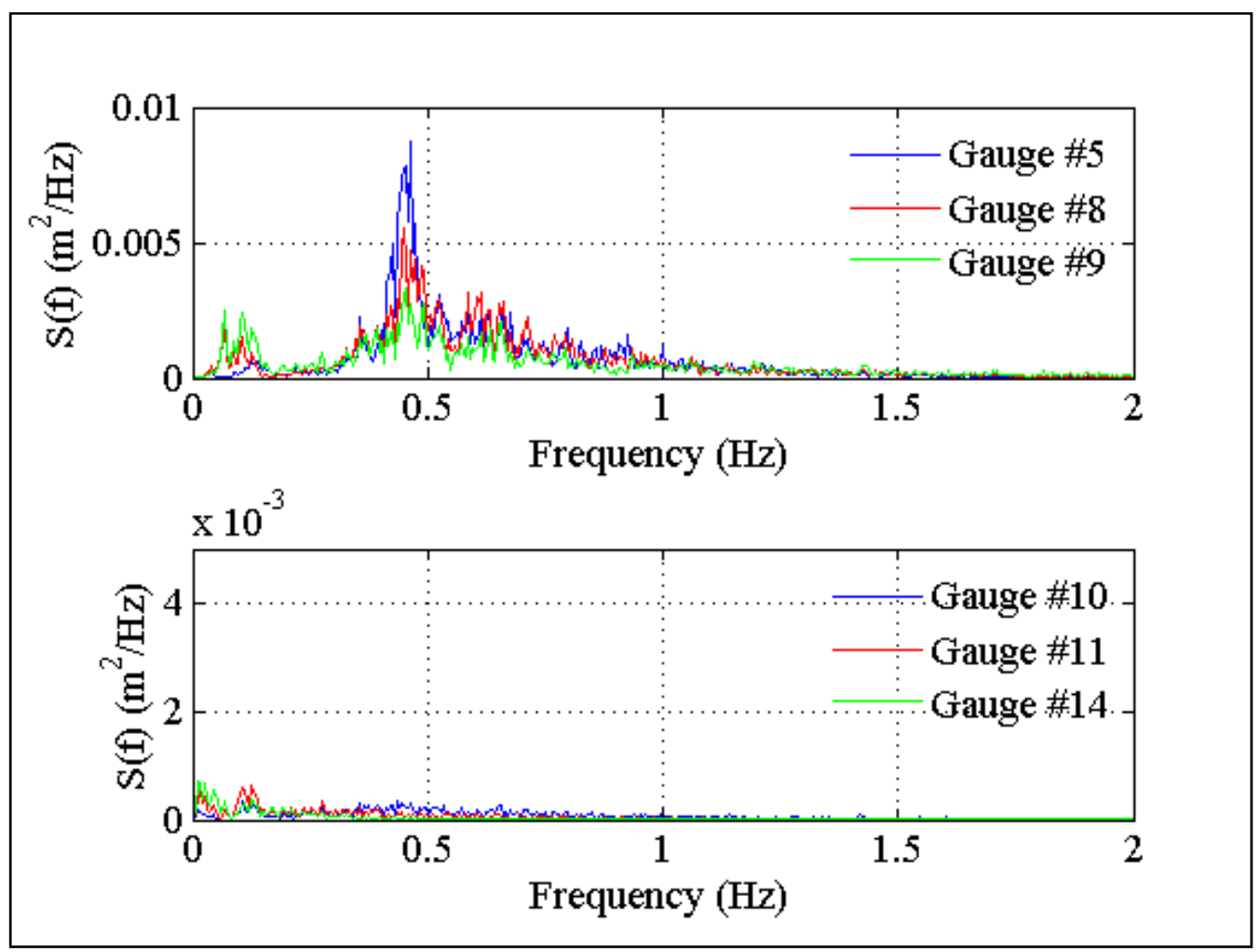

Figure 25. Energy density spectrum transformation of $2.3-\mathrm{sec}, 0.13-\mathrm{m}$ waves at low water.

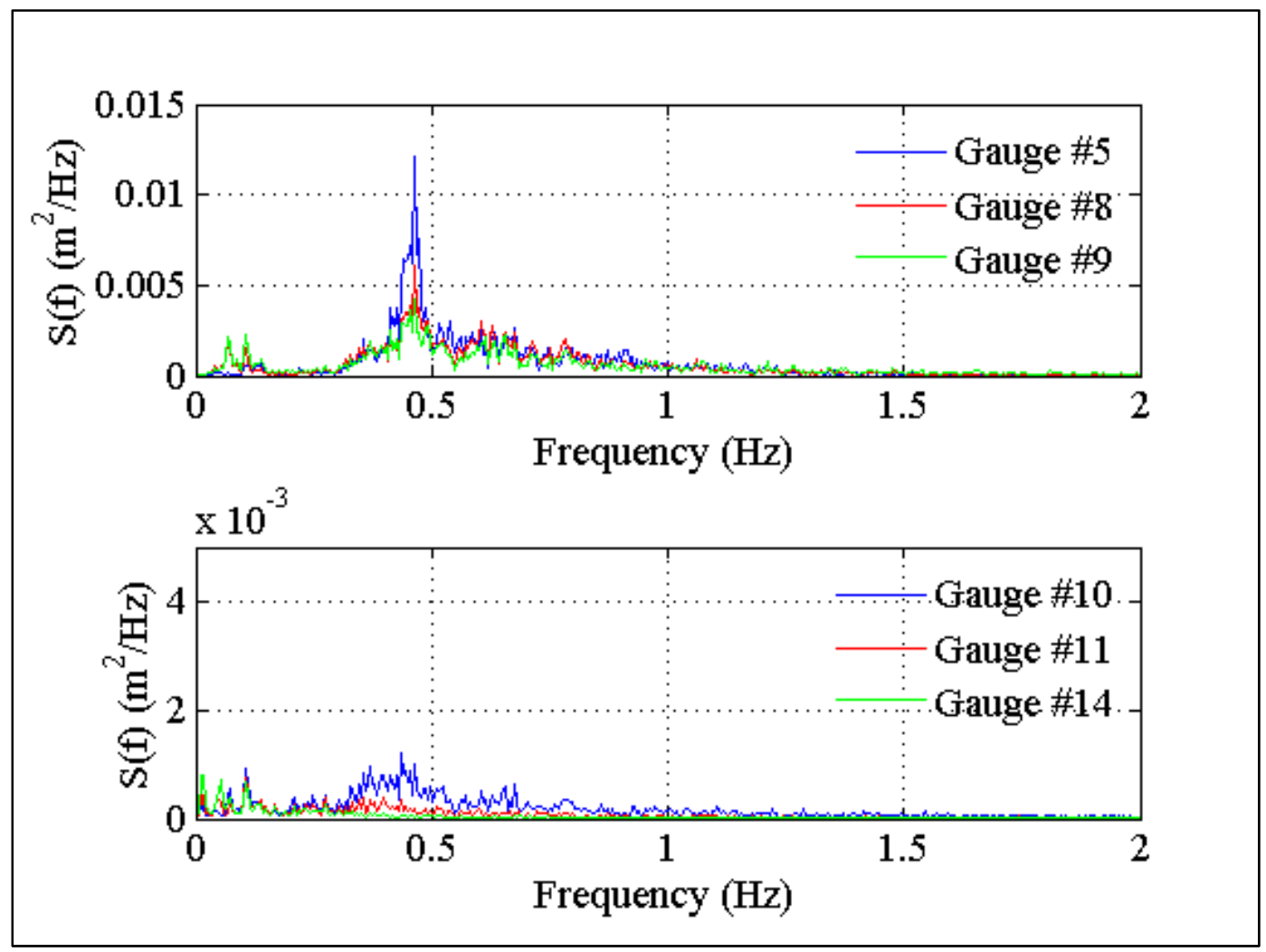

Figure 26. Energy density spectrum transformation of 2.3-sec, 0.14-m waves at mid water. 


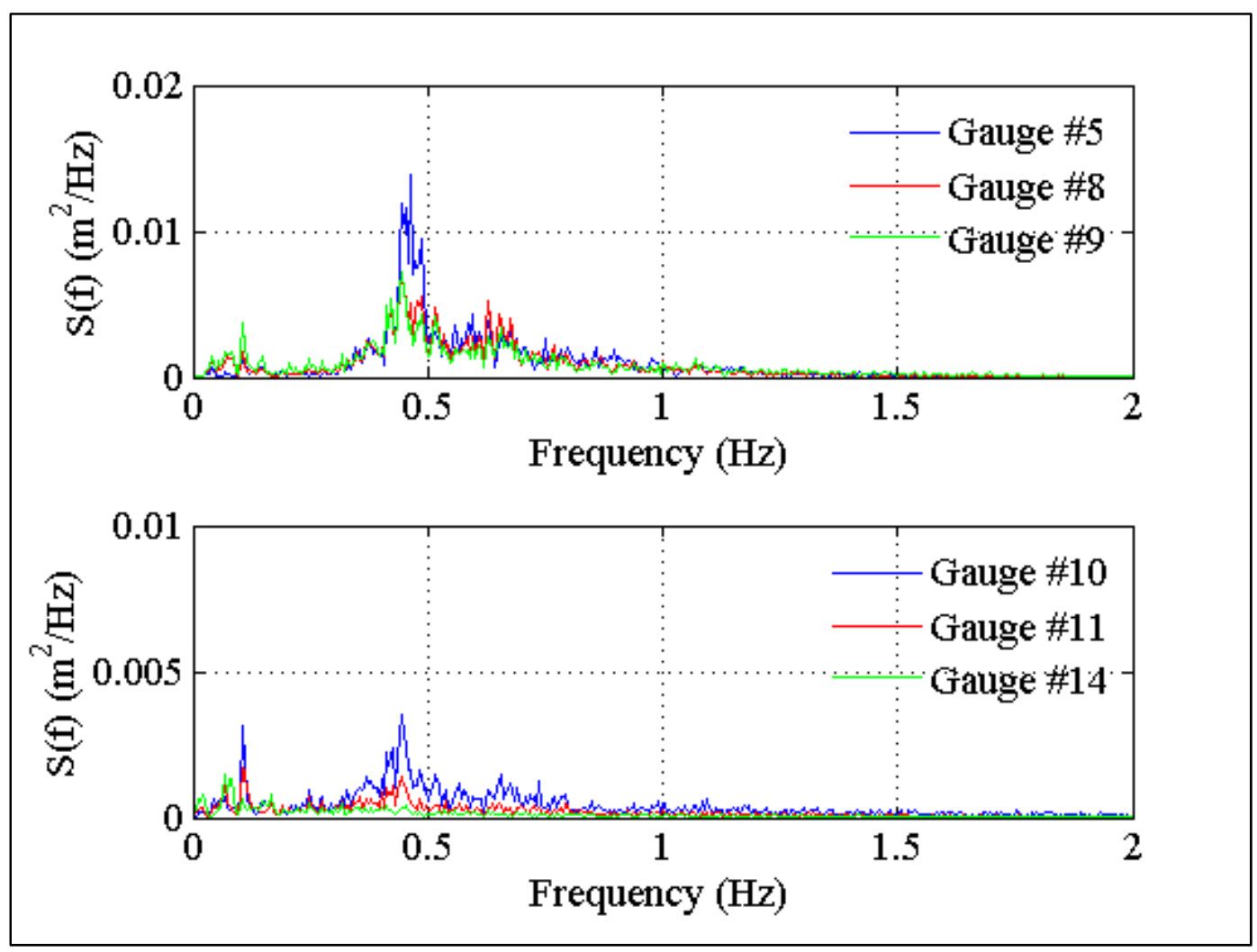

Figure 27. Energy density spectrum transformation of $2.3-\mathrm{sec}, 0.16-\mathrm{m}$ waves at high water.

Low Frequency Spectral Transformation. The low-frequency energy spectral transformation at all three water levels is shown in Figures 28 to 30 for similar incident waves. Low-frequency energy peaks at the offshore gauges (top panels) were a result of reflection and seiching in the basin. The offshore region of the basin could be assumed to be a rectangular basin. The period of oscillations for seiching in a rectangular basin as given by Dean and Dalrymple (1984) is:

$$
T=\frac{2 l}{n \sqrt{g h_{1}}}
$$

where $T$ is the period of seiching, $l$ is the length of the basin, $h_{1}$ is the depth of the basin, and $n$ is mode of seiching. The basin floor was flat between the wavemaker and the base of the reef slope, and the reef slopes are considered steep. Therefore, $l$ was the distance between the wavemaker and top of the reef slopes, $30 \mathrm{~m}$, and $h_{1}$ was the elevation of the reef slope crest above the floor, $0.354 \mathrm{~m}$. The first mode of seiching, $n=1$, has a period of oscillation equal to 30 seconds, or a fundamental frequency of $0.03 \mathrm{~Hz}$. There was no peak measured at $0.03 \mathrm{~Hz}$ at any of the water levels. 

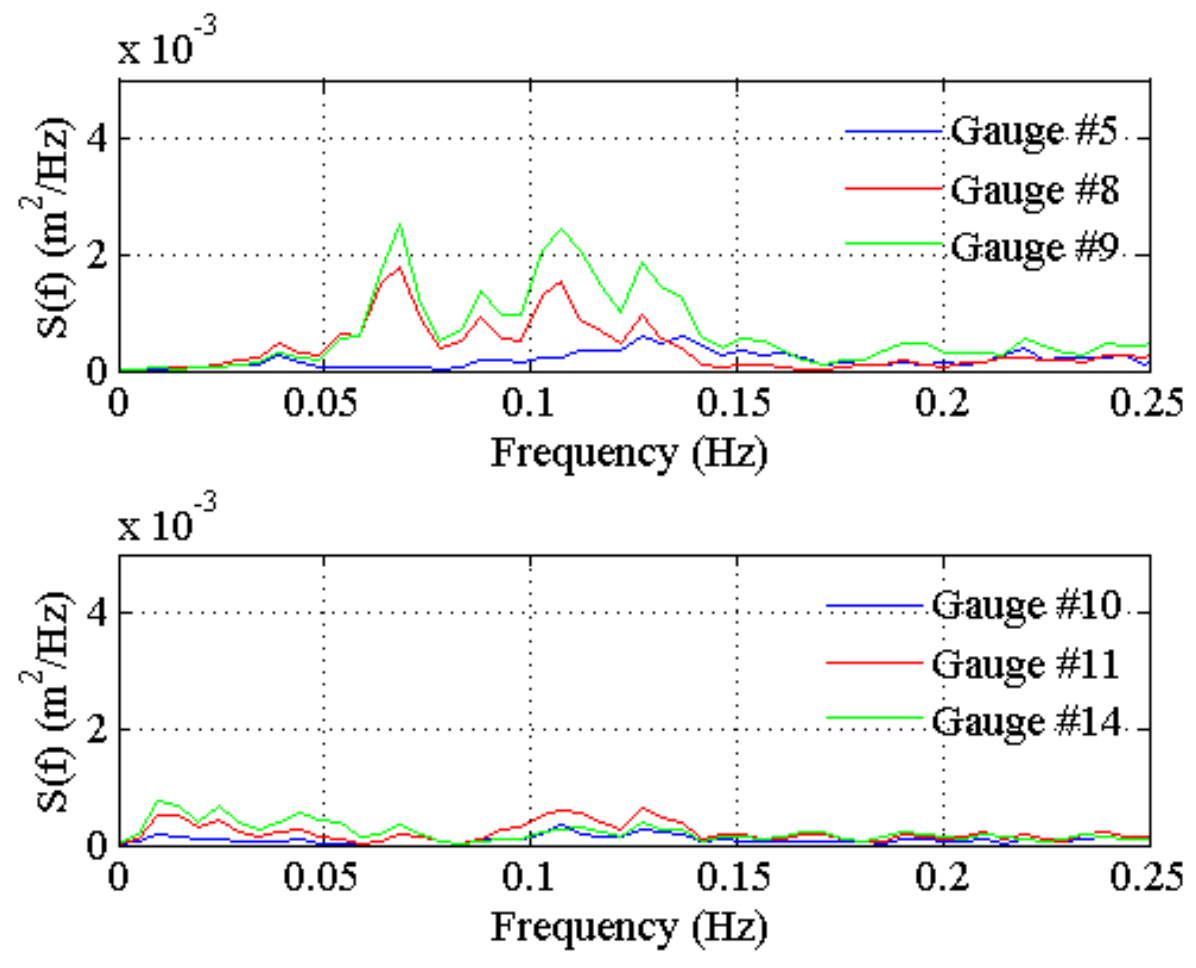

Figure 28. Low frequency energy density spectrum for $2.3-\mathrm{sec}, 0.13-\mathrm{m}$ waves at low water.
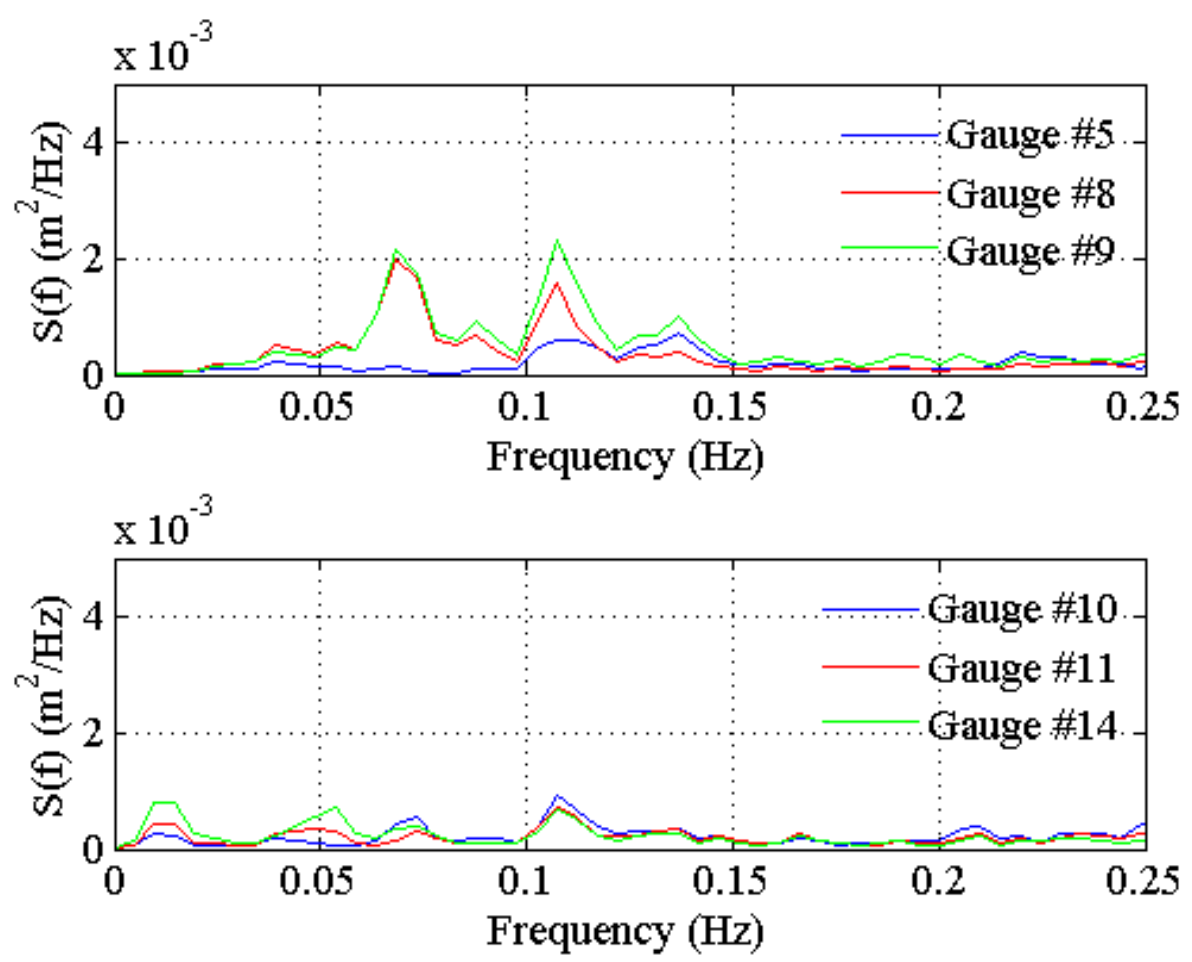

Figure 29. Low frequency energy density spectrum for $2.3-\mathrm{sec}, 0.14-\mathrm{m}$ waves at mid water. 


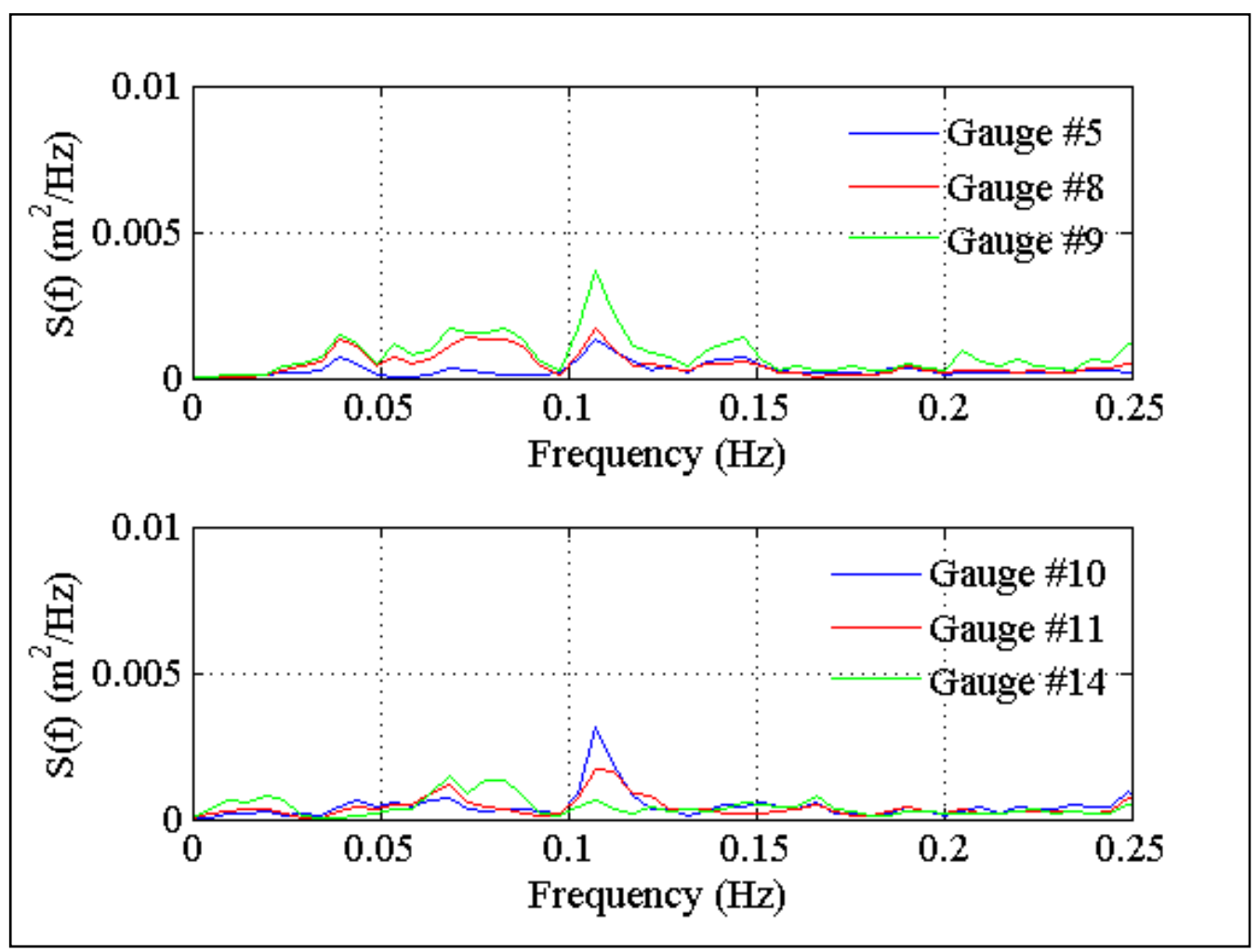

Figure 30. Low frequency energy density spectrum for $2.3-\mathrm{sec}, 0.16-\mathrm{m}$ waves at high water.

However, the $2^{\text {nd }}$ mode of seiching, $n=2$, was equal to $15 \mathrm{sec}$, or a frequency of $0.07 \mathrm{~Hz}$, where a peak was measured in the energy spectrum in all three water levels. The $3^{\text {rd }}$ mode occurred at a frequency of $0.1 \mathrm{~Hz}$ near the location of the second peak measured in the low-frequency spectrum.

Transformation of the low-frequency energy spectrum on the reef is shown in the bottom panels of Figures 28 to 30 . Peaks associated with seiching on the reef centerline were not apparent in the 3 -D tests, which could be caused by the lateral dimension in the basin. The lateral freedom of the basin could create spreading of the energy across the lower frequencies, which would minimize the peaks at expected seiching frequencies. One gauge in the high-water tests recorded energy in the low-frequency associated with seiching (Figure 30). The transmission of low-frequency energy was more apparent in the high water tests because of the deeper water over the reef crest. 


\subsubsection{Runup}

Runup is defined as the elevation above the initial water level reached by an incident wave, and was obtained from the time series of water elevations of the five runup gauges (Gauges 20 through 24). The initial water level of the low-water tests was lower than the elevation of the runup gauges; therefore the elevation difference between the initial water level and gauges was added to the measured values. A common expression of runup is twopercent runup, $R_{2}$, which is defined as the elevation exceeded by two percent of the total runups. Figure 31 shows $R_{2}$ plotted by water level and surface roughness versus wave power. It shows a trend of increasing runup with wave power. Runup does not appear to be influenced by water level. The runup gauges were not functioning during most of the rough surface experiments, but roughness does not appear to influence runup based on the available data. There is scatter in $R_{2}$ for common values of wave power, which is part due to the alongshore variation of runup. Two-percent runup versus wave power is plotted in Figure 32 with symbols representing the distance between each runup gauge and the alongshore centerline, $\Delta Y_{C L}$. The highest runup generally occurs at the centerline (Gauge 22) and at $\Delta Y_{C L}=-2.4 \mathrm{~m}$ (Gauge 21). The gauges farthest from center (Gauges 20 and 24) typically had the lowest runup values. This is consistent with the wave heights measured from the alongshore gauges (Gauges 12 through 16), where highest waves occurred in relatively deeper water near the centerline of the reef.

\subsubsection{Reflection}

Wave reflection analysis was performed to separate incident and reflected waves from the reef. Reflected waves interacting with incident waves are often present in recorded wave data from laboratory experiments (Hughes 1993), especially when structures or steep slopes are present. In this laboratory study, reflection analysis was performed to provide more accurate incident wave characteristics for numerical modeling.

Goda and Suzuki (1976) developed a separation technique using two gauges separated by an exact distance. The incident and reflected wave components were separated using a Fast Fourier Transform. The method developed by Goda and Suzuki, commonly called the Goda method, is based on linear wave theory, so nonlinear interactions were excluded. In the present research, the Goda method was employed with three gauges which allows for redundancy. 


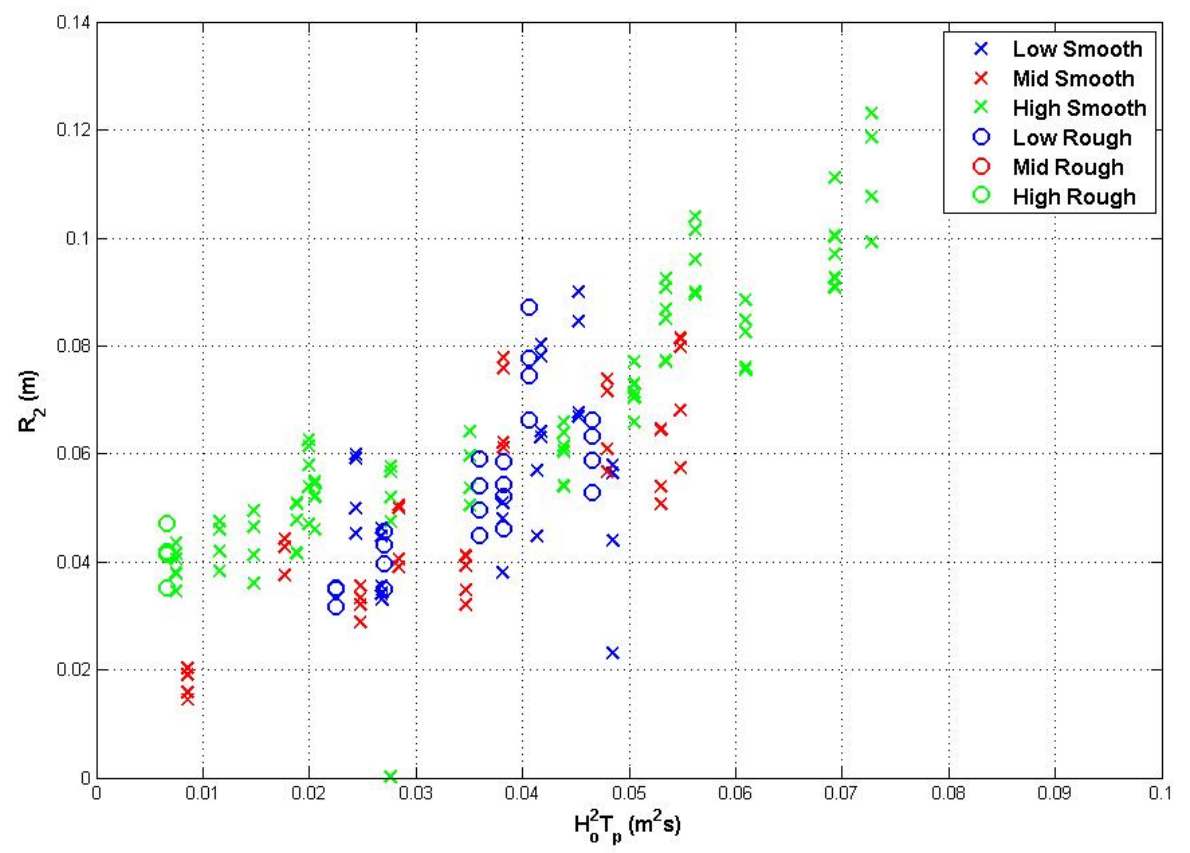

Figure 31. Two-percent runup as a function of wave power grouped by water level and surface roughness.

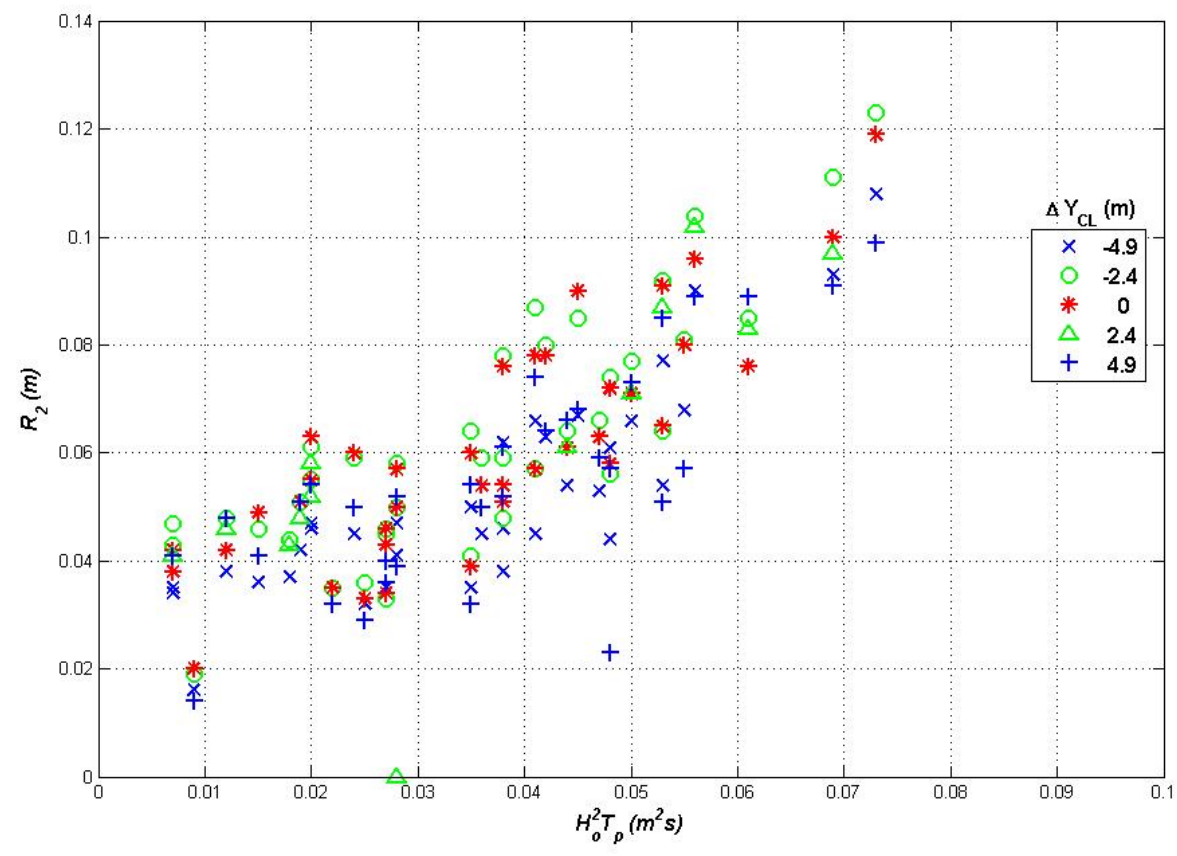

Figure 32. Two-percent runup as a function of wave power grouped by alongshore location. 
Battjes (1974) found correlation between the Iribarren number, commonly called the surf similarity parameter, and wave reflection off a beach. The surf similarity parameter is defined as:

$$
\xi_{o}=\frac{m}{\sqrt{\frac{H_{o}}{L_{o}}}}
$$

where $\xi_{o}$ is the surf similarity parameter in which the subscript denotes deepwater conditions, $m$ is the slope of the bottom, and $H_{o}$ and $L_{o}$ are deepwater height and wave length, respectively. The reef in the 3-D study consisted of three slopes of 1:4.3, 1:13.6, and 1:6. An average slope of approximately $1: 6.5$ was used to calculate the surf similarity parameter.

The Battjes (1974) relationship is expressed as:

$$
K_{r}=0.1 \xi_{o}^{2}
$$

where $K_{r}$ is the reflection coefficient.

Seelig and Ahrens (1981) developed the following equation also based on $\xi$ for plane slopes, beaches and breakwaters:

$$
K_{r}=\frac{\alpha}{1+\frac{\chi}{\xi^{2}}}
$$

in which $\alpha$ and $\chi$ are empirical coefficients, the values of which are dependent on the reflecting surface such as plane slopes, sandy beaches, or rubble breakwaters. Values of $\alpha=1.0$ and $\chi=6.2$ were given for a 1:2.5 slope.

Smith and Kraus (1990) performed laboratory experiments to study wave breaking over breakpoint bars and artificial reefs. They found that reflection was weakly dependent on the seaward slope of the bar and independent of wave steepness. Based on a 1:6.5 slope for the 3-D study, Smith and Kraus estimate a constant $K_{r}$-value of 0.15 .

Smith and Kraus (1992) re-analyzed the data of Smith and Kraus (1990) and modified the Seelig and Ahrens (1981) coefficients to apply to beach slopes having breakpoint bars. The equations to calculate $\alpha$ and $\chi$ are 
solely a function of the breakpoint bar's shoreward slope. In the case of reefs, the shoreward slope is approximately zero, and the resulting coefficients are $\alpha=0.19$ and $\chi=0.23$.

Incident and reflected waves were calculated by the Goda method from water surface elevations recorded at Gauges 4, 5, and 6. Reflection coefficient, $K_{r}$, versus $\xi_{o}$ for all wave conditions and water levels is shown in Figure 33. Also shown in the figure are the equations of Battjes (1974) (Equation 2-4), Seelig and Ahrens (1981), (Equation 2-5), and Smith and Kraus (1992) (Equation 2-5 with modified coefficients). Measured reflection coefficients do not vary with surf similarity and all values are 21 percent or less, with exception of one point. The outlying point with $K_{r}$ greater than 30 percent was likely a result of a faulty gauge in the array.

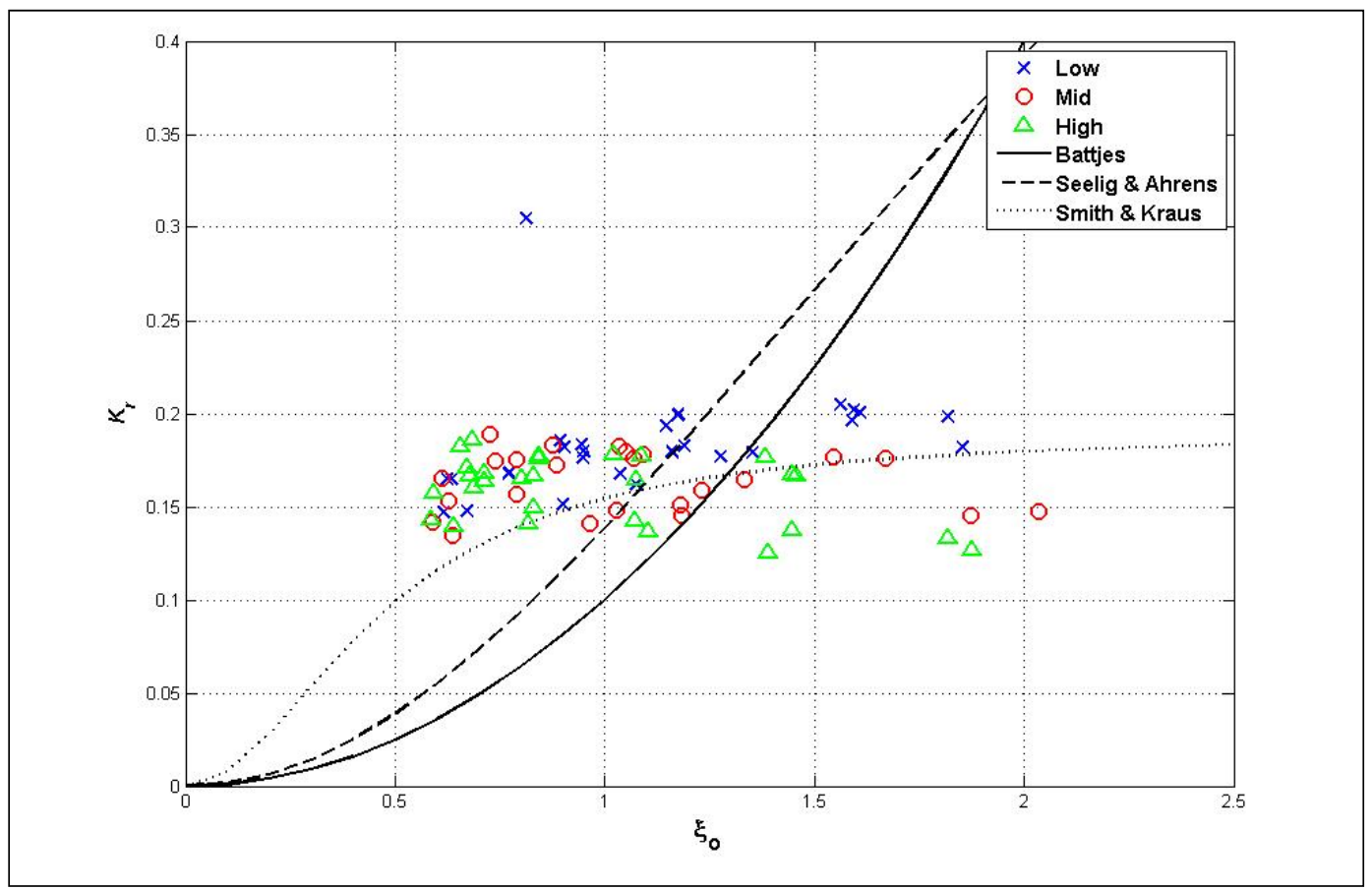

Figure 33. Reflection coefficient as a function of surf similarity parameter.

The equations of Battjes (1974) and Seelig and Ahrens (1981) under predict $K_{r}$ for $\xi_{o}>1$ and overpredict $K_{r}$ for $\xi_{o}>1.5$. The Smith and Kraus (1992) relationship gives reasonable reflection values, but underpredicts at lower surf parameters. The constant reflection coefficient predicted by Smith and Kraus (1990) of 0.15 gives a reasonable, although low, prediction of the data. 


\subsubsection{Channel experiment}

Following the experiment with the smooth and rough surfaces, a channel was cut into the bathymetry at an angle to incident waves (Figure 34). The channel was $5.5 \mathrm{~m}$ long and $0.15 \mathrm{~m}$ wide with vertical sides that extended to the underlying steel platform (0.1-m deep with respect to the mid-water level). The shoreward end of the channel contained a 1:1 slope which transitioned to the reef surface.

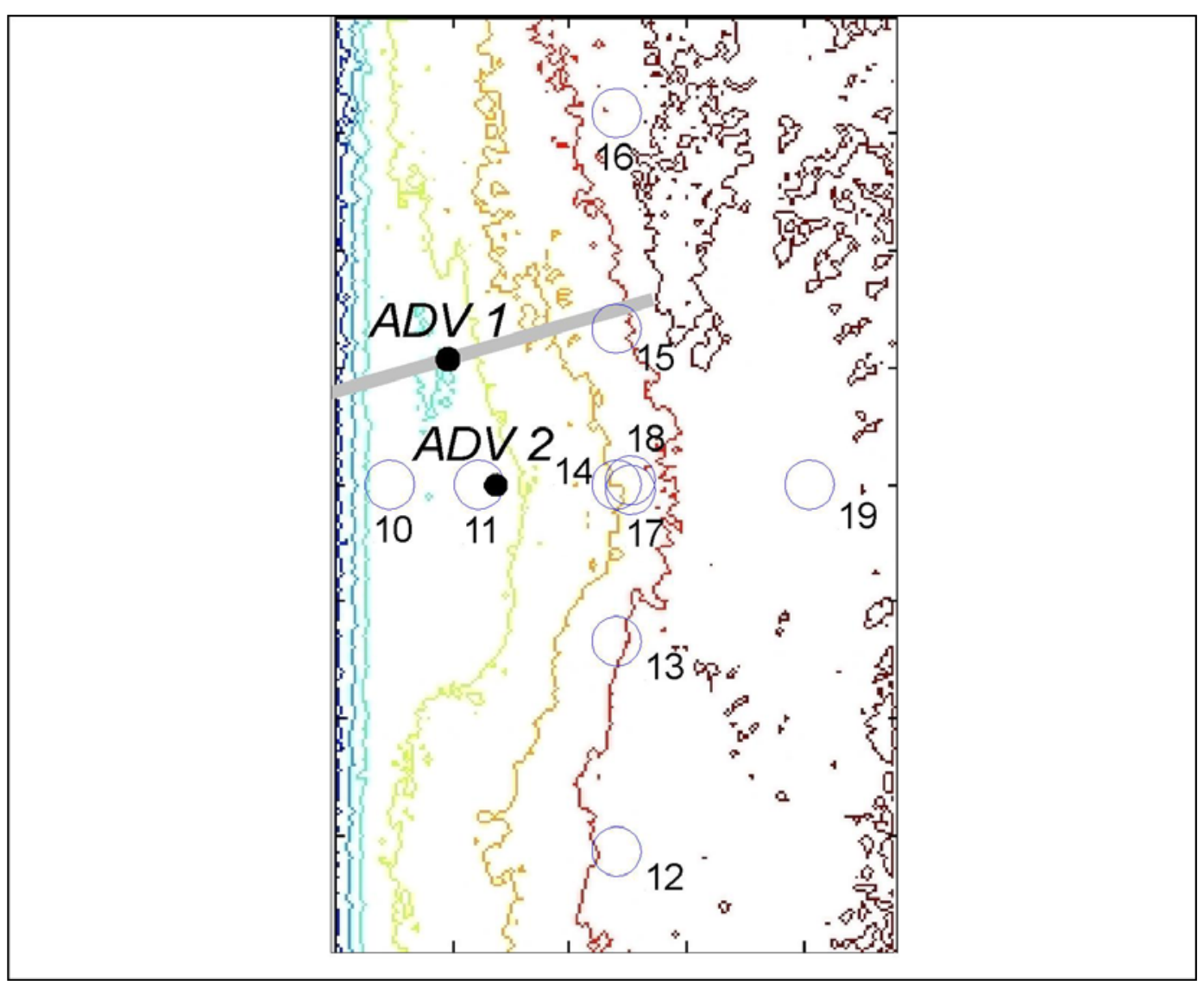

Figure 34. Location of channel and ADVs during channel experiment.

Two Acoustic Doppler Velocimeters (ADVs) were placed on the reef to measure currents; ADV 1 was placed at the midpoint of the channel with its sampling volume measuring in the center of the channel and ADV 2 was placed near the center of the reef. The ADVs were positioned vertically at one-third of the water depth from the bottom to measure velocities that approximated the mean velocities (Hamilton et al. 2001). Both ADVs were 2-dimensional, side-looking instruments and were sampled at $20 \mathrm{~Hz}$. Figure 34 shows the two ADV locations, shown as solid circles in relation to wave gauges placed on the reef, shown as open circles. The model coordinates for 
the ADVs are given in Table 4. Wave and velocity measurements were taken for the highest wave height for each wave period at the mid-water level, and for all wave conditions at the high-water level excluding the highest waves for the 2.3-sec and 2.8-sec periods.

Table 4. SWIMS 3-D reef ADV locations (see Figure 7 for coordinate system).

\begin{tabular}{|l|l|l|}
\hline ADV & $x(m)$ & $y(m)$ \\
\hline 1 & 31.24 & 7.73 \\
\hline 2 & 31.85 & 6.10 \\
\hline
\end{tabular}

Measurements were made for selected conditions in Table 2 at the midand high-water levels. Tests with the channel included the highest waves for each period at the mid-water level and all wave conditions at the high water level except for the highest wave at the 1.4-, 2.3- and 2.8-sec wave periods.

Appendix C contains figures generated from the channel experiment, which include wave height transformation, rose plots of currents taken from the ADVs, and alongshore current plots. The following paragraphs discuss selected figures from the channel experiment.

Centerline $H_{m o}$ and $\eta$ from the channel experiment are plotted with distance from the wave generator in Figures $\mathrm{C} 1$ to $\mathrm{C} 17$ and Figures 35 to 38 for select tests. Data collected from the rough surface tests without the channel are shown in the figures for comparison (although both surfaces were rough, references to the rough surface tests imply no channel was installed). Also plotted are initial water levels and reef bathymetry (scaled down by a factor of 10) for reference. The figures include the highest waves generated for 1.0and 2.8-sec periods at the mid-water level and 1.0- and 2.3-sec at the highwater level for the channel and rough cases. Despite identical input wave signals, the incident wave height generated during the channel test with the 2.8-sec wave at high water was much lower than the incident heights with the rough condition. Therefore, the 2.3 -sec wave was selected to provide a better comparison for discussion. The figures show that wave transformation is similar with and without the channel. The 1.0-sec period incident wave height with the channel is slightly lower than the rough surface test height at the mid-water level, but wave heights at breaking and in the surf zone are comparable (Figure 35). Figure 36 shows that $H_{m o}$ with 2.8-sec waves at mid water are similar between the two cases, with the exception to waves immediately offshore of breaking, where heights are slightly higher as the waves approach the break point with the channel in place. 


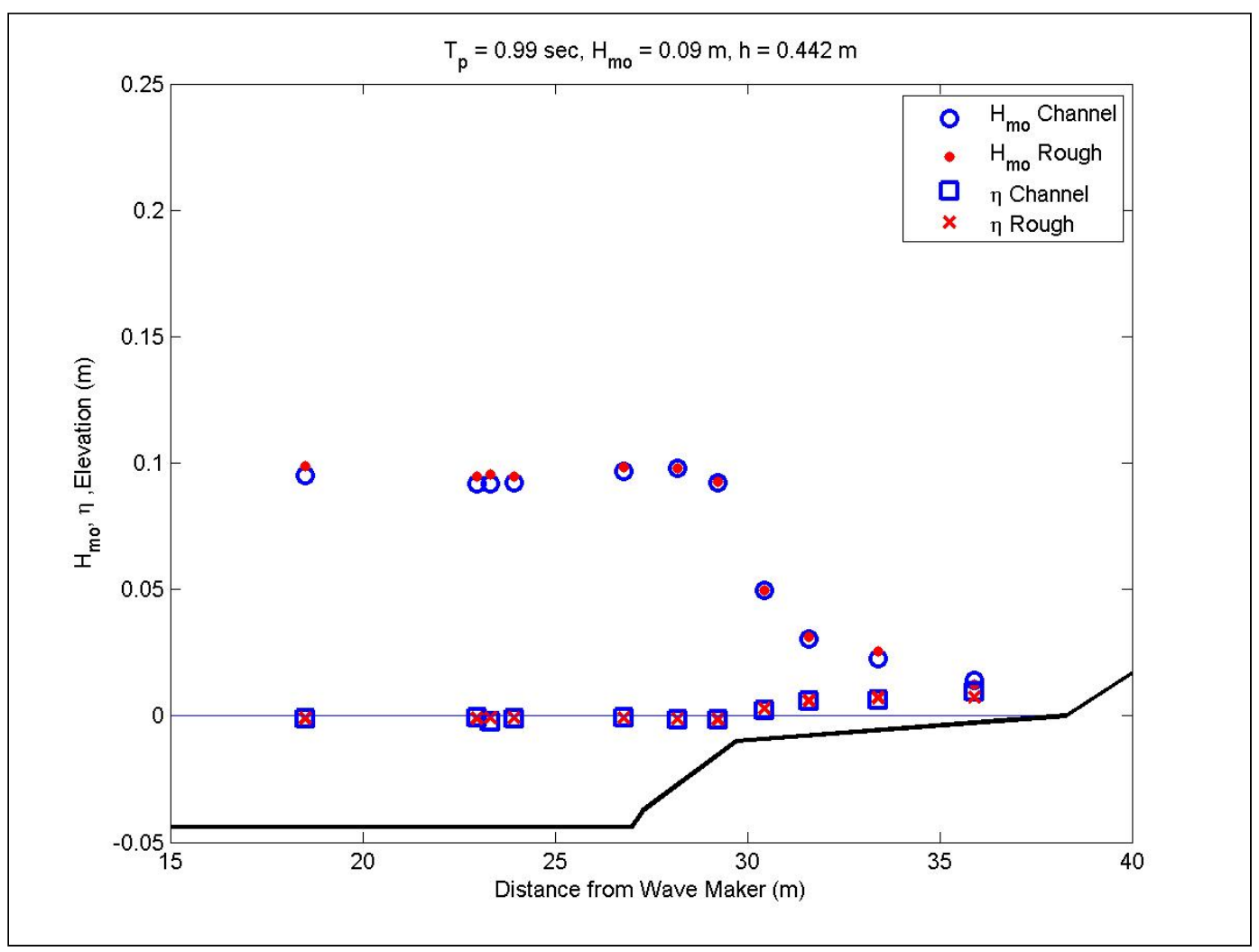

Figure 35. Wave transformation for 1-sec, 0.1-m waves at mid water.

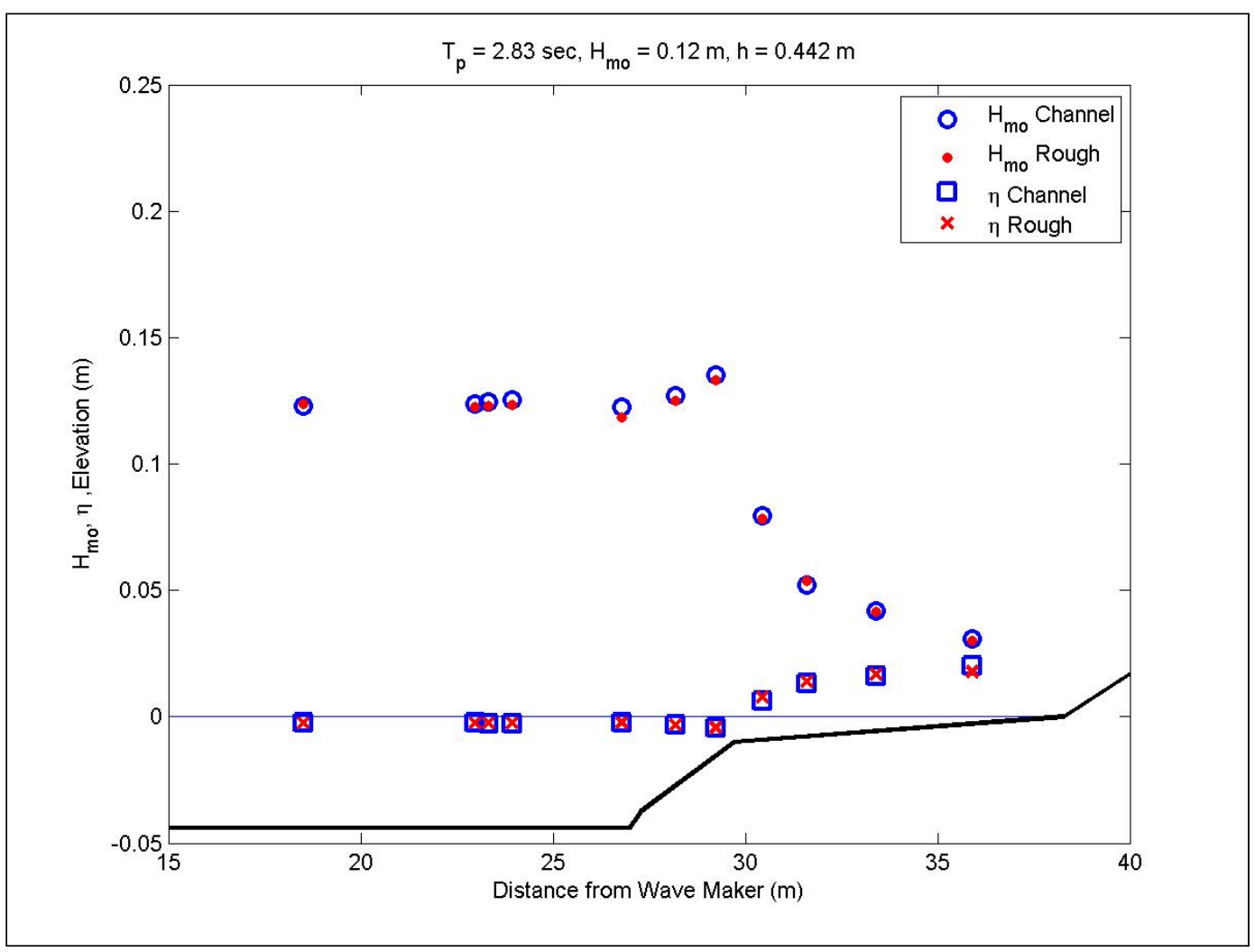

Figure 36. Wave transformation for $2.8-\mathrm{sec}, 0.12-\mathrm{m}$ waves at mid water. 


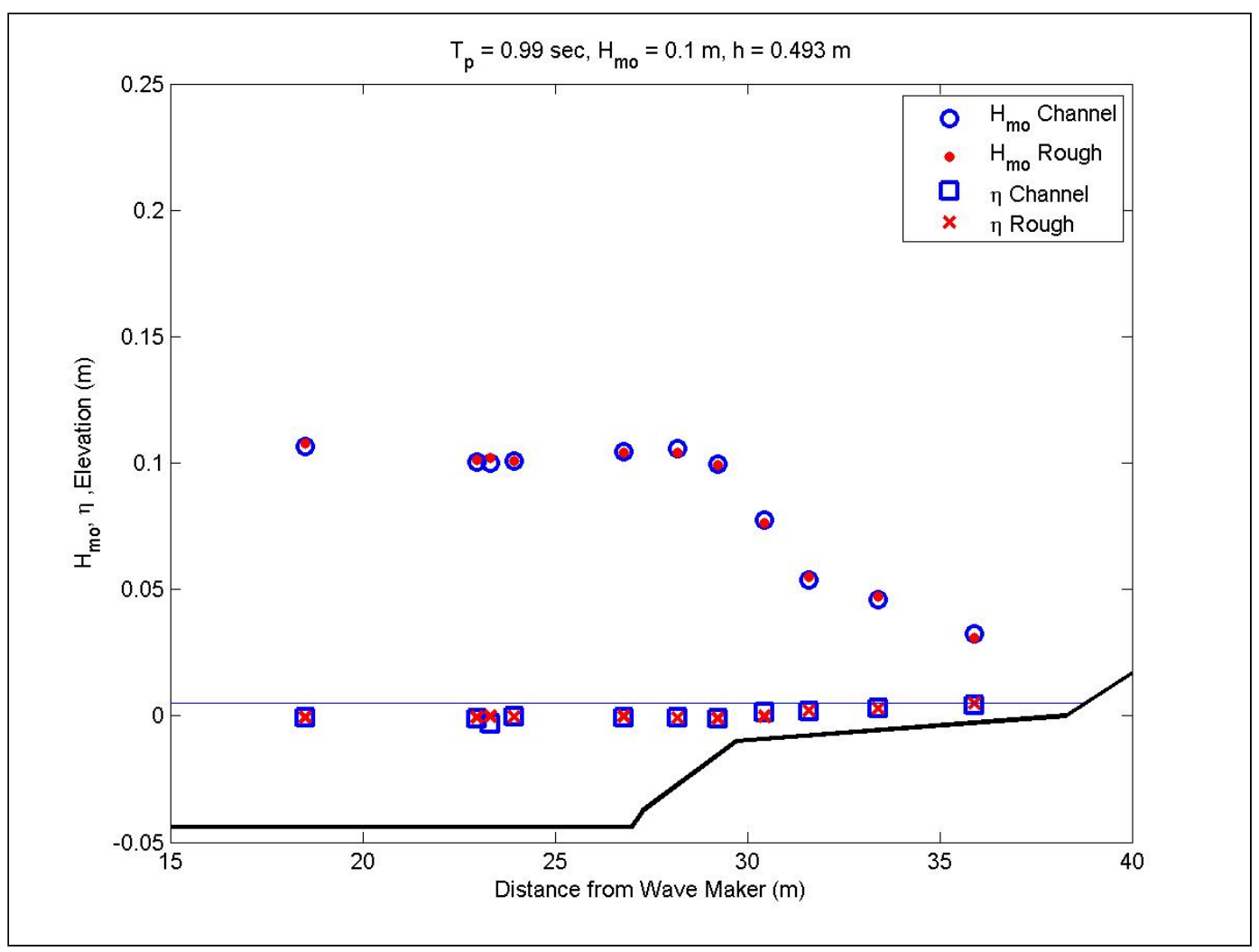

Figure 37. Wave transformation for 1-sec, 0.1-m waves at high water.

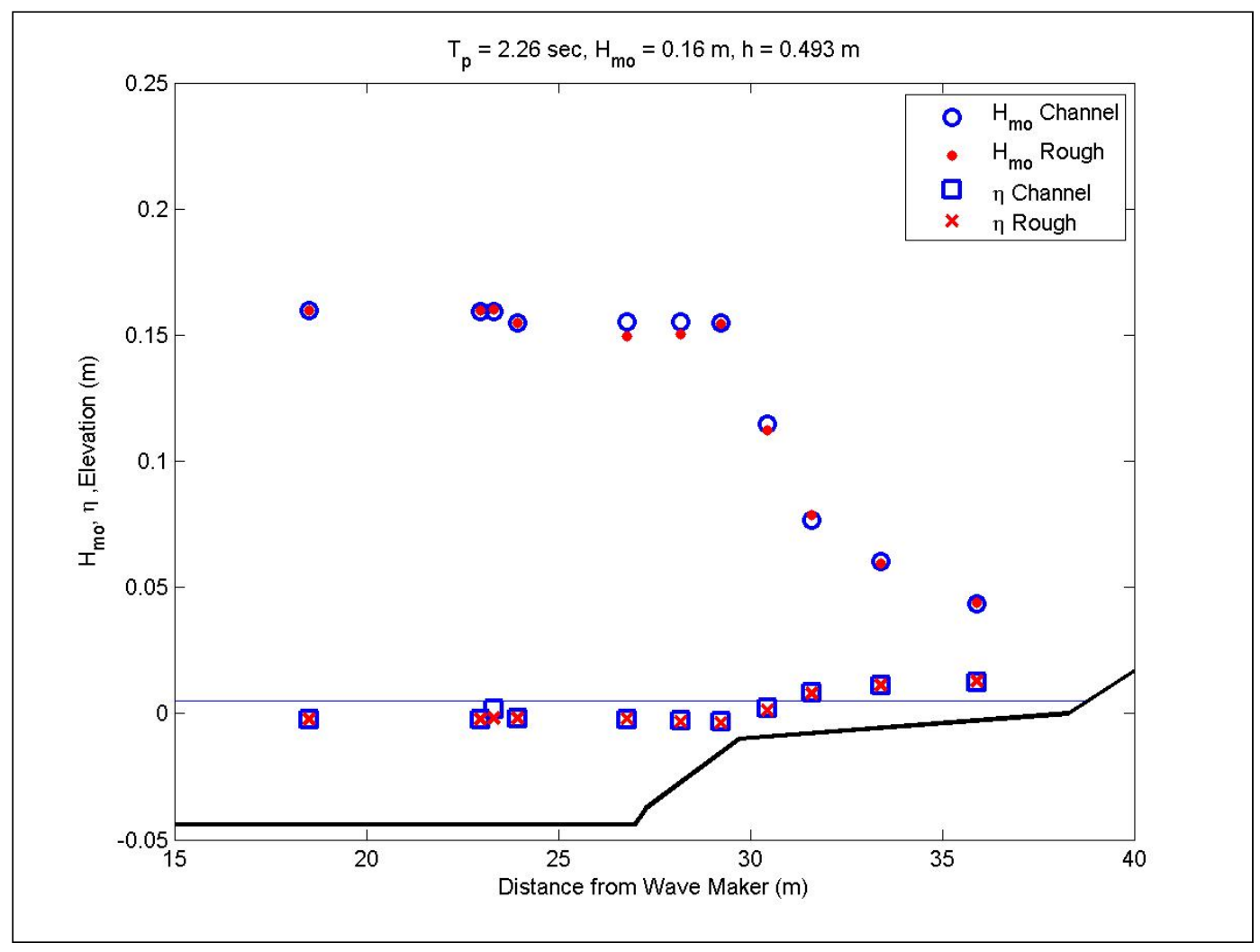

Figure 38. Wave transformation for 2.3-sec, 0.16-m waves at high water. 
Little variation is seen between wave heights for 1.0-sec waves at high water between the channel and non-channel tests (Figure 37). However, 2.3-sec waves measured with the channel installed are higher than the rough surface test waves directly offshore of breaking (Figure 38).

Wave height comparisons between the channel and rough surface tests showed similar trends as observed in Figures 35 to 38 . Wave heights were similar between the two tests, but longer-period waves and higher incident waves generally showed greater heights in the shoaling region directly offshore of breaking with the channel.

Rose plots showing velocities from the ADVs are presented in Figures $\mathrm{C} 18$ to $\mathrm{C}_{34}$ and in Figures 39 to 42 for the same conditions discussed for wave transformation. The rose plots represent cumulative velocity by direction. Each $20-\mathrm{Hz}$ velocity measurement was separated into 20 directional bins and the velocities were summed by bin. The origin of the rose-plot figures is the lower offshore corner of the acrylic reef and shoreward to the right. For ease of discussion, the lower part of the figure will be referred to as downdrift, with updrift toward upper alongshore locations.

The figures show that currents in the channel are, expectedly, mainly bi-directional in the direction of the channel but with a net flow directed offshore. Figure 39 shows cumulative velocities at ADV 2 are mostly offshore directed, but also are skewed downdrift for the 1.0-sec wave condition at mid water. Velocities at ADV 2 with the 2.8 -sec wave at mid water also show slight net flows offshore and downdrift, but the rose plot is more symmetrical in the onshore-offshore direction than the 1.0-sec wave at the same water level (Figure 40). Velocities at ADV 2 with the 1.0-sec wave condition at high water show a strong offshore net flow and a net downdrift velocity (Figure 41). Figure 42 shows that, like the 2.8 -sec wave at mid water, onshore-offshore velocities are nearly equal at ADV 2 with the 2.3-sec waves, but longshore velocities are directed downdrift.

The rose plots show that cumulative velocities in the channel were directed offshore. It is possible that the offshore-directed velocities in the channel led to increased height on waves as they approached breaking through wave-current interaction. It is interesting that currents on the reef have a downdrift tendency with shore-normal incident waves. Reef elevations were unintentionally constructed higher on the "updrift" portion of the reef due to anomalies of the underlying steel platform, which may have 


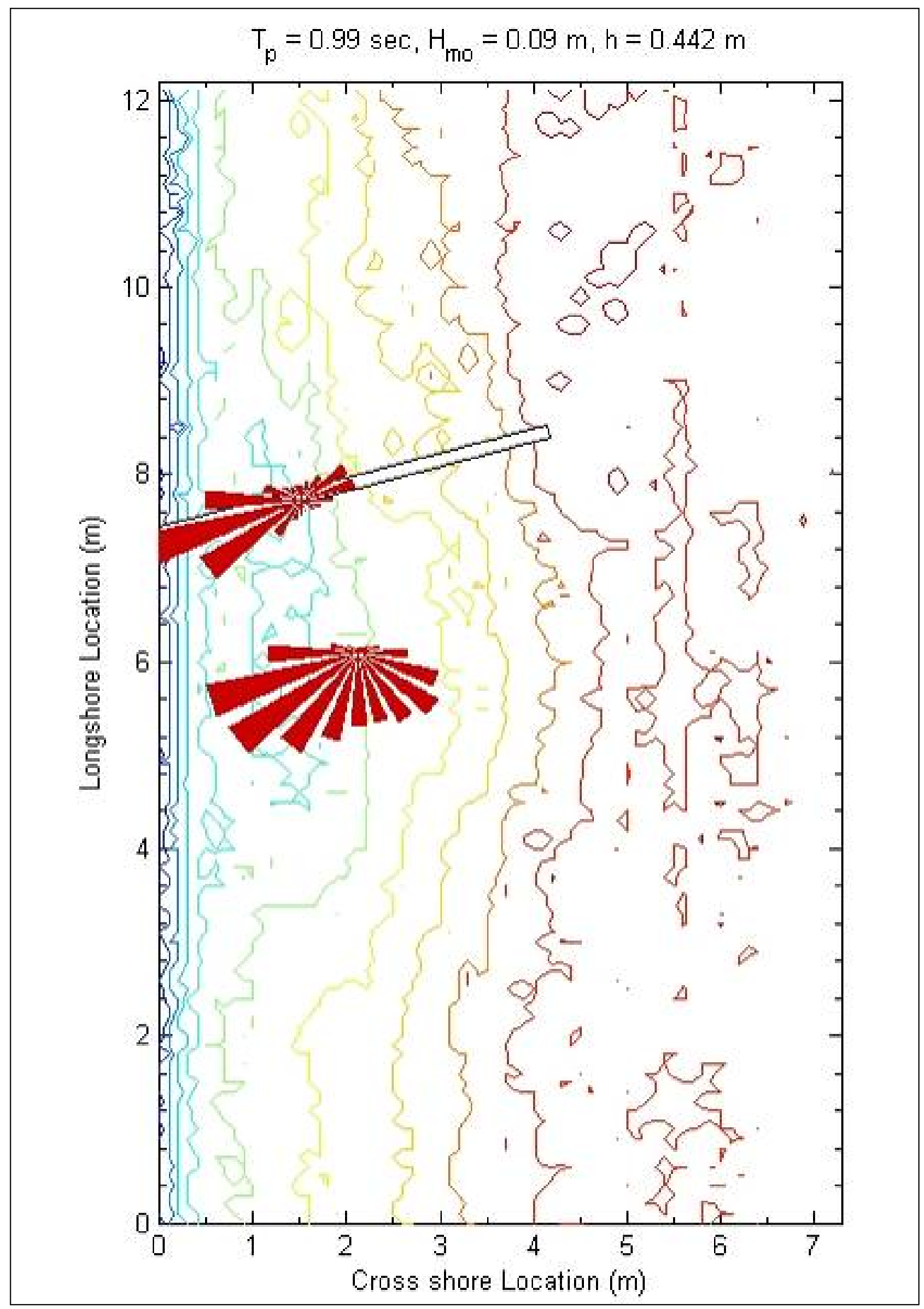

Figure 39. Current rose patterns for 1-sec, 0.1-m waves at mid water. 


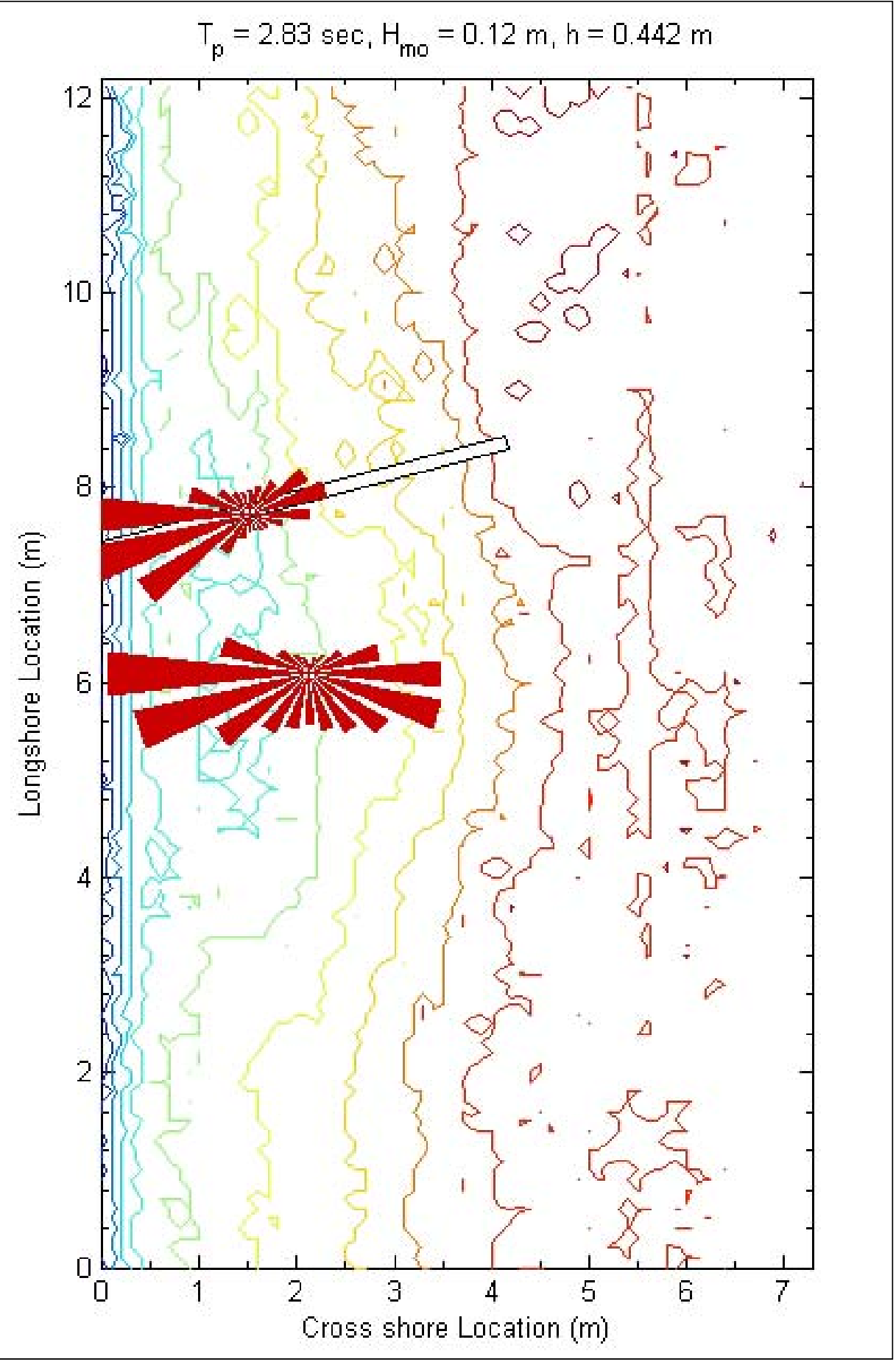

Figure 40 . Current rose patterns for $2.8-\mathrm{sec}, 0.12-\mathrm{m}$ waves at mid water. 


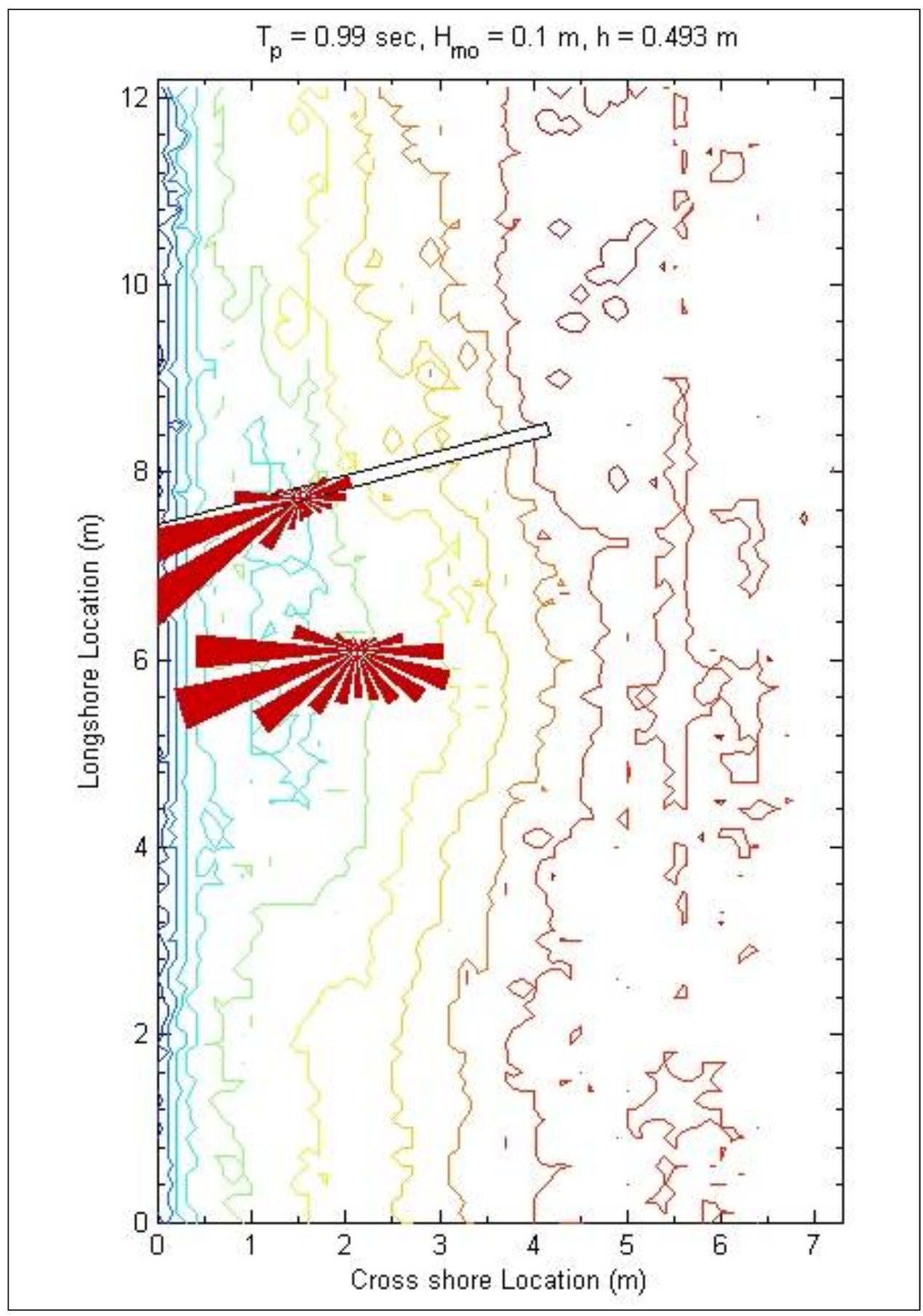

Figure 41. Current rose patterns for 1-sec, 0.1-m waves at high water. 


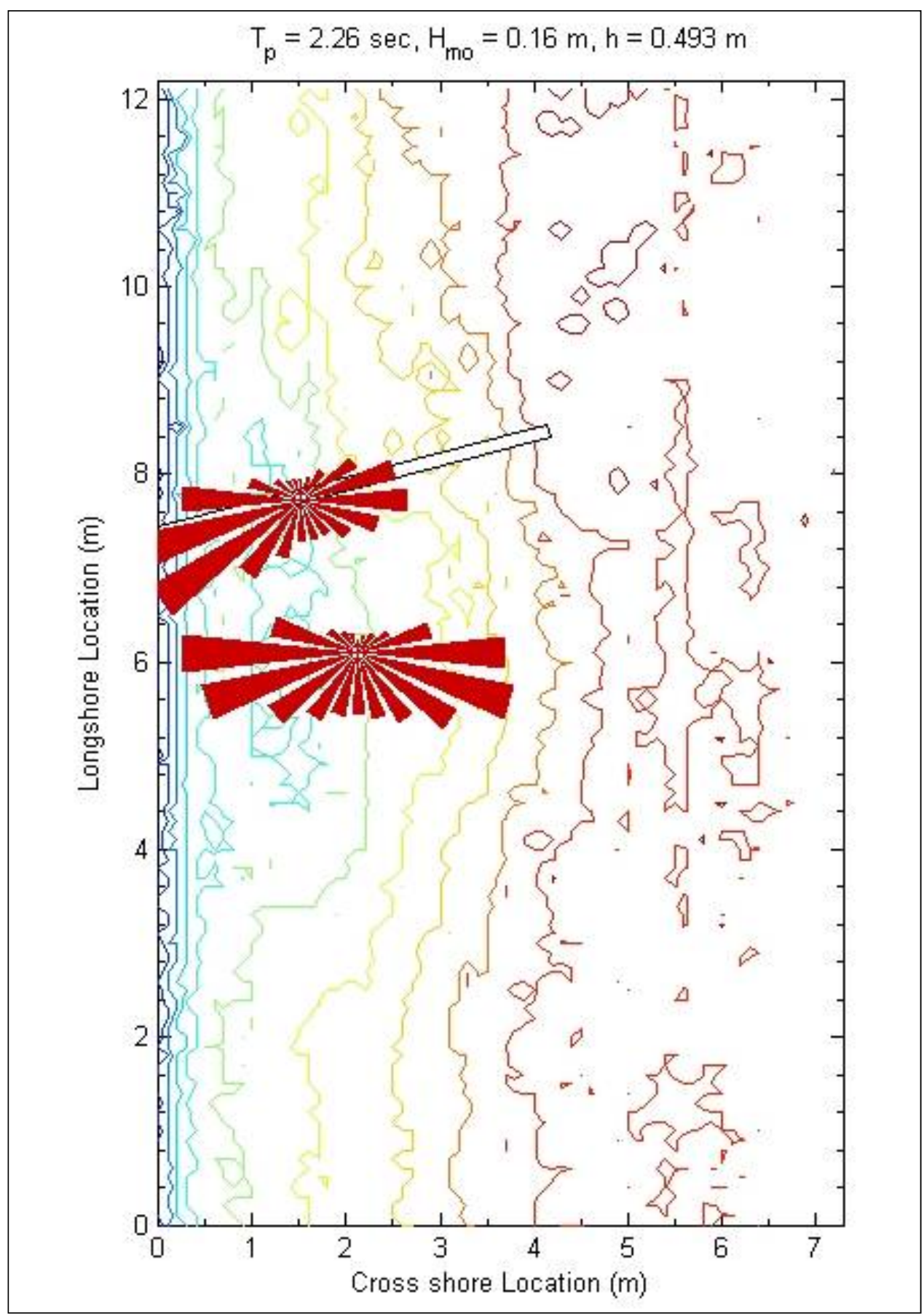

Figure 42. Current rose patterns for $2.3-\mathrm{sec}, 0.16-\mathrm{m}$ waves at high water.

produced a downdrift water surface gradient. Shorter periods produced a strong net offshore-directed flow at ADV 2, whereas onshore-offshore directed currents were more balanced with longer periods.

Figures of $H_{m o}$ and $\eta$ measured at the alongshore reef gauges (Gauges 12 through 16) for the channel and rough surface conditions are given in 
Figures $\mathrm{C}_{35}$ to $\mathrm{C}_{51}$. For reference, the figures include the initial water level and measured bathymetry elevations at the gauge locations.

Alongshore wave heights measured with the channel installed are presented for discussion in Figures 43 to 46 for the same wave conditions discussed previously in this section. Wave heights with the rough surface also are plotted to provide a comparison with the channel condition. Wave heights generally are comparable between the channel and rough surface condition, except at Gauge 15 (alongshore location $\approx 8 \mathrm{~m}$ ), where height is consistently lower with the channel condition. Gauge 15 was located next to the channel, where the influence of deeper water probably affected the heights at Gauge 15. Variability in heights between the two conditions also is evident at other locations, particularly for the shorter period tests (Figures 43 and 45), but most of the differences are slight and within measurement error. Wave setup is very similar for channel and rough surface tests for all conditions.

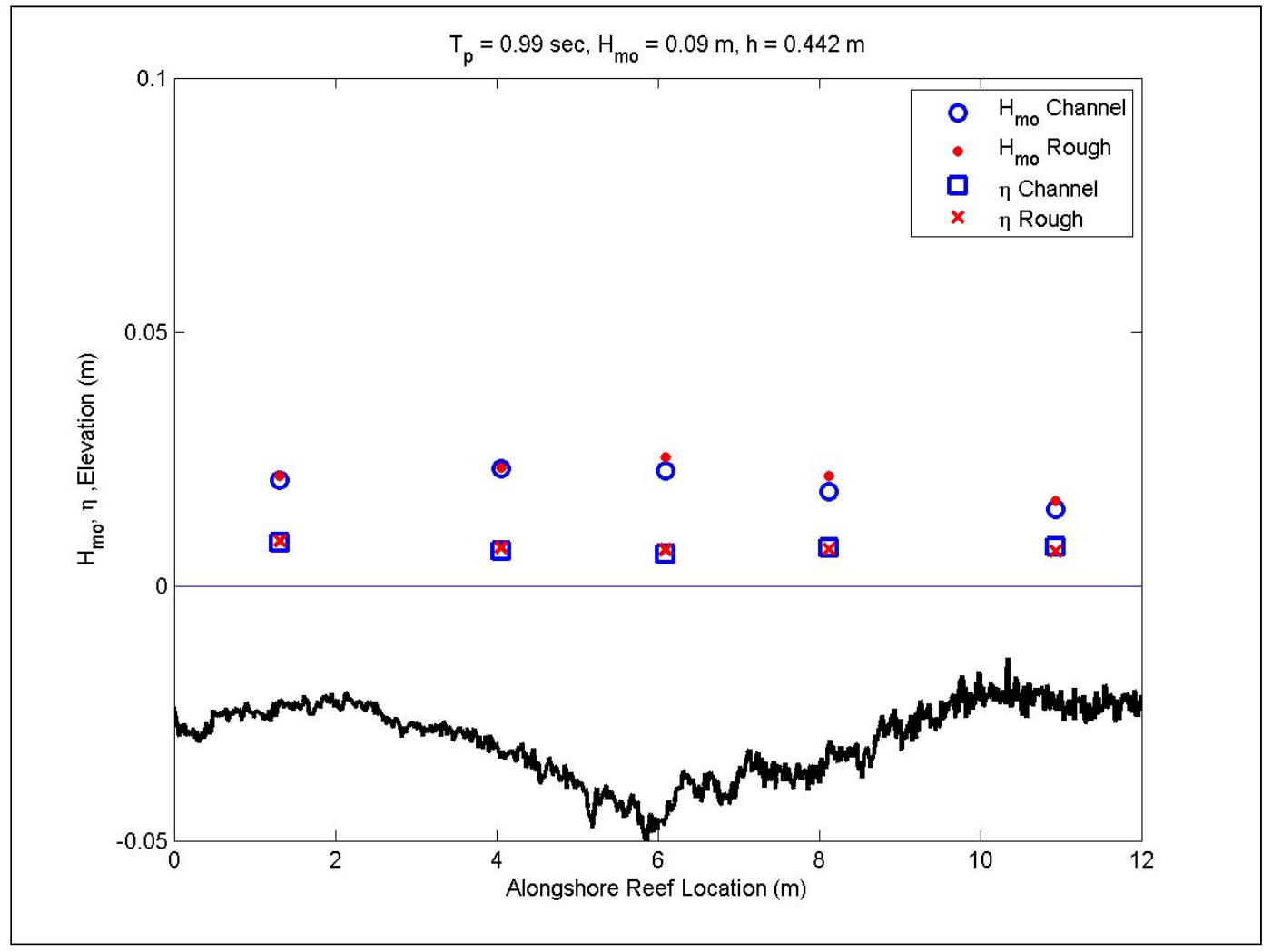

Figure 43. Alongshore wave heights for 1-sec, 0.1-m waves at mid water. 


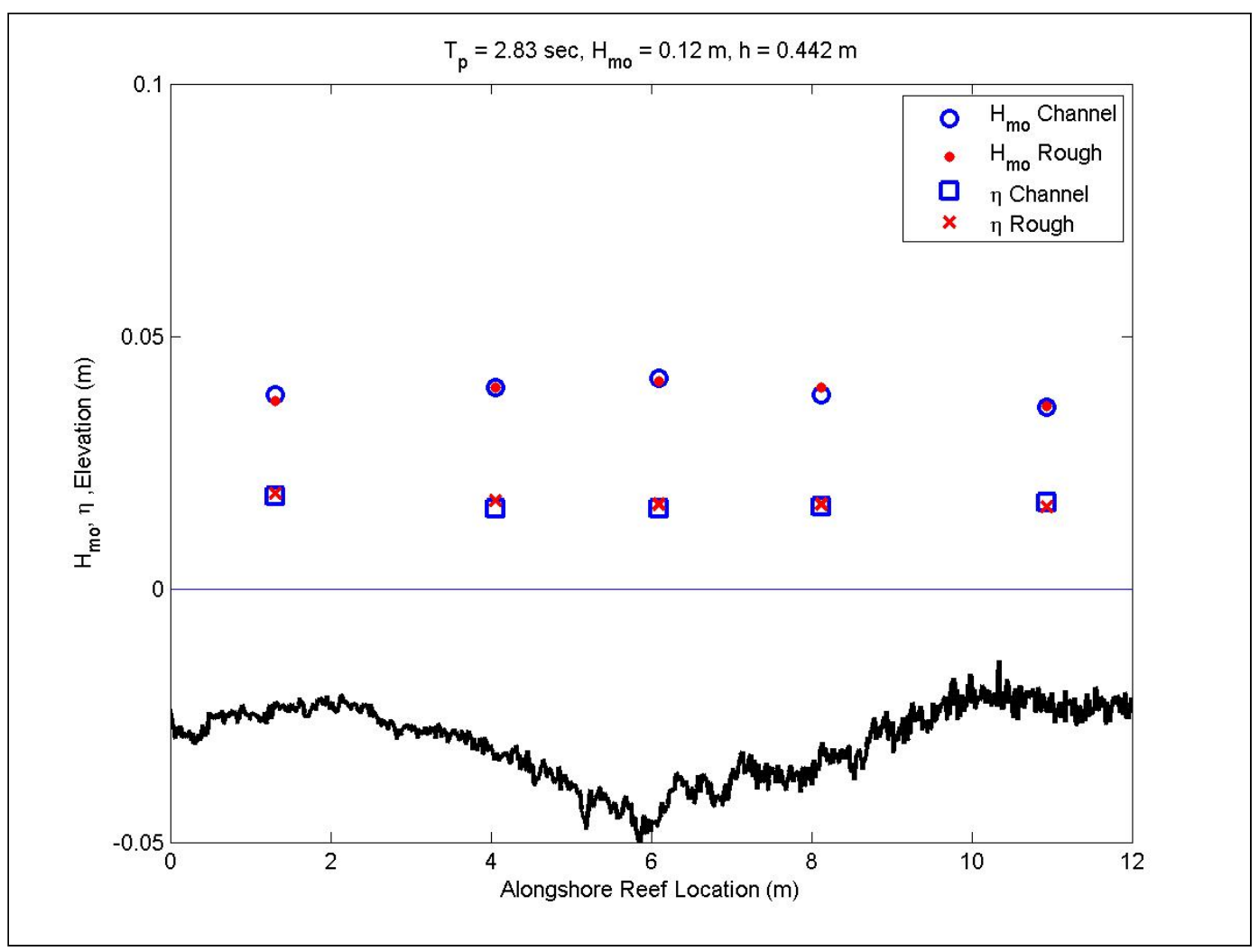

Figure 44. Alongshore wave heights for $2.8-\mathrm{sec}, 0.12-\mathrm{m}$ waves at mid water.

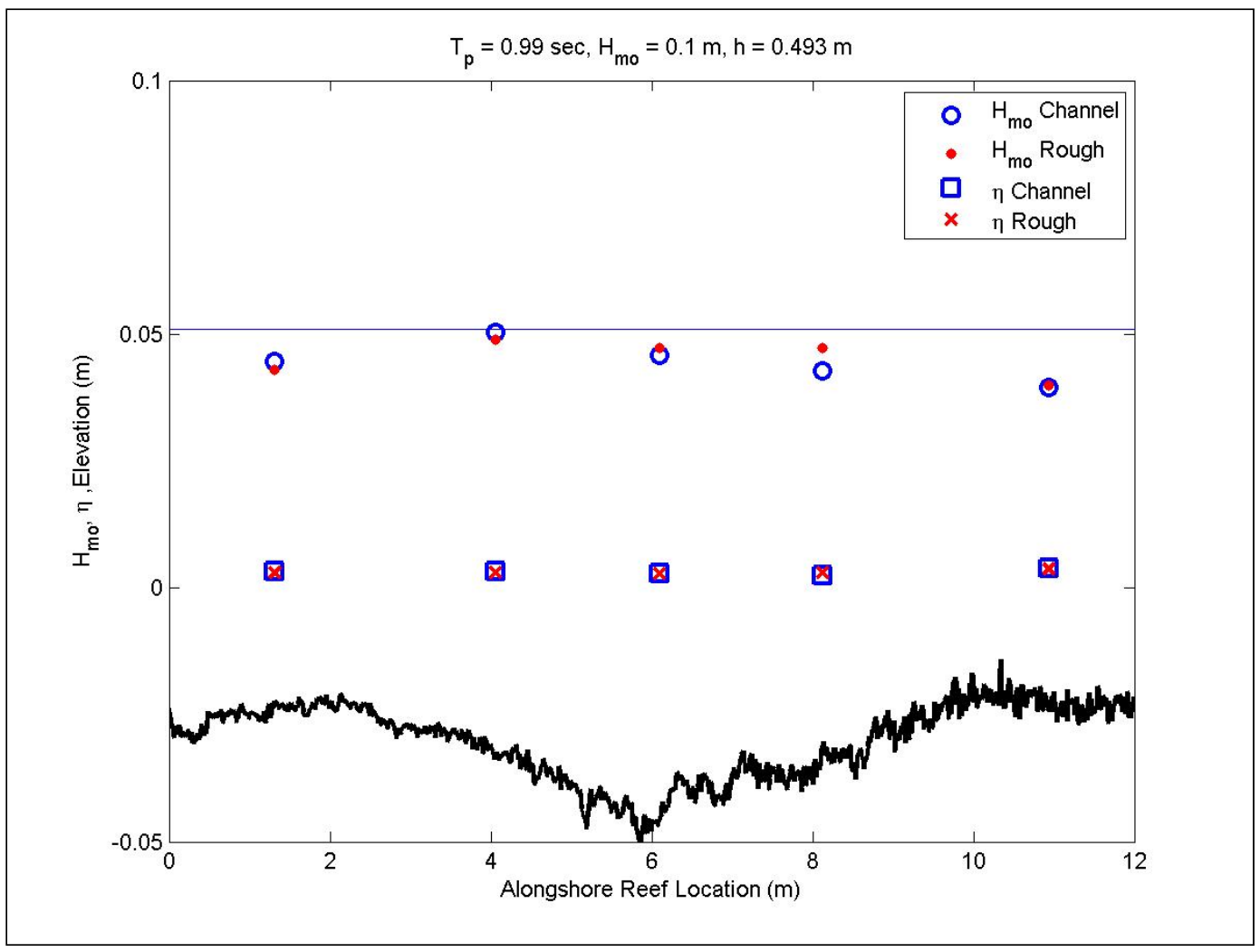

Figure 45. Alongshore wave heights for 1-sec, 0.1-m waves at high water. 


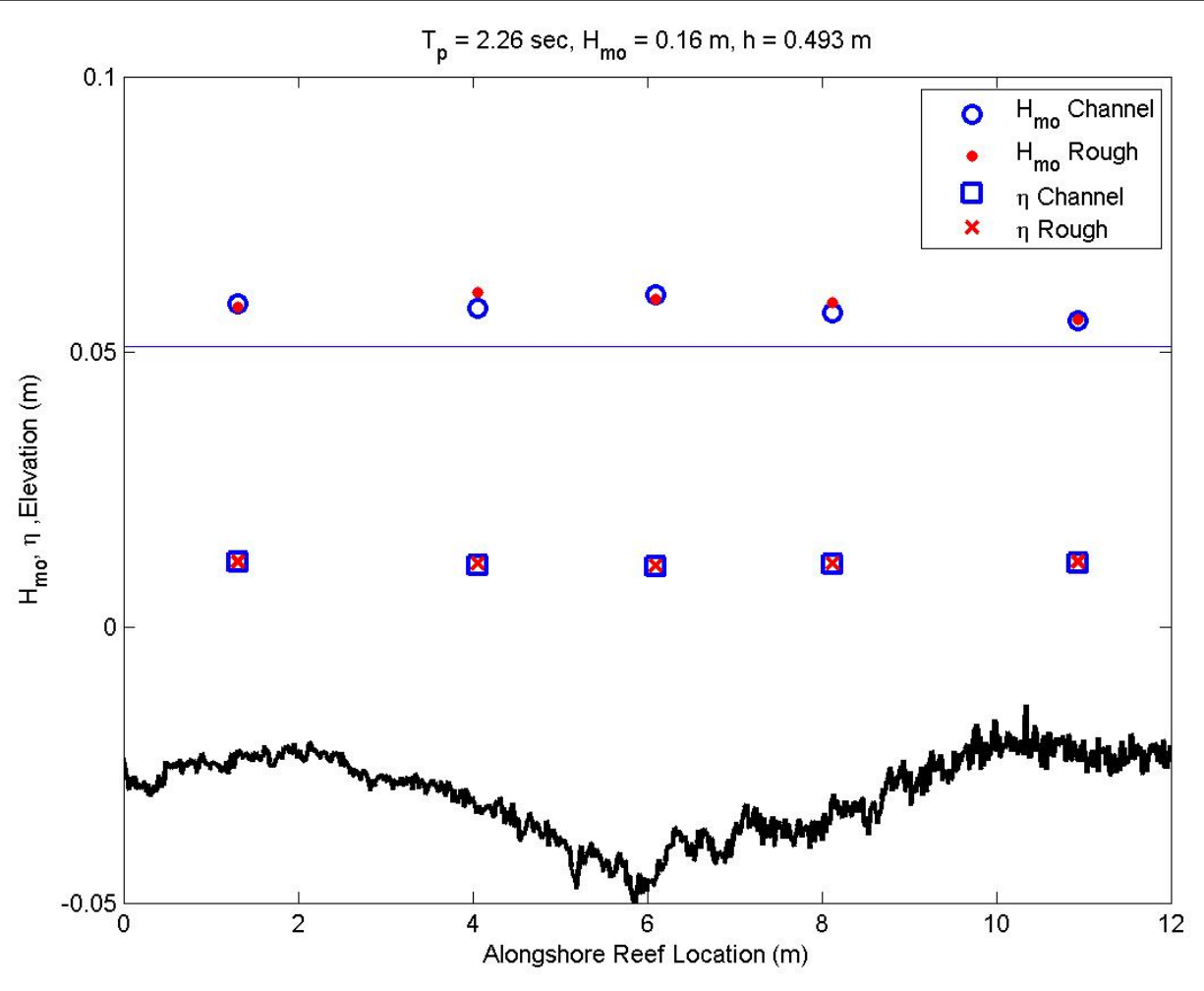

Figure 46. Alongshore wave heights for 2.3-sec, 0.16-m waves at high water. 


\section{Two-Dimensional Physical Model Experiments}

This chapter describes the design and construction of the two-dimensional reef experiments, which included a duplicate cross-shore section from the three-dimensional experiment. The purpose of these experiments was to obtain wave, setup and runup data over an idealized reef for a range of wave heights, periods, water levels and reef roughnesses with minimized three-dimensional effects.

\subsection{Test facilities and equipment}

Two-dimensional experiments were performed in a 45.7-m long, 0.91-m wide, and 0.91-m deep wave tank. The bottom configuration of the wave tank included an 11-m horizontal bottom, a 3.4-m long section at a 1:20 slope, and a 21.6-m long section at a 1:100 slope.

An electronically controlled hydraulic system controlled the position of a piston-type wave board and produced waves by periodic motion of the board. Displacement of the wave board was controlled by a command signal transmitted to the board by computer. A TMA shallow-water wave spectrum (Bouws et al. 1985) with a gamma value of 3.3 was used to define the spectral shape in the command signal for all wave conditions.

\subsection{Model design}

A 0.61-m wide cross-shore section of the three-dimensional acrylic reef was duplicated and installed in the wave tank as the reef surface. It was desired to choose a section of the 3 -D reef that had uniform alongshore contours, yet was near the center of the reef to allow comparison of wave heights with the 3-D model. The area chosen is shown in Figure 47, of which the longshore edges ranged between 1.22 and $1.83 \mathrm{~m}$ from the $3-\mathrm{D}$ model centerline.

Fronting slopes of reefs are much steeper than the slopes that were present in the 2-D tank, and it was desired that the waves encounter the reef slope without influence of the gentler slopes. Therefore, the reef was constructed so that the crest of the offshore slope was positioned over the toe of the initial flume slope. 


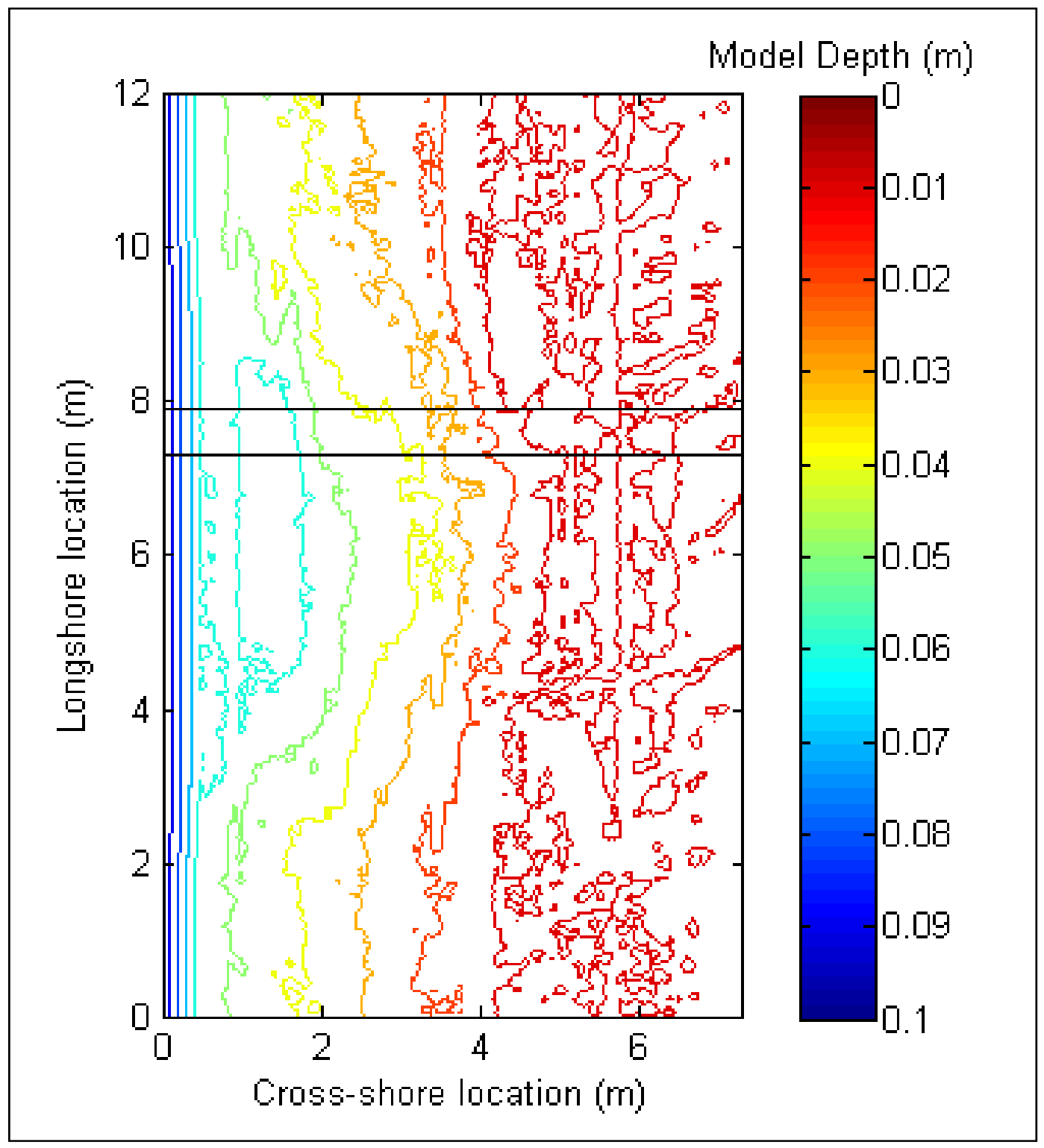

Figure 47. Area of bathymetry installed in 2-D flume.

Experiments were conducted with two reef-front slopes, 1:2.5 and 1:5. The reef was designed to allow installation and removal of the 1:5 slope without removing the 1:2.5 slope. The toe of the reef was located $9.26 \mathrm{~m}$ and 10.15 $\mathrm{m}$ from the wave board for the 1:5 and 1:2.5 slopes, respectively. The reef crest was $11.0 \mathrm{~m}$ from the wave board and the reef shelf extended $7.3 \mathrm{~m}$ shoreward, where a 1:10 foreshore slope was constructed. Figure 48 shows side and plan views of the reef and wave gauges in the 2-D wave tank.

A wall was installed to divide the flume horizontally into 0.61 and $0.30 \mathrm{~m}$ sections, and extended from the toe of the reef slope to the top of the 1:10 foreshore slope. The acrylic reef surface was placed in the $0.61 \mathrm{~m}$ wide section and rubberized absorber was placed in the 0.30-m-wide section. 


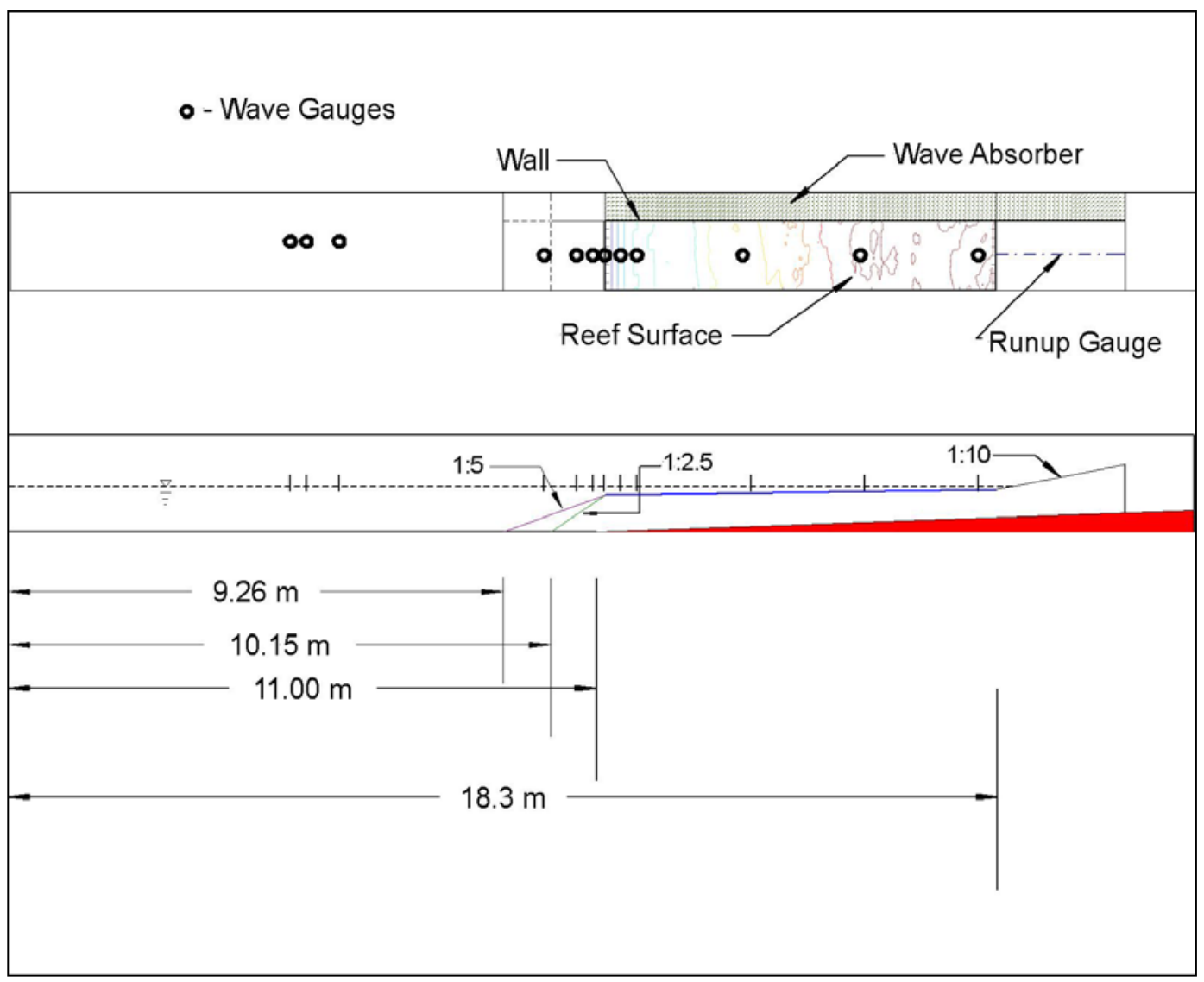

Figure 48. Sketch of 2-D wave flume and reef.

Installation of the 2-D reef surface differed from the 3 -D, which was attached to legs that were attached to the underlying surface. In the 2-D experiments, a flat marine plywood floor was constructed above the concrete slope. The floor sides were sealed to the glass and dividing walls to prevent motion from wave action or buoyancy. The acrylic sections were mounted on 0.005- by 0.01-m wood cross-sectional pieces attached every $0.61 \mathrm{~m}$ along the plywood floor. Each cross-sectional piece was adjusted to an elevation that would maintain a smooth surface between acrylic sections. The acrylic sections were sealed to the cross-section pieces, the dividing wall, glass wall and each other with silicone.

The same procedure used in the 3-D tests was employed to compare reef roughness. Initial tests were performed with the bathymetry unpainted to provide a smooth surface. Following the smooth-surface experiments, the panels were roughened by applying paint mixed with the roughening agent used in the 3 -D experiment $\left(d_{50} \approx 0.33 \mathrm{~mm}\right)$. 


\subsection{Wave and water level conditions}

Waves generated in the 2-D flume were similar to the conditions generated in the 3-D basin. The experiments were performed at a 1:50 scale with target periods ranging from 7 to $20 \mathrm{sec}$ for the maximum wave height the wave generator could produce at each period. Waves were generated for three water levels, representing low- , mid-, and high-tide conditions. The water line of the mid-tide water level coincided with the base of the 1:10 foreshore slope and the shoreward end of the reef.

Two data sets were collected in the flume. The first data set was a collaboration of ERDC and the University of Florida (UF) to measure unidirectional waves and currents over steep slopes characteristic of reef environments. A description of the tests and wave transformation figures are given in Appendix D. Following the UF experiments, the design waves shown in Table 5 were generated for both smooth and rough reef bathymetry with 1:2.5 and 1:5 reef slopes. The table lists values in prototype units with model units in parenthesis.

Table 5. SWIMS 2-D reef design wave conditions in prototype, $m$ (Model, $m$ ).

\begin{tabular}{|l|l|l|l|}
\hline \multirow{2}{*}{$\begin{array}{l}T_{p} \\
\text { sec }\end{array}$} & $h=20.8 \mathrm{~m}(0.415 \mathrm{~m})$ & $h=21.9 \mathrm{~m}(0.439 \mathrm{~m})$ & $h=24.5 \mathrm{~m}(0.490 \mathrm{~m})$ \\
\cline { 2 - 4 } & $H_{i}(\mathrm{~m})$ & $\begin{array}{l}H_{i} \\
\mathrm{~m}(\mathrm{~m})\end{array}$ & $\begin{array}{l}H_{i} \\
\mathrm{~m}(\mathrm{~m})\end{array}$ \\
\hline $7(1.0)$ & $4.2(0.084)$ & $3.9(0.079)$ & $4.1(0.081)$ \\
\hline $7(1.0)$ & $5.2(0.103)$ & $5.2(0.104)$ & $5.2(0.104)$ \\
\hline $10(1.4)$ & $4.5(0.090)$ & $3.8(0.076)$ & $4.6(0.091)$ \\
\hline $10(1.4)$ & $5.7(0.115)$ & $6.0(0.121)$ & $6.1(0.122)$ \\
\hline $10(1.4)$ & $6.9(0.137)$ & $6.8(0.137)$ & $6.9(0.138)$ \\
\hline $13(1.8)$ & $5.5(0.110)$ & $4.4(0.088)$ & $4.6(0.092)$ \\
\hline $13(1.8)$ & $6.1(0.122)$ & $6.0(0.120)$ & $6.1(0.122)$ \\
\hline $13(1.8)$ & $7.5(0.149)$ & $7.5(0.149)$ & $7.7(0.154)$ \\
\hline $16(2.3)$ & $5.5(0.109)$ & $4.6(0.091)$ & $4.6(0.092)$ \\
\hline $16(2.3)$ & $6.1(0.122)$ & $6.0(0.120)$ & $6.1(0.122)$ \\
\hline $16(2.3)$ & $7.6(0.152)$ & $7.7(0.155)$ & $7.7(0.155)$ \\
\hline $20(2.3)$ & $5.2(0.104)$ & $4.2(0.084)$ & $4.4(0.088)$ \\
\hline $20(2.8)$ & $6.2(0.124)$ & $6.0(0.120)$ & $6.1(0.122)$ \\
\hline $20(2.8)$ & $8.0(0.160)$ & $6.9(0.138)$ & $8.0(0.160)$ \\
\hline $20(2.8)$ & - & $8.0(0.160)$ & - \\
\hline
\end{tabular}

Values in parenthesis refer to values in model seconds and meters 
Water surface elevations were measured with twelve capacitance-type gauges sampled at $20 \mathrm{~Hz}$. Gauges 1 through 3 were located seaward of the reef and positioned to measure wave reflection by the method of Goda and Suzuki (1976). Positions of Gauges 4 through 6 varied depending on the reef slope installed to capture wave heights in the breaking region. Gauge 4 was positioned immediately seaward of the reef toe, Gauge 5 at midslope of the reef slope, and Gauge 6 midway between Gauge 5 and the reef crest. Gauges 7 through 12 were placed on the reef, with Gauge 7 located at the reef crest and the remaining gauges spaced at increasingly greater distances. Gauge locations for both slopes are listed in Table 6.

Table 6. SWIMS 2-D reef wave gauge locations.

\begin{tabular}{|l|l|l|}
\hline \multirow{2}{*}{ Gauge } & \multicolumn{2}{|c|}{ Distance from Wavemaker $(\mathrm{m})$} \\
\cline { 2 - 3 } & $1: 2.5$ slope & $1: 5$ slope \\
\hline 1 & 4.82 & 4.82 \\
\hline 2 & 5.13 & 5.13 \\
\hline 3 & 5.74 & 5.74 \\
\hline 4 & 9.72 & 8.77 \\
\hline 5 & 10.64 & 10.19 \\
\hline 6 & 10.84 & 10.63 \\
\hline 7 & 11.02 & 11.02 \\
\hline 8 & 11.35 & 11.35 \\
\hline 9 & 11.66 & 11.66 \\
\hline 10 & 12.83 & 12.83 \\
\hline 11 & 14.68 & 14.68 \\
\hline 12 & 17.73 & 17.73 \\
\hline
\end{tabular}

A 2-m long capacitance wire runup gauge was installed on the centerline of the foreshore slope. The gauge was positioned with the bottom at the base of the slope with the length extending $2 \mathrm{~m}$ up the slope. Because the gauge was placed on the slope, it was required to convert the runup gauge values from distance along the 1:10 slope to vertical elevations.

\subsection{Test procedures}

Wave gauges were calibrated daily before performing experiments. For the mid- and low-water levels, some gauges that were positioned on the reef had insufficient depth to perform a calibration. Under these circumstances, 
the last calibration obtained at the high-water level was applied for these gauges. At a minimum, all gauges were calibrated once per week, which required raising the water level to high water, performing wave gauge calibration, and then lowering the water to the desired level to perform experiments. On days that calibration was skipped for gauges on the reef, a previously tested wave condition was generated and the results were compared to the previous run. Waves shoaled on the reef slope, and wave heights were high in comparison to the local water depth. Consequently, the calibration ranges necessary to capture wave heights on and immediately shoreward of the reef slope were greater than the local water depths. Therefore, Gauges 4 through 8 were calibrated in the flat portion of the wave tank and moved to position prior to tests.

Prior to each run of a test case, the water level of the basin was checked and adjusted as needed. Water level could be adjusted to within $0.03 \mathrm{~cm}$ of the target value. The desired wave condition file was selected on the control computer and waves were started. Each time series was 20-min. Data collection was synchronized with the start of the wave generator and ended at the conclusion of the wave time series.

The experiments also were documented through photos and video taken from the side of the wave tank near breaking and along the reef surface.

\subsection{Results}

Excluding the UF experiment, data were collected for a total of 172 runs, which include 43 runs on each reef surface and reef slope configuration. This section includes results from the wave and runup gauges, including presentation of wave transformation, analysis of spectral transformation, wave setup, runup and reflection.

\subsubsection{Wave height transformation}

Figures of $H_{m o}$ and $\eta$ measured in the flume are plotted with distance from the wave generator in Appendix E. Data collected for both the smooth and rough surfaces are shown for each wave and water level condition. Initial water level and reef bathymetry also are presented as a reference, but both are scaled down by a factor of 10 for the plot.

The initial water level for the low-water experiments was lower than the elevations of Wave Gauge 12 and the runup gauge; i.e., the gauges were out 
of the water at the onset of the test. Mean water levels were adjusted in the same manner as the 3-D experiment: the elevation difference between the particular gauge and the initial water elevation were added to the recorded measurement.

The highest waves generated for 1.0- and 2.8-sec periods at each water level for both reef surfaces are provided in Figures 49 to 54 for the 1:2.5 slope and Figures 55 to 60 for the 1:5 slope. The 1.0-sec period waves with the 1: 2.5 slope (Figures 49, 51, and 53) remained relatively constant and showed slight shoaling leading to breaking. A slight decrease in wave height offshore of the breakpoint also is observed. The 2.8-sec wave heights (Figures 50, 52, and 54) decreased as they approached the slope, followed by sizeable shoaling to the breakpoint. At breaking, both the 1.0- and 2.8-sec waves dissipated rapidly, followed by a moderate decrease in height across the reef.

Similar wave transformation trends occurred for wave conditions with the 1:5 reef slope. Heights of the 1.0-sec period waves are relatively uniform between the wavemaker and the breakpoint for all water levels (Figures 55, 57, and 59). Waves broke near the top of the reef slope, and heights decreased sharply and propagated over the reef with a moderate decrease in height. Shoaling was more apparent at the low water level and the plots indicate that the shorter period waves were less effected by the reef slope, and didn't shoal appreciably until shallower water was encountered at the top of the slope. Conversely, the 2.8 -sec waves (Figures 56,58 , and 60) shoaled significantly over the reef slope, broke with a sharp decrease in height, and propagated over the reef with a more moderate decrease in height. Figures 56 and 59 indicate a subtle height decrease immediately offshore of the reef slope, which was observed in many of the 3-D experiments. Also, heights varied at Gauge 3 during the smooth reef tests with the 1:5 slope and the measurement is suspect. However, measurements at the gauge look reasonable for experiments performed on the 1:2.5 slope.

Wave heights were similar over the reef bathymetry between the smooth and rough surfaces. Differences between smooth and rough wave heights are generally small and within measurement error, and no consistent trend was found to show that the roughness applied to the reef had a significant influence on wave height. As was stated in the 3-D section of this report, this does not imply that roughness does not affect wave height, but the roughness selected for the present experiment did not have significant influence on heights. 


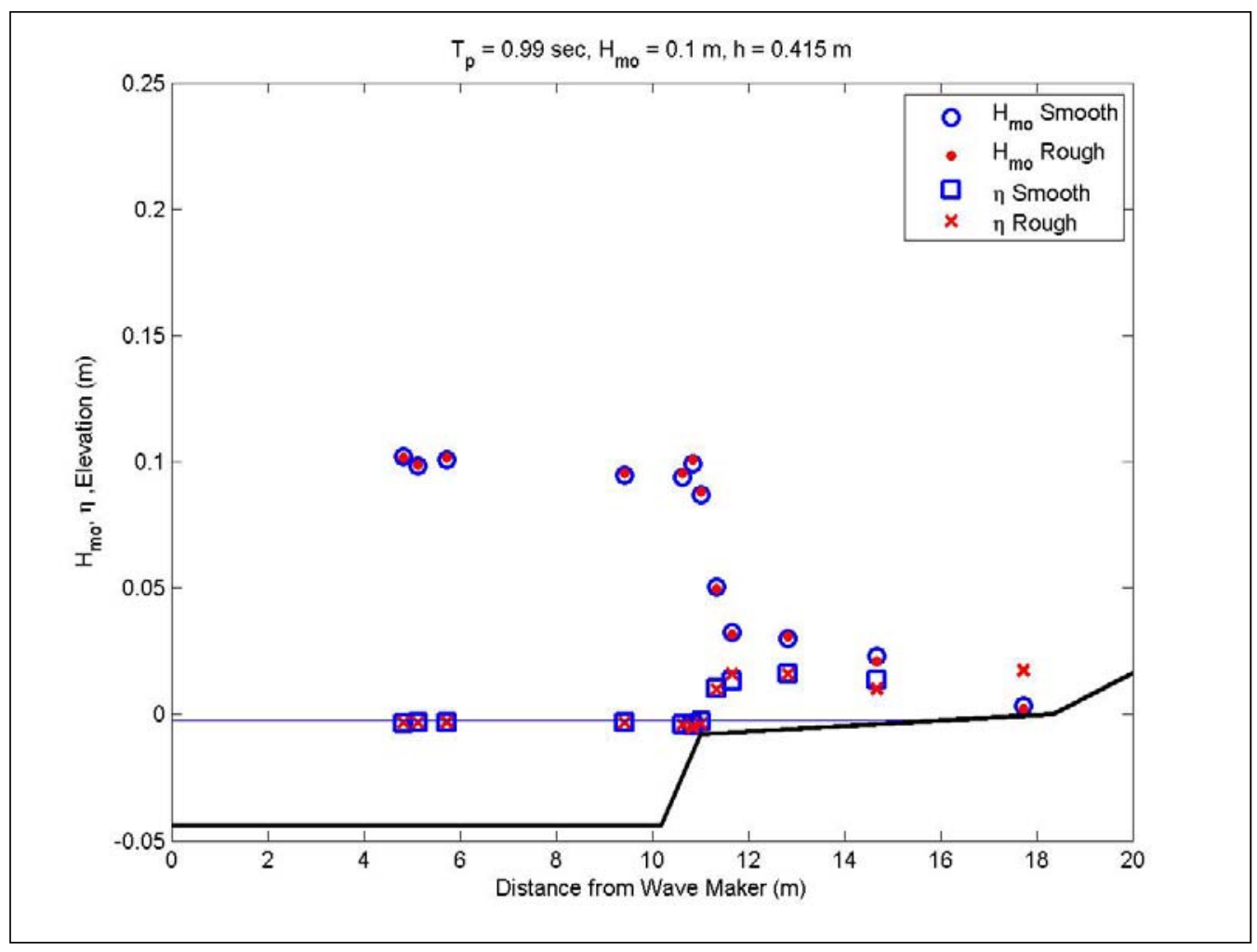

Figure 49. Wave transformation for 1-sec, $0.1 \mathrm{~m}$ waves at low water with a 1:25 reef slope.

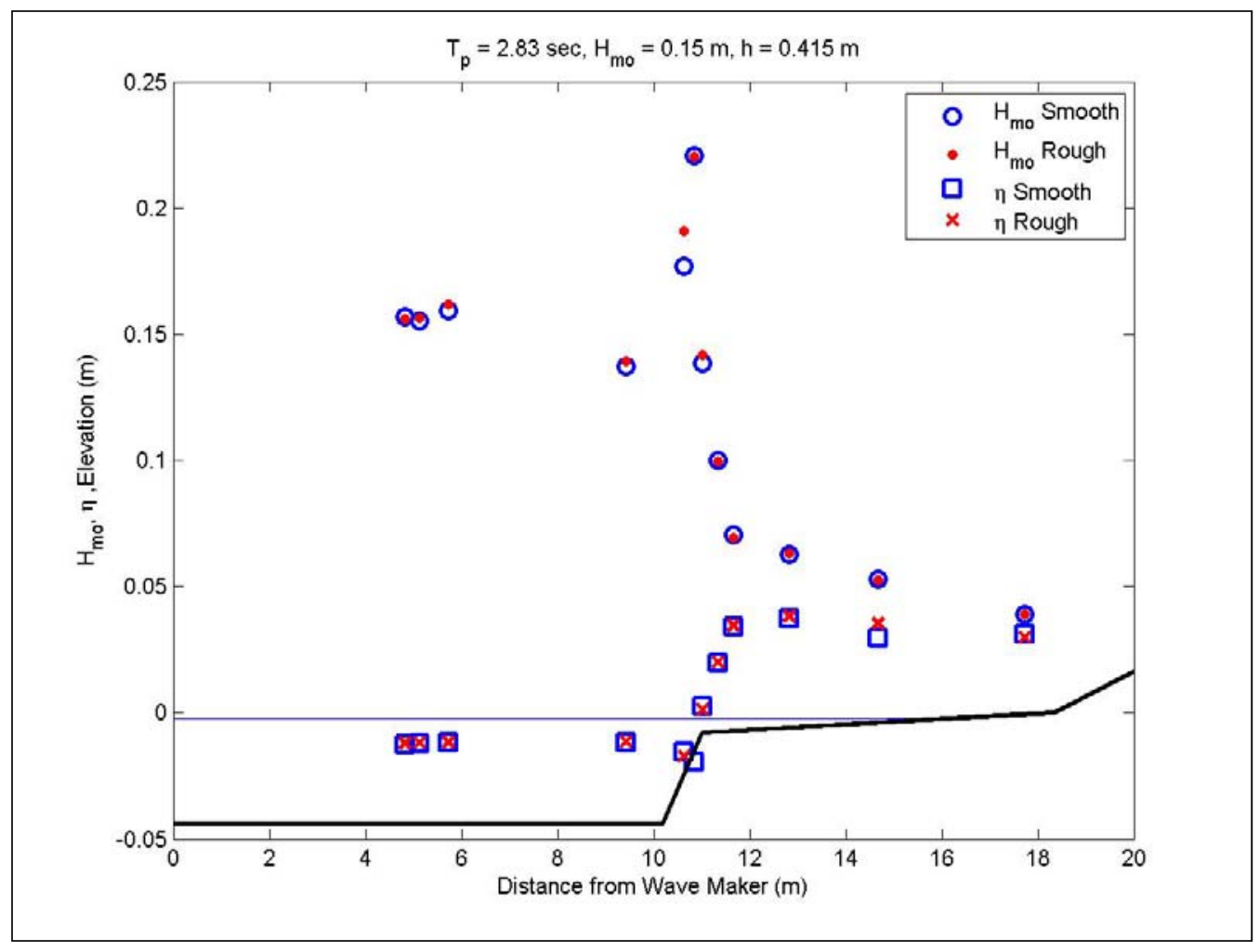

Figure 50. Wave transformation for $2.8-\mathrm{sec}, 0.15 \mathrm{~m}$ waves at low water with a 1:25 reef slope. 


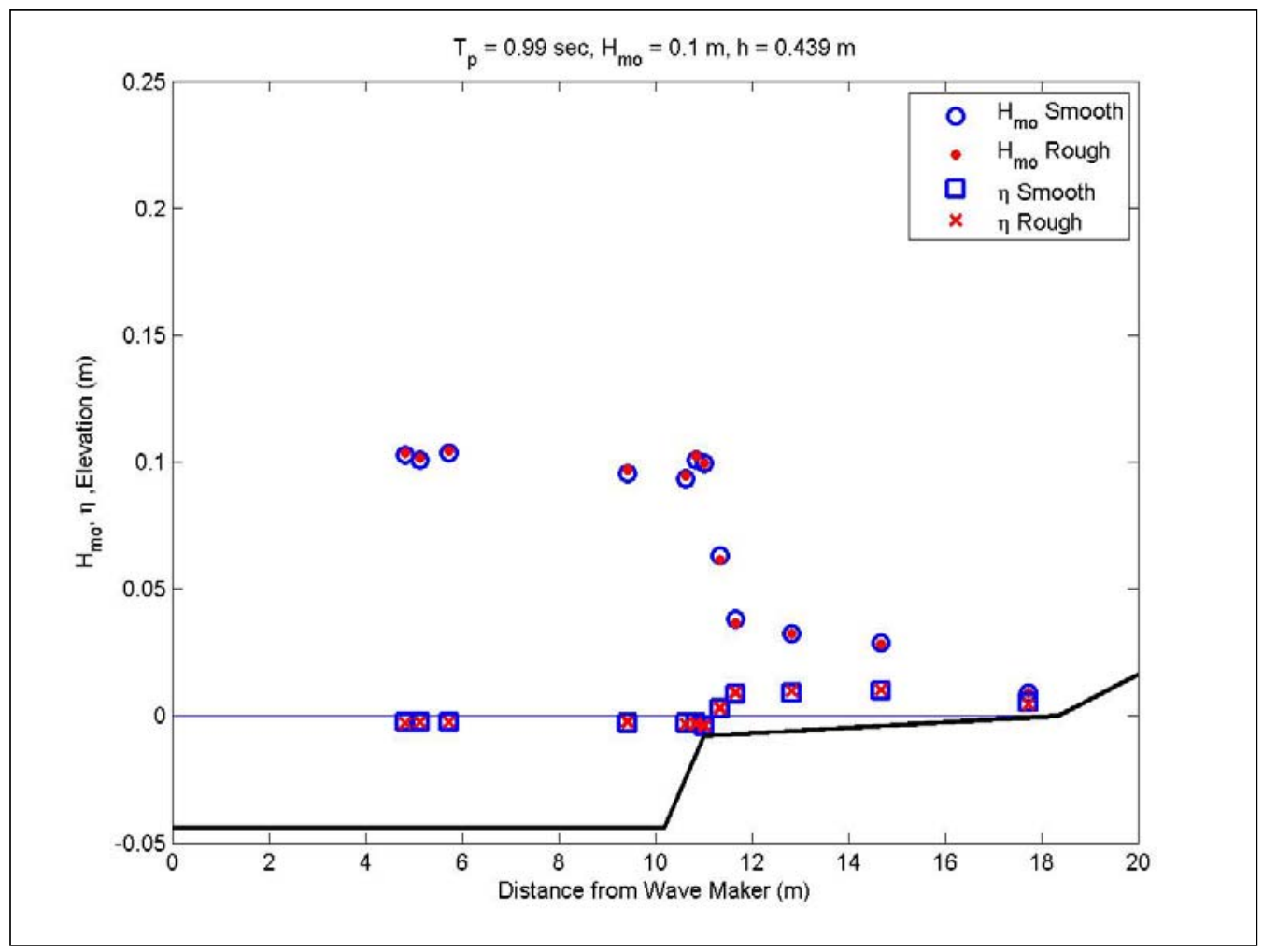

Figure 51. Wave transformation for 1-sec, $0.1 \mathrm{~m}$ waves at mid water with a 1:25 reef slope.

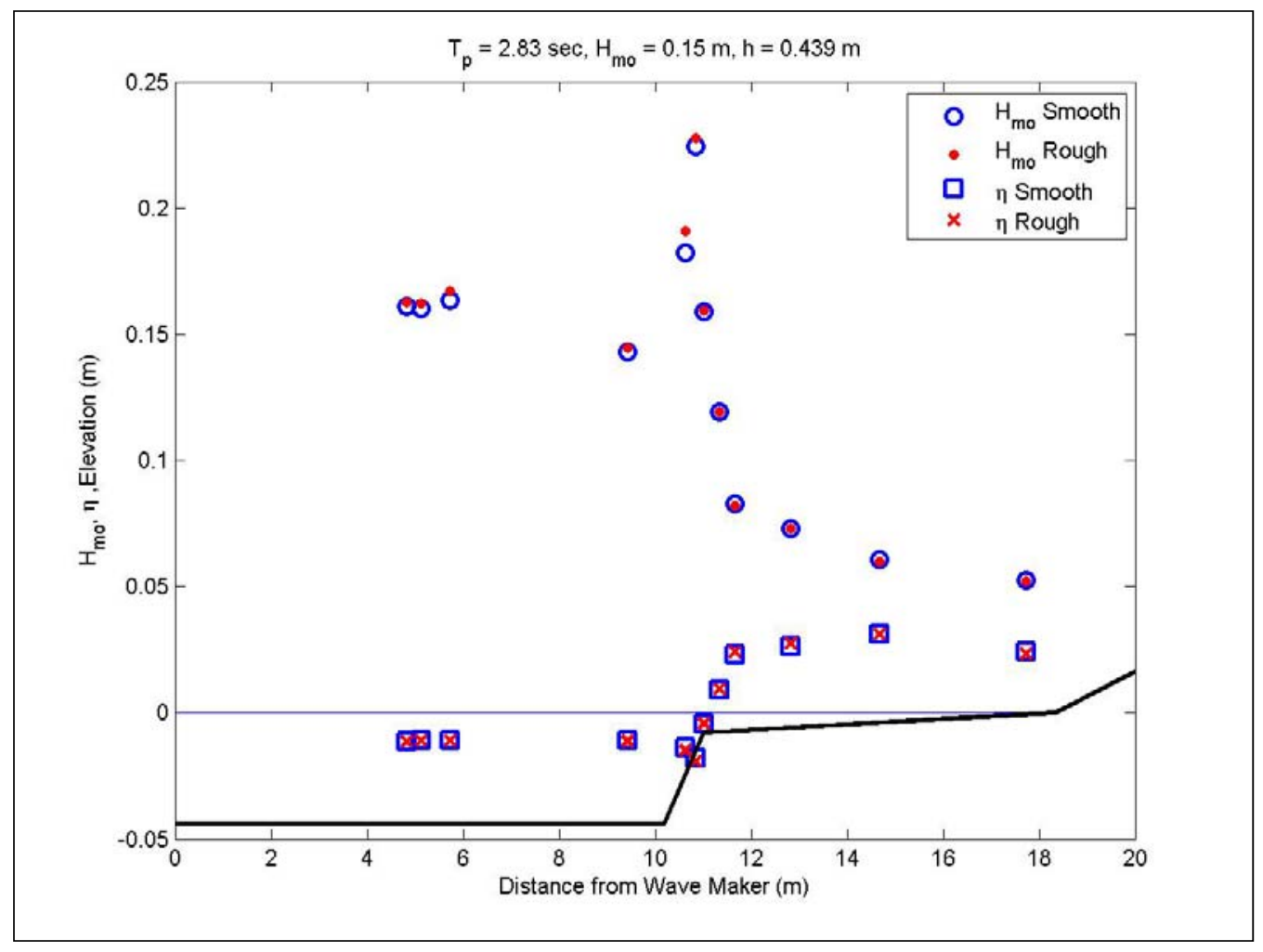

Figure 52 . Wave transformation for 2.8 -sec, $0.15 \mathrm{~m}$ waves at mid water with a 1:25 reef slope. 


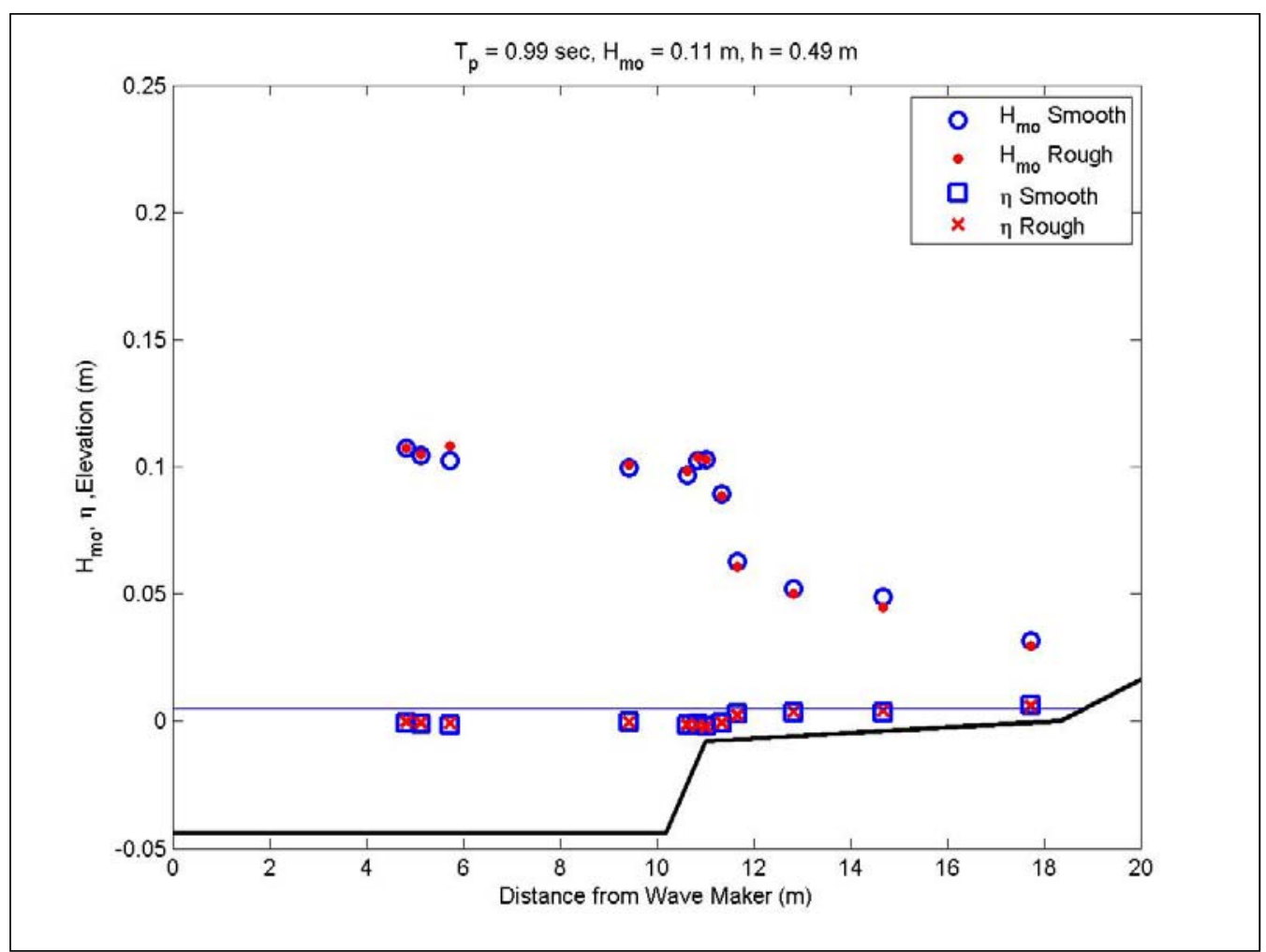

Figure 53. Wave transformation for 1-sec, $0.11 \mathrm{~m}$ waves at high water with a 1:25 reef slope.

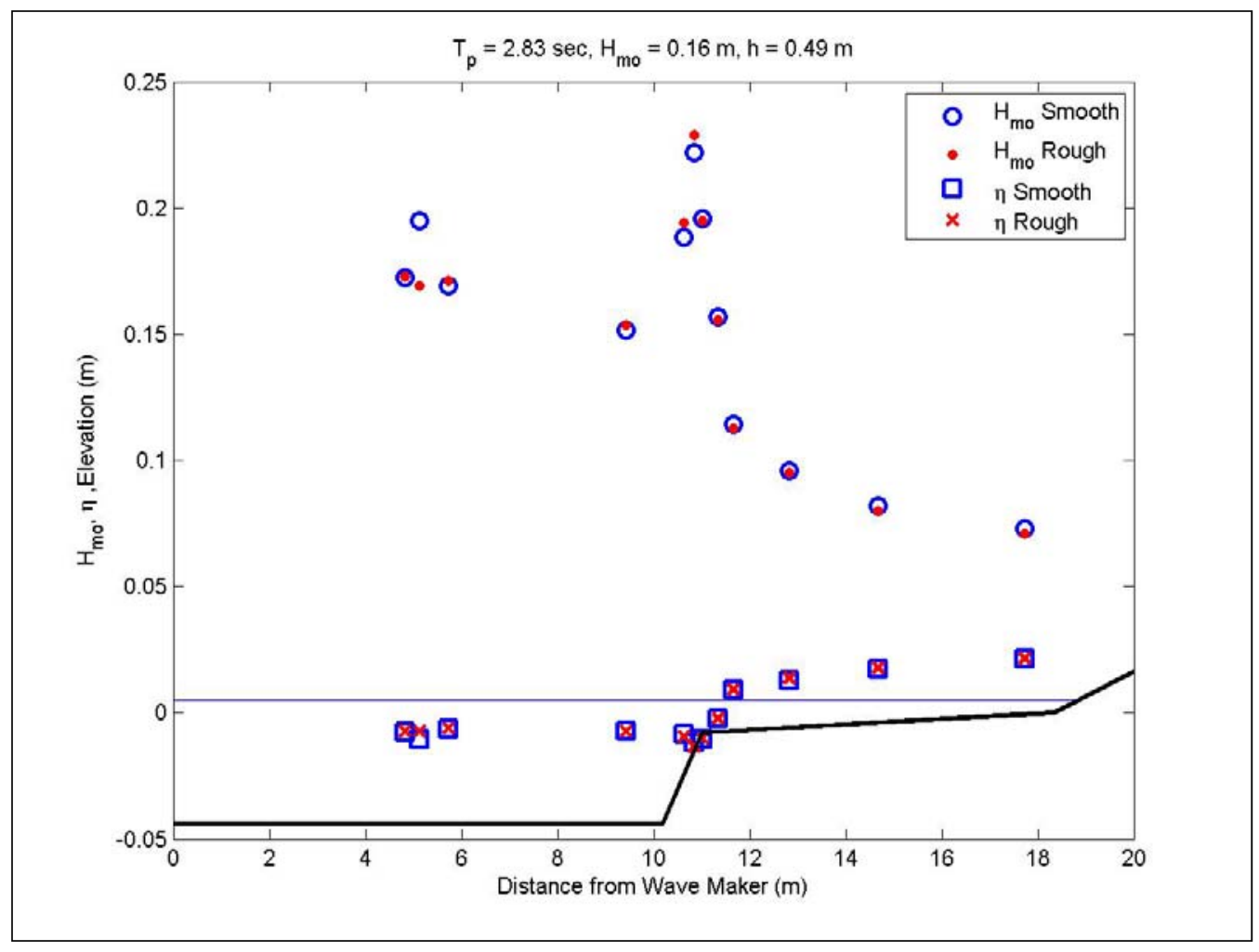

Figure 54. Wave transformation for 2.8-sec, $0.16 \mathrm{~m}$ waves at high water with a 1:25 reef slope. 


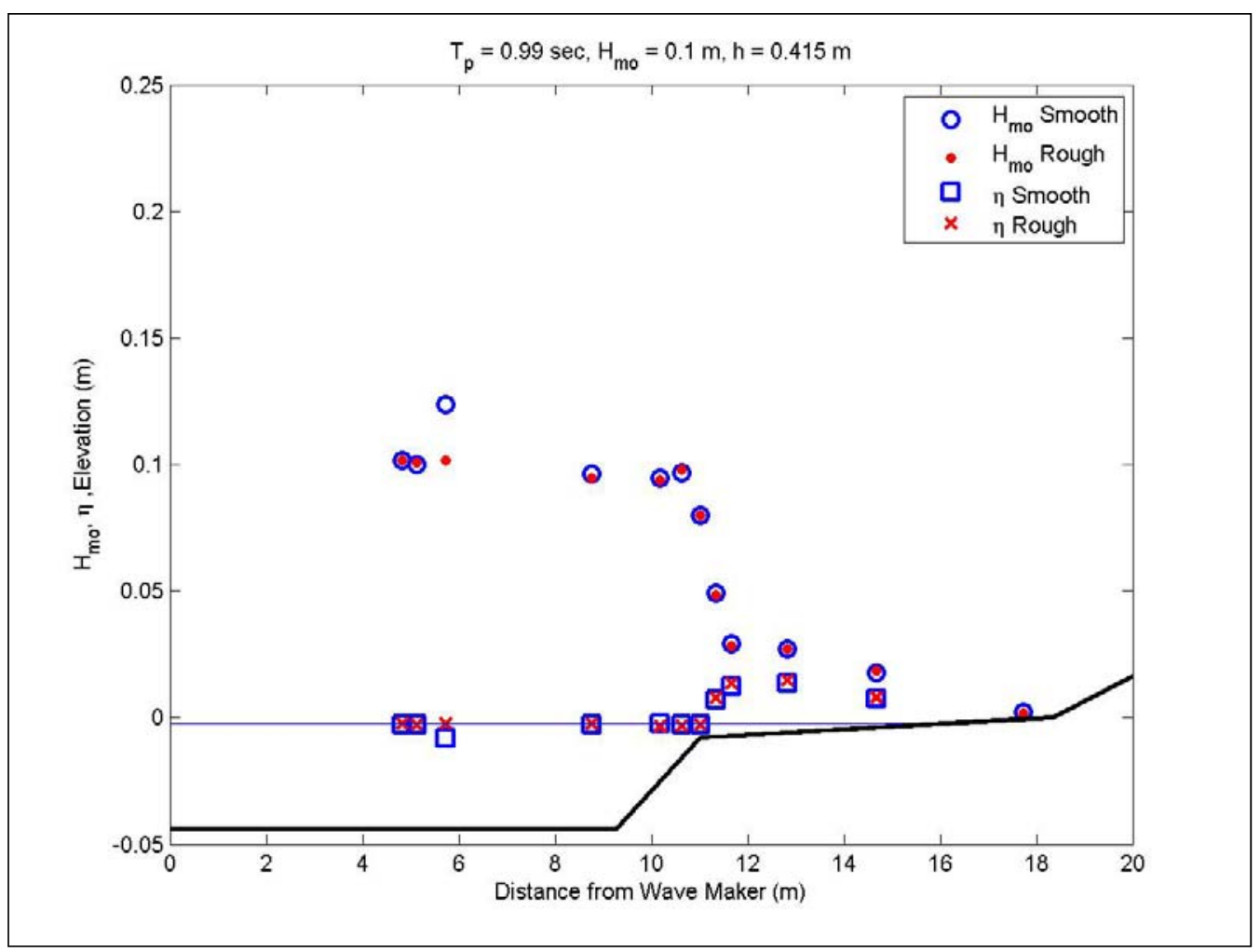

Figure 55. Wave transformation for $1-\mathrm{sec}, 0.1 \mathrm{~m}$ waves at low water with a 1:5 reef slope.

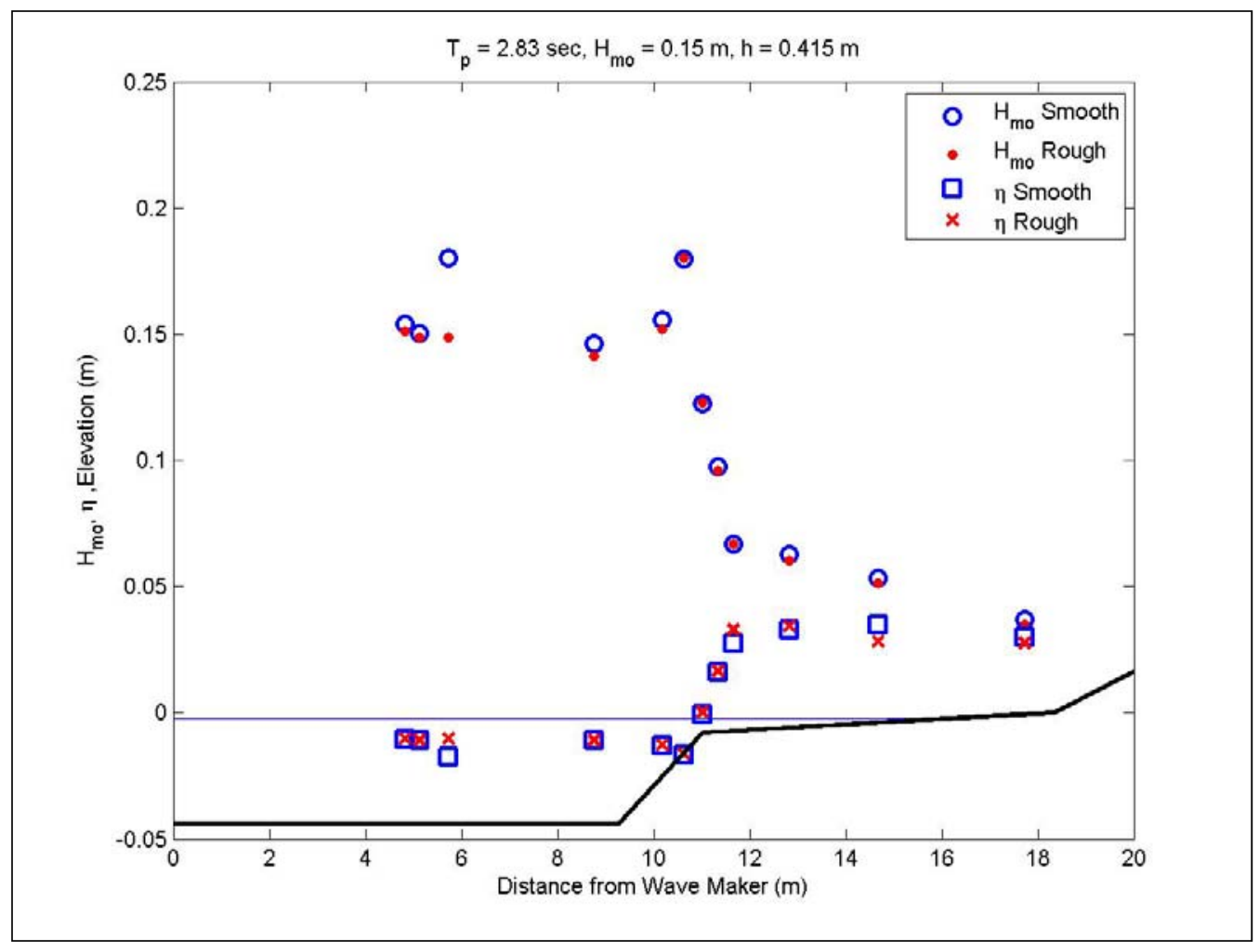

Figure 56. Wave transformation for $2.8-\mathrm{sec}, 0.15 \mathrm{~m}$ waves at low water with a 1:5 reef slope. 


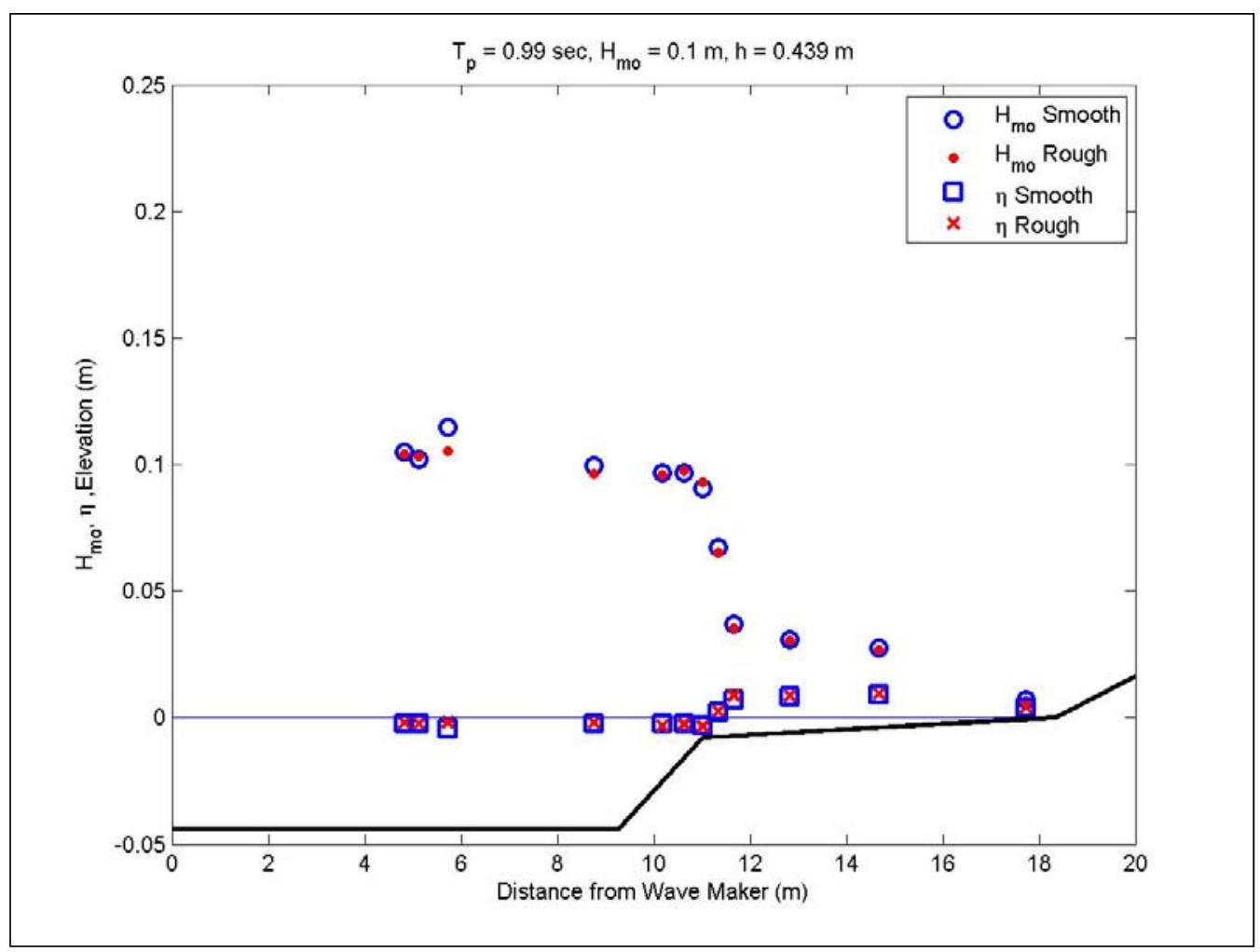

Figure 57 . Wave transformation for $1-\mathrm{sec}, 0.1 \mathrm{~m}$ waves at mid water with a 1:5 reef slope.

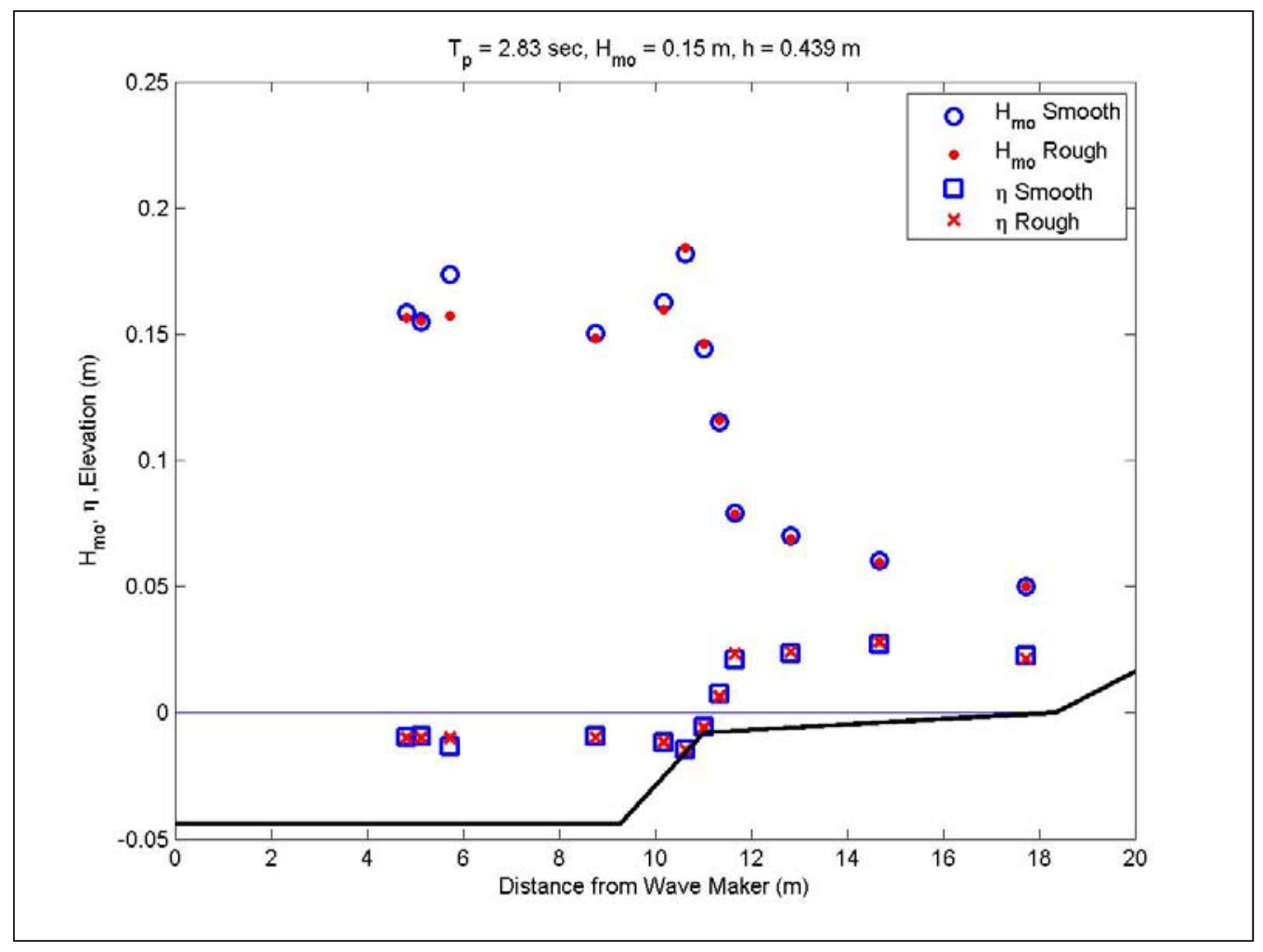

Figure 58. Wave transformation for 2.8 -sec, $0.15 \mathrm{~m}$ waves at mid water with a $1: 5$ reef slope. 


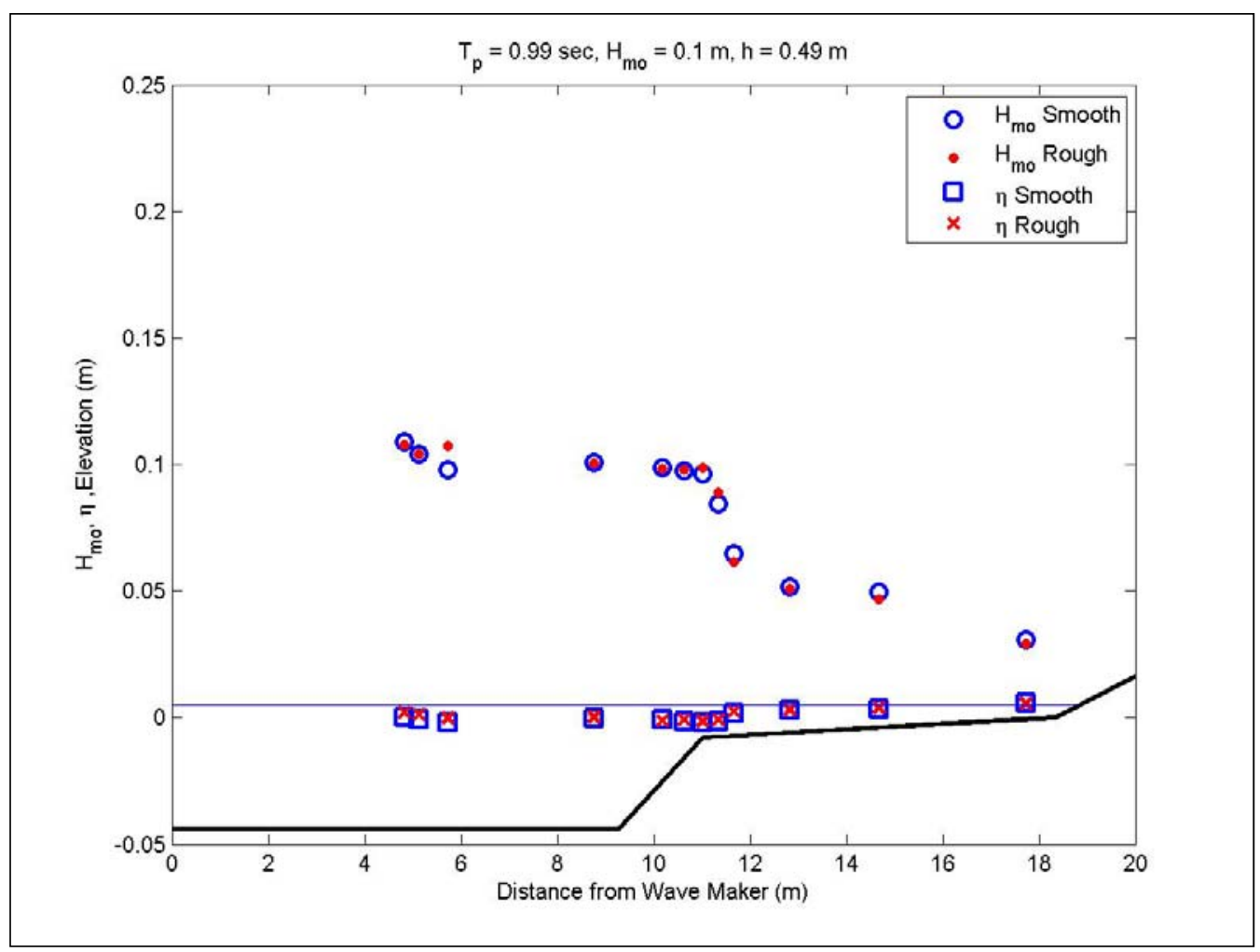

Figure 59. Wave transformation for 1-sec, $0.1 \mathrm{~m}$ waves at high water with a 1:5 reef slope.

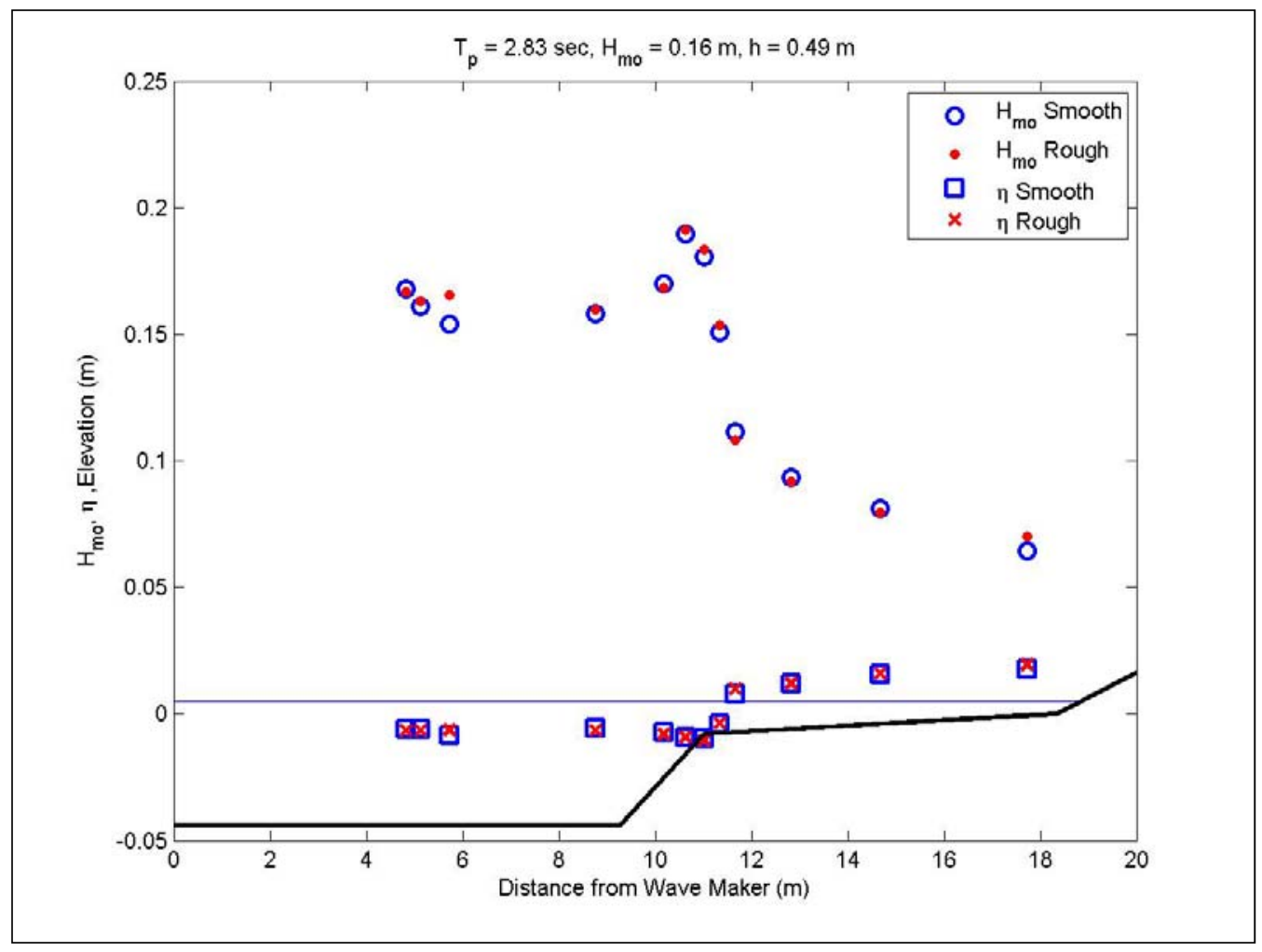

Figure 60 . Wave transformation for $2.8-\mathrm{sec}, 0.16 \mathrm{~m}$ waves at high water with a 1:5 reef slope. 
Comparisons of zero-moment wave heights for the two reef slopes are shown in Figures 61 through 66 for the previously discussed wave conditions with the roughened reef surface. The bottom elevations, reduced by a factor of 10, are plotted for reference. It should be noted that Gauges 4 through 6 for the 1:2.5 slope tests are positioned shoreward of the 1:5 slope locations (Table 6). As a result, the location of Gauge 5 for the 1:2.5 slope tests $(10.64 \mathrm{~m})$ is approximately the same location as Gauge 6 for the 1:5 slope tests $(10.63 \mathrm{~m})$. This location was used to compare waves between slopes near breaking. For low- and mid-water tests, 1.0-sec waves show consistent wave heights offshore of the reef slope with slightly higher waves near breaking for the 1:5 slope (Figures 61 and 63). Wave heights are similar shoreward of breaking but are generally higher with the 1:2.5 slope. Wave heights are very similar throughout the flume between the two slopes for 1.0-sec waves at high water with exception at breaking, where heights with the 1:2.5 slope are slightly higher (Figure 65).

Differences between heights are more apparent with the 2.8-sec waves, particularly for the low- and mid-water tests (Figures 62 and 64). Waves are slightly higher near breaking in the outer surf zone for the 1:2.5 slope. Wave heights remain higher in the inner surf zone for the 1:2.5 slope, but appear to converge with heights on the 1:5 slope. A similar trend is shown at the high water level (Figure 66), but differences between heights are less. Also, the first three gauges measure higher waves with the 1:2.5 slope, which may be due to reflection from the steeper reef slope.

The breaking wave height to depth ratio for the 2.8-sec waves is large; ratios range from 0.85 to 1.5, which is much higher than that expected on sandy coastlines. Breaking wave height is influenced by several factors, particularly wave period and bottom slope. Larger breaking wave height-to-depth ratios generally occur for longer periods and steeper slopes. Therefore, a higher breaker height would be expected with the 2.8 -sec wave than the 1.0-sec wave. The present experiments simulated shallow fringing reefs that transition from deeper ocean depths over a relatively short distance, which requires a steep slope and would increase wave height at breaking. Although the reef slope was common to the 1.0-sec and 2.8-sec waves, the depth at which the shorter period waves are affected by the bottom is much shallower. Therefore, the steep bottom slope had more of an effect on the 2.8-sec waves. 


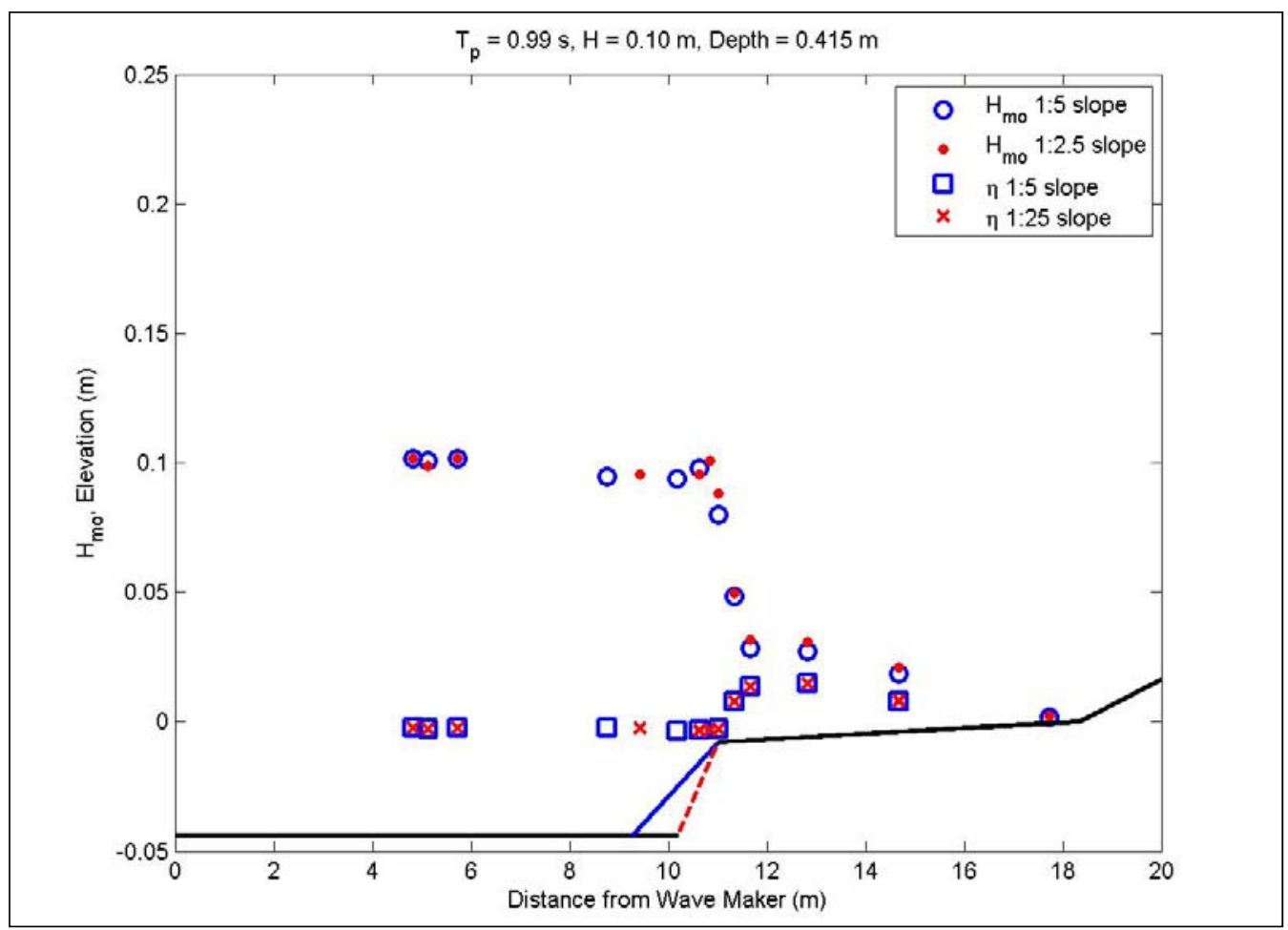

Figure 61. Wave transformation comparison between reef slopes for 1-sec, $0.1 \mathrm{~m}$ waves at low water with rough reef surface.

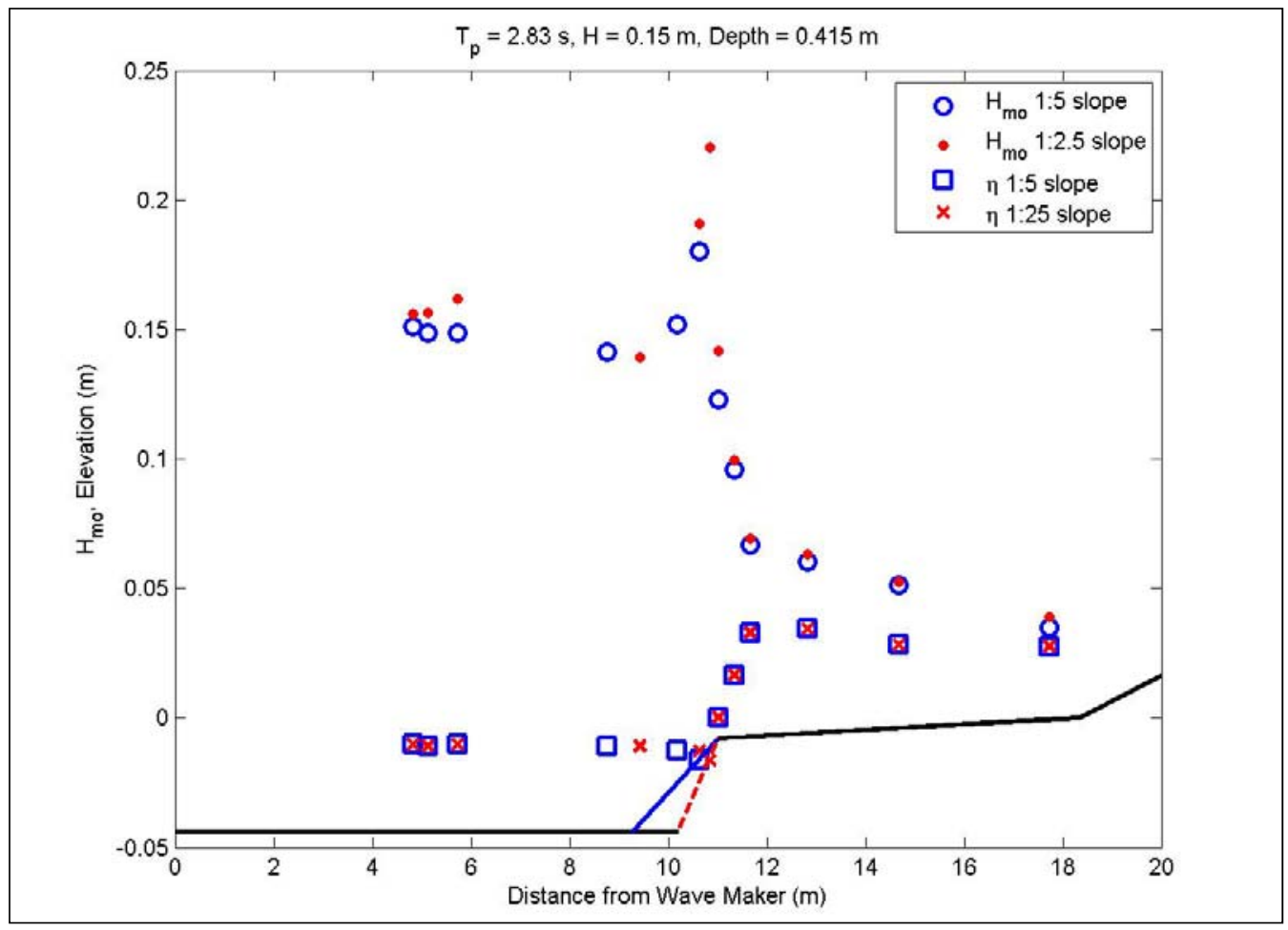

Figure 62. Wave transformation comparison between reef slopes for 2.8 -sec, $0.15 \mathrm{~m}$ waves at low water with rough reef surface. 


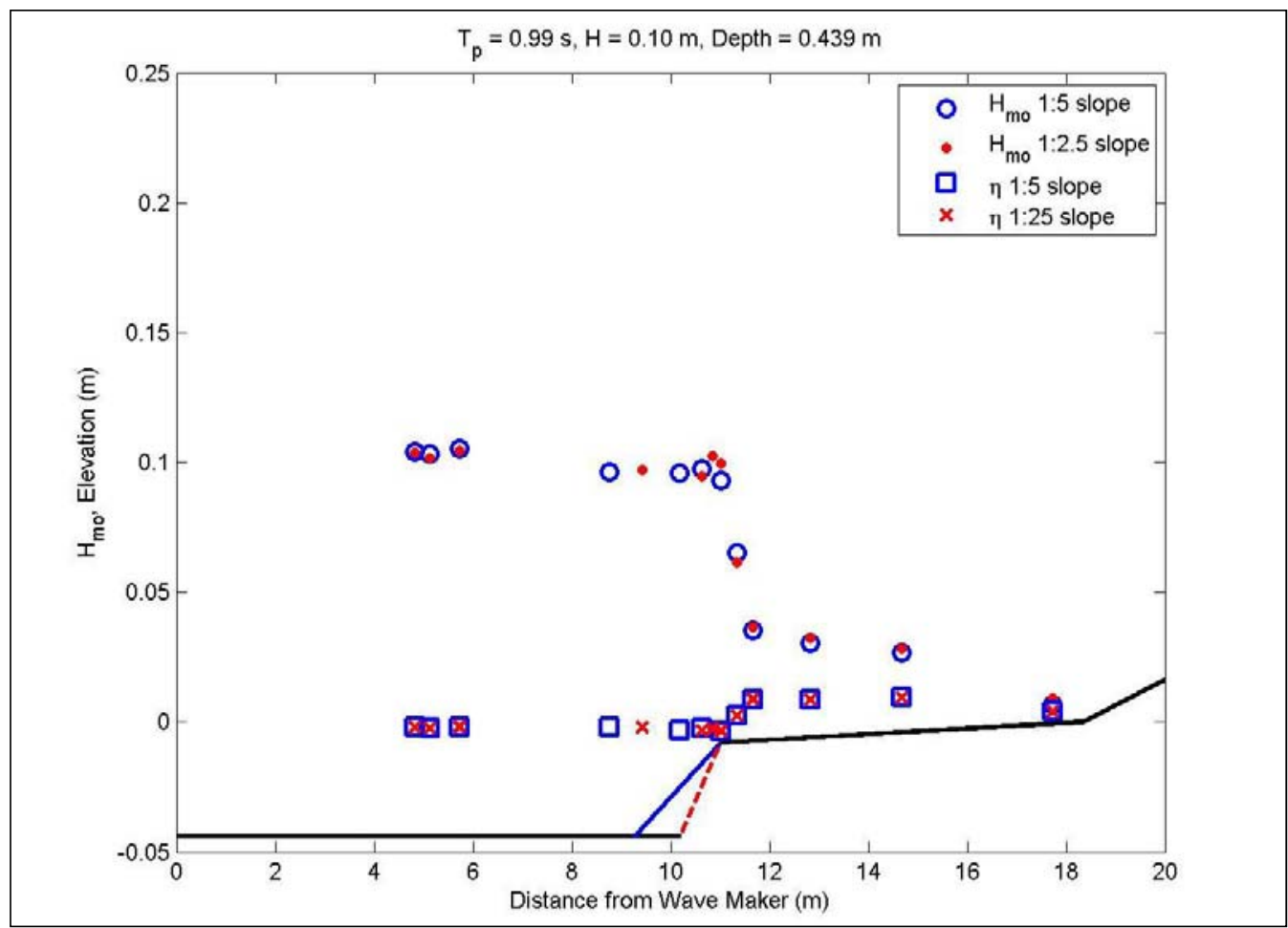

Figure 63. Wave transformation comparison between reef slopes for 1-sec, $0.1 \mathrm{~m}$ waves at mid water with rough reef surface.

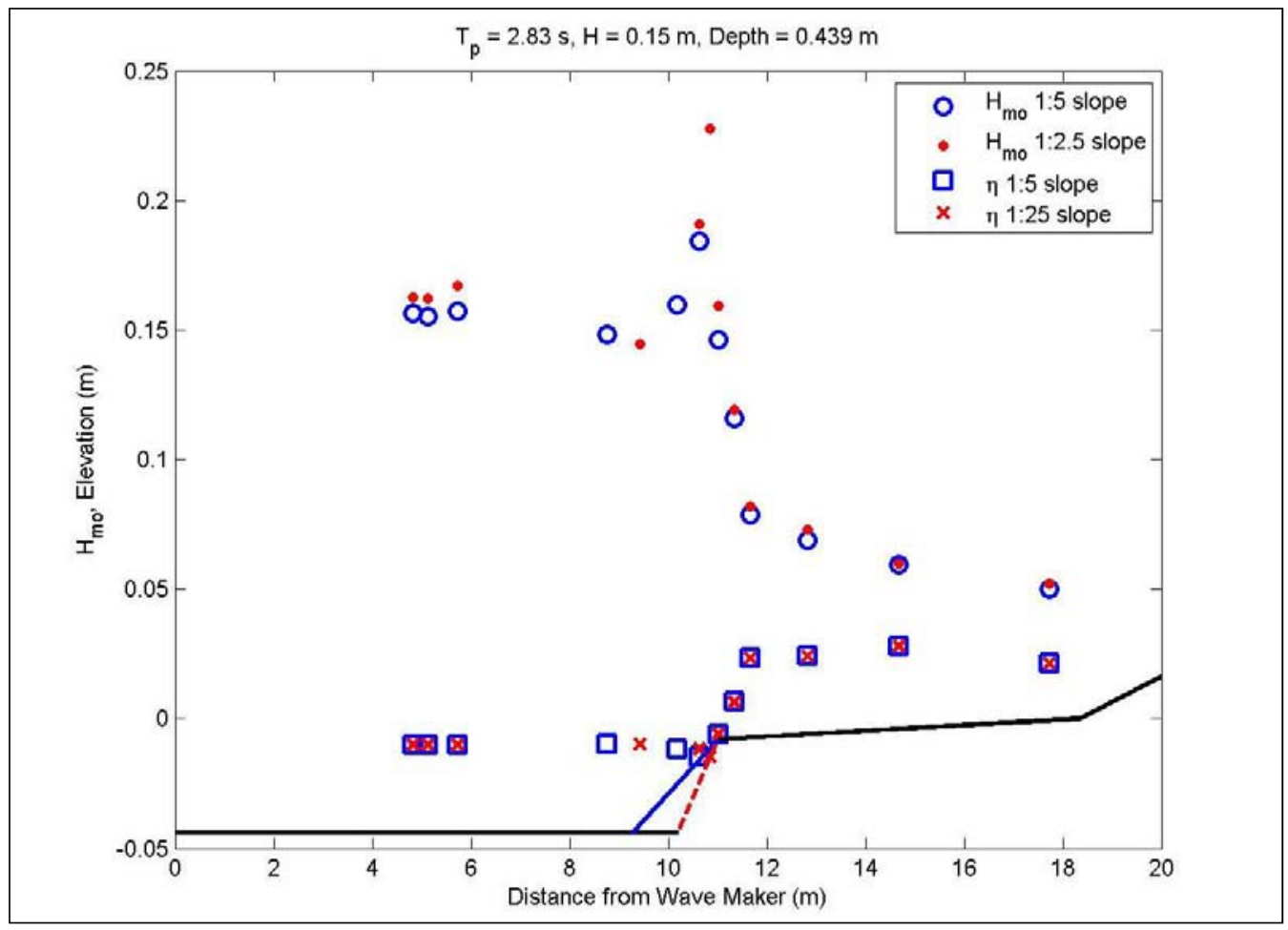

Figure 64. Wave transformation comparison between reef slopes for 2.8 -sec, $0.15 \mathrm{~m}$ waves at mid water with rough reef surface. 


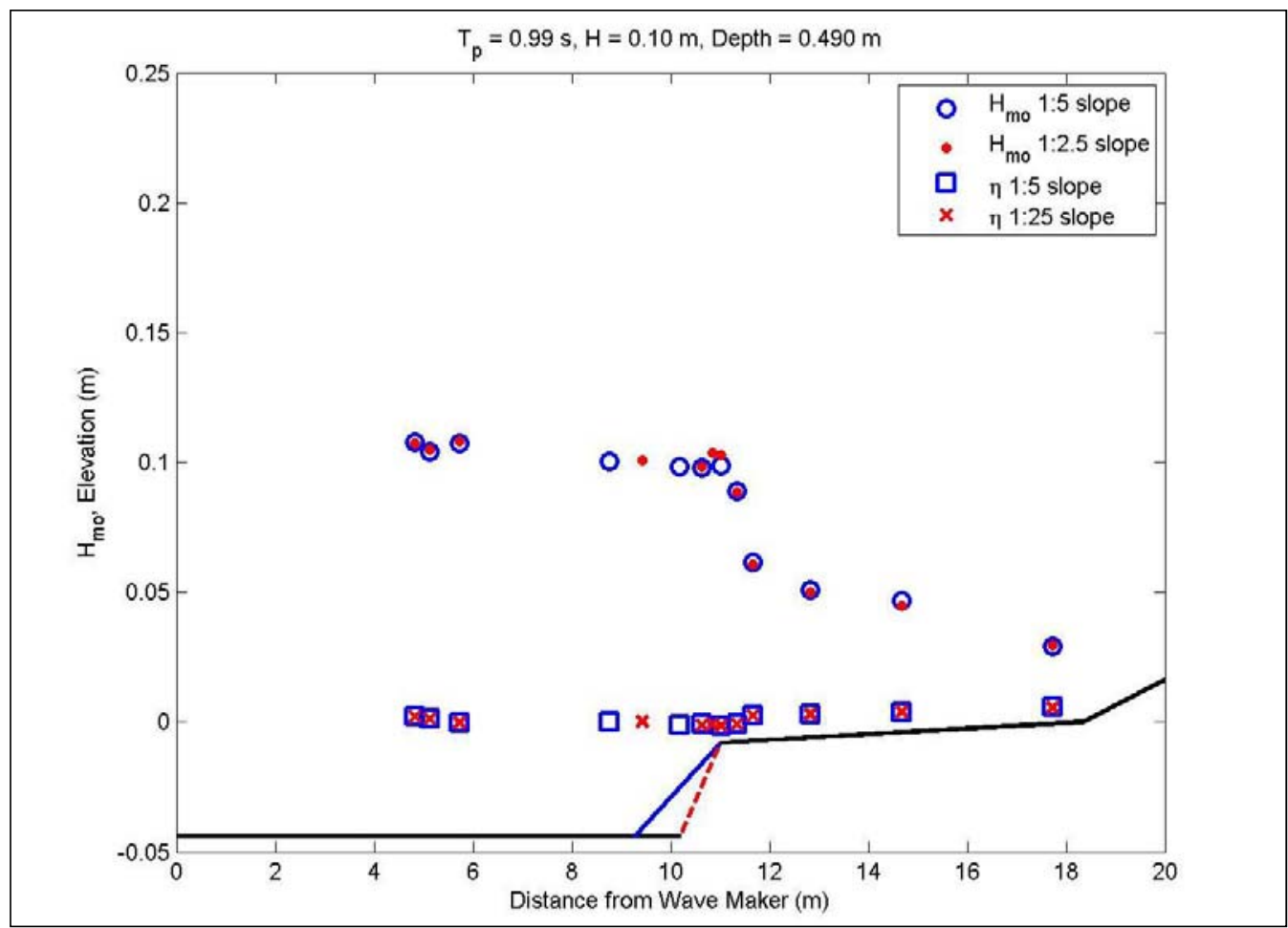

Figure 65. Wave transformation comparison between reef slopes for 1-sec, $0.1 \mathrm{~m}$ waves at high water with rough reef surface.

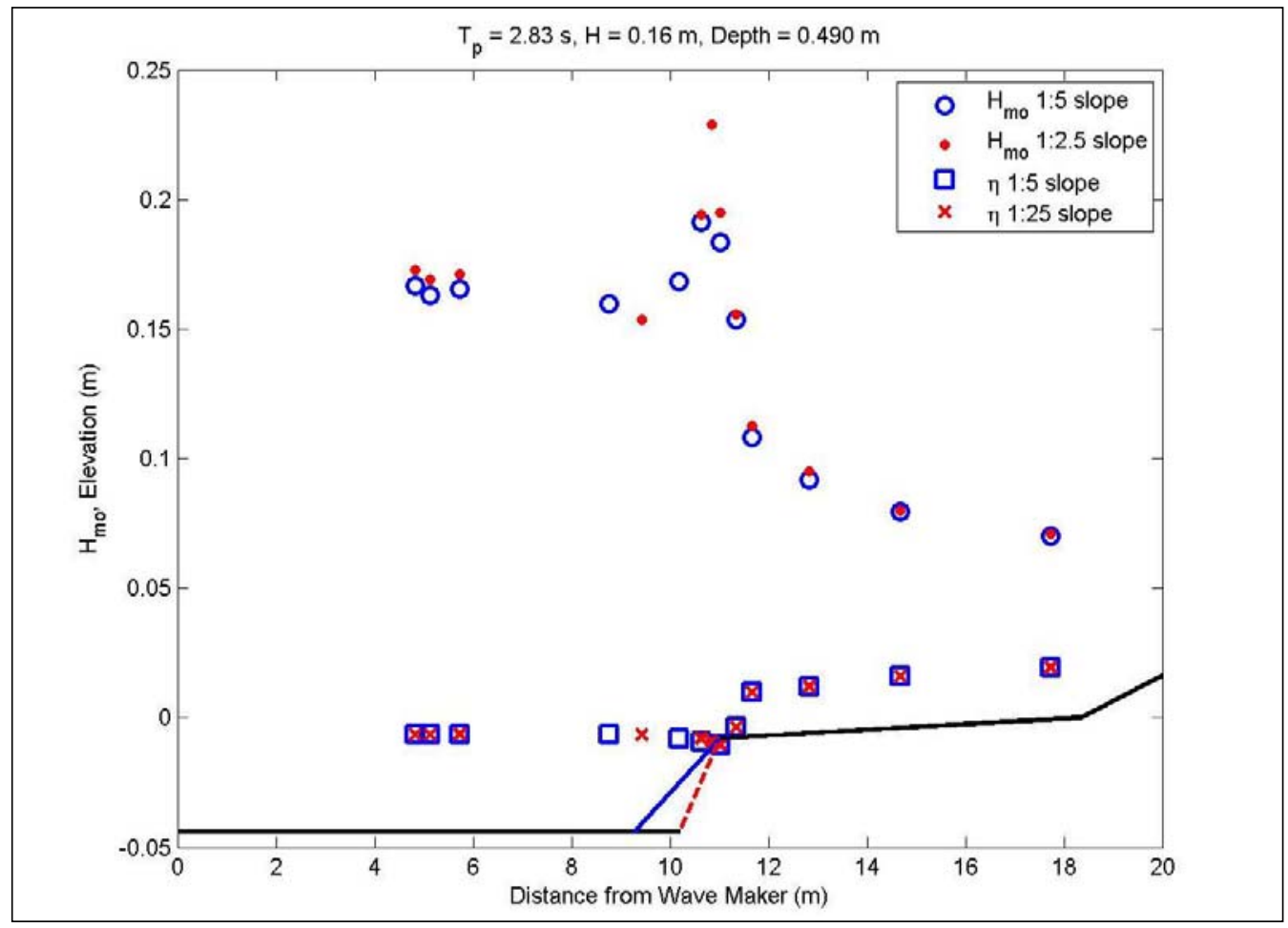

Figure 66. Wave transformation comparison between reef slopes for 2.8 -sec, $0.16 \mathrm{~m}$ waves at high water with rough reef surface. 
In addition to the above factors, breaker height also is greater in the presence of an opposing flow. A strong return flow was observed during the experiment over both reef slopes, and the flow occasionally extended to the midpoint of the reef slope similar to a weir flow. Higher return flow is expected with longer periods because orbital wave velocities are higher and may explain the high breaker heights with the 2.8-sec waves. Furthermore, the reef encompassed a large distance that was shallow, and through continuity, return flow would be higher in shallow water than monotonically-increasing deeper water found in surf zones along typical coastal beaches.

\subsubsection{Wave setup}

Setup was very similar between roughnesses (Figures 49 to 60) and reef slopes for the 1.0- and 2.8-sec waves (Figures 61 to 66) at all water levels. Set down is observed as waves approach the break point followed by setup due to breaking.

The highest mean water level measurement on the reef, i.e., the maximum $\eta$ recorded from Gauges 9 through 12, is shown for each water level and reef slope as a function of wave power in Figure 67 (also compare to Figure 14). The figure shows increasing setup with wave power and higher setup values for the lower water levels. The high water tests had the lowest setup on the reef, which is consistent with previous results. Gourlay (1996b) observed similar trends in setup measured during tests of coral reef structures with various water levels.

\subsubsection{Spectral wave energy}

Changes in energy density spectra were measured from the offshore gauges across the entire bathymetric profile for all water levels and both reef slopes in Figures 68 to 73. In the top window of all the figures, energy density change is shown from an offshore gauge (Gauge 2) to Gauge 5 (located in the middle of the reef slope) to Gauge 6 (the breakpoint for most waves). Spectra changes are shown in the middle window for Gauge 7, immediately offshore of the reef and shoreward of breaking to Gauges 8 and 9, which are on the seaward portion of the reef. The bottom window shows spectra from the three gauges located on the shoreward portion of the reef (Gauge 10, 11, and 12). Downshifting in the peak frequency was very noticeable for the three most shoreward gauges in most test cases. 


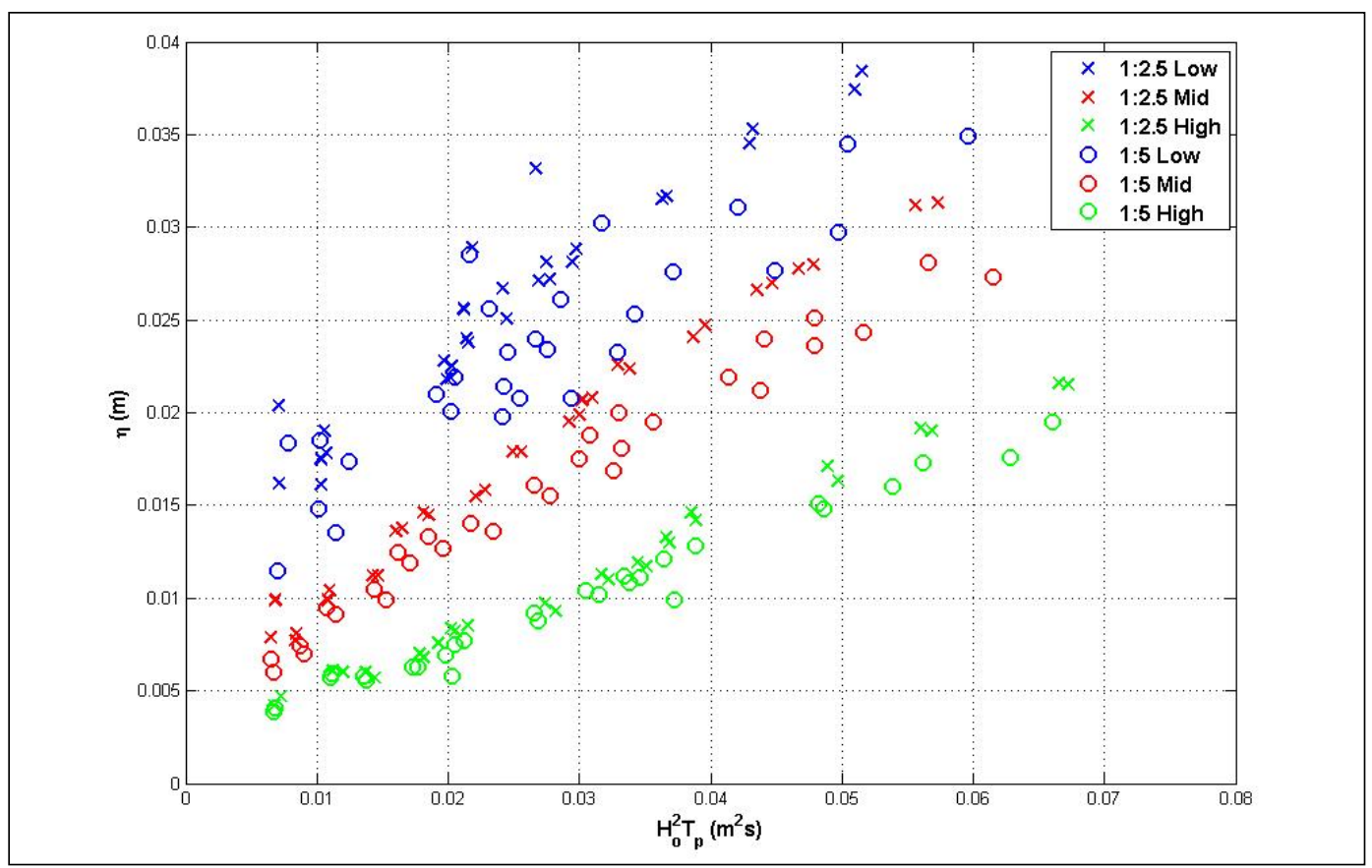

Figure 67. Highest mean water level recorded for each wave condition.

Some differences were observed in the energy density spectra transformation between the three different water levels on the 1:2.5 slope (Figures 68 to 70). The cases shown in these figures were of similar incident wave height and peak period. In the top window of all three figures, the increase in energy at the peak frequency differed for the three water levels between Gauge 2 and Gauge 6. The low water case had the greatest increase in energy at the peak frequency (Figure 68), and the high water case had the lowest (Figure 70).

This is expected due to shoaling. Also, more energy resided in the lower frequencies with the low-water wave condition than with the mid- (Figure 69) and high-water levels (Figure 70). Increases in energy in the lower frequencies were caused by reflection and seiching off the reef slope and growth of infragravity waves. Low frequency oscillations will be discussed in the next section.

Dissipation of energy at the peak frequency was measured for all three water levels and is shown in the middle window of Figures 68 to 70 . The highest energy shoreward of breaking occurred with the high-water case (Figure 70). The low-water (Figure 68) and the mid-water case (Figure 69) had a greater energy decrease between Gauges 6 and 7. The lower water levels resulted in less wave energy transfer onto the reef; therefore, there was less energy at all frequencies. The deeper water at the top of the reef 

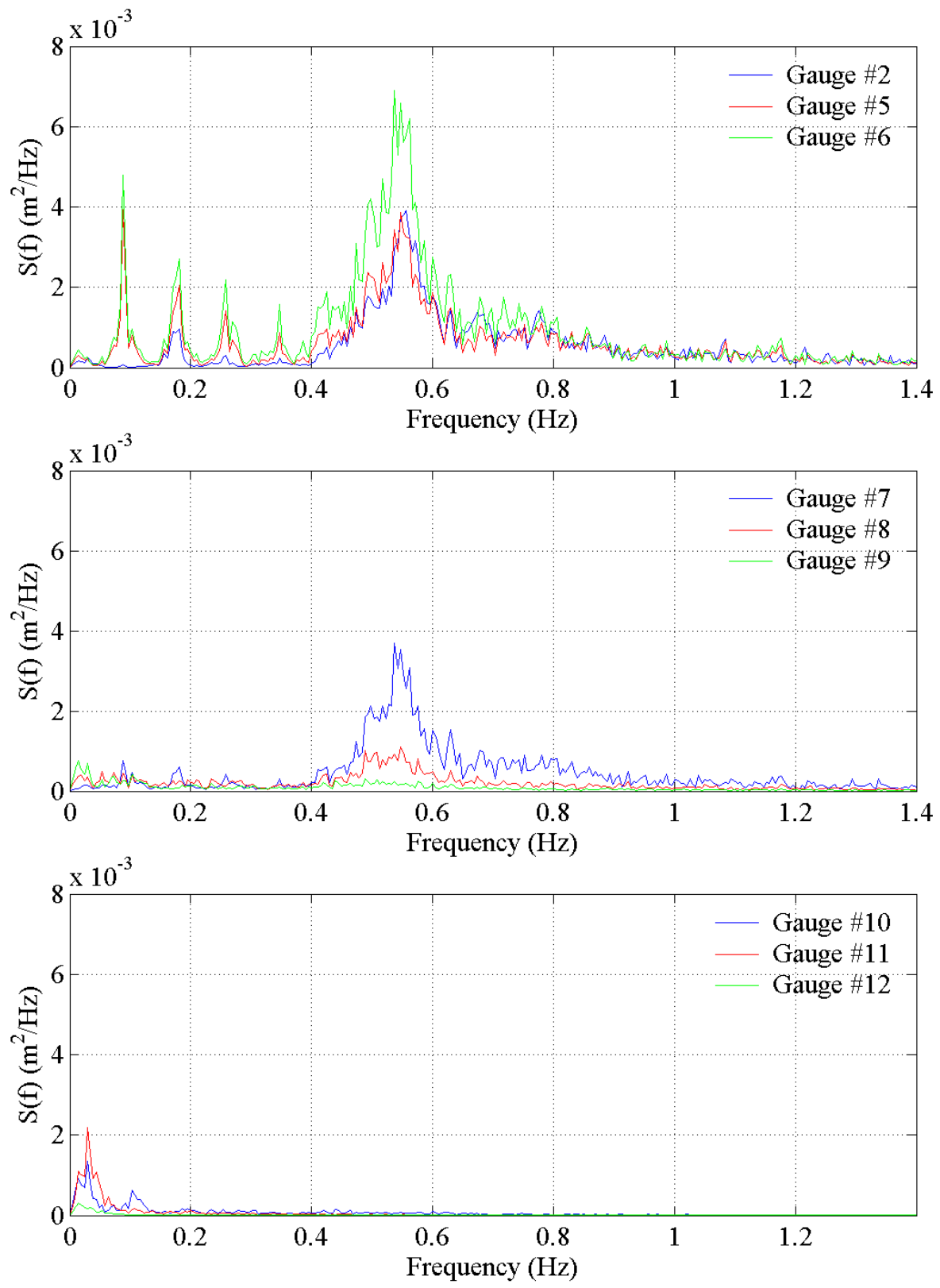

Figure 68. Energy density spectrum transformation of $1.8-\mathrm{sec}, 0.11-\mathrm{m}$ waves at low water with a 1:2.5 reef slope and smooth reef surface. 

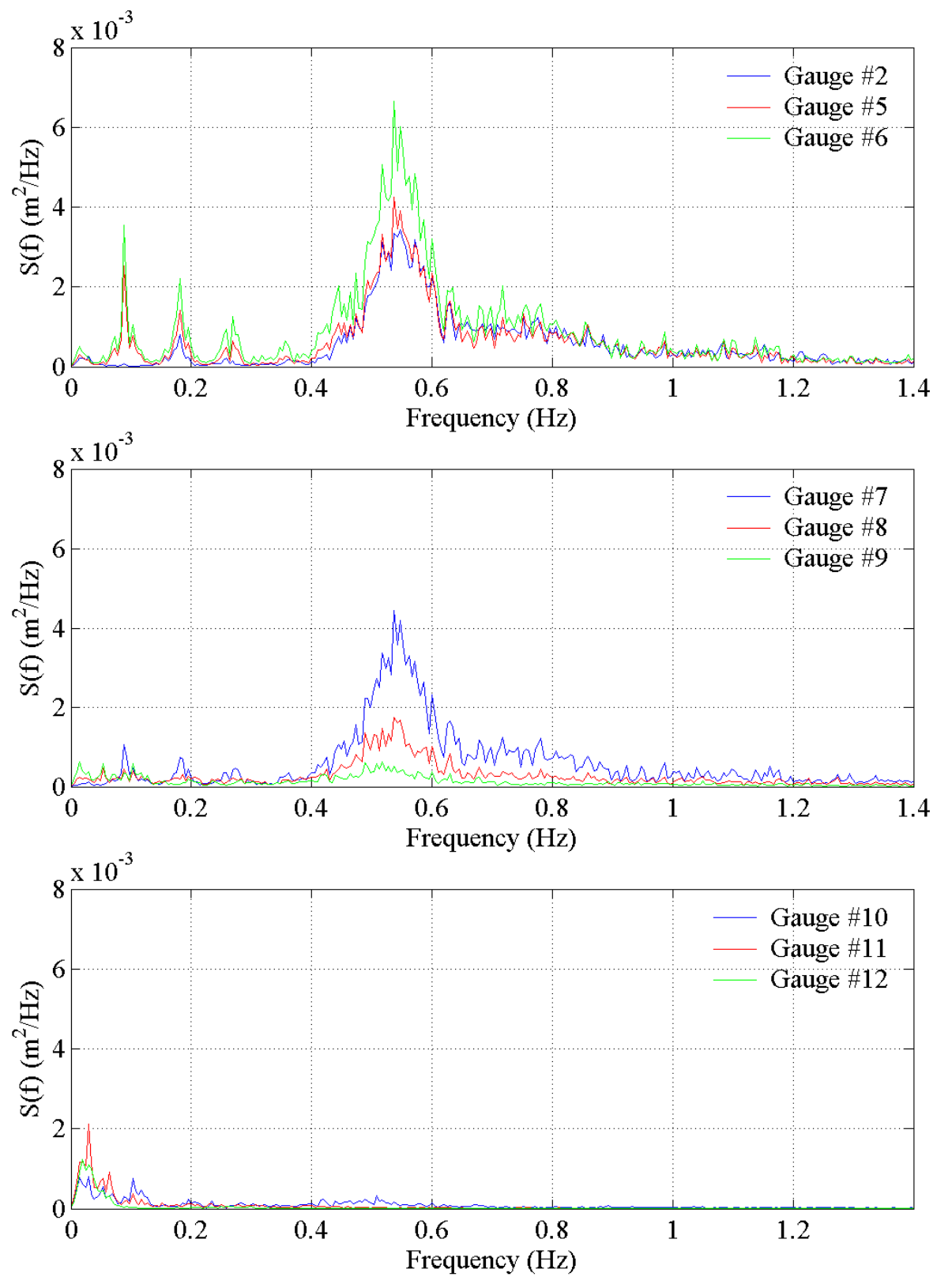

Figure 69. Energy density spectrum transformation of 1.8-sec, 0.11-m waves at mid water with a 1:2.5 reef slope and smooth reef surface. 

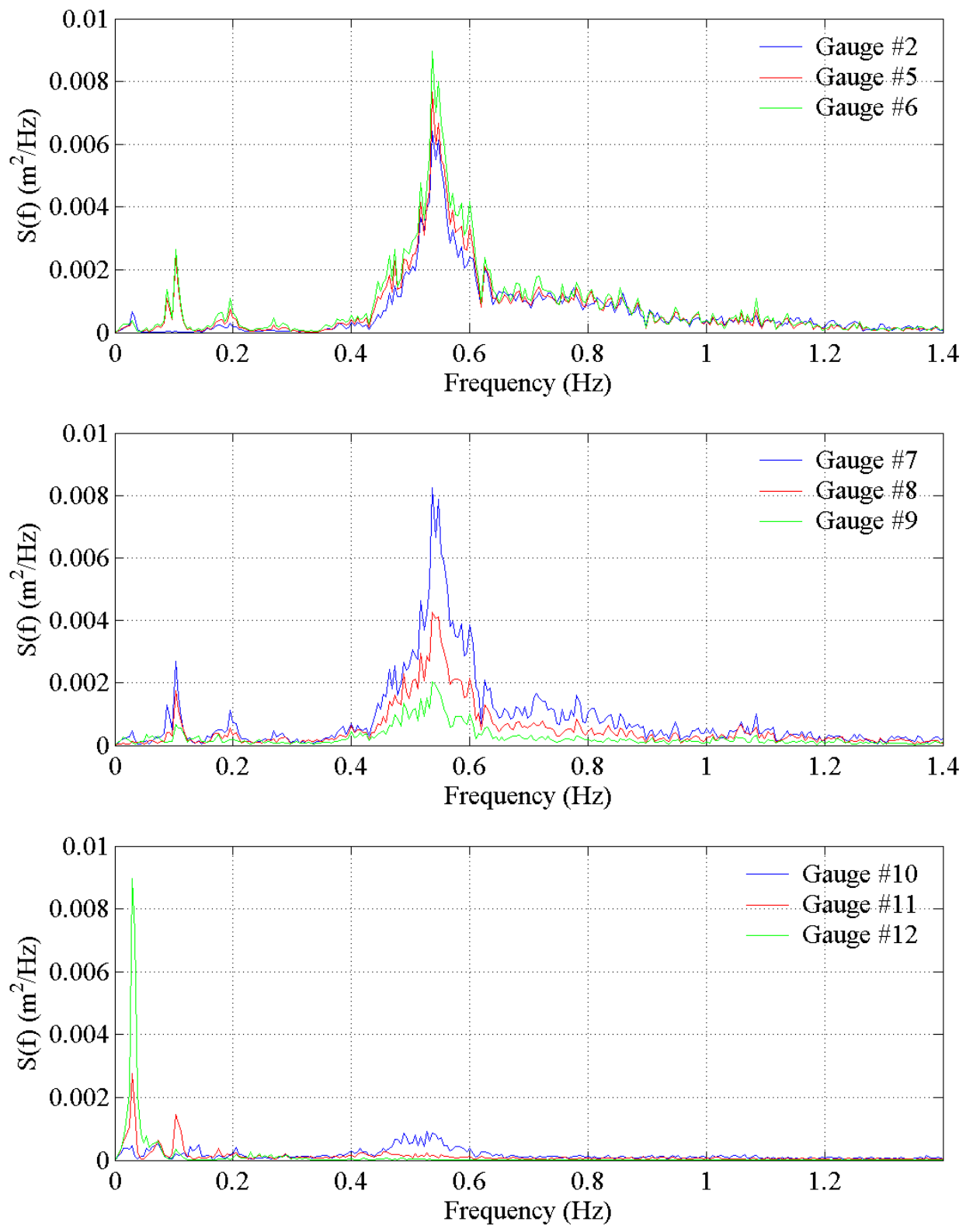

Figure 70. Energy density spectrum transformation of 1.8-sec, $0.12-\mathrm{m}$ waves at high water with a 1:2.5 reef slope and smooth reef surface. 

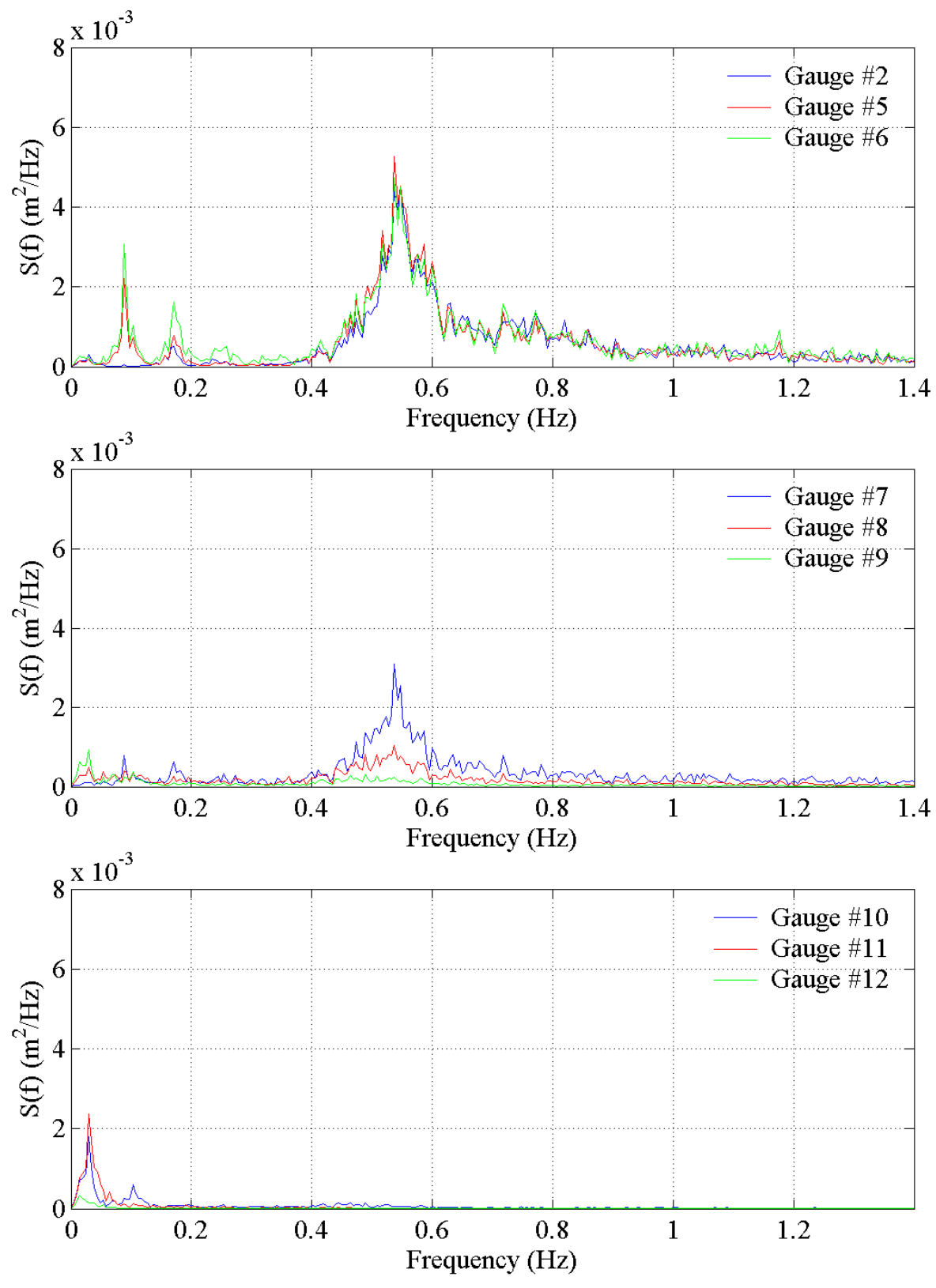

Figure 71. Energy density spectrum transformation of $1.8-\mathrm{sec}, 0.12-\mathrm{m}$ waves at low water with a 1:5 reef slope and smooth reef surface. 

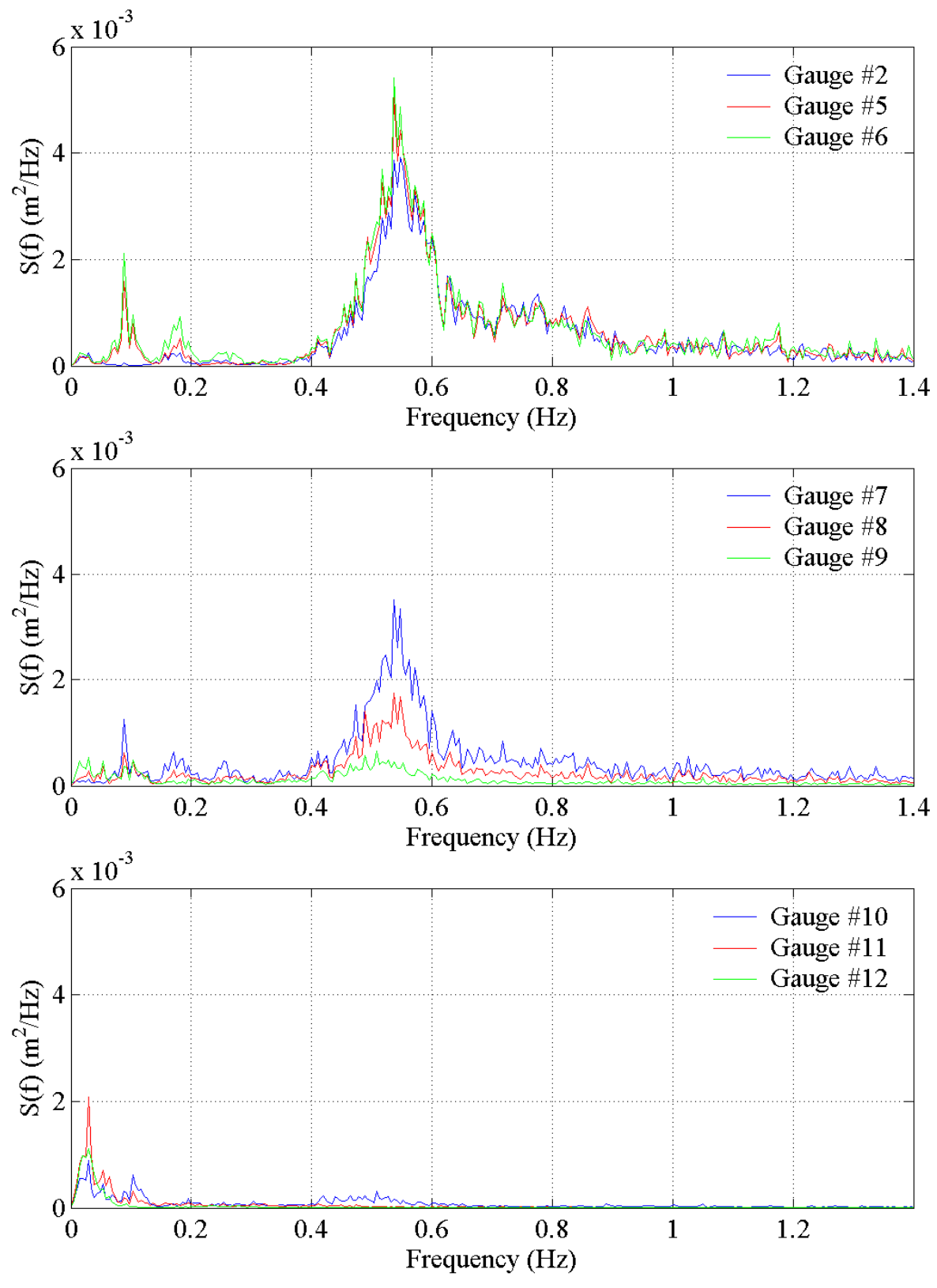

Figure 72. Energy density spectrum transformation of 1.8-sec, $0.12-\mathrm{m}$ waves at mid water with a 1:5 reef slope and smooth reef surface. 

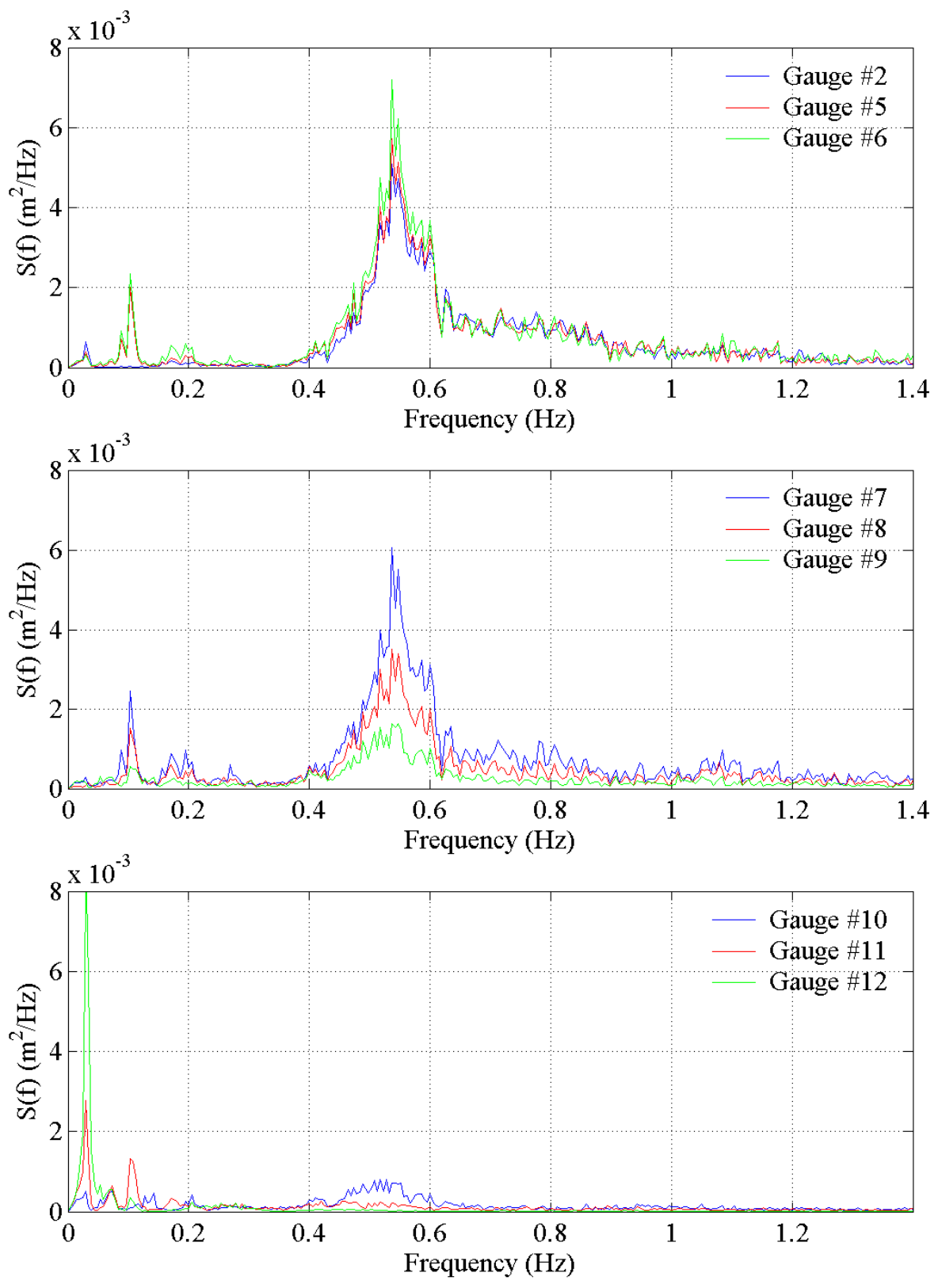

Figure 73. Energy density spectrum transformation of 1.8-sec, 0.12-m waves at high water with a 1:5 reef slope and smooth reef surface.

slope and on the reef with the high-water case allowed more energy to transfer to the gauges on the shoreward portion of the reef. The decrease in energy at the peak frequency between Gauges 7 and 9 was likely a combination of depth-limited breaking and friction. More dissipation of 
energy at the peak frequency was observed in the shallower water level for the low- and mid-water tests compared to the high-water tests.

The bottom window in Figures 68 to 70 shows the continued dissipation of energy at the peak frequency on the reef as well as increase in low frequency energy. In the low-water tests (Figure 68), all energy is shifted to the lower frequencies. Gauge 12 was not always in the water for the low water tests; therefore, the energy density spectrum at all frequencies was near zero. Very little energy remained at the initial peak frequency at Gauge 10 during the mid-water test (Figure 69), and that energy dissipated between Gauges 10 and 11. All significant energy at Gauge 11 and Gauge 12 was located in the lower frequencies with the mid-water tests. Gauge 12 was in the water for most of the mid-water tests, but the water was very shallow. Low-frequency energy increased between Gauge 10 and Gauge 11, but less energy reached Gauge 12. In the high-water tests (Figure 70), energy at the initial peak frequency was dissipated by Gauge 11. The low-frequency energy increased between Gauge 10 and Gauge 12.

Similar trends in transformation of the energy density spectra across the flume profile were measured in the 1:5 tests (Figure 71 to 73 ) as the 1:2.5 tests. The biggest difference observed between the two slopes was wave energy transformation at the peak frequency at Gauge 6 . The highest energy at the peak frequency for the 1:2.5 tests occurred at Gauge 6, but less of an energy increase occurred between Gauge 2 and Gauge 6 with the 1:5 tests. This result was consistent for all three water levels with the 1:5 slope tests. The amount of low frequency energy in the top windows of Figures 71 to 73 was less than the amount measured with the 1:2.5 slope. Overall, the changes in the transformation of wave energy between the low- (Figure 71), mid-(Figure 72), and high-(Figure 73) water levels were similar to the results described for the 1:2.5 tests.

Low-Frequency Spectral Wave Energy. The low-frequency spectral wave energy is shown for select gauges through the laboratory profile for both the 1:2.5 (Figures 74 to 76) and 1:5 (Figures 77 to 79) reef slopes. The test cases shown in the figures all have an initial $T_{p}=1.8 \mathrm{sec},\left(f_{p}\right.$ of $\left.0.56 \mathrm{~Hz}\right)$, and are representative of the runs for the three water levels and the two reef slopes. Based on the method described in the Chapter 2 for separating the low-and high-frequency oscillations, the low-frequency cut off was set at $0.37 \mathrm{~Hz}$. 

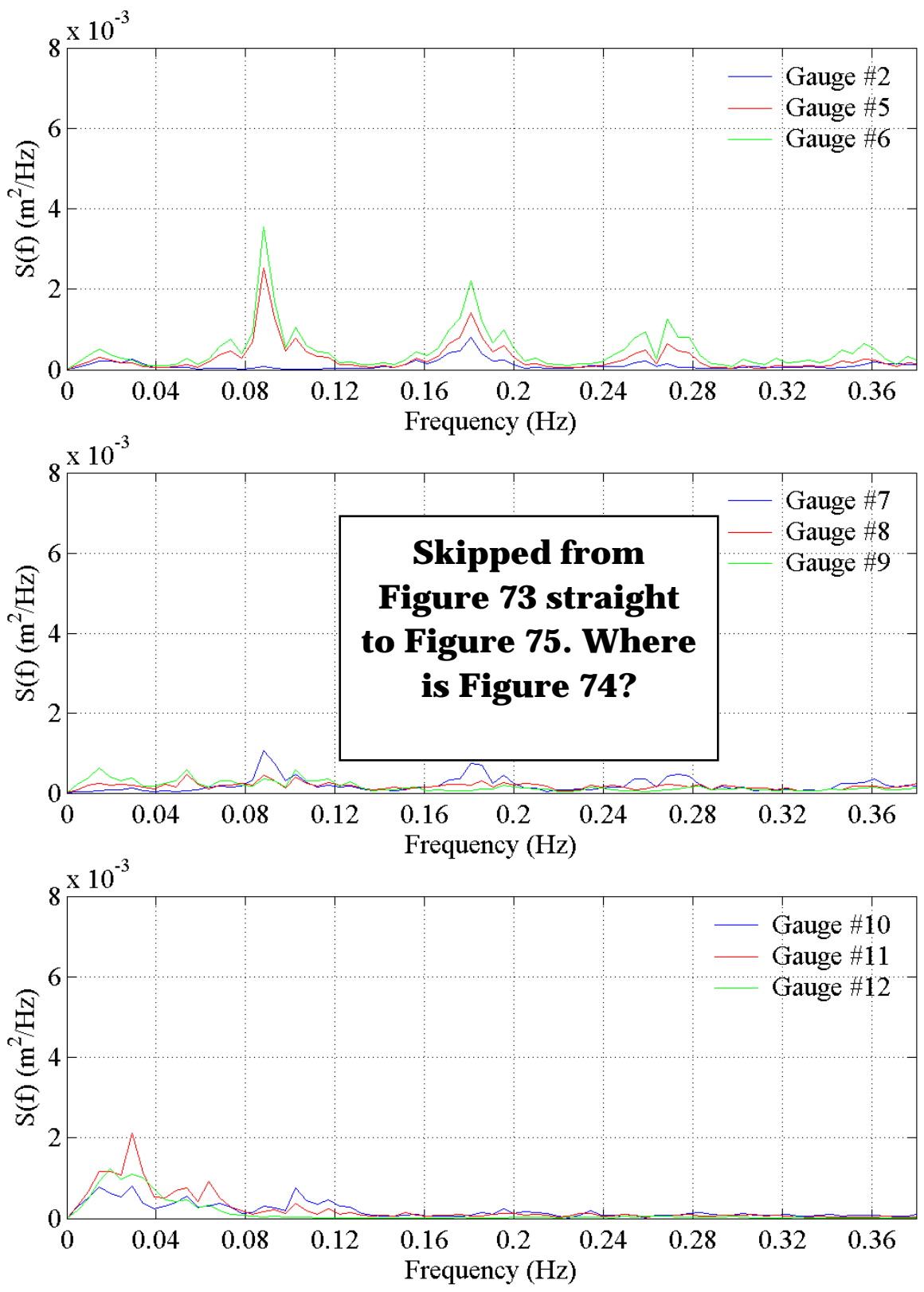

Figure 75 . Low frequency energy density spectrum for $1.8-\mathrm{sec}, 0.11-\mathrm{m}$ waves at mid water with a 1:2.5 reef slope and smooth reef surface. 

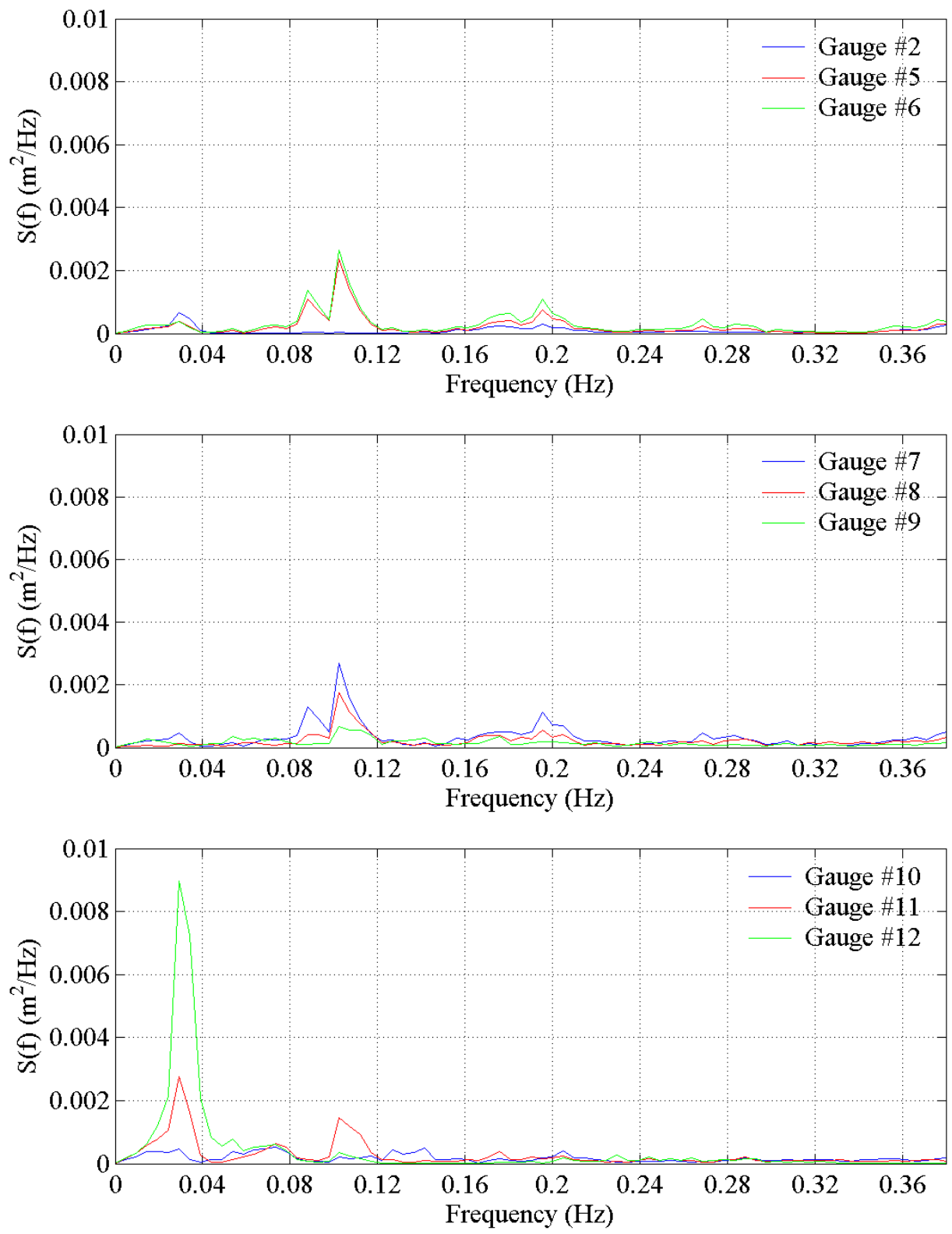

Figure 76. Low frequency energy density spectrum for $1.8-\mathrm{sec}, 0.12-\mathrm{m}$ waves at high water with a 1:2.5 reef slope and smooth reef surface. 

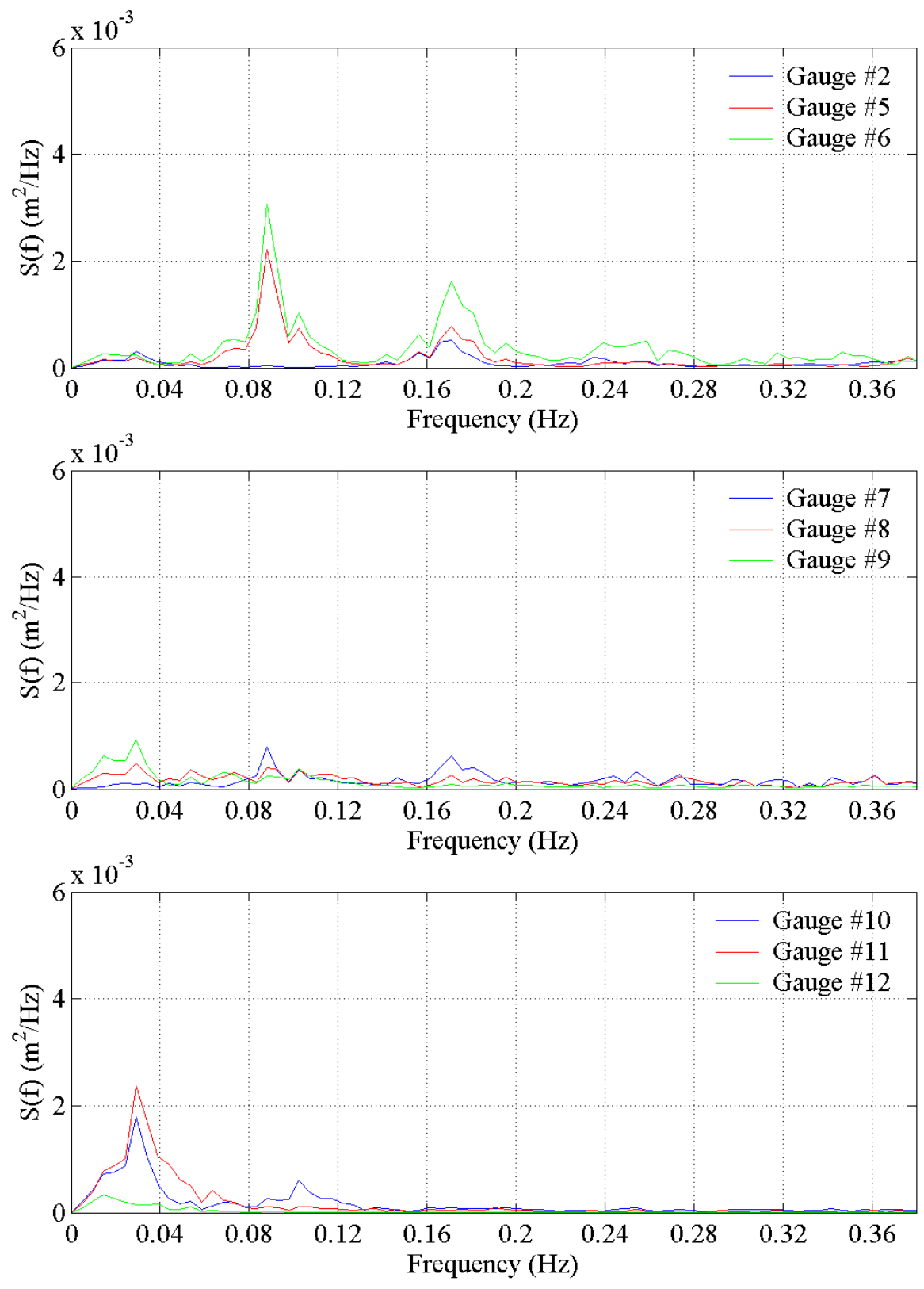

Figure 77. Low frequency energy density spectrum for $1.8-\mathrm{sec}, 0.12-\mathrm{m}$ waves at low water with a 1:5 reef slope and smooth reef surface. 

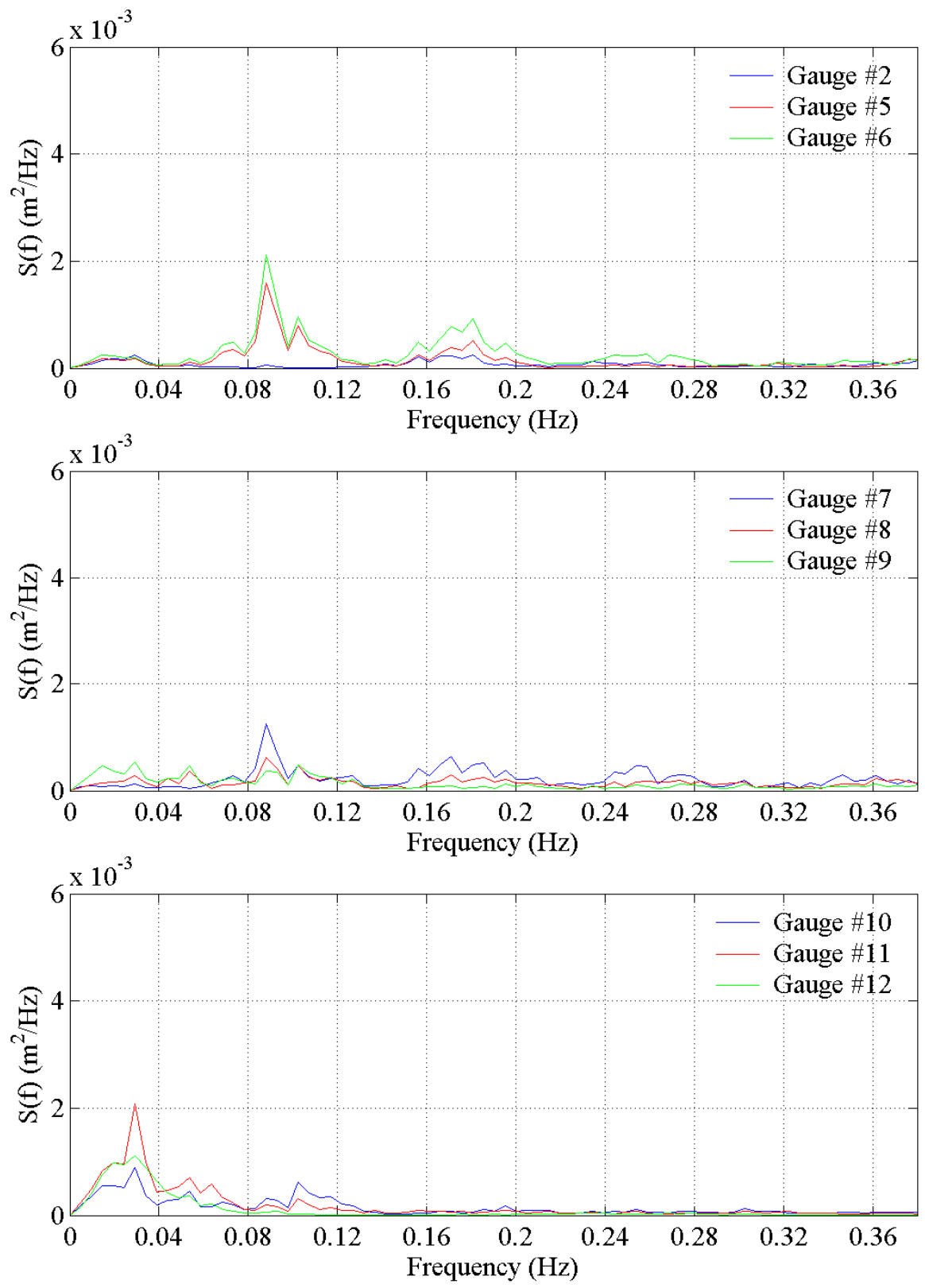

Figure 78. Low frequency energy density spectrum for $1.8-\mathrm{sec}, 0.12-\mathrm{m}$ waves at mid water with a 1:5 reef slope and smooth reef surface. 

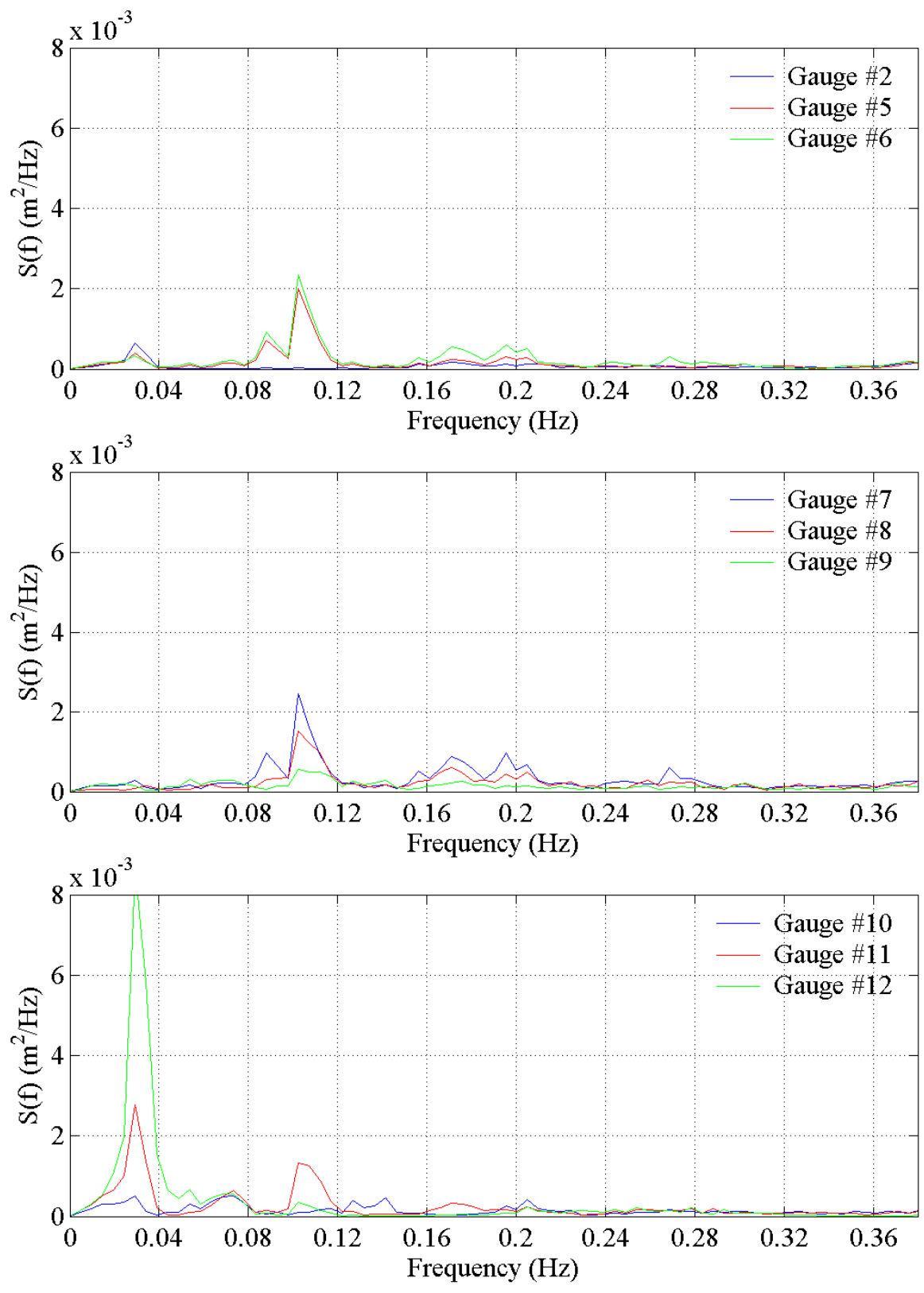

Figure 79. Low frequency energy density spectrum for 1.8-sec, 0.12-m waves at high water with a 1:5 reef slope and smooth reef surface.

Peaks in the low-frequency spectra observed offshore of the reef for all three water levels and reef slopes can be explained by seiching. The shape of the offshore section of the flume was flat for approximately $10 \mathrm{~m}$ for the 1:2.5 slope and $9 \mathrm{~m}$ for the 1:5 slope. Both of the reef slopes are considered very steep; therefore, the shape of the offshore region can be approximated as a 
rectangular basin. Periods of oscillation for seiching were calculated with Equation 2-2 for $l=9.5 \mathrm{~m}$, the distance from the wavemaker to the reef mid-slope, and $h_{1}=0.341 \mathrm{~m}$, the distance from the top of the reef slope to the flume floor. The fundamental frequency of seiching $(n=1)$ was $0.09 \mathrm{~Hz}$. This is consistent with the peaks measured at a similar frequency in the top window of Figures 74 to 79 . The second peak was measured at $0.18 \mathrm{~Hz}$ which is similar to the value of the $2^{\text {nd }}$ mode of oscillation. The third and fourth modes of oscillation were only measured in the low water tests of the 1:2.5 slope, and the values of 0.27 and $0.37 \mathrm{~Hz}$ are consistent with the observed values (Figure 74). Thus seiching which occurred in the offshore section of the tests could have affected wave height measurements between Gauges 2 and 7 due to the location of the nodes and antinodes.

\subsubsection{Runup}

Runup from the 2-D experiments is plotted versus wave power in Figure 80 with symbols indicating reef slope and water level. The 2-D runup values show an increasing trend of runup with wave power, which was observed in the 3 -D tests (Figure 31). The 2-D $R_{2}$-values appear slightly higher than those of the 3-D experiment, which is probably due to energy restricted to the cross shore direction. Figure 80 also indicates that $R_{2}$ was independent of reef slope and water level.

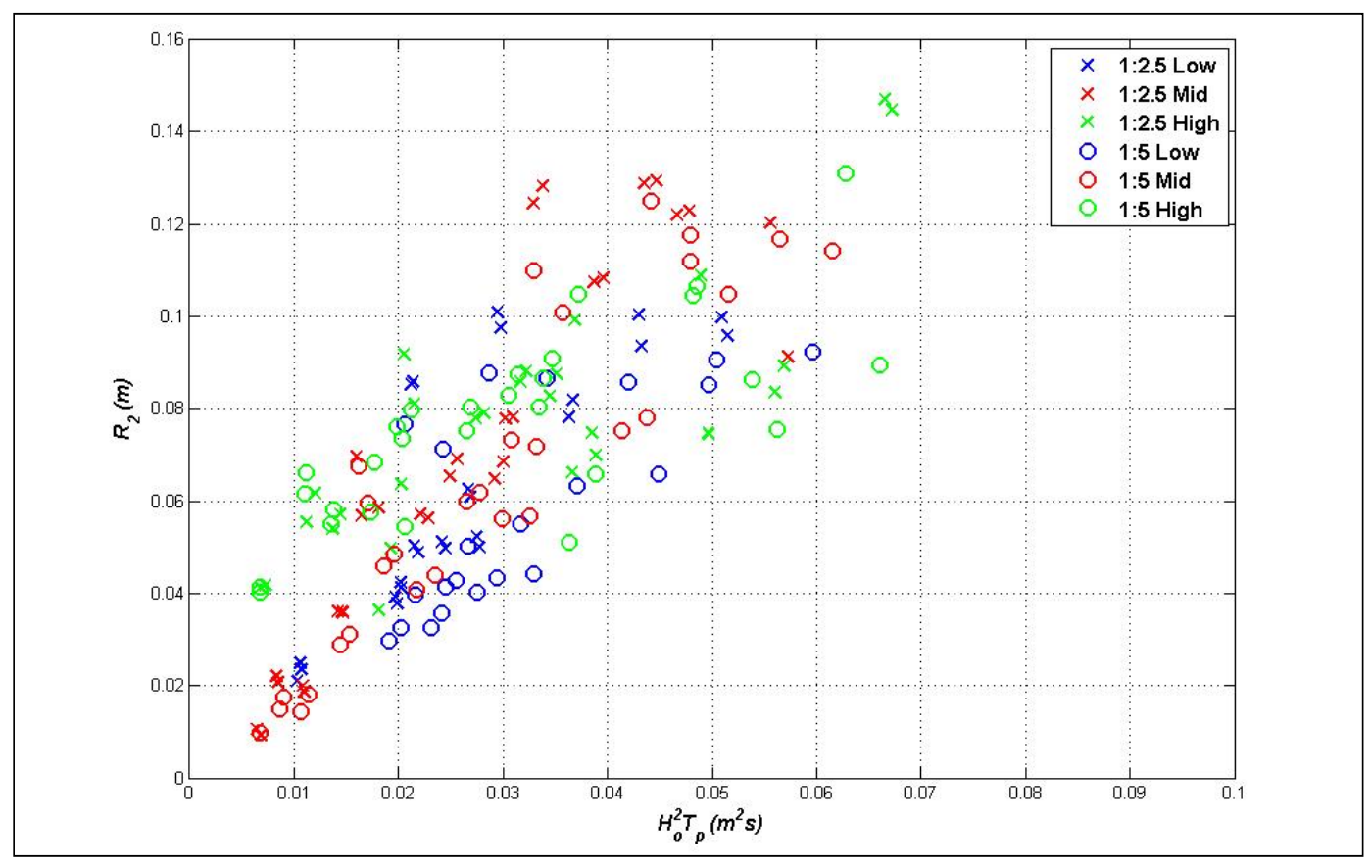

Figure 80. Two-percent runup as a function of wave power. 


\subsubsection{Reflection}

The water surface elevation time series from Gauges 1, 2, and 3 were used to calculate incident and reflected waves by the Goda method discussed in Chapter 2. The reflection coefficients for all wave conditions at each water level are shown as a function of $\xi_{0}$ in Figure 81 with the relationships of Battjes (1974), Seelig and Ahrens (1981), and Smith and Kraus (1992). Reflection coefficients show a scattered, but slightly increasing trend with $\xi_{o}$, and range from $K_{r} \approx 0.08$ to 0.32. Battjes, Seelig and Ahrens give reasonable $K_{r}$ estimates between $\xi_{0}$-values of 0.8 and 1.5 , but greatly overpredict the lab data for higher surf similarity parameters. The Smith and Kraus predictions are reasonable for $\xi_{o}<2$, but generally underpredict $K_{r}$ at higher values of $\xi_{0}$. The Smith and Kraus (1990) relationship discussed in Chapter 2 estimates $K_{r}$ as a function of only seaward bar slope. For the 2-D slopes, constant values of 0.18 for the 1:2.5 slope and 0.16 for the 1:5 slope were calculated. These values fall within the range of $K_{r}$ at lower $\xi_{o}$-values, but are lower than $K_{r}$ at high values of $\xi_{o}$.

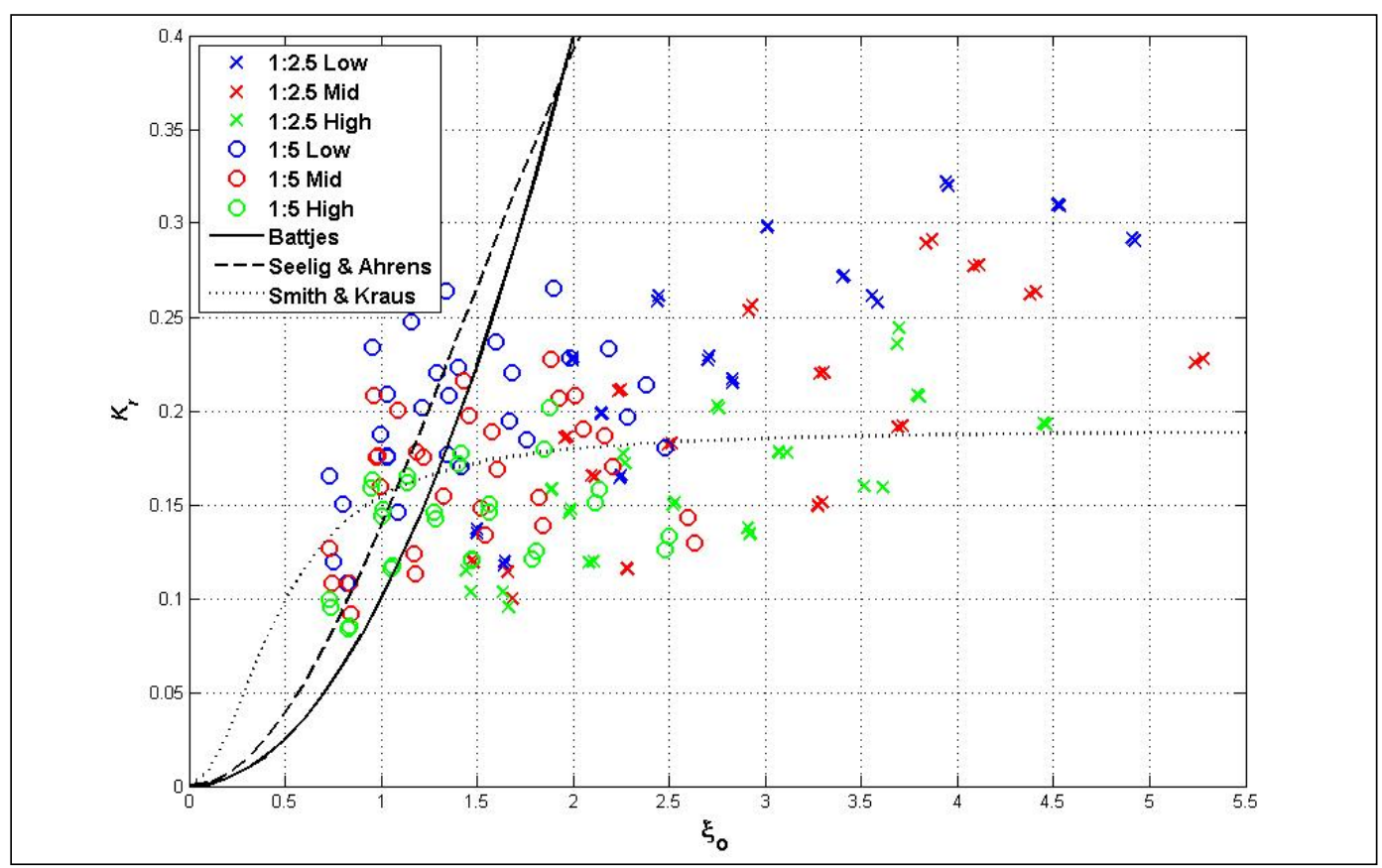

Figure 81. Reflection coefficient as a function of surf similarity parameter.

Although reflection coefficients didn't vary with surf similarity in the 3-D experiments, $K_{r}$ shows a slight increasing trend with $\xi_{o}$ in the 2-D tests. The Smith and Kraus (1992) relation, which used modified coefficients with the Seelig and Ahrens (1981) equation, generally follows the trend of the data. Smith and Kraus derived the coefficients from laboratory data 
performed with breakpoint bars on a 1:30 plane slope, which may explain the underprediction of the relationship.

The similar bathymetry between the 2-D laboratory study and the study by Demirbilek et al. (2007) make comparison of reflection coefficients beneficial. The Demirbilek study was performed in a wave tank with three reef slopes of 1:5, 1:18.8, and 1:10.6, with an average slope of 1:9. Demirbilek measured $K_{r}$ less than 0.1 for all tests, less than those in the present study. The greater $K_{r}$-values in the present study were likely a result of steeper reef slopes of 1:2.5 and 1:5 compared to 1:9 in Demirbilek study. 


\section{Summary}

As part of the Surge and Wave Island Modeling Studies project, 3-D and 2-D physical model studies were performed to measure wave transformation, setup, runup, and reflection on steep bathymetry and fringing reefs to improve the understanding of the processes and to assist in validation of numerical models and modeling systems. Wave conditions generated in both the 3-D and 2-D studies included three water levels and five wave periods that encompassed the range of conditions that are representative of locations with fringing waves. This chapter summarizes the results from the experiments.

\subsection{Three-dimensional study}

The 3-D experiment was conducted in a 51.8-m-long, 29.0-m-wide, and 1.5-m-deep wave basin. The 3-D model was constructed of acrylic glass to simulate an idealized reef. The idealized bathymetry was based on LIDAR data taken in southeast Guam. The acrylic glass was table-routed in 0.61-m by $0.61-\mathrm{m}$ panels to a $0.5 \mathrm{~m}$ horizontal resolution at a $1: 50$ scale, and provided a durable surface to mount instruments. Adjustable legs were attached to each reef panel and then attached to the horizontal portion of a compound-slope platform with marine urethane adhesive.

A total of 78 runs were performed on the 3 -D model, which include 40 tests on a smooth surface (the reef panels were unpainted) and 38 tests on a roughened surface (the panels were painted with a roughening agent included in the paint). Tests on each surface were conducted with three water levels and five wave periods ranging from 1.0 to $2.8 \mathrm{sec}$ at model scale (7 to $20 \mathrm{sec}$ at prototype scale).

Wave heights measured on the centerline profile of the basin showed the general trend of waves breaking on the front slope and then further dissipating on the reef top. Wave height remained relatively uniform in the flat portion of the test basin, but several tests, particularly for the longer period waves, showed a small decrease in wave height offshore of the breakpoint and fronting reef slope. The incident and higher frequency wave energy dissipated along the cross-shore profile of the basin, whereas low frequency wave energy remained nearly constant. On the inner reef, energy in the low-frequency band dominated the spectra for the low- and mid- 
water levels. At high water levels, incident and high-frequency wave energy penetrated further along the reef profile. Setup measured on the reef was found to be proportional to the offshore wave power, with higher setup associated with the low-water tests and less setup for higher water levels.

Wave heights recorded in the alongshore direction on the reef were generally symmetrical with the highest in the center of the reef where the depth was greatest and lowest on the reef edges where depths were shallowest. However, three-dimensional patterns were observed even though shore-normal incident waves were generated. The highest waves recorded in the alongshore direction for some cases were $2 \mathrm{~m}$ from the centerline. The three-dimensionality is attributed to non-symmetrical bathymetry in the longshore, wave-wave interactions, wave refraction, and reflection. Runup measured during the experiment exhibited similar results, with runup frequently higher at the gauges $\pm 2 \mathrm{~m}$ of the centerline.

Calculated $K_{r}$ was 21 percent or less with the exception of one outlying point. Reflection coefficients did not vary with surf similarity and were scattered between 12 and 21 percent.

The roughness applied to the reef had little effect on wave heights, runup, or reflection. Therefore, it was concluded a rougher surface would be required to significantly reduce wave height. Although the contribution of surface roughness was minor, the data provide numerical modelers with two roughnesses for model development.

Additional tests were performed with an angled channel cut into the reef bathymetry. Two ADVs were placed on the reef: one in the center of the channel and one near the reef centerline. Velocities in the channel were mainly bi-directional in the direction of the channel but with a net flow directed offshore. Net velocities at the centerline ADV also showed a net offshore flow. The centerline ADV also showed a net downdrift alongshore velocity, which may be caused by the non-uniformity in elevation of the underlying platform and the resulting variability in the wave setup. Wave heights were similar between the channel and rough surface tests without a channel, but longer-period waves and higher incident waves generally showed greater heights in the shoaling region directly offshore of breaking with the channel, possibly due to wave-current interaction. Wave heights and setup were similar between channel and non-channel tests except at 
the gauge located next to the channel, which recorded consistently lower wave heights.

\subsection{Two-dimensional study}

The 2-D experiments were performed in a 45.7-m long, 0.91-m wide, and 0.91-m deep wave tank. A wall was installed to divide the flume horizontally into 0.61 and $0.30 \mathrm{~m}$ sections adjacent to the reef. A 0.61-m wide crossshore section of the three-dimensional acrylic reef was duplicated and installed in the wave tank as the reef surface. Installation of the 2-D reef surface differed from the 3 -D. In the 2-D experiments, acrylic sections were mounted on cross-sectional pieces of a horizontal floor. The acrylic sections were sealed to the floor cross-sections, the dividing wall, glass wall and adjoining acrylic sections with silicone.

Two data sets were acquired during the 2-D experiments. A set of tests were conducted in collaboration with the University of Florida to measure waves and currents on and in the vicinity of the reef slope. The study included 84 tests that were performed with the reef unpainted, or smooth. Results from UF data set can be found in Sheremet et al. 2011 and figures from the study are given in Appendix D.

The second set of 2-D experiments included 172 runs also for three water levels, five periods between 1.0 and $2.8 \mathrm{sec}$, reef slopes of 1:2.5 and 1:5, but with smooth and roughened reef surfaces. The reef surface was roughened in the same manner as in the 3 -D experiments, with the same roughening agent.

Wave height transformation showed a similar trend as in the 3-D study. Shorter period waves shoaled slightly prior to breaking, and a small decrease in height was observed with the 1:2.5 slope. Longer period wave heights decreased offshore of the breakpoint and then shoaled significantly, with greater shoaling occurring with the 1:2.5 slope. A strong return flow was observed during the tests, which influenced breaker height. Setup measurements on the reef were proportional to the offshore wave power and similar to the 3-D study results; higher setup occurred with the lowwater level tests and less setup for higher water level tests. Runup results also were similar to the 3 -D study in that runup was proportional to offshore wave power. 
Reflection coefficients were higher in the 2-D study than the 3-D experiments, with several measurements exceeding 20 percent. Although the data were scattered, $K_{r}$ increased with increasing surf similarity, which was not observed in the 3-D experiment. However, predictive equations of Battjes (1974) and Seelig and Ahrens (1981) overpredicted $K_{r}$-values for $\xi_{o}$ greater than 1.5. The modified coefficients proposed by Smith and Kraus (1992) followed the general trend of the data for lower values of $\xi_{o}$, but underpredicted $K_{r}$ at higher surf similarity parameters.

Wave heights, setup, runup, and reflection were not affected by the different surface conditions, an observation also made in the 3-D study. It was concluded a rougher surface would be required to significantly reduce wave height.

\subsection{Reef construction}

The idealized reef bathymetry in both the 3-D and 2-D studies was constructed of 0.61 by $0.61 \mathrm{~m}$ acrylic panels. However, the method to attach the panels differed between the studies. The adjustable legs affixed to 3-D panels were attached to the underlying platform with a marine adhesive, which resulted in a gap between the panel and platform. Problems with the panels becoming detached occurred frequently in the 3-D study and it is believed that a combination of buoyant forces, back pressure caused by breaking and propagating waves, and the small adhesive surface area available on the legs couldn't secure the panels in place. Conversely, the panels in the 2-D study were secured directly to the underlying platform, and also to the dividing wall and glass. The 2-D reef panels remained secure throughout the experiment. The method used to attach the panels in the 2-D study was clearly better and should be utilized in future studies. 


\section{References}

Battjes, J. A. 1974. Surf similarity. Proceedings of the $14^{\text {th }}$ Coastal Engineering Conference, ASCE, 1993-2004.

Bouws, E., H. Gunther, W. Rosenthal, and C.L. Vincent. 1985. Similarity of the wind wave spectrum in finite depth water. 1. Spectral form. Journal of Geophysical Research 90(C1), 975-986.

Dean, R. G. and R. Dalrymple, R. 1984. Water wave mechanics for engineers and scientists. Prentice-Hall, Inc., Englewood Cliffs, New Jersey.

Demirbilek, Z., O. G. Nwogu, and D. L. Ward. 2007. Laboratory study of wind effect on runup over fringing reefs, Report 1: Data report. ERDC/CHL-TR-07-4. Vicksburg, MS: U.S. Army Engineer Research and Development Center.

Goda, Y., and Y. Suzuki. 1976. Estimation of incident and reflected waves in random wave experiments. Proceedings of the $15^{\text {th }}$ Coastal Engineering Conference, ASCE, 828-845.

Gourlay, M. R. 1994. Wave transformation on a coral reef. Coastal Engineering 23:17-42. 1996a. Wave setup on coral reefs. 1. Setup and wave-generated flow on an idealized two dimensional horizontal reef. Coastal Engineering, 27:161-193.

. 1996b. Wave setup on coral reefs. 2. Setup on reefs with various profiles. Coastal Engineering, 28:17-55.

Hamilton, D.G., B.A. Ebersole, E.R. Smith, and P. Wang. 2001. Development of a largescale laboratory facility for sediment transport study. Technical Report ERDC/CHL TR-01-22, U.S. Army Engineer Waterways Experiment Station, Vicksburg, Mississippi.

Hardy, T. A., I.R. Young, R.C. Nelson, and M.R. Gourlay. 1991. Wave attenuation on coral reefs. Australian Civil Engineering Transactions, 33:17-22.

Headquarters, U.S. Army Corps of Engineers. 2002. Coastal Engineering Manual. EM 1110-2-1100. Washington, DC; U.S. Army Corps of Engineers (in 6 volumes).

Hughes, S. A. 1993. Physical models and laboratory techniques in coastal engineering. World Scientific, JBW Printers \& Binders Pte. Ltd., Singapore.

Massel, S. R., and M. R. Gourlay. 2000. On the wave modeling of wave breaking and wave setup on coral reefs. Coastal Engineering, 39:1-27.

Nwogu, O. G., and Z. Demirbilek. 2006. Nonlinear wave interaction with submerged and surface-piercing porous structures. Proceedings of the $30^{\text {th }}$ Coastal Engineering Conference, ASCE, 287-299. 
Nwogu, O. and Z. Demirbilek. 2010. Infragravity wave motions and runup over shallow fringing reefs. Journal of Waterway, Port, Coastal, and Ocean Engineering, ASCE, 136: 295:305.

Seelig, W. N. 1983. Laboratory study of reef-lagoon system hydraulics, Journal of Waterway, Port, Coastal and Ocean Engineering, ASCE, 109: 380-391.

Seelig, W. N., and J.P. Ahrens. 1981. Estimation of wave reflection and energy dissipation coefficients for beaches, revetments, and breakwaters. Technical Paper No. 91-1. U.S. Army Engineer Waterways Experiment Station, Coastal Engineering Research Center, Vicksburg, MS.

Sheremet, A., J.M. Kaihatu, S-F. Su, E.R. Smith, and J.M. Smith. 2011. Modeling of nonlinear wave propagation over fringing reefs, Coastal Engineering, 58, $1125-1137$.

Smith, E. R., and N.C. Kraus. 1990. Laboratory study on macro-features of wave breaking over bars and artificial reefs. Technical Report CERC-90-12. U.S. Army Engineer Waterways Experiment Station, Coastal Engineering Research Center, Vicksburg, MS.

Smith, E. R., and N.C. Kraus. 1992. Laboratory study on wave transformation on barred beach profiles. Proceedings of the $23^{\text {rd }}$ Coastal Engineering Conference, ASCE, 630-643.

Stevens, J. C., C.E. Bardsley, E.W. Lane, and L.G. Straub. 1942. Hydraulic models. Manuals on Engineering Practice No. 25, ASCE. 


\section{Appendix A: Wave transformation figures from the three-dimensional experiments}

The figures presented in this appendix show zero-moment wave heights and mean water levels as a function of distance along the centerline in the 3-D basin. Each figure includes wave height and mean water level measured on the smooth and roughened bathymetry for similar wave conditions. The model bottom also is presented as a reference, but is scaled by a factor of 10 .

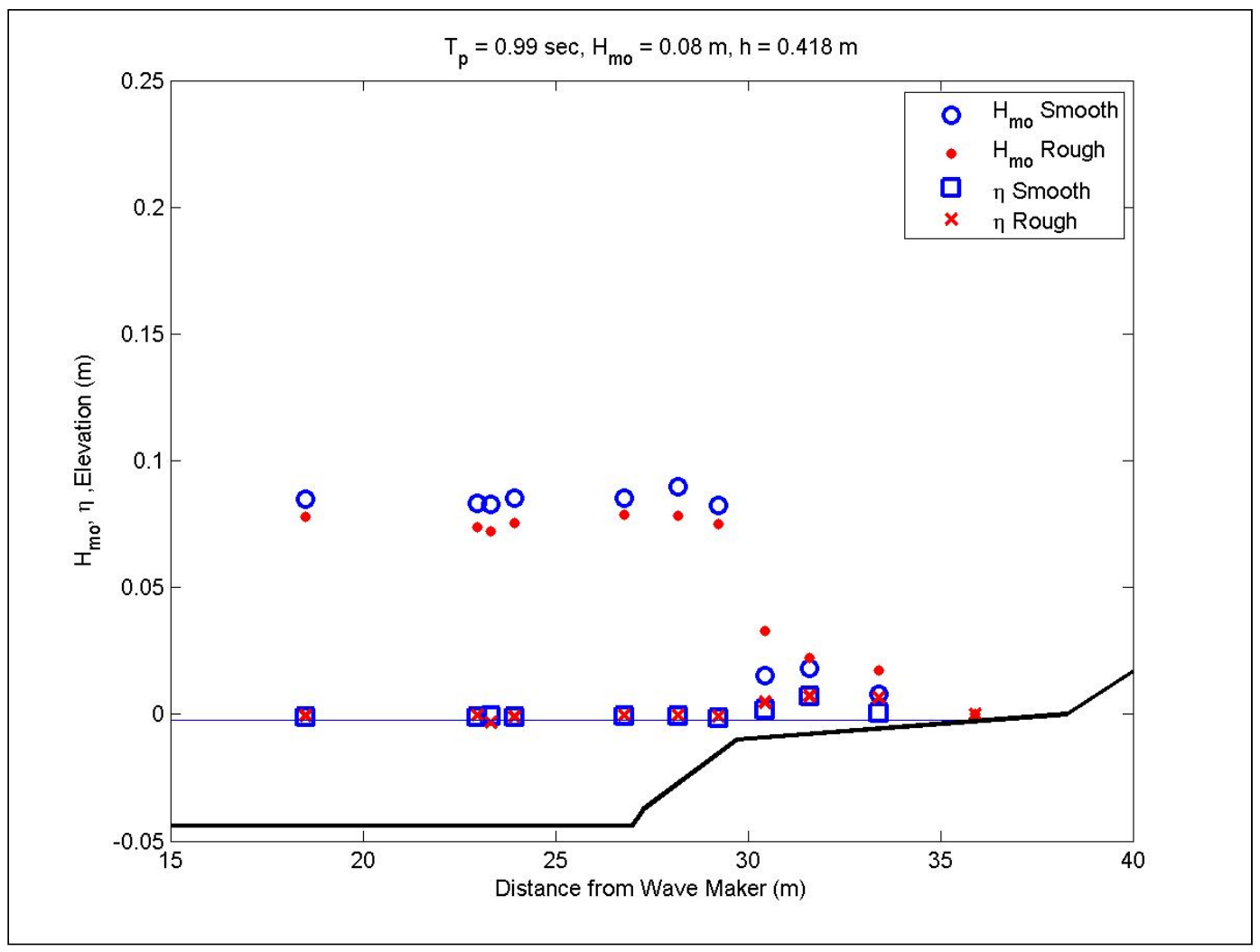

Figure A1. Wave transformation for 1-sec, 0.08-m waves at low water. 


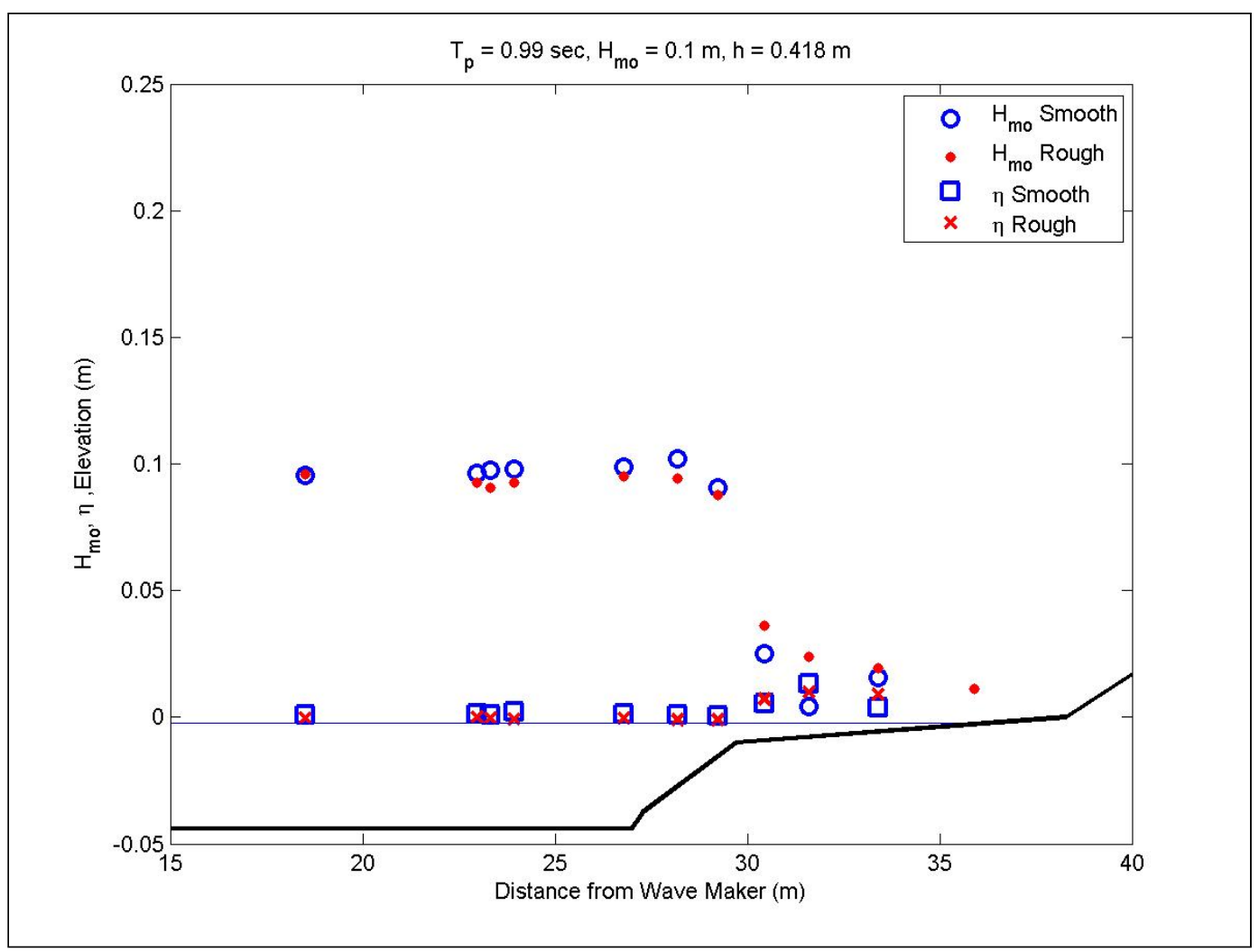

Figure A2. Wave transformation for 1-sec, 0.1-m waves at low water.

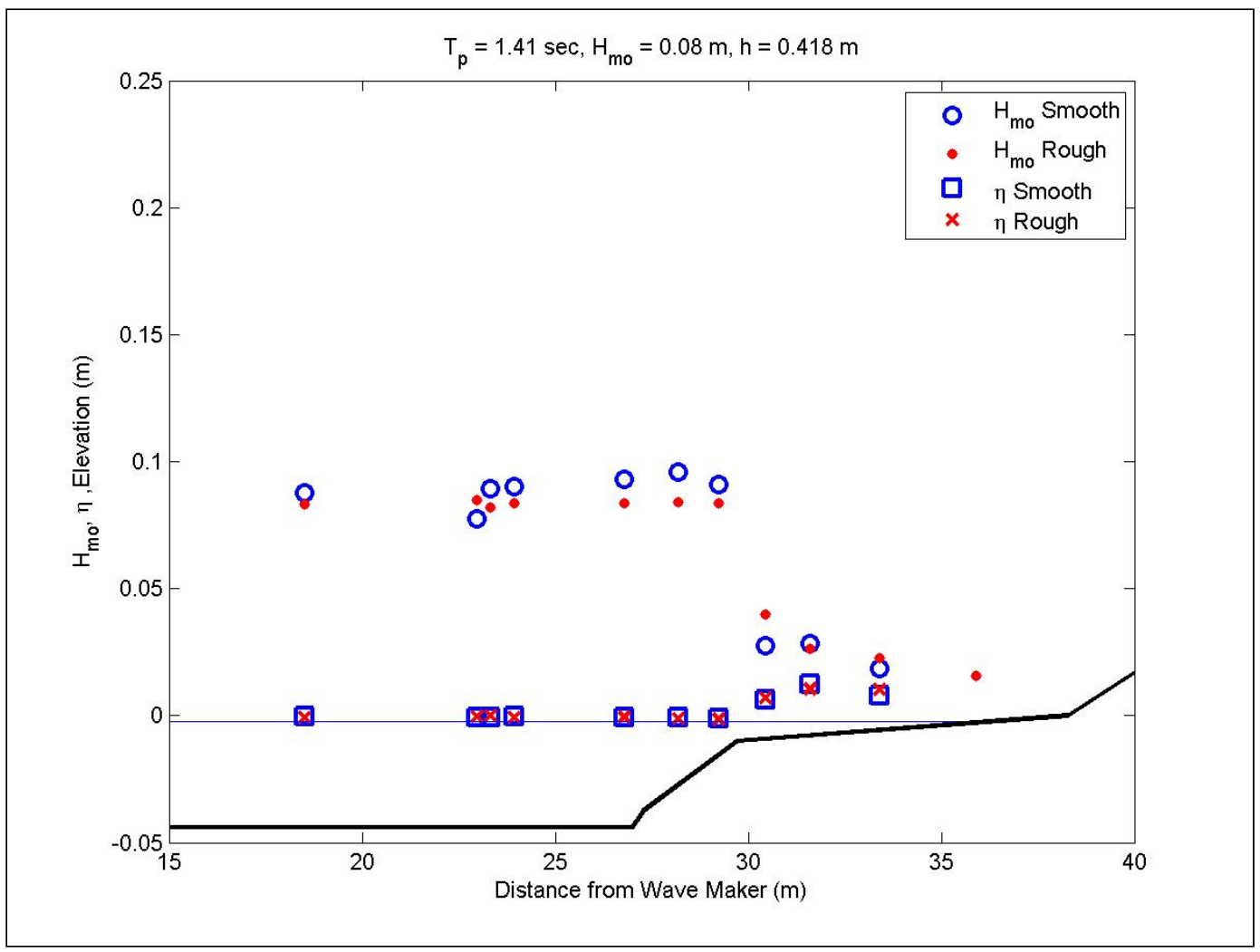

Figure A3. Wave transformation for $1.4-\mathrm{sec}, 0.08-\mathrm{m}$ waves at low water. 


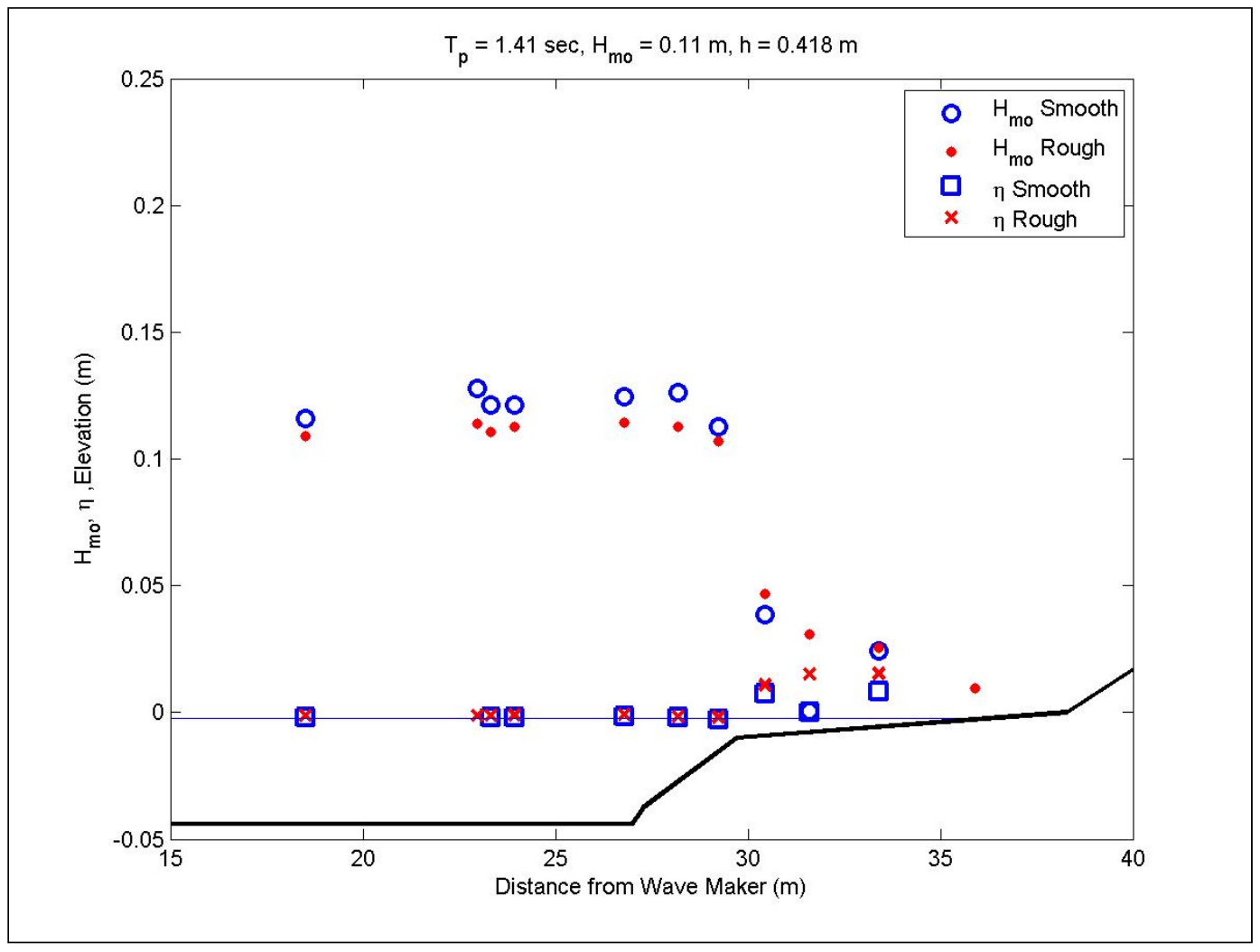

Figure A4. Wave transformation for 1.4-sec, 0.11-m waves at low water.

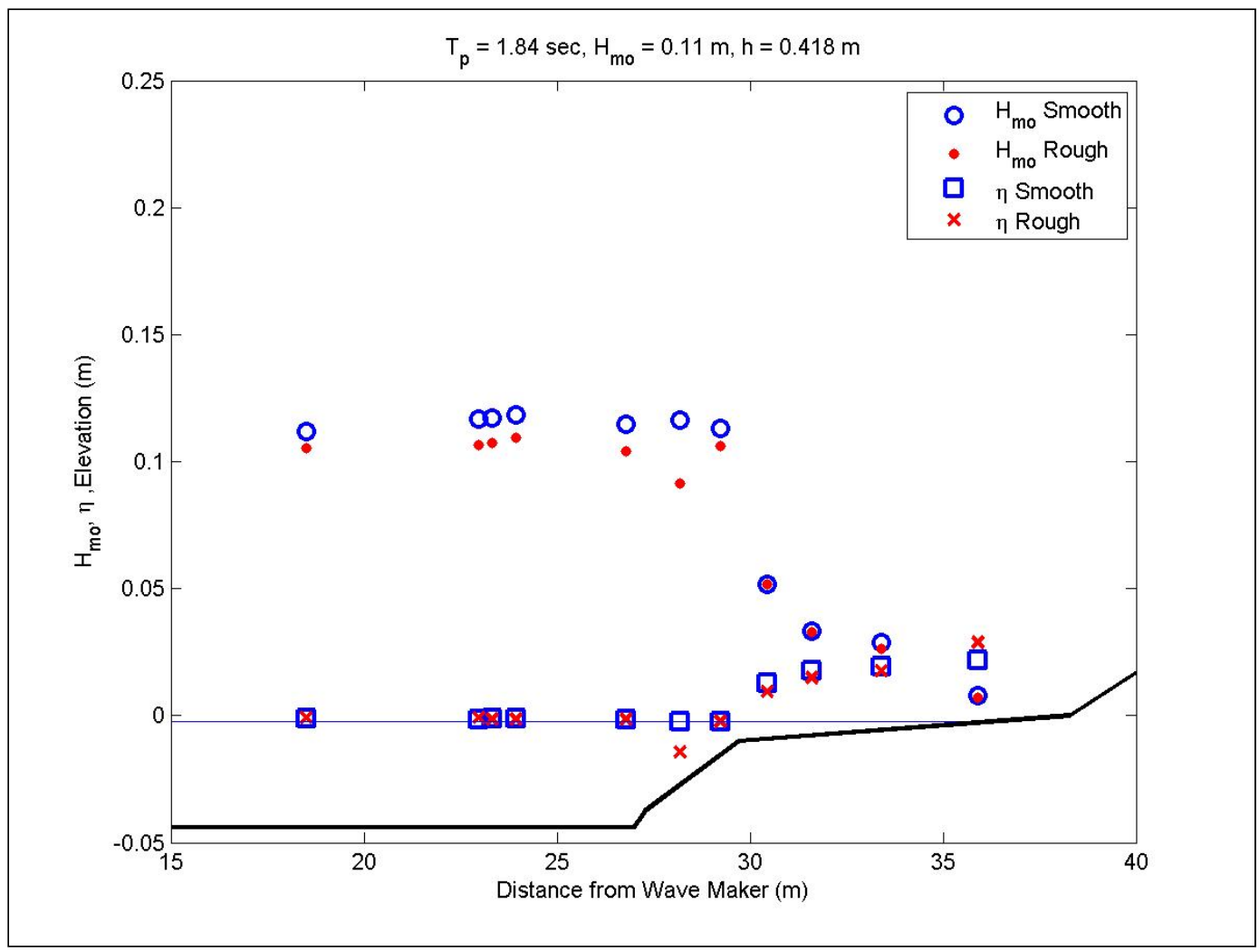

Figure A5. Wave transformation for $1.8-\mathrm{sec}, 0.11-\mathrm{m}$ waves at low water. 


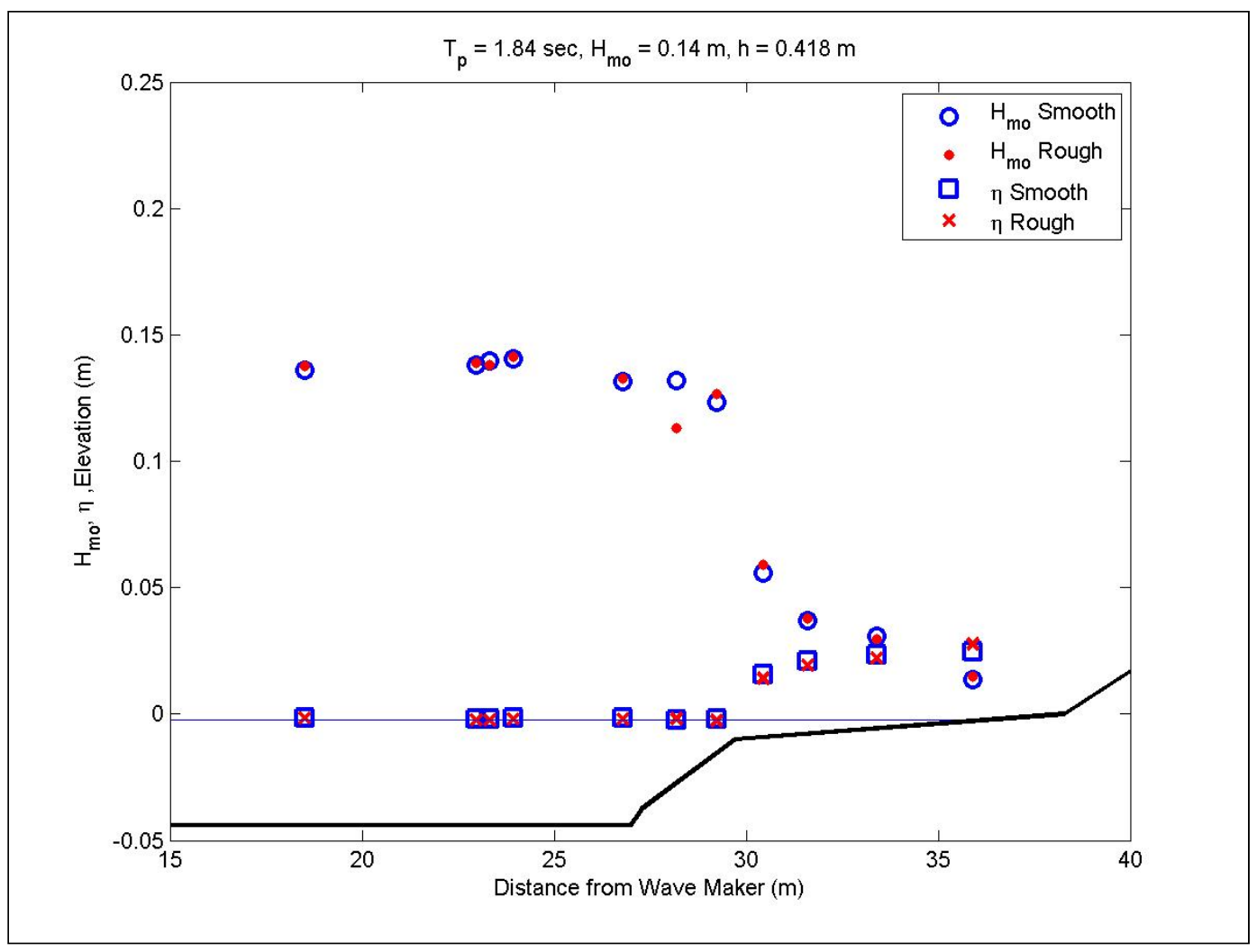

Figure A6. Wave transformation for 1.8-sec, 0.14-m waves at low water.

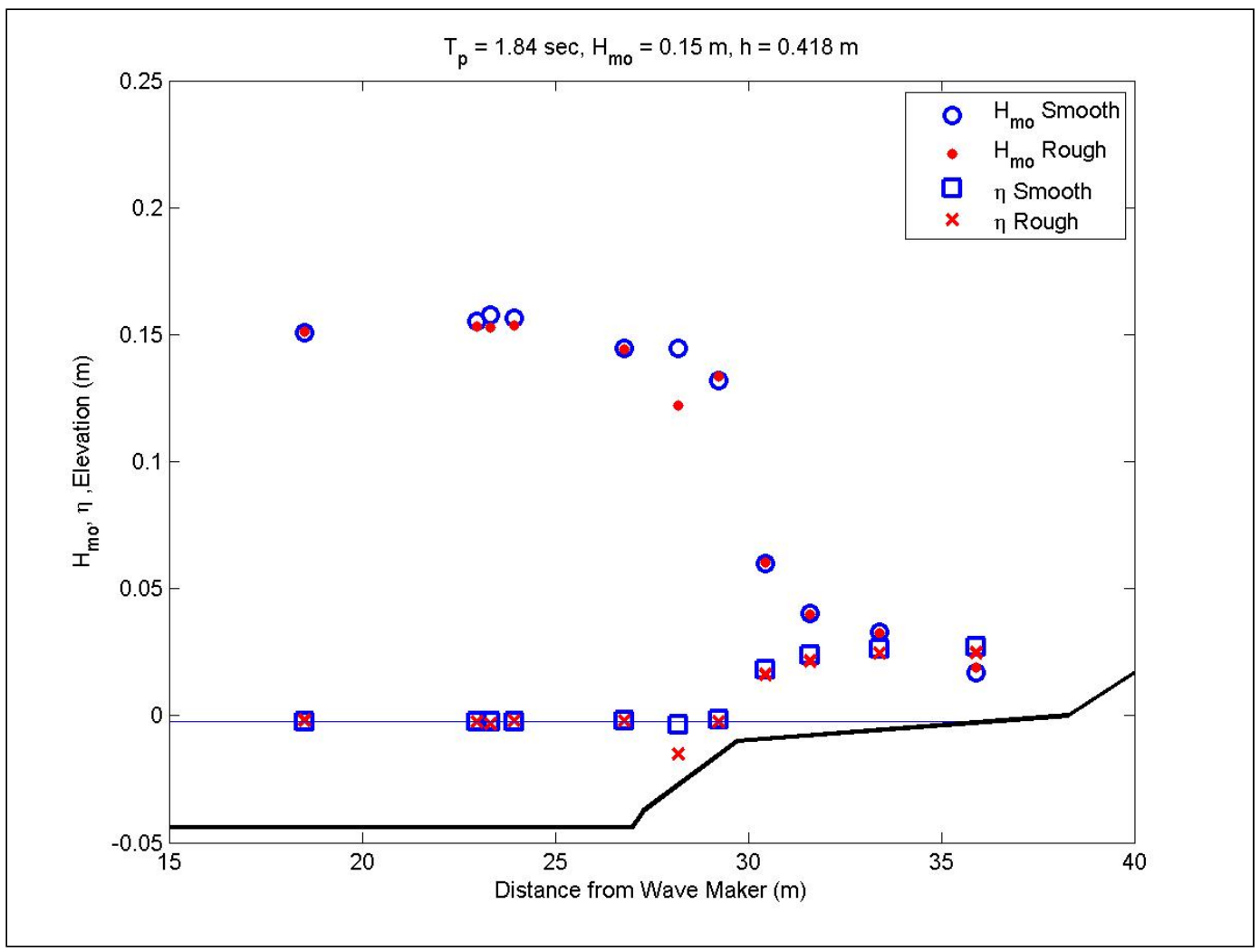

Figure A7. Wave transformation for $1.8-\mathrm{sec}, 0.15-\mathrm{m}$ waves at low water. 


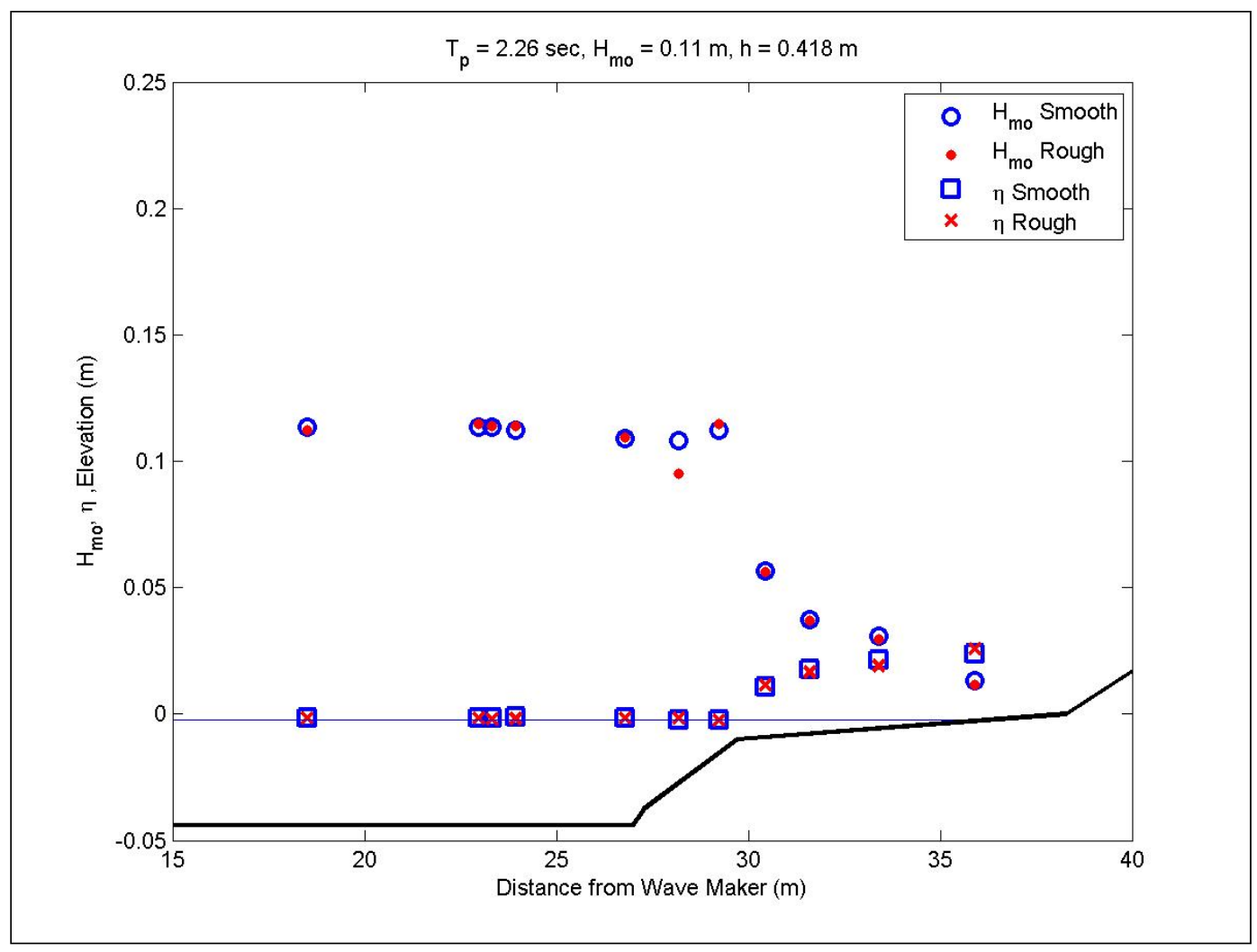

Figure A8. Wave transformation for 2.3-sec, 0.11-m waves at low water.

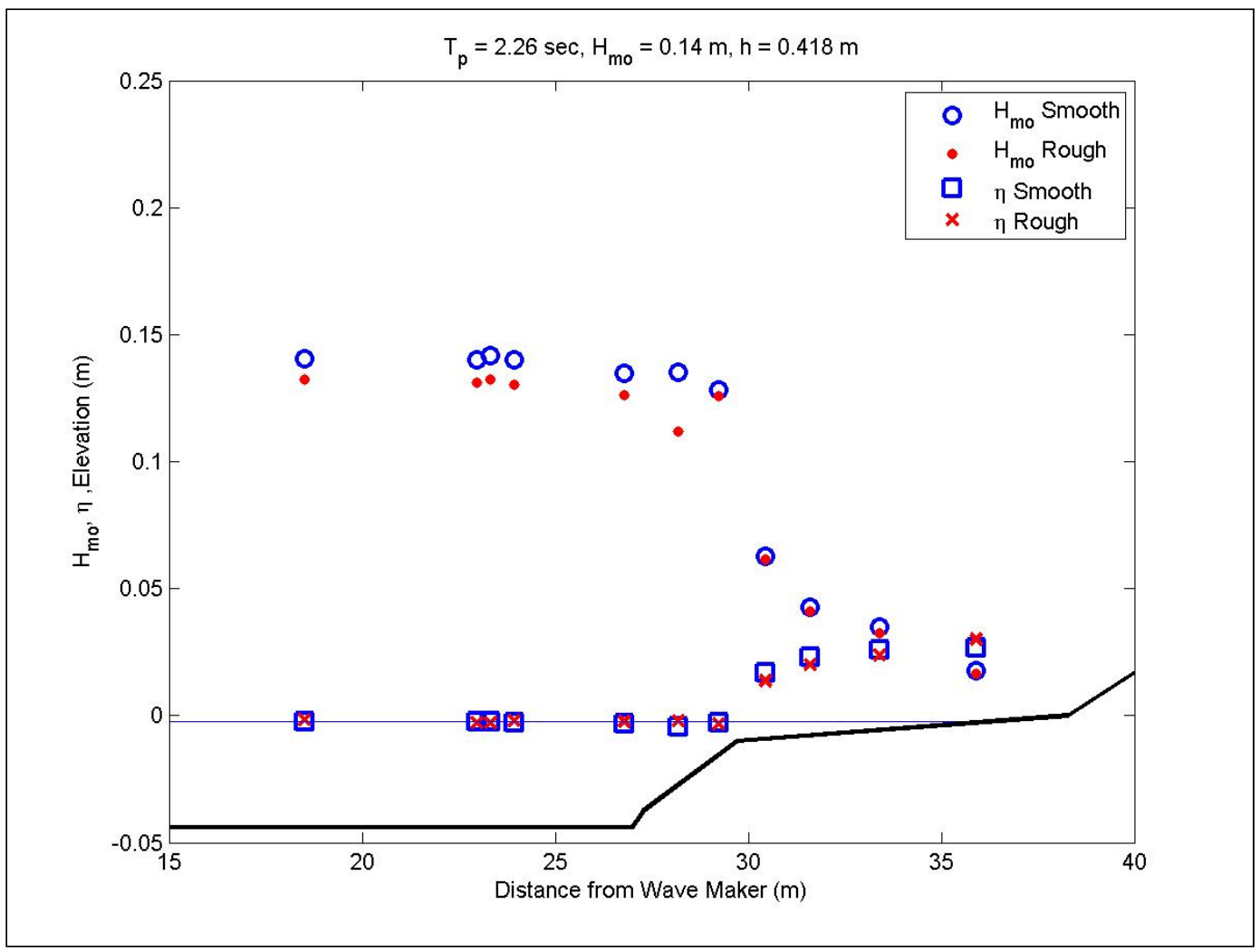

Figure A9. Wave transformation for $2.3-\mathrm{sec}, 0.14-\mathrm{m}$ waves at low water. 


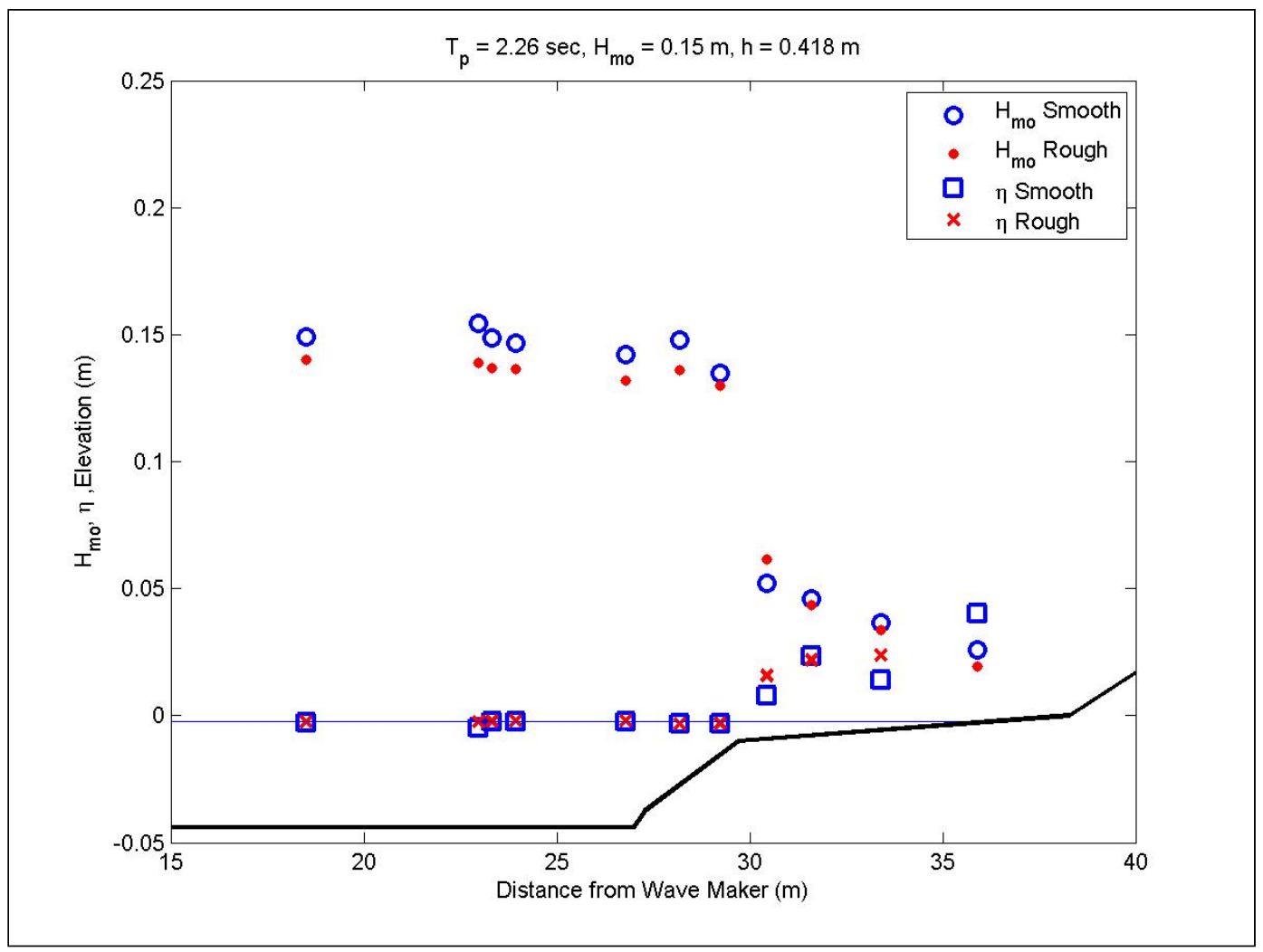

Figure A10. Wave transformation for 2.3-sec, $0.15-\mathrm{m}$ waves at low water.

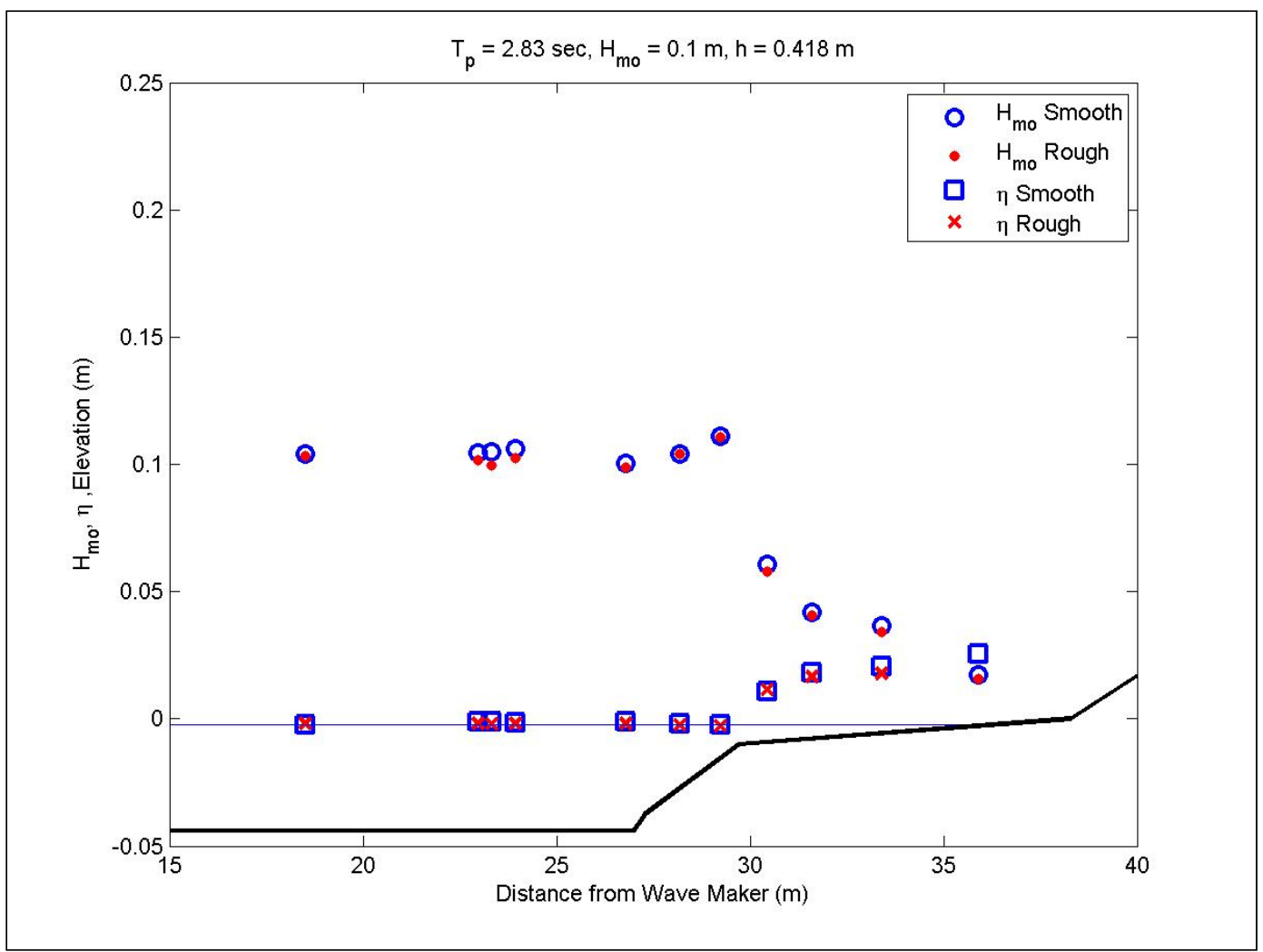

Figure A11. Wave transformation for 2.8-sec, 0.1-m waves at low water. 


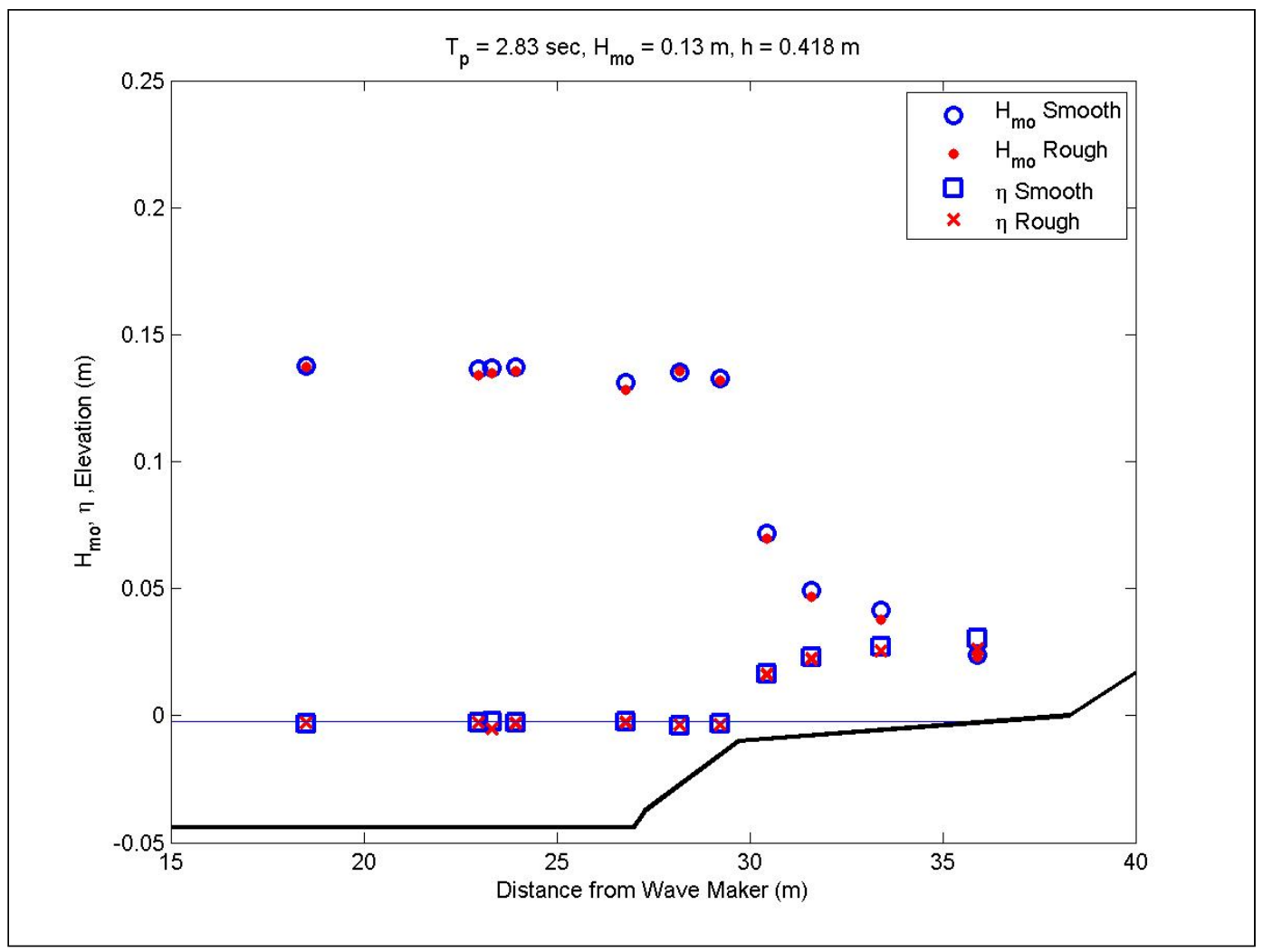

Figure A12. Wave transformation for 2.8-sec, 0.13-m waves at low water.

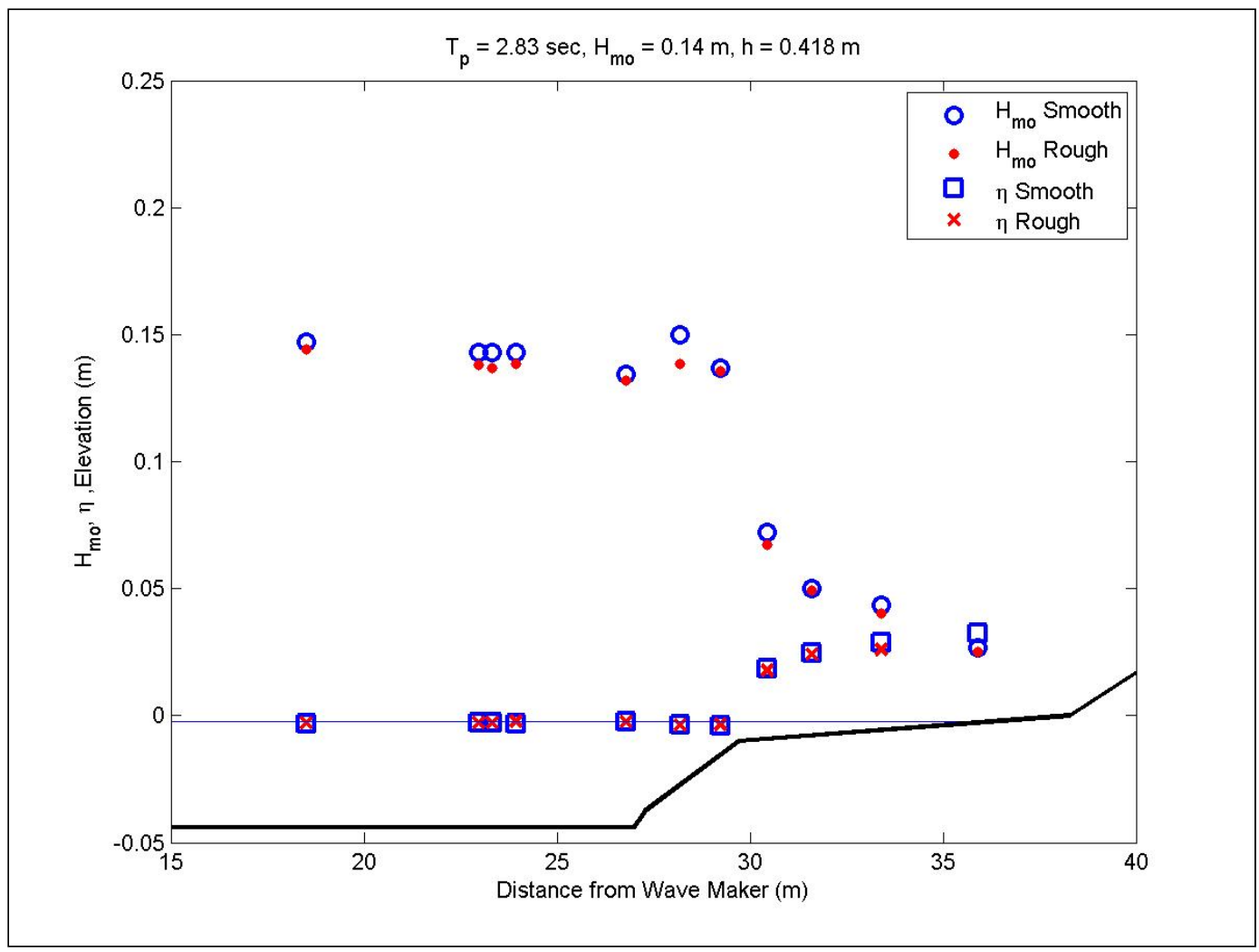

Figure A13. Wave transformation for 2.8-sec, 0.14-m waves at low water. 


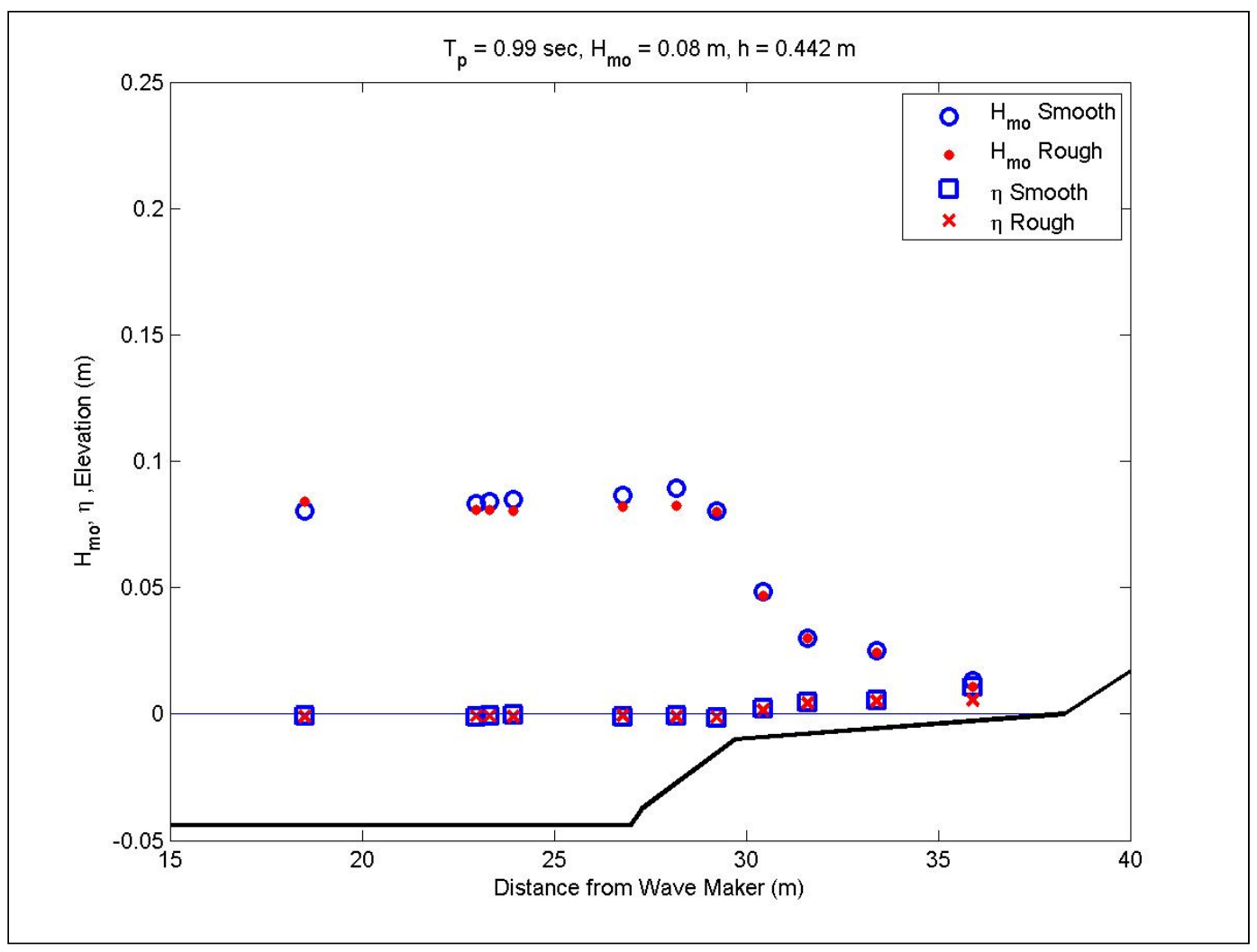

Figure A14. Wave transformation for 1-sec, 0.08-m waves at mid water.

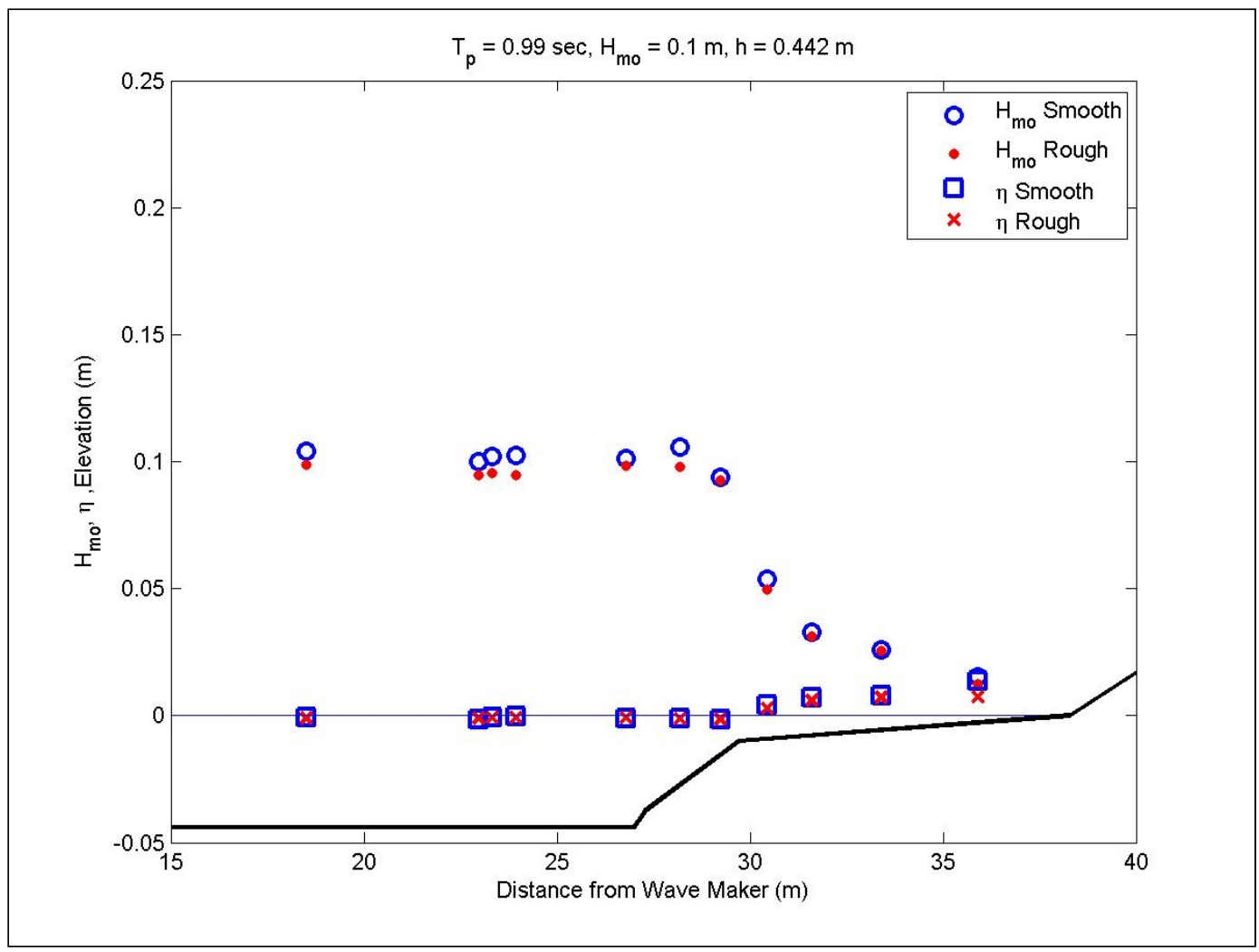

Figure A15. Wave transformation for 1-sec, 0.1-m waves at mid water. 


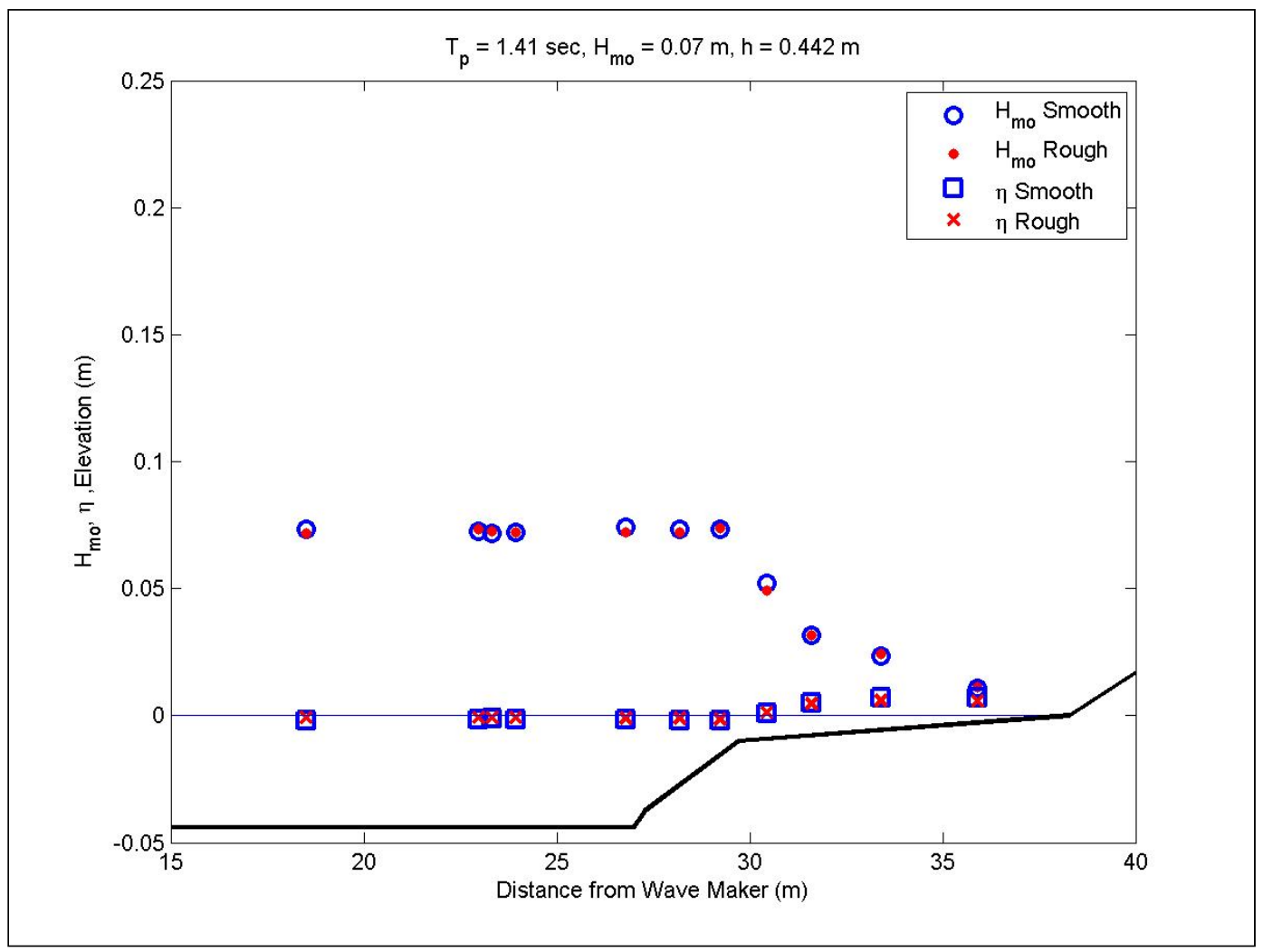

Figure A16. Wave transformation for 1.4-sec, 0.07-m waves at mid water.

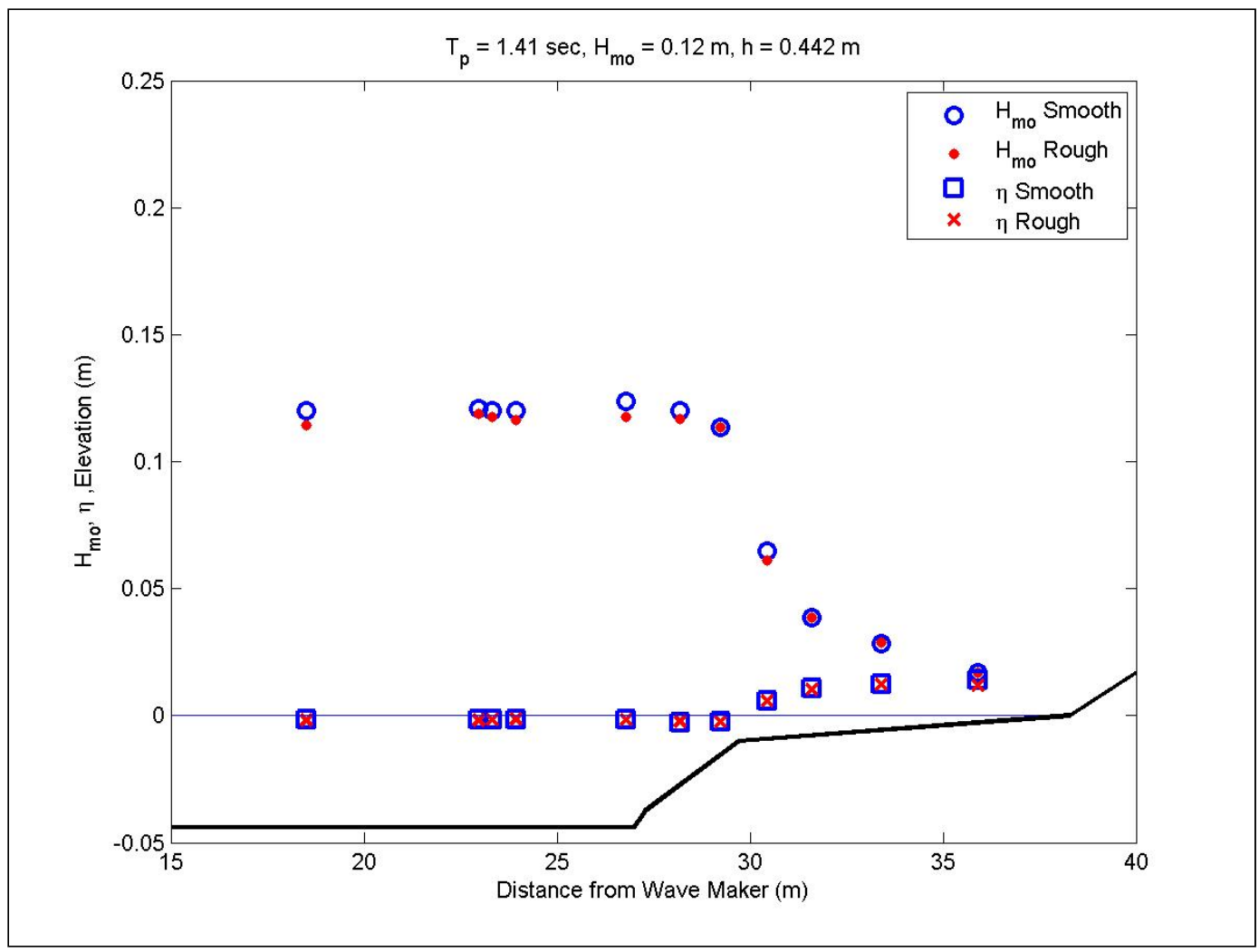

Figure A17. Wave transformation for 1.4-sec, 0.12-m waves at mid water. 


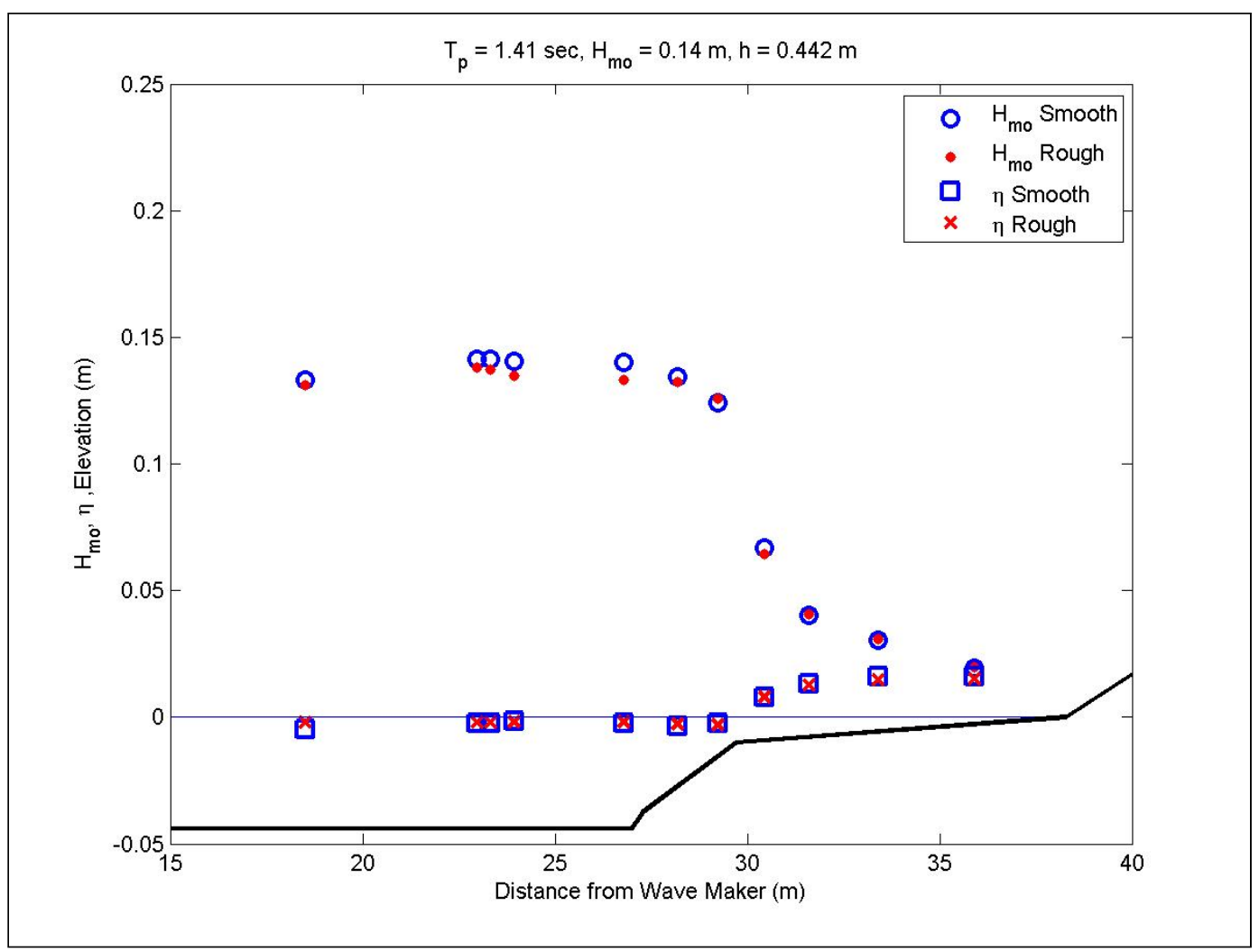

Figure A18. Wave transformation for 1.4-sec, 0.14-m waves at mid water.

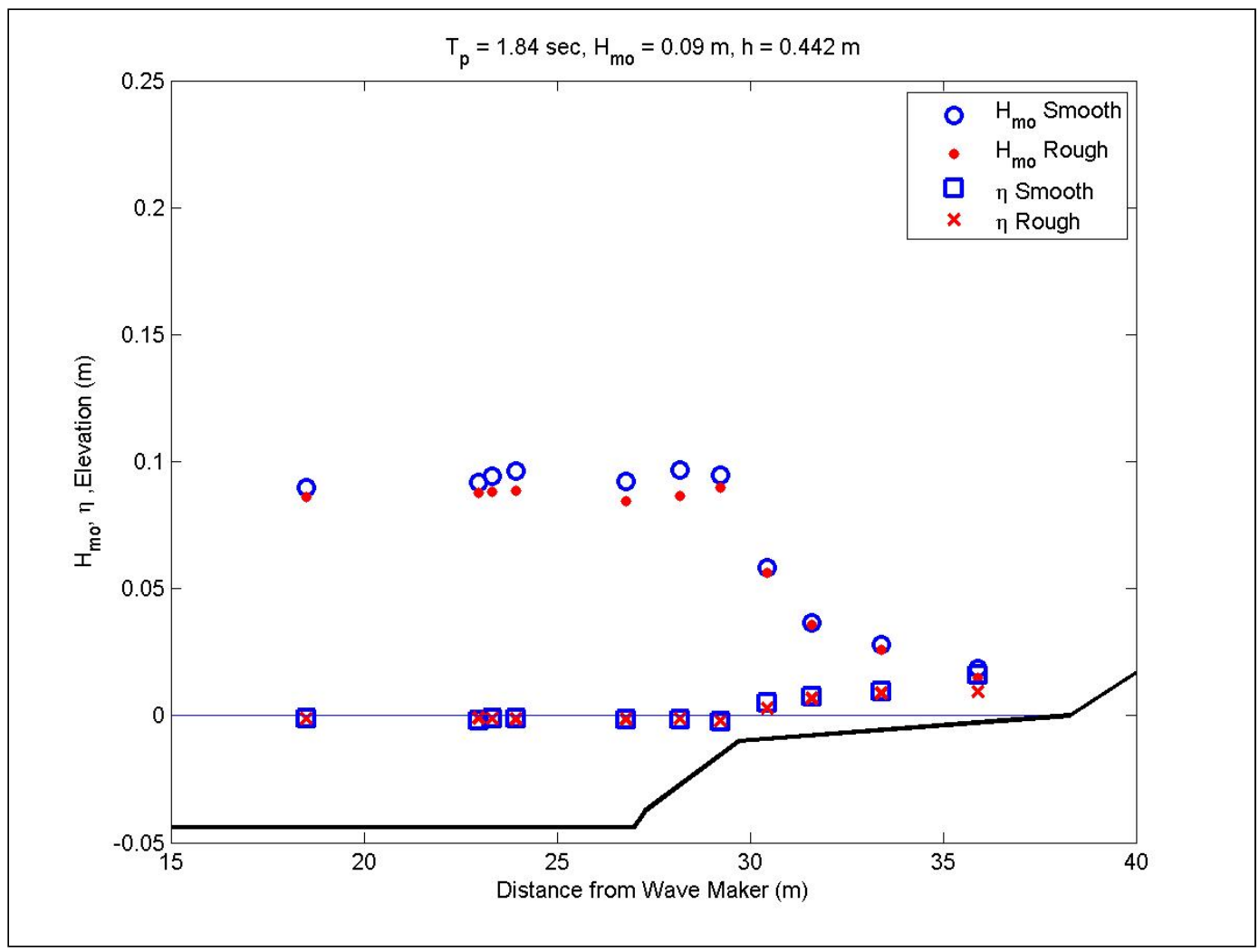

Figure A19. Wave transformation for 1.8-sec, 0.09-m waves at mid water. 


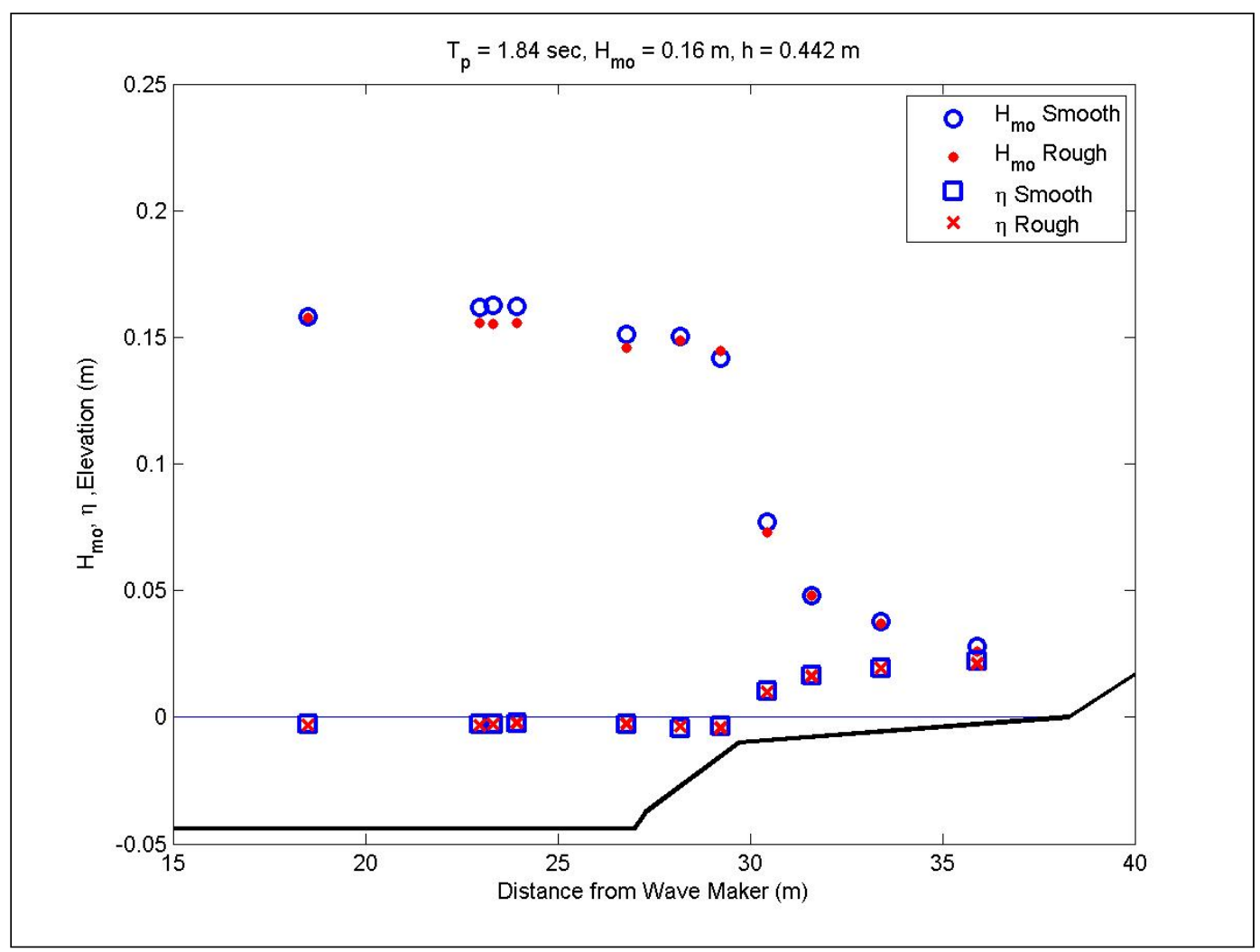

Figure A20. Wave transformation for 1.8-sec, 0.16-m waves at mid water.

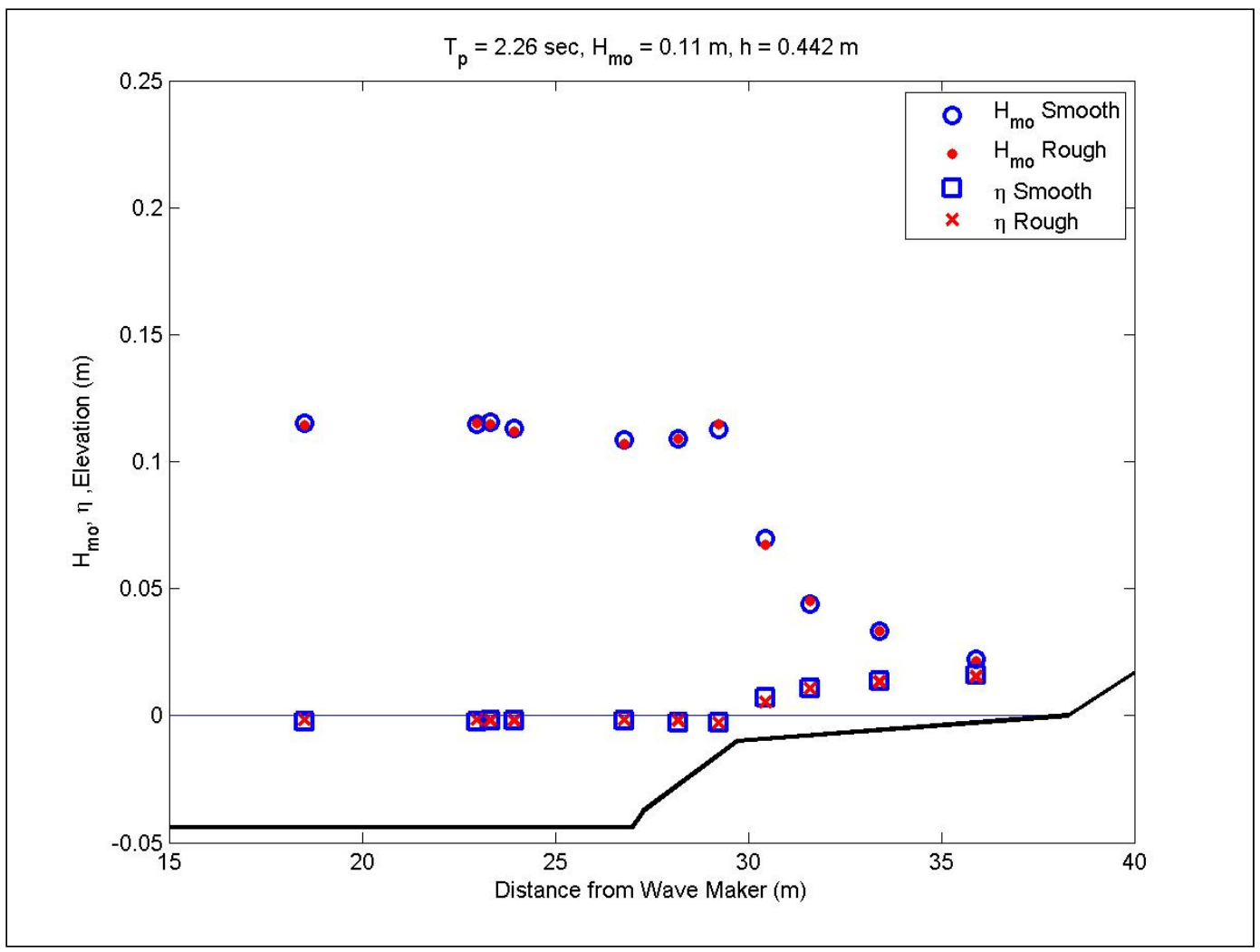

Figure A21. Wave transformation for 2.3-sec, 0.11-m waves at mid water. 


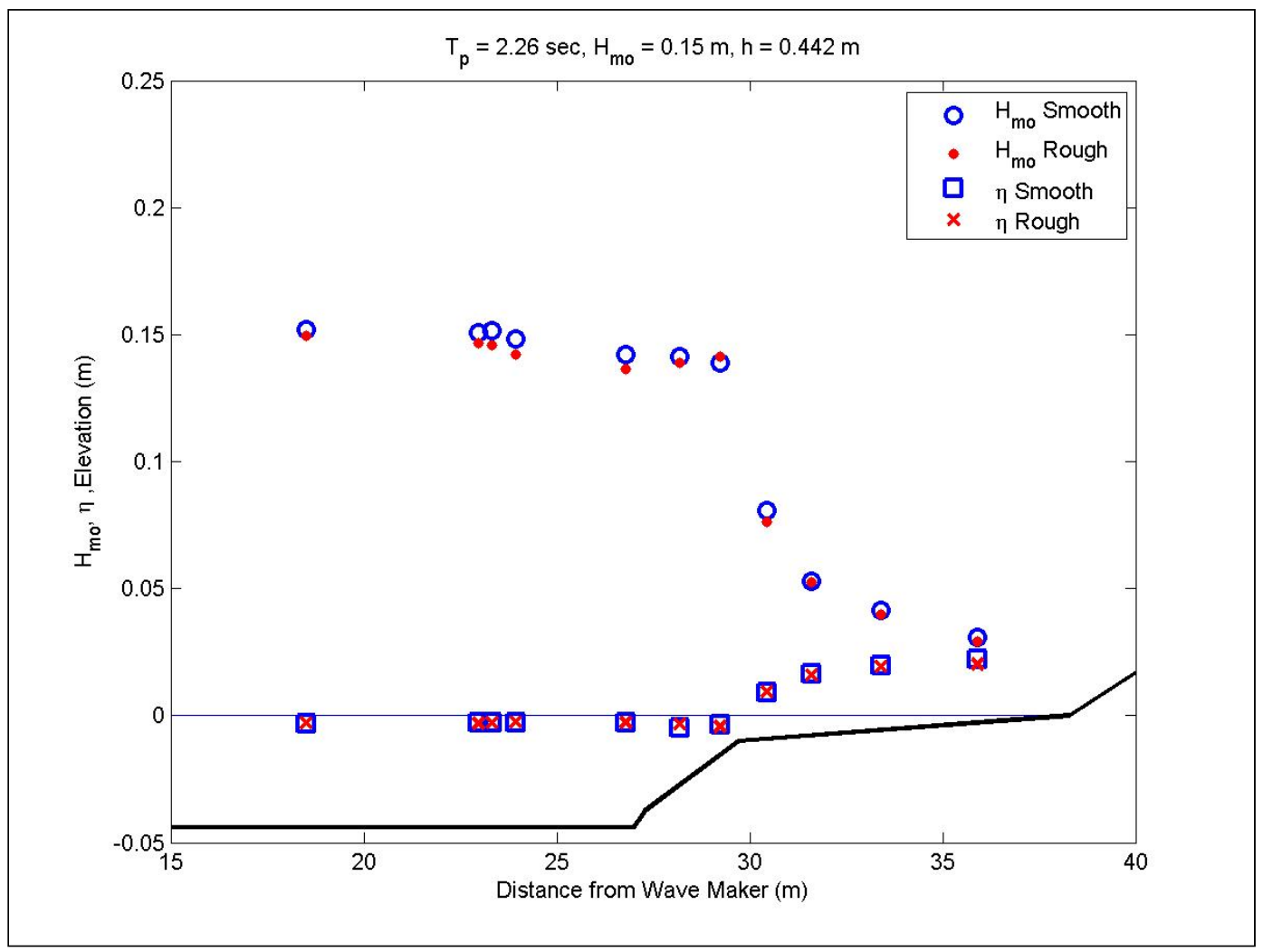

Figure A22. Wave transformation for 2.3-sec, 0.15-m waves at mid water.

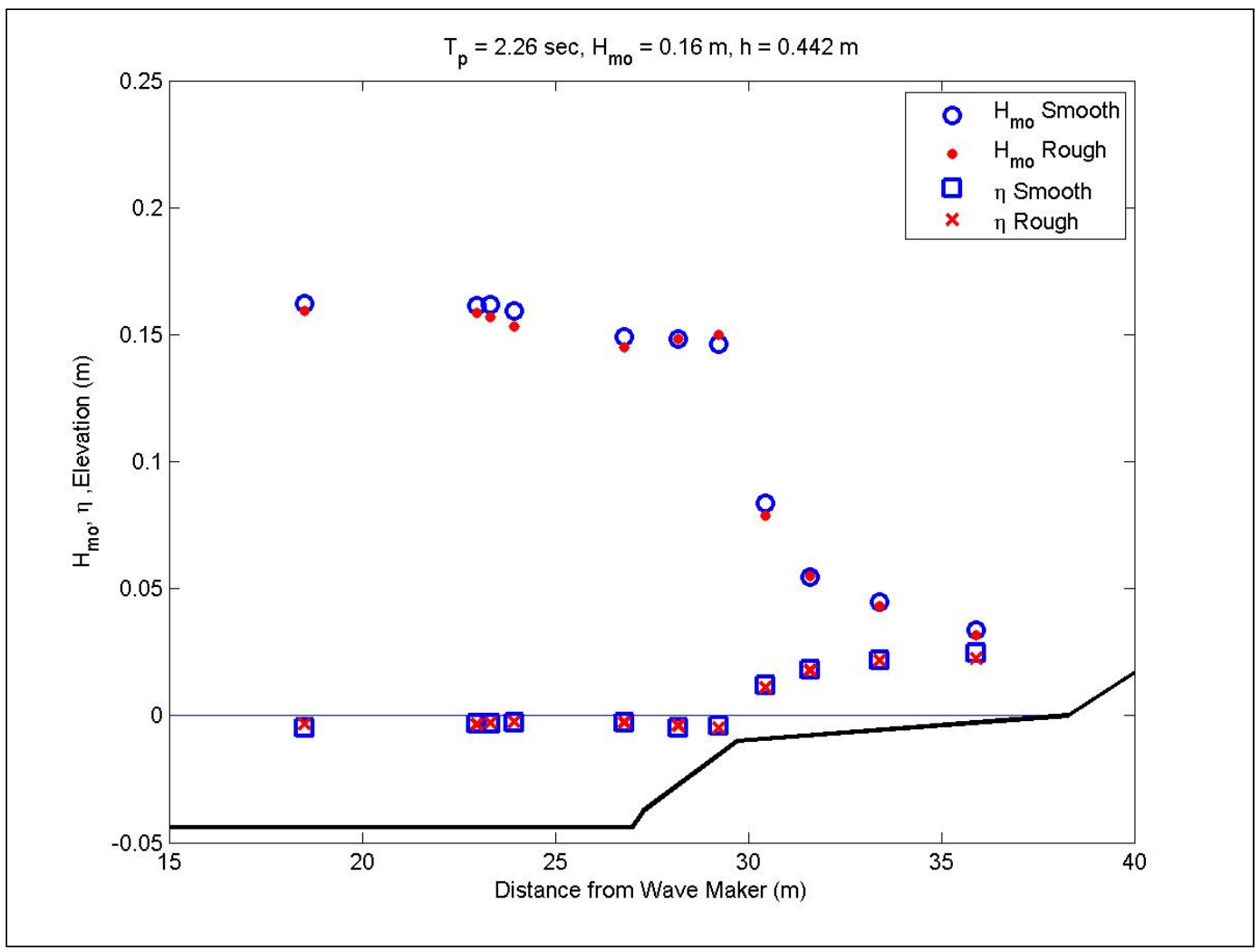

Figure A23. Wave transformation for 2.3-sec, 0.16-m waves at mid water. 


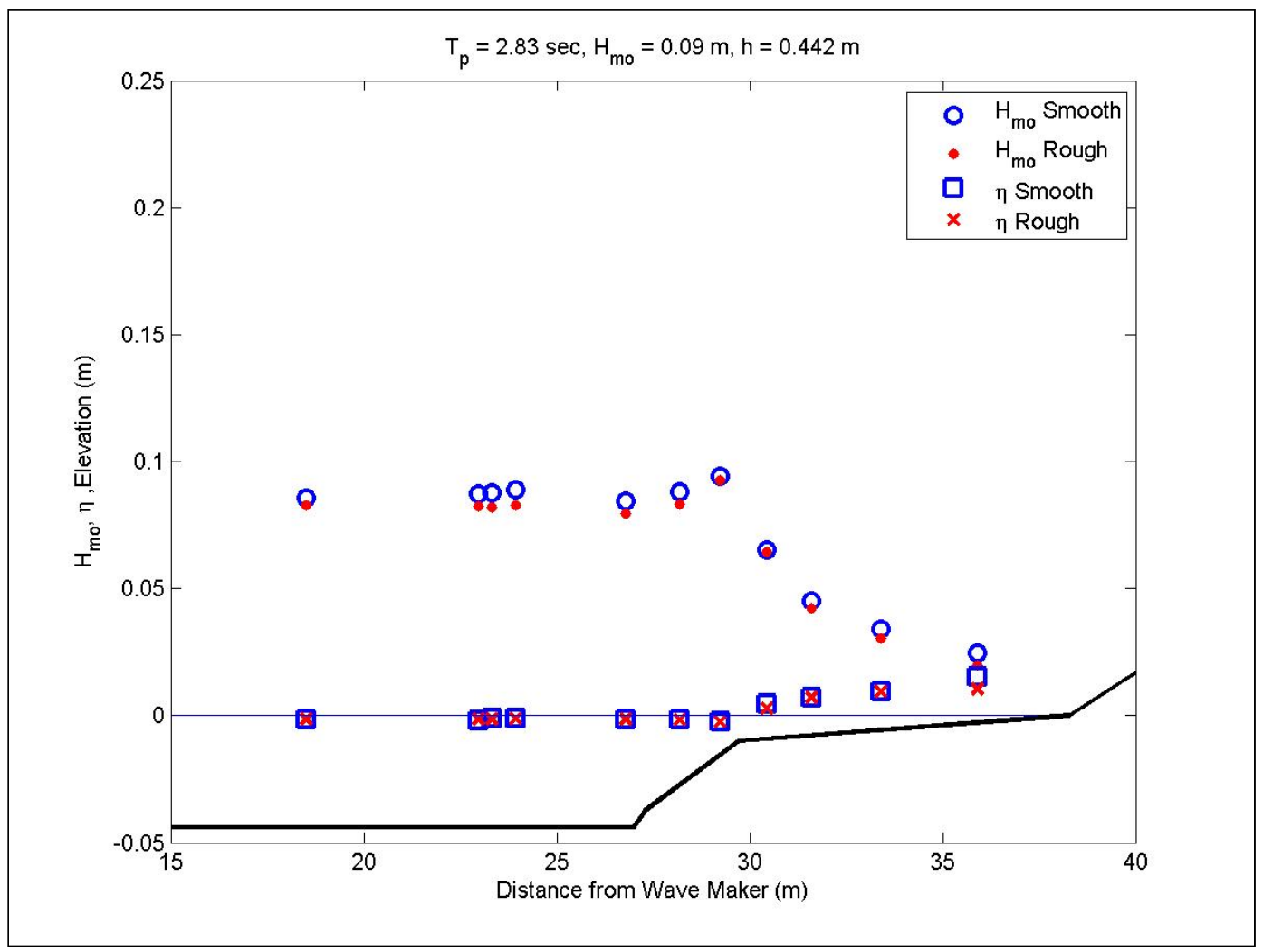

Figure A24. Wave transformation for 2.8-sec, 0.09-m waves at mid water.

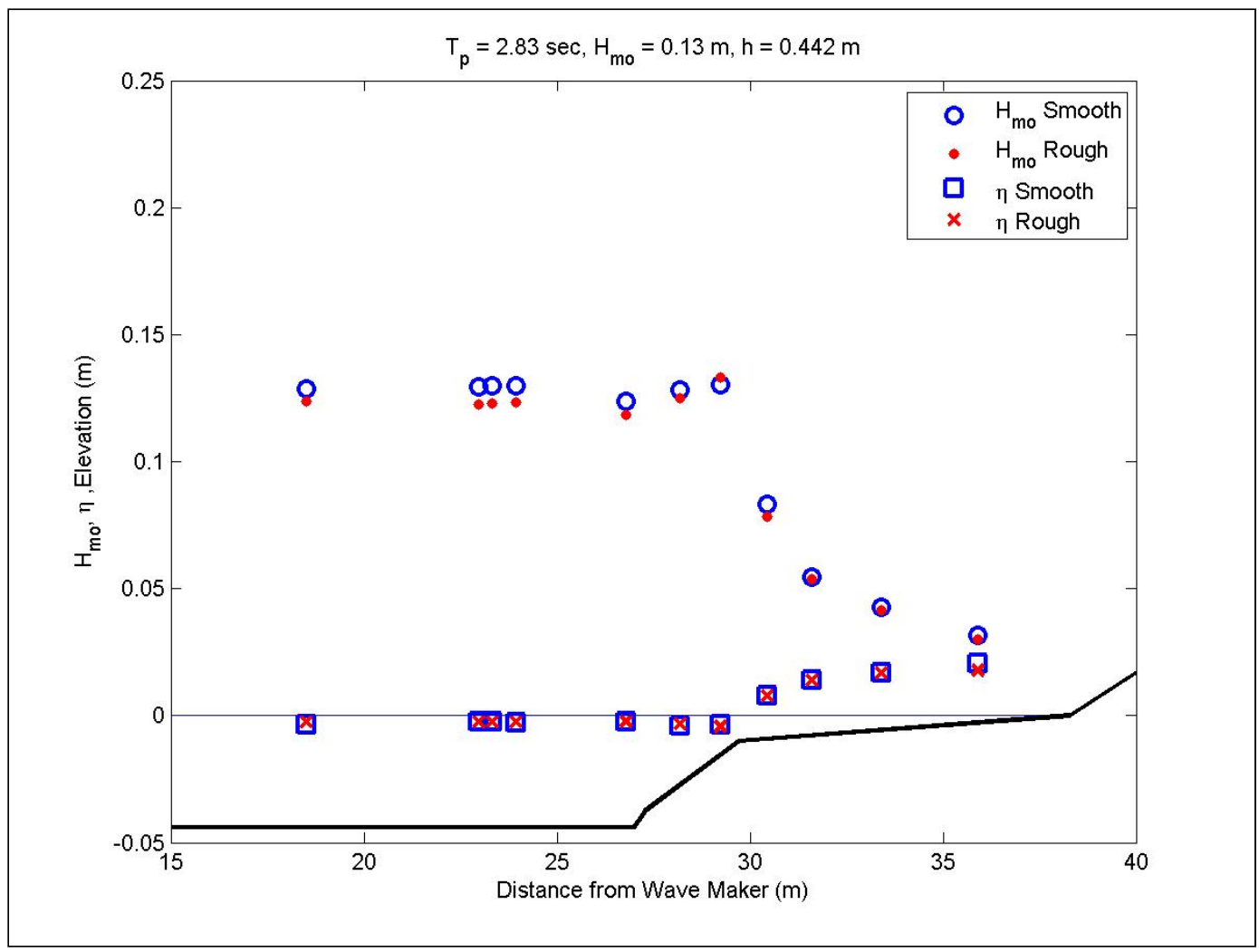

Figure A25. Wave transformation for 2.8-sec, 0.13-m waves at mid water. 


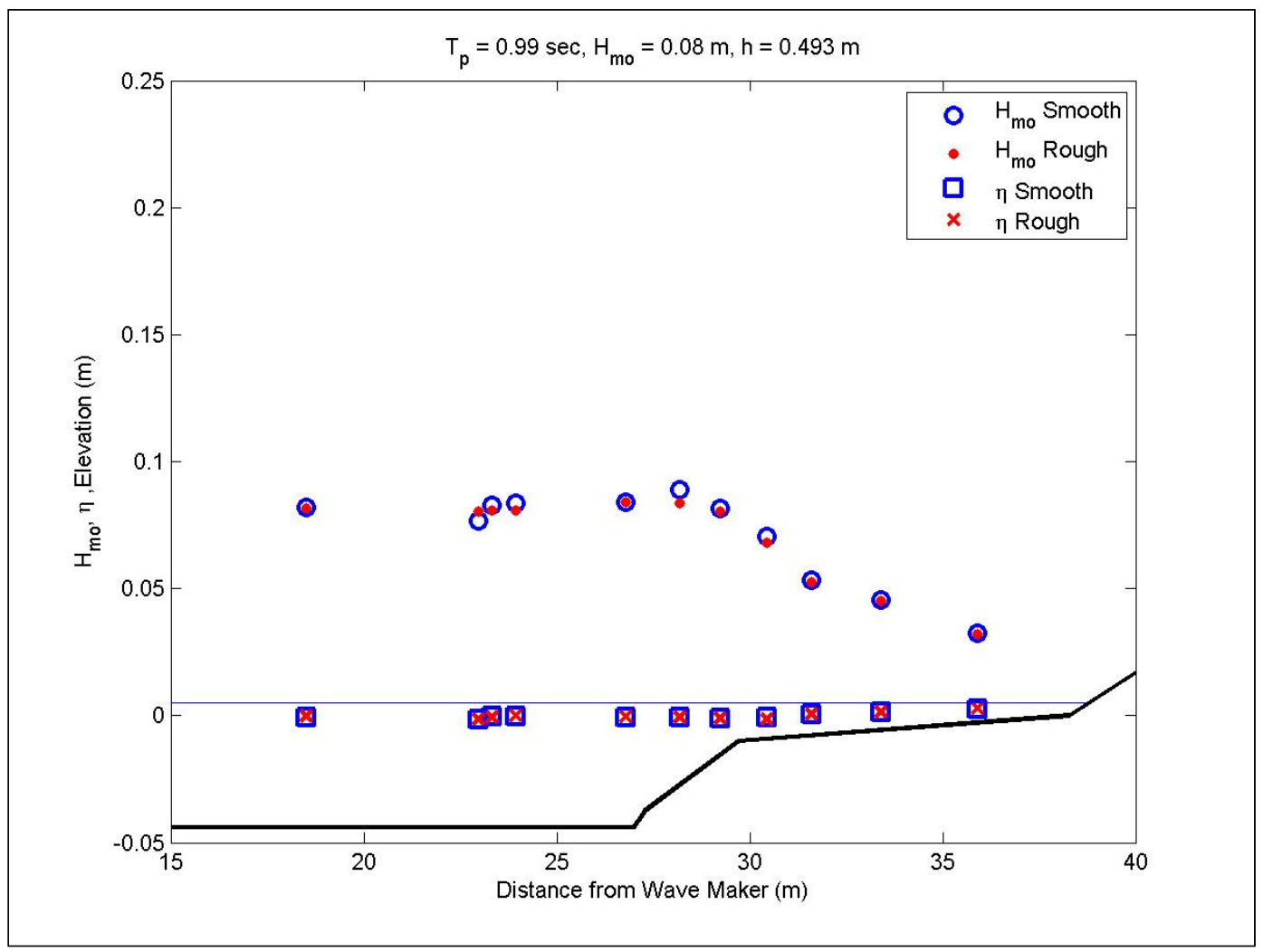

Figure A26. Wave transformation for 1-sec, 0.08-m waves at high water.

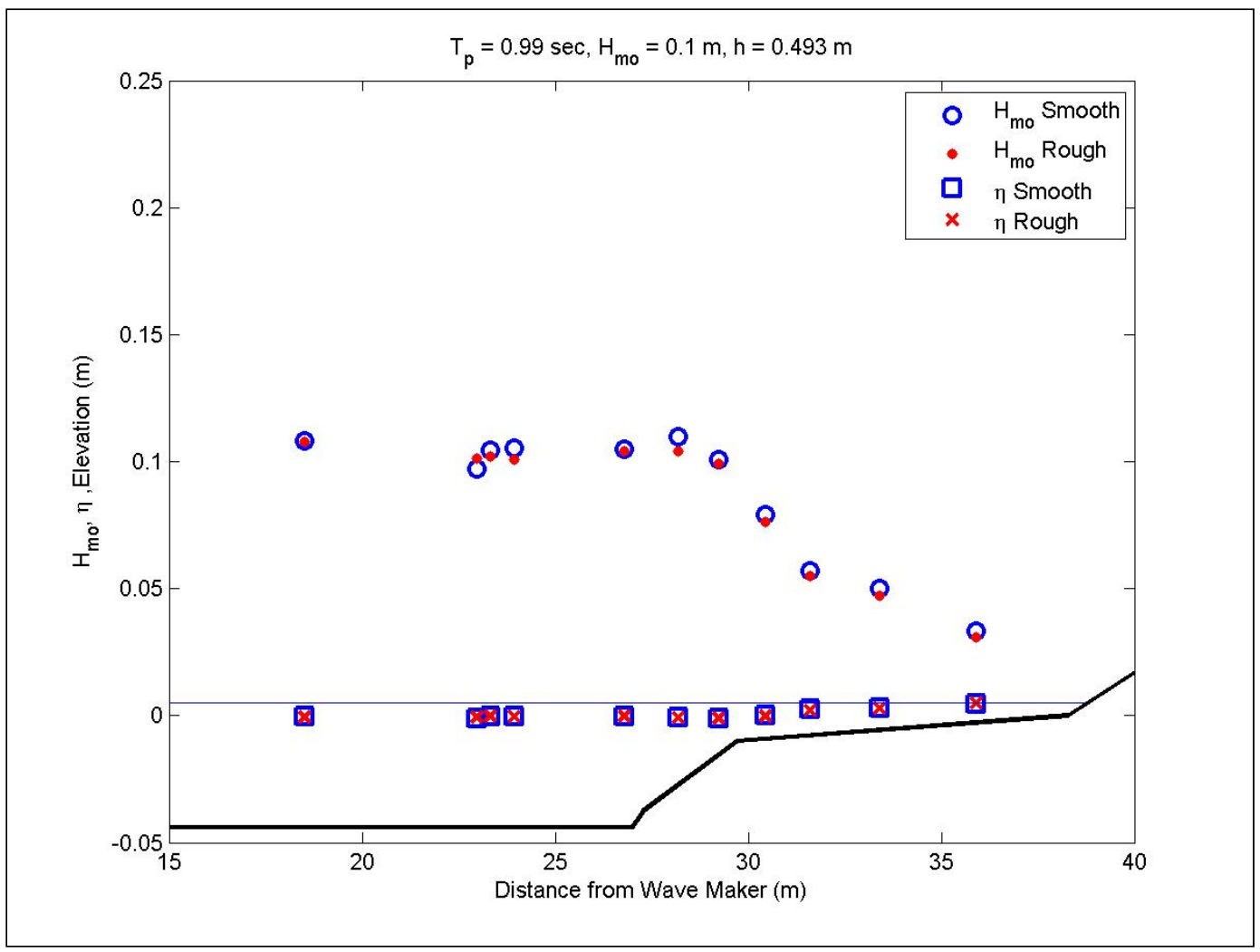

Figure A27. Wave transformation for 1-sec, 0.1-m waves at high water. 


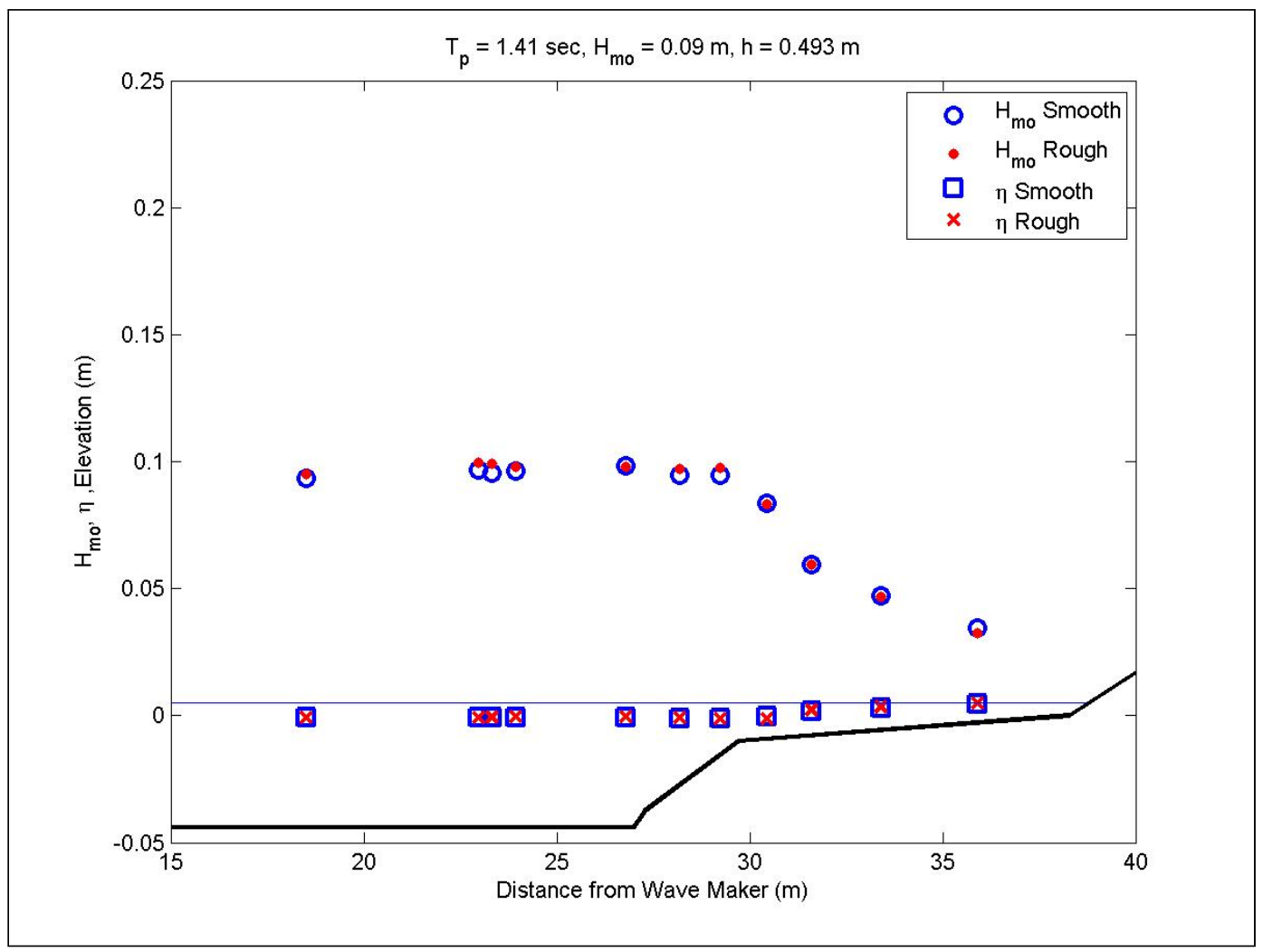

Figure A28. Wave transformation for 1.4-sec, 0.09-m waves at high water.

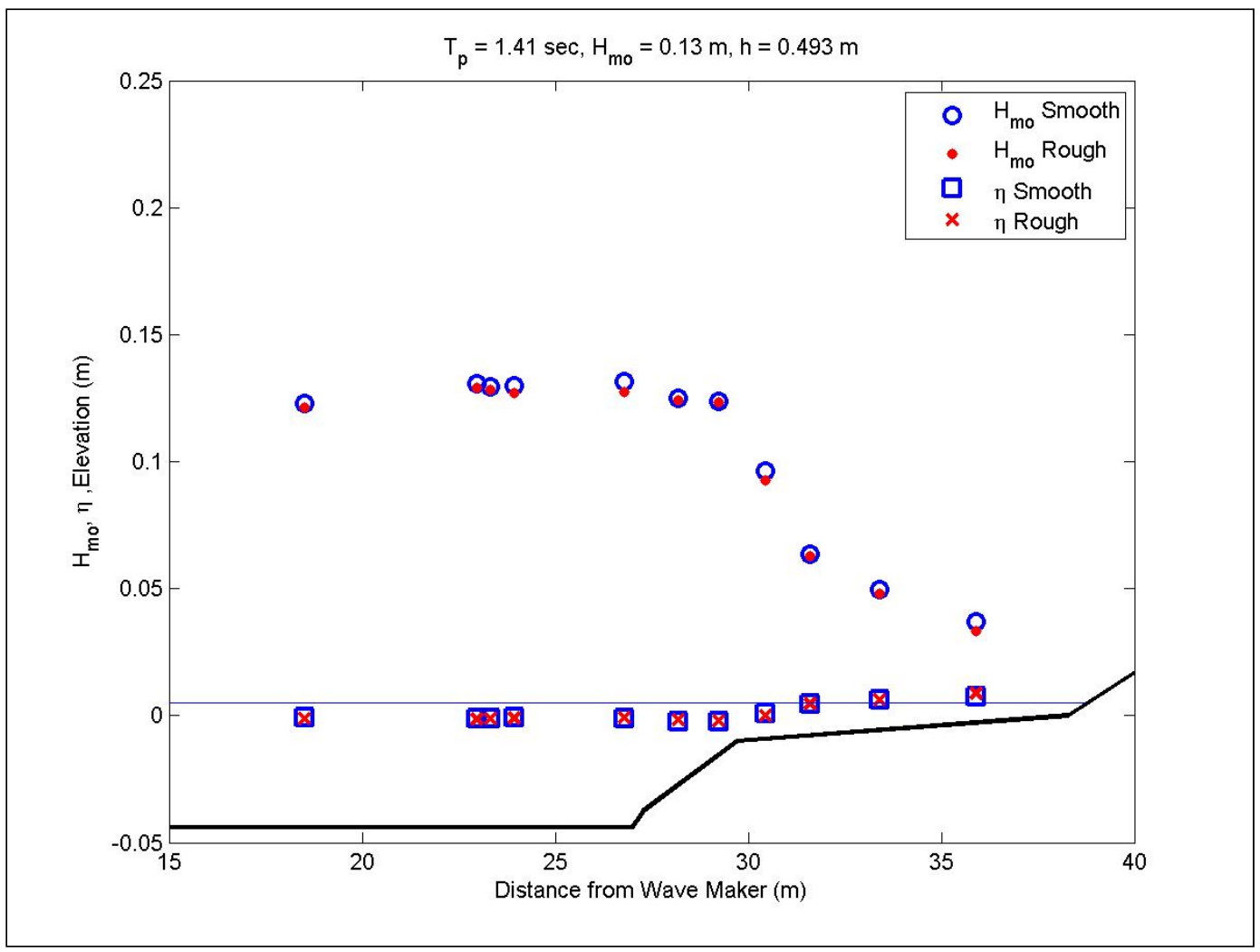

Figure A29. Wave transformation for 1.4-sec, 0.13-m waves at high water. 


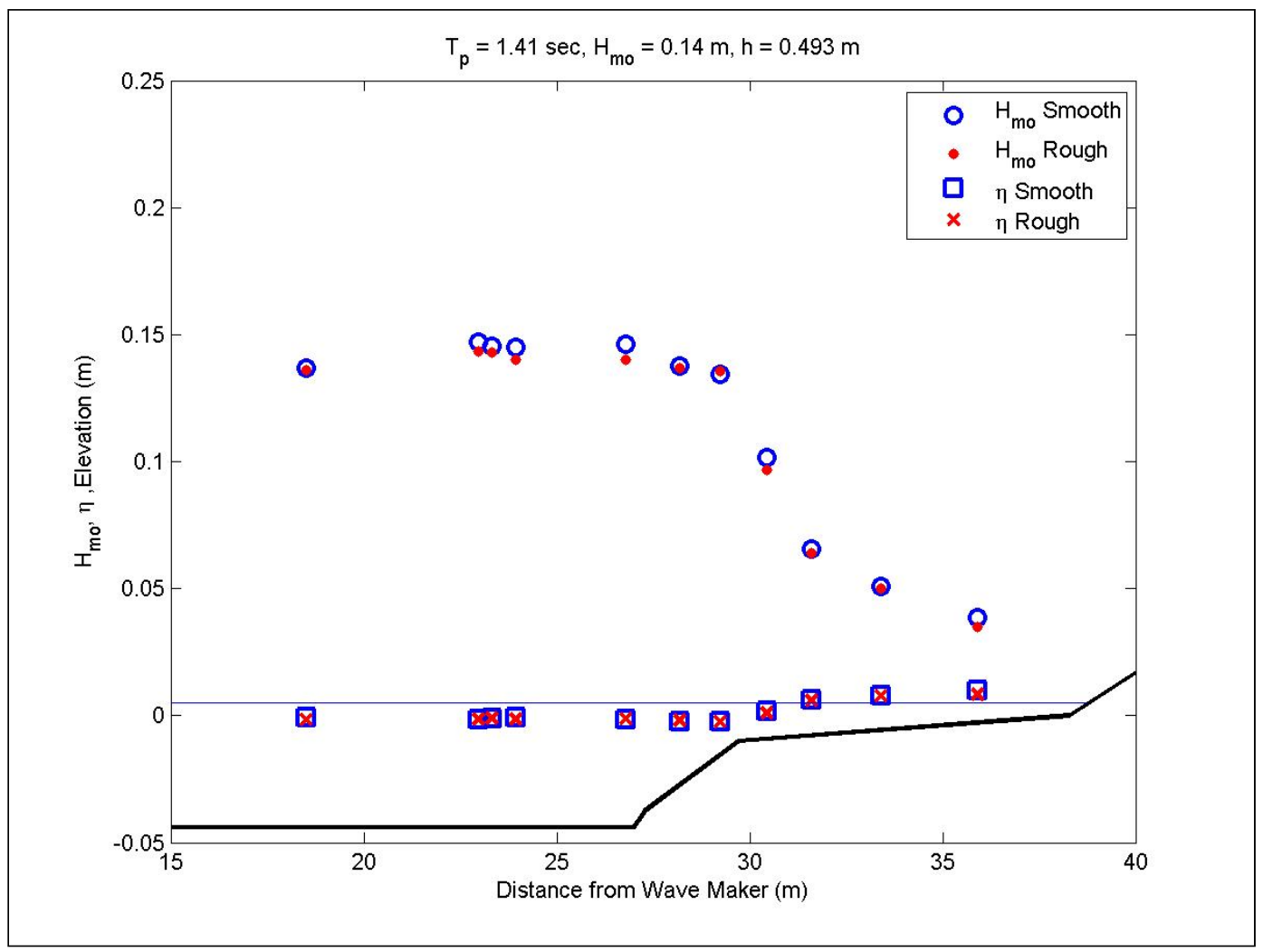

Figure A30. Wave transformation for 1.4-sec, 0.14-m waves at high water.

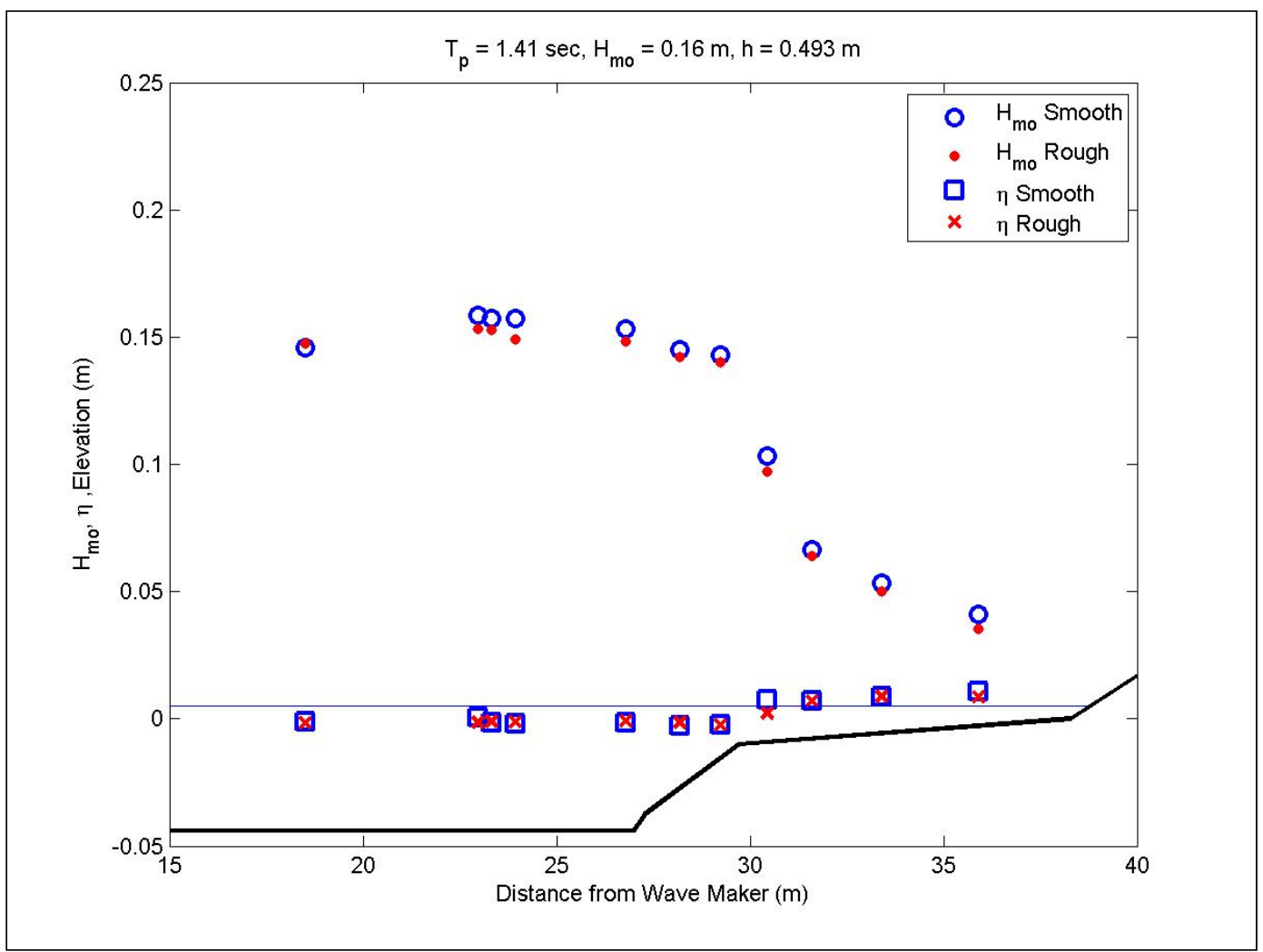

Figure A31. Wave transformation for 1.4-sec, 0.16-m waves at high water. 


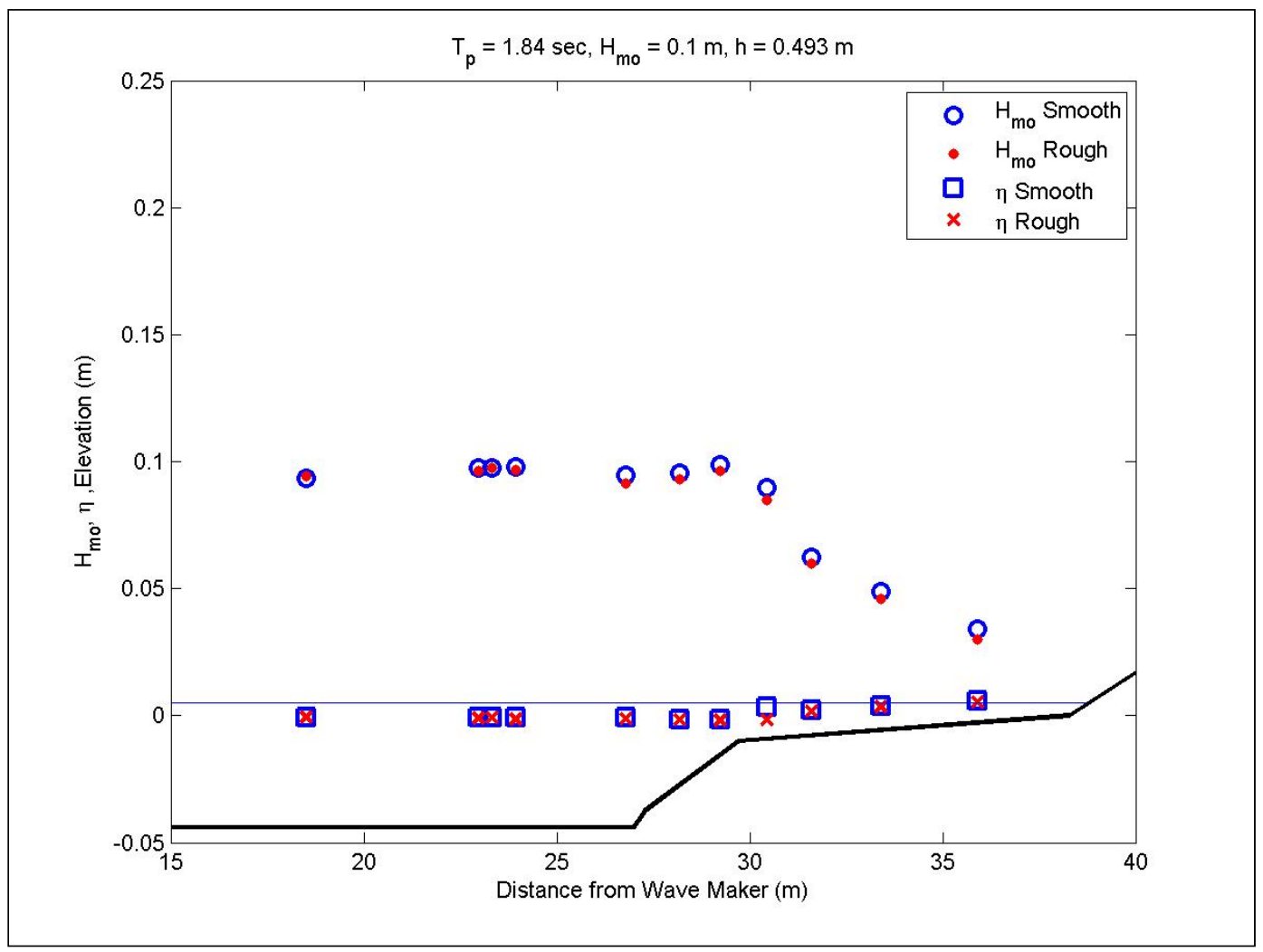

Figure A32. Wave transformation for 1.8-sec, 0.1-m waves at high water.

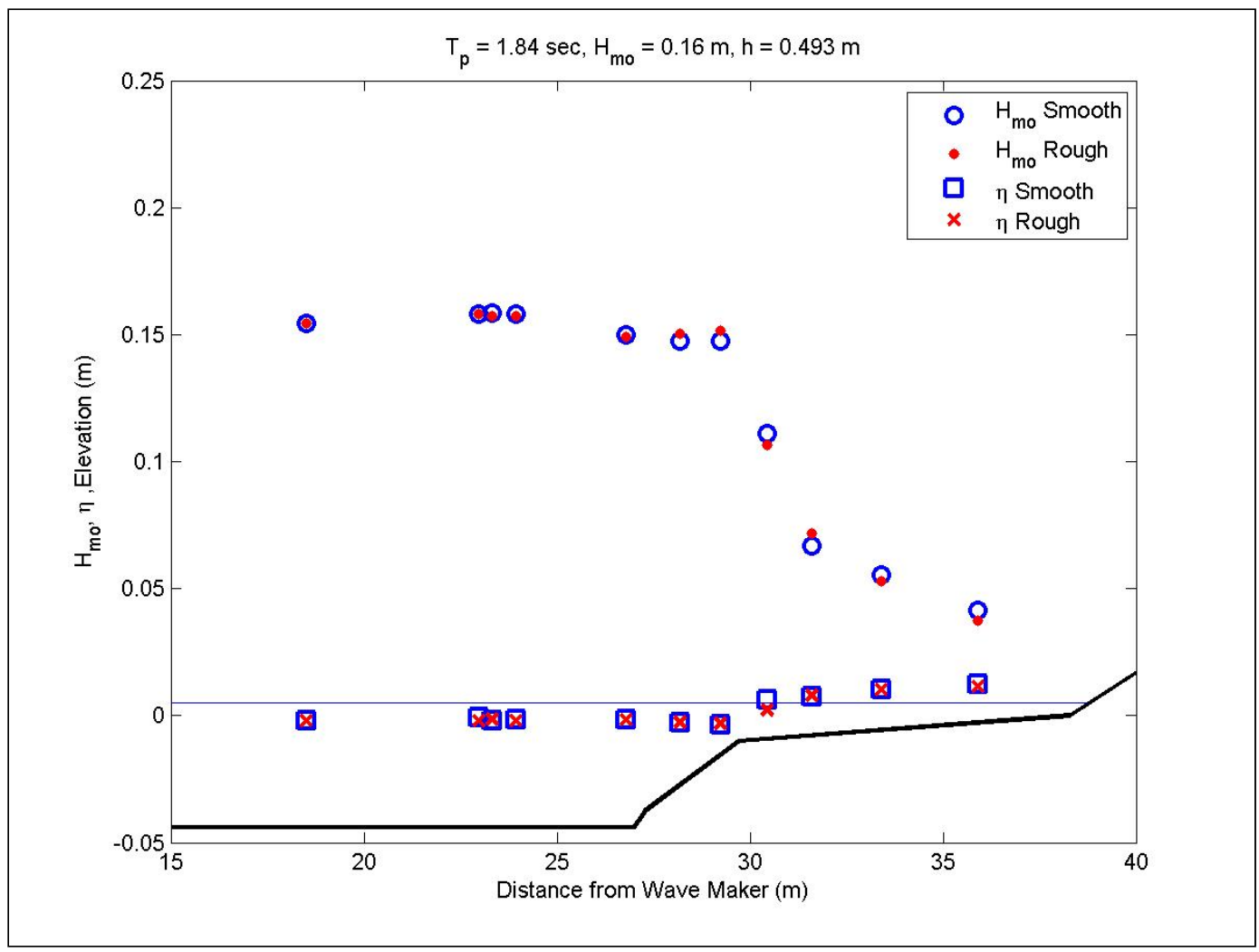

Figure A33. Wave transformation for 1.8-sec, 0.16-m waves at high water. 


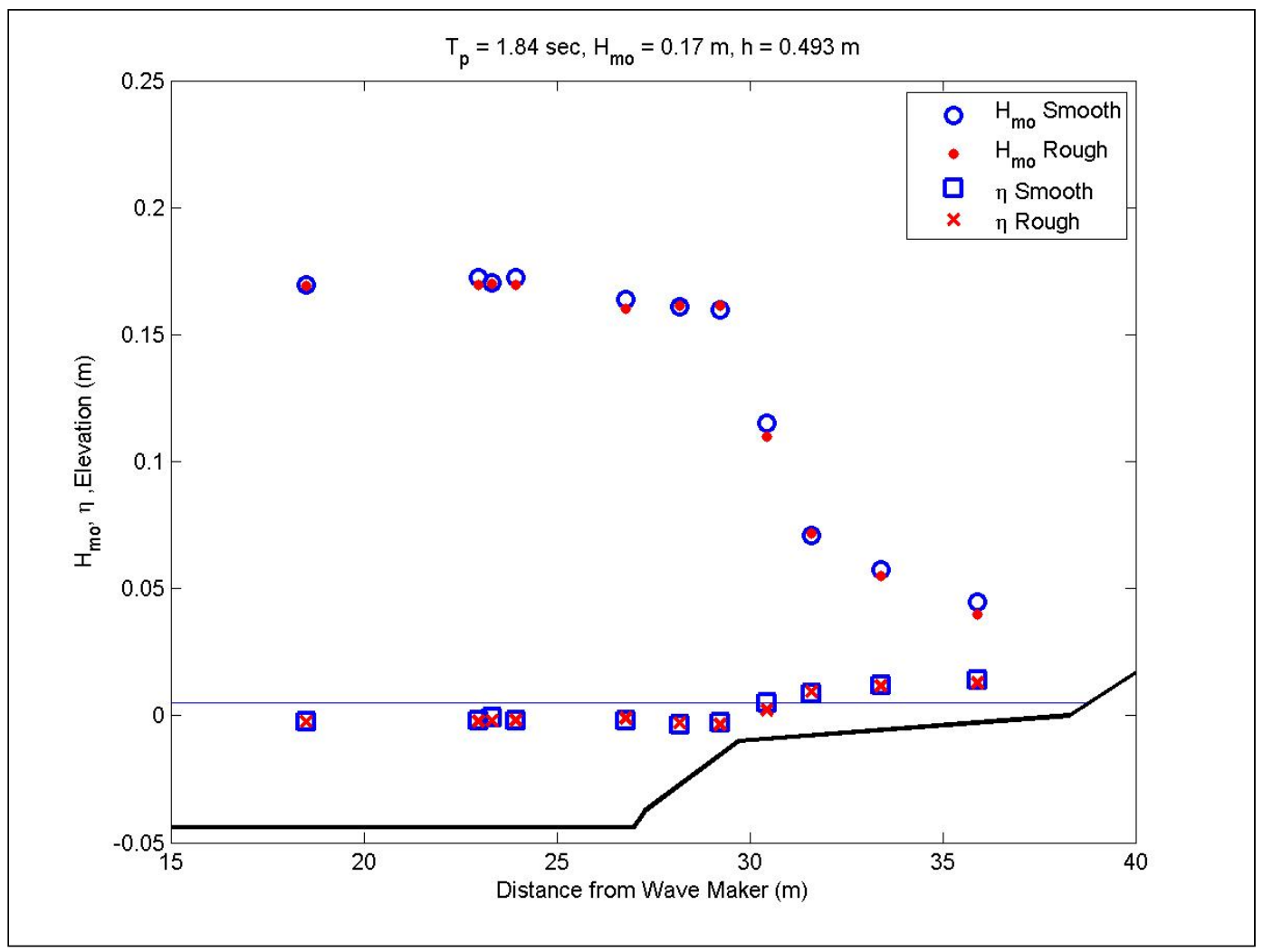

Figure A34. Wave transformation for 1.8-sec, 0.17-m waves at high water.

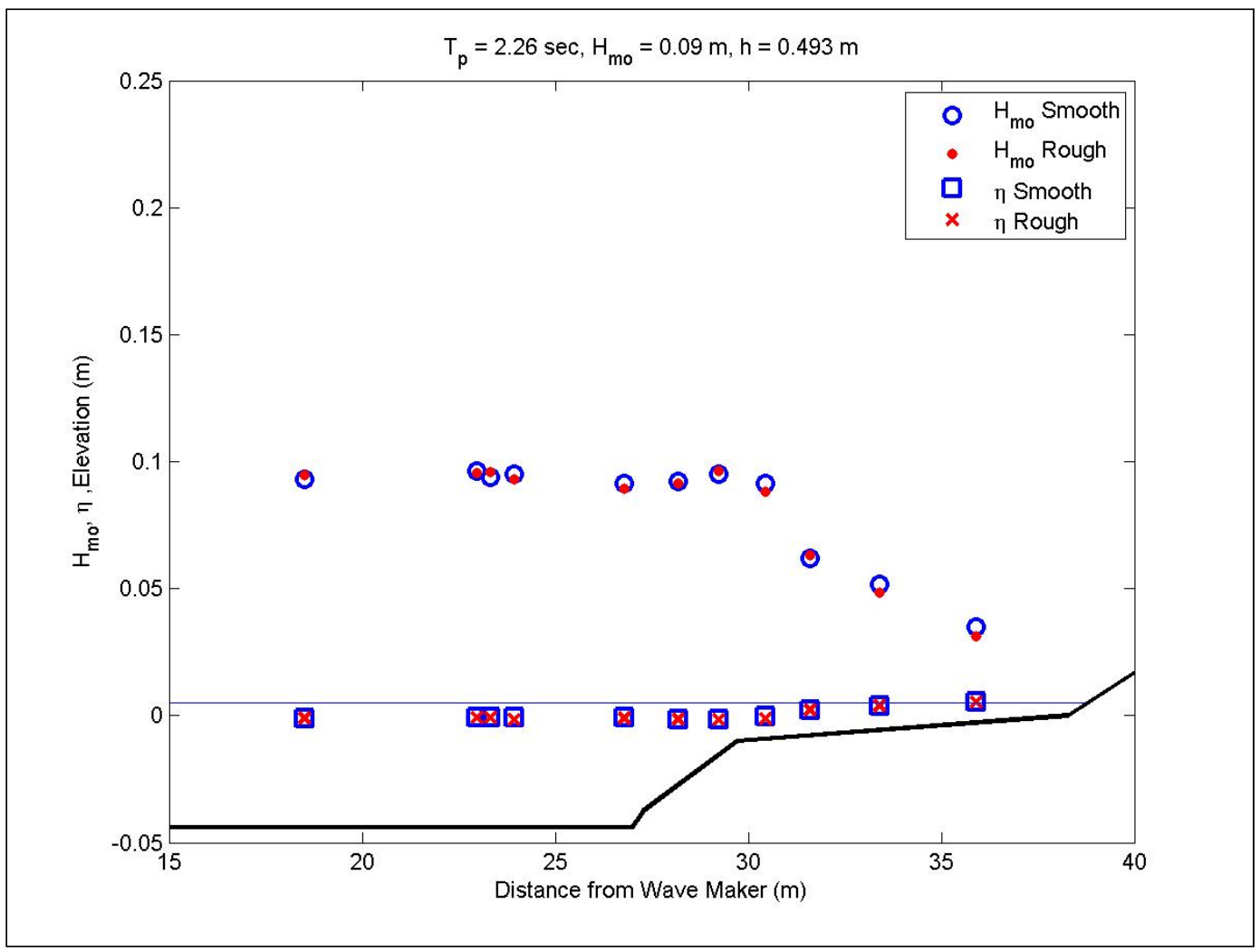

Figure A35. Wave transformation for 2.3-sec, 0.09-m waves at high water. 


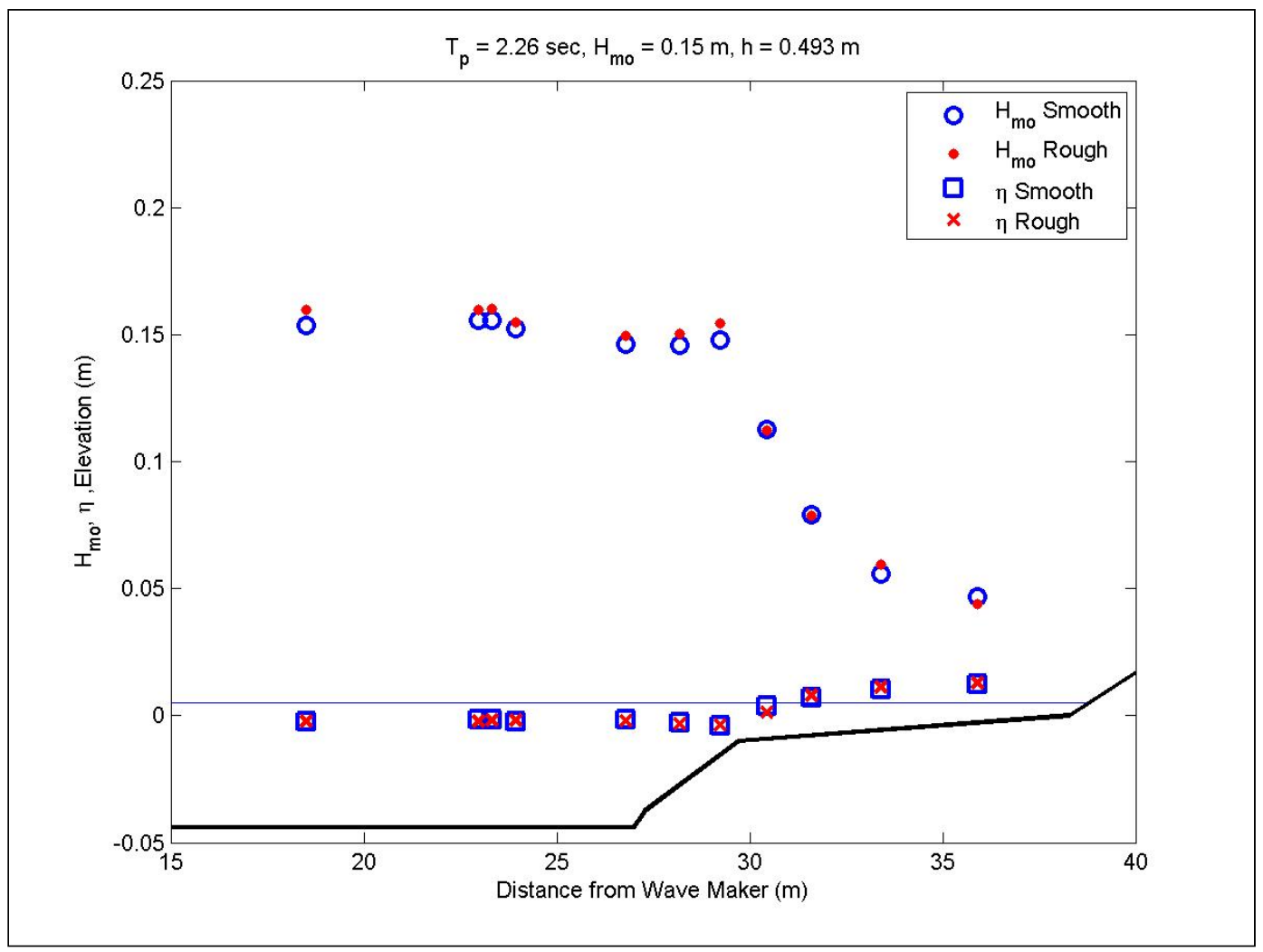

Figure A36. Wave transformation for 2.3-sec, 0.15-m waves at high water.

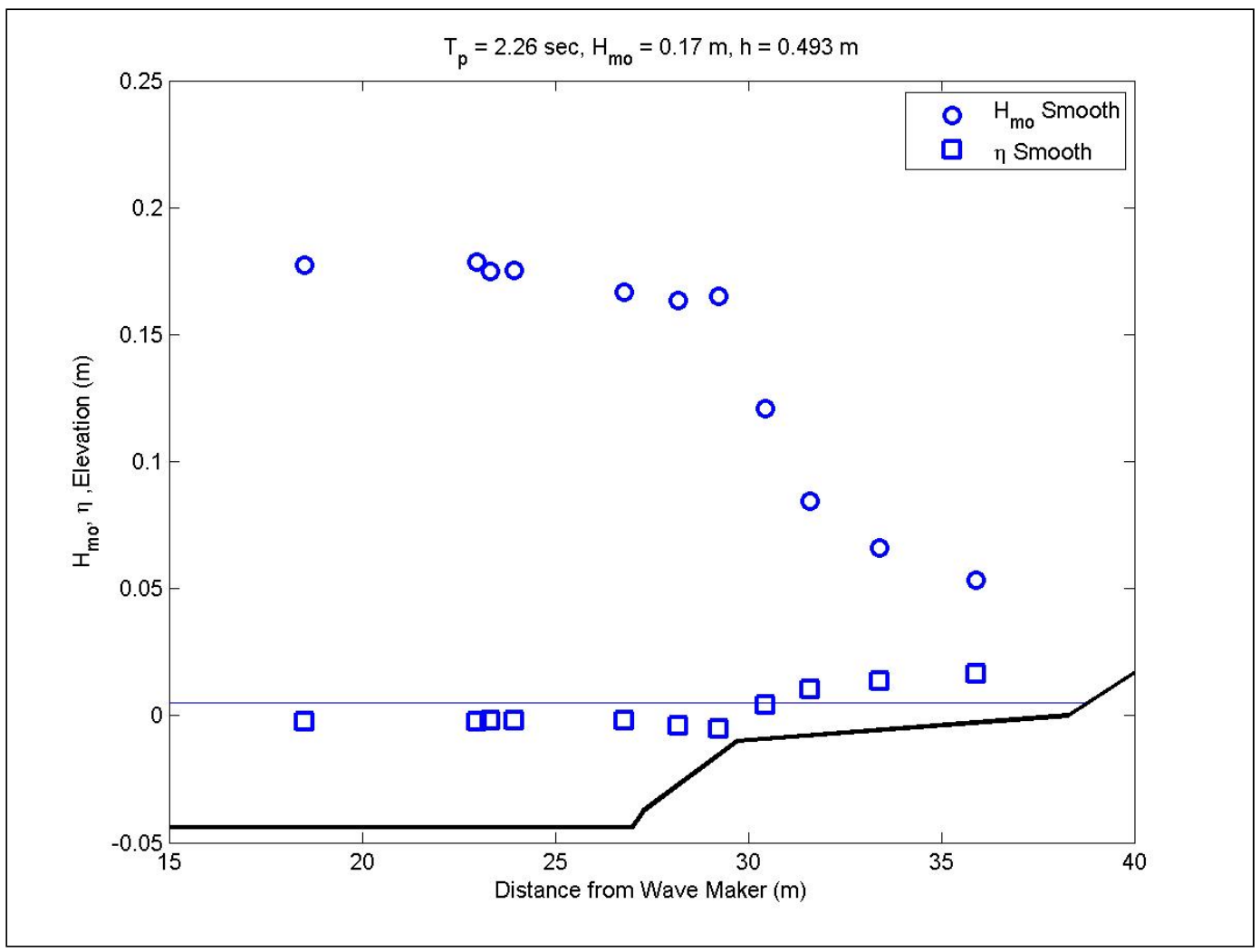

Figure A37. Wave transformation for 2.3-sec, 0.17- $\mathrm{m}$ waves at high water. 


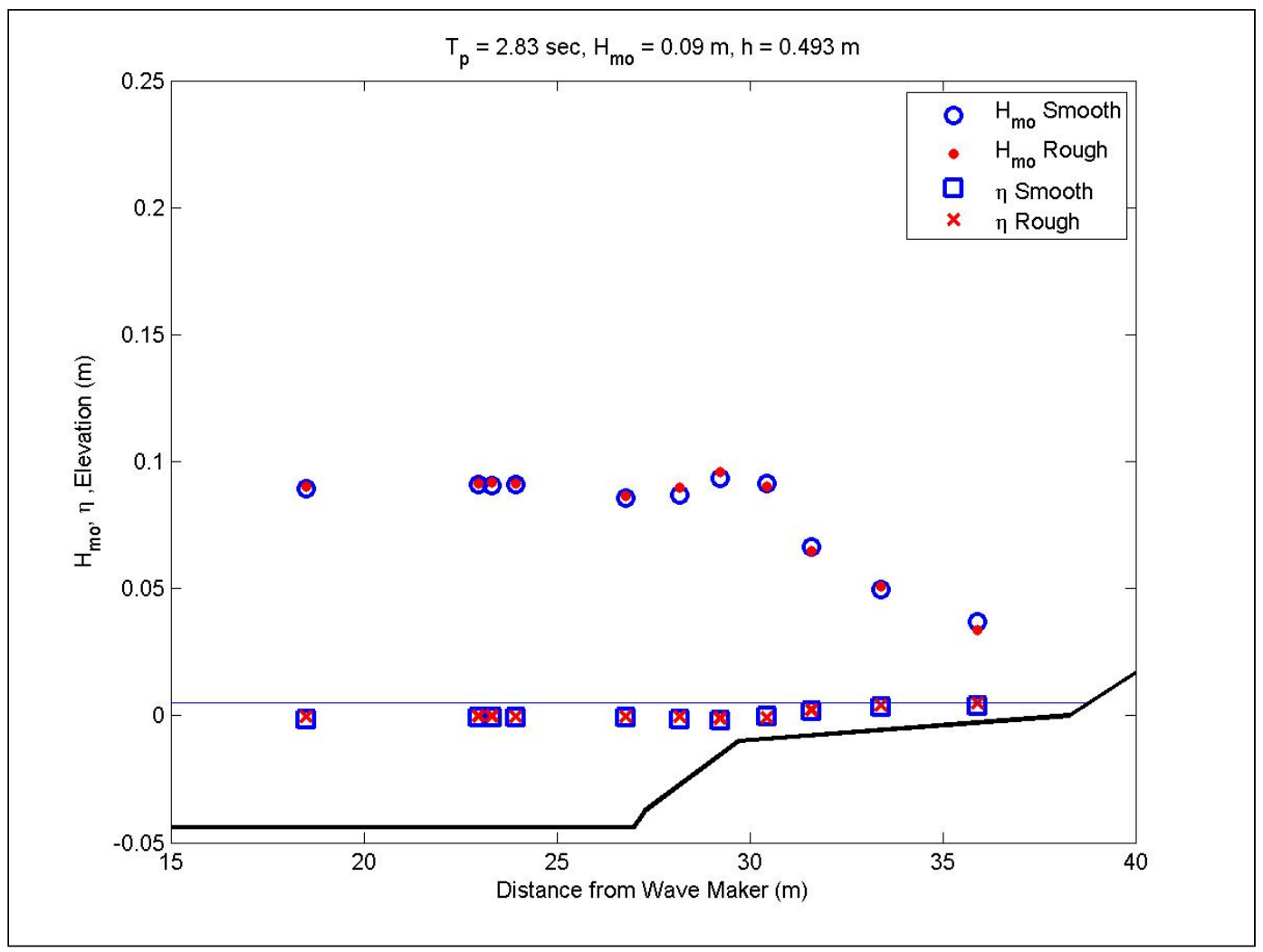

Figure A38. Wave transformation for 2.8-sec, 0.09-m waves at high water.

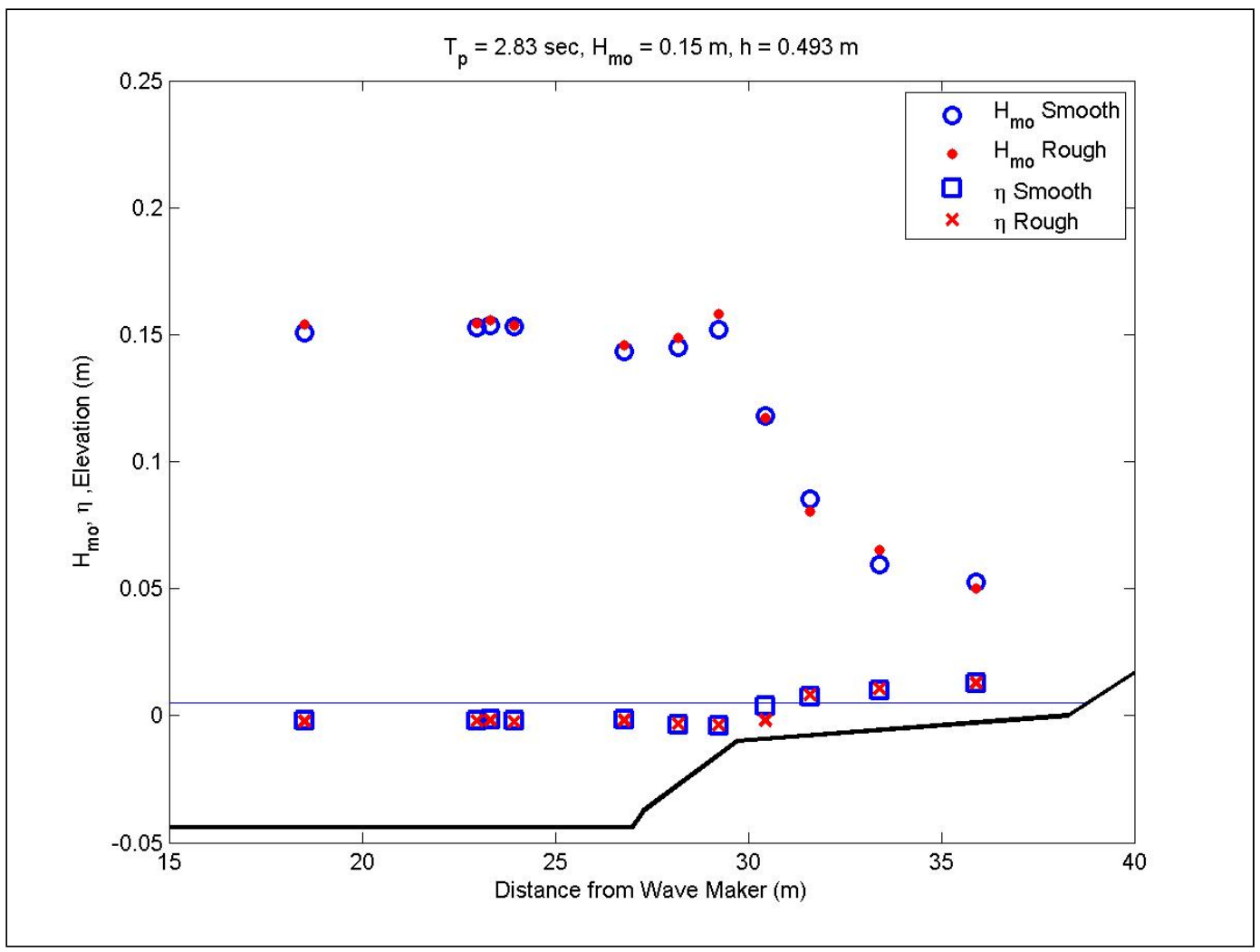

Figure A39. Wave transformation for 2.8-sec, 0.15-m waves at high water. 


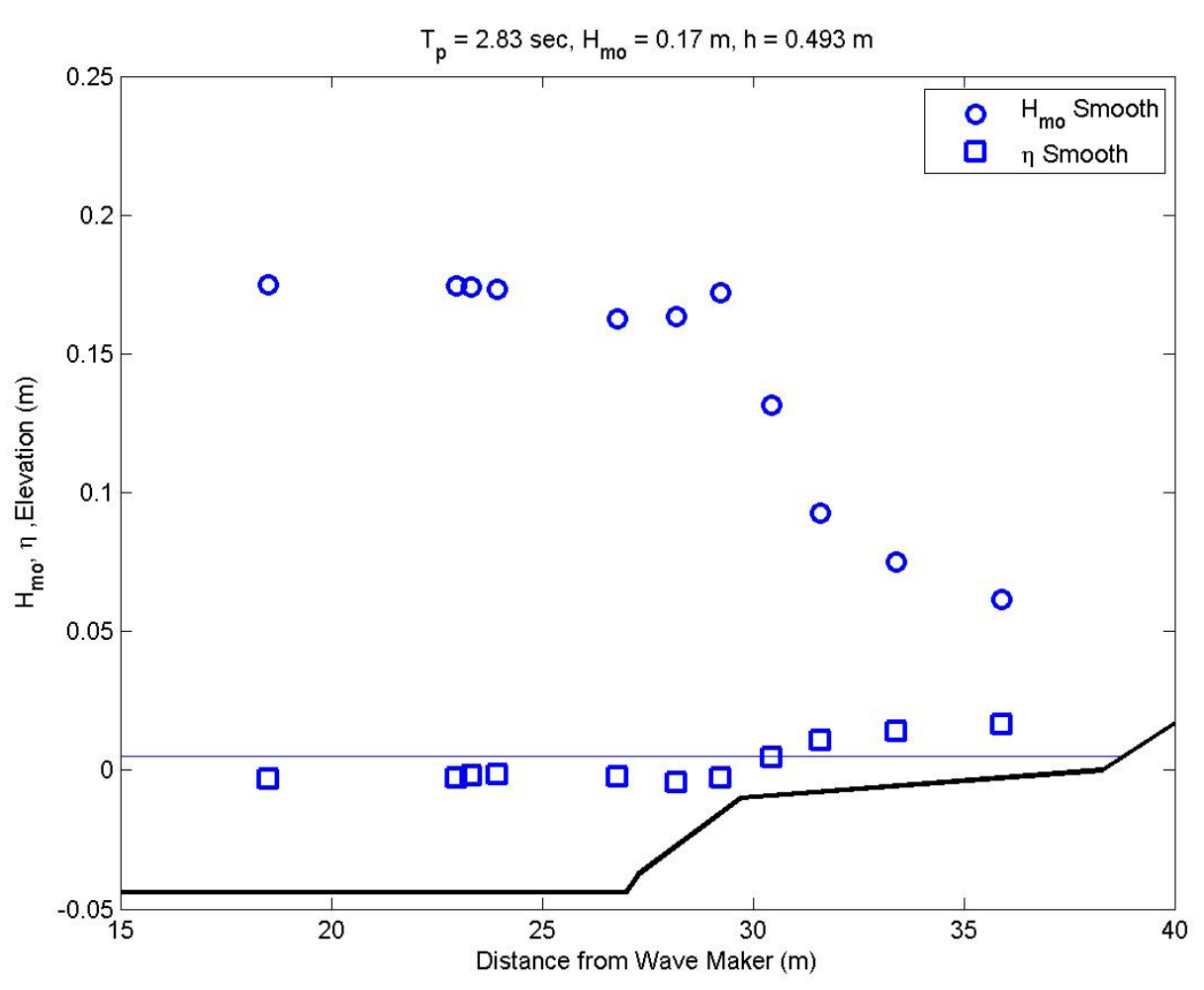

Figure A40. Wave transformation for 2.8-sec, 0.17- $\mathrm{m}$ waves at high water. 


\section{Appendix B: Alongshore wave height figures from the three-dimensional experiments}

The figures presented in Appendix B show zero-moment wave heights and mean water levels at the five alongshore gauges (Gauges 12 to 16) on the reef bathymetry. Each figure includes wave height and mean water level measured on the smooth and roughened bathymetry for similar wave conditions. The measured model bathymetry along the line of gauges also is plotted.

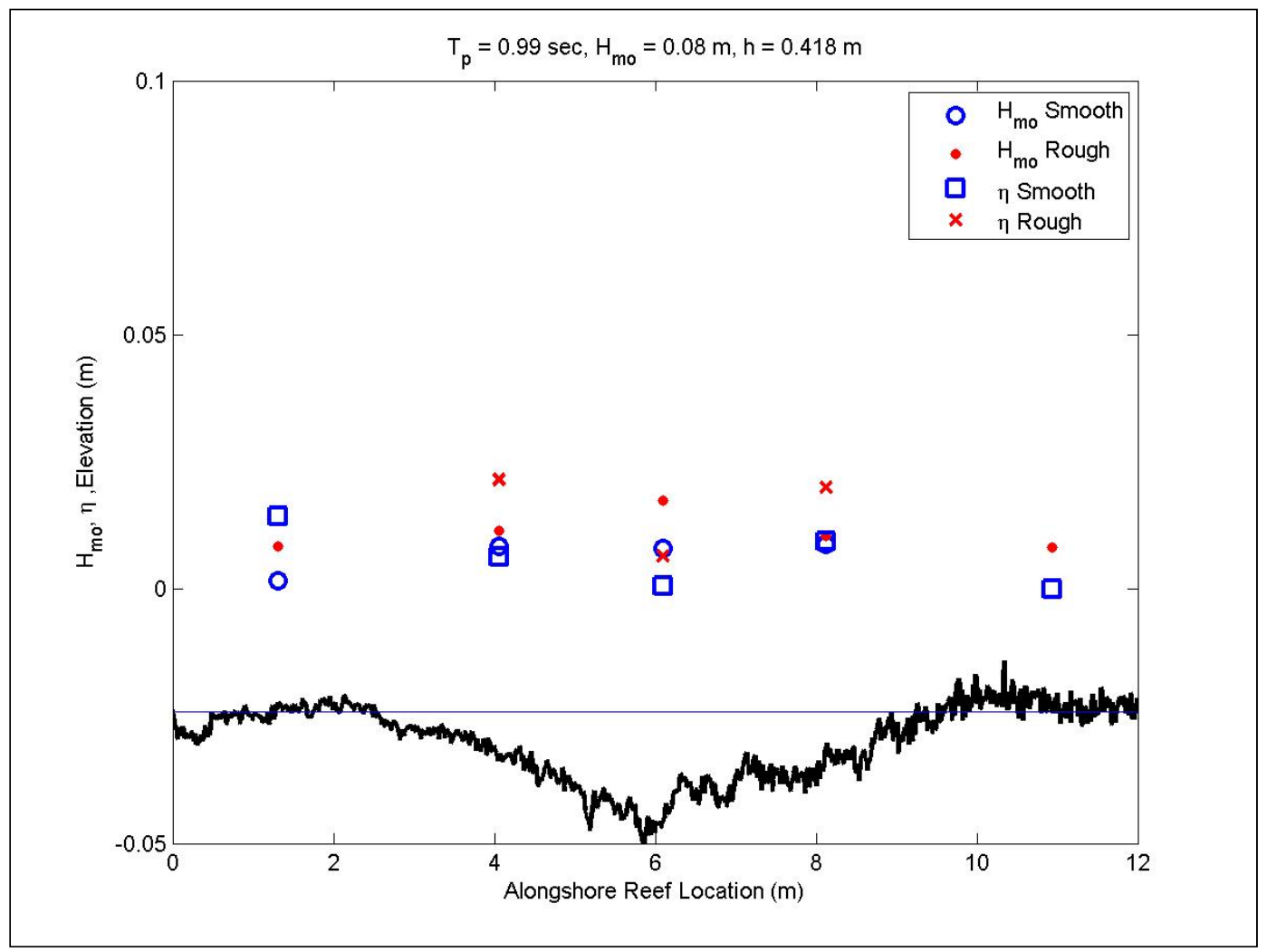

Figure B1. Alongshore wave heights for 1.0-sec, 0.08-m waves at low water. 


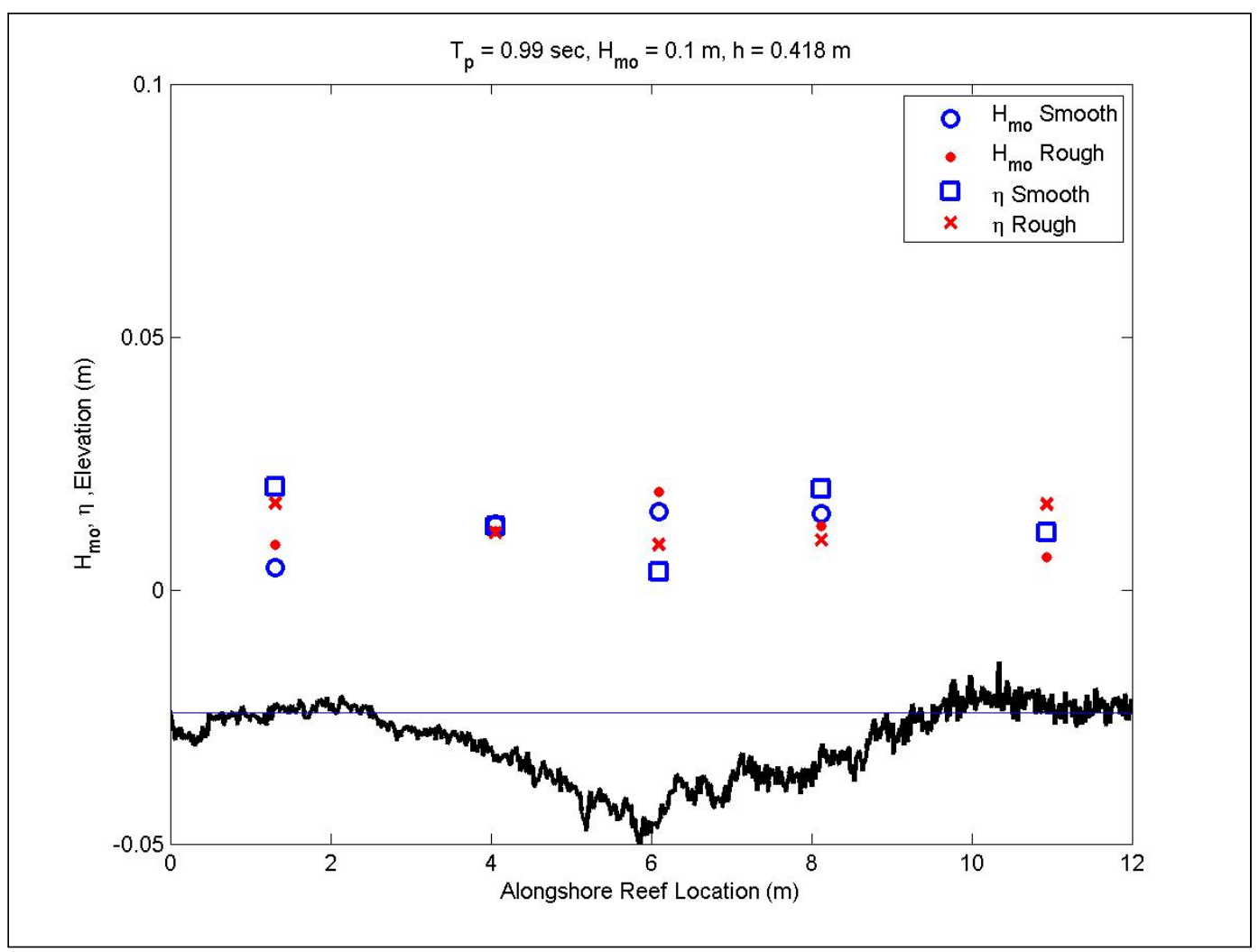

Figure B2. Alongshore wave heights for 1.0-sec, 0.1-m waves at low water.

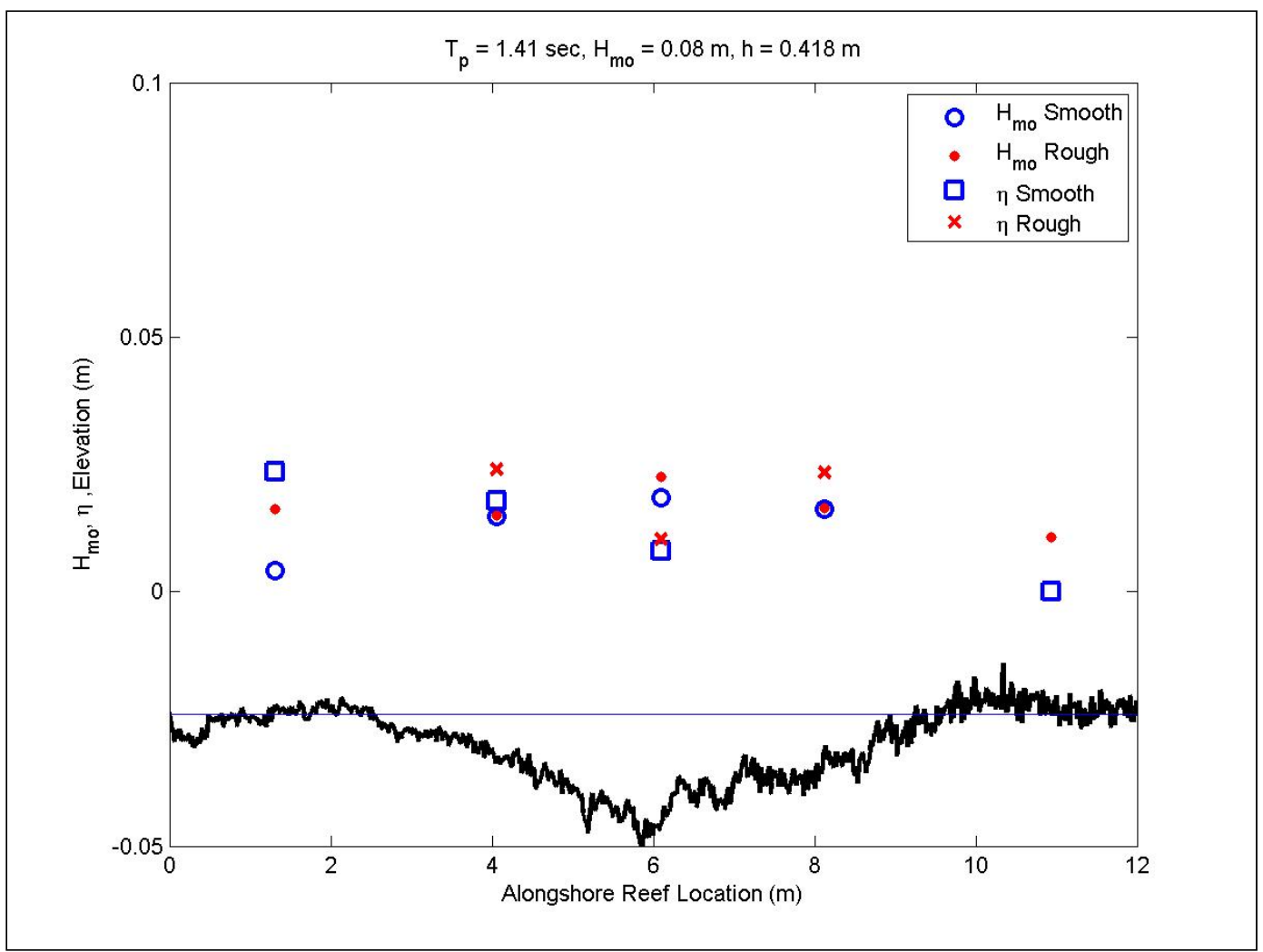

Figure B3. Alongshore wave heights for 1.4-sec, 0.08-m waves at low water. 


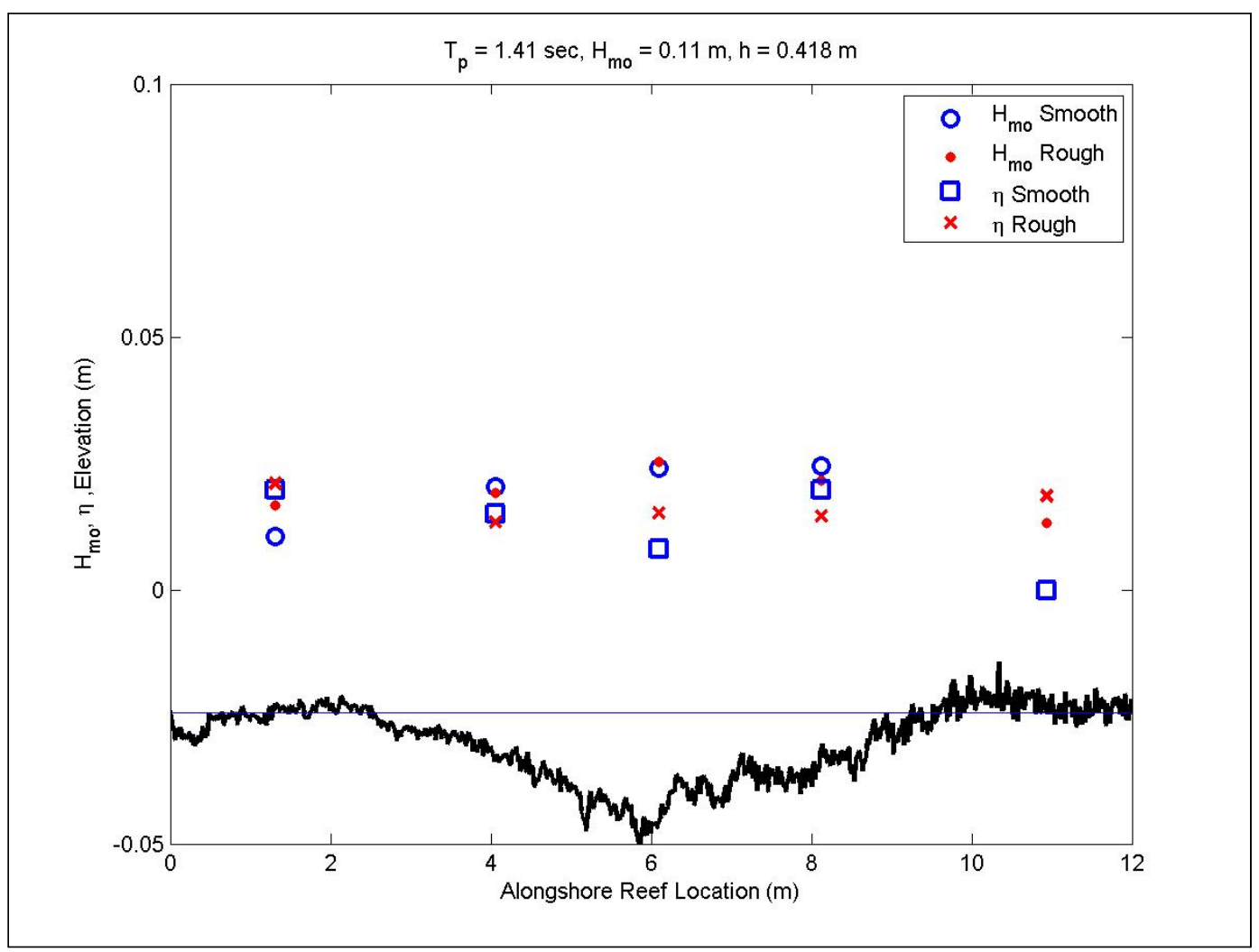

Figure B4. Alongshore wave heights for 1.4-sec, 0.11-m waves at low water.

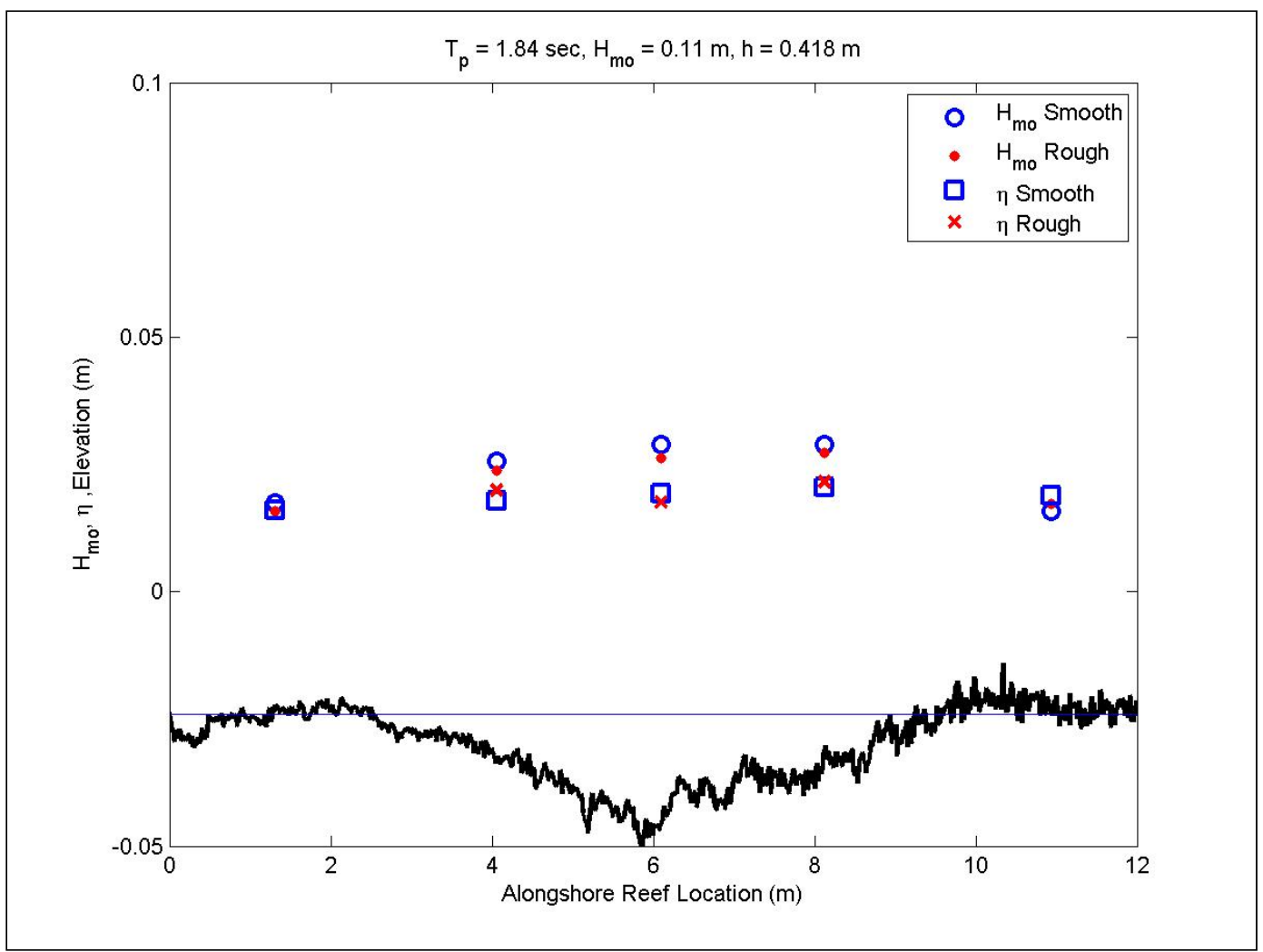

Figure B5. Alongshore wave heights for 1.8-sec, 0.11-m waves at low water. 


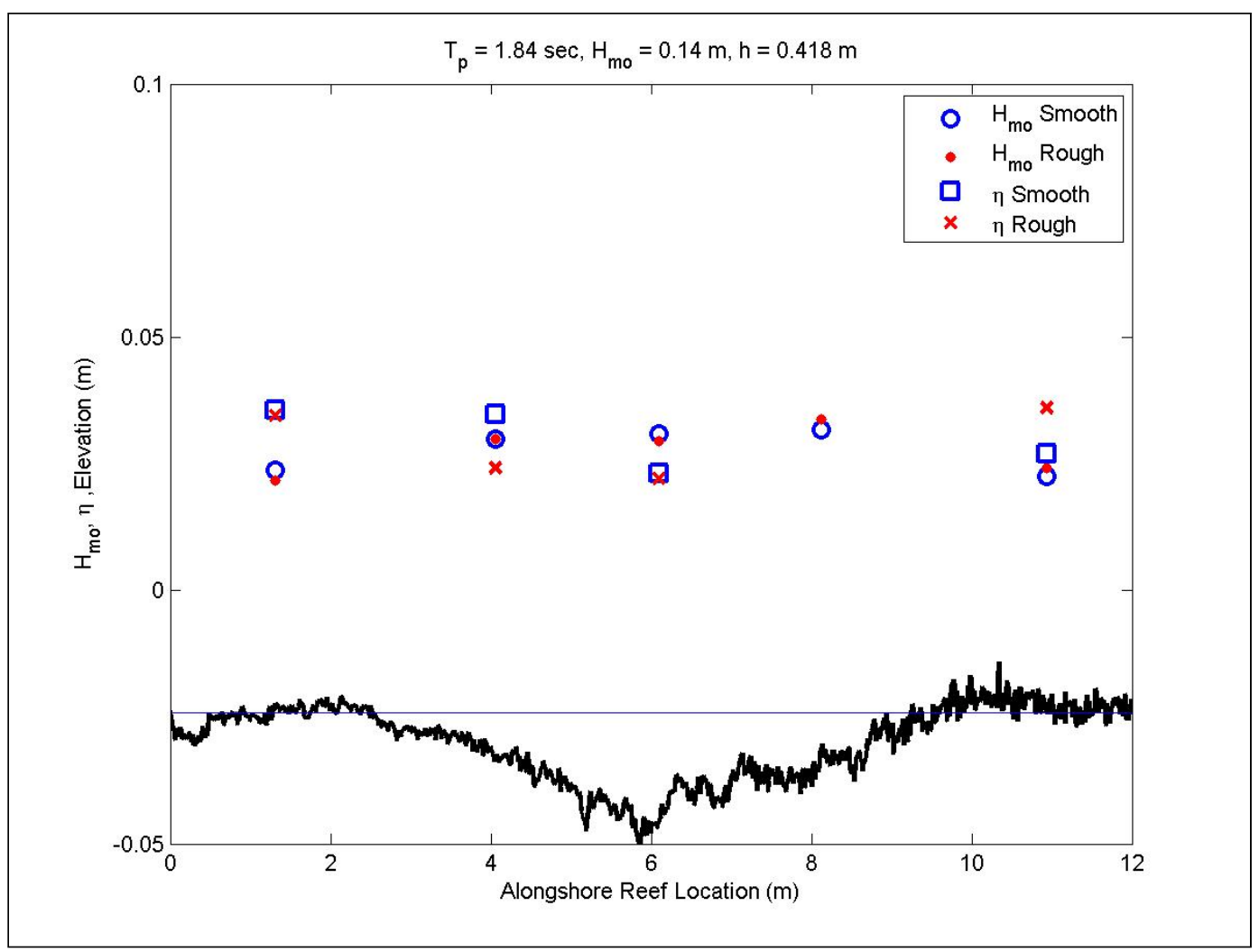

Figure B6. Alongshore wave heights for 1.8-sec, 0.14-m waves at low water.

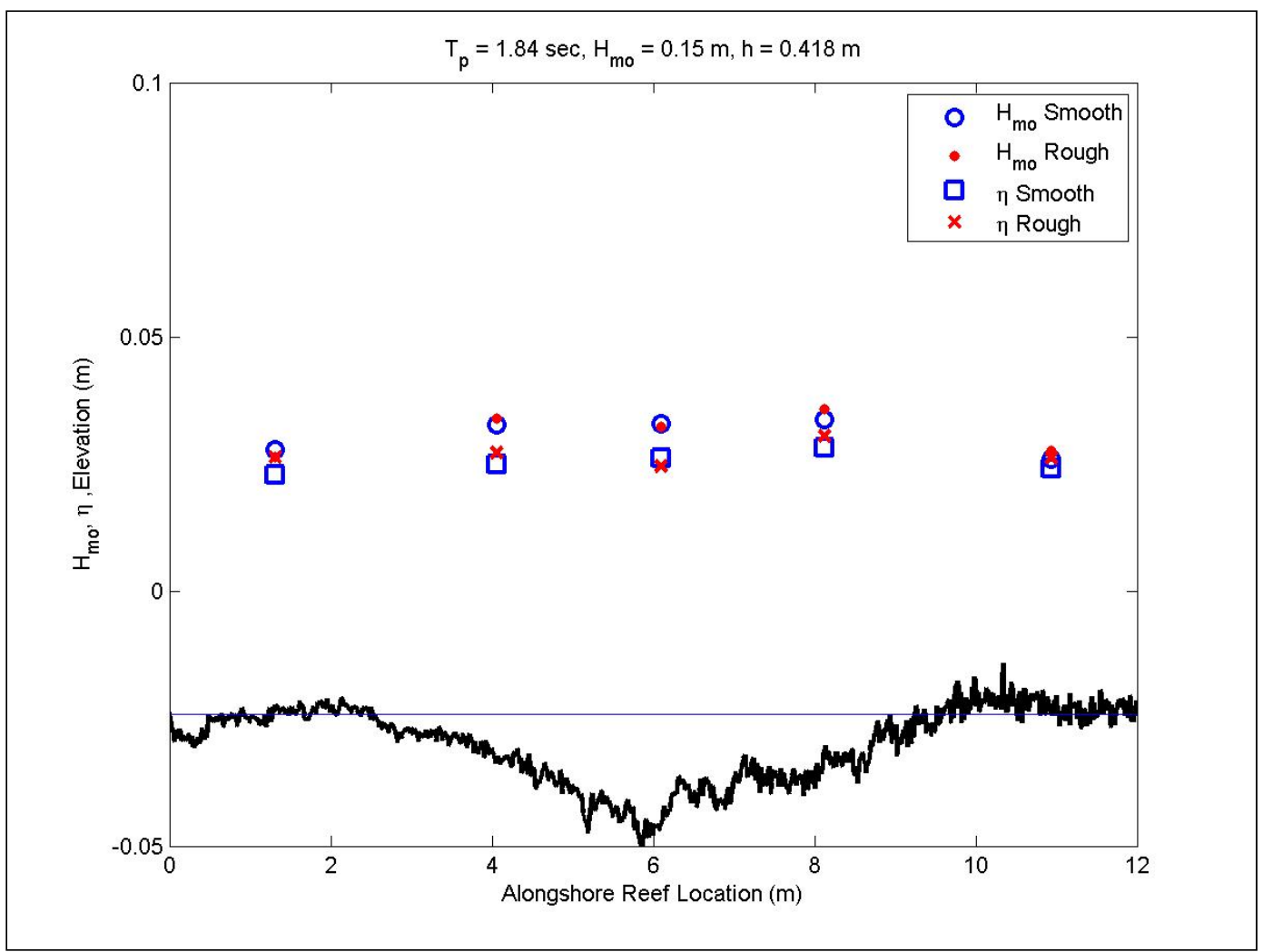

Figure B7. Alongshore wave heights for 1.8-sec, 0.15-m waves at low water. 


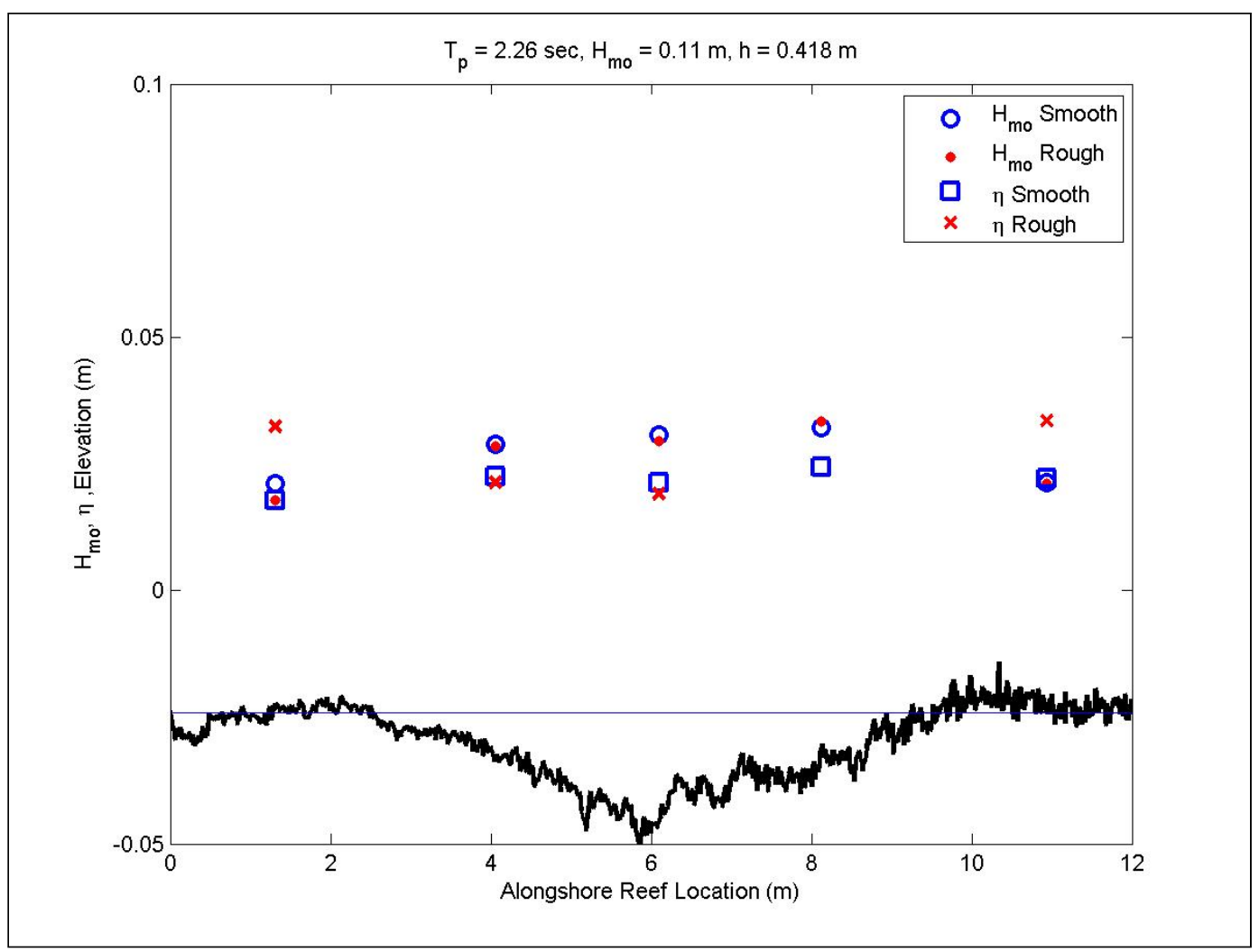

Figure B8. Alongshore wave heights for 2.3-sec, 0.11-m waves at low water.

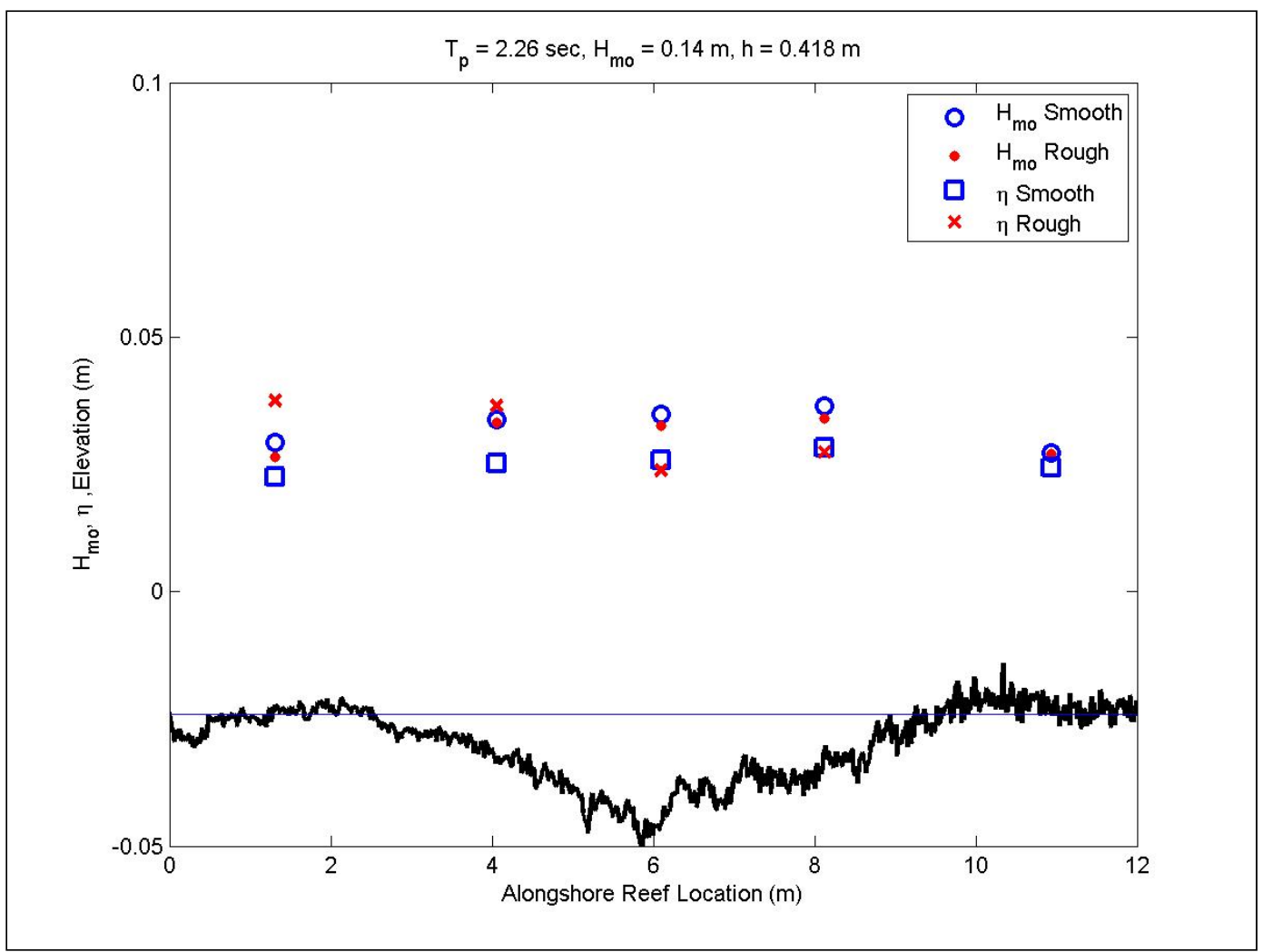

Figure B9. Alongshore wave heights for 2.3-sec, 0.14-m waves at low water. 


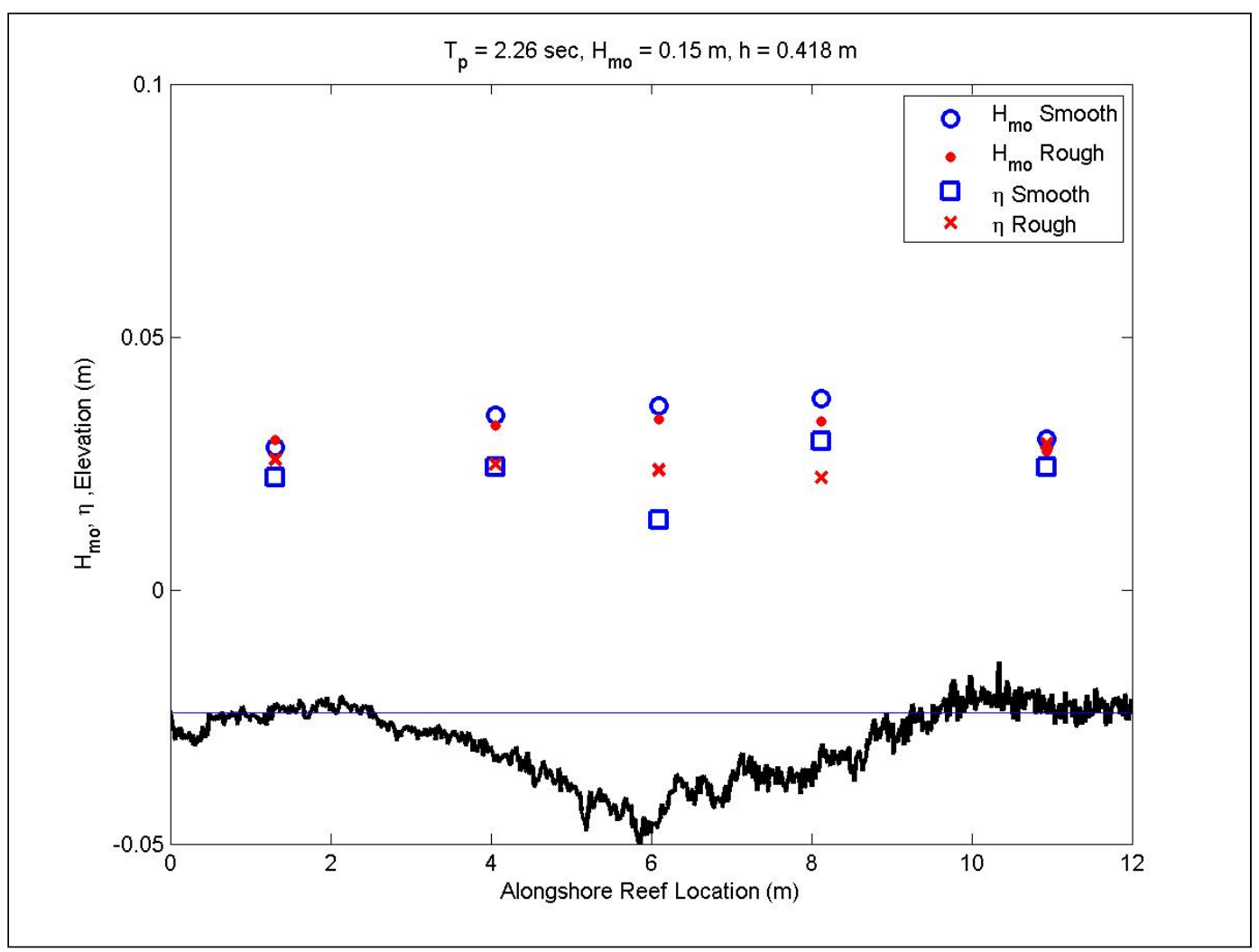

Figure B10. Alongshore wave heights for 2.3-sec, 0.15-m waves at low water.

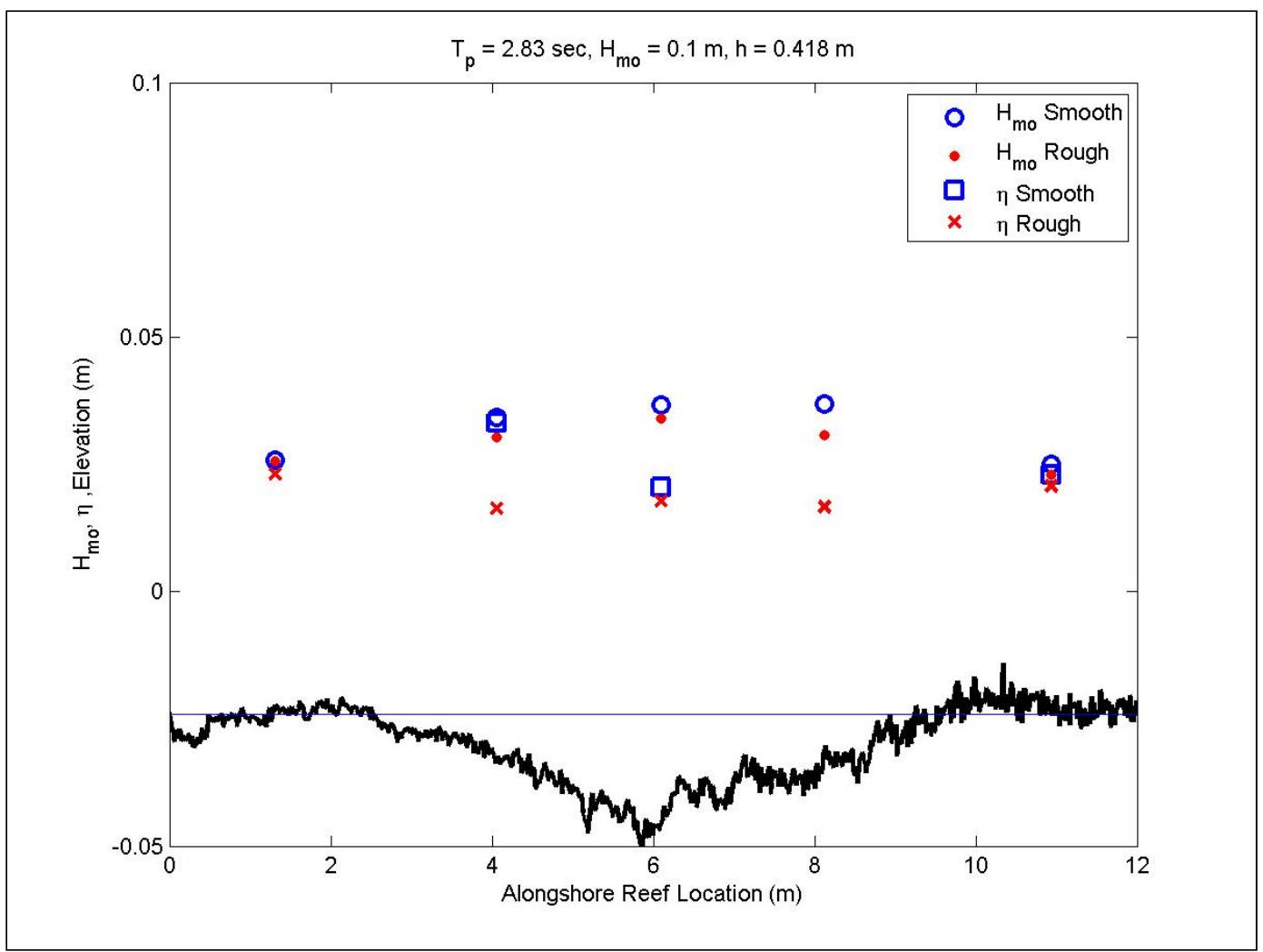

Figure B11. Alongshore wave heights for 2.8-sec, 0.1-m waves at low water. 


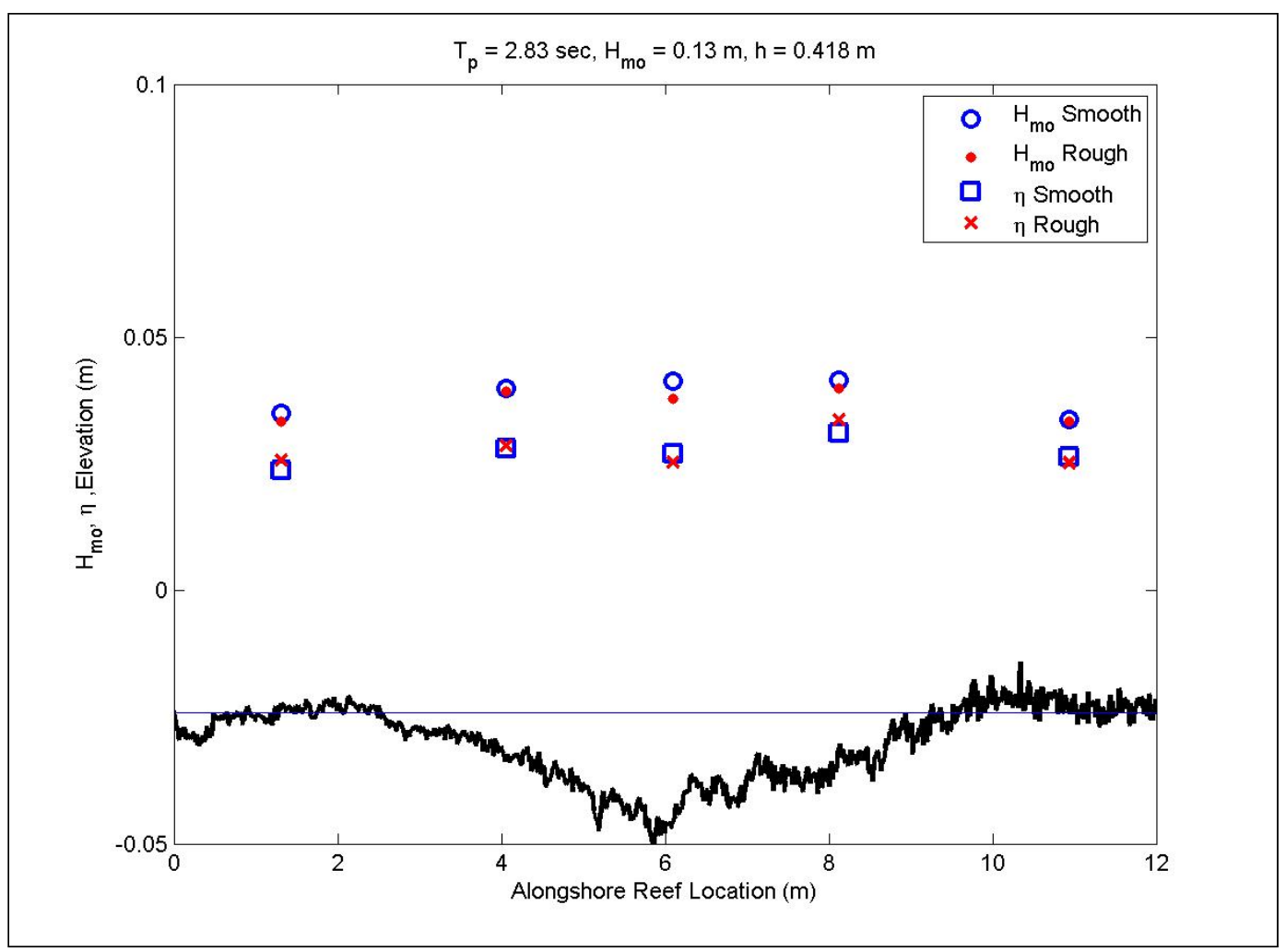

Figure B12. Alongshore wave heights for 2.8-sec, 0.13-m waves at low water.

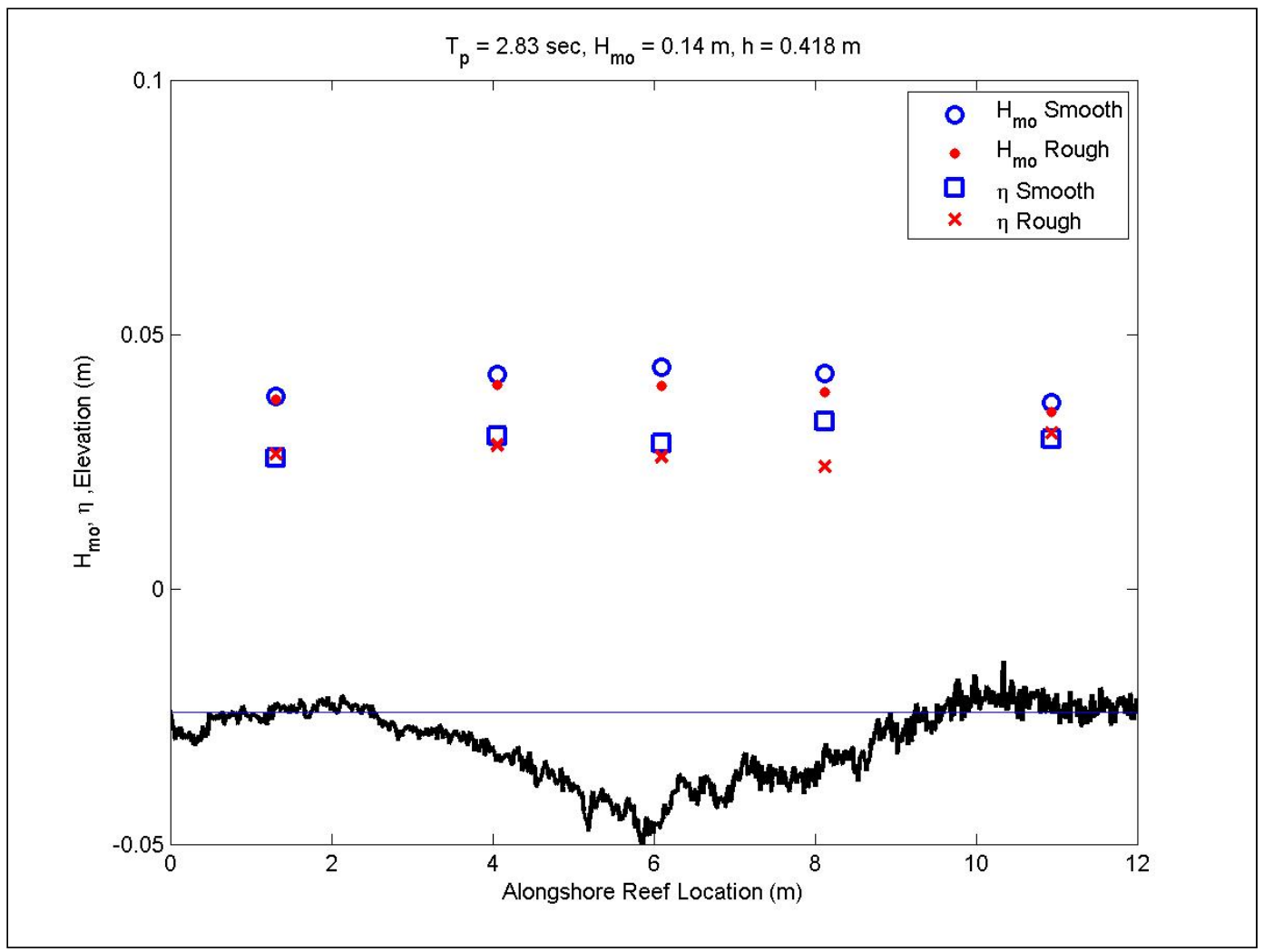

Figure B13. Alongshore wave heights for 2.8-sec, 0.14-m waves at low water. 


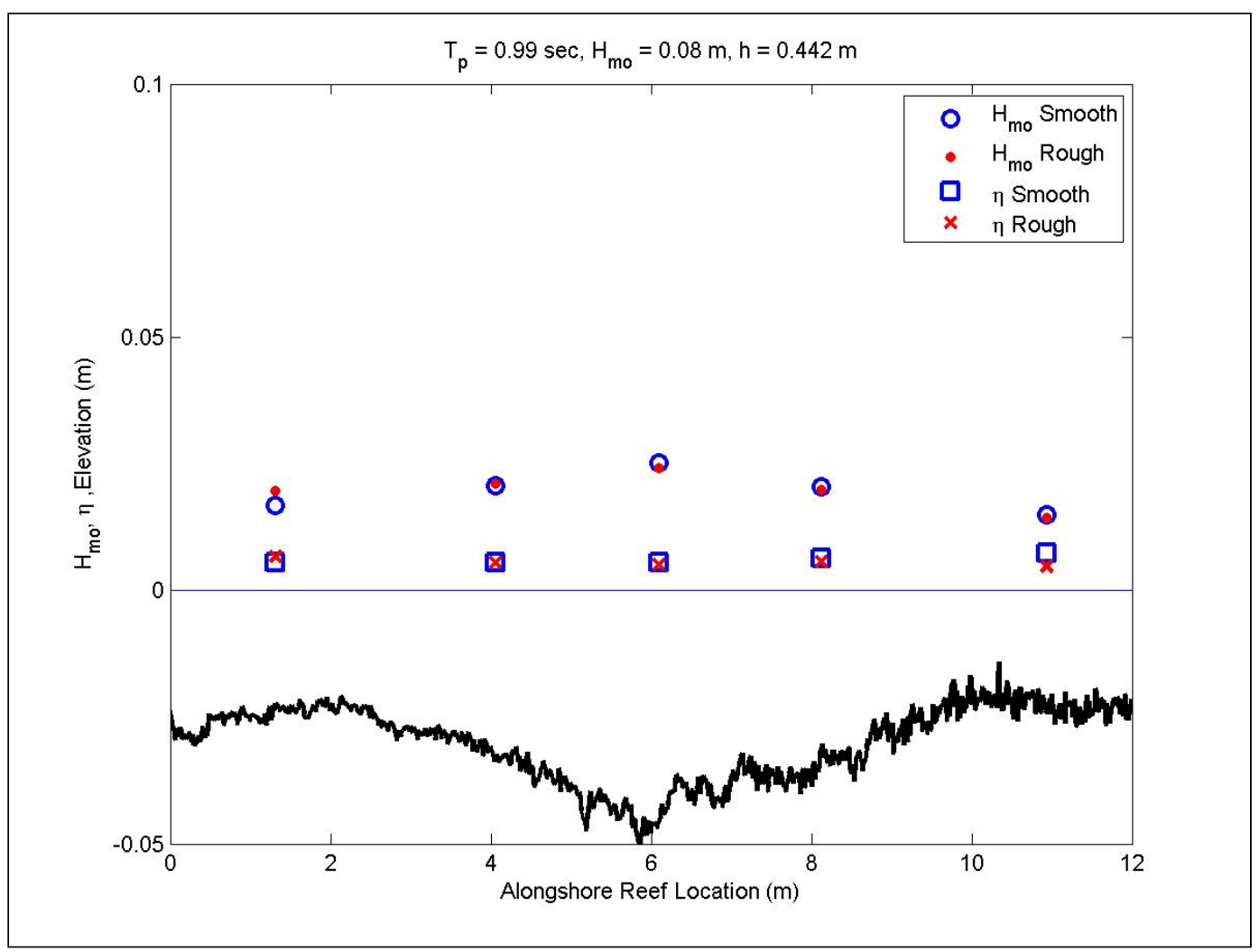

Figure B14. Alongshore wave heights for 1.0-sec, 0.08-m waves at mid water.

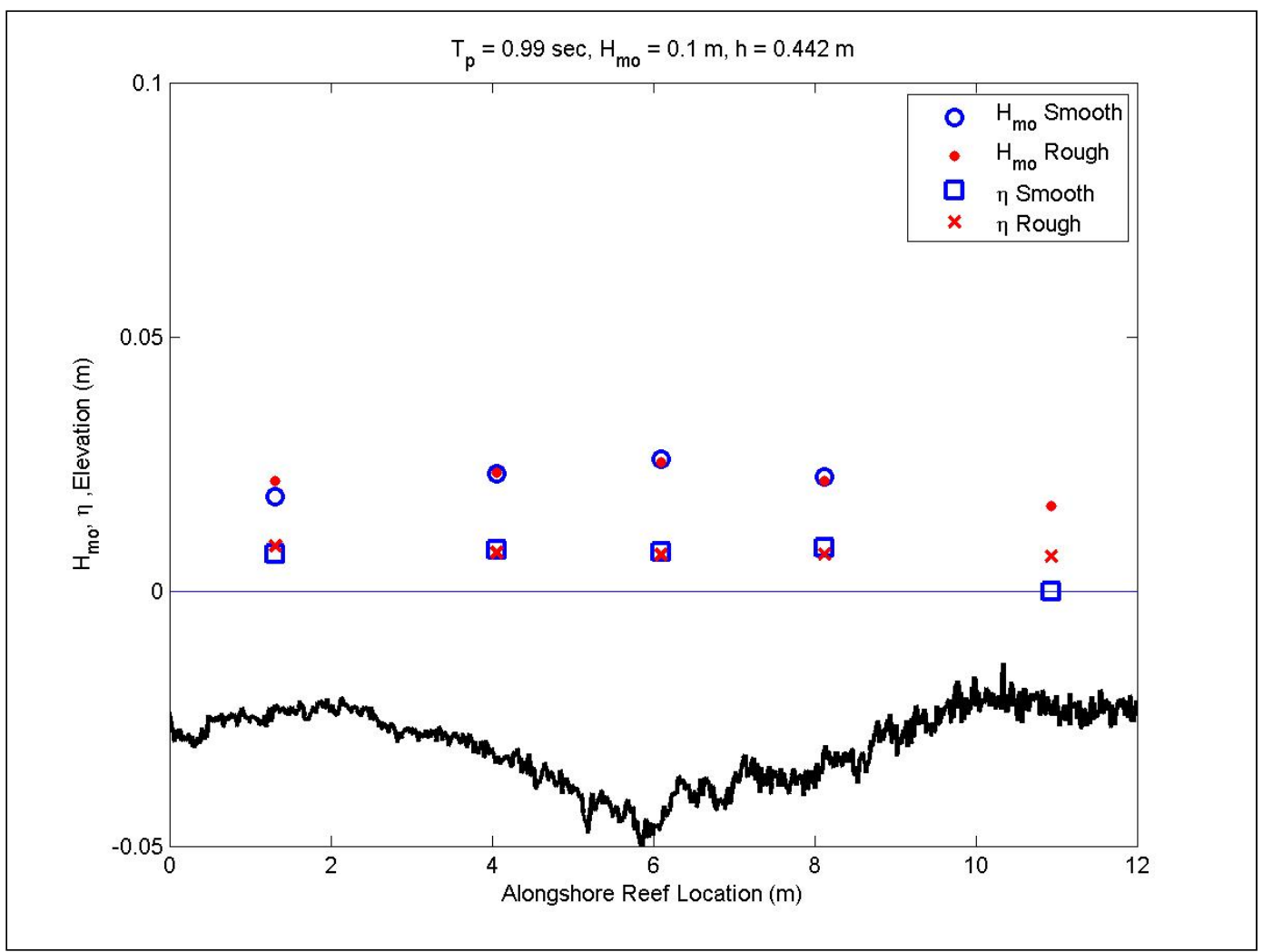

Figure B15. Alongshore wave heights for 1.0-sec, 0.1-m waves at mid water. 


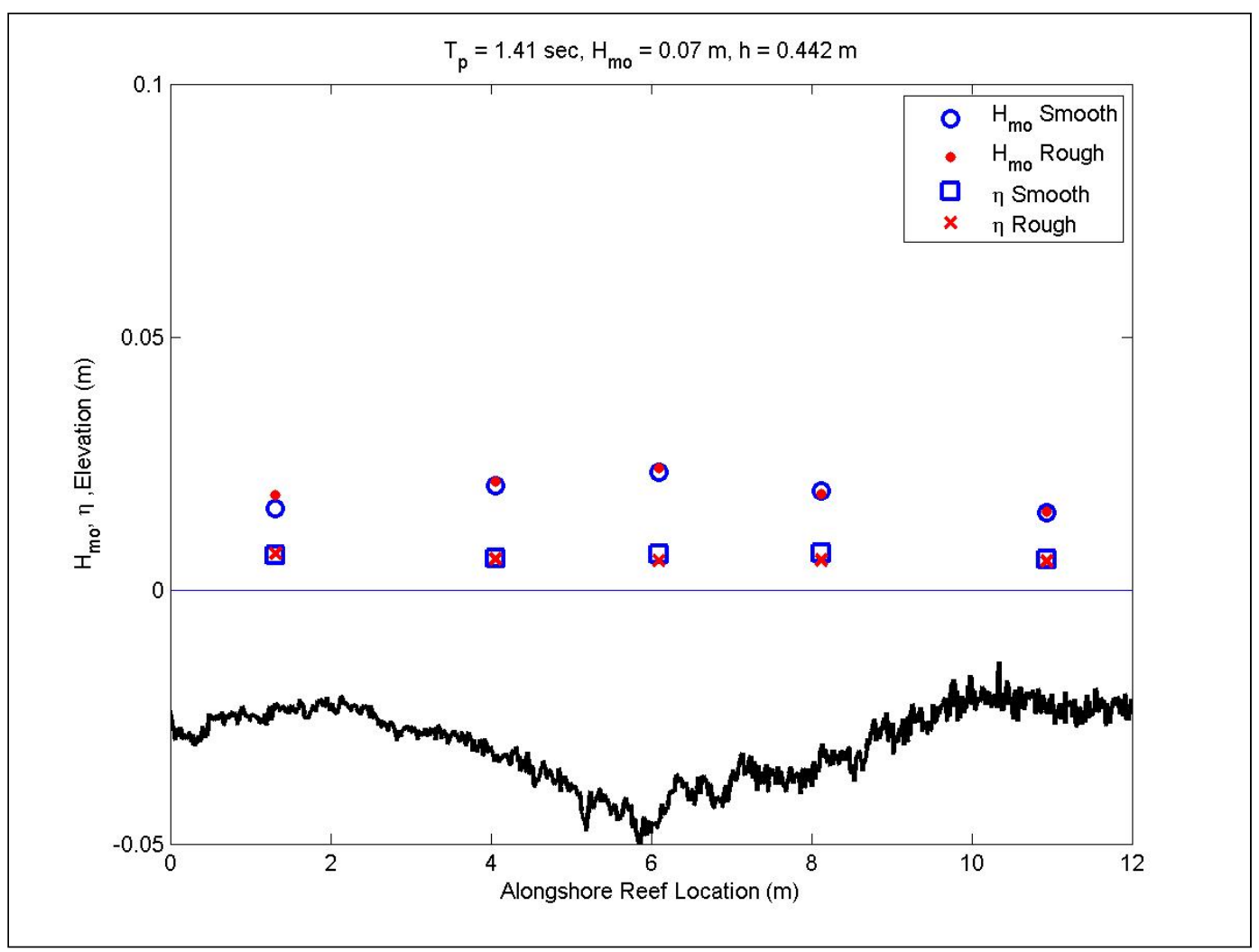

Figure B16. Alongshore wave heights for 1.4-sec, 0.07-m waves at mid water.

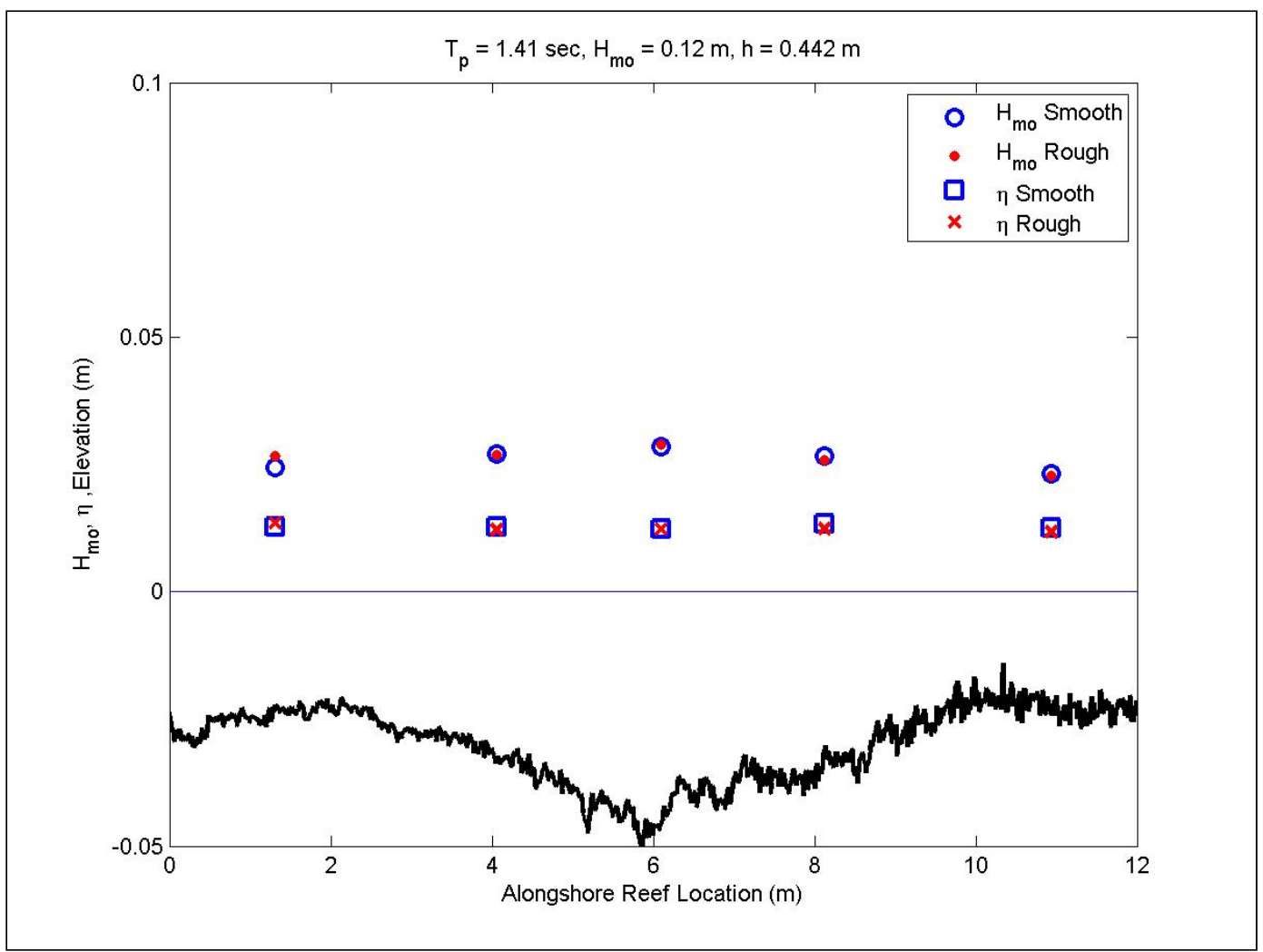

Figure B17. Alongshore wave heights for 1.4-sec, 0.12-m waves at mid water. 


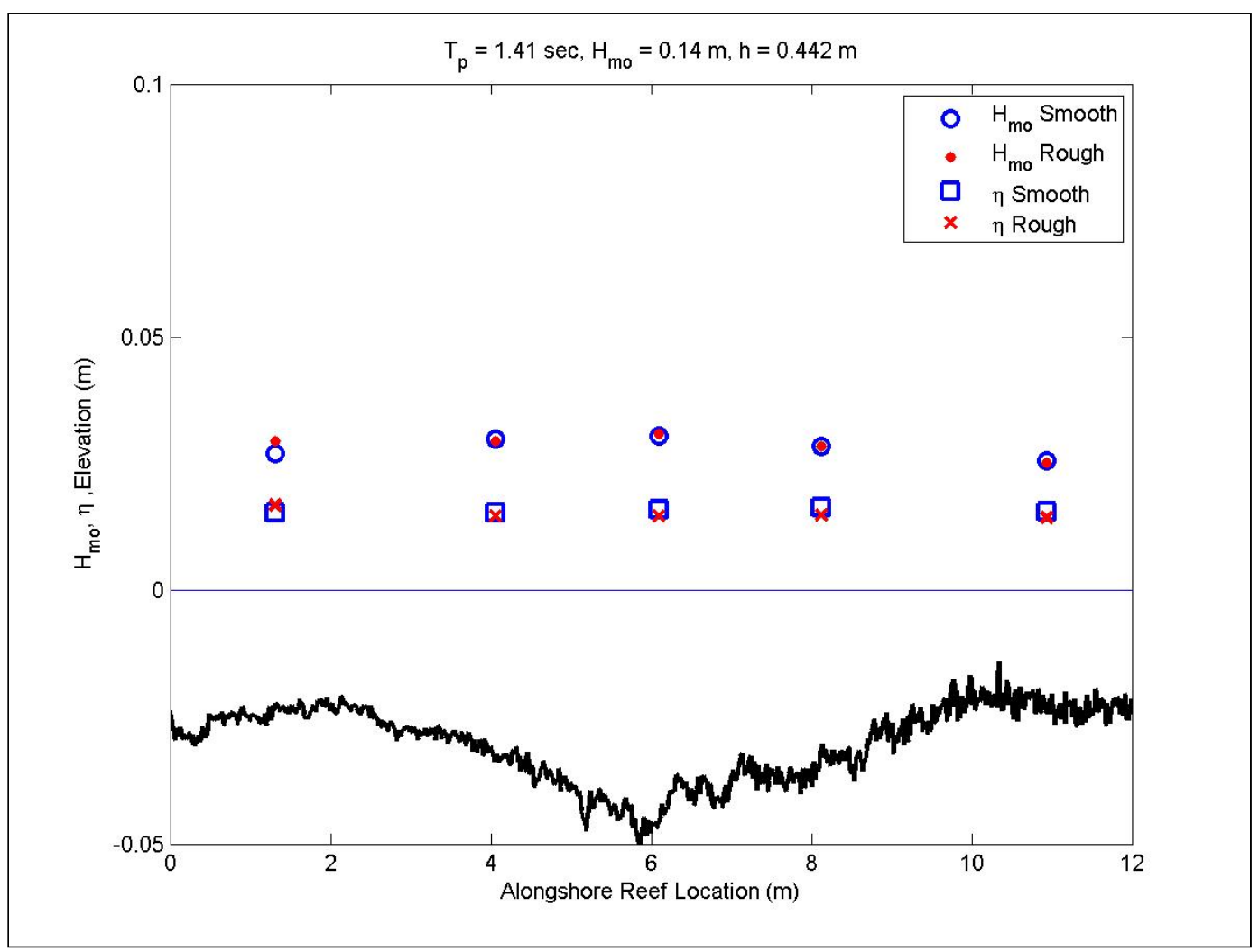

Figure B18. Alongshore wave heights for 1.4-sec, 0.14-m waves at mid water.

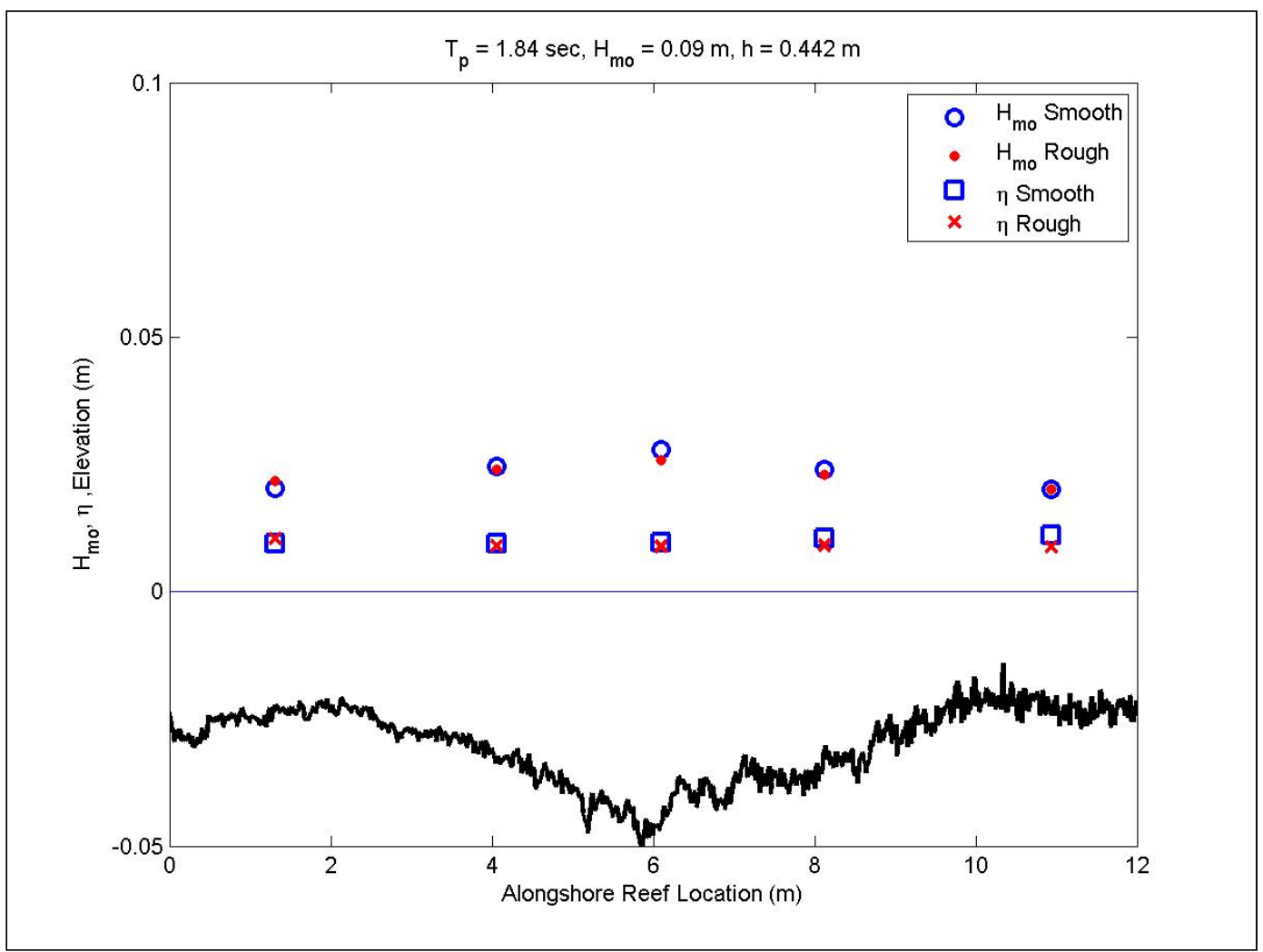

Figure B19. Alongshore wave heights for 1.8-sec, 0.09-m waves at mid water. 


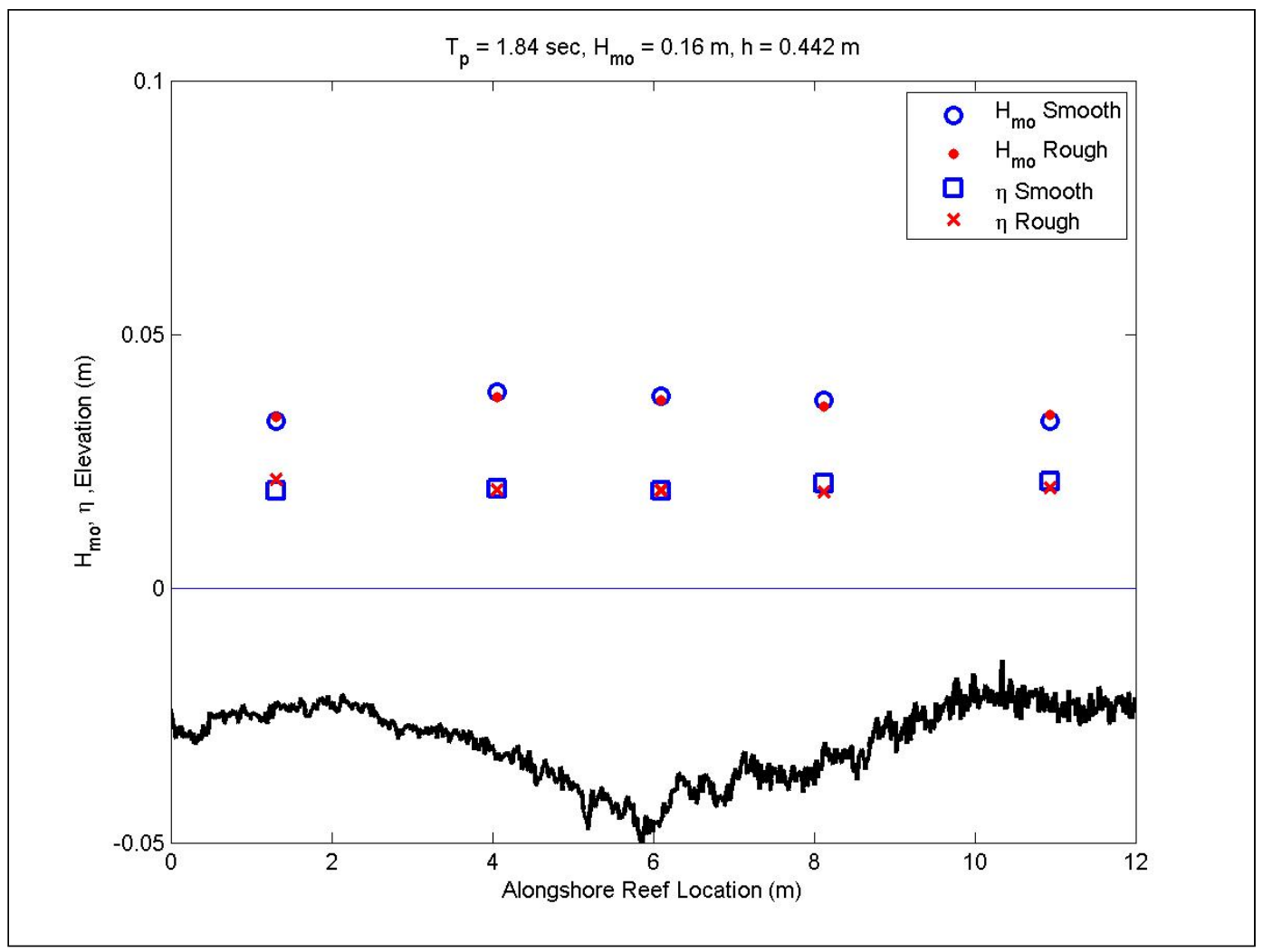

Figure B20. Alongshore wave heights for 1.8-sec, 0.16-m waves at mid water.

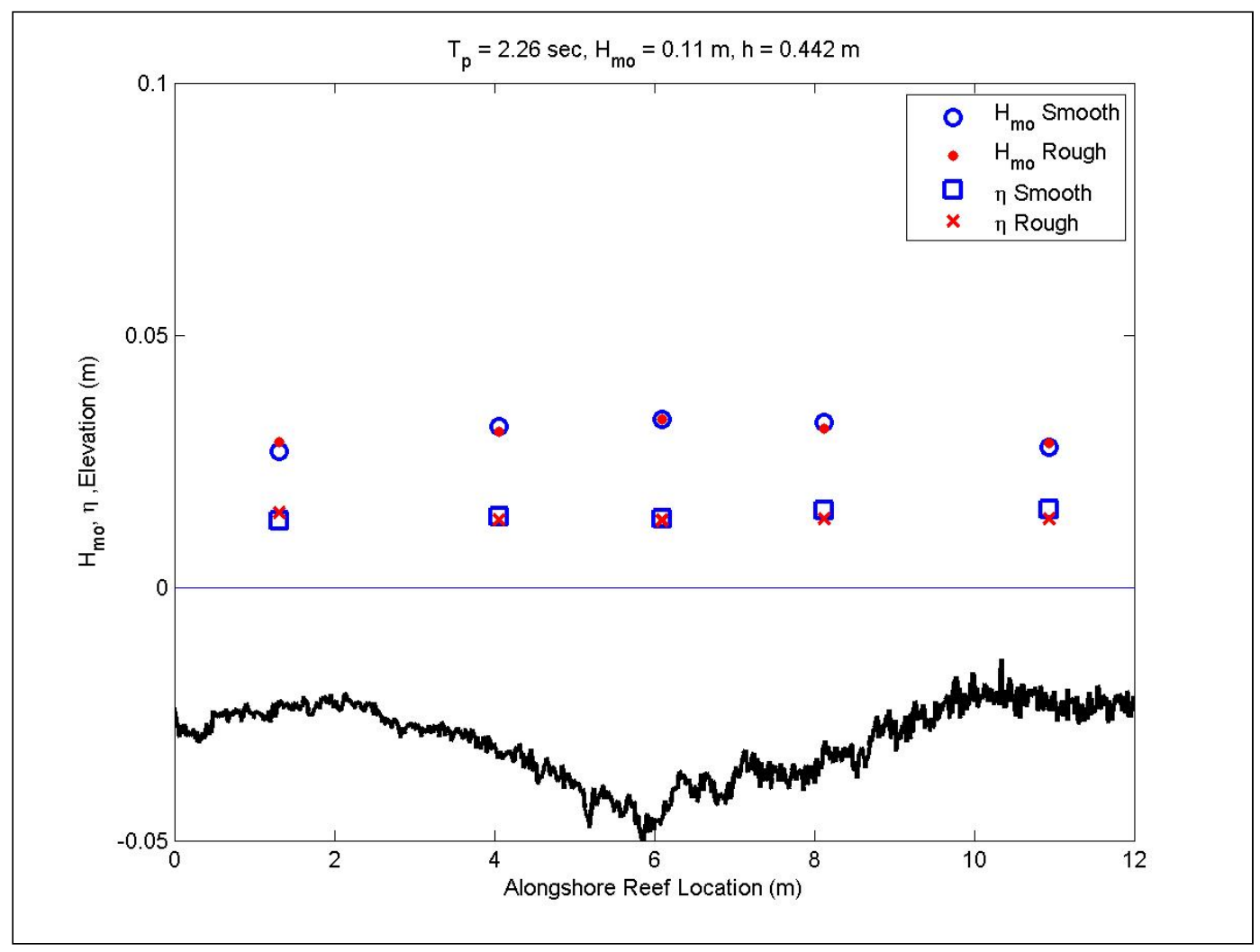

Figure B21. Alongshore wave heights for 2.3-sec, 0.11-m waves at mid water. 


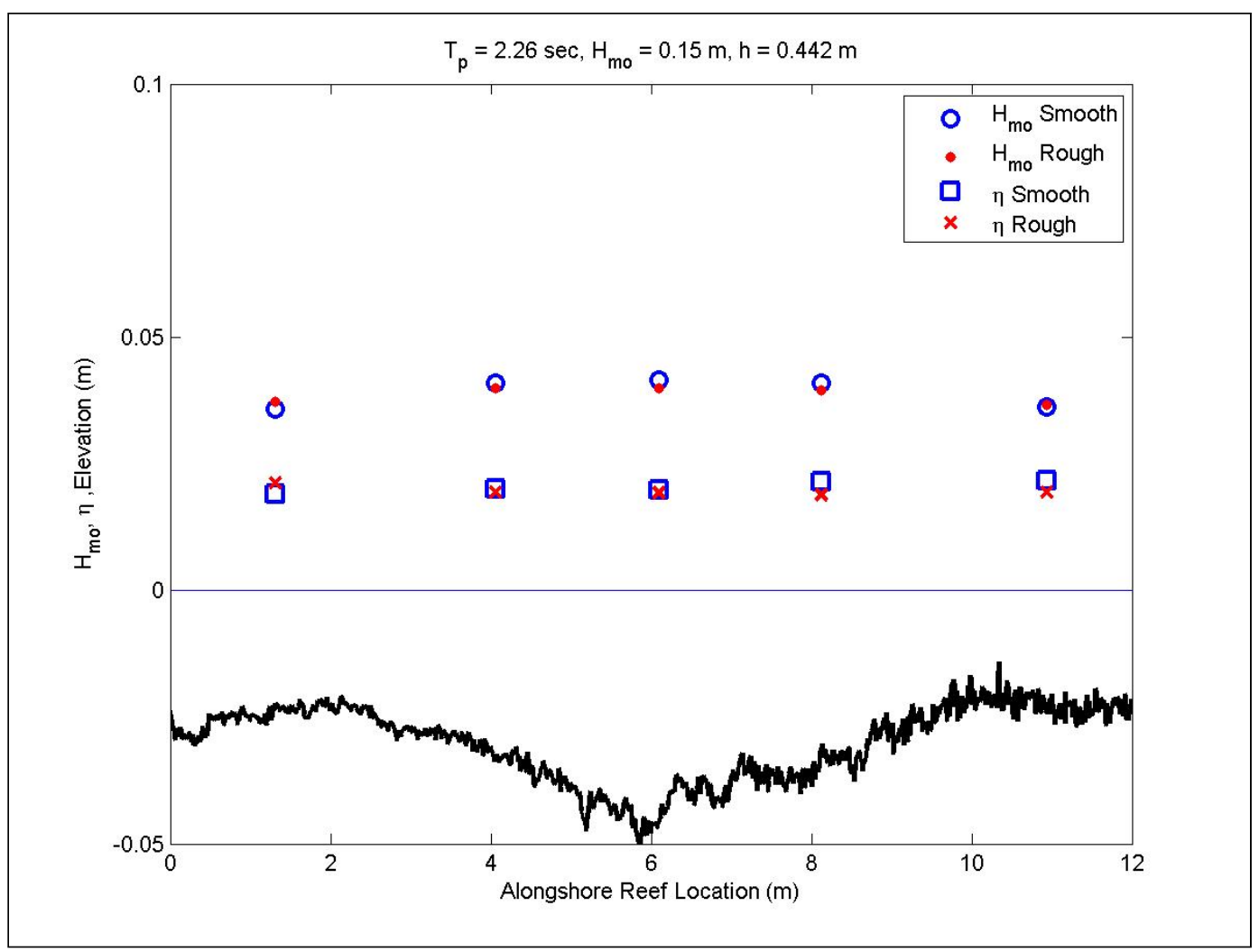

Figure B22. Alongshore wave heights for 2.3-sec, 0.15-m waves at mid water.

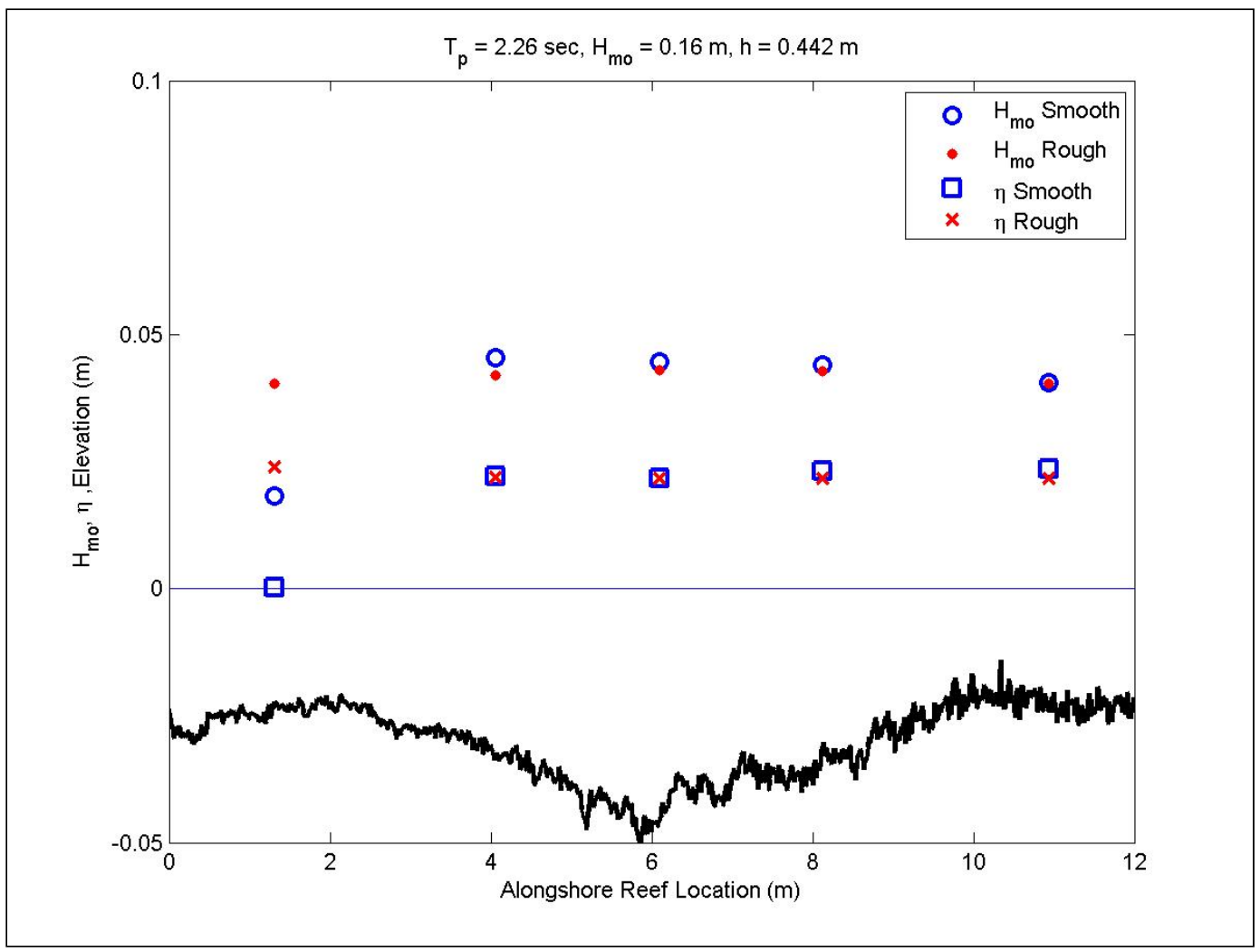

Figure B23. Alongshore wave heights for 2.3-sec, 0.16-m waves at mid water. 


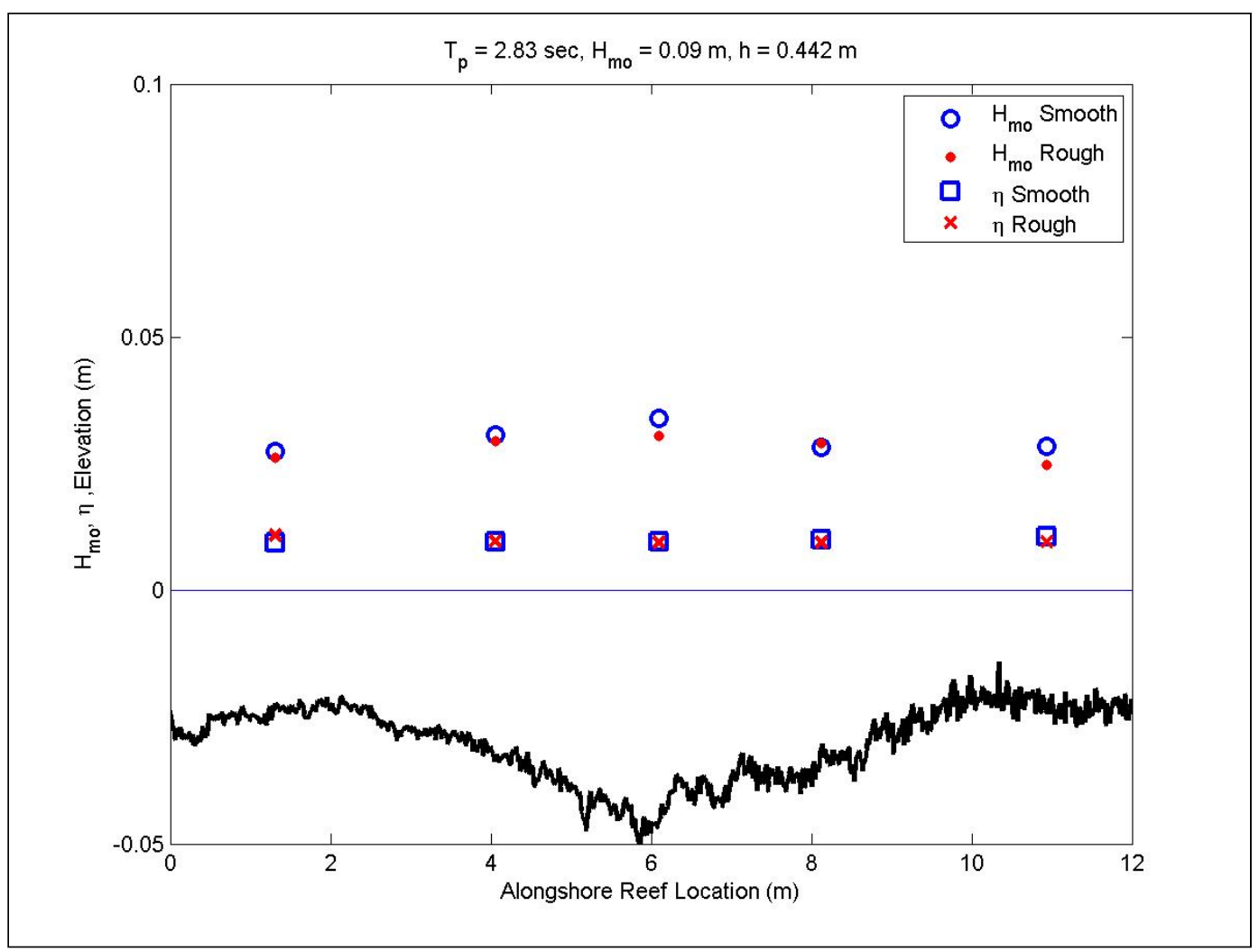

Figure B24. Alongshore wave heights for 2.8-sec, 0.09-m waves at mid water.

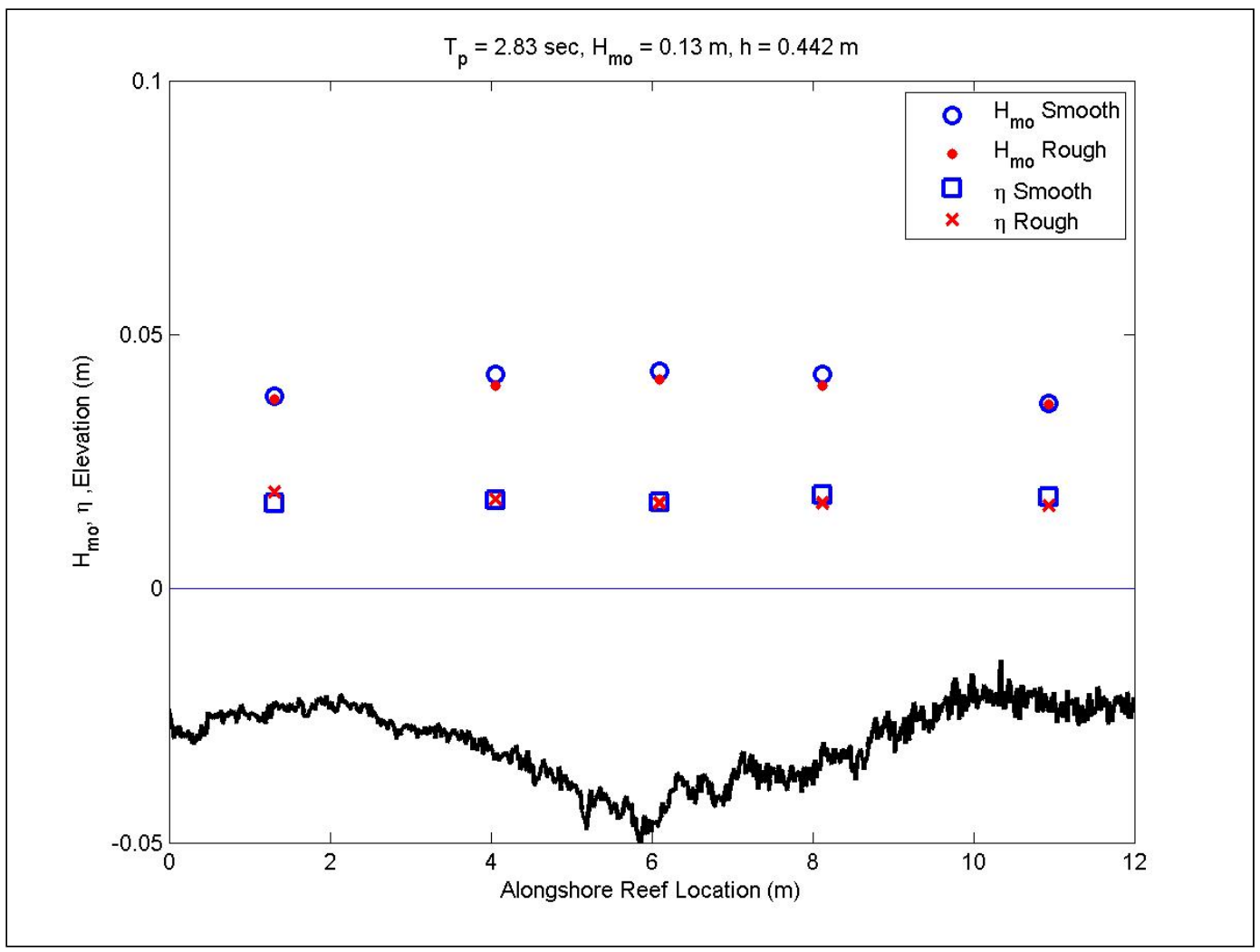

Figure B25. Alongshore wave heights for 2.8-sec, 0.13-m waves at mid water. 


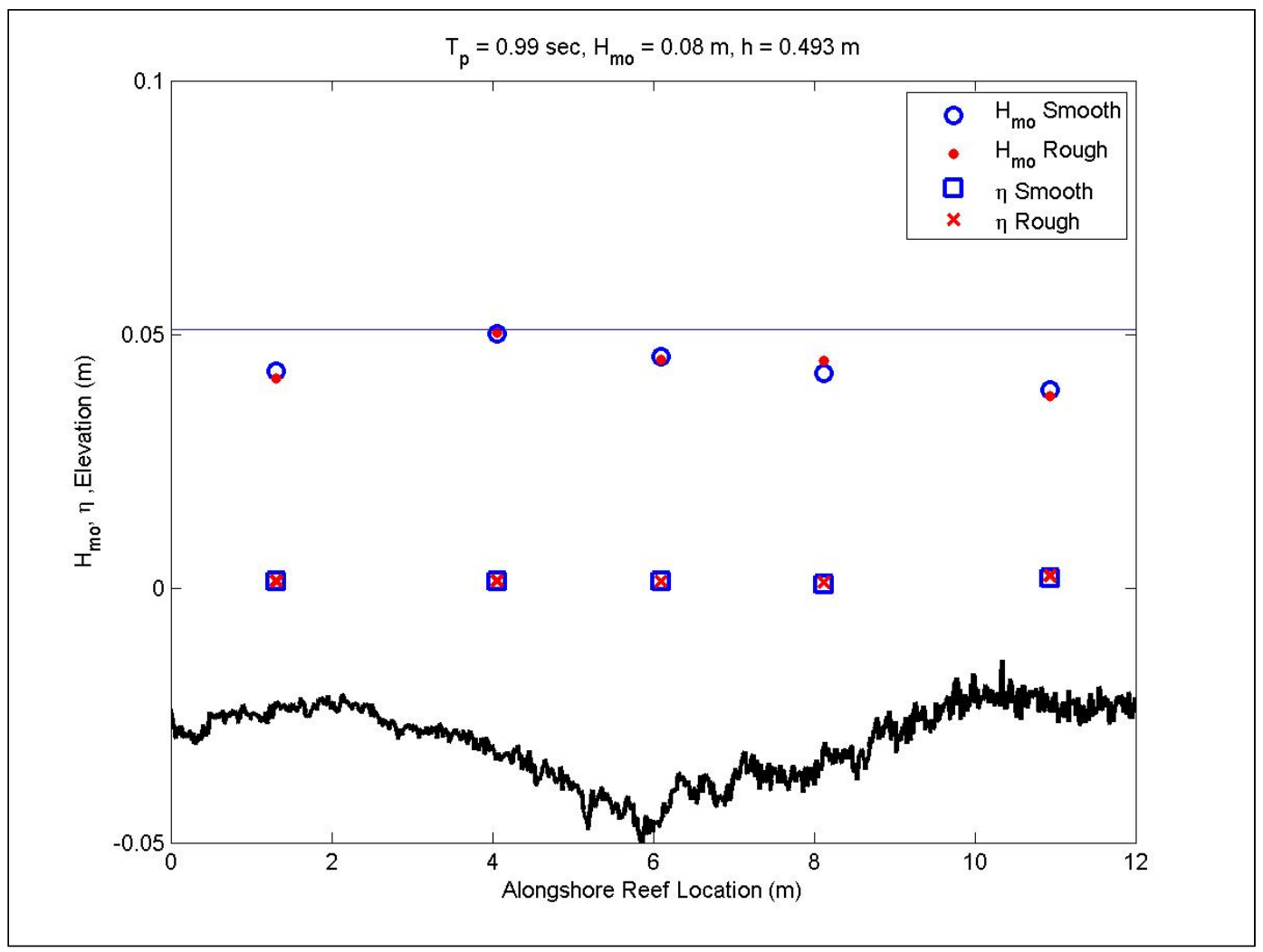

Figure B26. Alongshore wave heights for 1.0-sec, 0.08-m waves at high water.

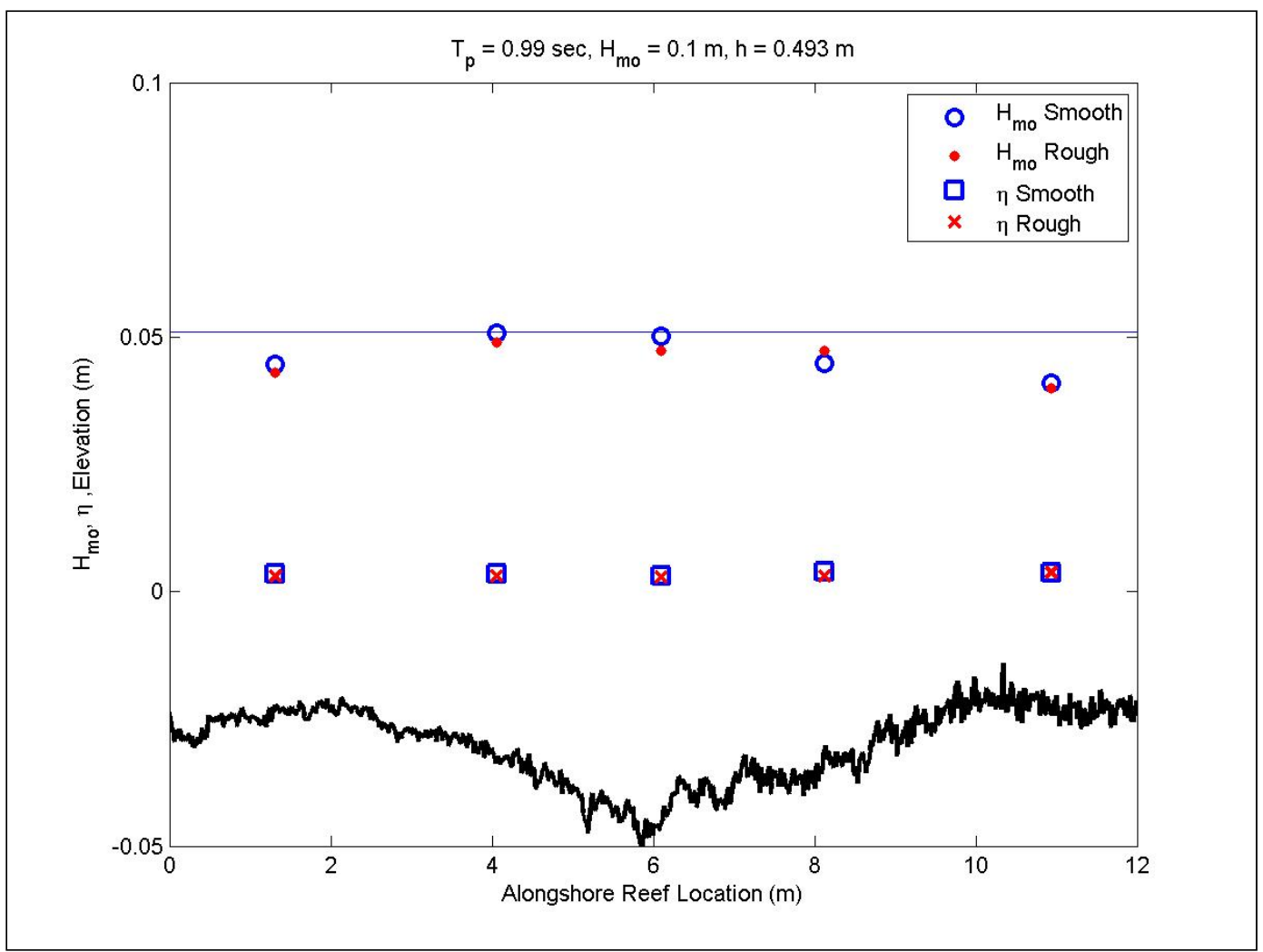

Figure B27. Alongshore wave heights for 1.0-sec, 0.1-m waves at high water. 


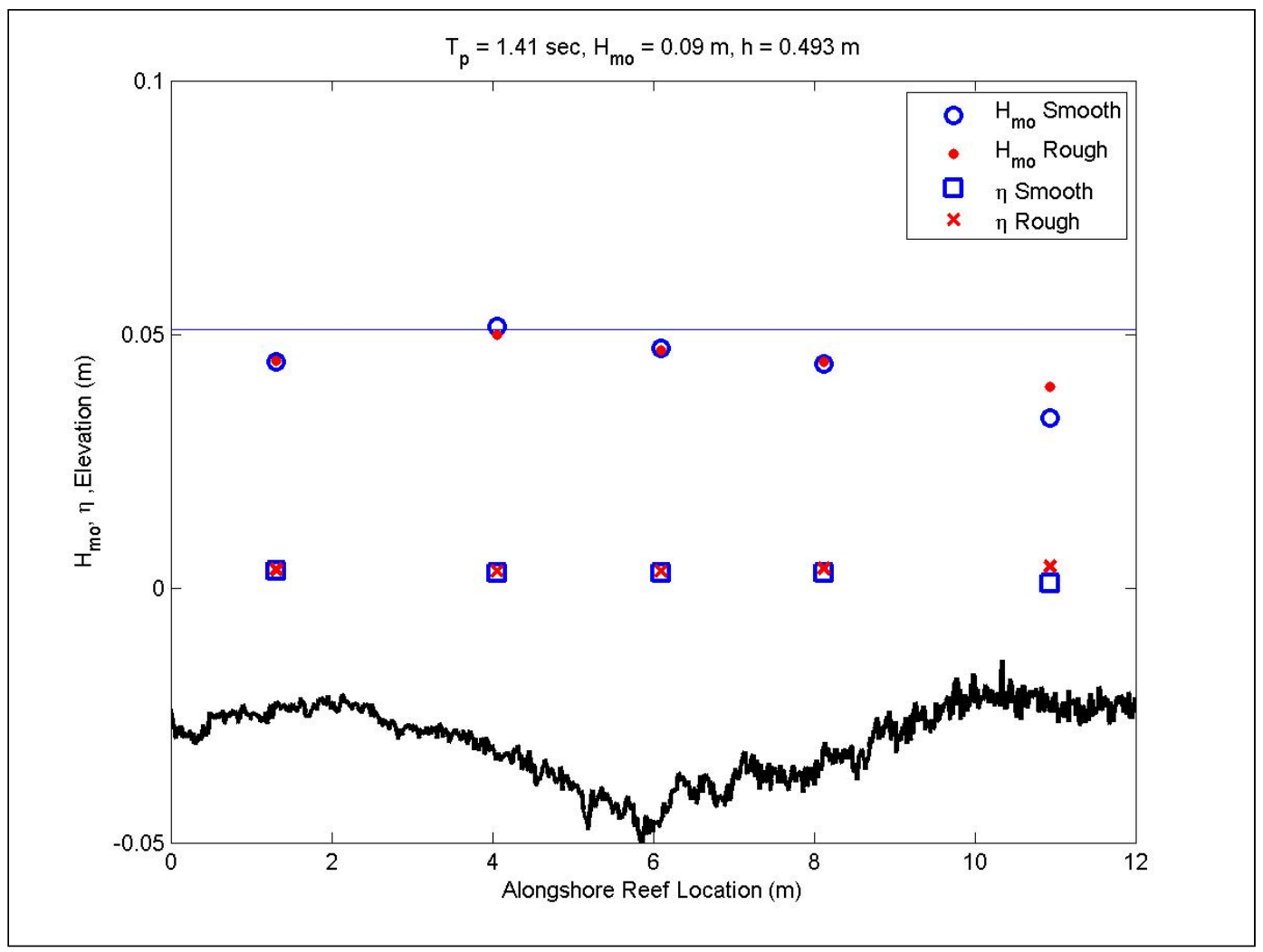

Figure B28. Alongshore wave heights for 1.4-sec, 0.09-m waves at high water.

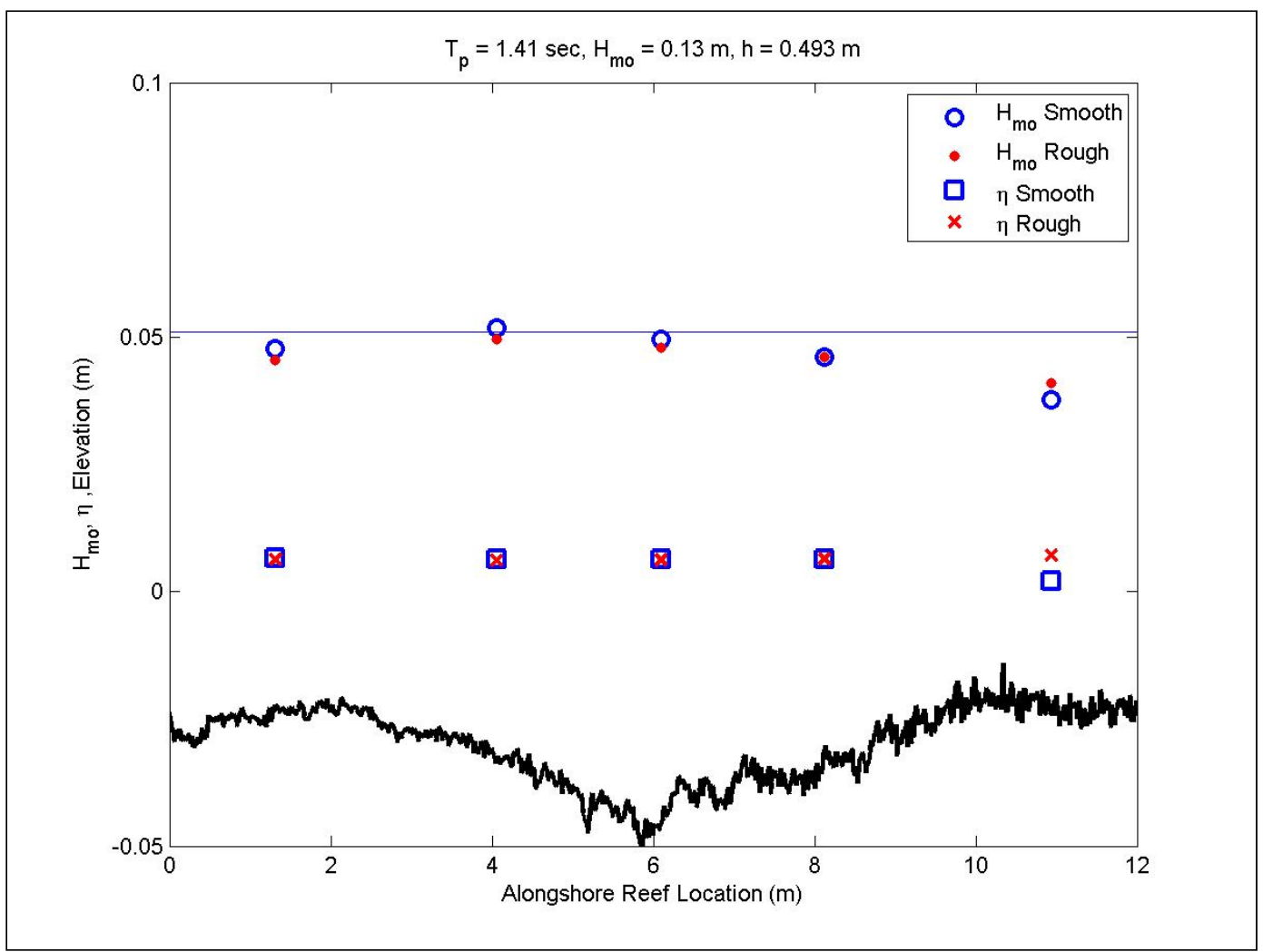

Figure B29. Alongshore wave heights for 1.4-sec, 0.13-m waves at high water. 


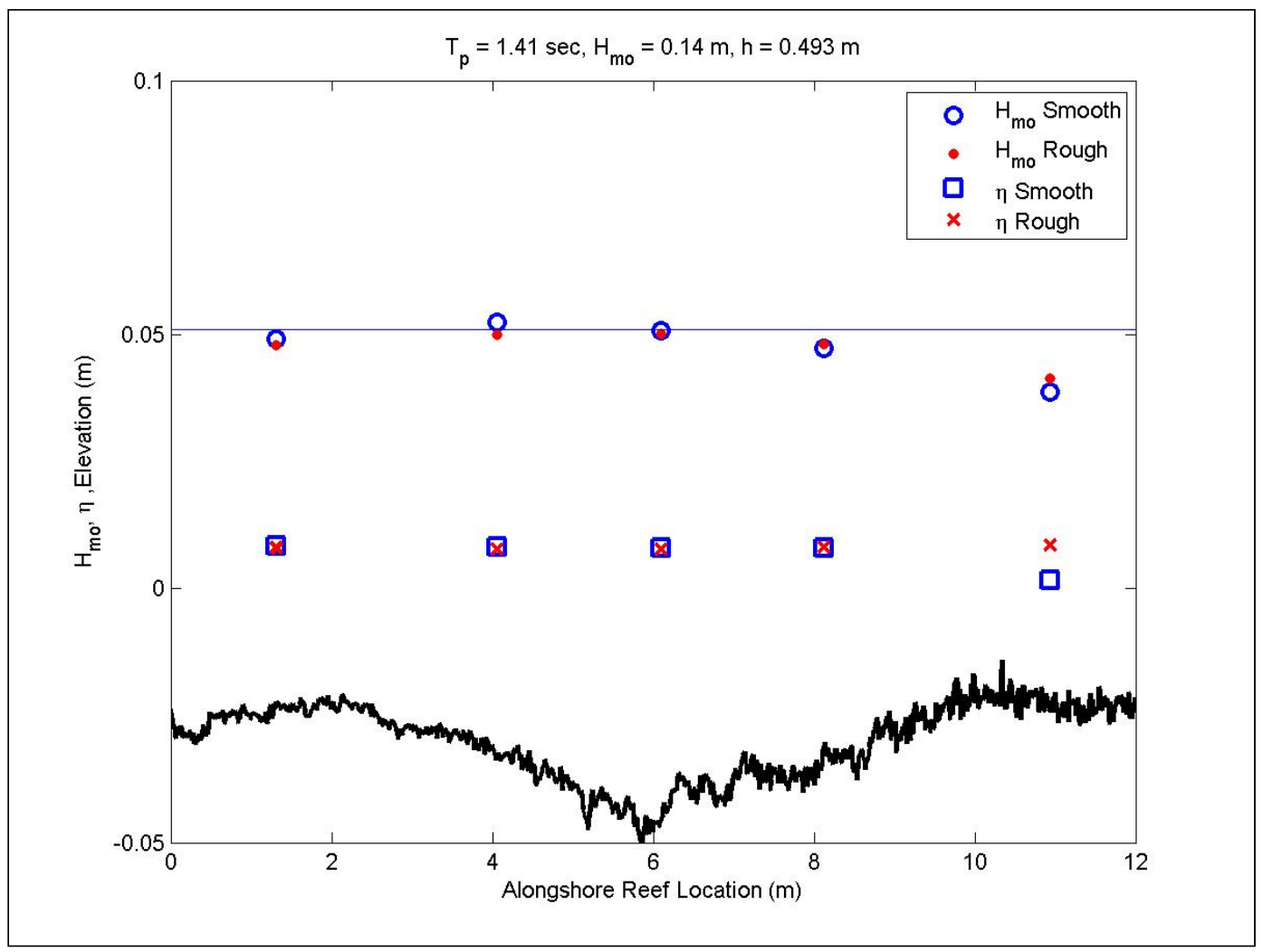

Figure B30. Alongshore wave heights for 1.4-sec, 0.14-m waves at high water.

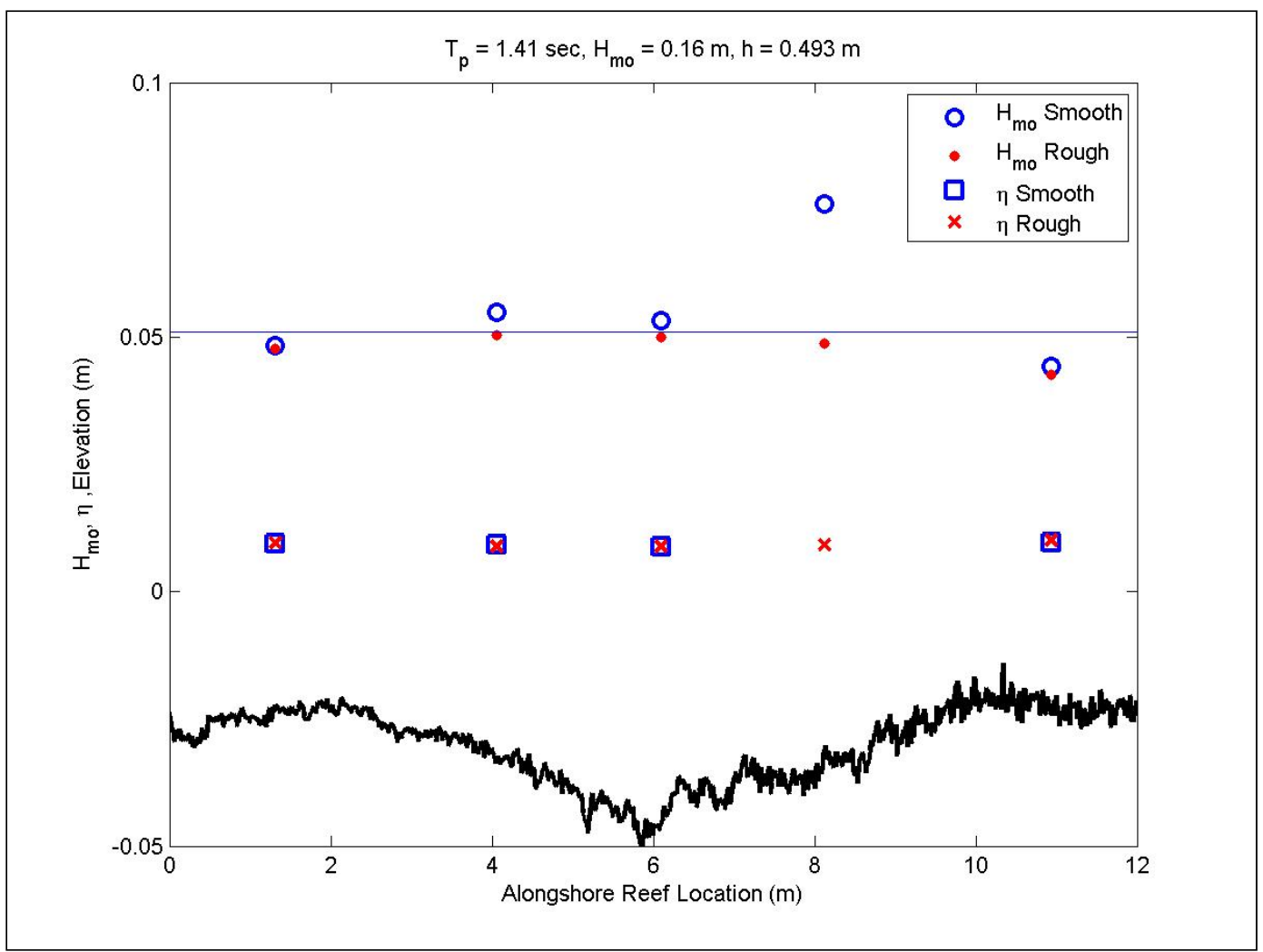

Figure B31. Alongshore wave heights for 1.4-sec, 0.16-m waves at high water. 


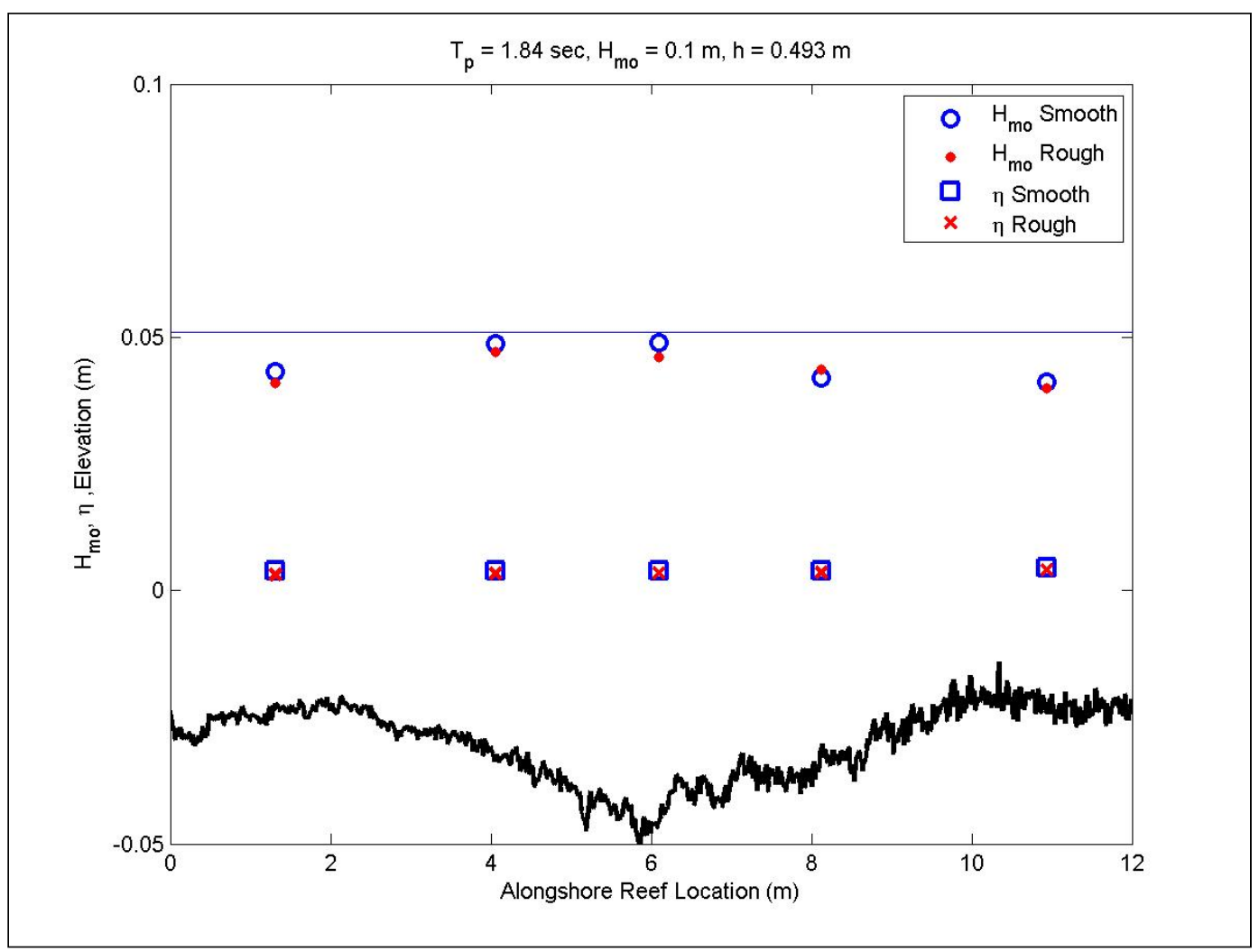

Figure B32. Alongshore wave heights for 1.8-sec, 0.1-m waves at high water.

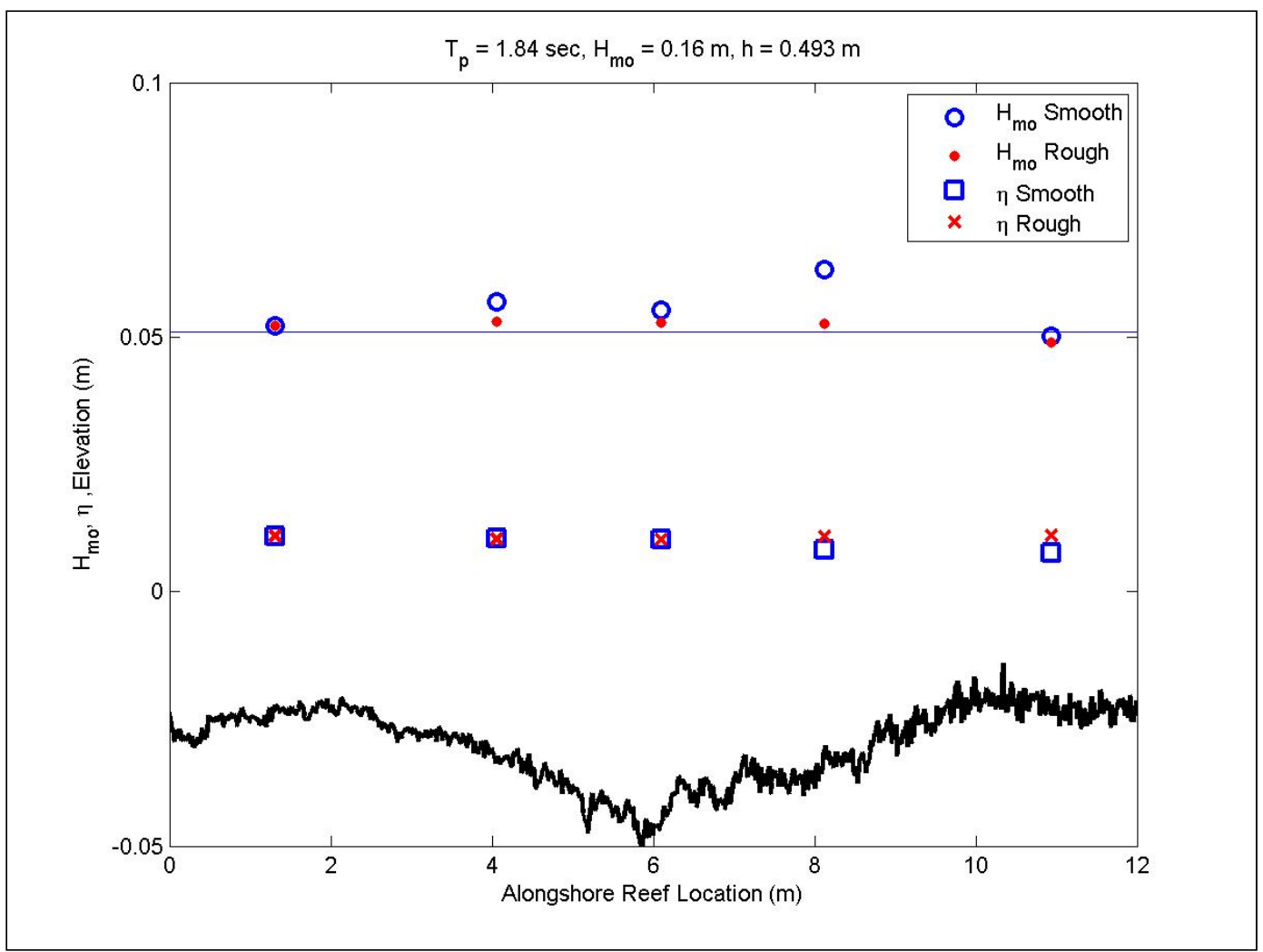

Figure B33. Alongshore wave heights for 1.8-sec, 0.16-m waves at high water. 


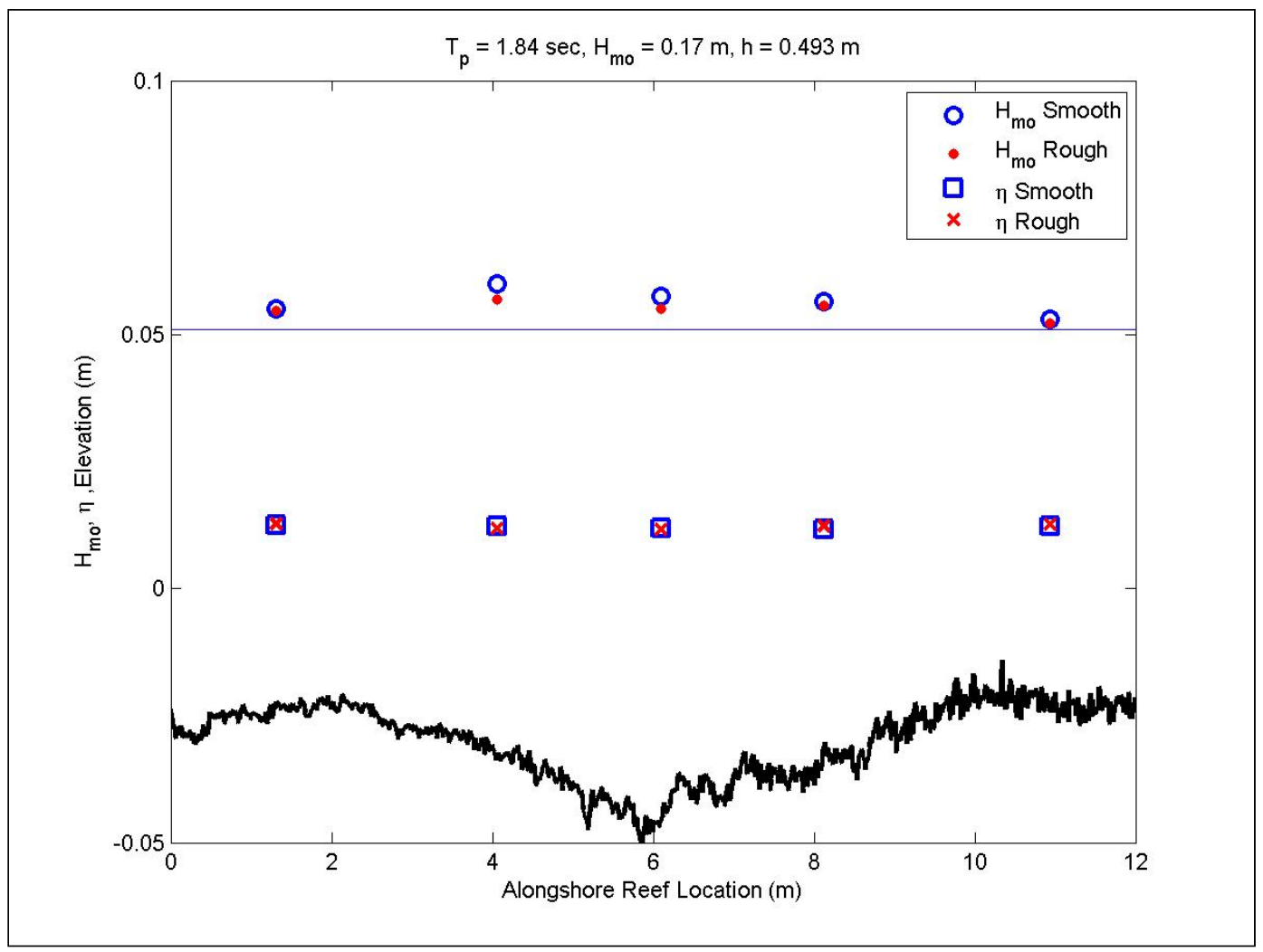

Figure B34. Alongshore wave heights for 1.8-sec, 0.17-m waves at high water.

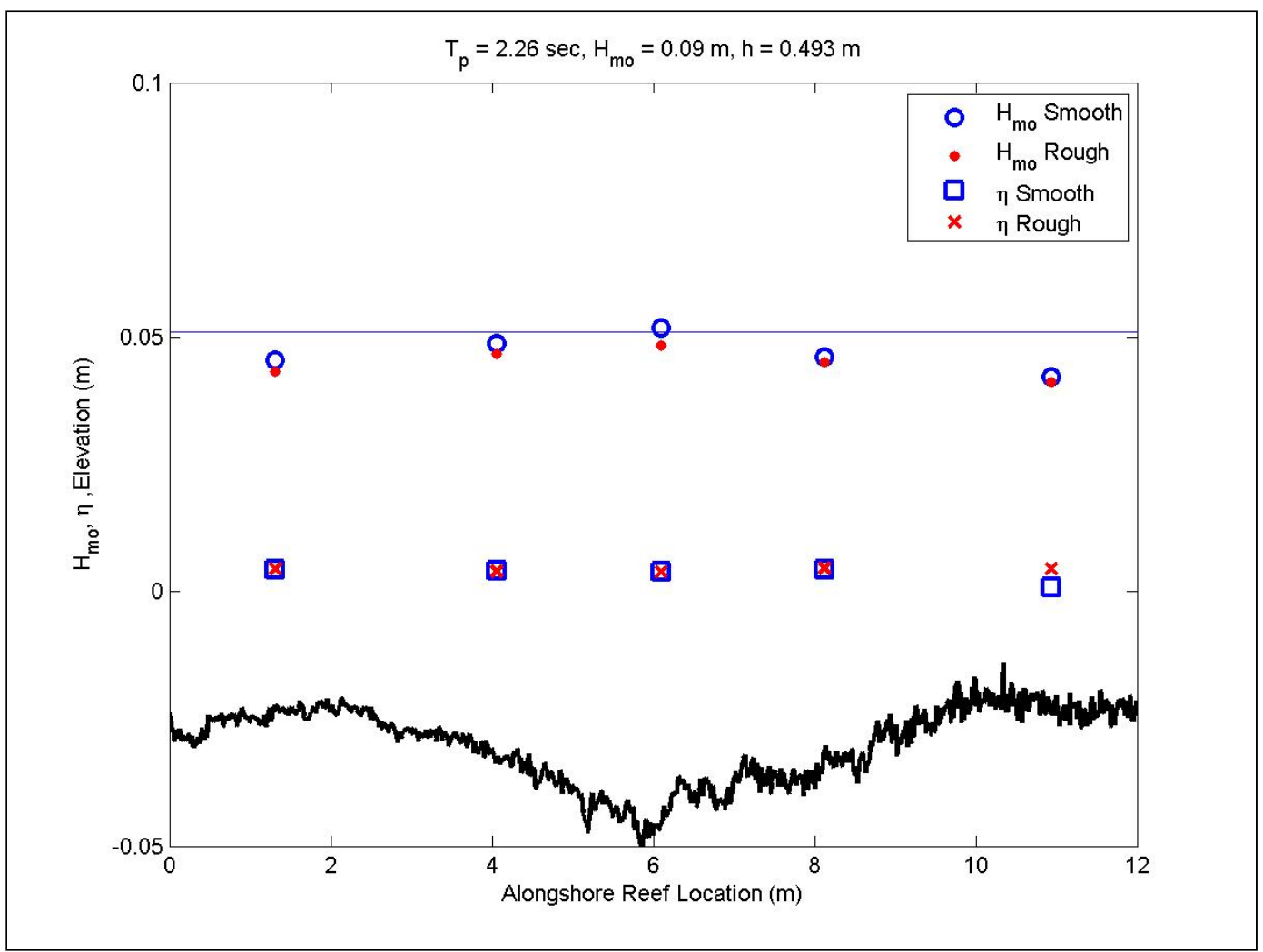

Figure B35. Alongshore wave heights for 2.3-sec, 0.09-m waves at high water. 


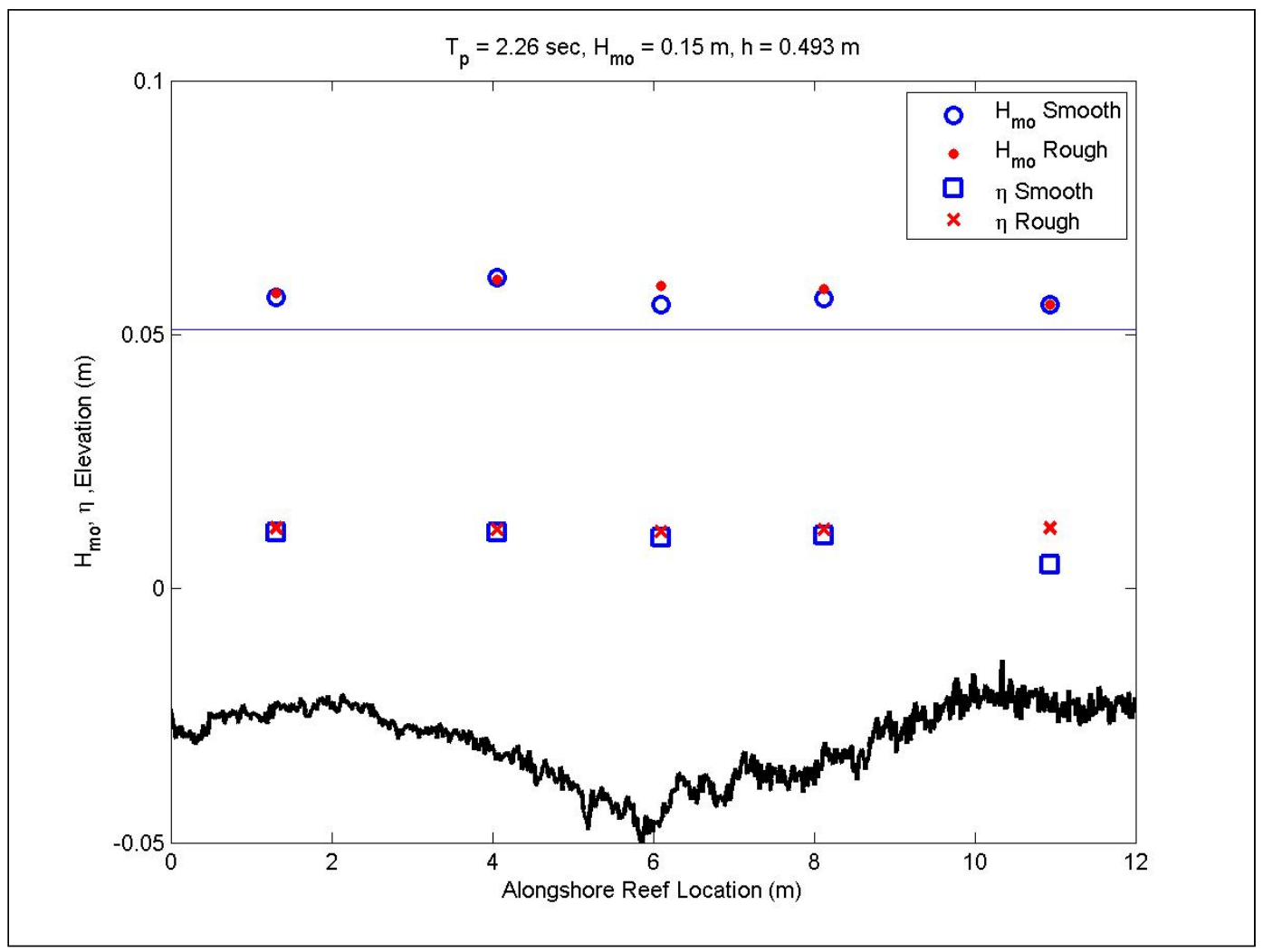

Figure B36. Alongshore wave heights for 2.3-sec, 0.15-m waves at high water.

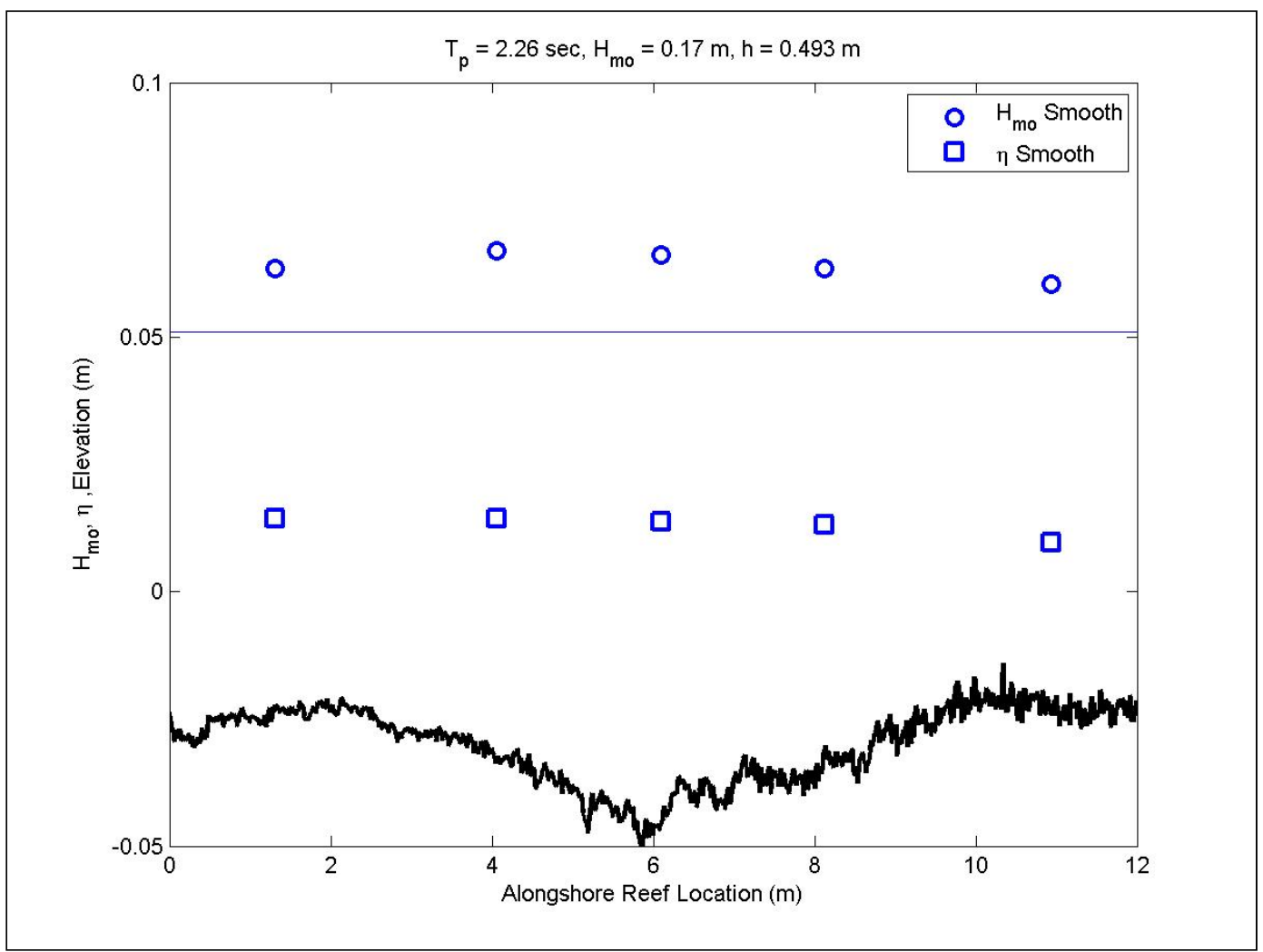

Figure B37. Alongshore wave heights for 2.3-sec, 0.17-m waves at high water. 


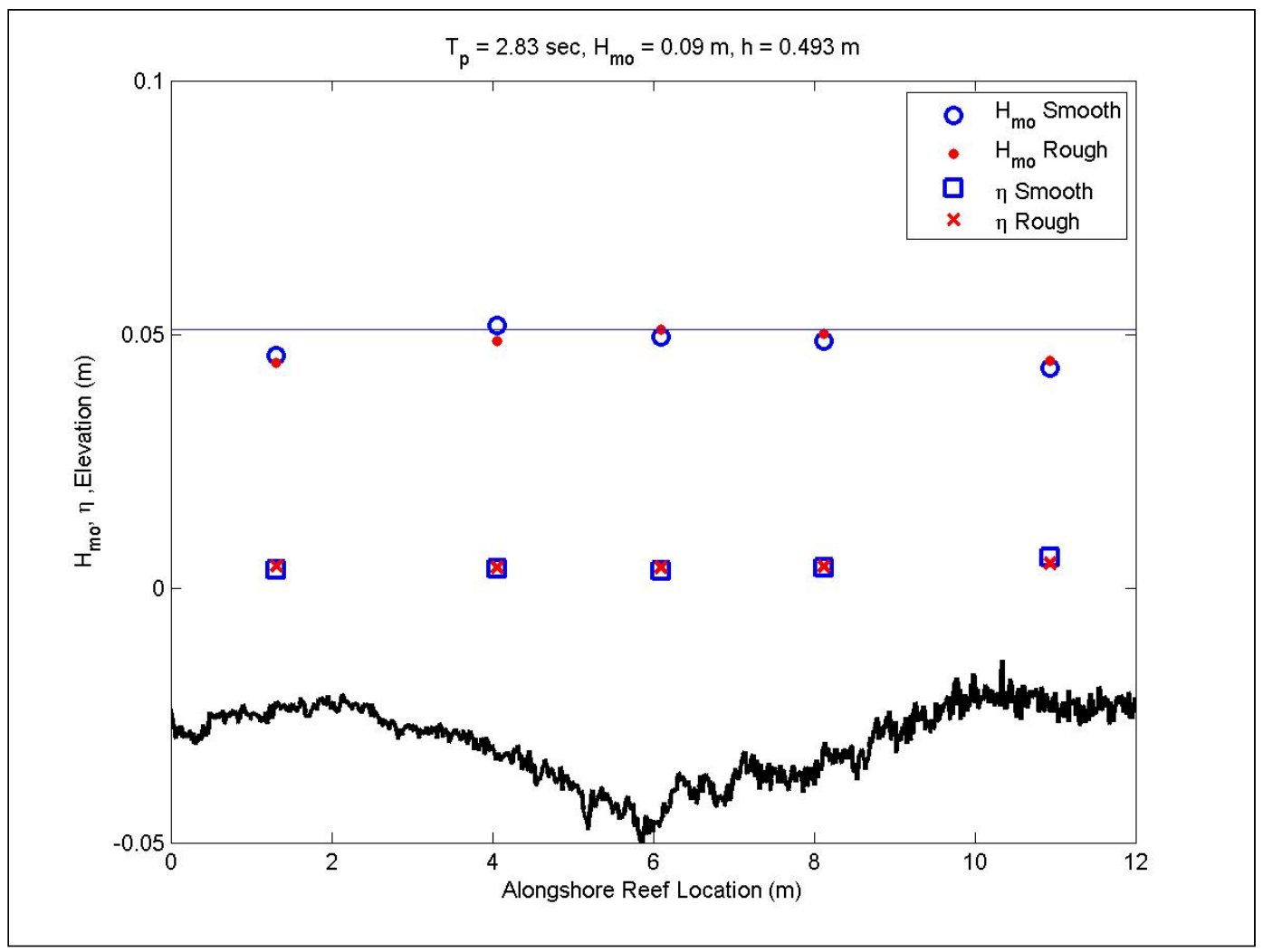

Figure B38. Alongshore wave heights for 2.8-sec, 0.09-m waves at high water.

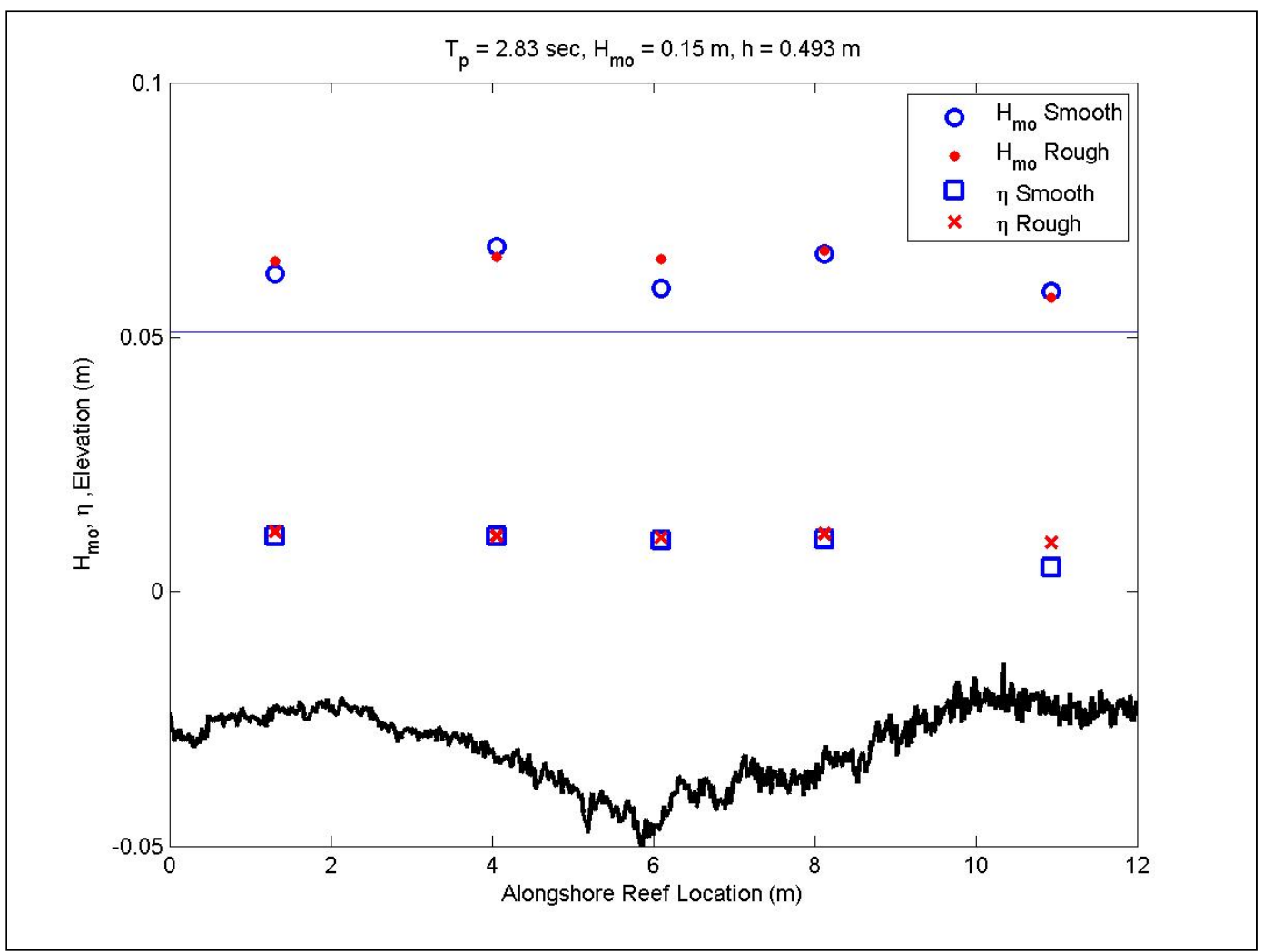

Figure B39. Alongshore wave heights for 2.8-sec, 0.15-m waves at high water. 


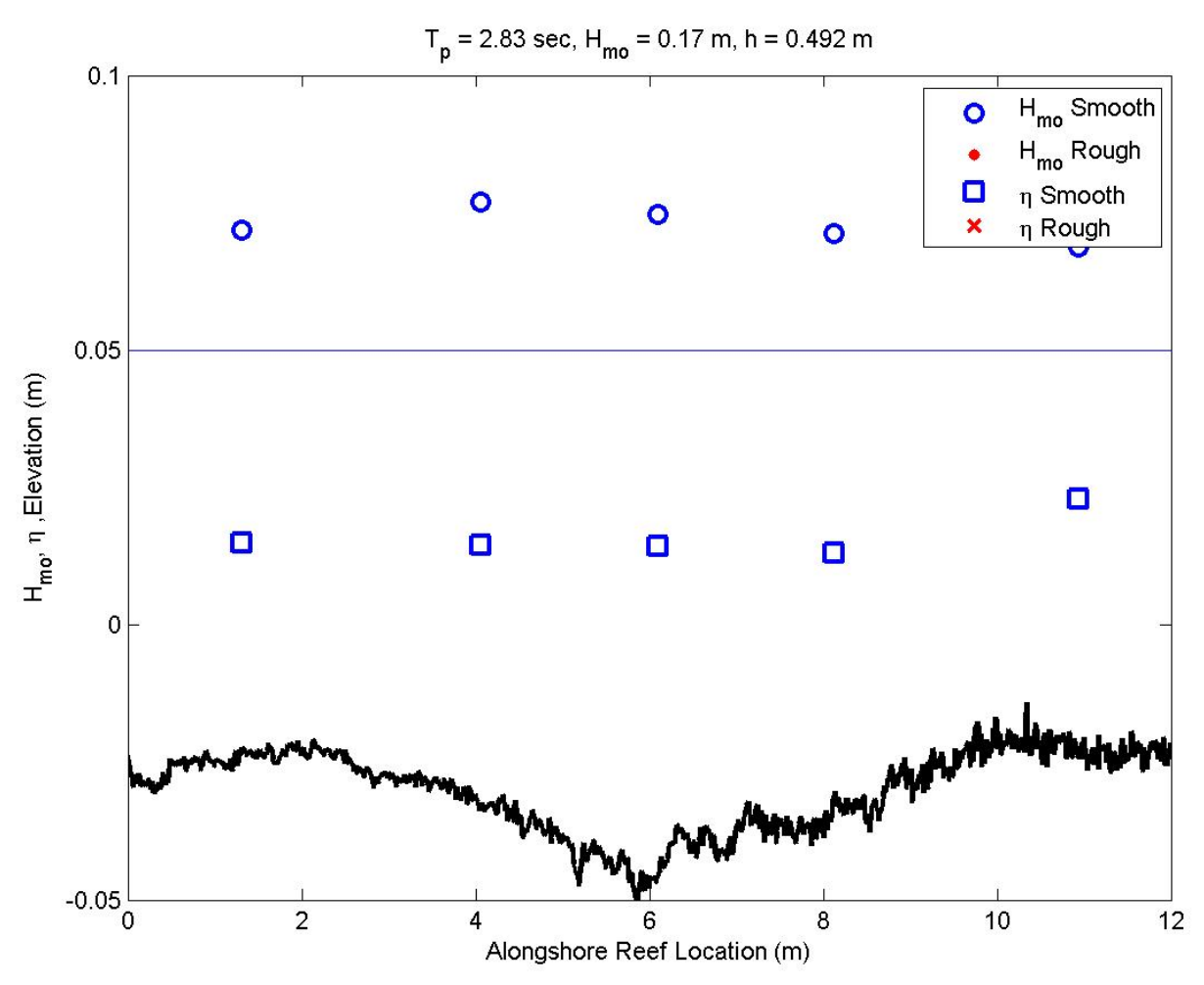

Figure B40. Alongshore wave heights for 2.8-sec, 0.17-m waves at high water. 


\section{Appendix C: Channel wave-height and current figures from the three-dimensional experiments}

The figures presented in Appendix $\mathrm{C}$ show results from the channel experiment performed in the $3-\mathrm{D}$ basin. The figures are grouped by transformation plots, rose plots of currents from two ADVs placed on the reef, and alongshore wave heights.

Transformation plots (Figures $\mathrm{C} 1$ to $\mathrm{C} 17$ ) include zero-moment wave heights and mean water levels as a function of distance along the centerline of the 3-D basin. The figures include wave height and mean water level measured on the smooth and roughened bathymetry for similar wave conditions. The measured model bathymetry along the line of gauges also is plotted, but is scaled by a factor of 10 .

Rose plots (Figures $\mathrm{C} 18$ to $\mathrm{C} 34$ ) show cumulative velocity by direction with the center of the rose at the ADV location. Each velocity measurement was separated into 20 directional bins and velocities were summed by bin. ADV 1 was located at the channel midpoint with its sampling volume located in the center of the channel. ADV2 was placed near the center of the reef. The ADVs were positioned vertically at one-third of the water depth from the bottom to measure velocities that approximated the mean velocities (Hamilton, et al.

Figures $\mathrm{C}_{35}$ to $\mathrm{C}_{51}$ show zero-moment wave heights and mean water levels at the five alongshore gauges (Gauges 12 to 16) on the reef bathymetry. Each figure includes wave height and mean water level measured on the smooth and roughened bathymetry for similar wave conditions. The measured model bathymetry along the line of gauges also is plotted. 


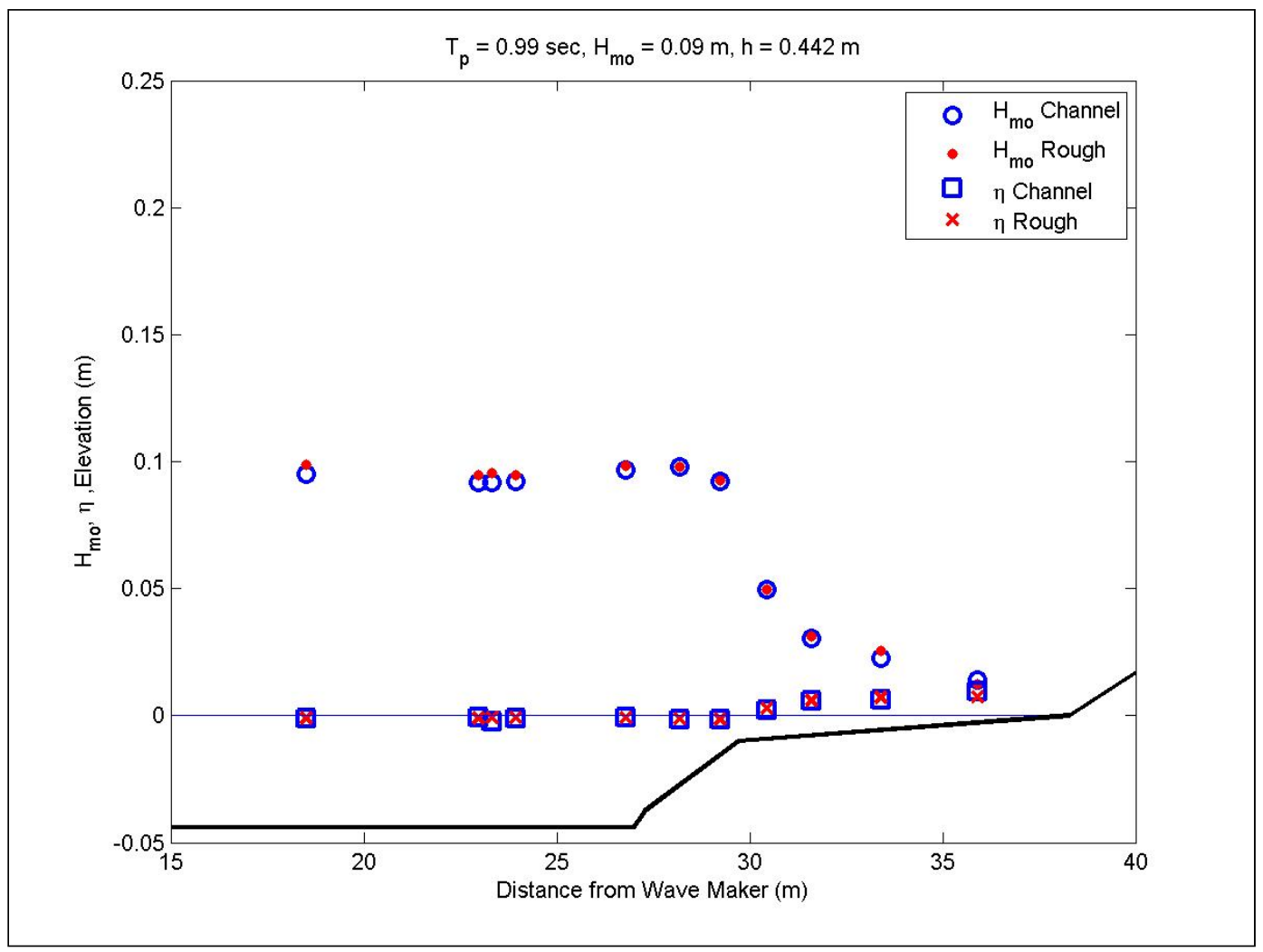

Figure C1. Wave transformation for $1-\mathrm{sec}, 0.09-\mathrm{m}$ waves at mid water.

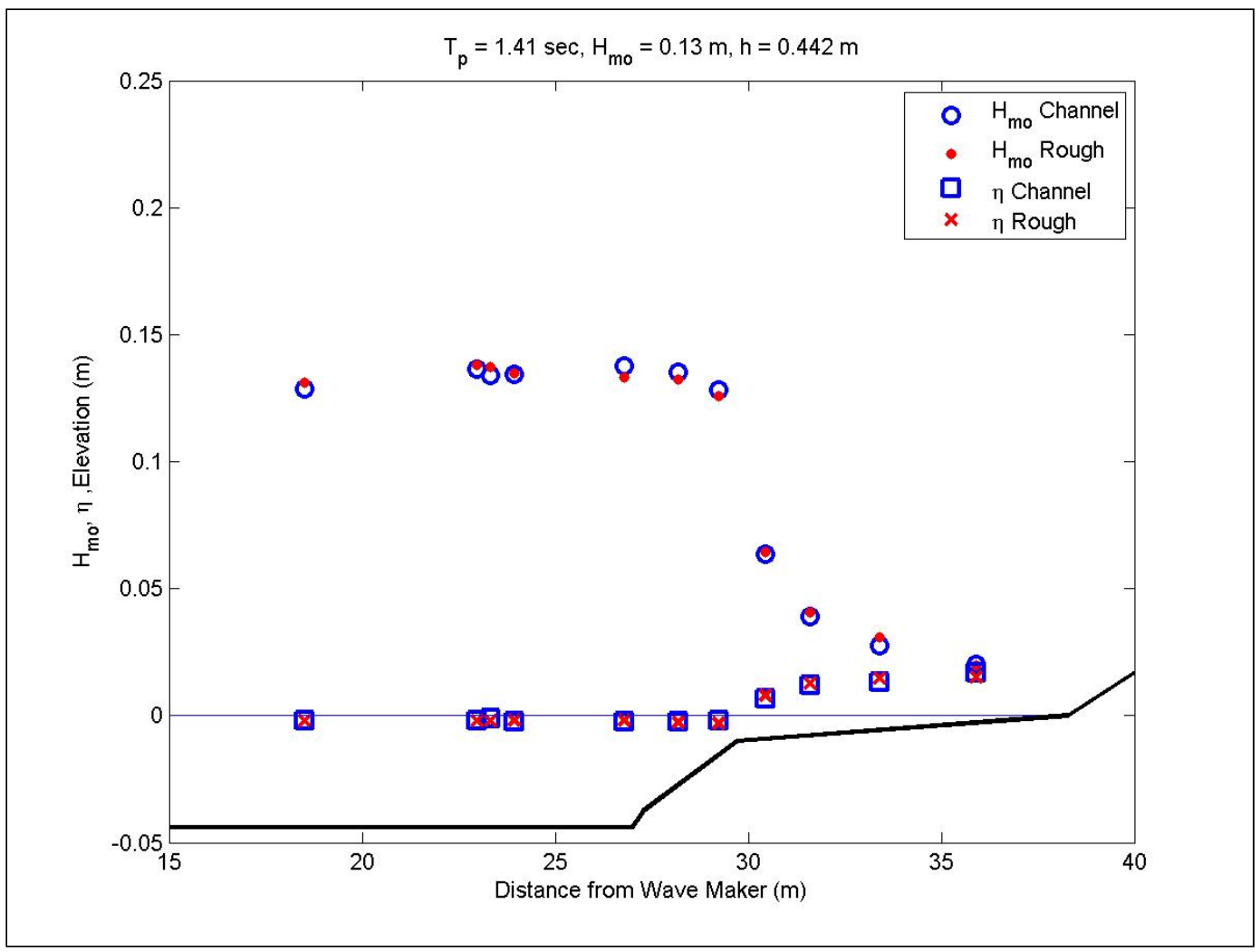

Figure C2. Wave transformation for $1.4-\mathrm{sec}, 0.13-\mathrm{m}$ waves at mid water. 


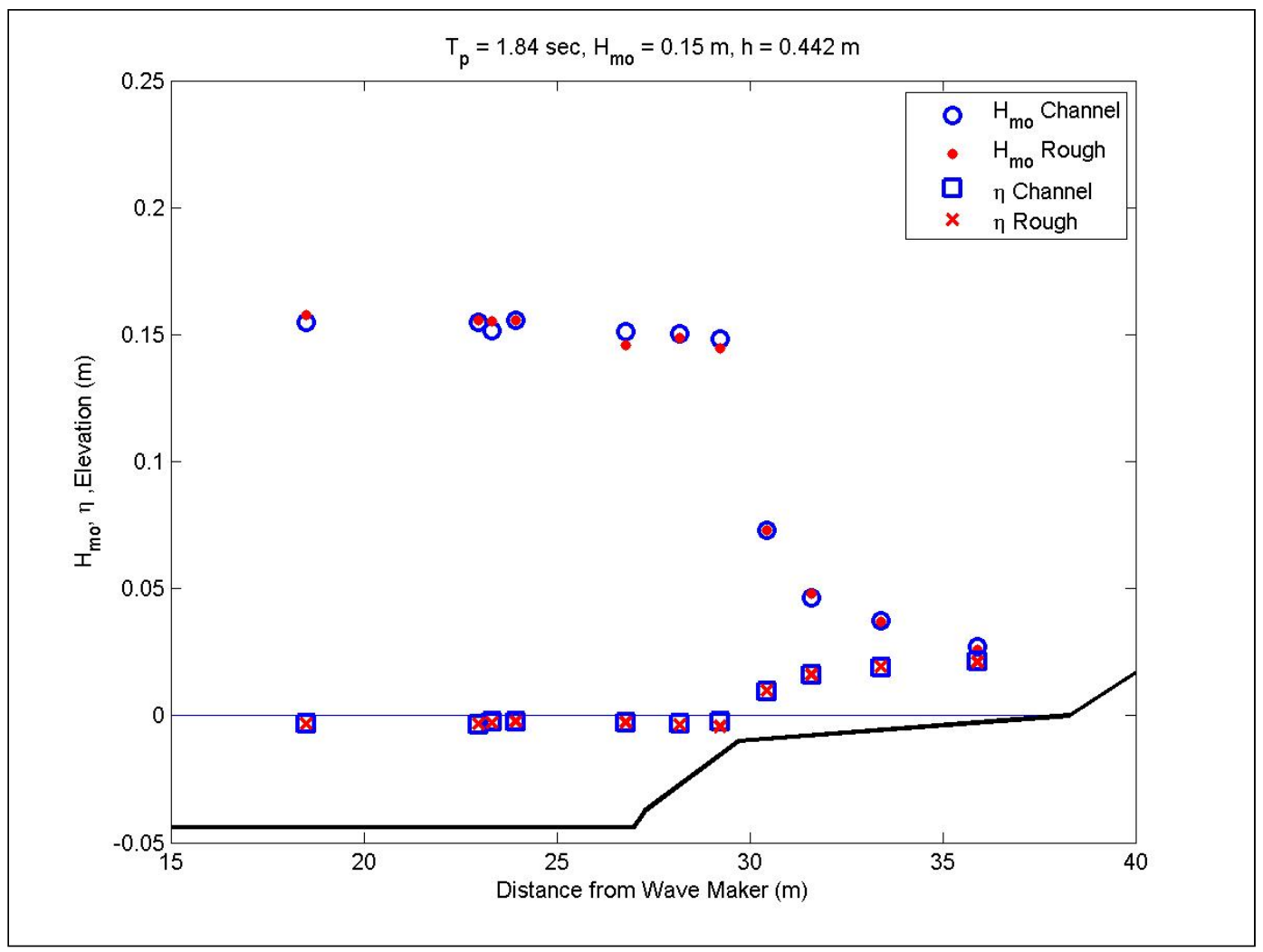

Figure C3. Wave transformation for $1.8-\mathrm{sec}, 0.15-\mathrm{m}$ waves at mid water.

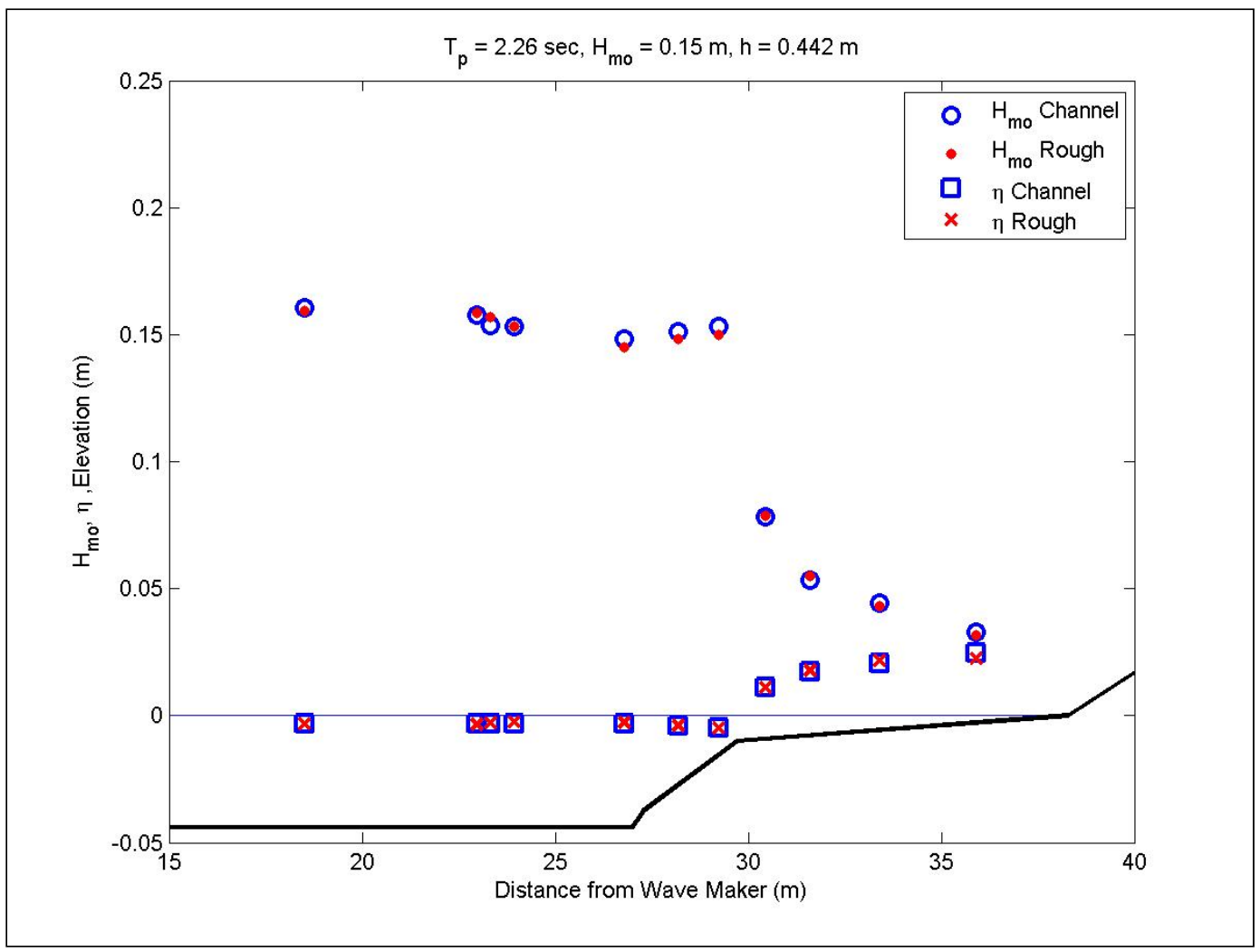

Figure C4. Wave transformation for $2.3-\mathrm{sec}, 0.15-\mathrm{m}$ waves at mid water. 


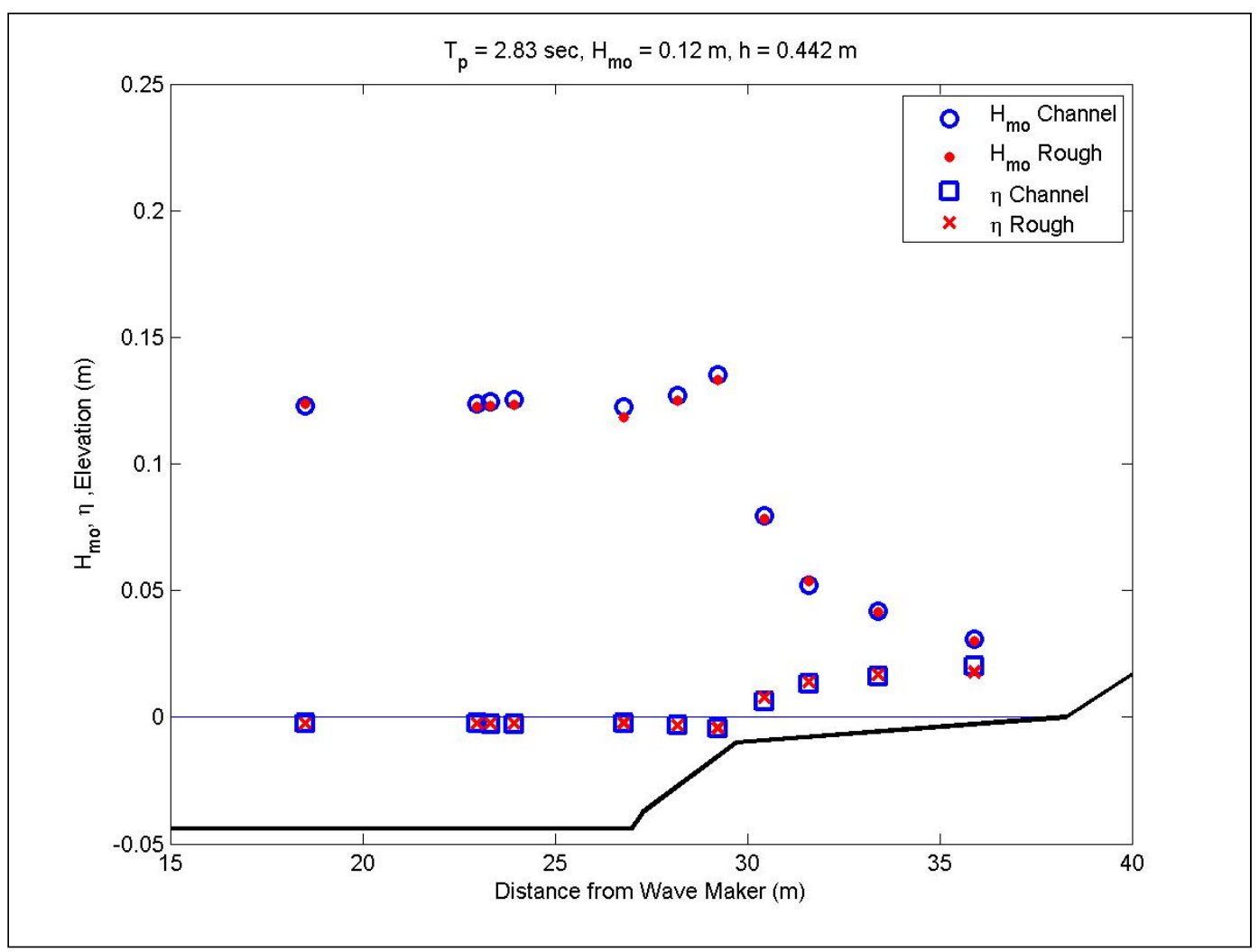

Figure C5. Wave transformation for $2.8-\mathrm{sec}, 0.12-\mathrm{m}$ waves at mid water.

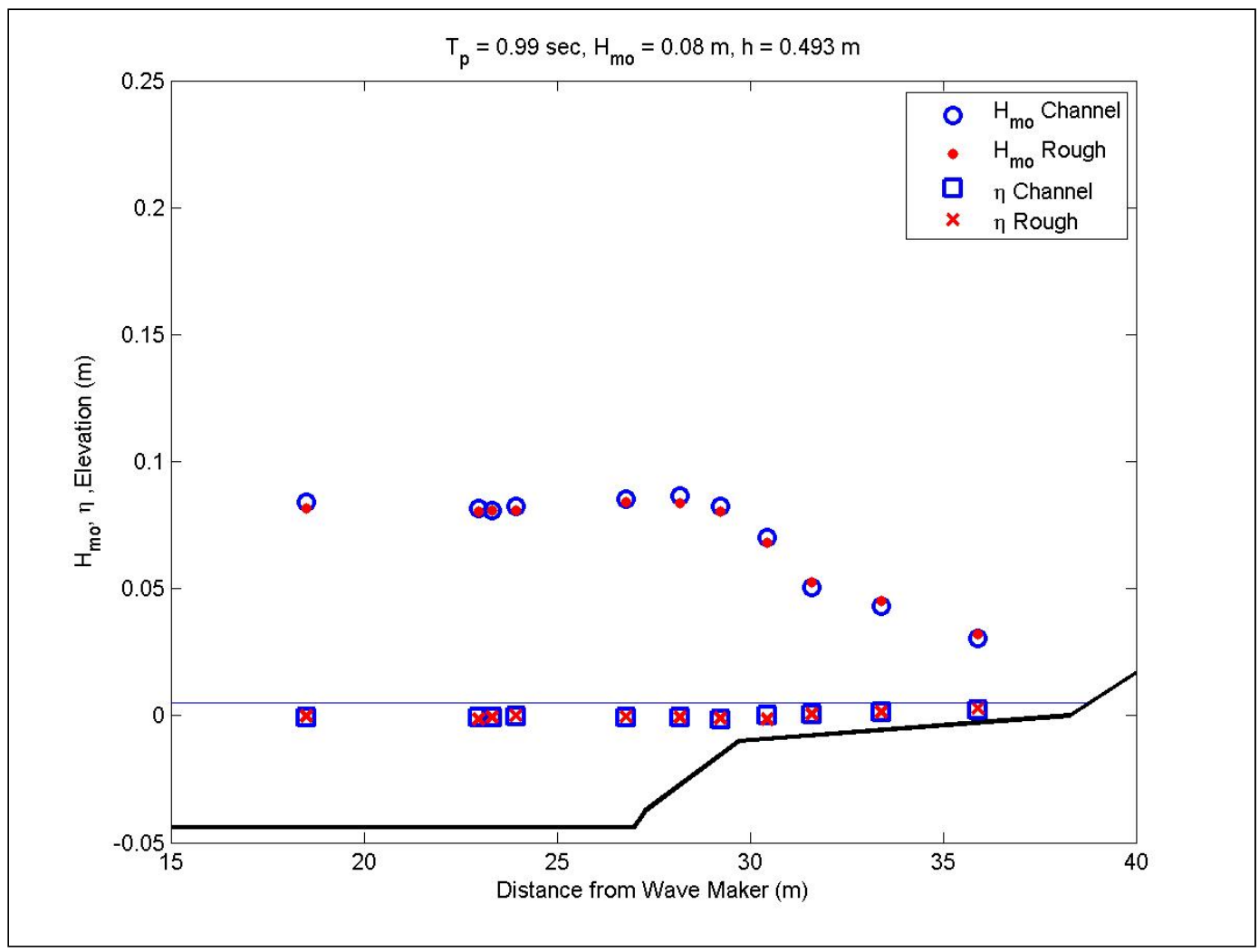

Figure C6. Wave transformation for 1 -sec, $0.08-\mathrm{m}$ waves at high water. 


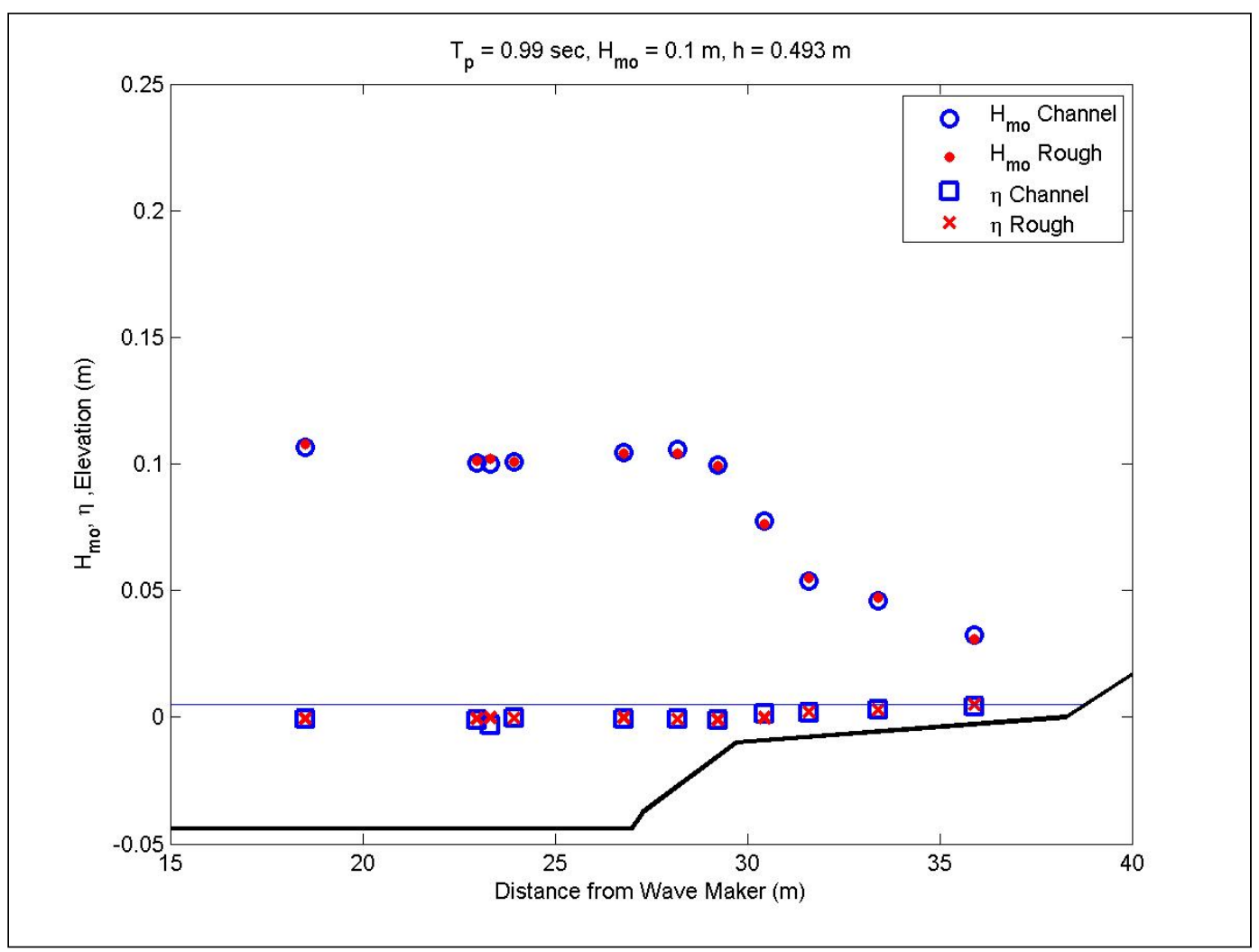

Figure $\mathrm{C} 7$. Wave transformation for 1-sec, 0.1-m waves at high water.

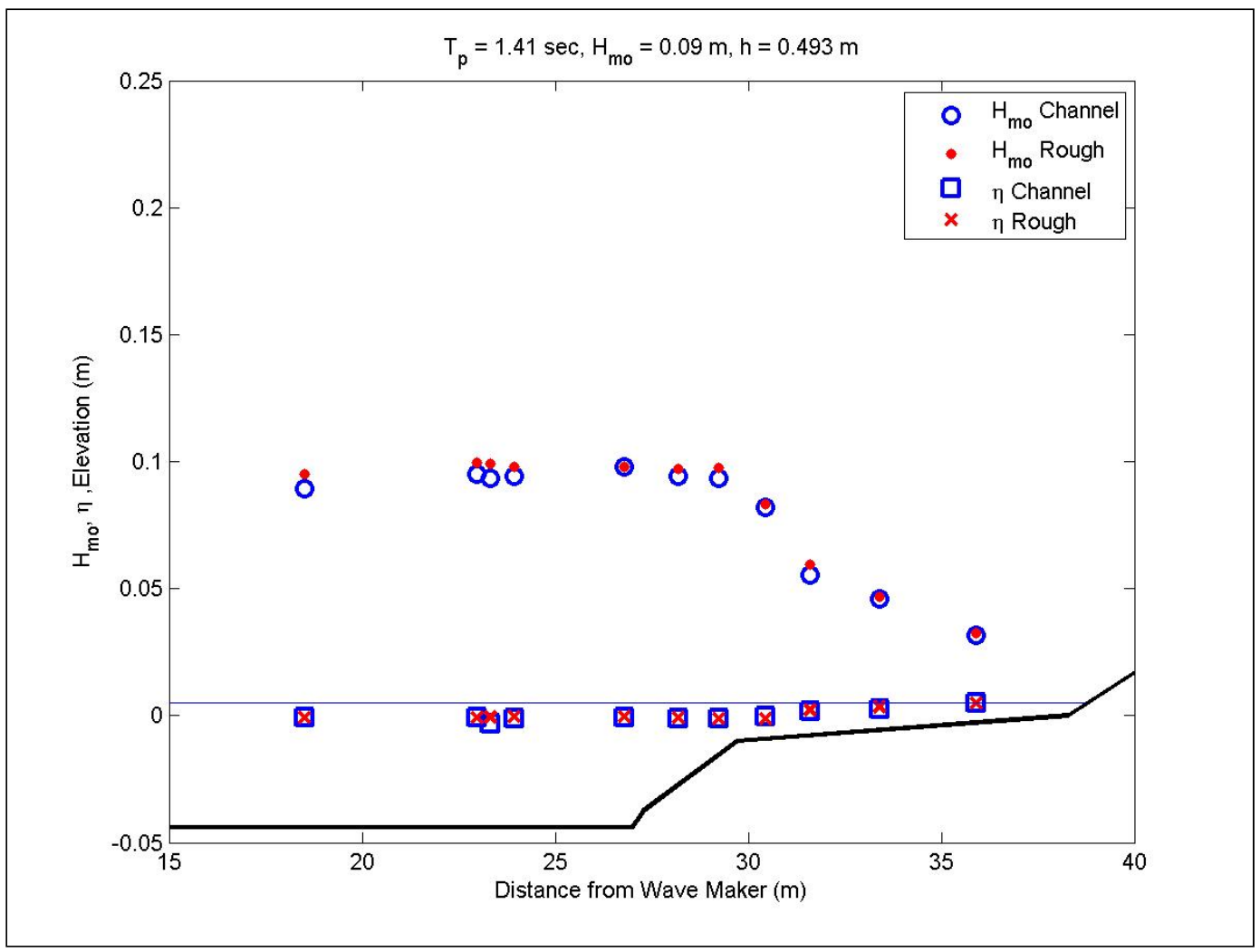

Figure C8. Wave transformation for 1.4-sec, 0.09-m waves at high water. 


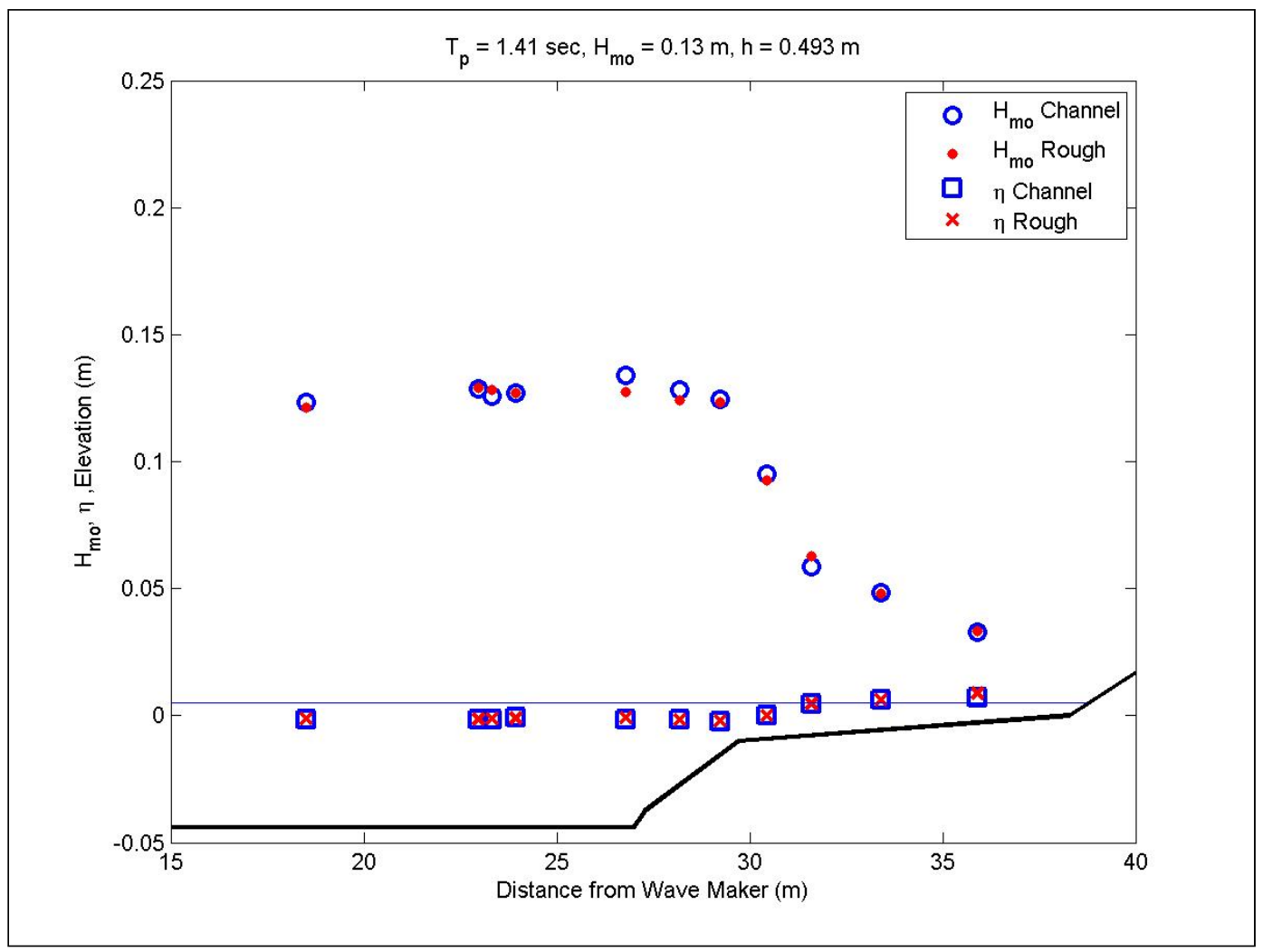

Figure C9. Wave transformation for 1.4-sec, 0.13-m waves at high water.

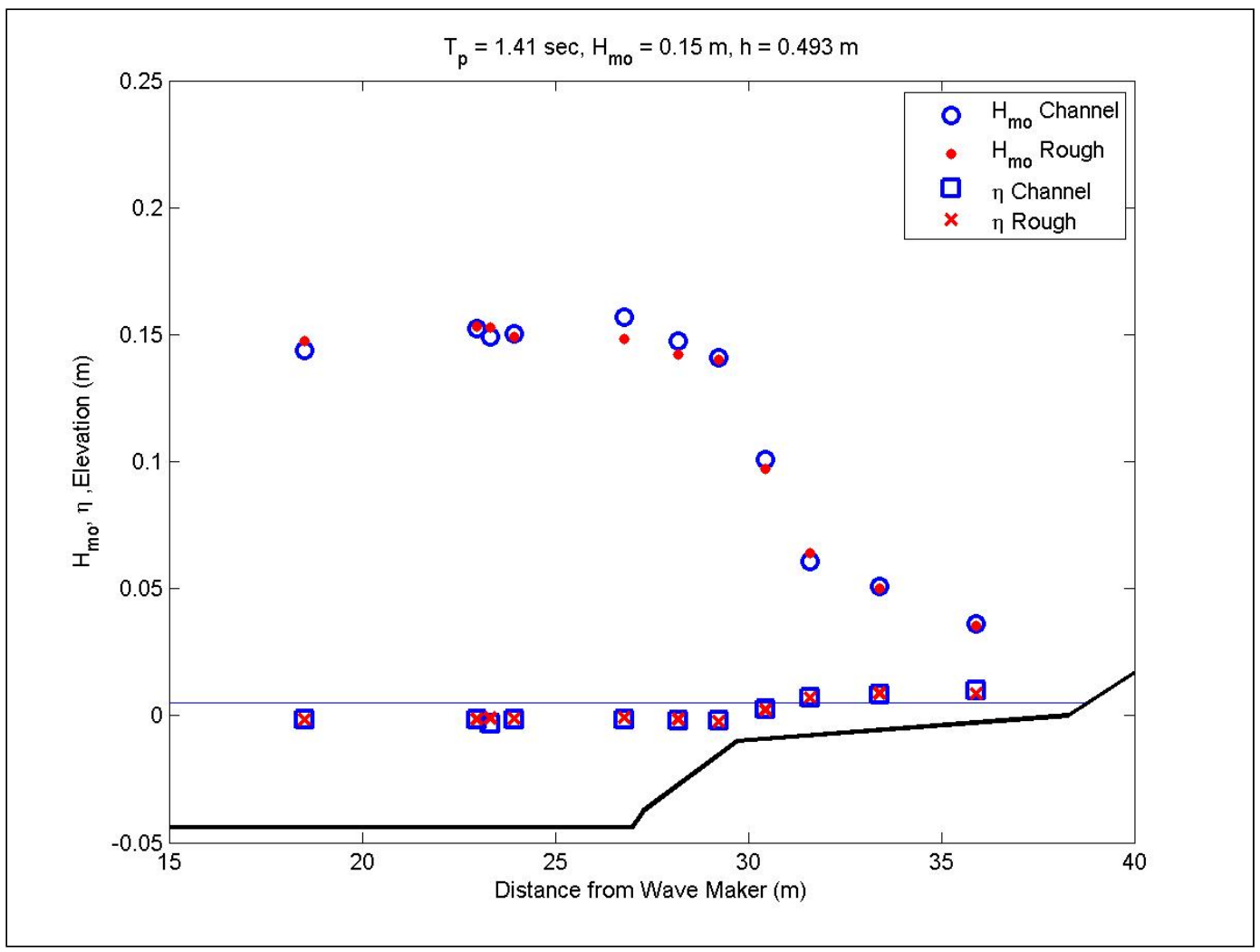

Figure C10. Wave transformation for $1.4-\mathrm{sec}, 0.15-\mathrm{m}$ waves at high water. 


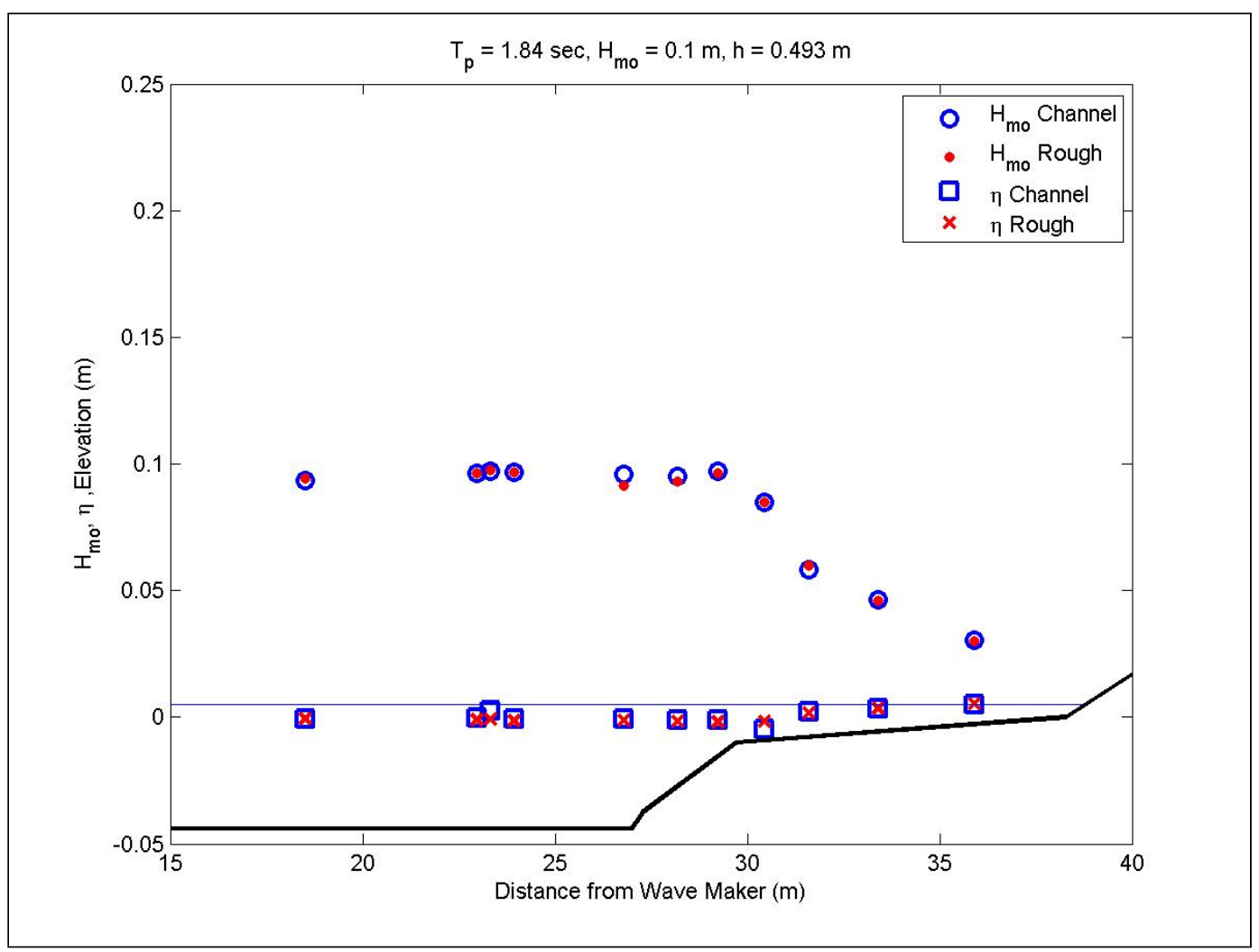

Figure C11. Wave transformation for 1.8-sec, 0.1-m waves at high water.

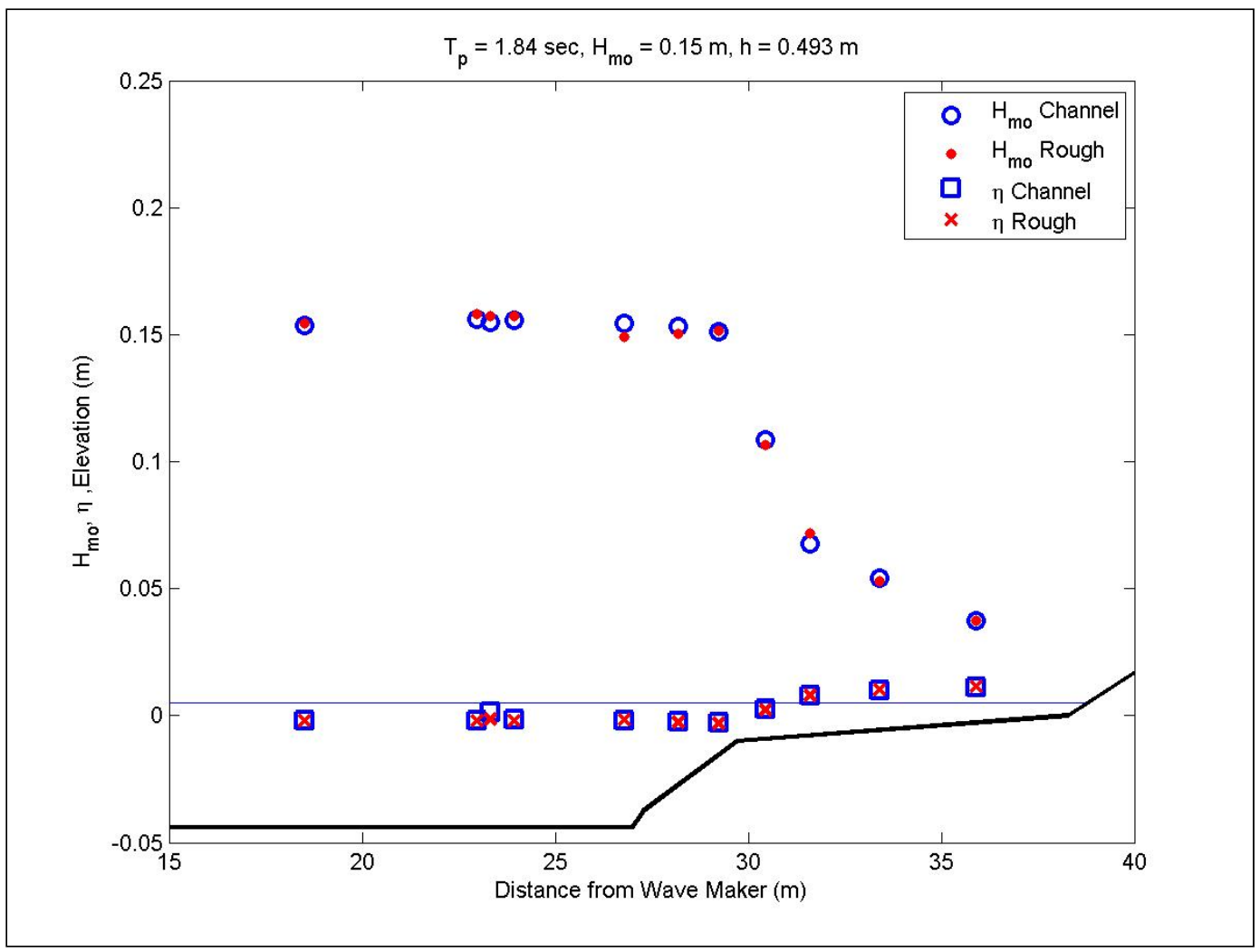

Figure C12. Wave transformation for $1.8-\mathrm{sec}, 0.15-\mathrm{m}$ waves at high water. 


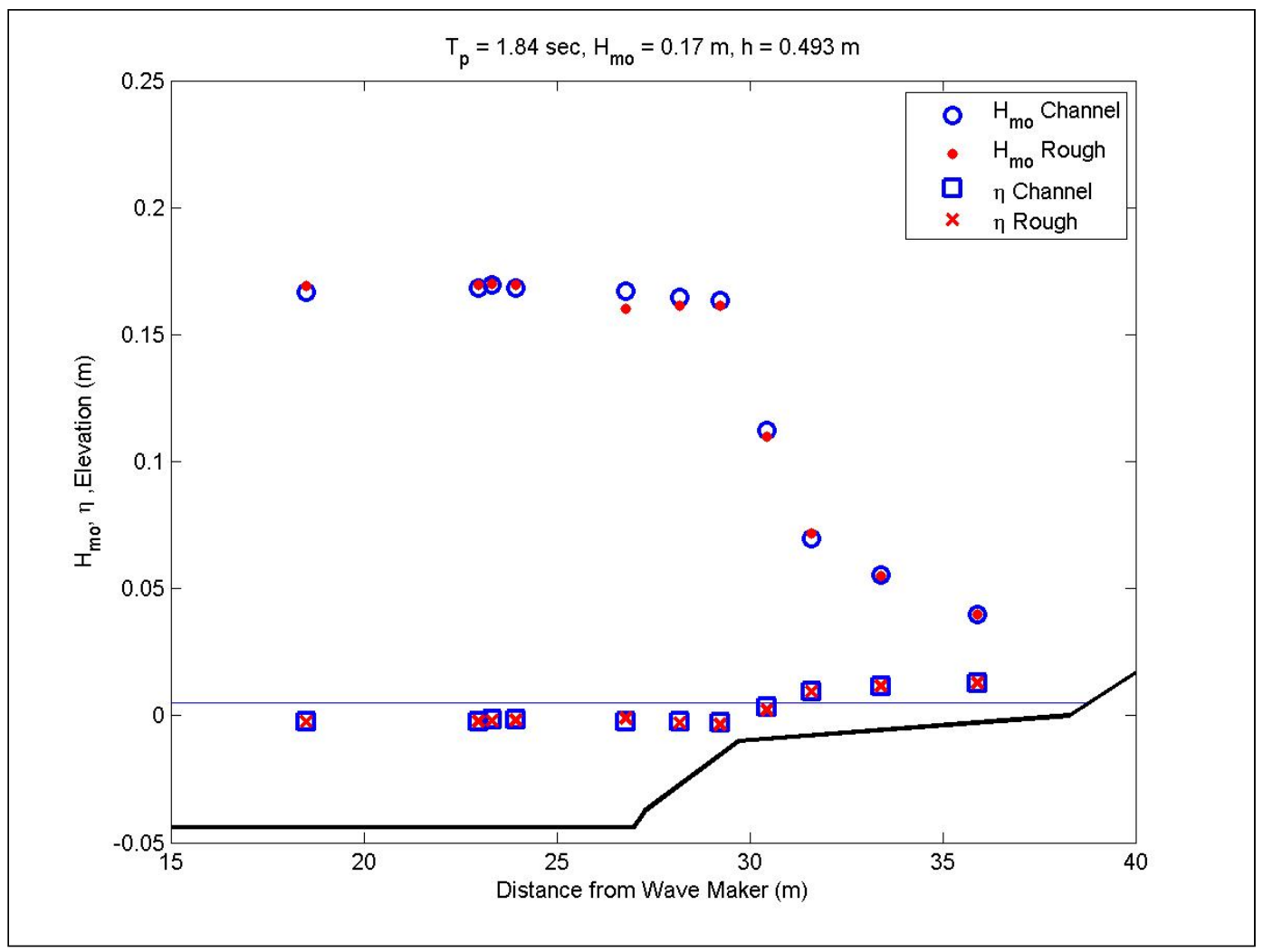

Figure C13. Wave transformation for 1.8-sec, 0.17-m waves at high water.

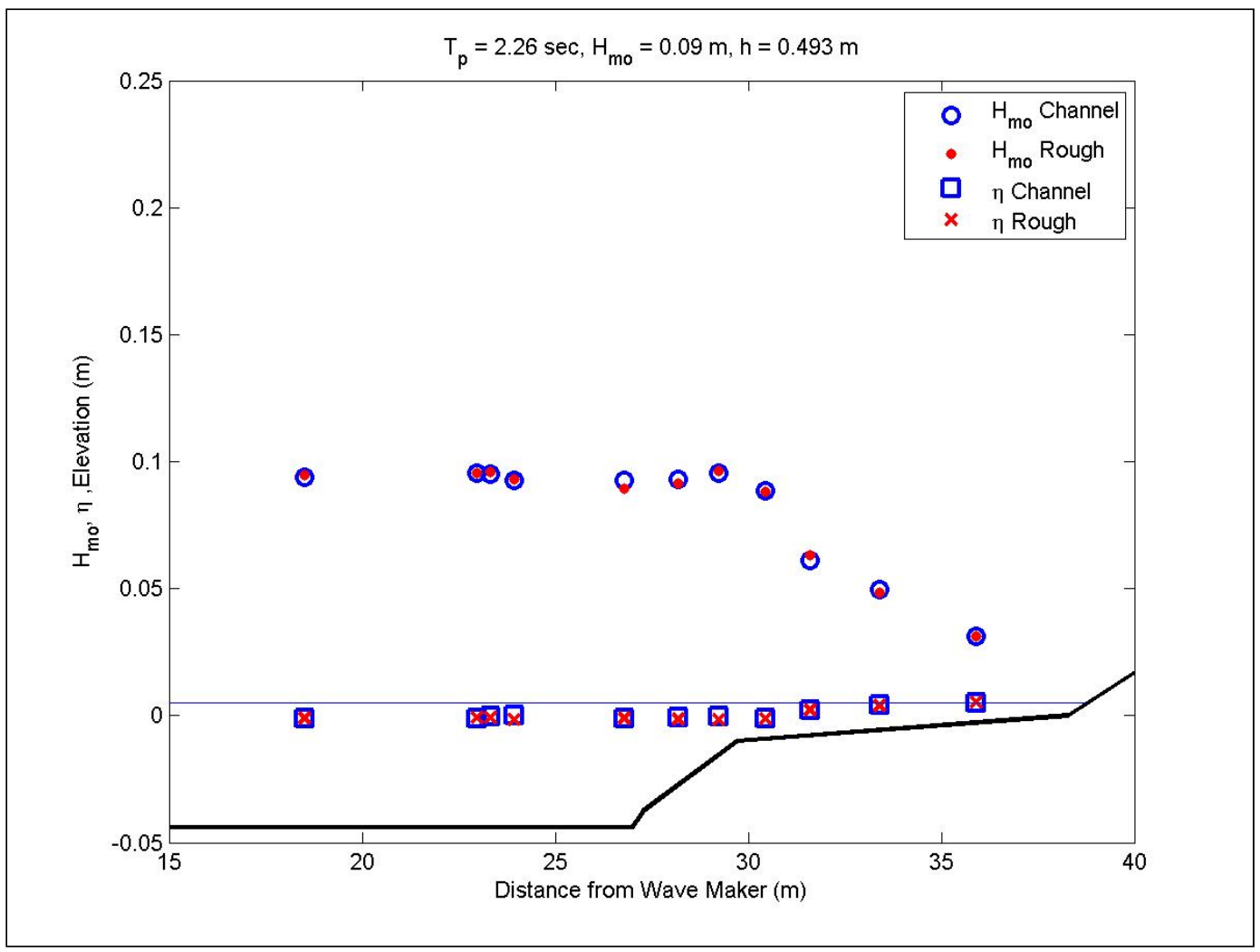

Figure C14. Wave transformation for 2.3-sec, 0.09-m waves at high water. 


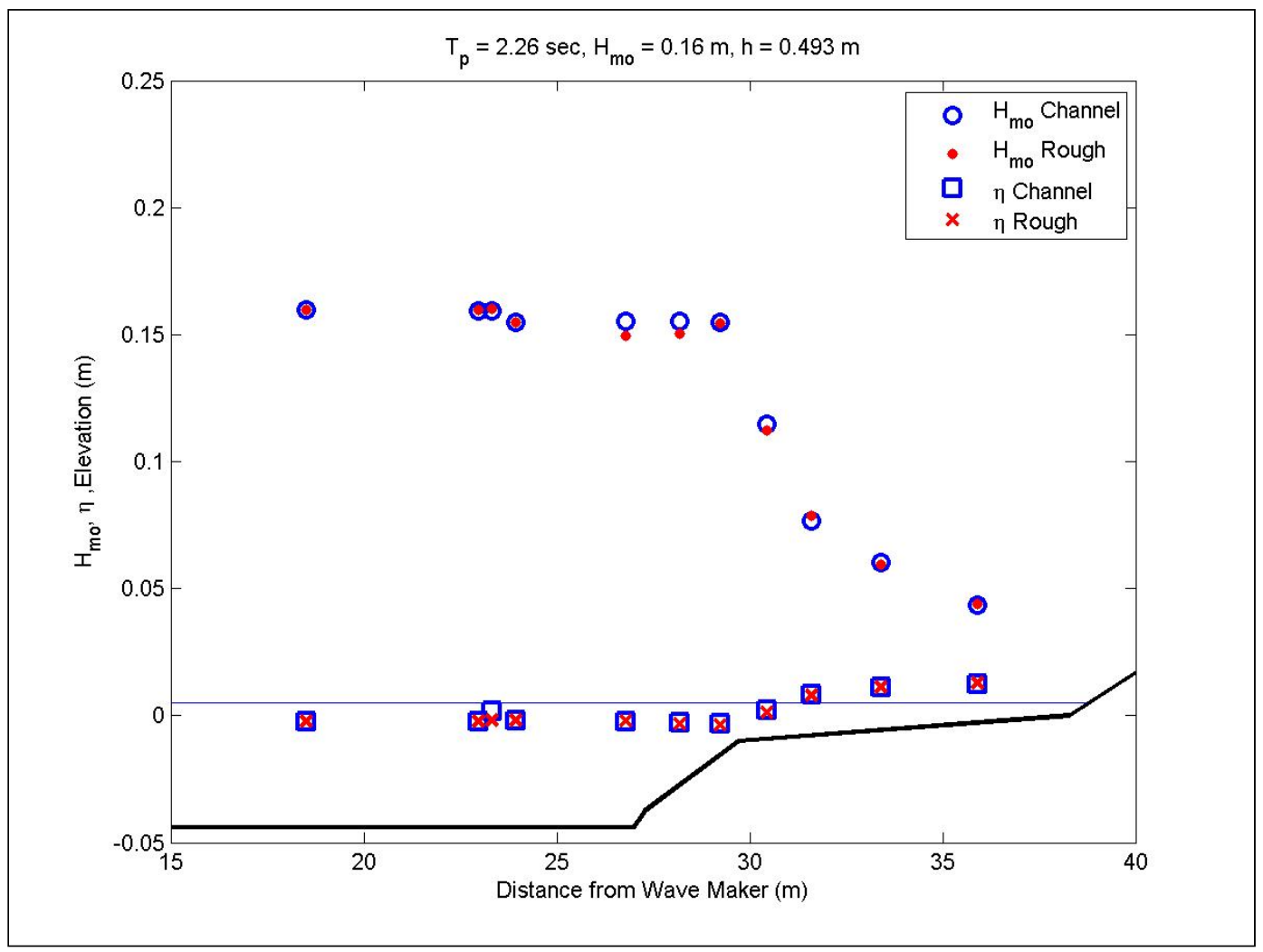

Figure C15. Wave transformation for 2.3-sec, 0.16-m waves at high water.

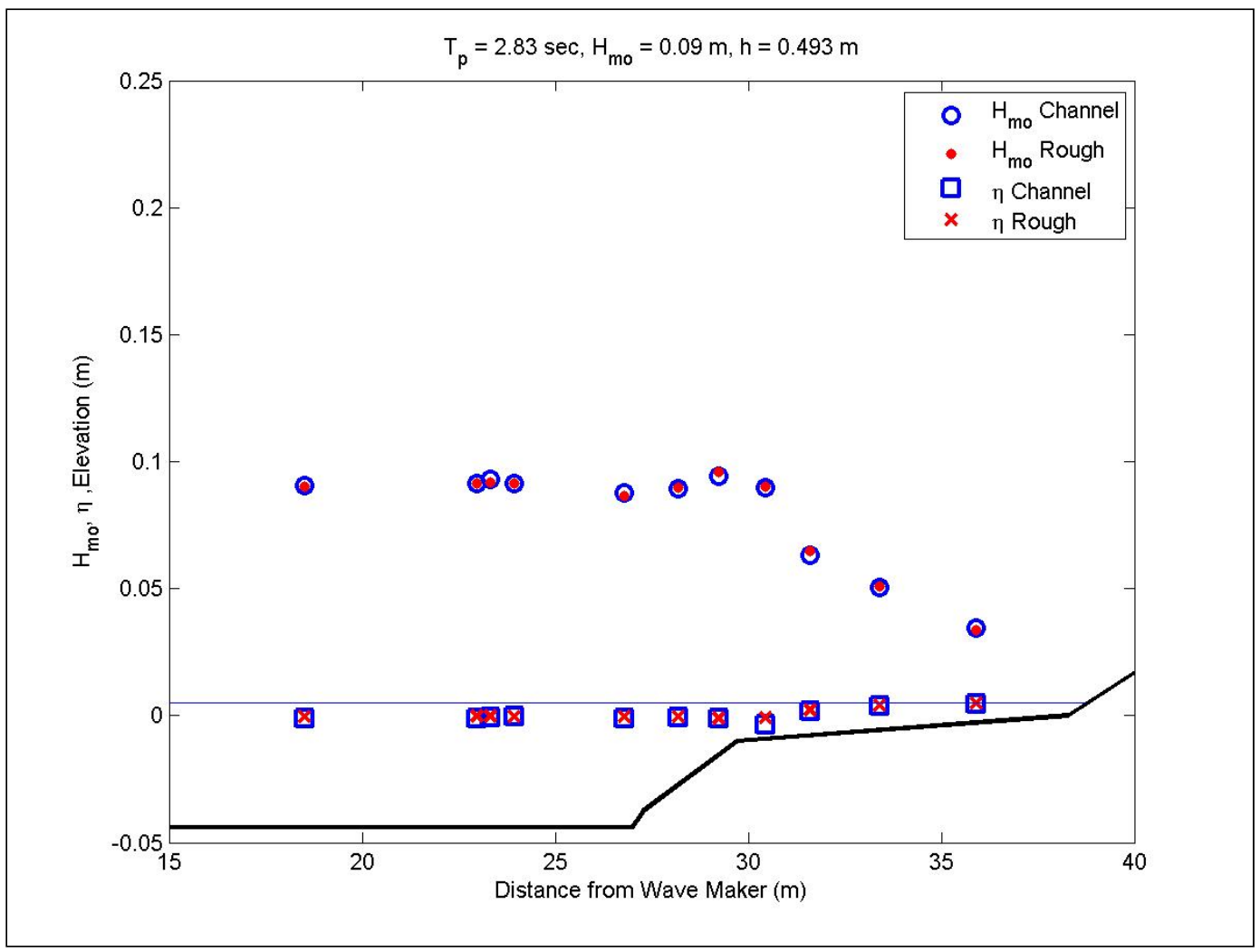

Figure C16. Wave transformation for 2.8-sec, 0.09-m waves at high water. 


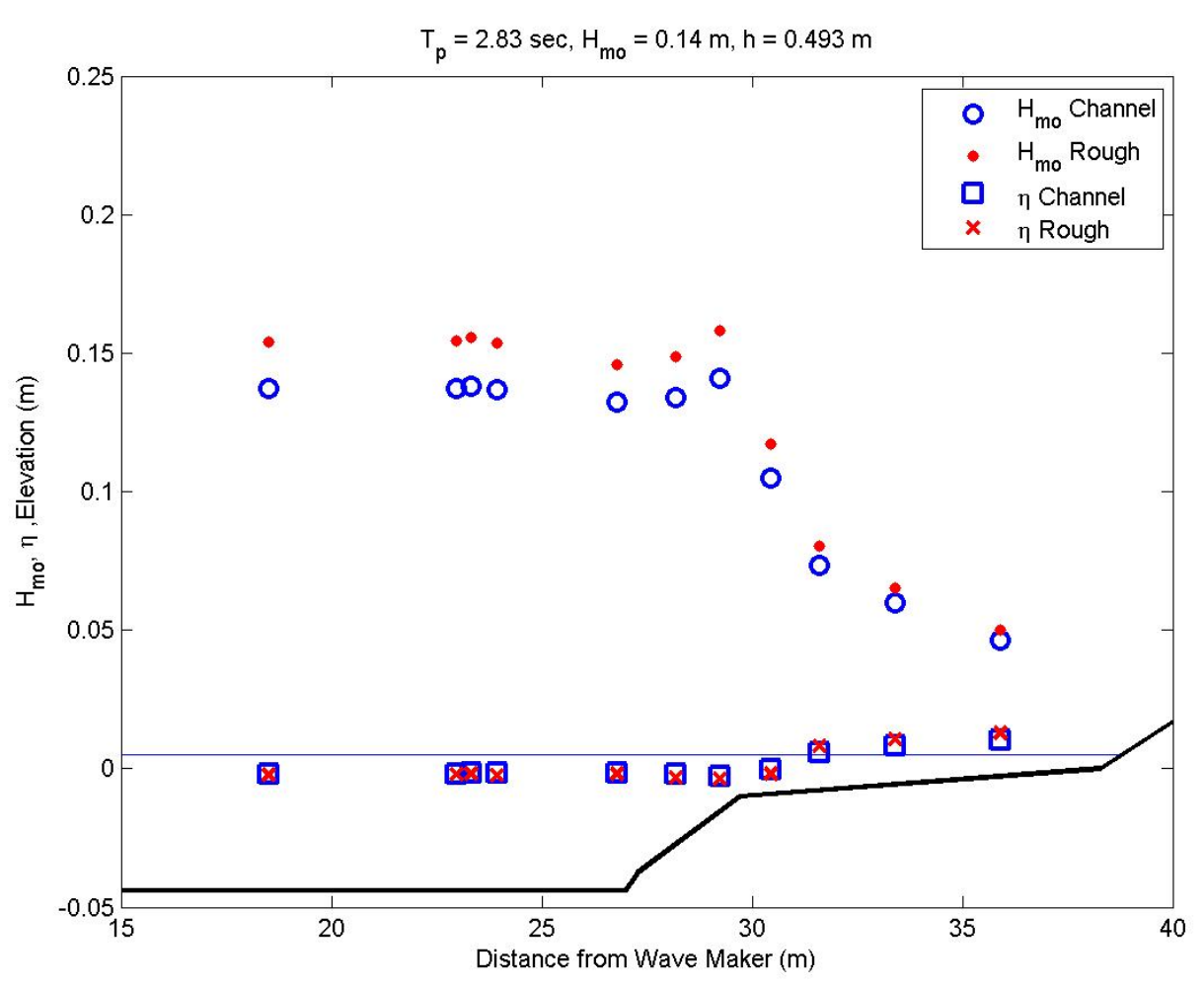

Figure $\mathrm{C} 17$. Wave transformation for 2.8 -sec, $0.14-\mathrm{m}$ waves at high water. 


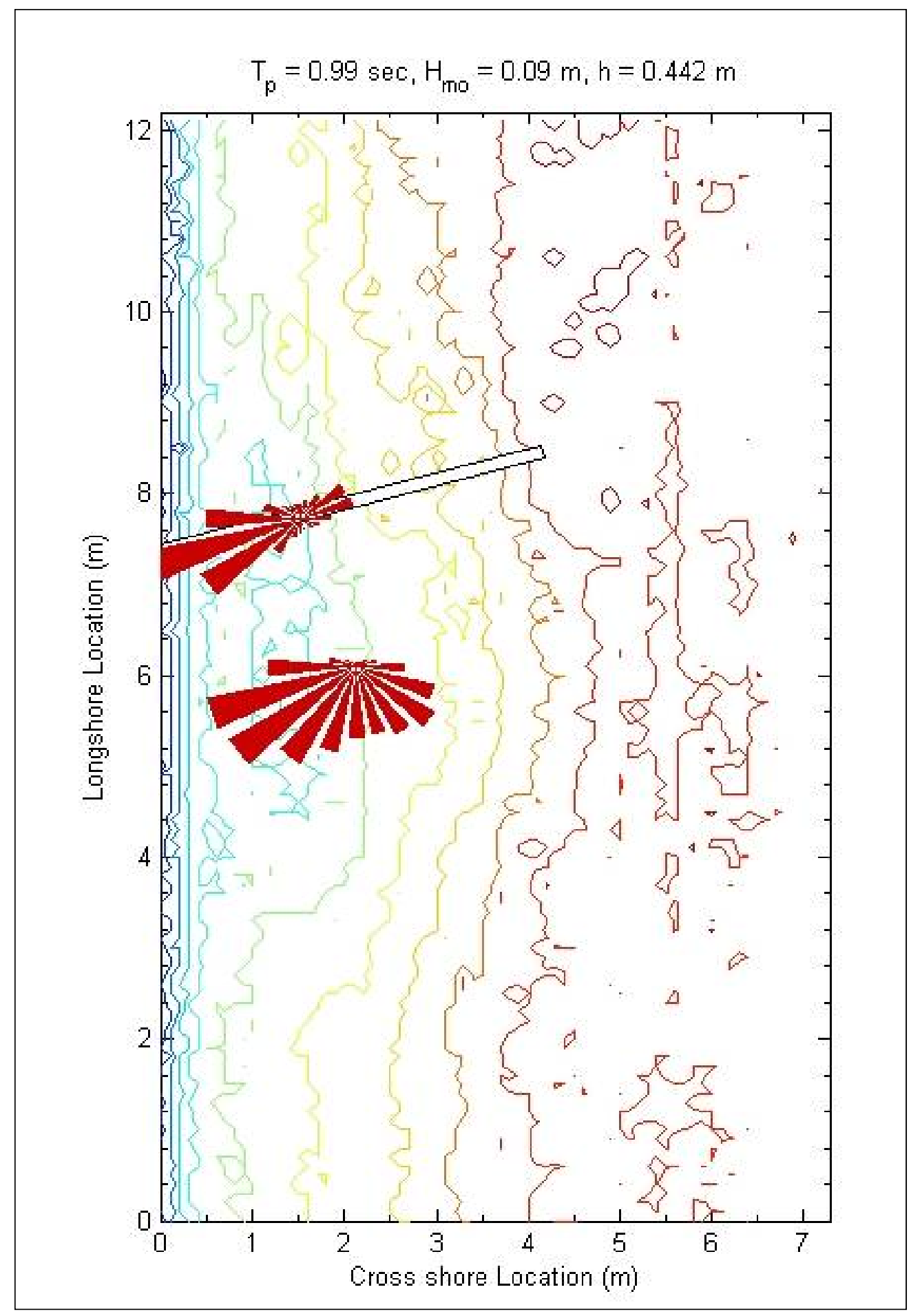

Figure C18. Current rose patterns for 1-sec, 0.09-m waves at mid water. 


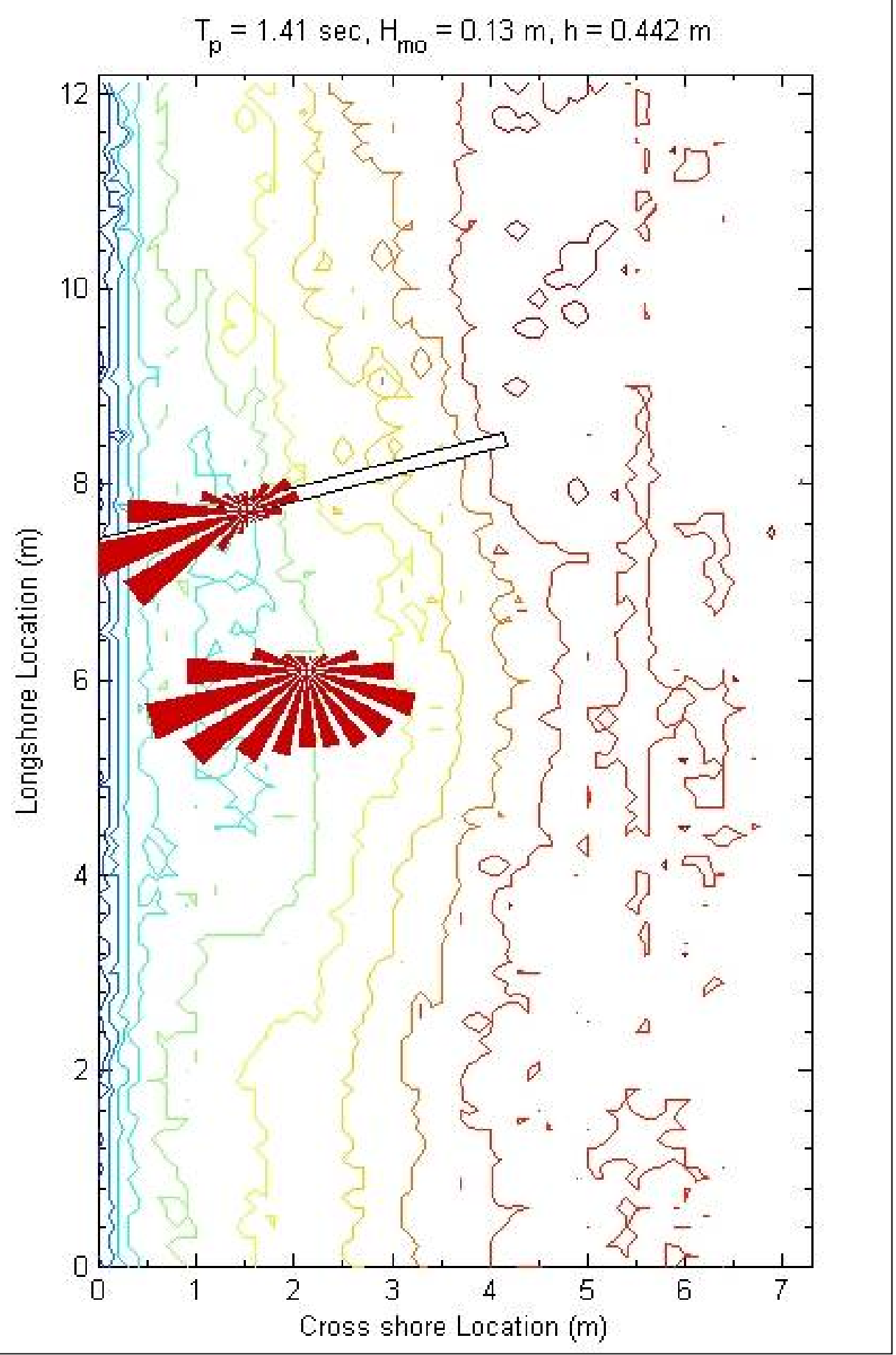

Figure C19. Current rose patterns for 1.4-sec, 0.13-m waves at mid water. 


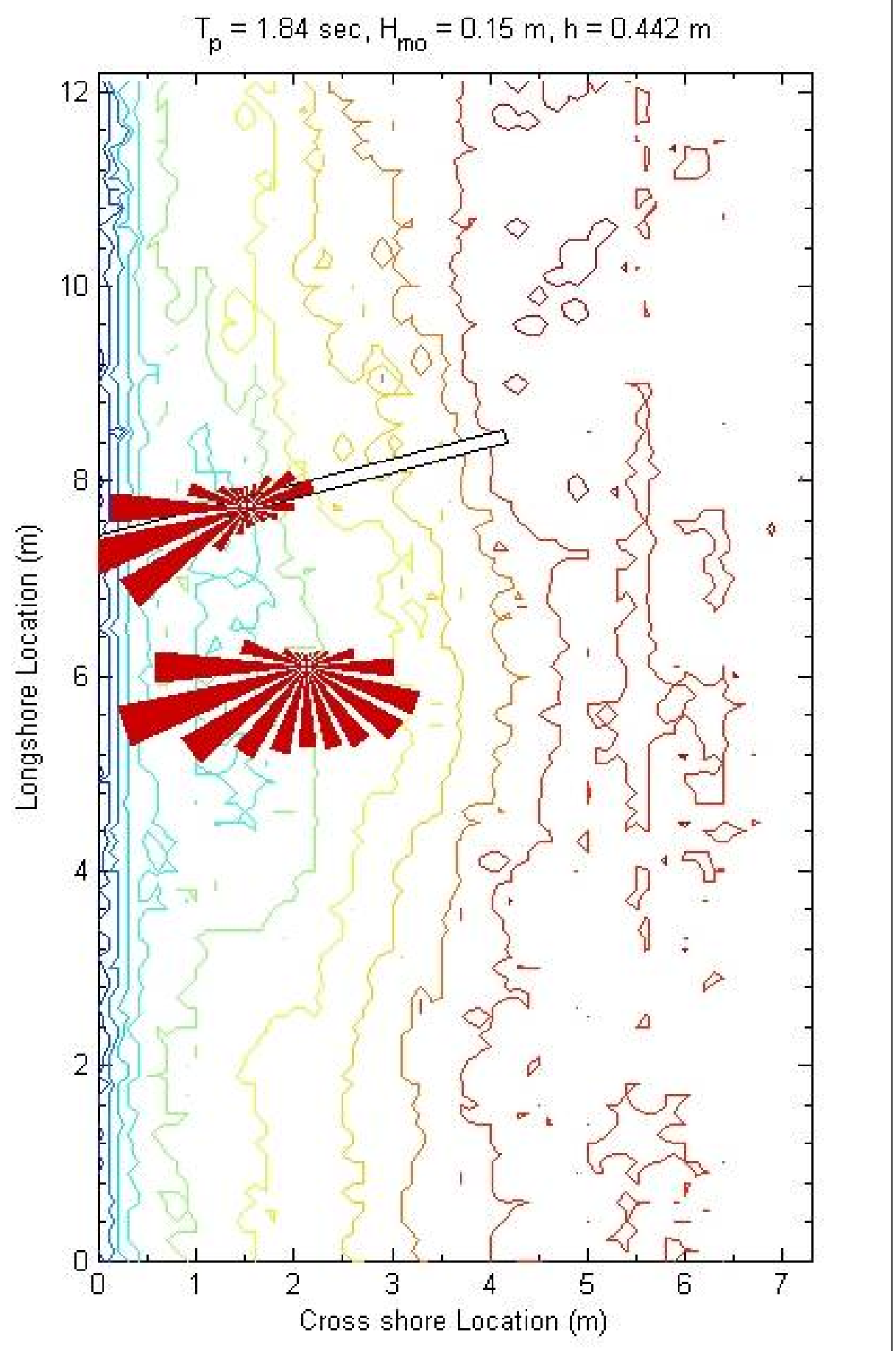

Figure C20. Current rose patterns for $1.8-\mathrm{sec}, 0.15-\mathrm{m}$ waves at mid water. 


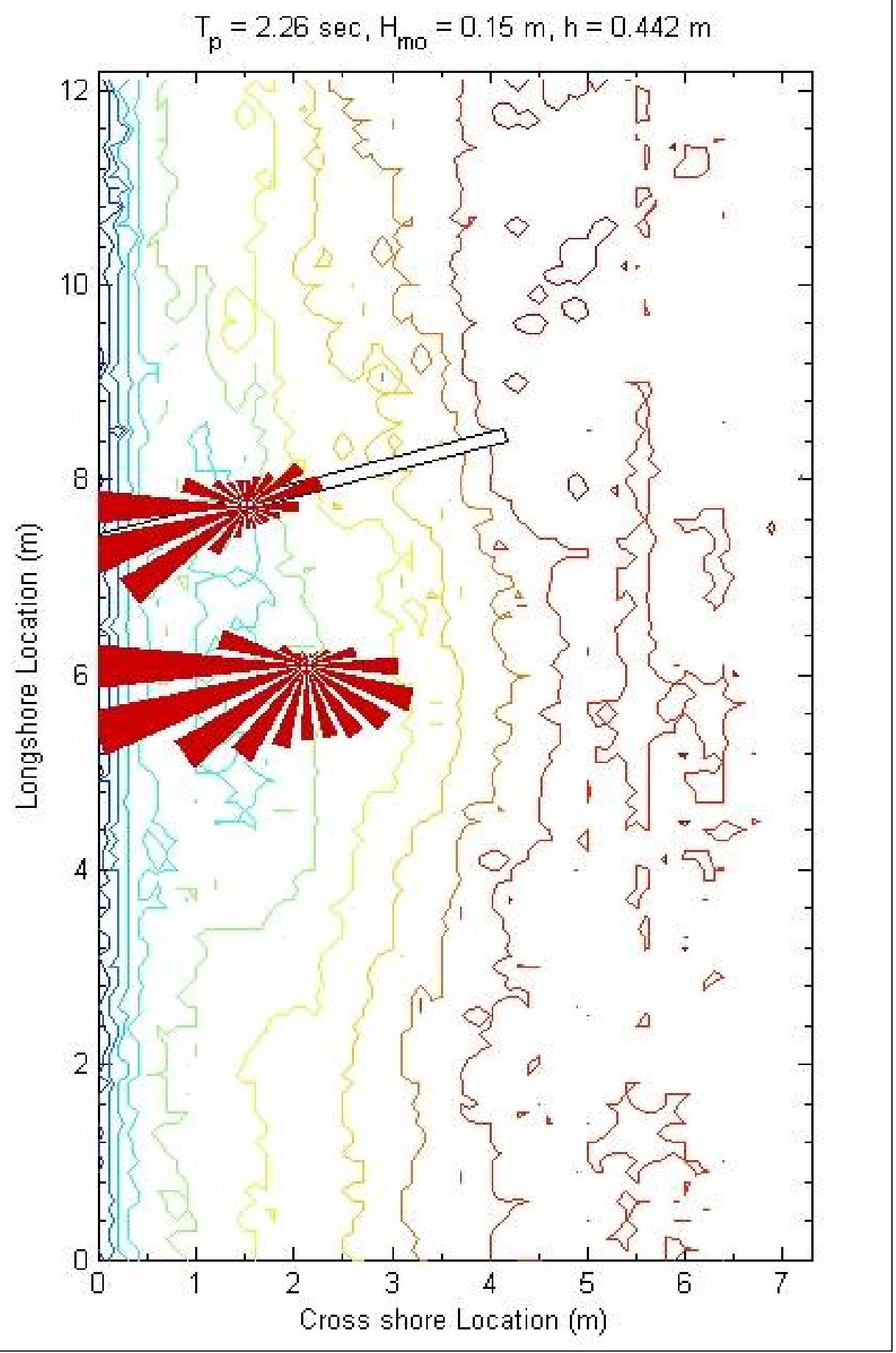

Figure C21. Current rose patterns for 2.3-sec, 0.15-m waves at mid water. 


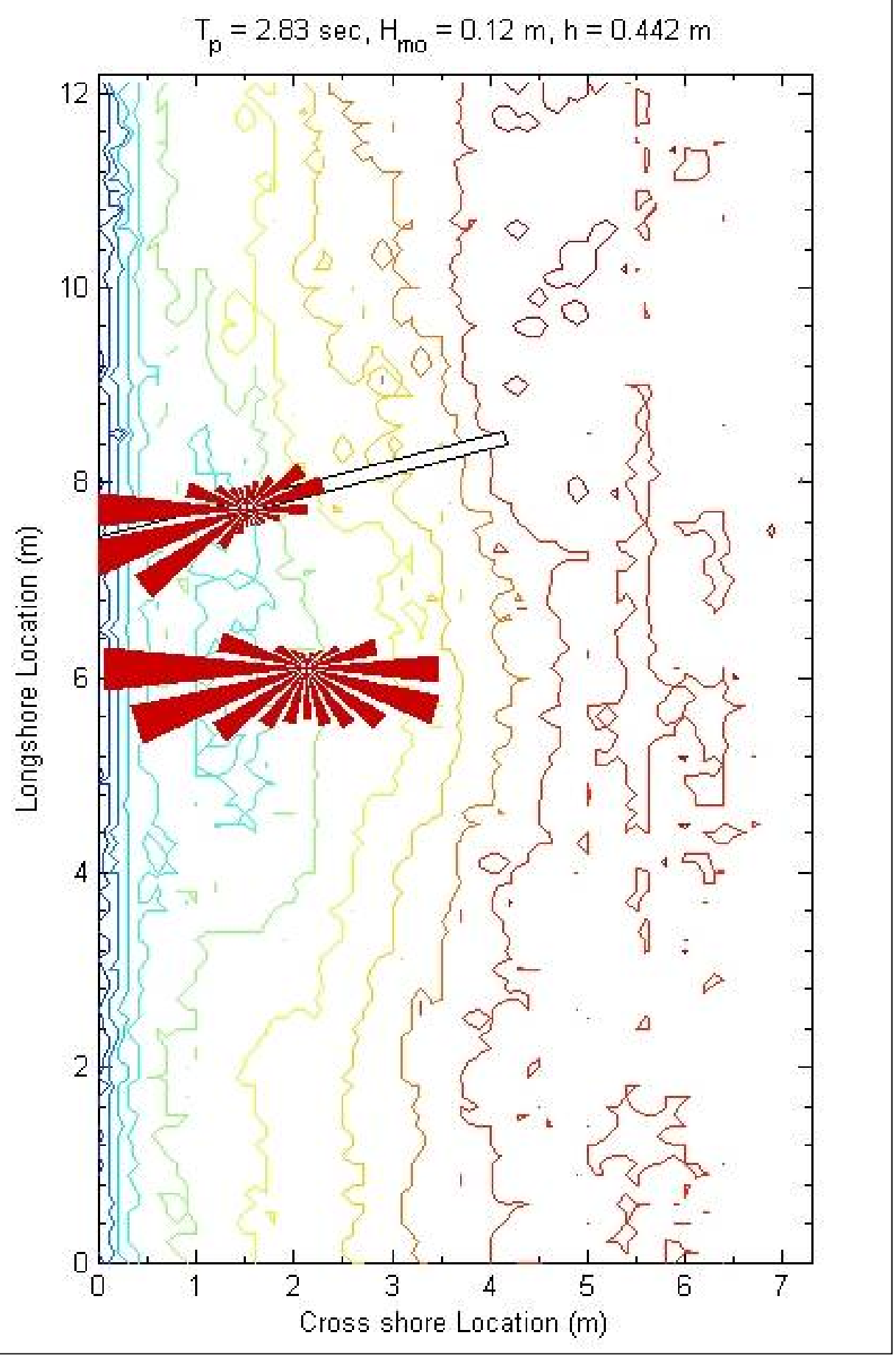

Figure C22. Current rose patterns for 2.8-sec, 0.12-m waves at mid water. 


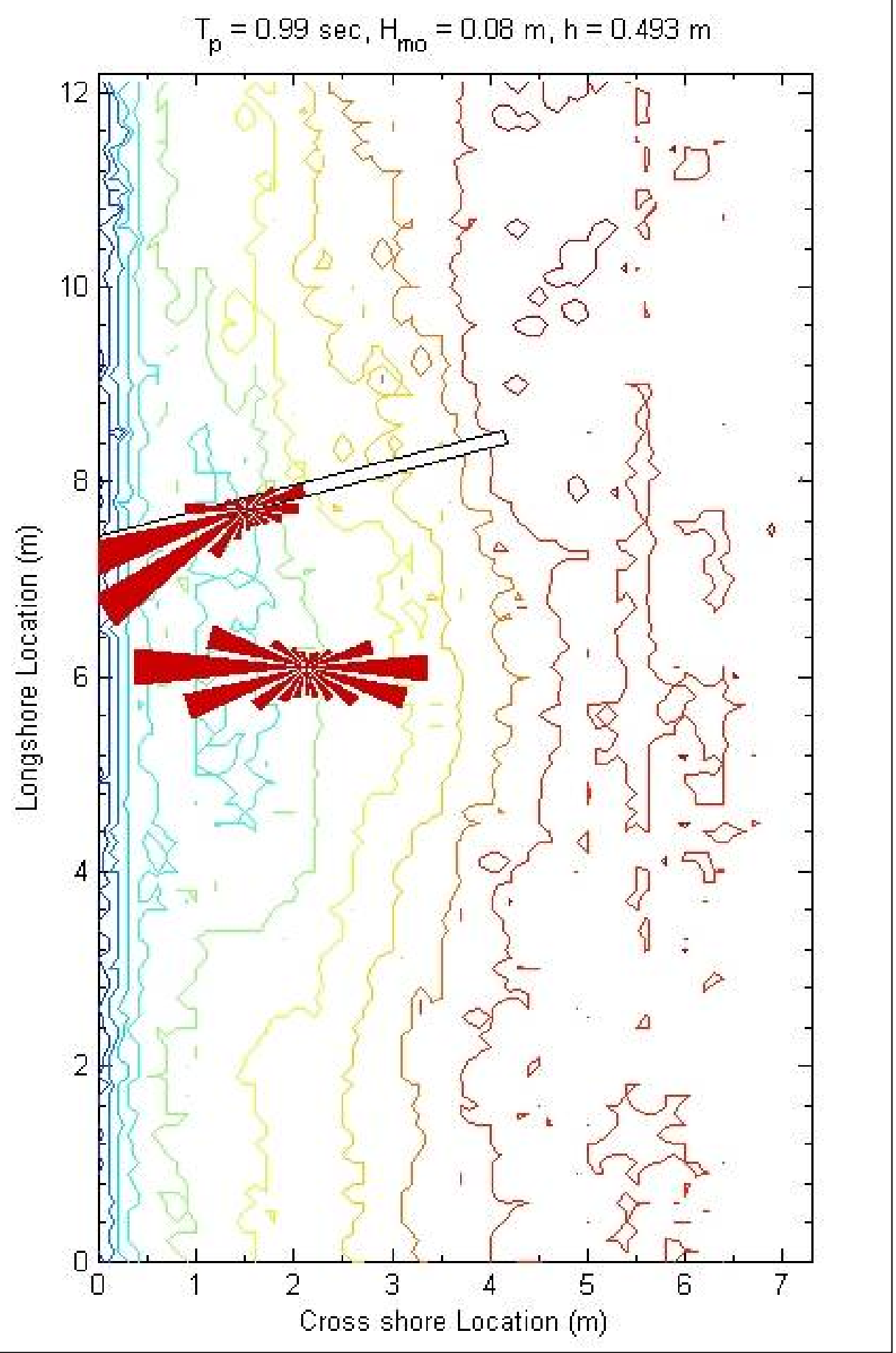

Figure C23. Current rose patterns for 1-sec, 0.08-m waves at high water. 


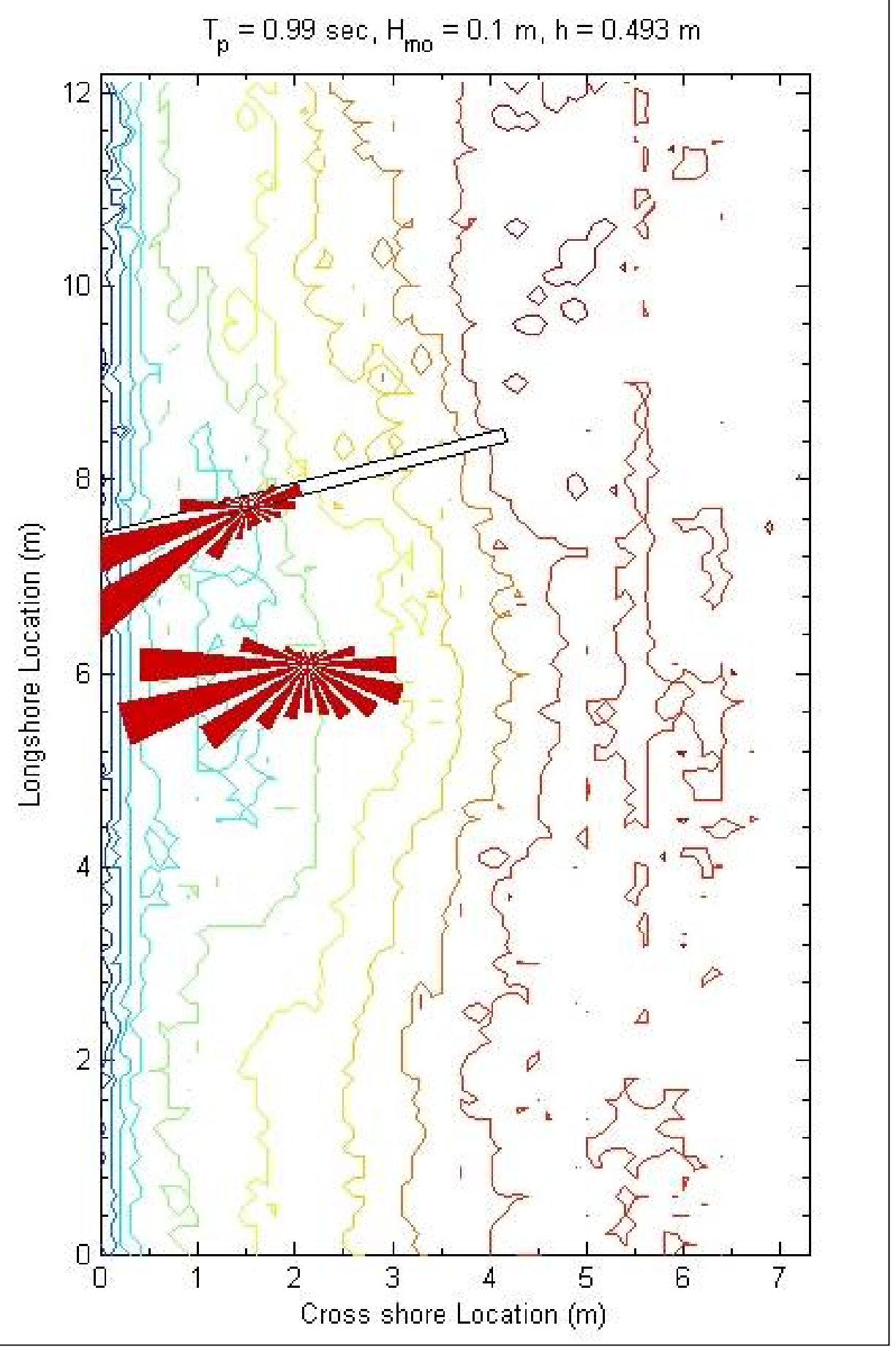

Figure C24. Current rose patterns for 1-sec, 0.1-m waves at high water. 


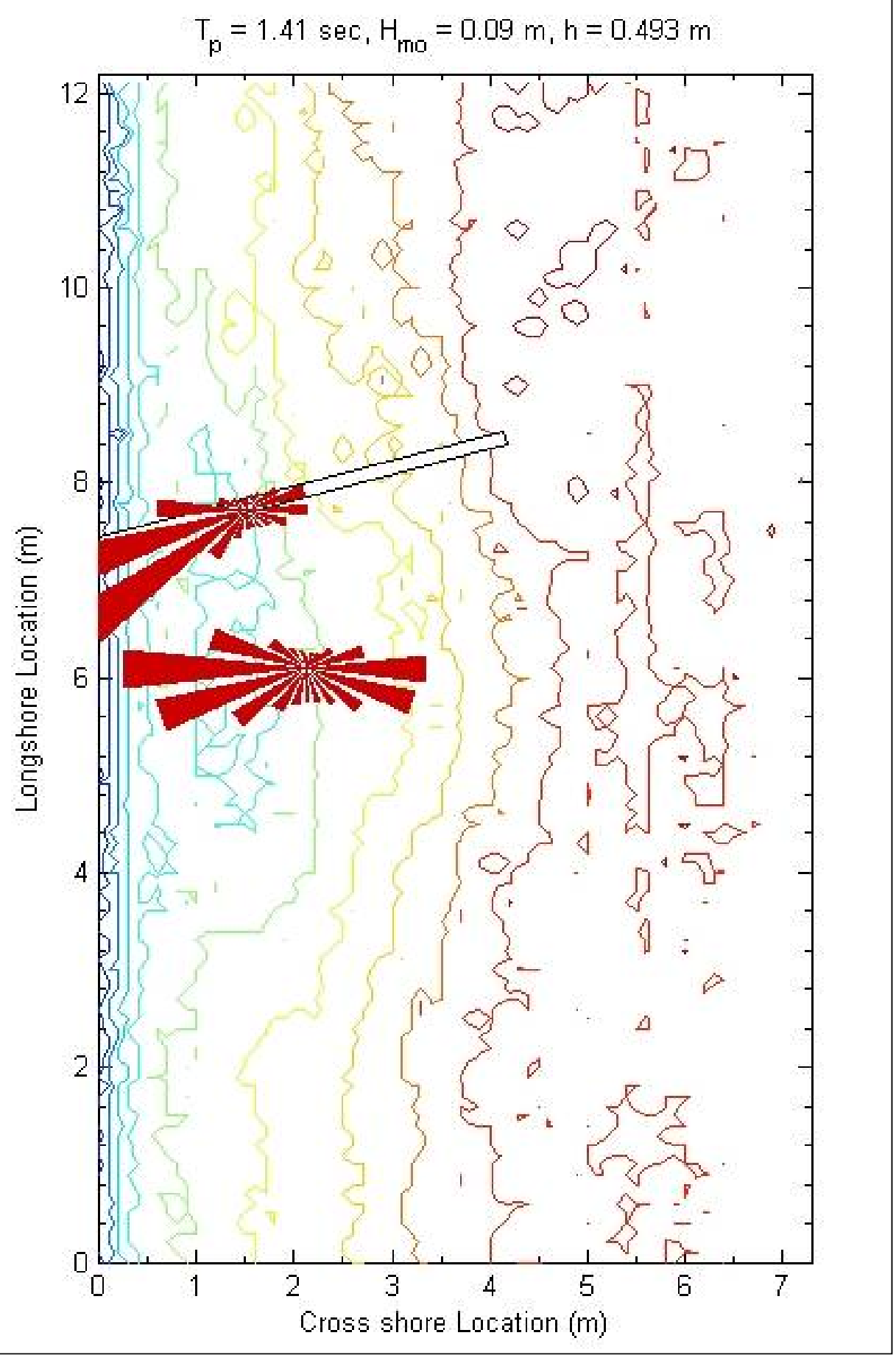

Figure C25. Current rose patterns for 1.4-sec, 0.09-m waves at high water. 


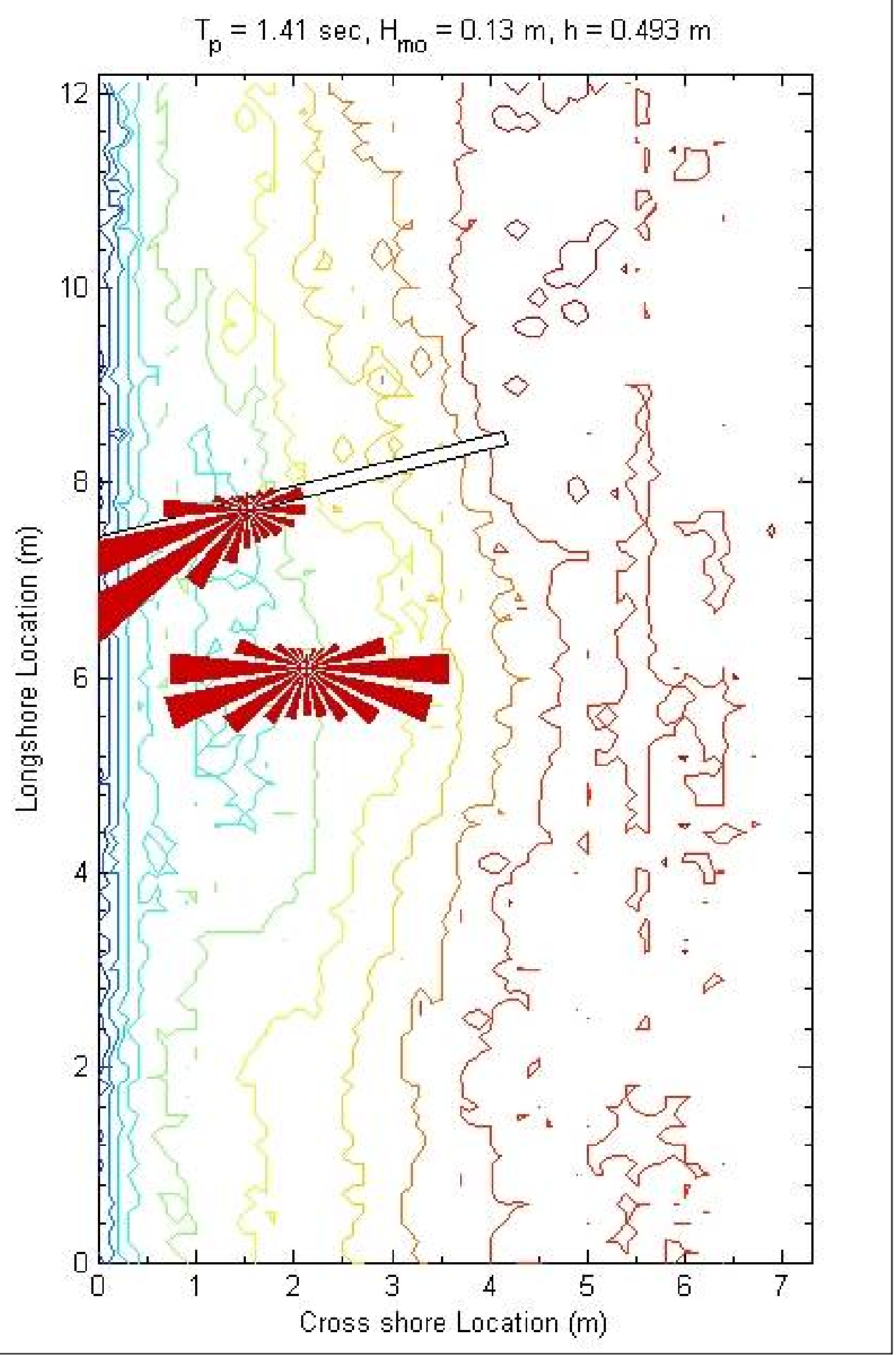

Figure C26. Current rose patterns for 1.4-sec, 0.13-m waves at high water. 


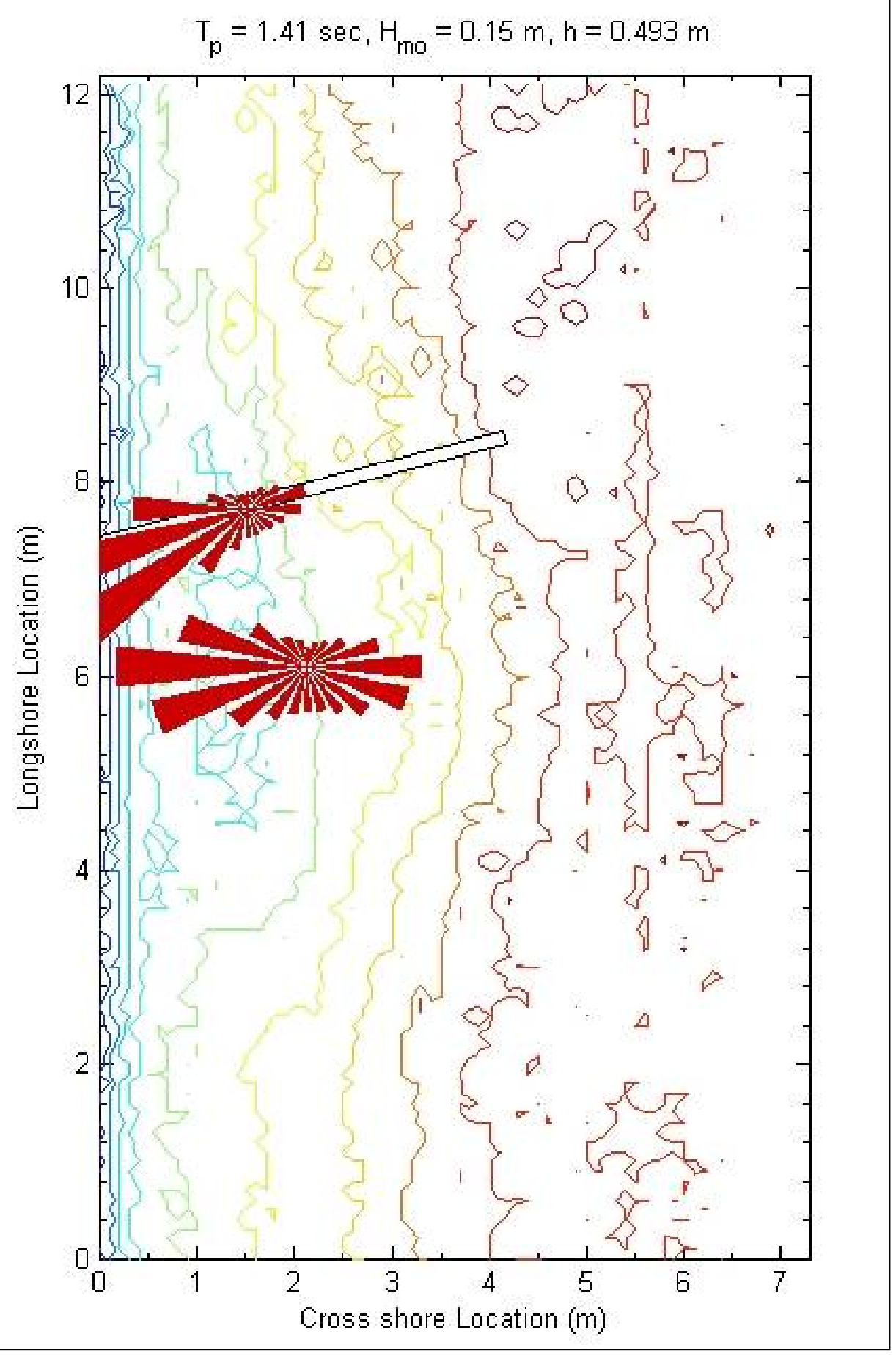

Figure C27. Current rose patterns for 1.4-sec, 0.15-m waves at high water. 


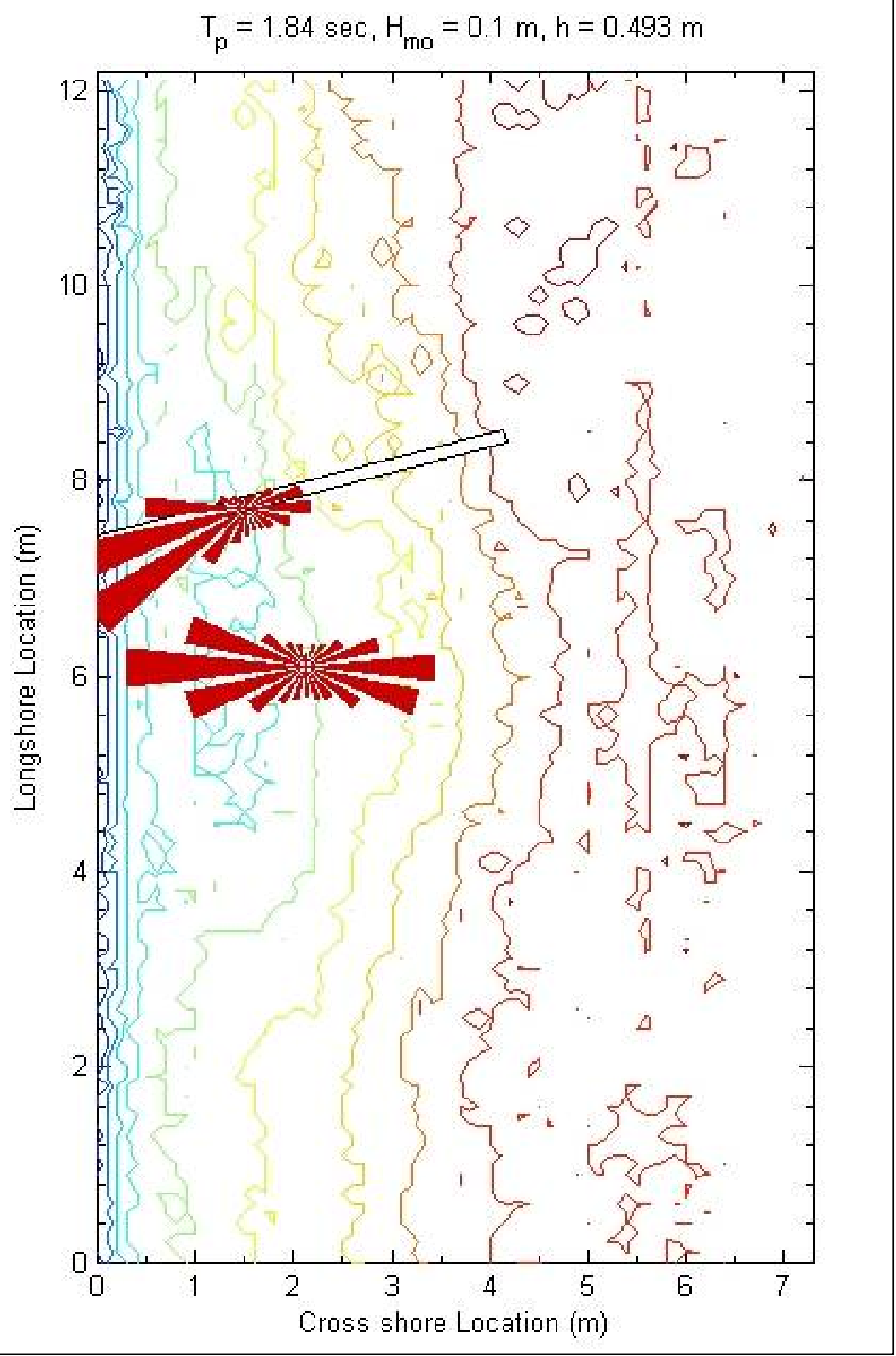

Figure C28. Current rose patterns for $1.8-\mathrm{sec}, 0.1-\mathrm{m}$ waves at high water. 


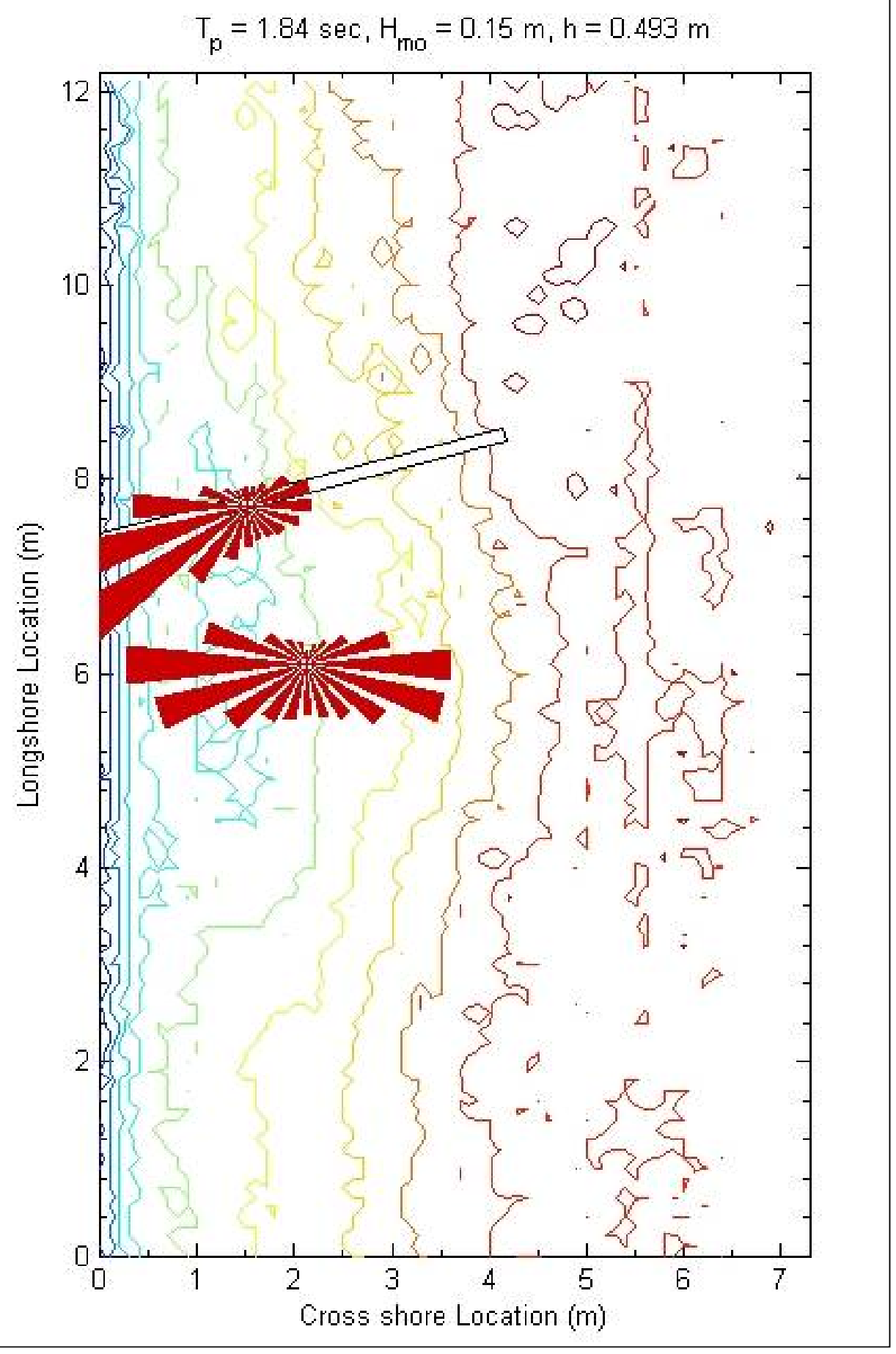

Figure C29. Current rose patterns for $1.8-\mathrm{sec}, 0.15-\mathrm{m}$ waves at high water. 


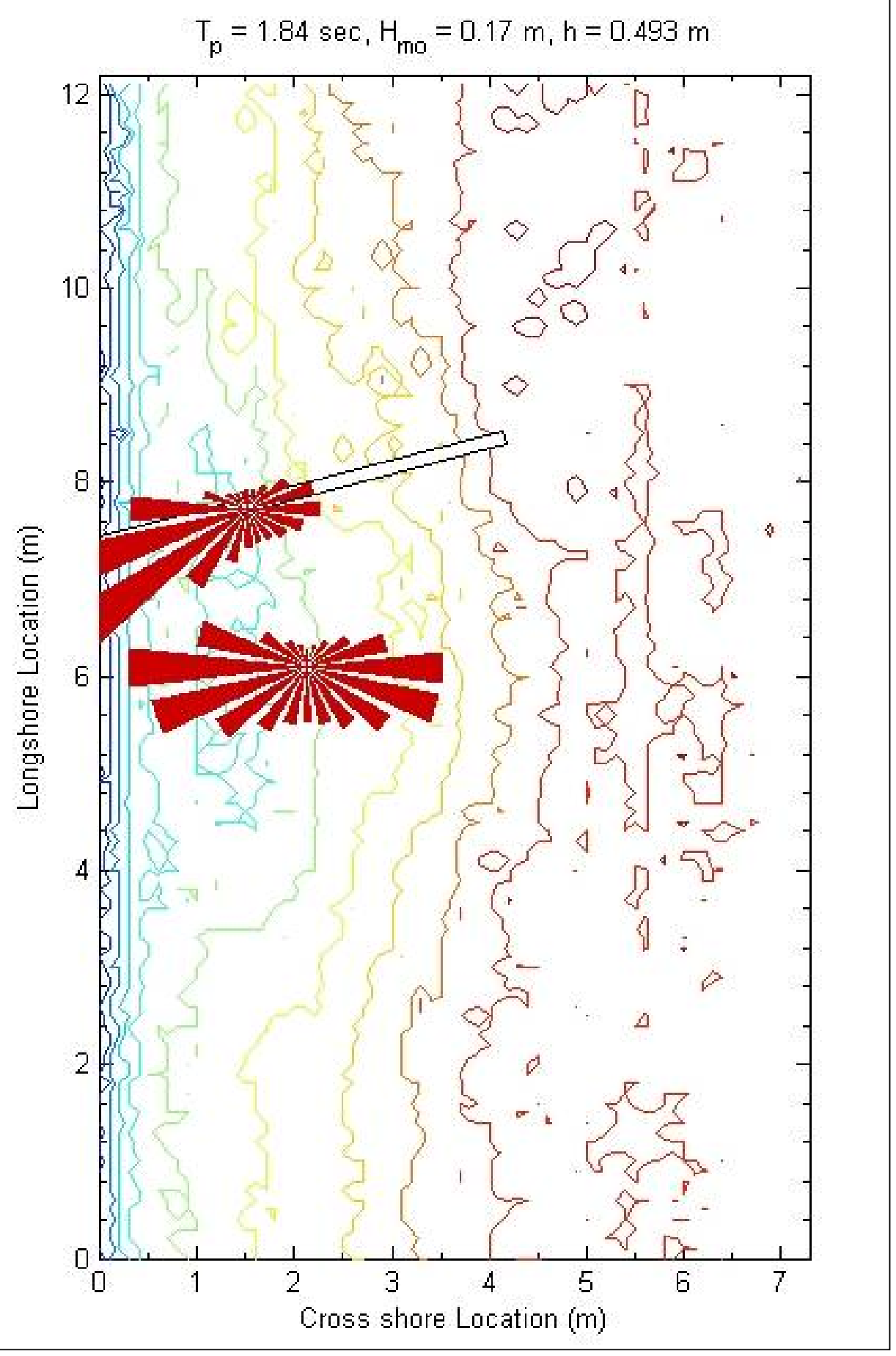

Figure C30. Current rose patterns for $1.8-\mathrm{sec}, 0.17-\mathrm{m}$ waves at high water. 


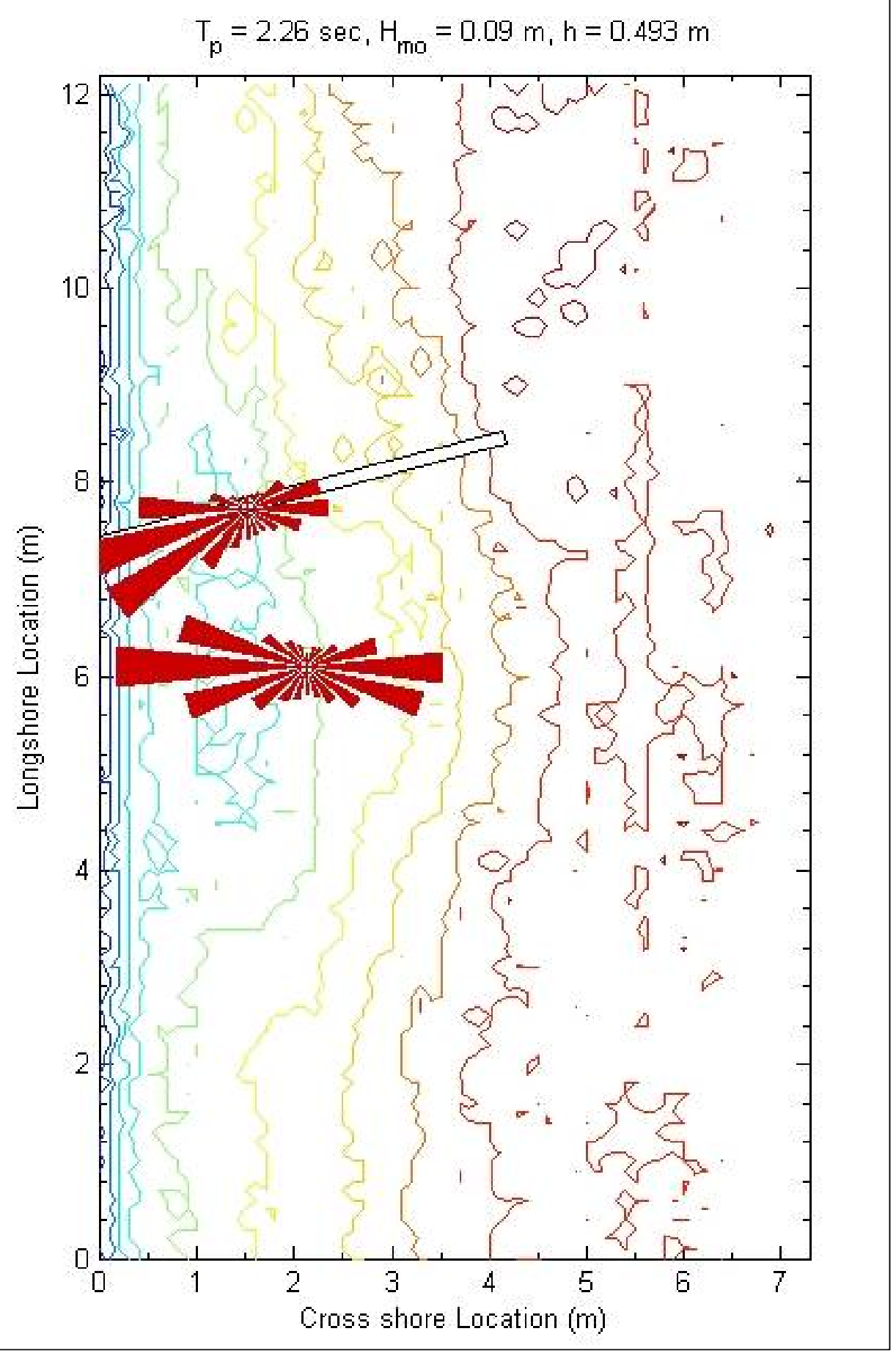

Figure C31. Current rose patterns for 2.3-sec, 0.09-m waves at high water. 


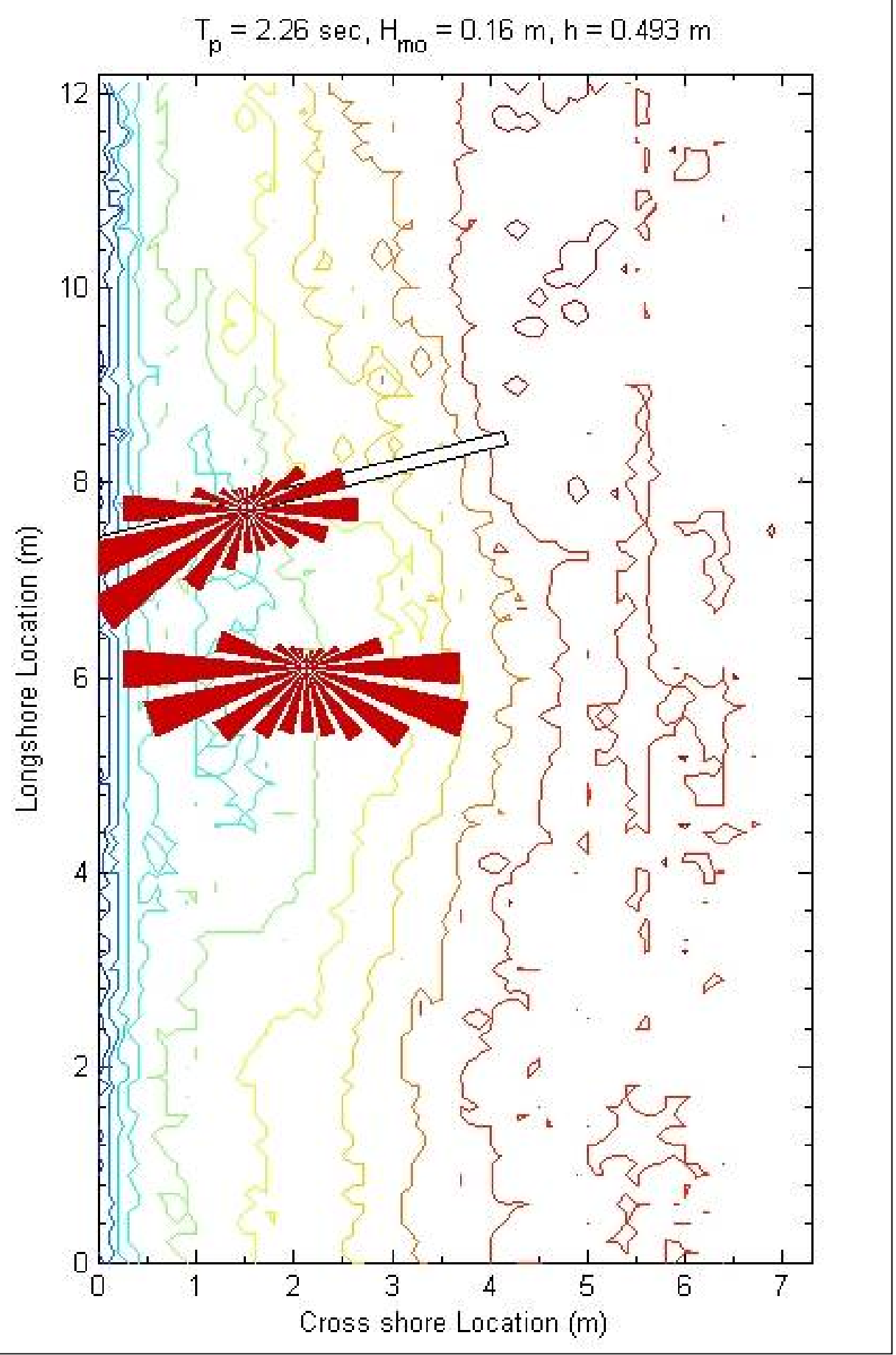

Figure C32. Current rose patterns for 2.3-sec, 0.16-m waves at high water. 


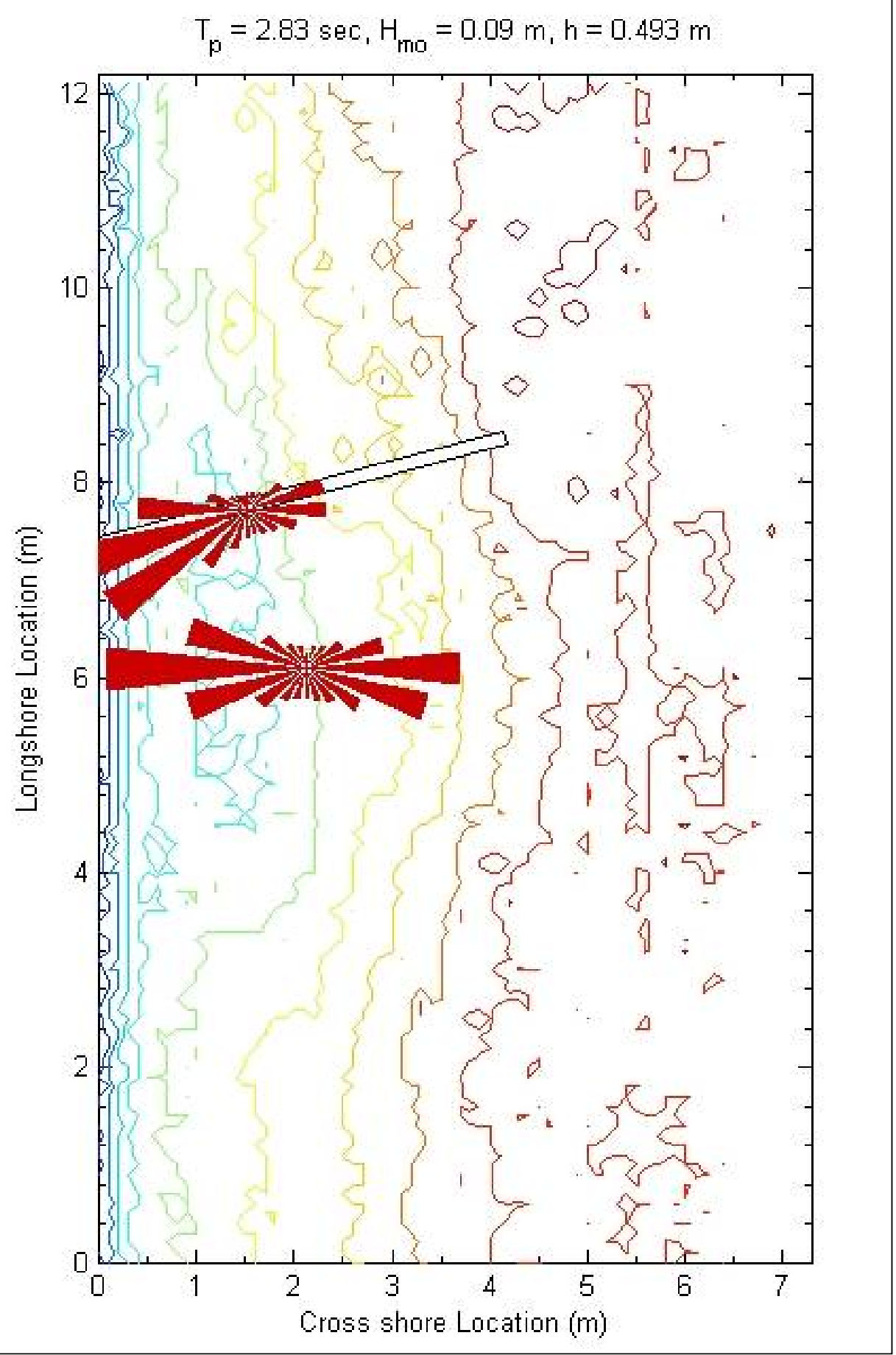

Figure C33. Current rose patterns for 2.8-sec, 0.09-m waves at high water. 


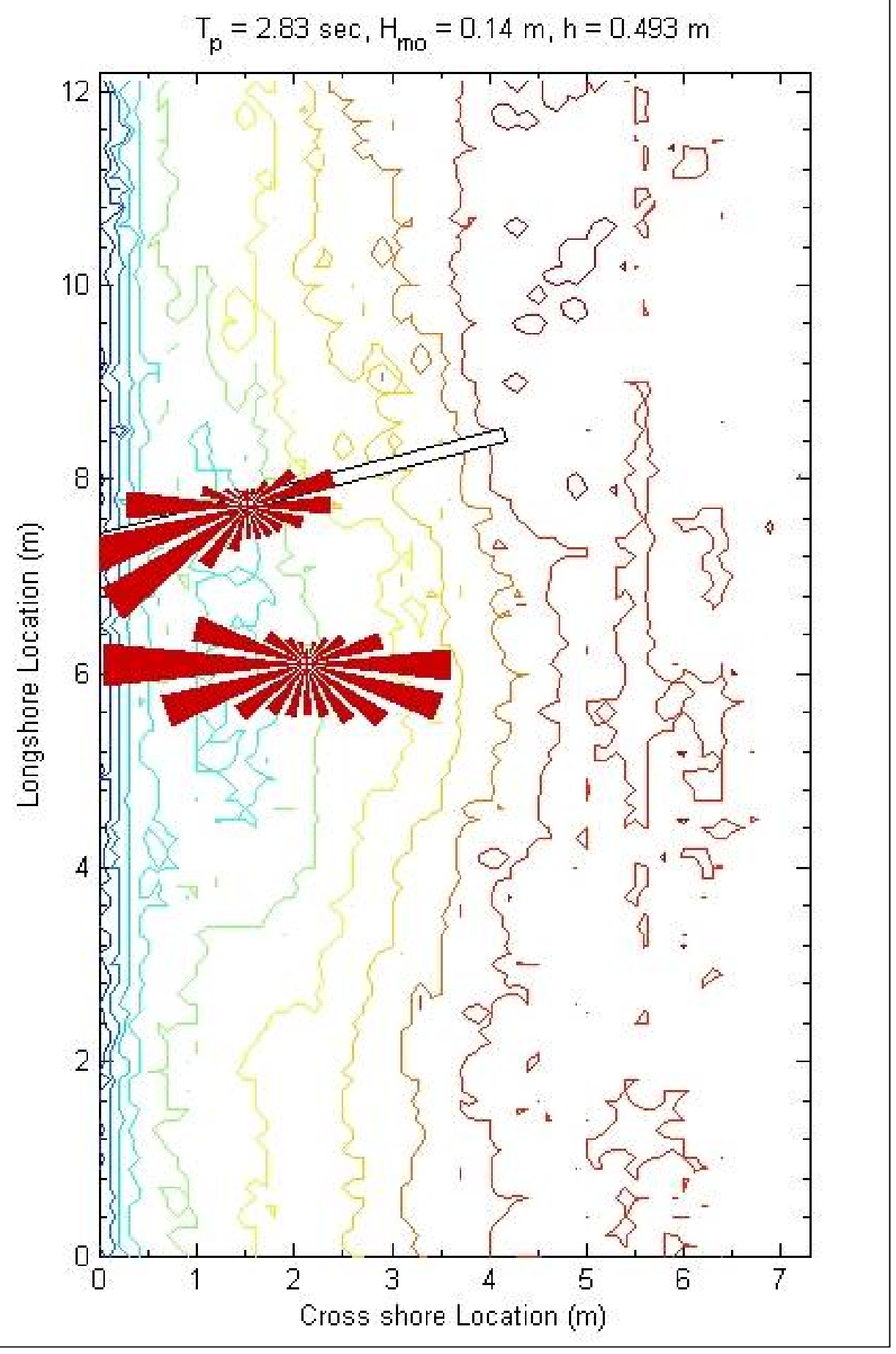

Figure C34. Current rose patterns for 2.8-sec, 0.14-m waves at high water. 


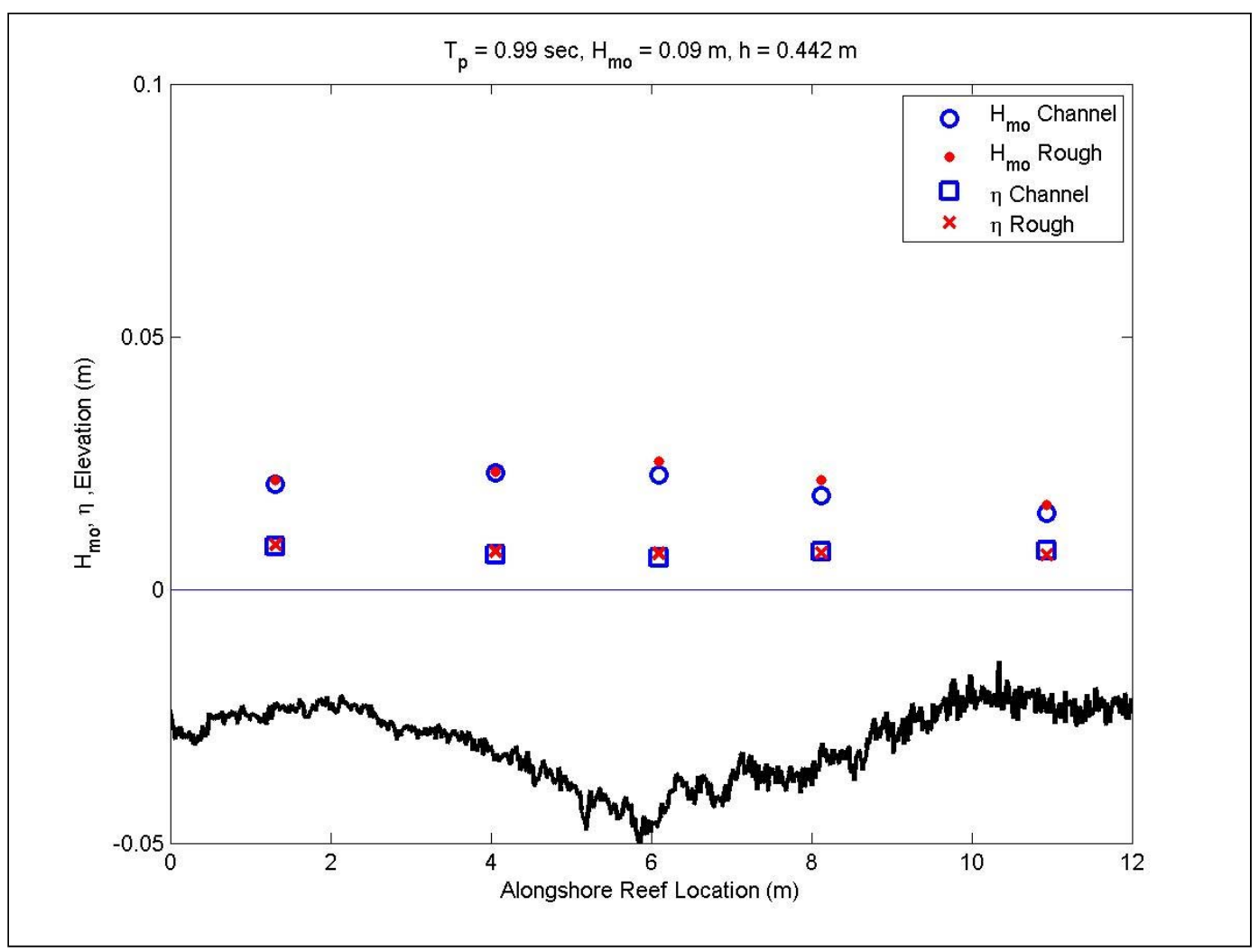

Figure C35. Alongshore wave heights for 1-sec, 0.09-m waves at mid water.

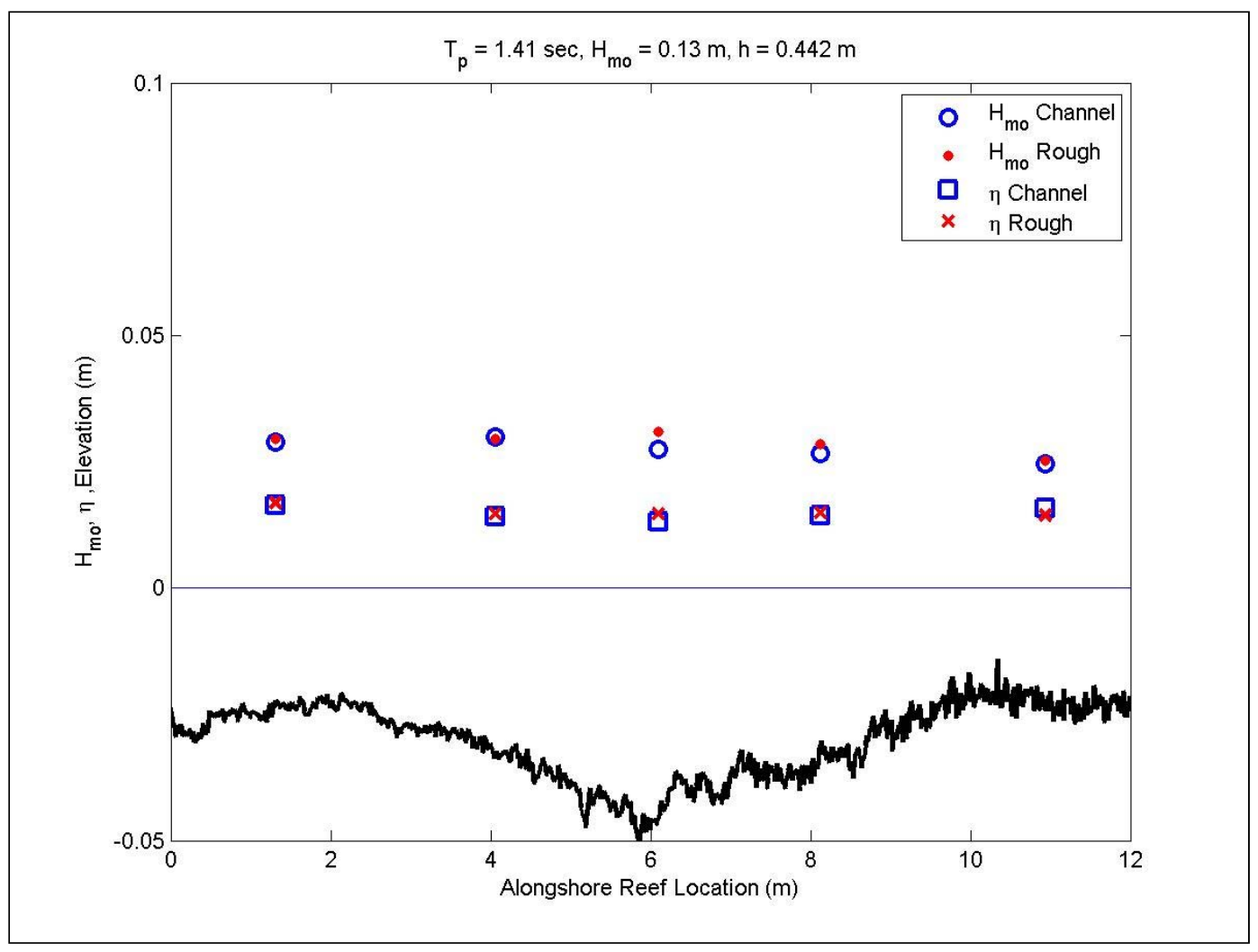

Figure C36. Alongshore wave heights for 1-sec, 0.13-m waves at mid water. 


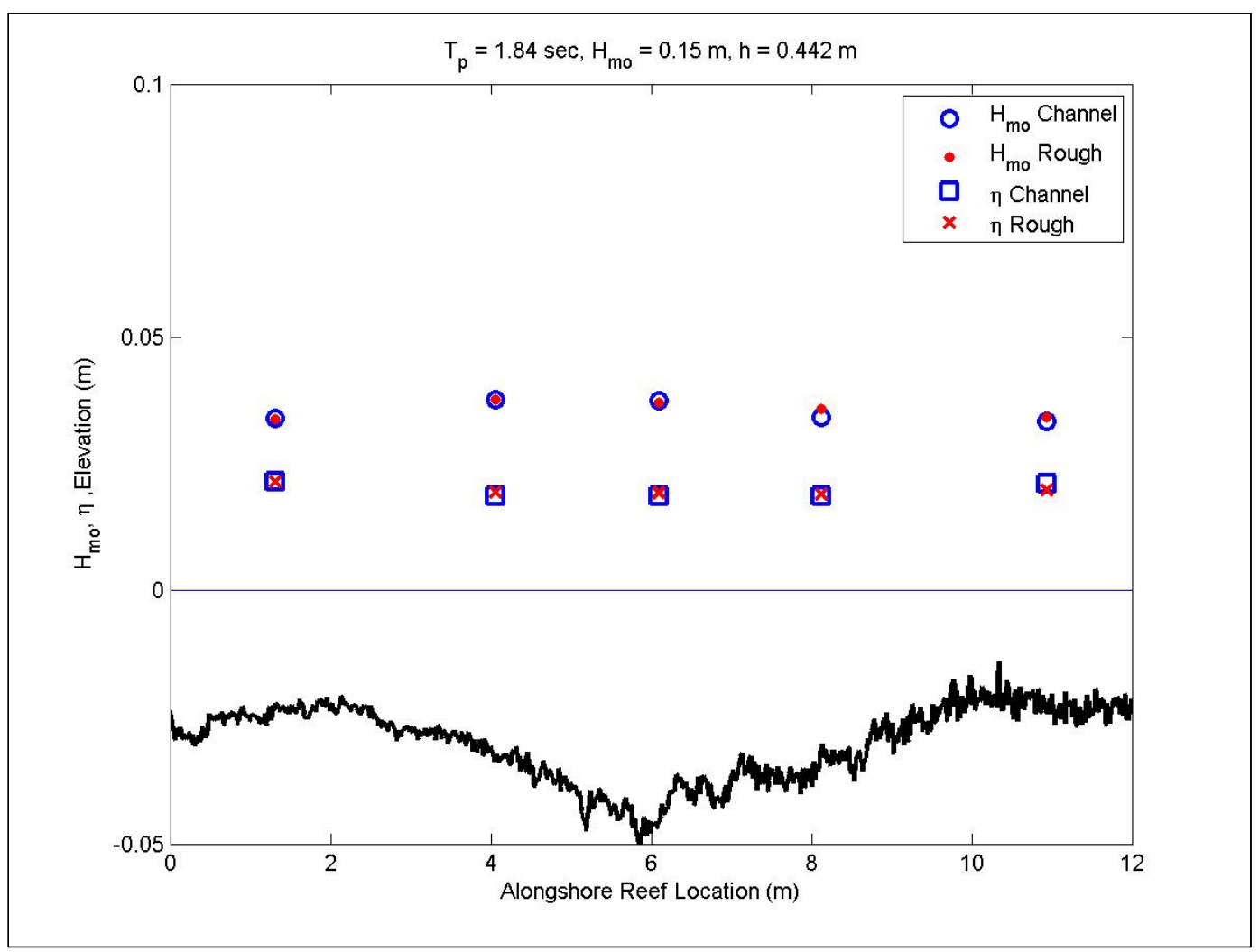

Figure C37. Alongshore wave heights for $1.84-\mathrm{sec}, 0.15-\mathrm{m}$ waves at mid water.

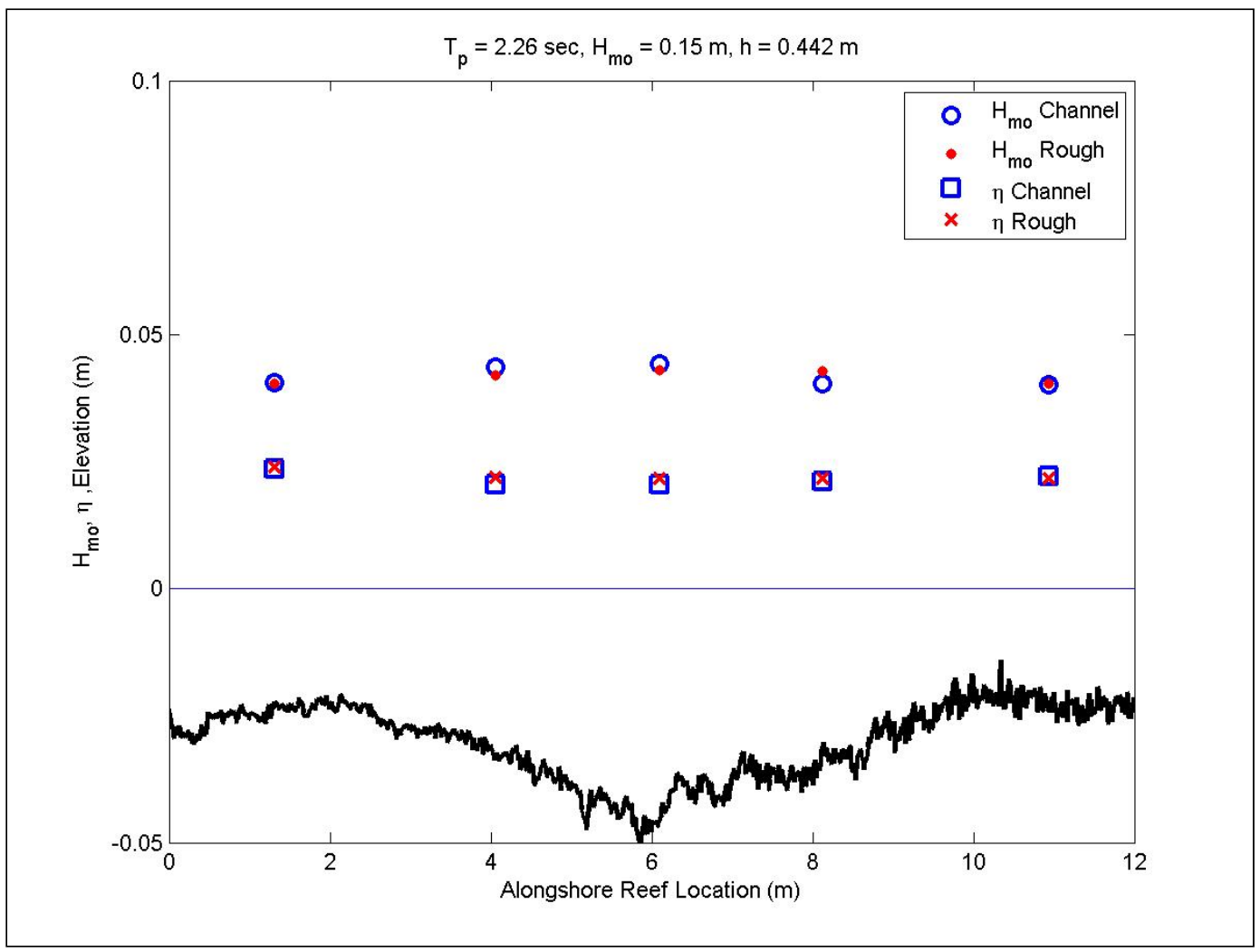

Figure C38. Alongshore wave heights for $2.3-\mathrm{sec}, 0.15-\mathrm{m}$ waves at mid water. 


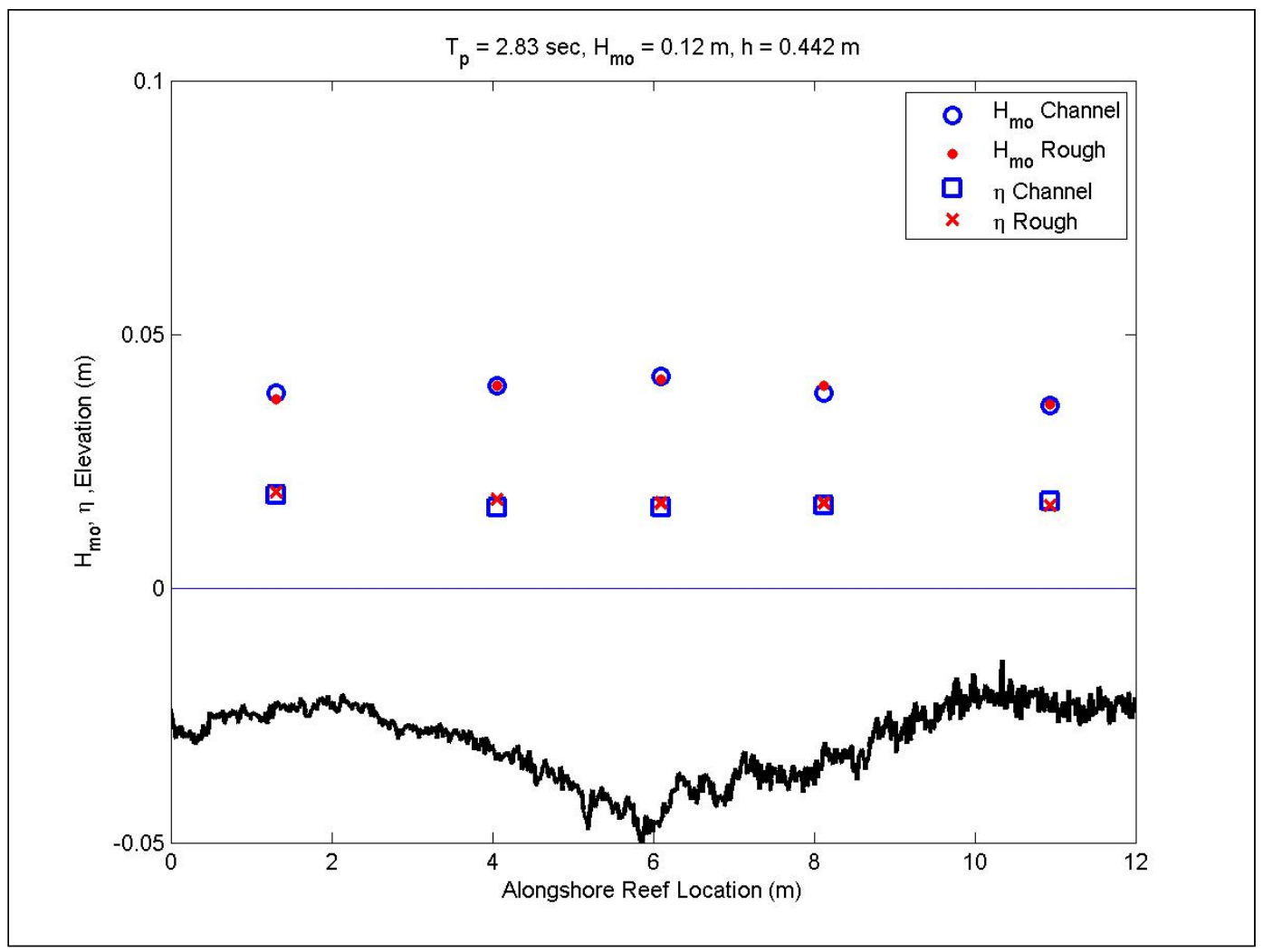

Figure C39. Alongshore wave heights for 2.8-sec, 0.12-m waves at mid water.

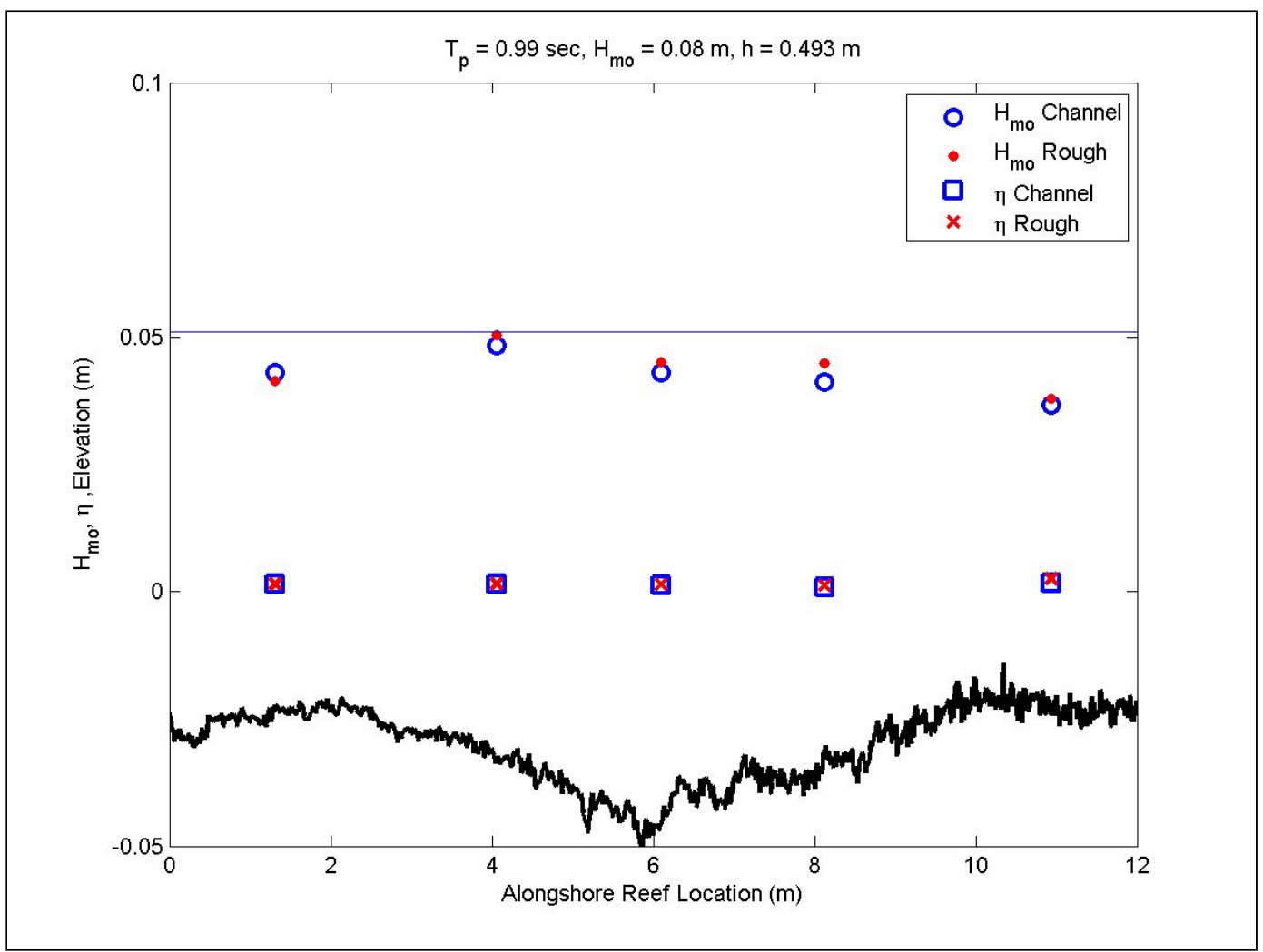

Figure C40. Alongshore wave heights for $1-\mathrm{sec}, 0.08-\mathrm{m}$ waves at high water. 


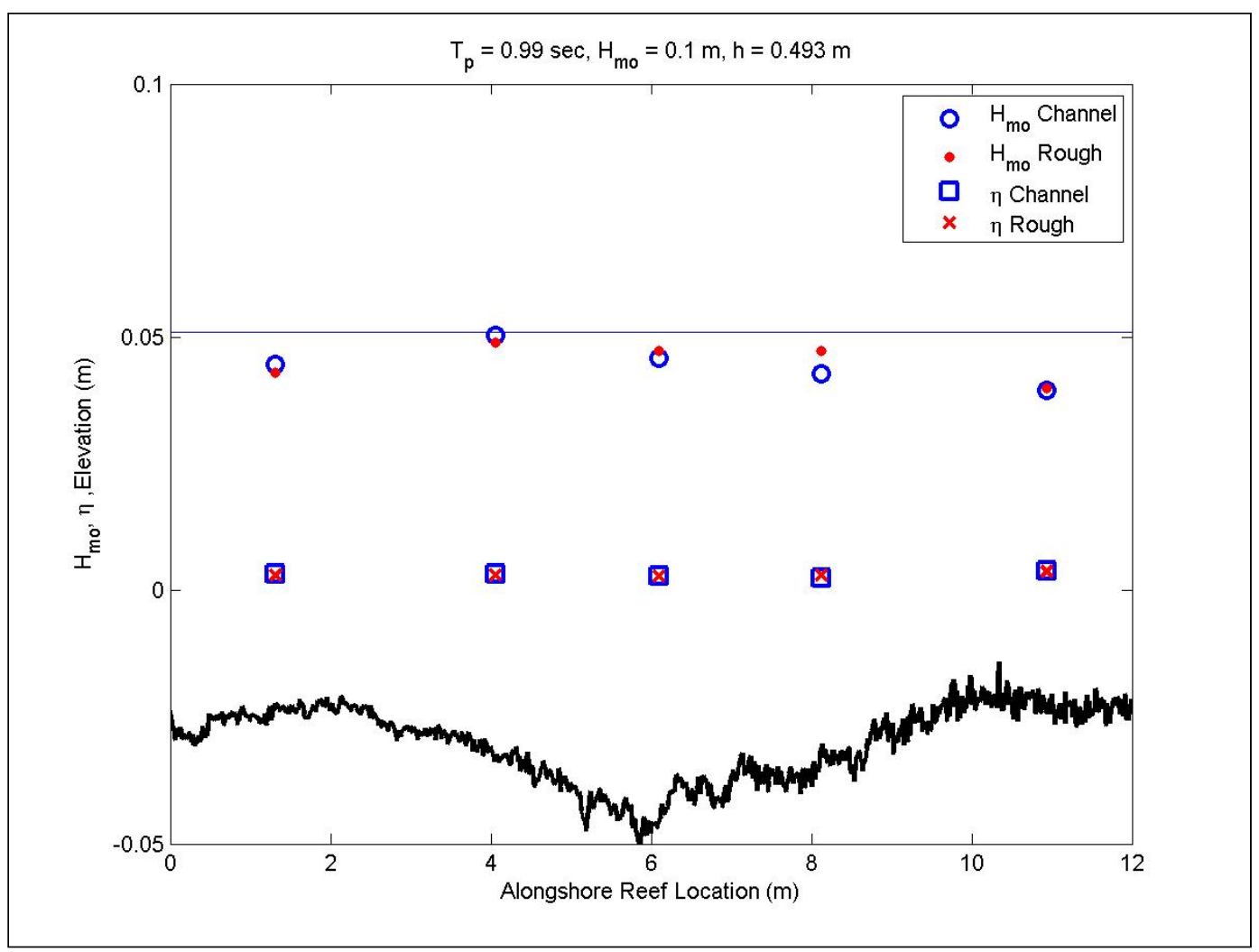

Figure C41. Alongshore wave heights for 1-sec, 0.1-m waves at high water.

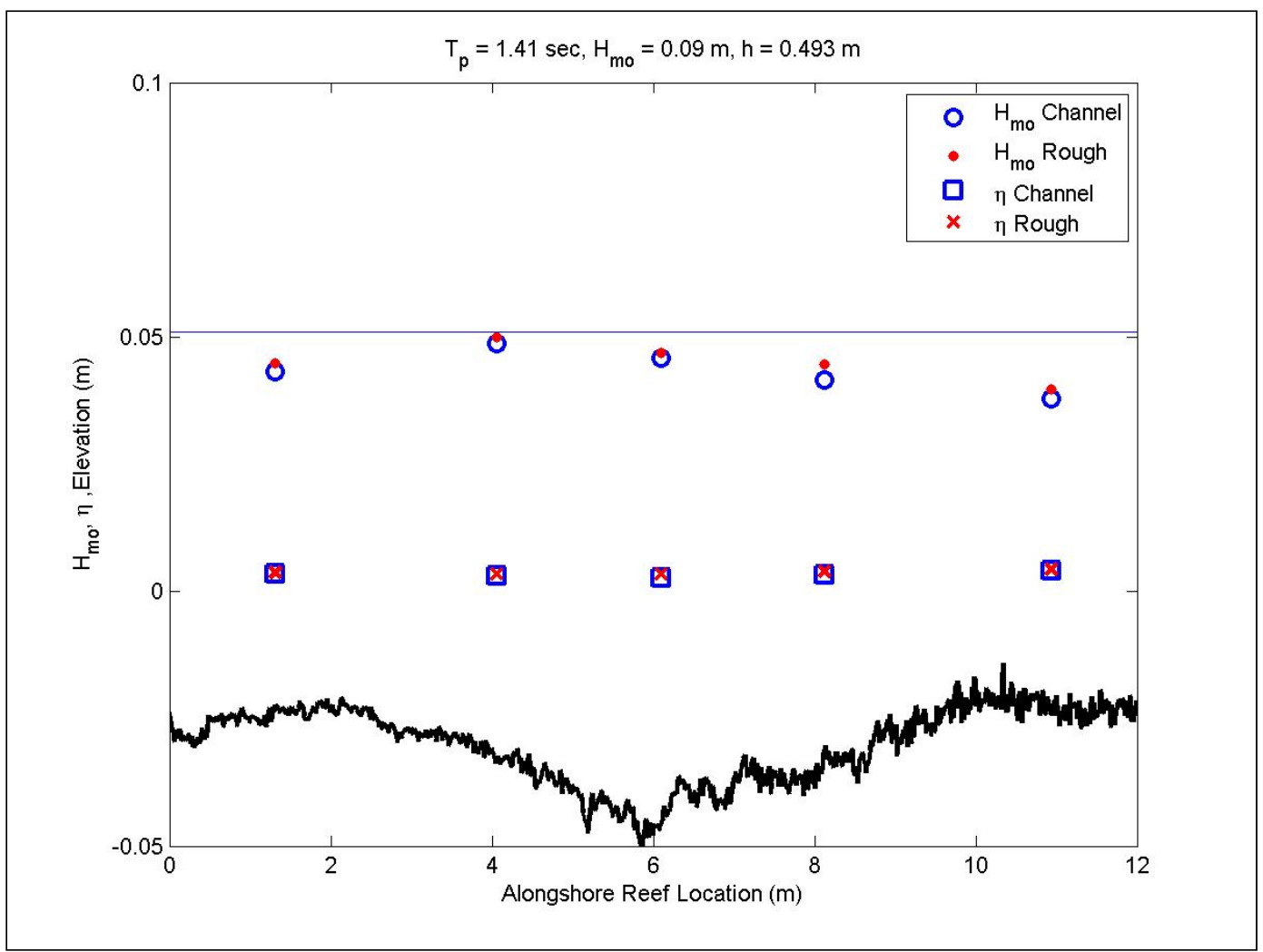

Figure C42. Alongshore wave heights for $1.4-\mathrm{sec}, 0.09-\mathrm{m}$ waves at high water. 


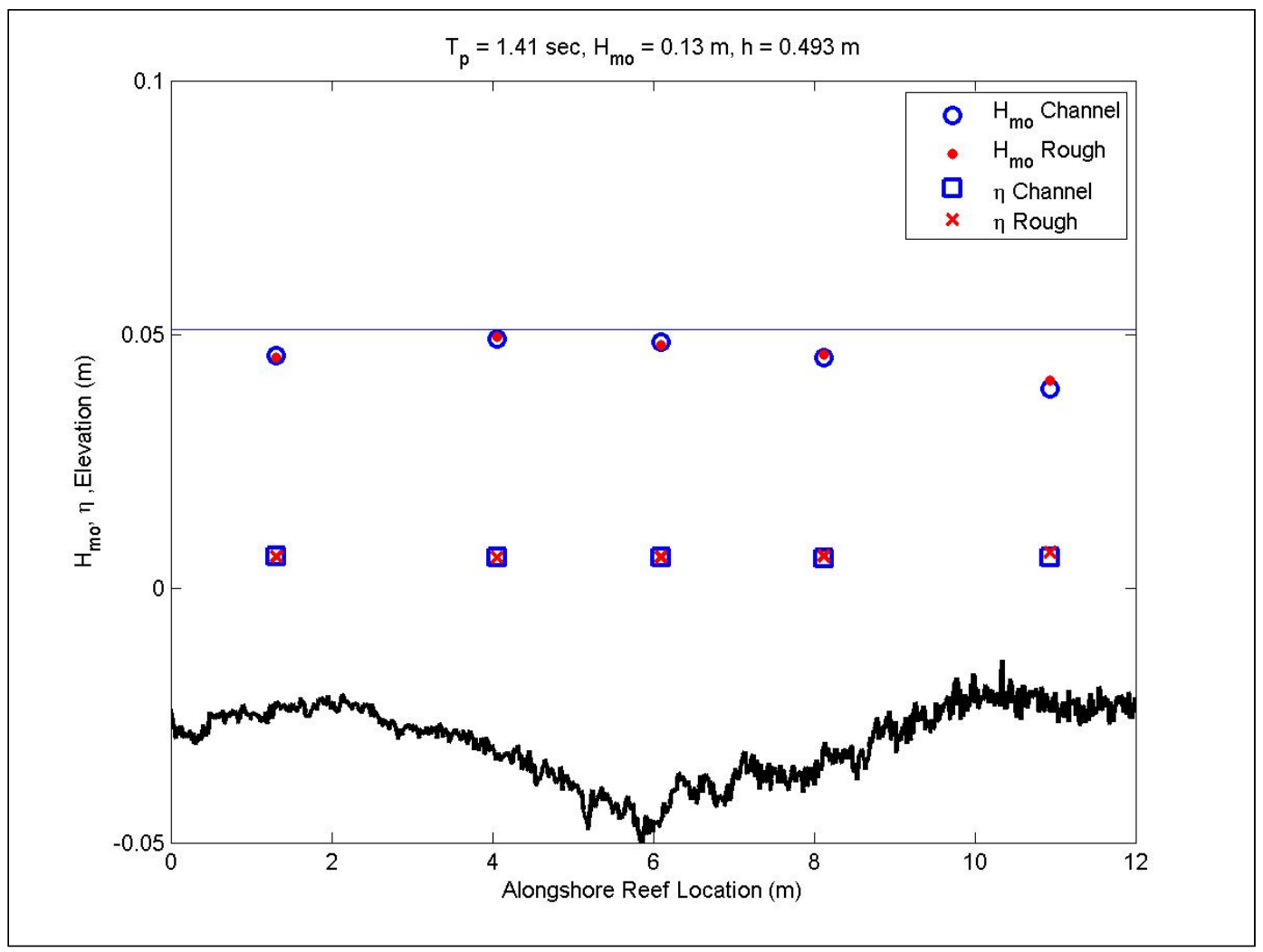

Figure C43. Alongshore wave heights for 1.4-sec, 0.13-m waves at high water.

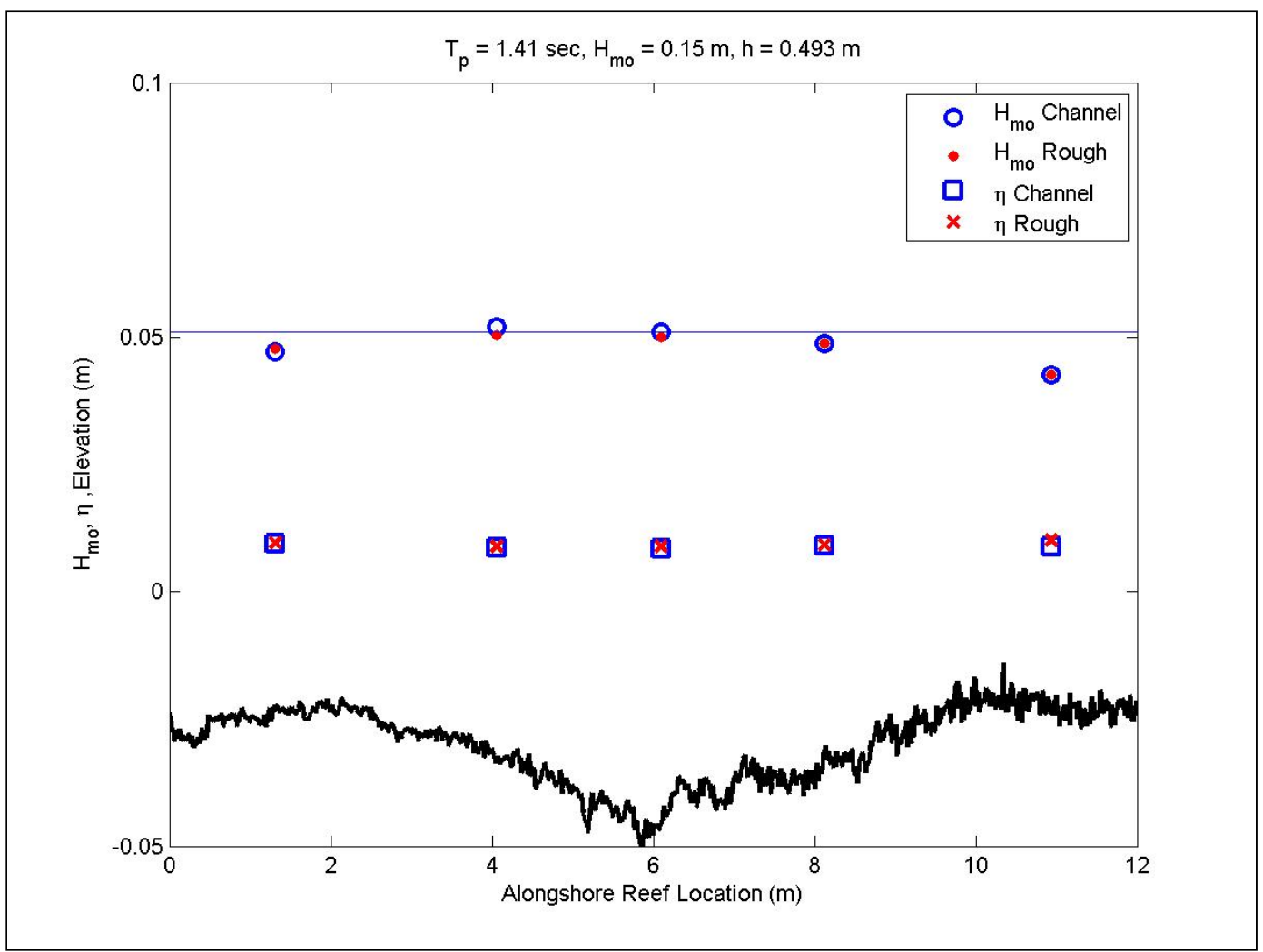

Figure C44. Alongshore wave heights for $1.4-\mathrm{sec}, 0.15-\mathrm{m}$ waves at high water. 


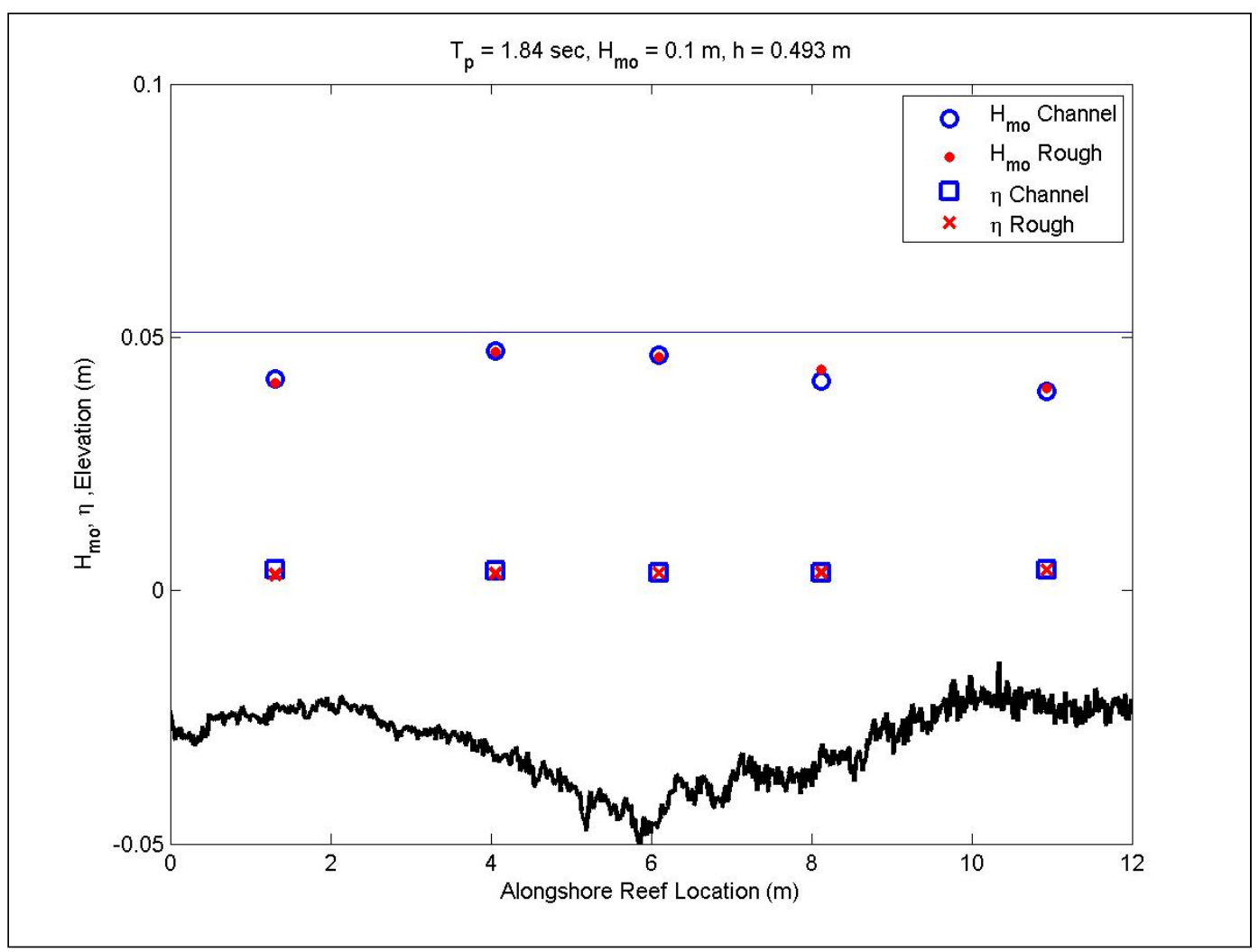

Figure C45. Alongshore wave heights for 1.8-sec, 0.1-m waves at high water.

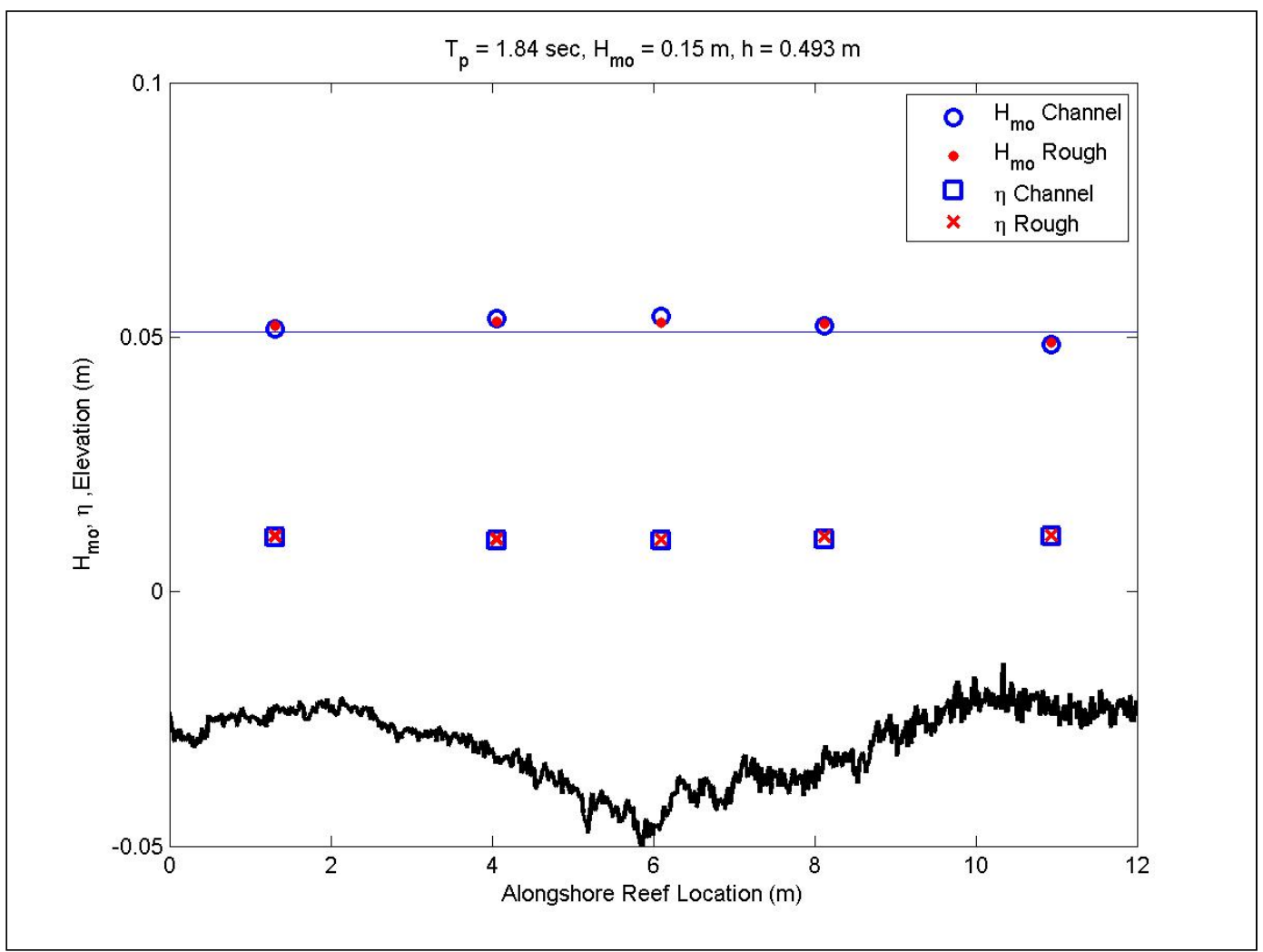

Figure C46. Alongshore wave heights for $1.8-\mathrm{sec}, 0.15-\mathrm{m}$ waves at high water. 


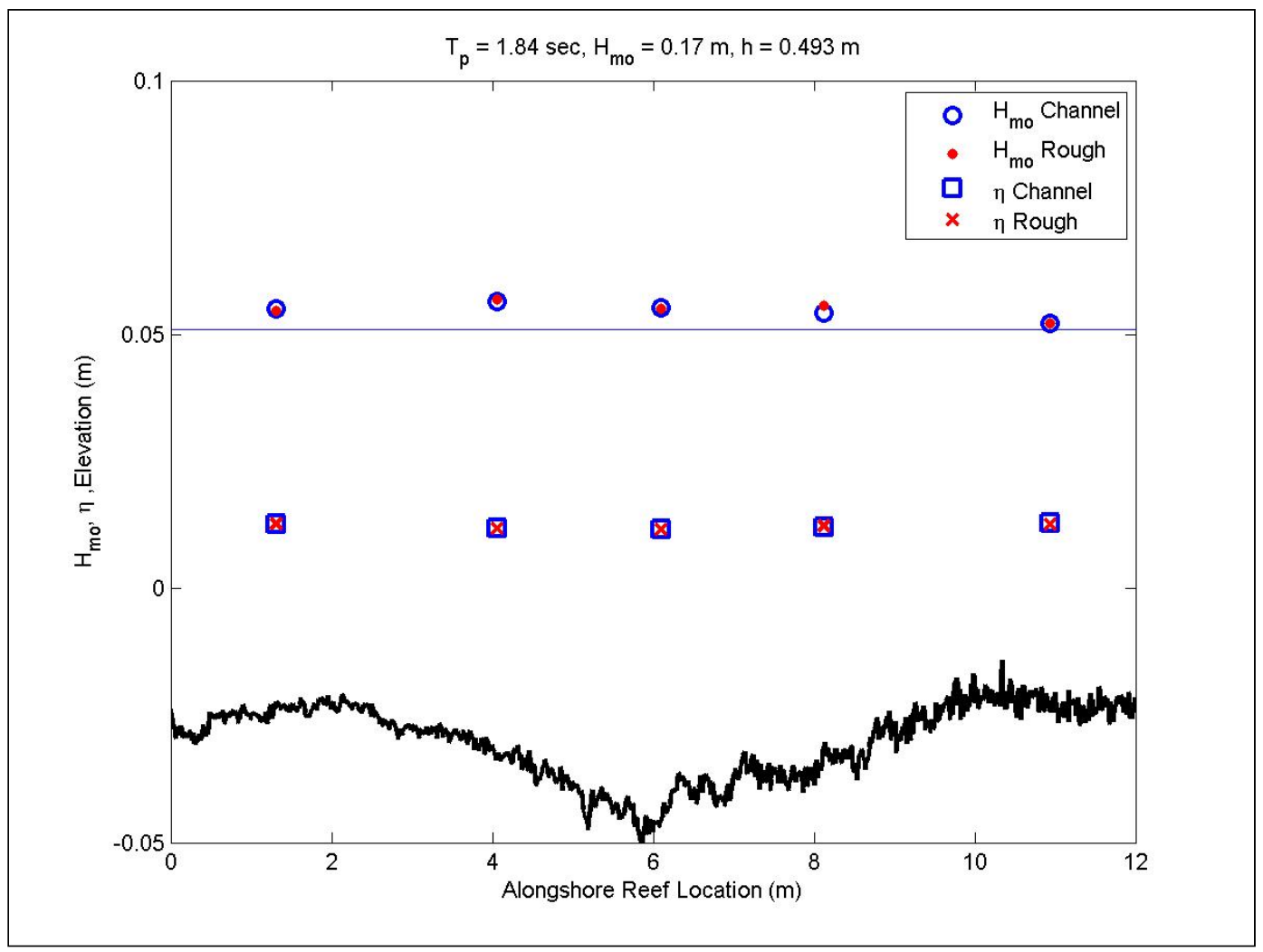

Figure $\mathrm{C} 47$. Alongshore wave heights for $1.8-\mathrm{sec}, 0.17-\mathrm{m}$ waves at high water.

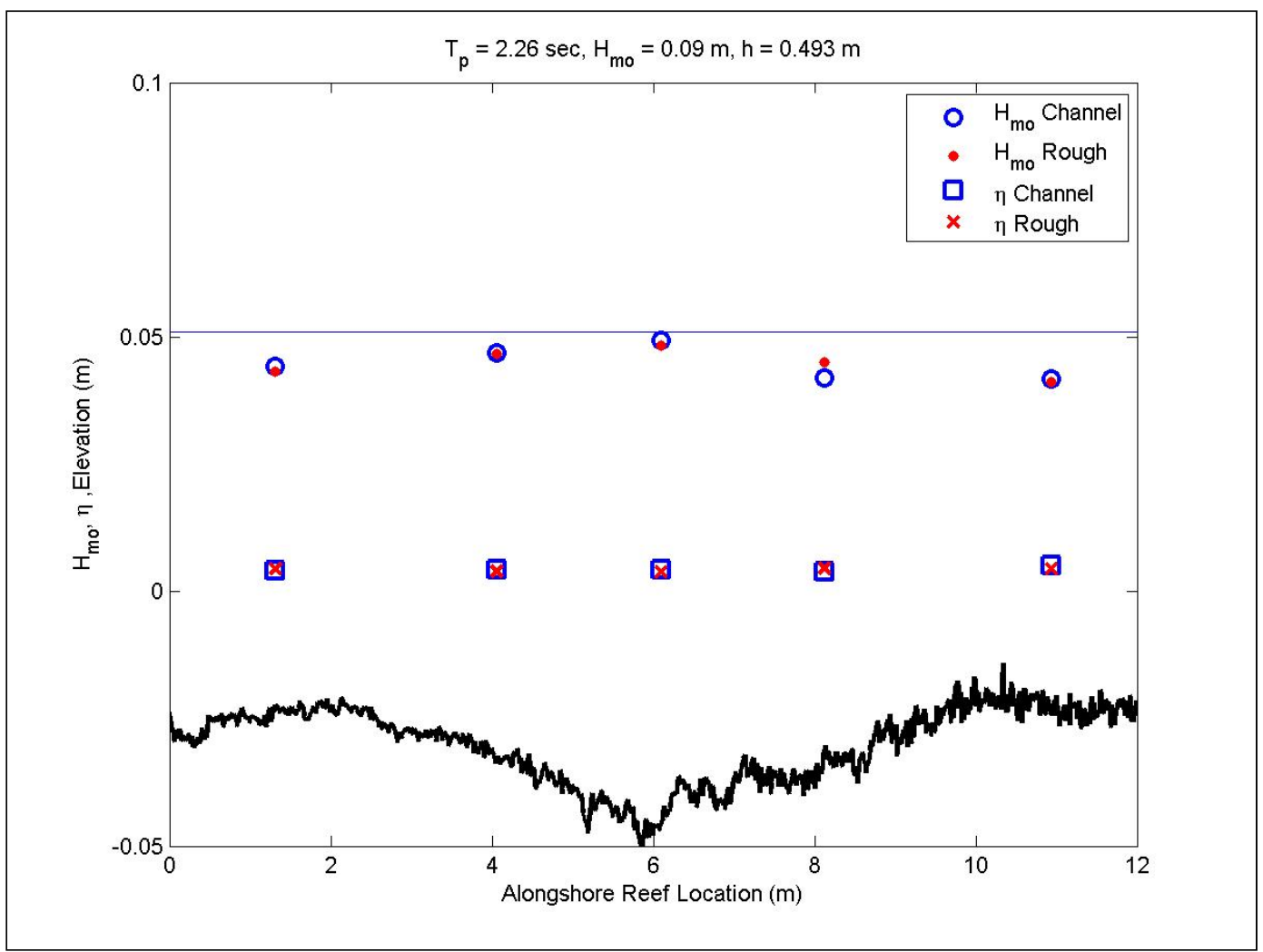

Figure C48. Alongshore wave heights for 2.3-sec, 0.09-m waves at high water. 


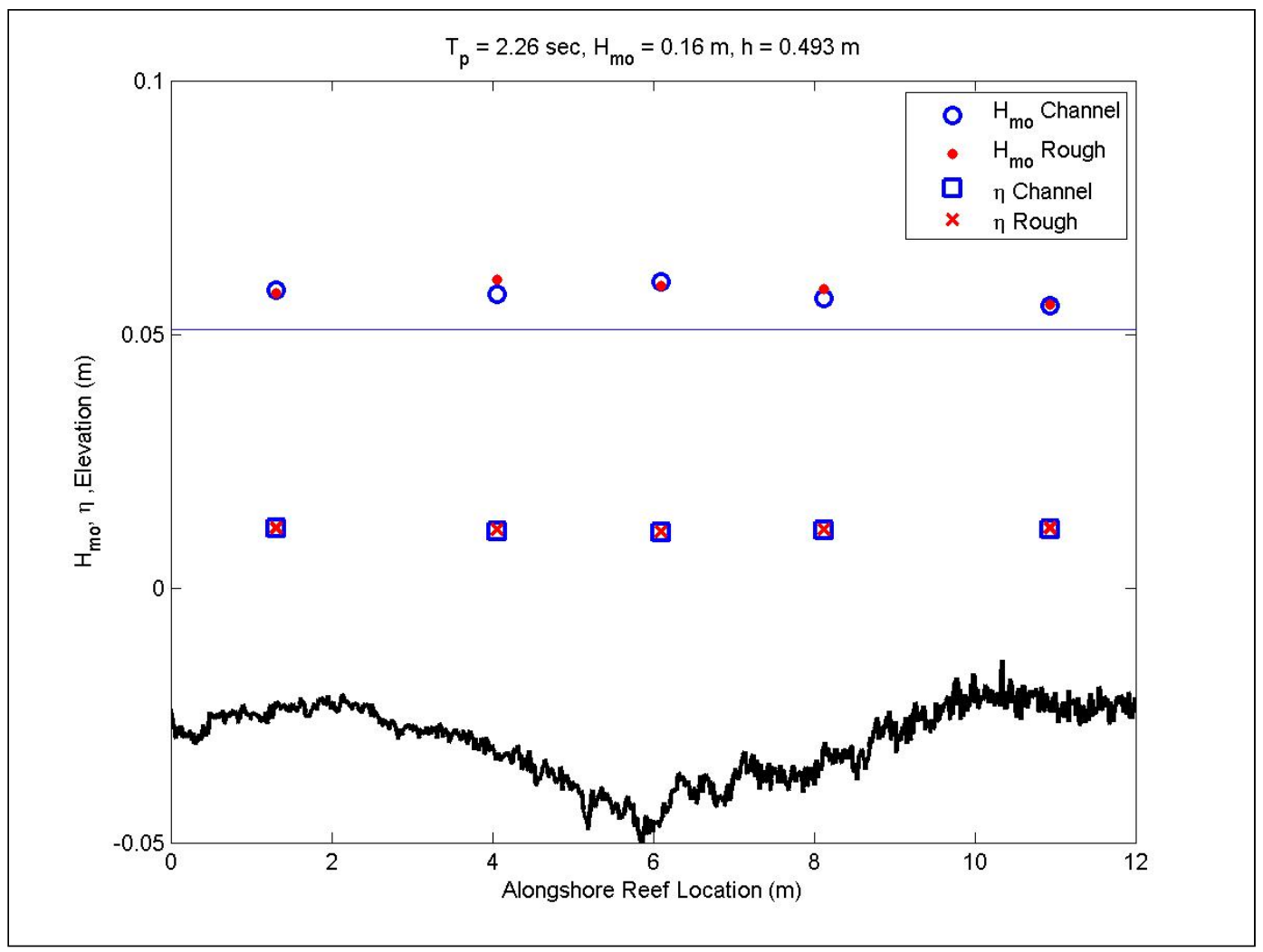

Figure C49. Alongshore wave heights for 2.3-sec, 0.16-m waves at high water.

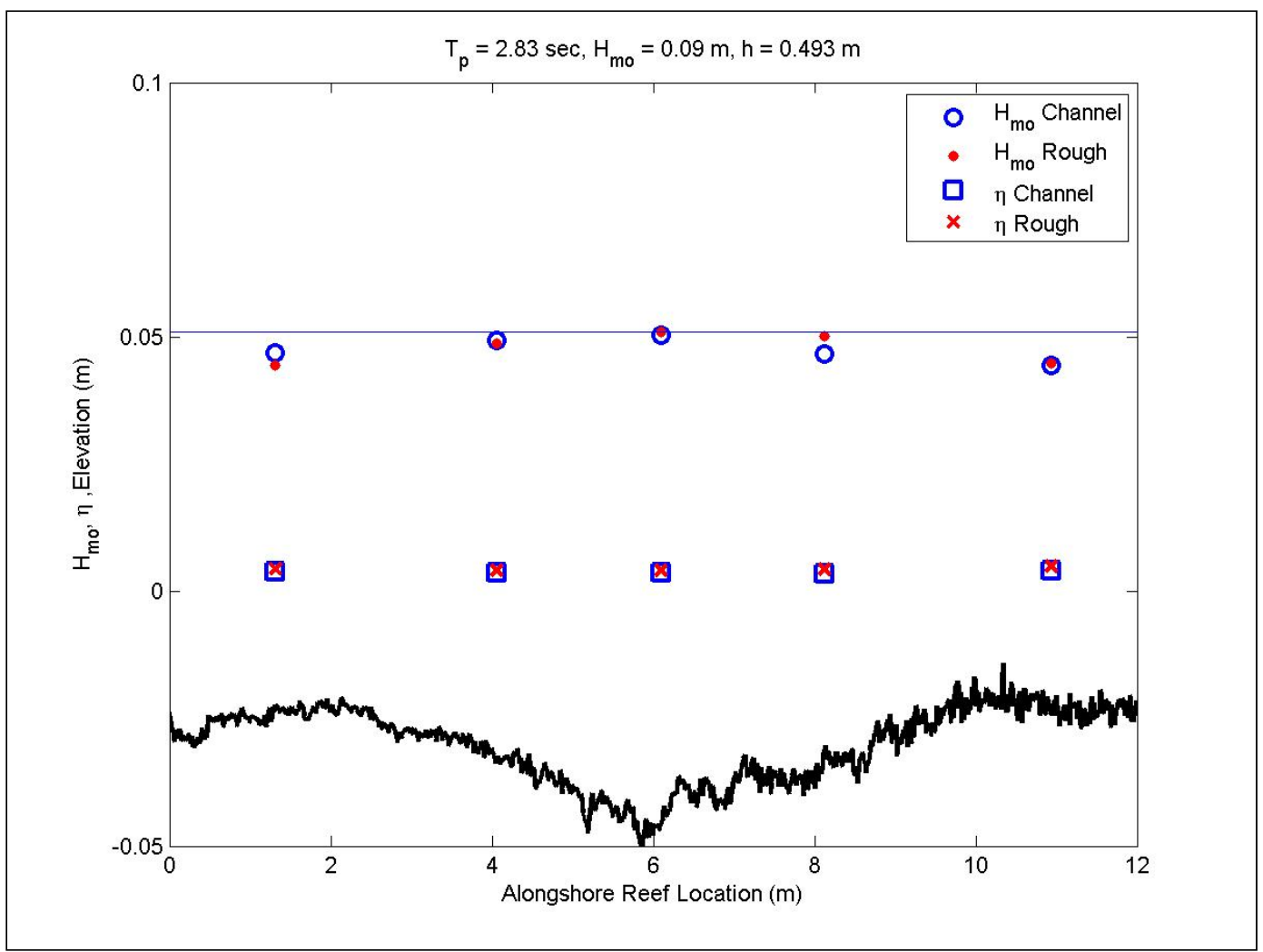

Figure C50. Alongshore wave heights for 2.8-sec, 0.09-m waves at high water. 


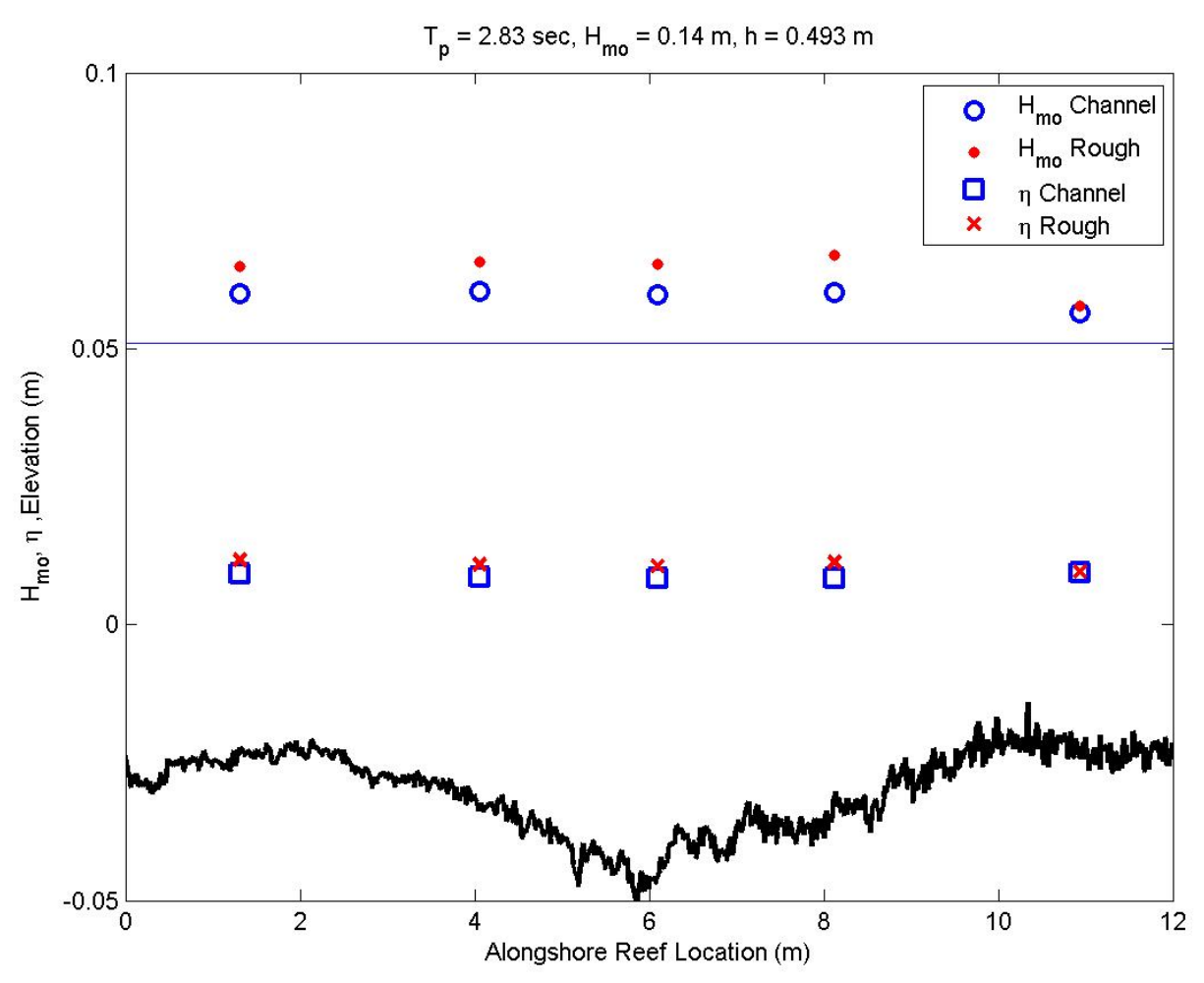

Figure C51. Alongshore wave heights for 2.8-sec, 0.14-m waves at high water. 


\section{Appendix D: Wave transformation figures from UF 2-D experiments}

Prior to conducted SWIMS 2-D experiments, collaboration between ERDC and the University of Florida (UF) waves and currents were collected to provide data for nonlinear spectral models over steep and smooth bathymetry profiles. Measurements were taken only on with the unpainted reef bathymetry with the 1 on 2.5 and 1 on 5 reef slopes installed. Four ADVs and an Acoustic Doppler Current Profiler (ADCP) provided by UF were placed in the flume near the reef slope (Figure D1). Data were collected for the conditions shown in Table D1 in prototype units with model units in parenthesis. A total of 84 tests were performed for the two reef slopes. Plots of $H_{m o}$ and $\eta$ as a function of distance from the wave generator are presented in this appendix. Numerical modeling of these experiments can be found in Sheremet et al. (2011).

Table D1. ERDC - UF 2-D reef design wave conditions in prototype, $m$ (Model, $m$ ).

\begin{tabular}{|c|c|c|c|}
\hline & $h=20.8 \mathrm{~m}(0.415 \mathrm{~m})$ & $h=21.9 \mathrm{~m}(0.439 \mathrm{~m})$ & $h=24.5 \mathrm{~m}(0.490 \mathrm{~m})$ \\
\hline $\begin{array}{l}T_{p} \\
\sec \end{array}$ & $\begin{array}{l}H_{i} \\
\mathrm{~m}(\mathrm{~m})\end{array}$ & $\begin{array}{l}H_{i} \\
\mathrm{~m}(\mathrm{~m})\end{array}$ & $\begin{array}{l}H_{i} \\
\mathrm{~m}(\mathrm{~m})\end{array}$ \\
\hline $7(1.0)$ & $4.2(0.084)$ & $4.2(0.079)$ & $4.1(0.081)$ \\
\hline $7(1.0)$ & $4.7(0.094)$ & $5.1(0.102)$ & $5.4(0.107)$ \\
\hline $10(1.4)$ & $4.4(0.088)$ & $3.8(0.076)$ & $6.1(0.122)$ \\
\hline $10(1.4)$ & $5.8(0.116)$ & $5.9(0.118)$ & $6.7(0.134)$ \\
\hline $10(1.4)$ & - & $6.6(0.133)$ & $7.2(0.144)$ \\
\hline $13(1.8)$ & $5.5(0.110)$ & $4.4(0.088)$ & $4.6(0.092)$ \\
\hline $13(1.8)$ & $7.0(0.140)$ & $6.0(0.120)$ & $7.6(0.152)$ \\
\hline $13(1.8)$ & $7.8(0.155)$ & $7.9(0.158)$ & $8.4(0.167)$ \\
\hline $16(2.3)$ & $5.5(0.110)$ & $6.0(0.120)$ & $4.6(0.092)$ \\
\hline $16(2.3)$ & $7.2(0.122)$ & $7.4(0.149)$ & $7.7(0.154)$ \\
\hline $16(2.3)$ & $7.4(0.149)$ & $8.2(0.164)$ & $8.8(0.177)$ \\
\hline $20(2.8)$ & $5.2(0.104)$ & $4.2(0.084)$ & $4.4(0.088)$ \\
\hline $20(2.8)$ & $6.2(0.124)$ & $6.0(0.120)$ & $7.6(0.151)$ \\
\hline $20(2.8)$ & $7.9(0.158)$ & $6.9(0.138)$ & $8.6(0.173)$ \\
\hline $20(2.8)$ & - & $8.0(0.160)$ & - \\
\hline
\end{tabular}

Values in parenthesis refer to values in model seconds and meters 


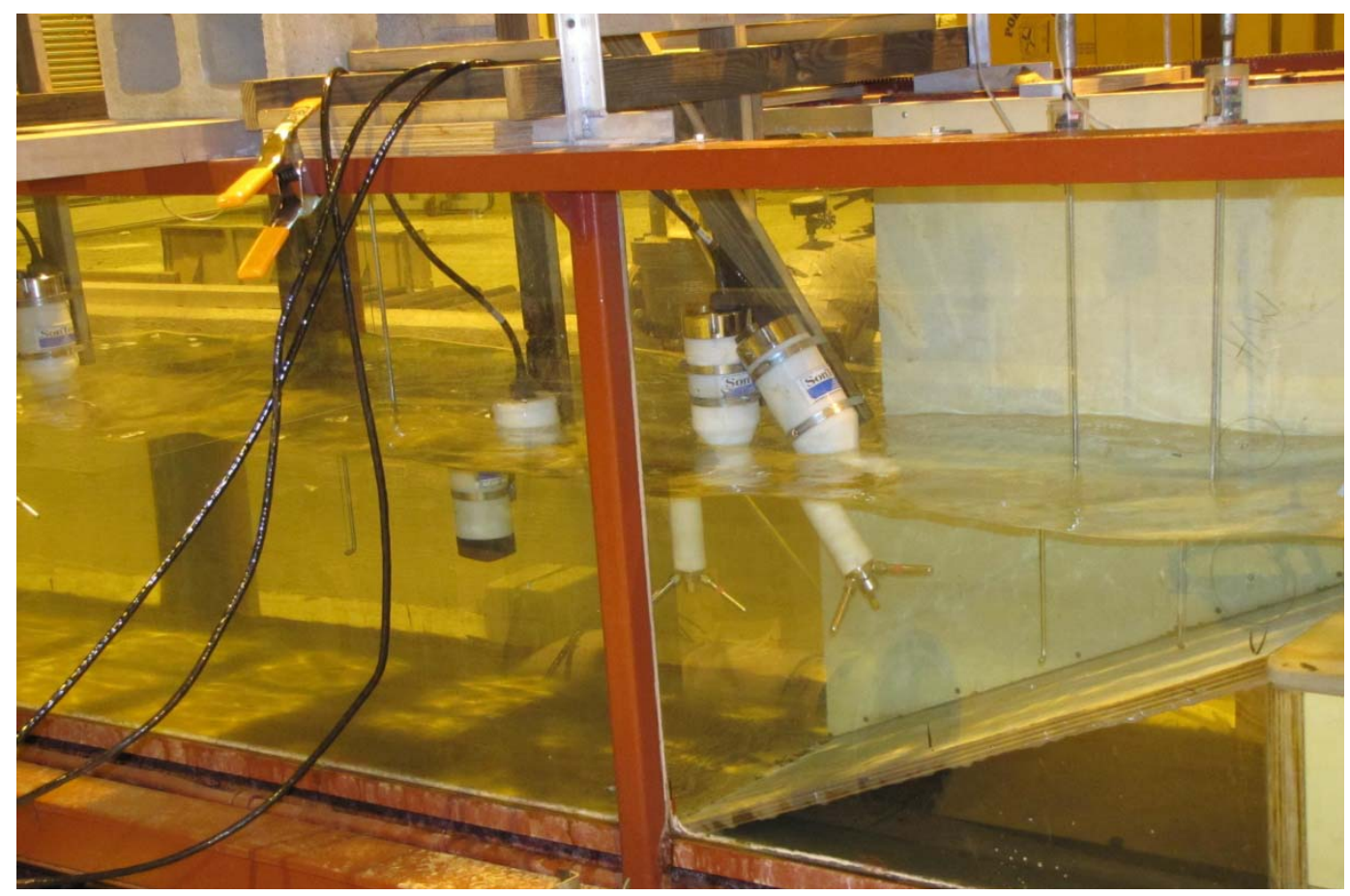

Figure D1. Photograph of UF ADVs and ADCP.

Figures D2 to D85 show $H_{m o}$ and $\eta$ as a function of distance in the 2-D wave tank. Circles in the figures represent the total $H_{m o}$, " $x$ " represents the incident $H_{m o}$ computed from incident and reflection analysis at the three-gauge array, and the asterisks denote $\eta$. The model bottom also is presented as a reference, but is scaled by a factor of 10 . Results of these experiments are described in Sheremet et al. 2011. 


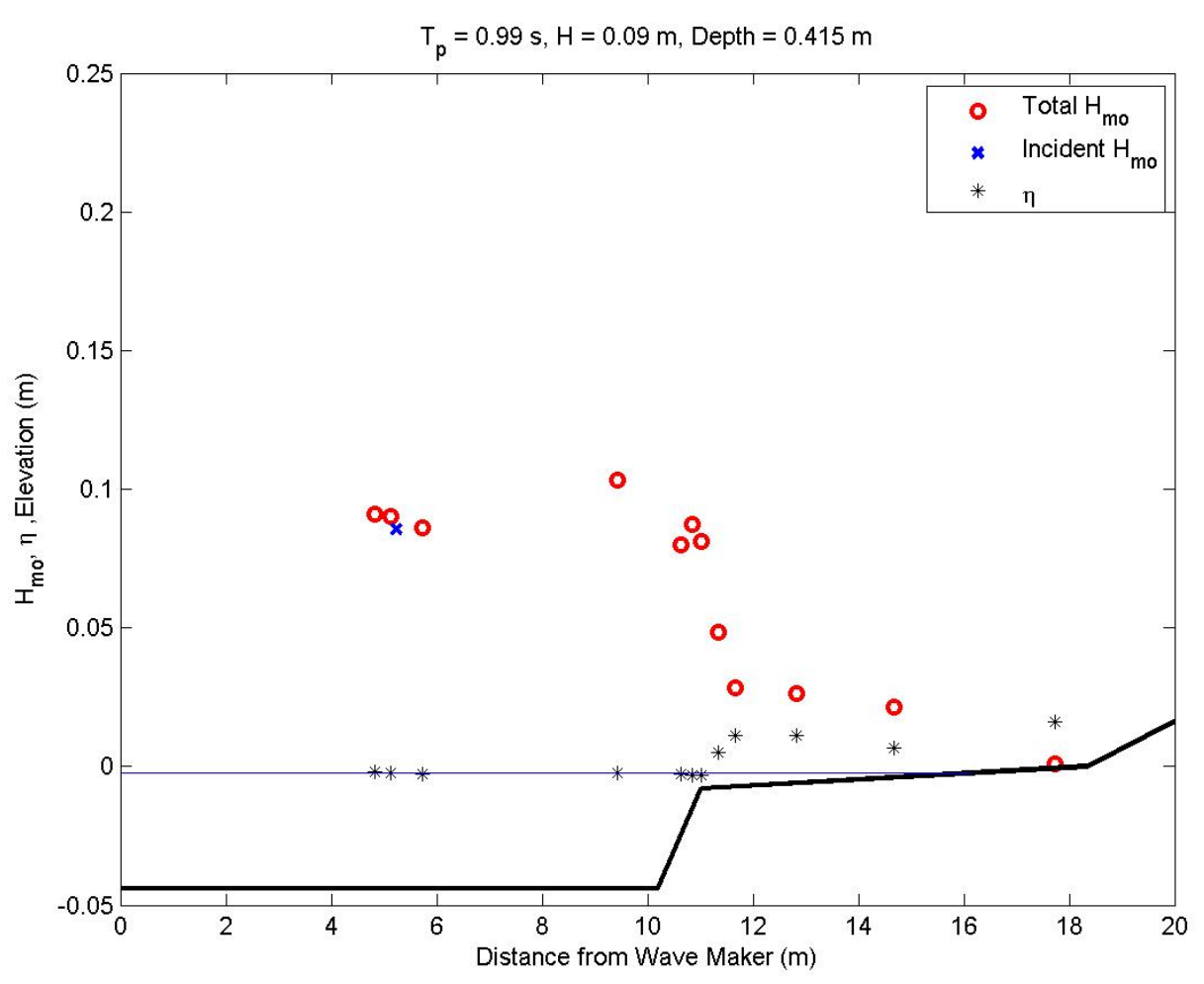

Figure D2. Wave transformation for 1-sec, $0.09 \mathrm{~m}$ waves at low water with a 1:25 reef slope.

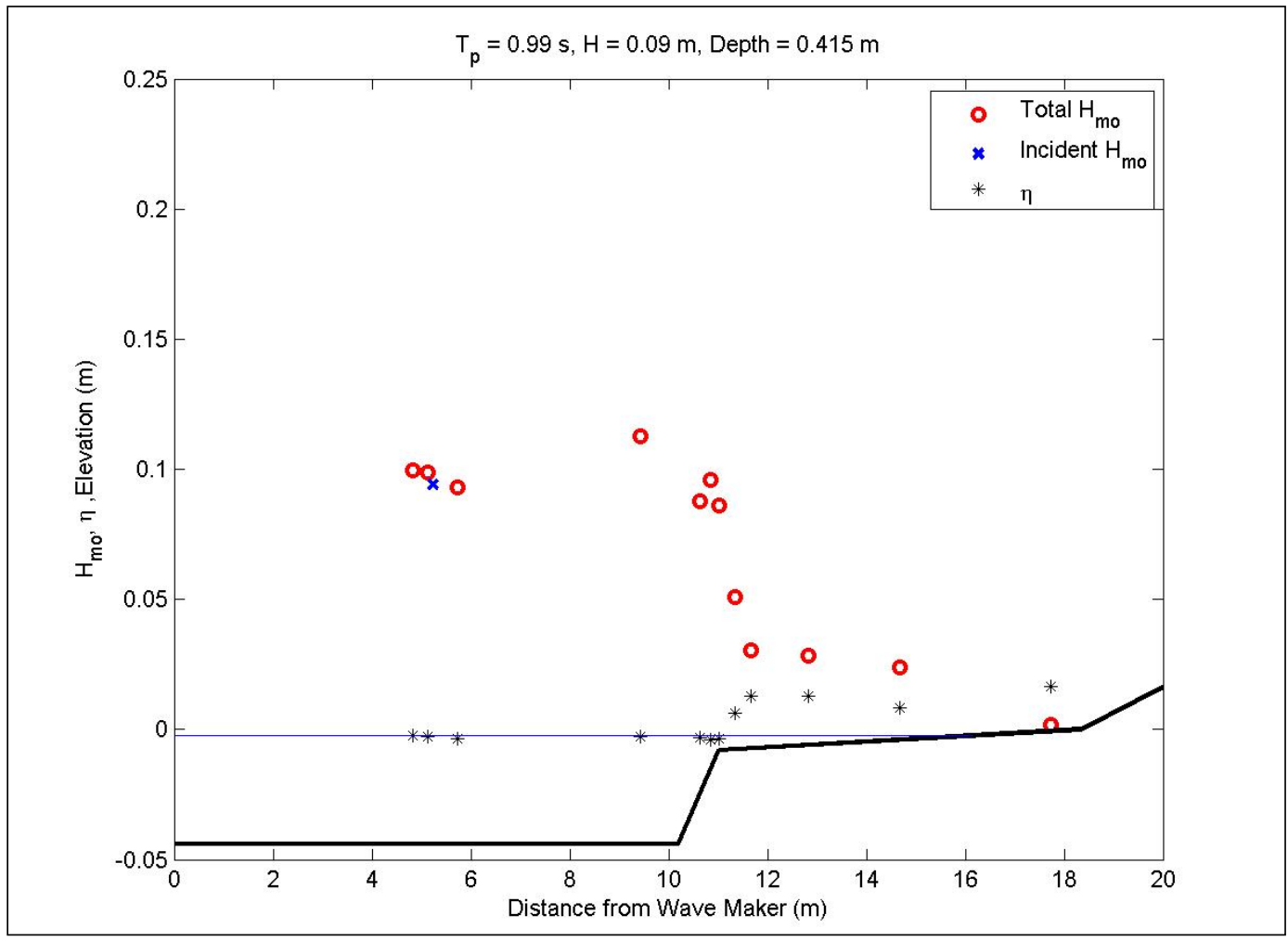

Figure D3. Wave transformation for 1-sec, $0.09 \mathrm{~m}$ waves at low water with a 1:25 reef slope. 


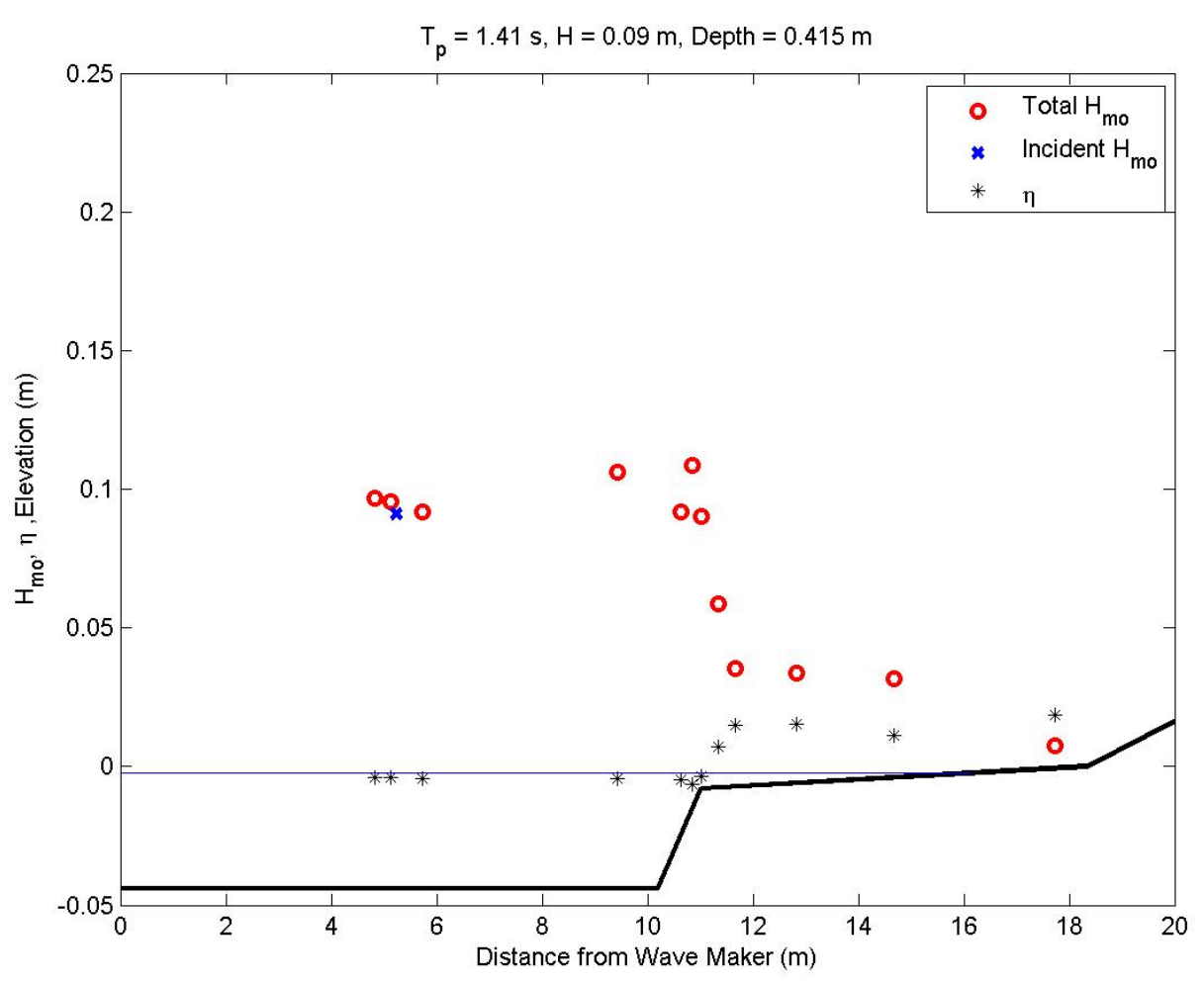

Figure D4. Wave transformation for $1.4-\mathrm{sec}, 0.09 \mathrm{~m}$ waves at low water with a 1:25 reef slope.

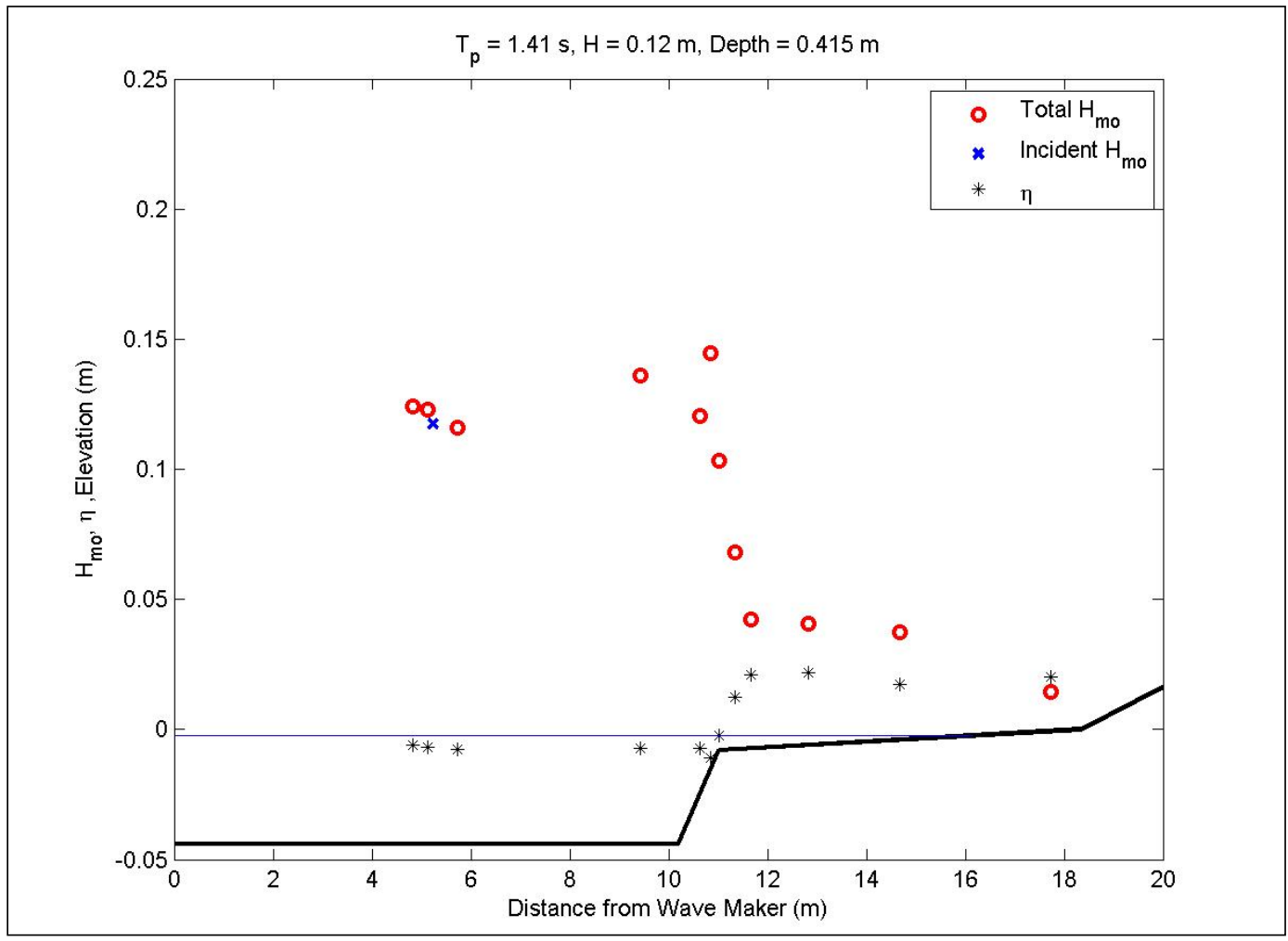

Figure D5. Wave transformation for $1.4-\mathrm{sec}, 0.12 \mathrm{~m}$ waves at low water with a 1:25 reef slope. 


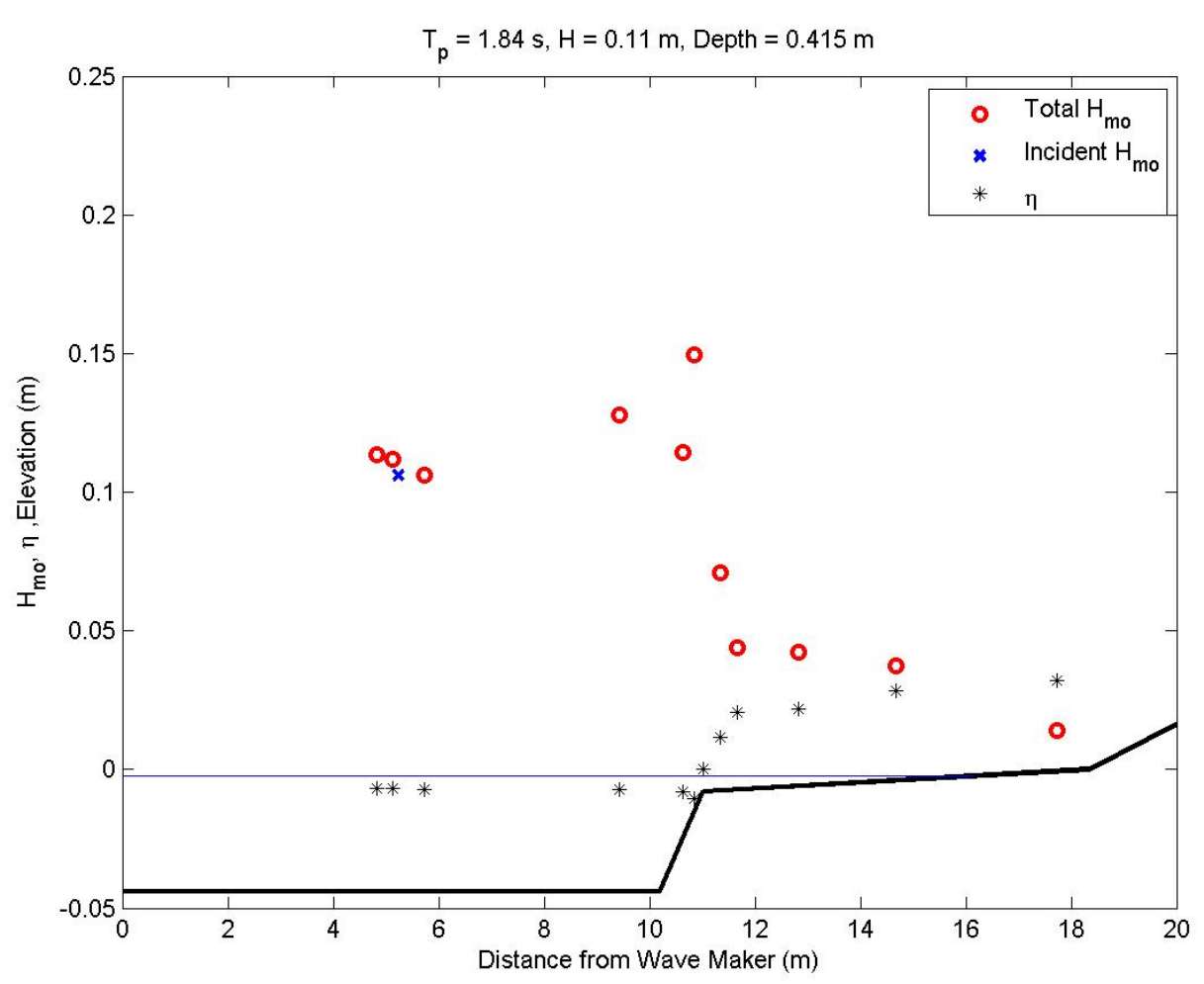

Figure D6. Wave transformation for 1.8-sec, $0.11 \mathrm{~m}$ waves at low water with a 1:25 reef slope.

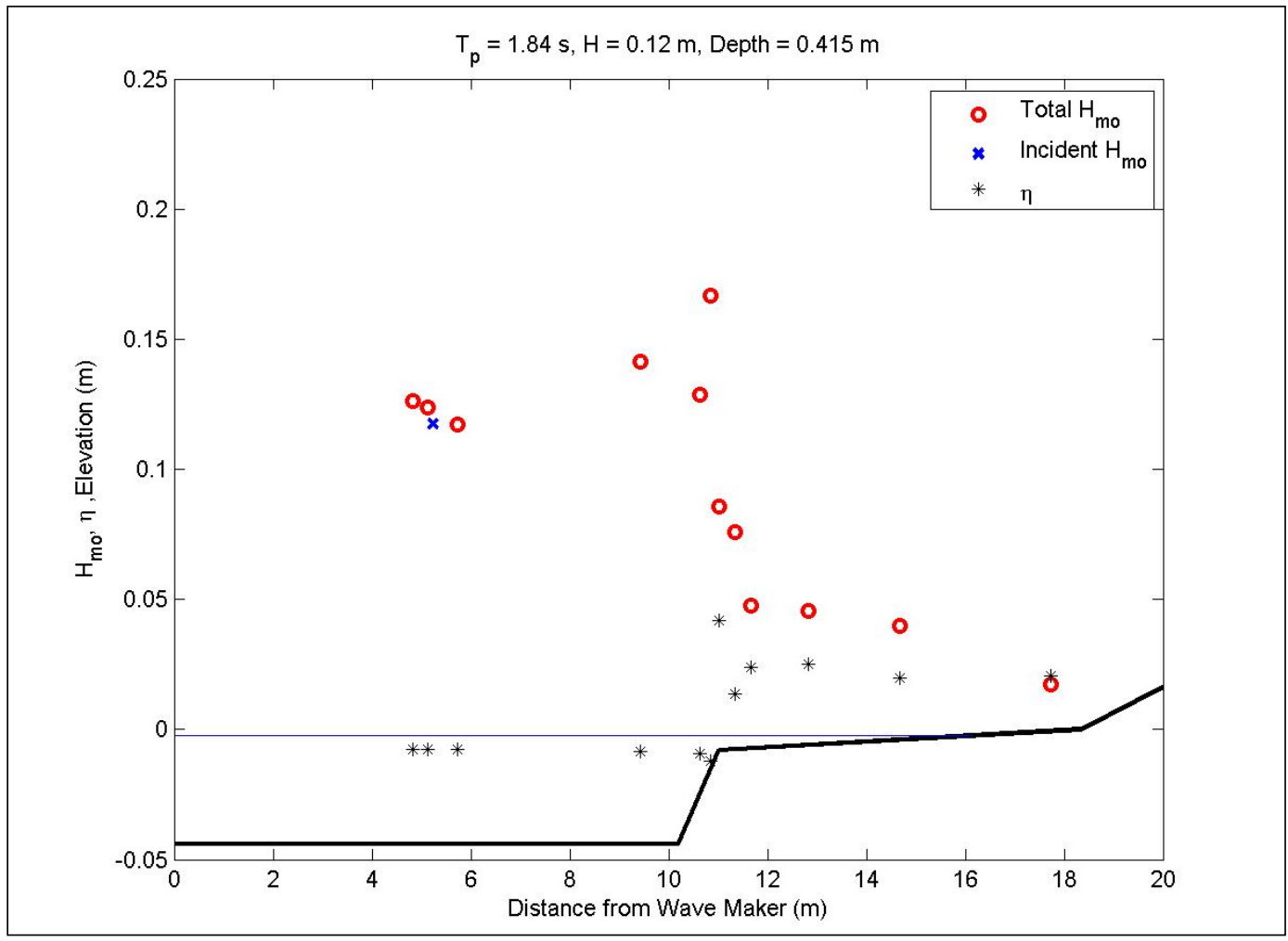

Figure D7. Wave transformation for $1.8-\mathrm{sec}, 0.12 \mathrm{~m}$ waves at low water with a 1:25 reef slope. 


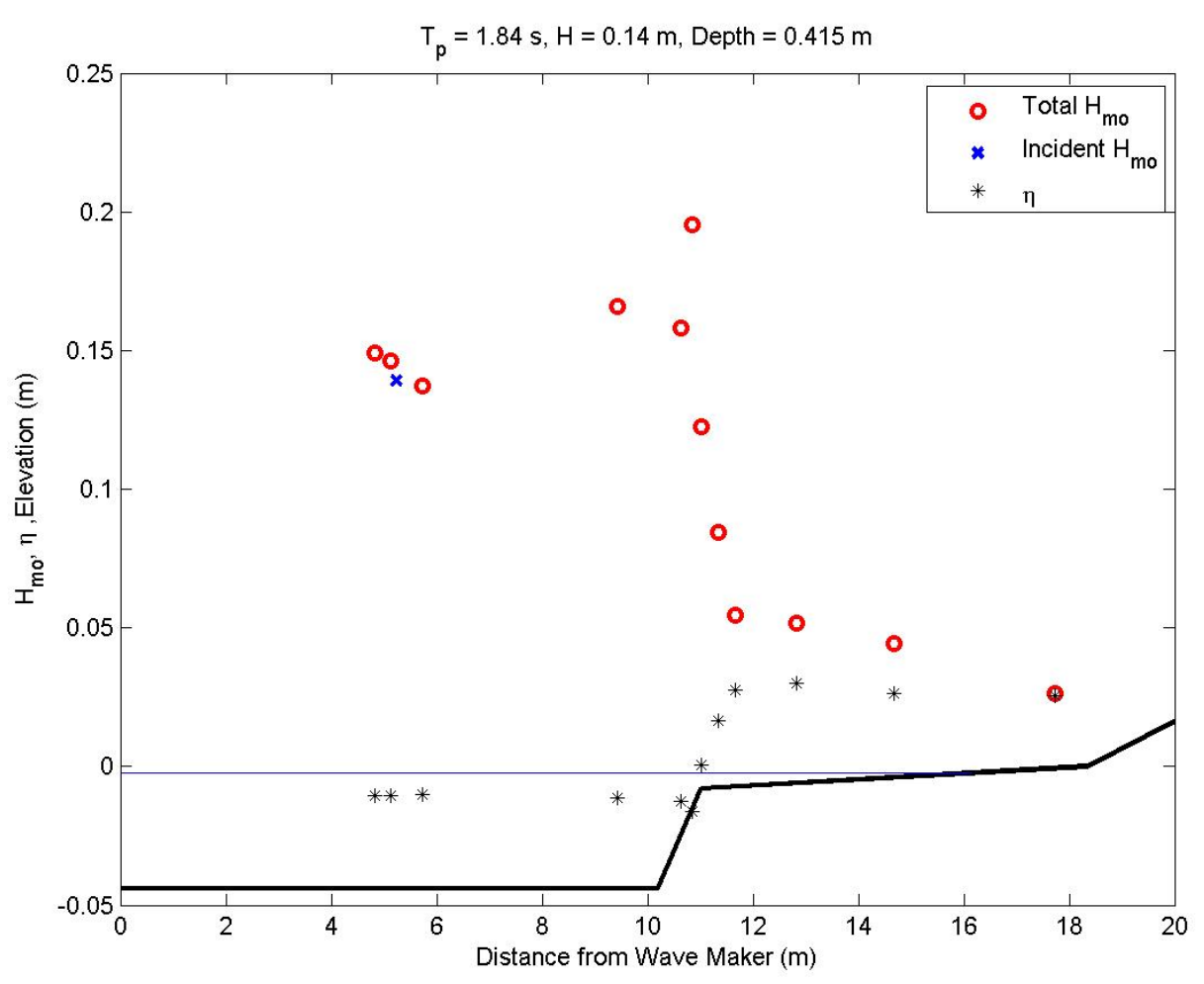

Figure D8. Wave transformation for 1.8-sec, $0.14 \mathrm{~m}$ waves at low water with a 1:25 reef slope.

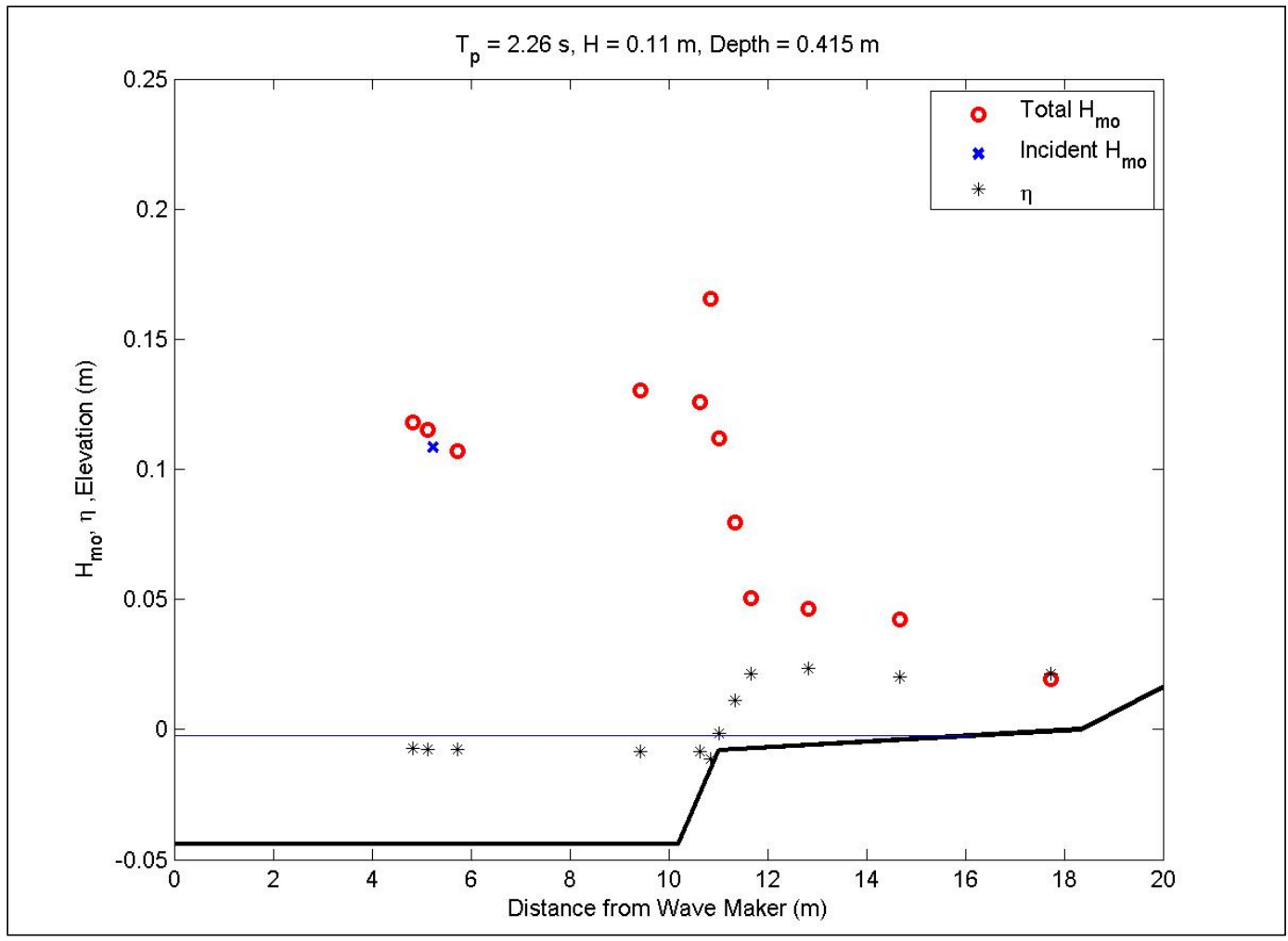

Figure D9. Wave transformation for 2.3-sec, $0.11 \mathrm{~m}$ waves at low water with a 1:25 reef slope. 


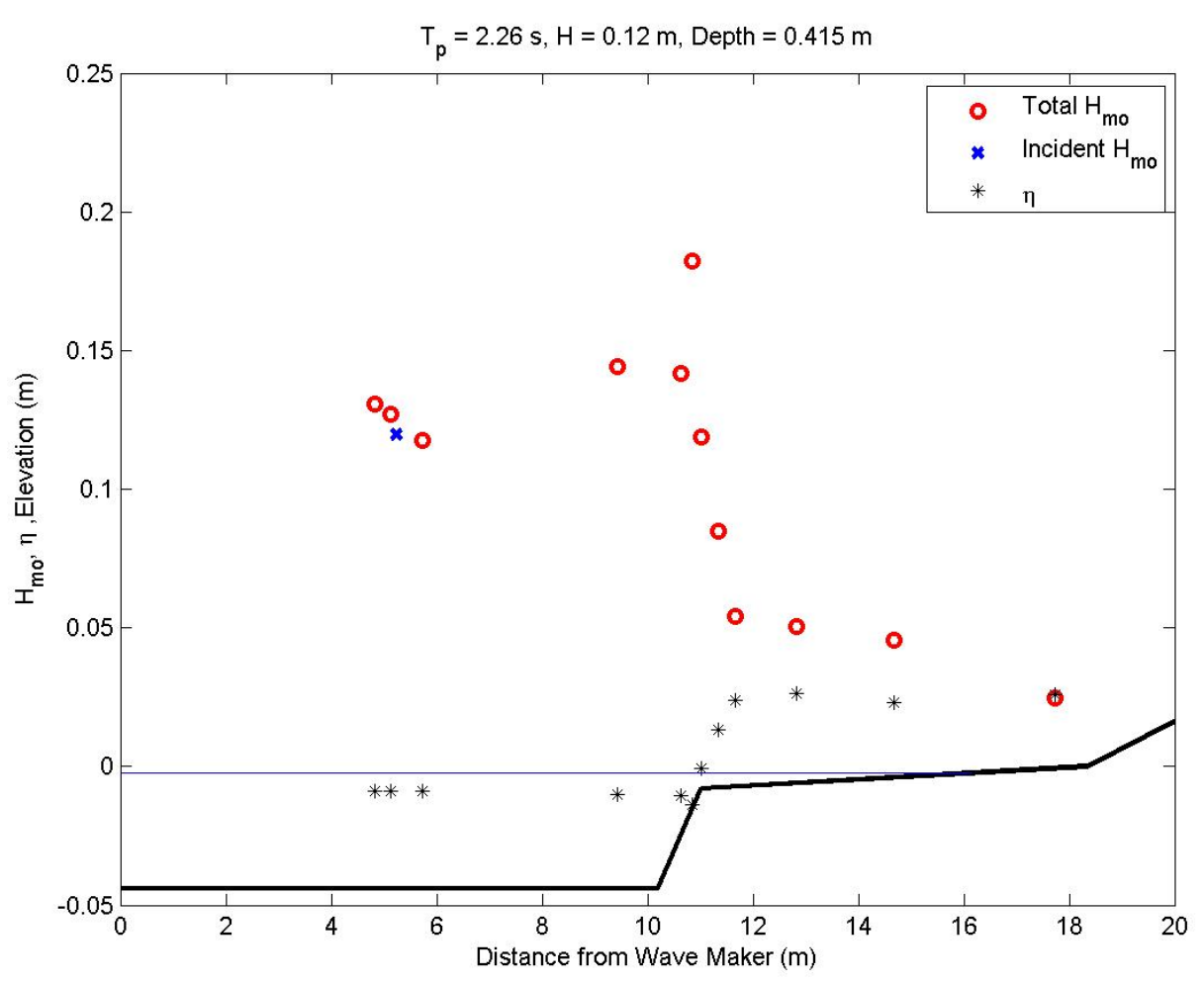

Figure D10. Wave transformation for 2.3-sec, $0.12 \mathrm{~m}$ waves at low water with a 1:25 reef slope.

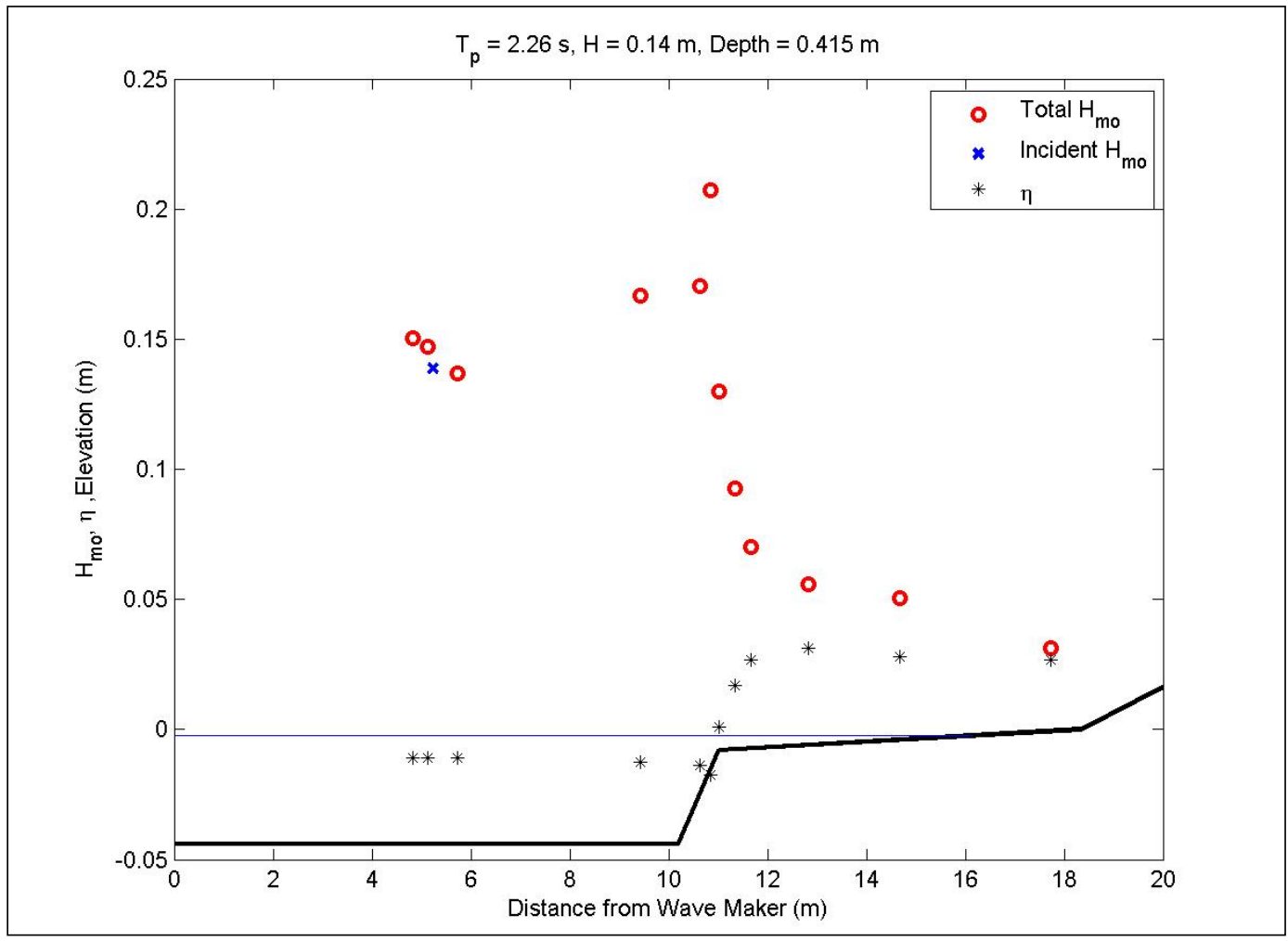

Figure D11. Wave transformation for 2.3-sec, $0.14 \mathrm{~m}$ waves at low water with a 1:25 reef slope. 


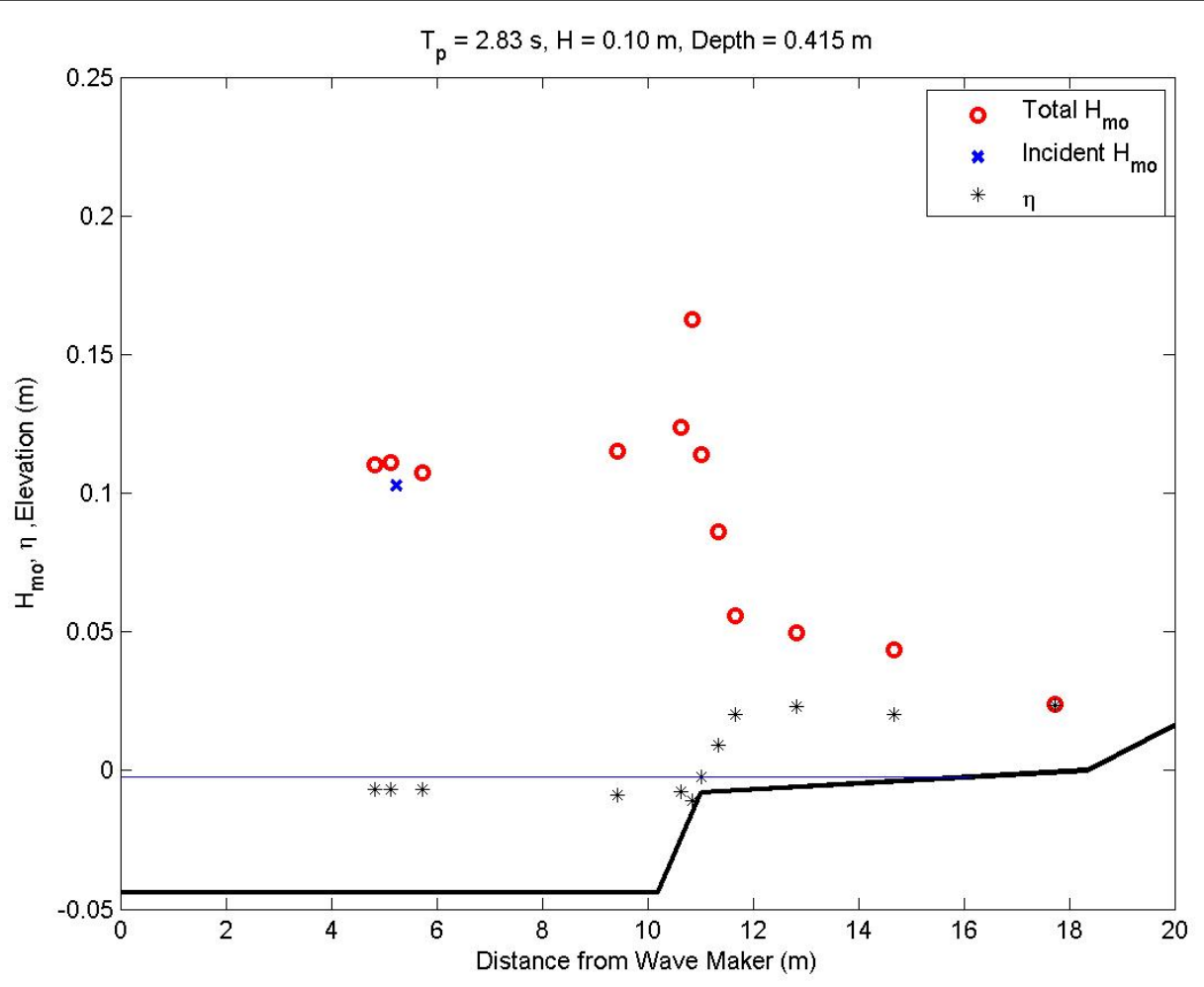

Figure D12. Wave transformation for 2.8-sec, $0.1 \mathrm{~m}$ waves at low water with a 1:25 reef slope.

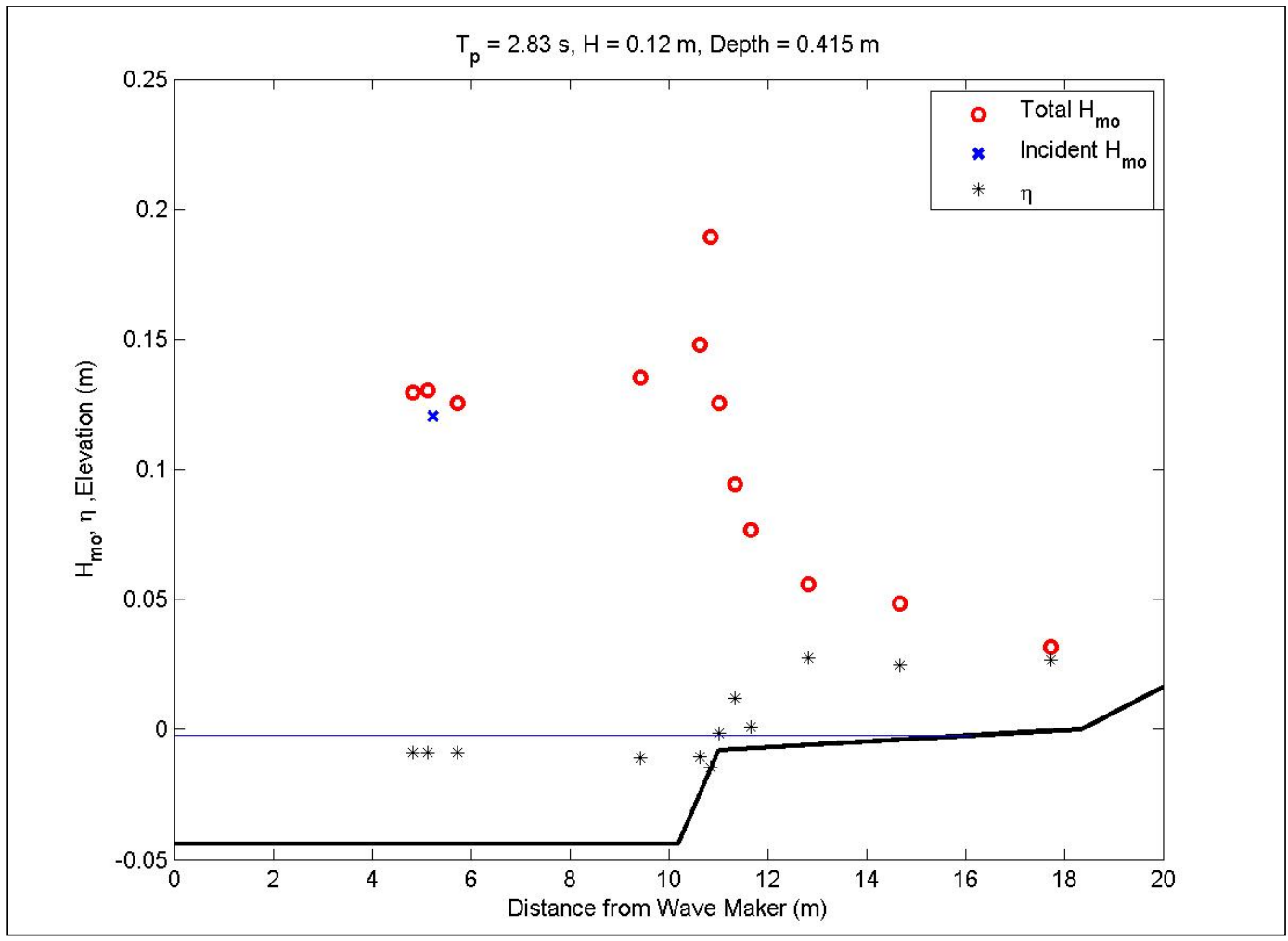

Figure D13. Wave transformation for 2.8-sec, $0.12 \mathrm{~m}$ waves at low water with a 1:25 reef slope. 


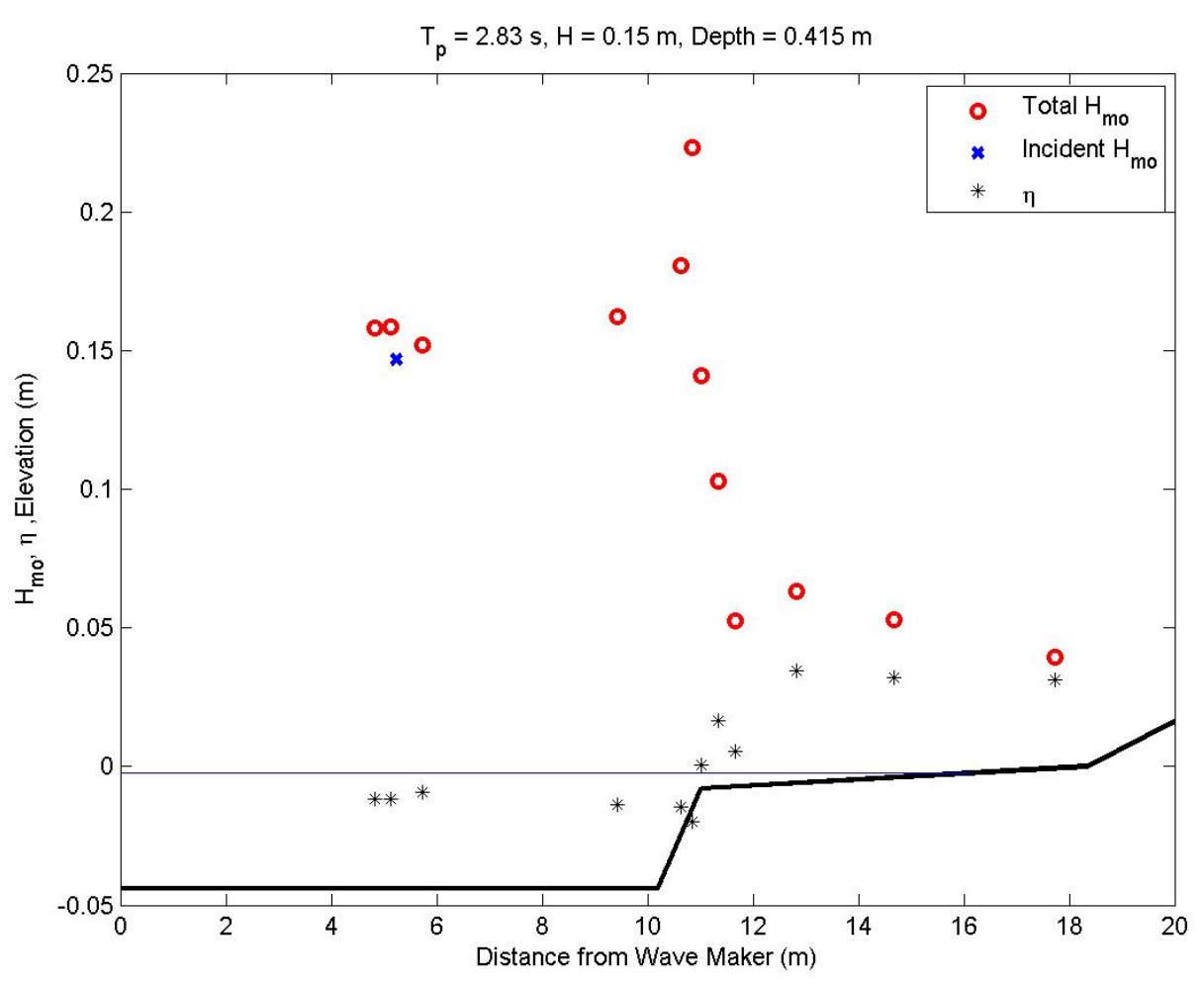

Figure D14. Wave transformation for 2.8-sec, $0.15 \mathrm{~m}$ waves at low water with a 1:25 reef slope.

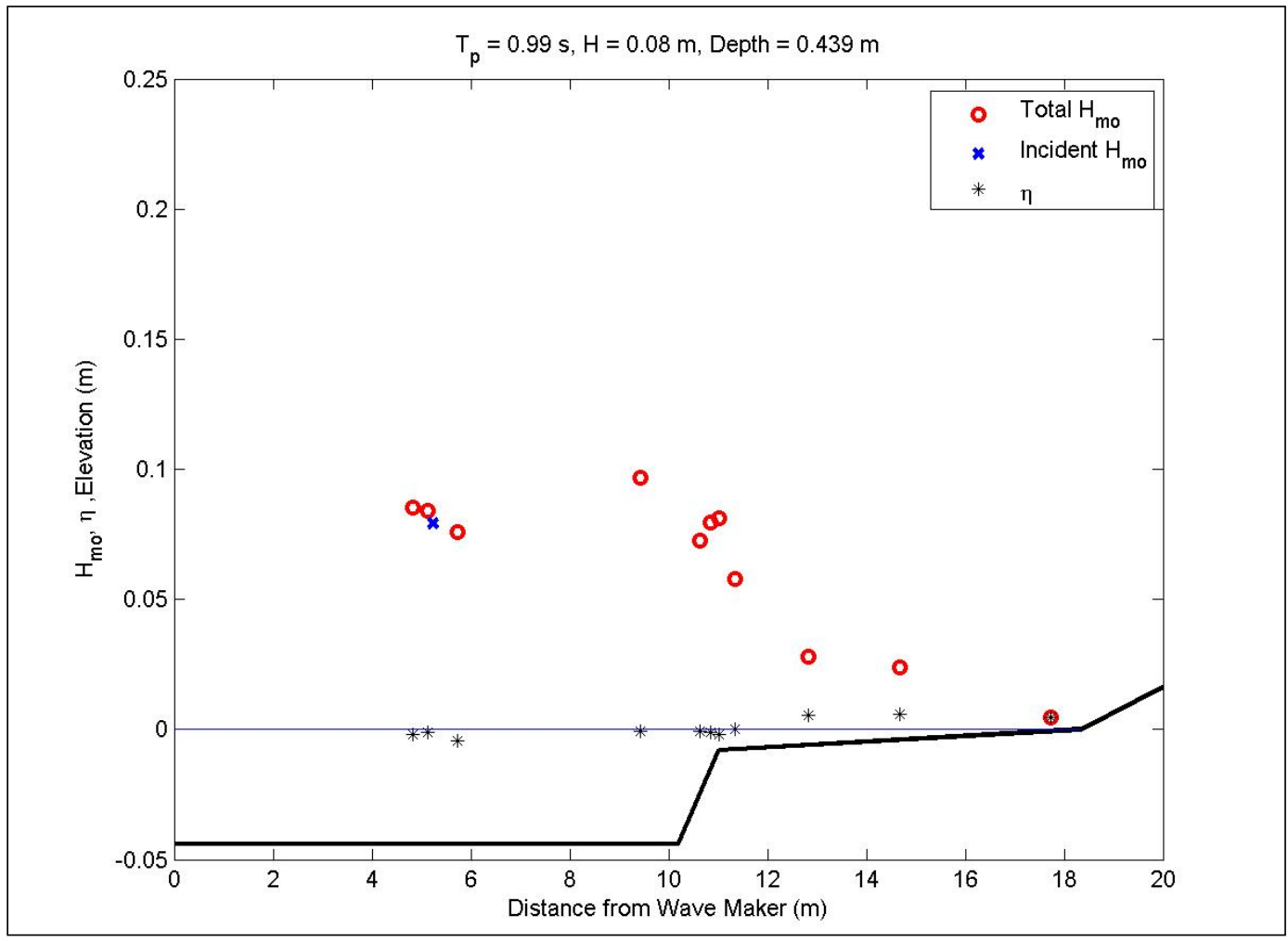

Figure D15. Wave transformation for 1-sec, $0.08 \mathrm{~m}$ waves at mid water with a 1:25 reef slope. 


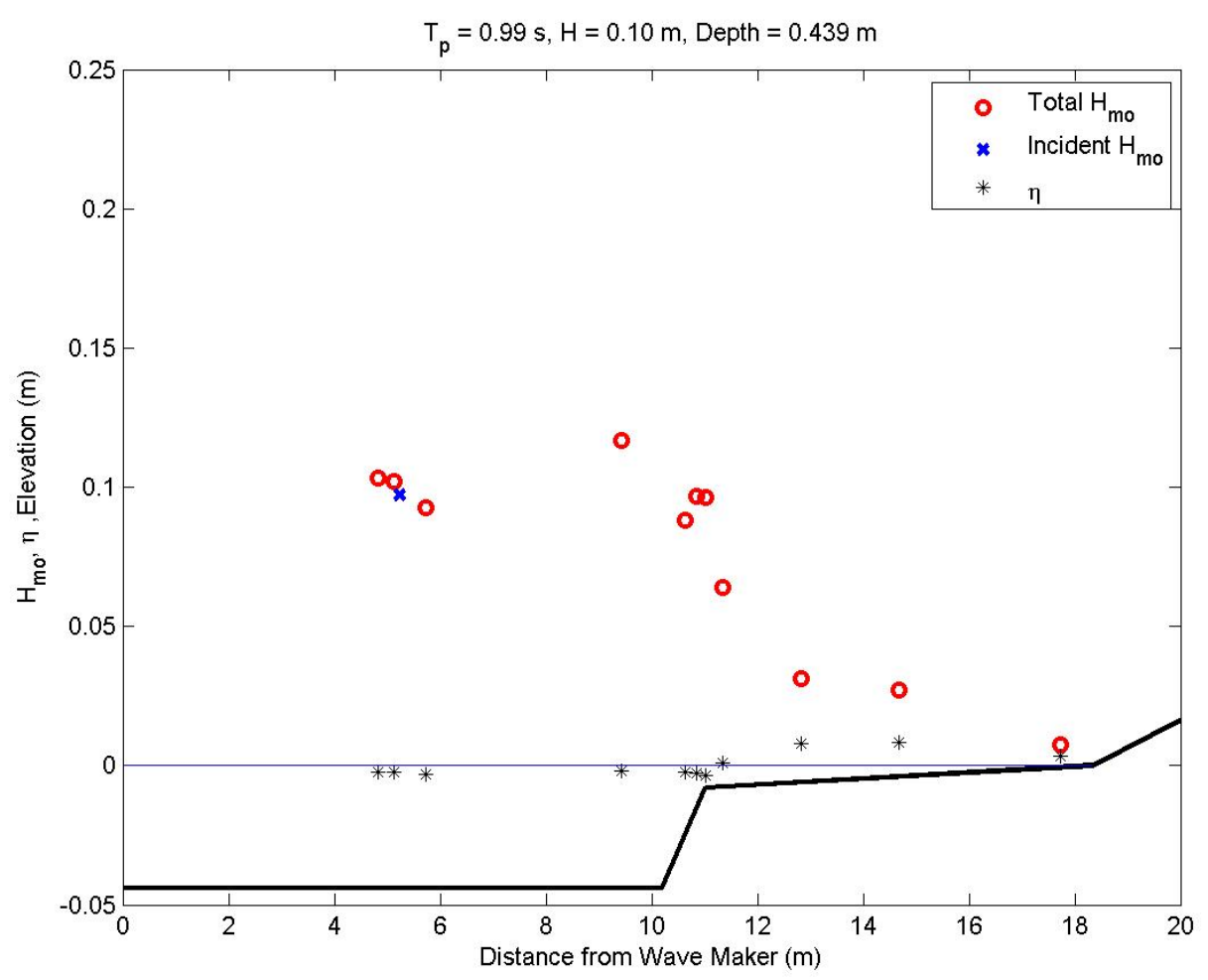

Figure D16. Wave transformation for 1-sec, $0.1 \mathrm{~m}$ waves at mid water with a 1:25 reef slope.

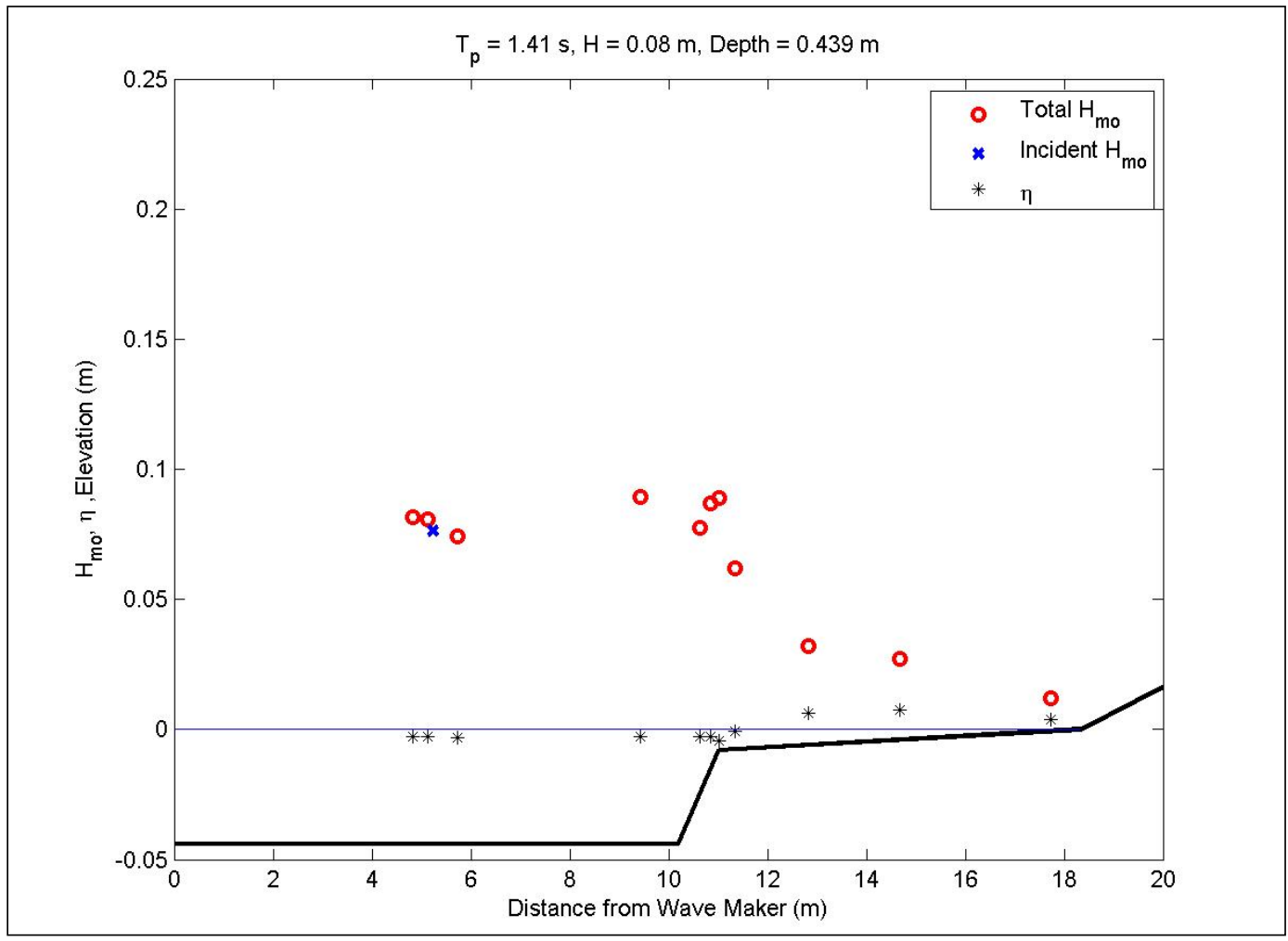

Figure D17. Wave transformation for 1.4-sec, $0.08 \mathrm{~m}$ waves at mid water with a 1:25 reef slope. 


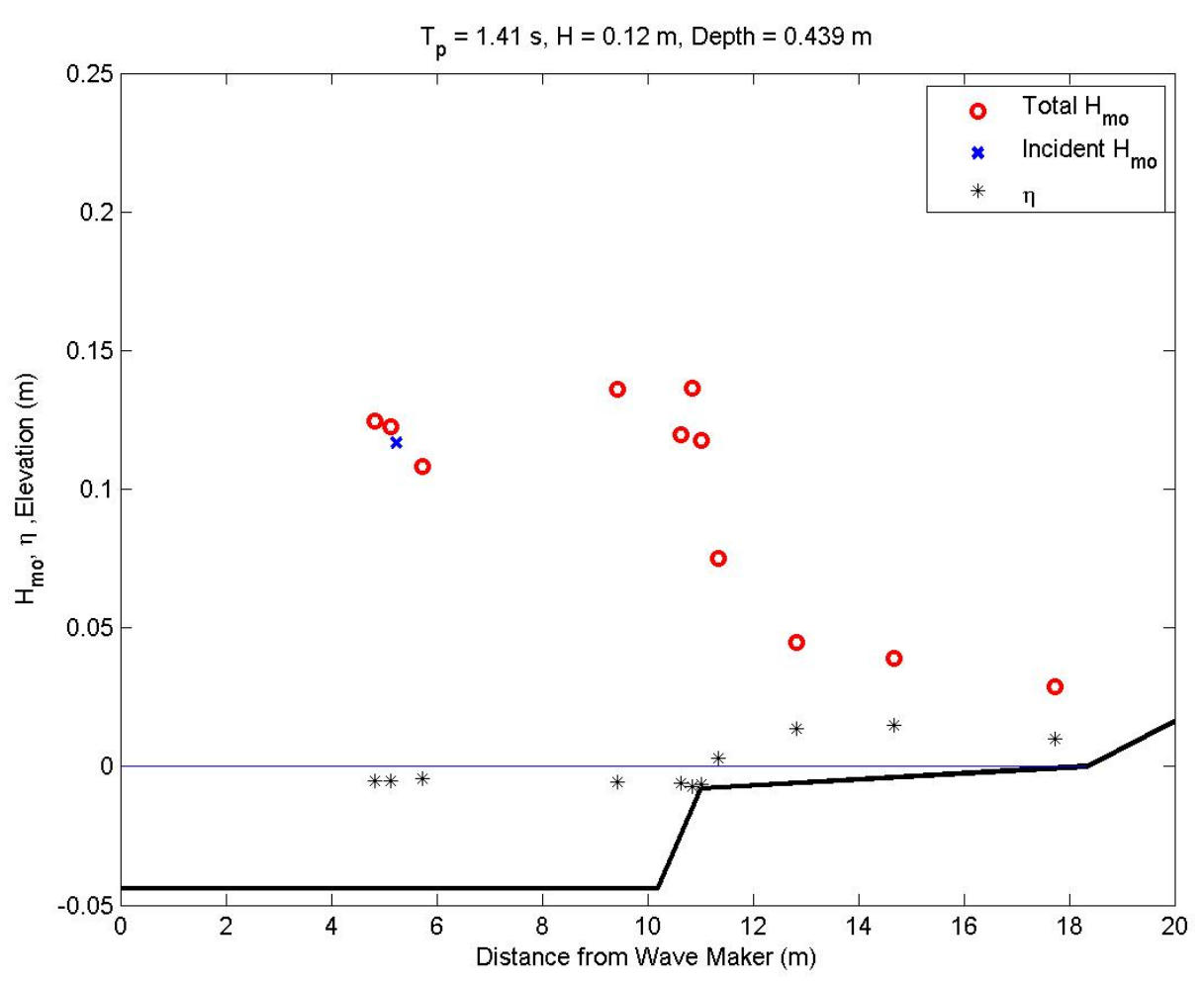

Figure D18. Wave transformation for $1-\mathrm{sec}, 0.08 \mathrm{~m}$ waves at mid water with a 1:25 reef slope.

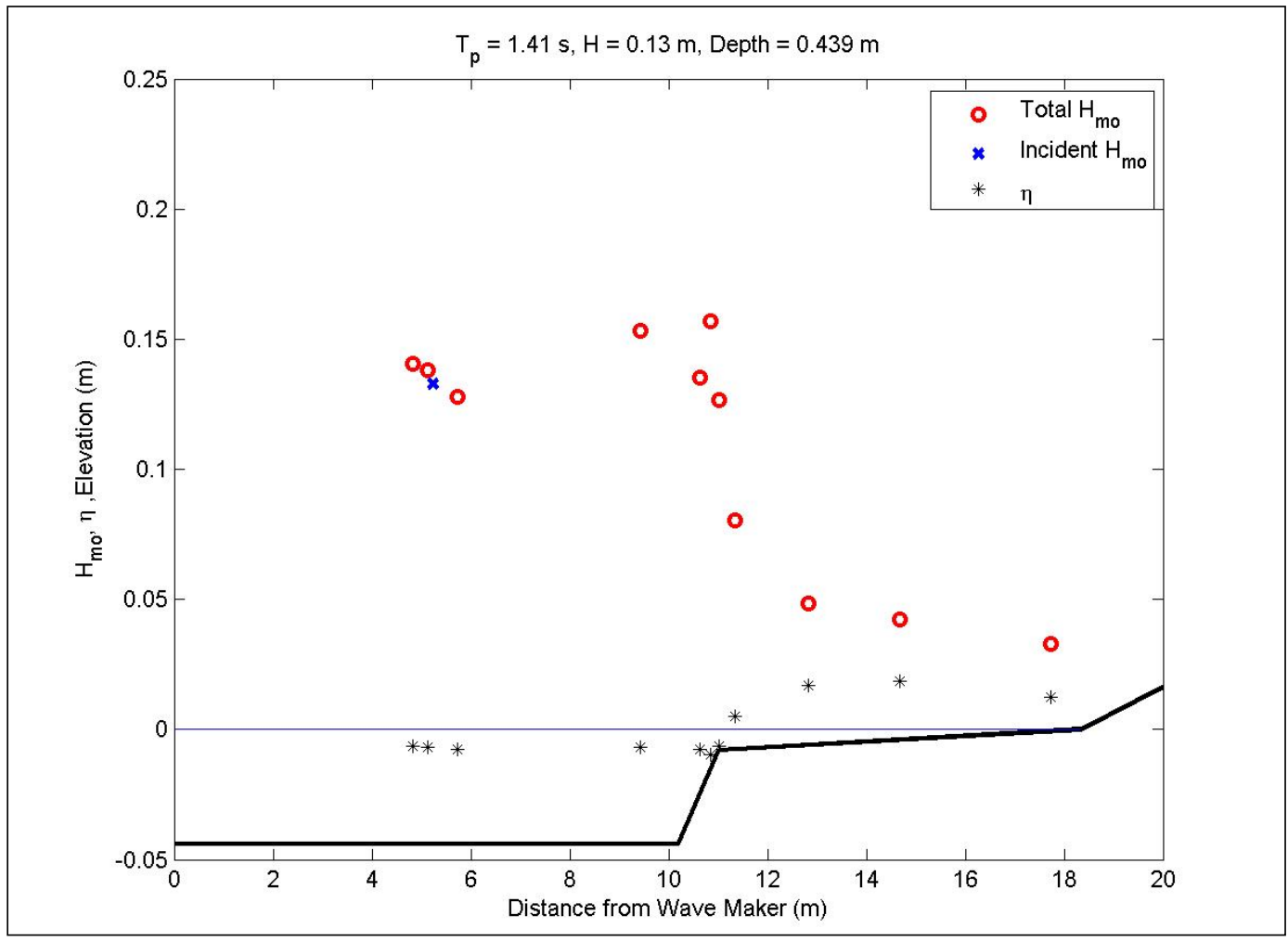

Figure D19. Wave transformation for 1.4-sec, $0.13 \mathrm{~m}$ waves at mid water with a 1:25 reef slope. 


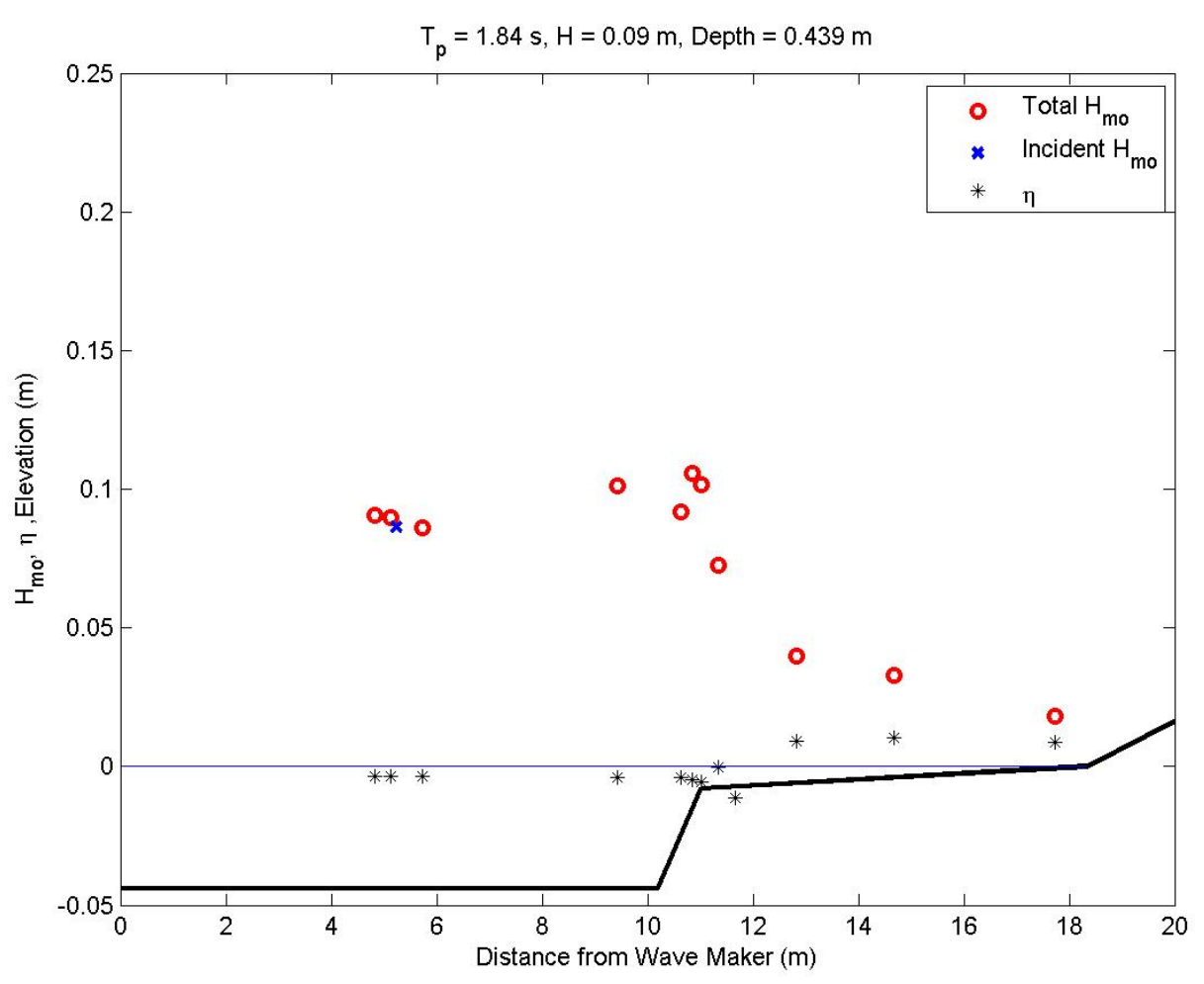

Figure D20. Wave transformation for 1.8-sec, $0.09 \mathrm{~m}$ waves at mid water with a 1:25 reef slope.

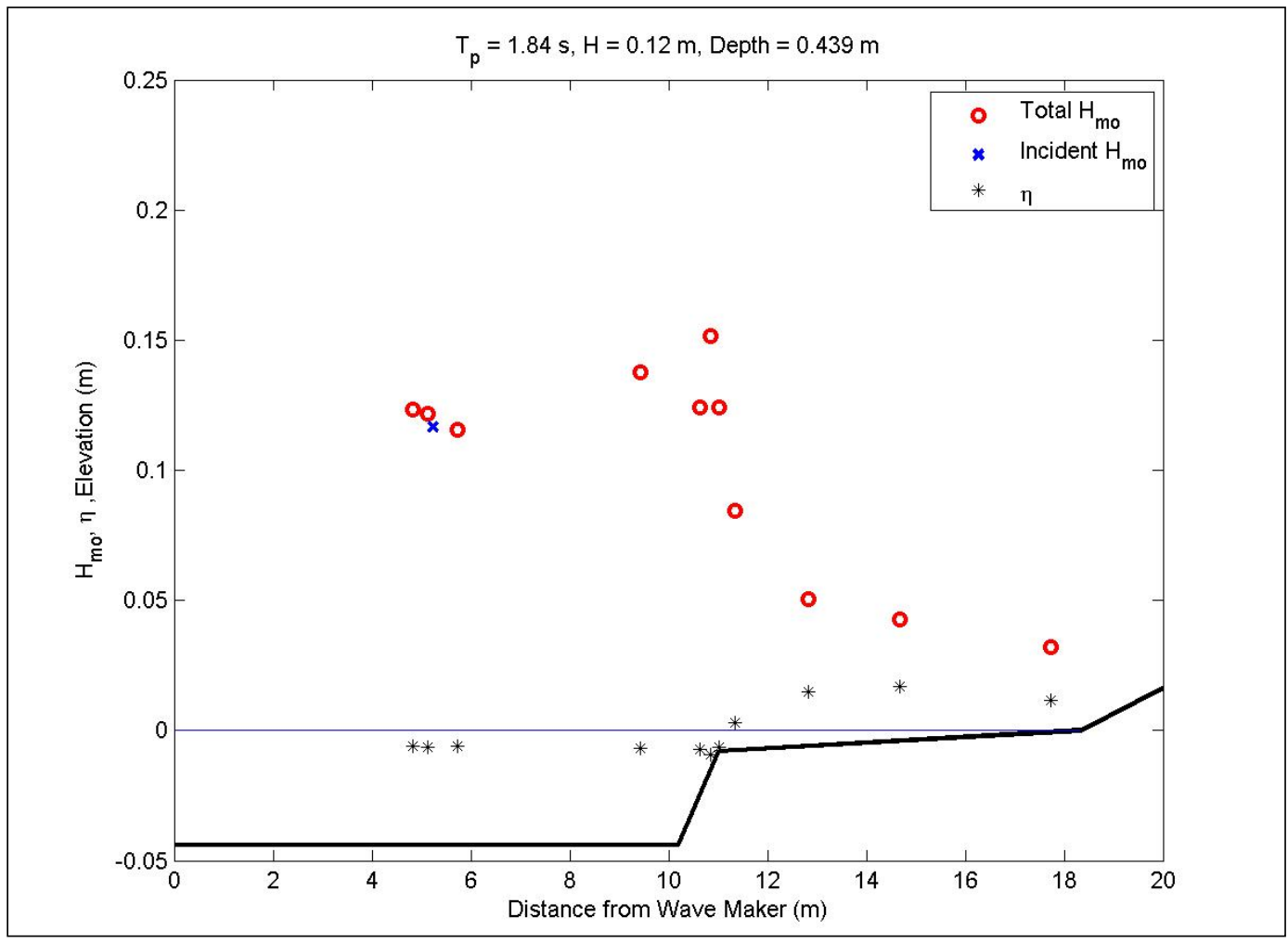

Figure D21. Wave transformation for 1.8-sec, $0.12 \mathrm{~m}$ waves at mid water with a 1:25 reef slope. 


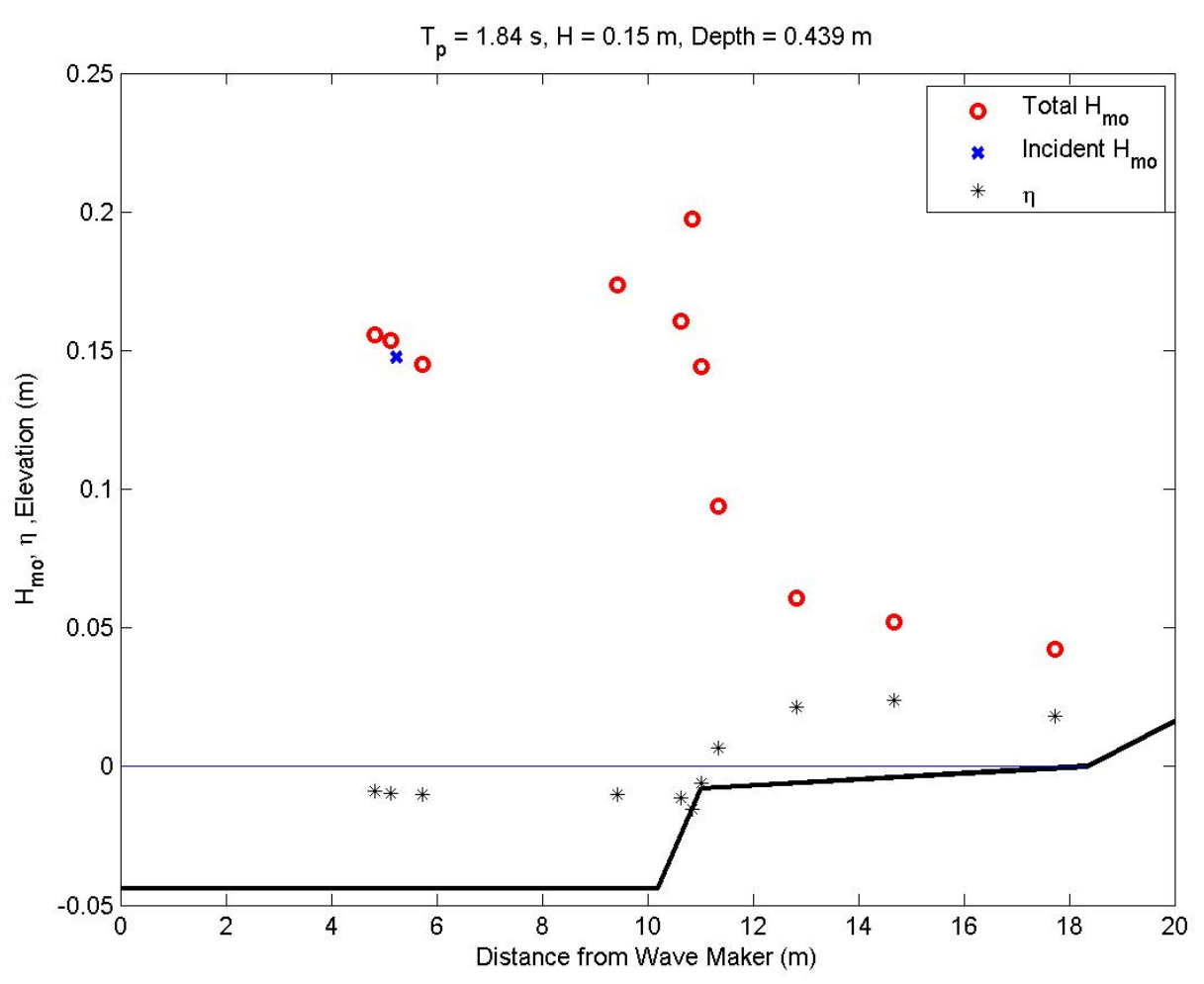

Figure D22. Wave transformation for $1.8-\mathrm{sec}, 0.15 \mathrm{~m}$ waves at mid water with a 1:25 reef slope.

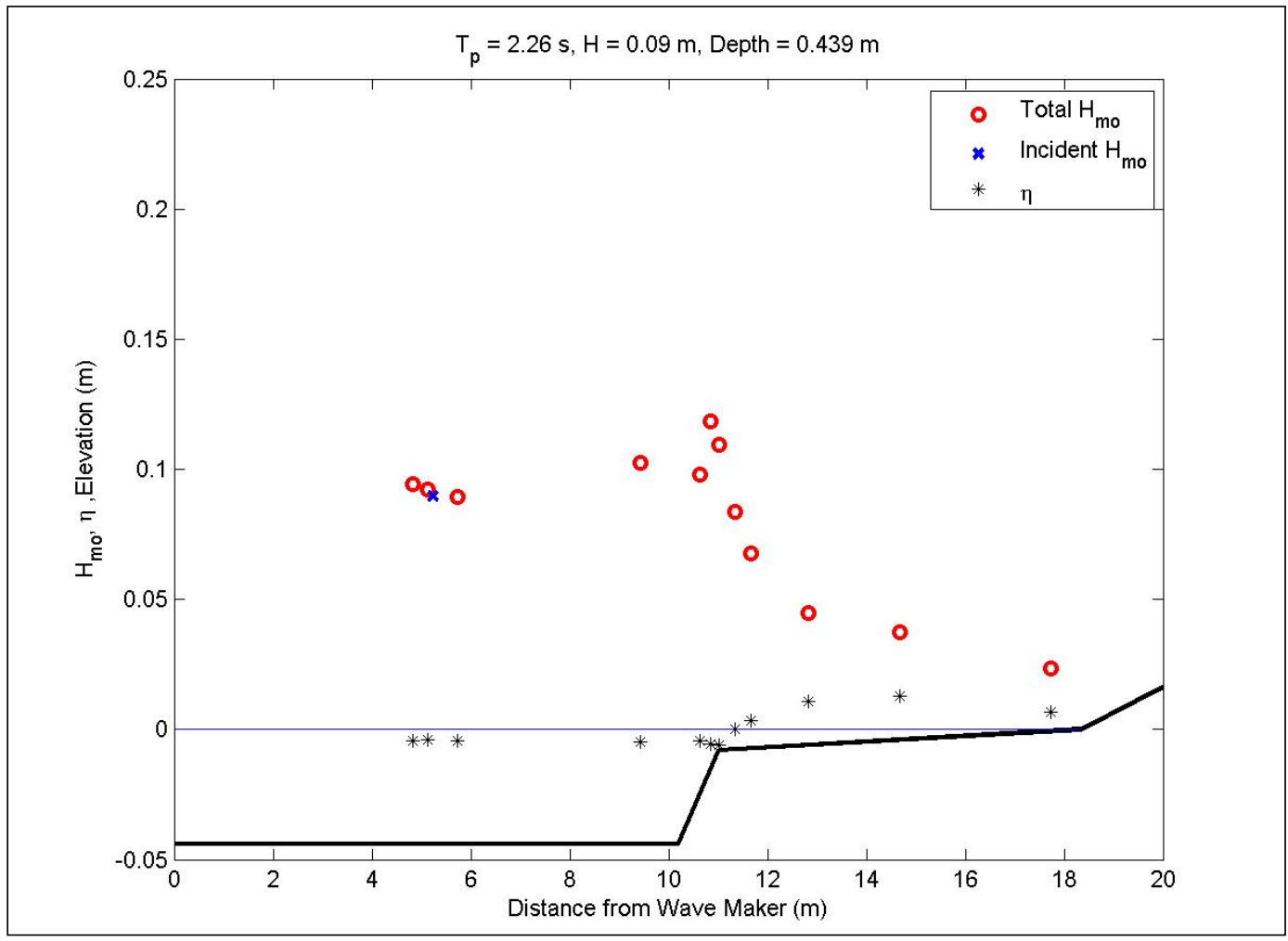

Figure D23. Wave transformation for 2.3-sec, $0.09 \mathrm{~m}$ waves at mid water with a 1:25 reef slope. 


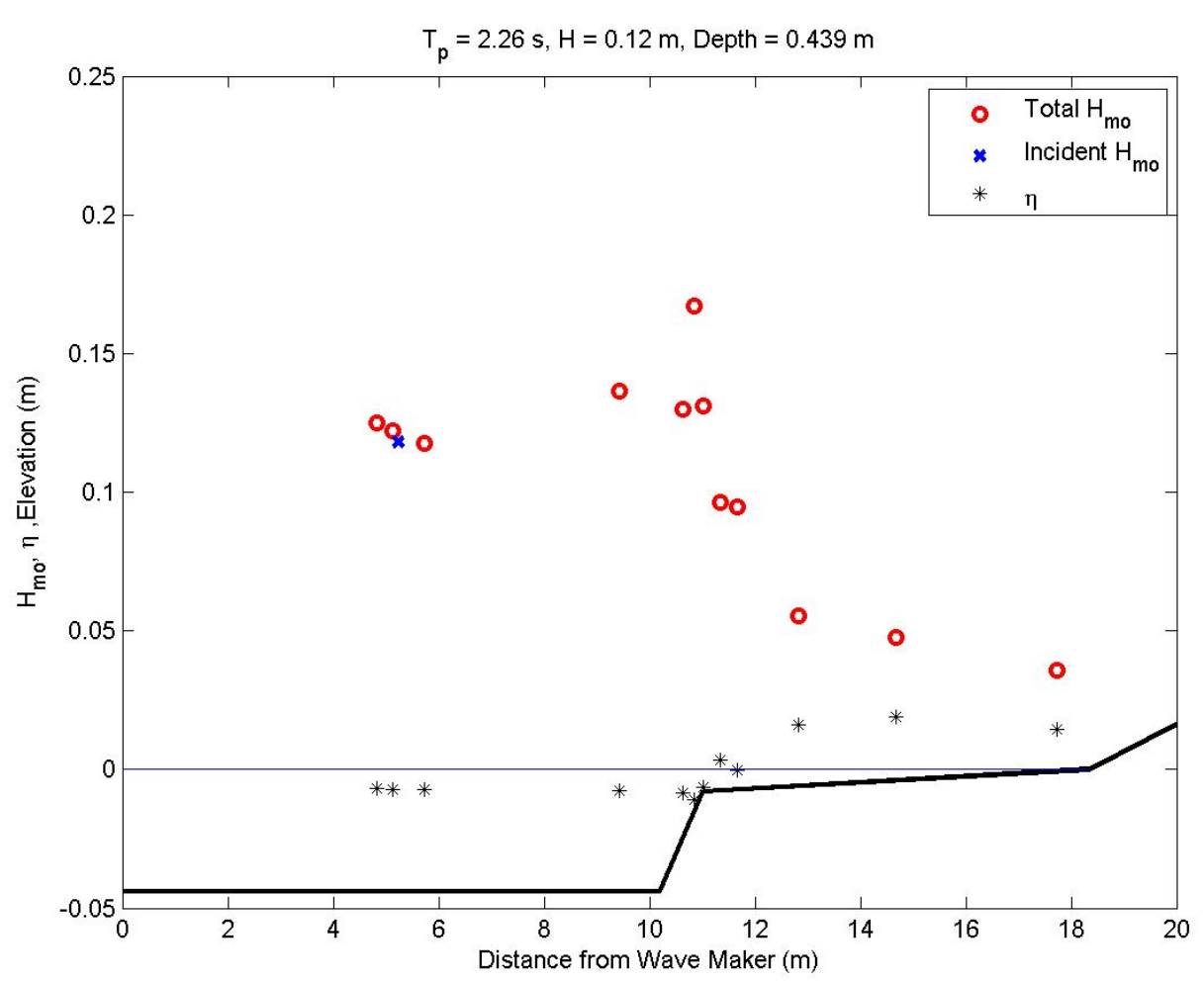

Figure D24. Wave transformation for 2.3-sec, $0.12 \mathrm{~m}$ waves at mid water with a 1:25 reef slope.

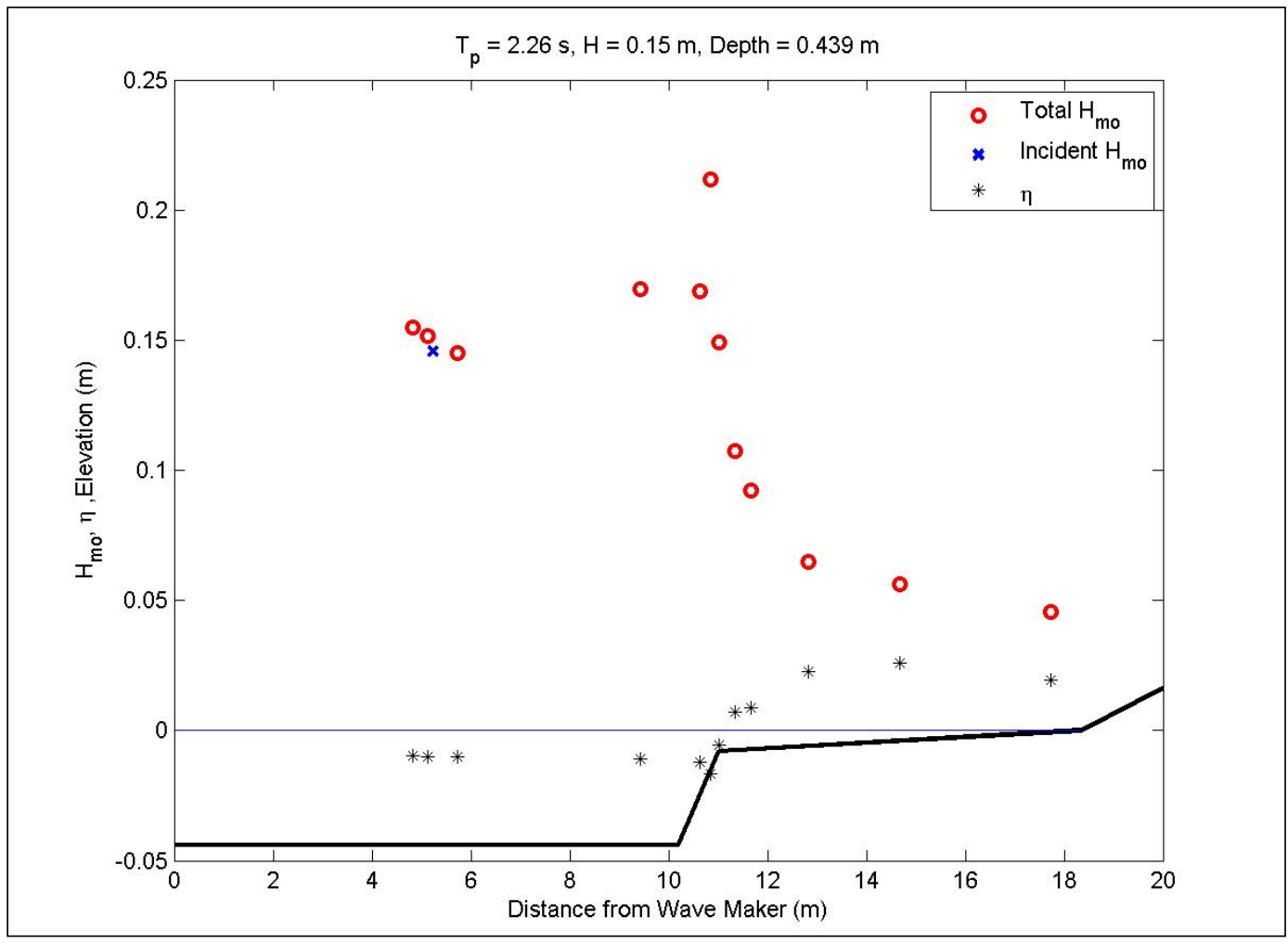

Figure D25. Wave transformation for 2.3-sec, $0.15 \mathrm{~m}$ waves at mid water with a 1:25 reef slope. 


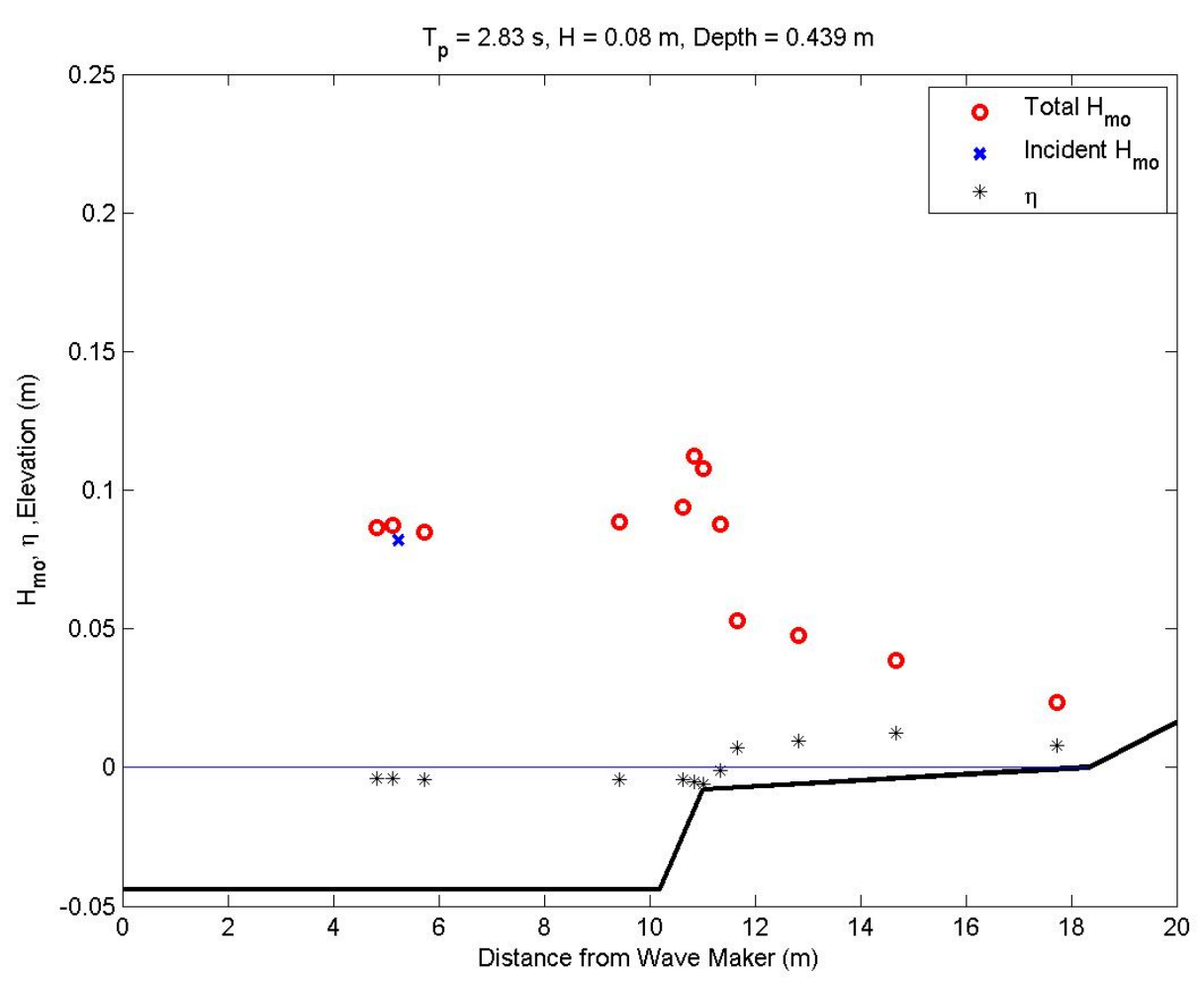

Figure D26. Wave transformation for $2.8-\mathrm{sec}, 0.08 \mathrm{~m}$ waves at mid water with a 1:25 reef slope.

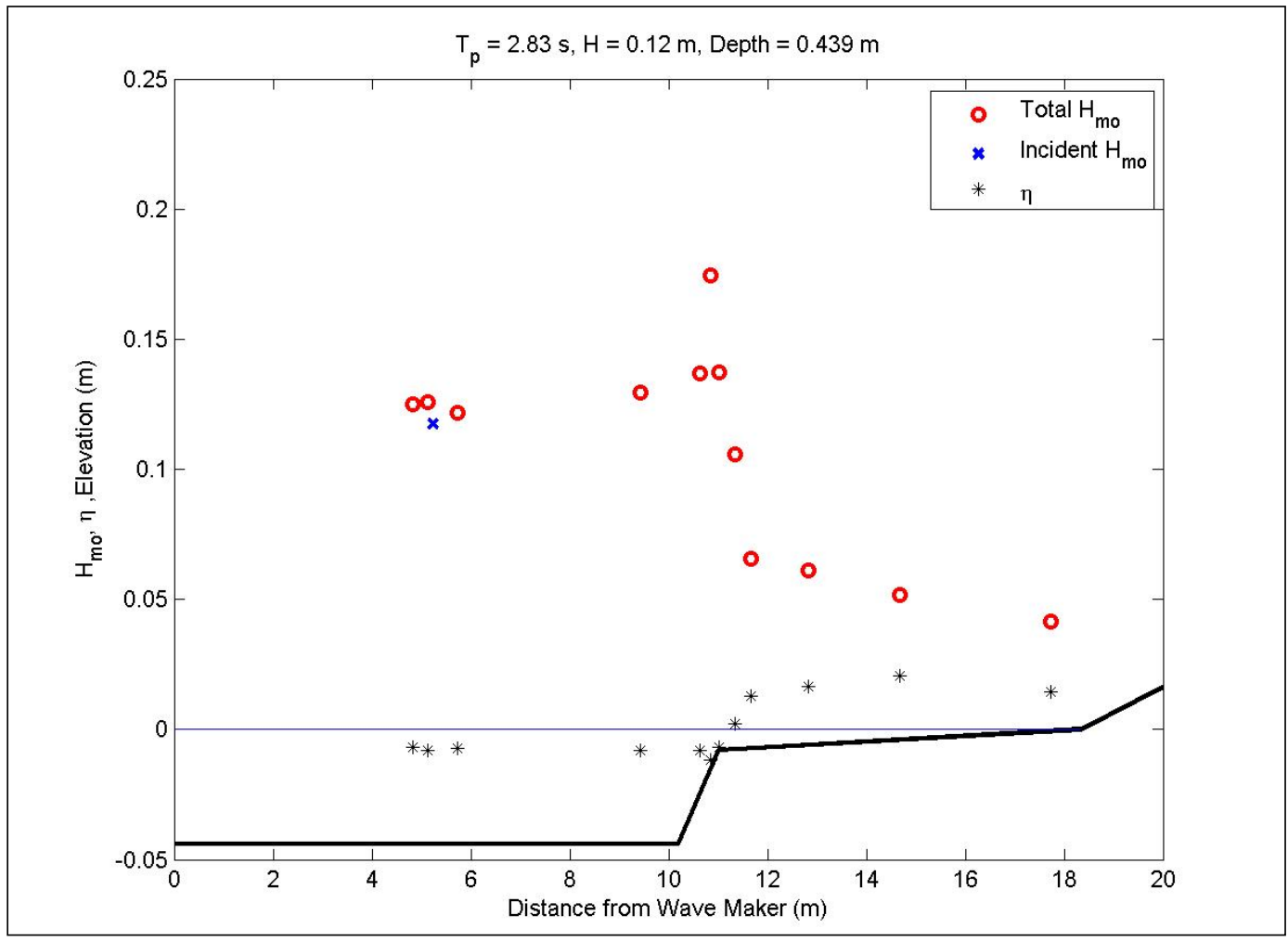

Figure D27. Wave transformation for 2.8-sec, $0.12 \mathrm{~m}$ waves at mid water with a 1:25 reef slope. 


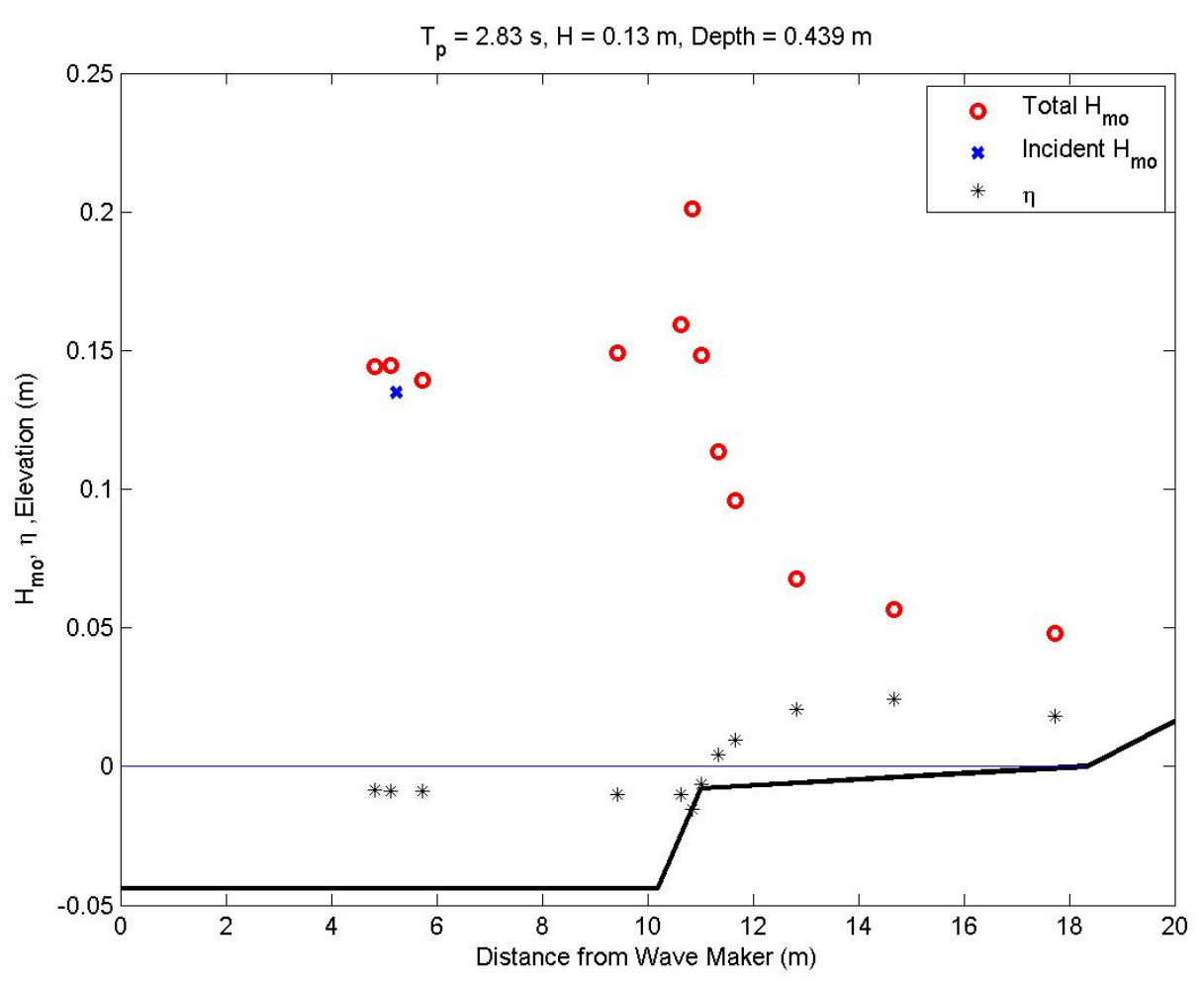

Figure D28. Wave transformation for $2.8-\mathrm{sec}, 0.13 \mathrm{~m}$ waves at mid water with a 1:25 reef slope.

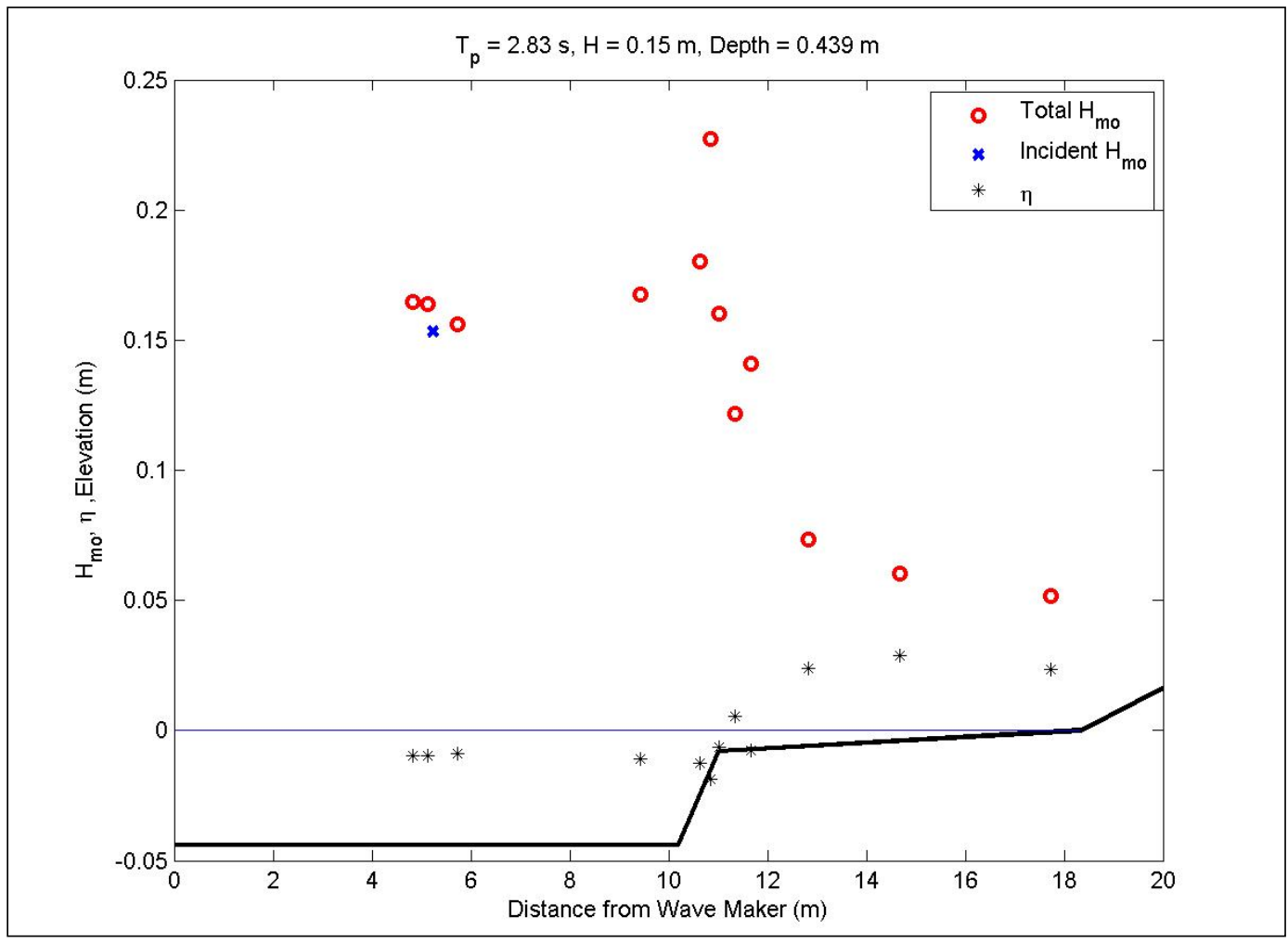

Figure D29. Wave transformation for 2.8-sec, $0.15 \mathrm{~m}$ waves at mid water with a 1:25 reef slope. 


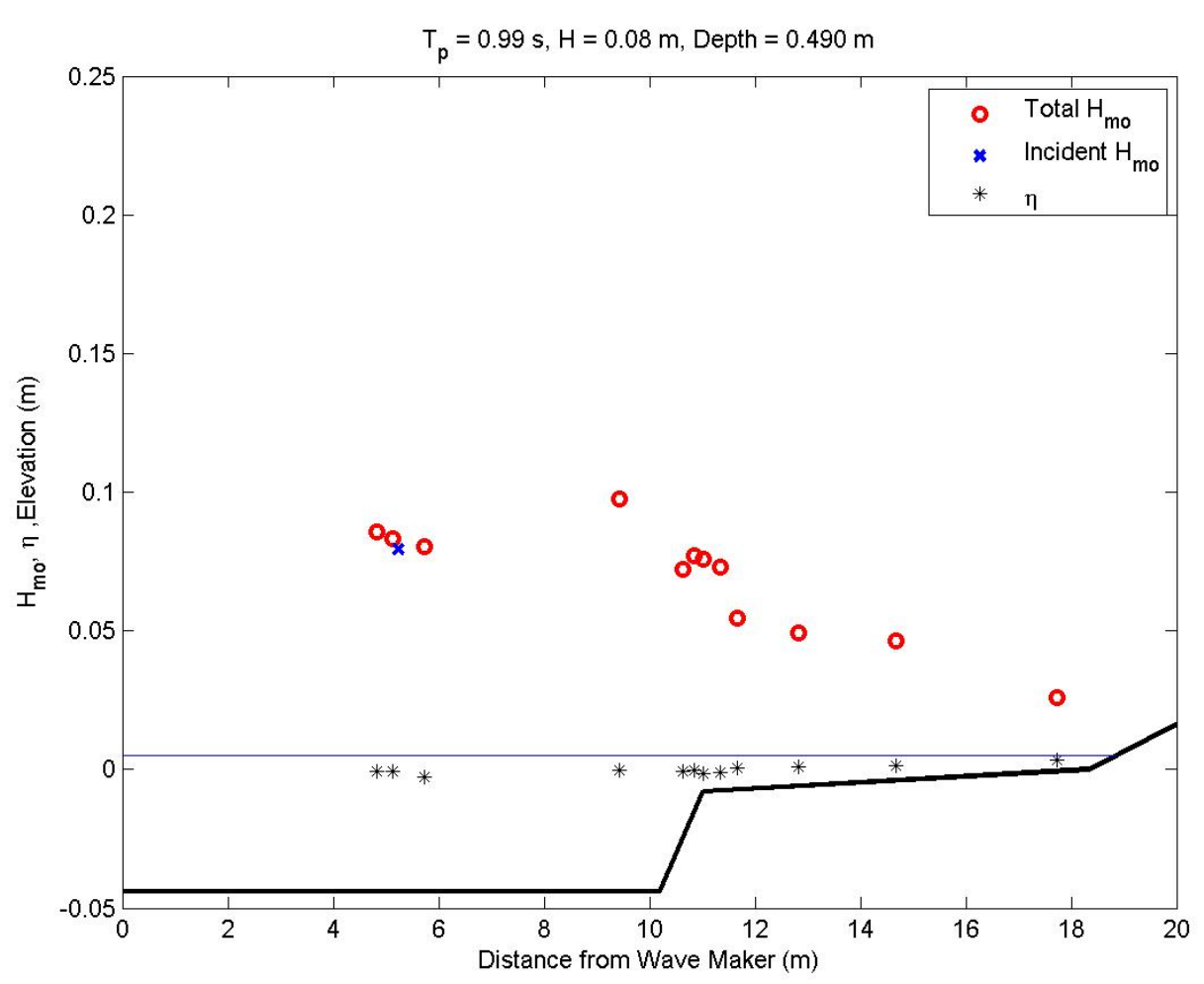

Figure D30. Wave transformation for 1-sec, $0.08 \mathrm{~m}$ waves at high water with a 1:25 reef slope.

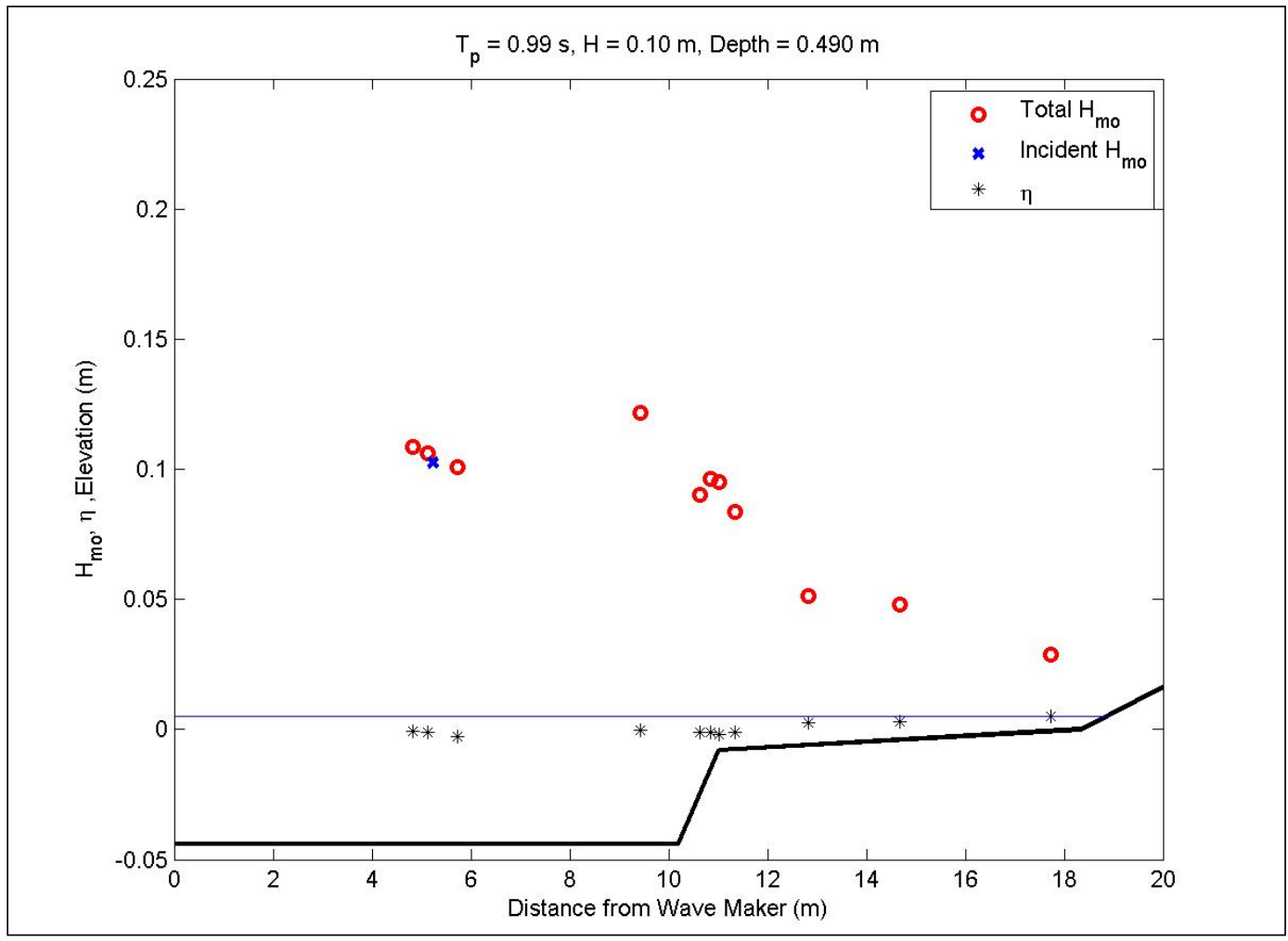

Figure D31. Wave transformation for 1-sec, $0.1 \mathrm{~m}$ waves at high water with a 1:25 reef slope. 


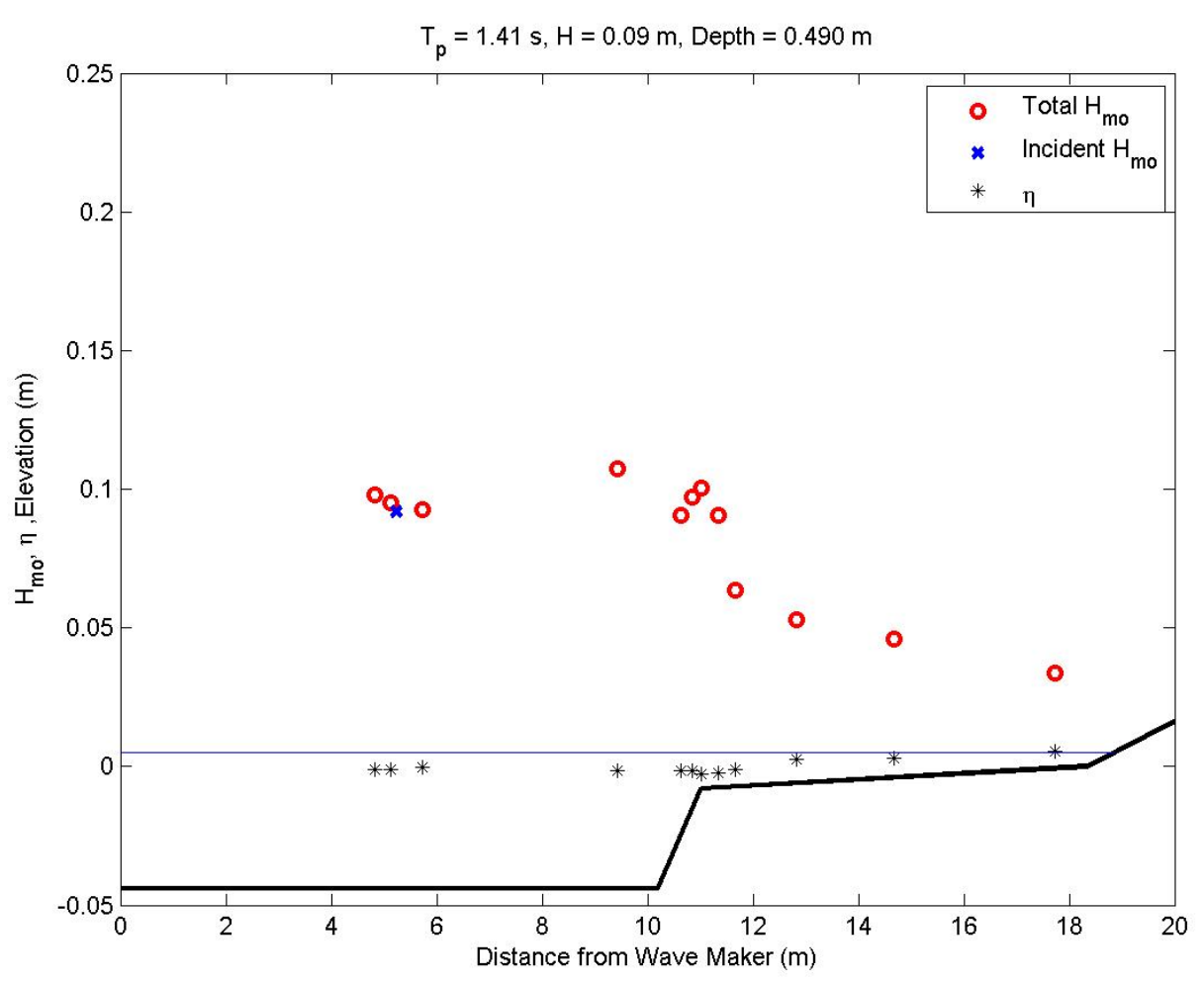

Figure D32. Wave transformation for 1.41-sec, $0.09 \mathrm{~m}$ waves at high water with a 1:25 reef slope.

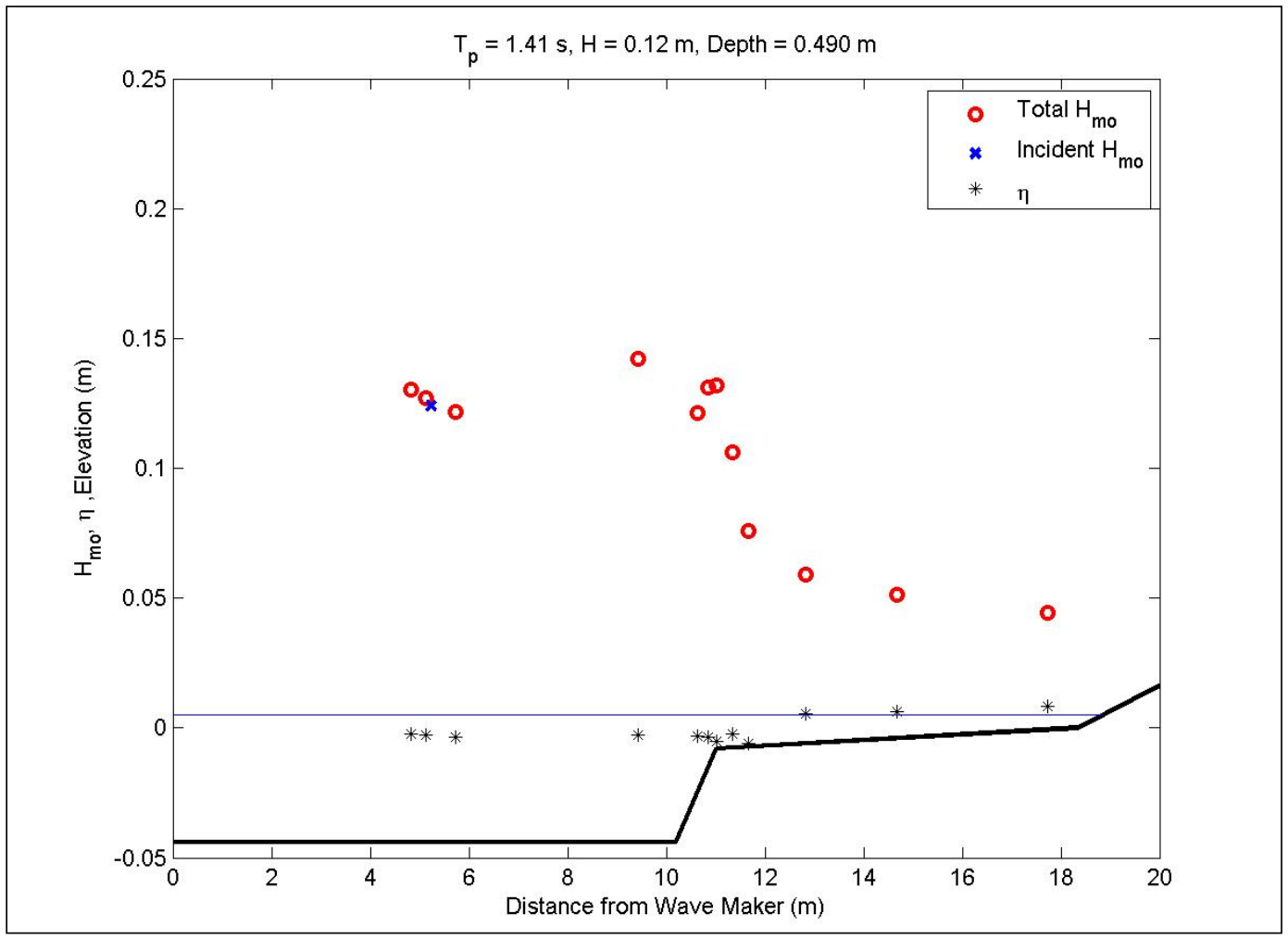

Figure D33. Wave transformation for 1.4-sec, $0.12 \mathrm{~m}$ waves at high water with a 1:25 reef slope. 


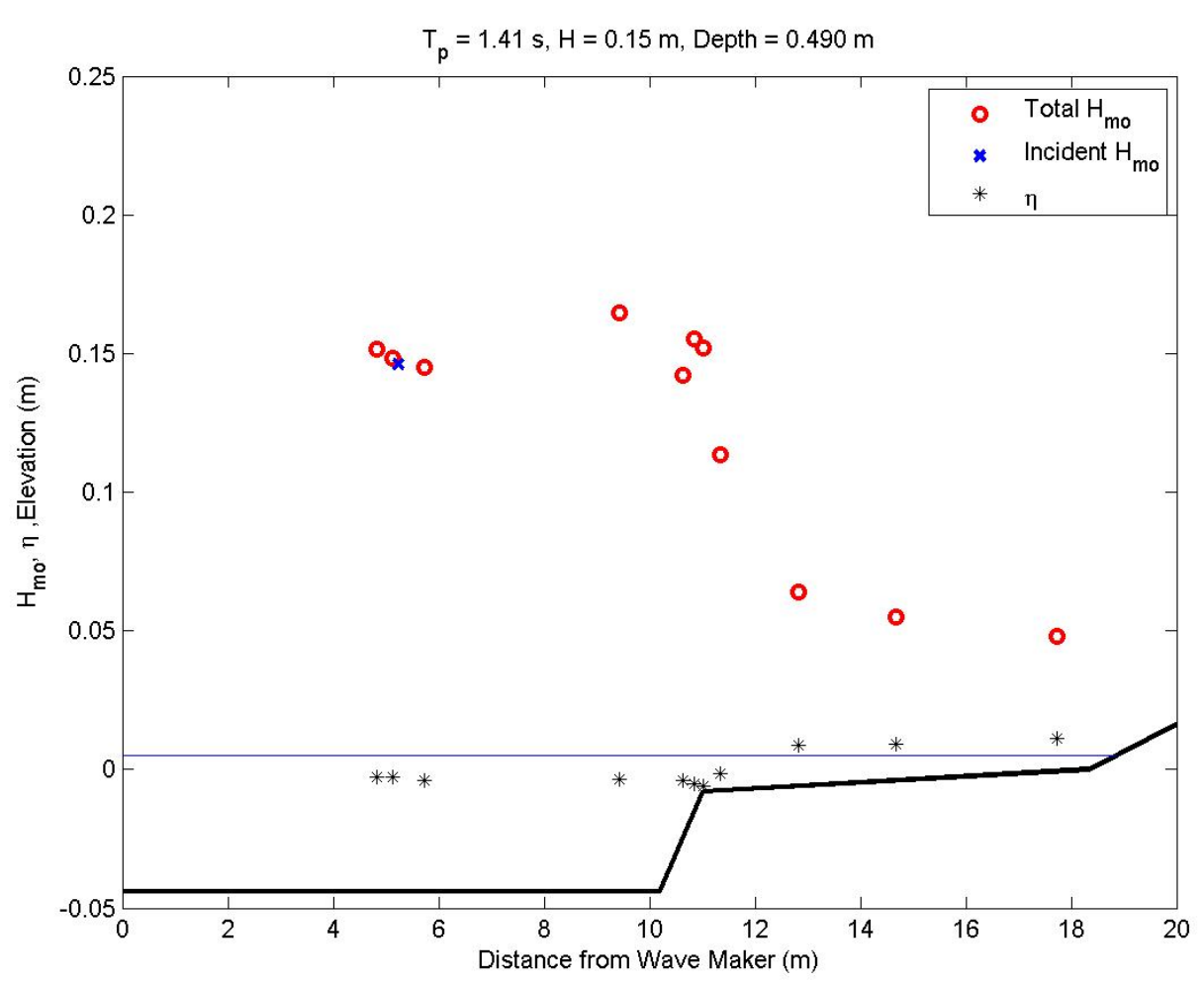

Figure D34. Wave transformation for 1.4-sec, $0.15 \mathrm{~m}$ waves at high water with a 1:25 reef slope.

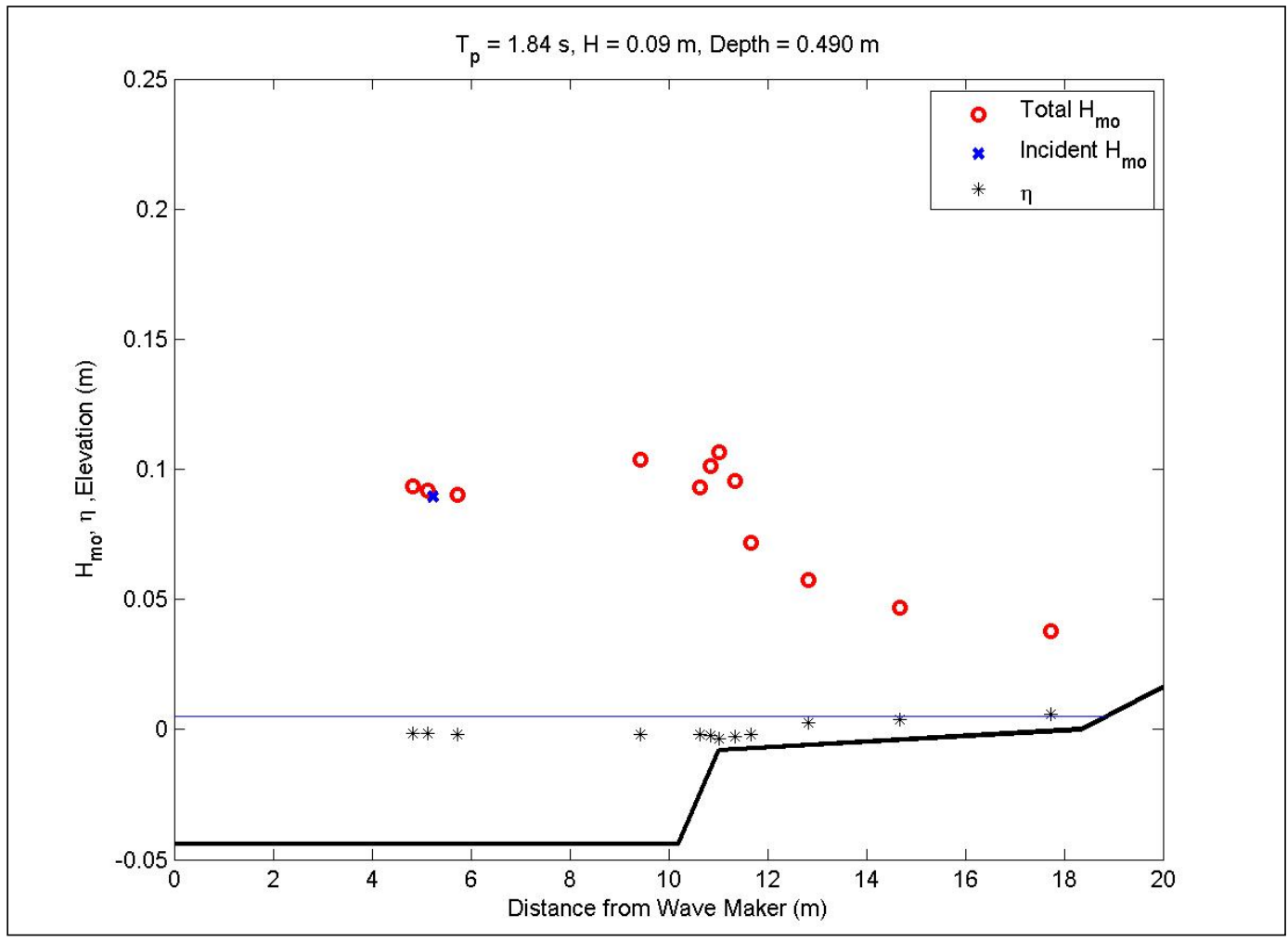

Figure D35. Wave transformation for 1.8-sec, $0.09 \mathrm{~m}$ waves at high water with a 1:25 reef slope. 


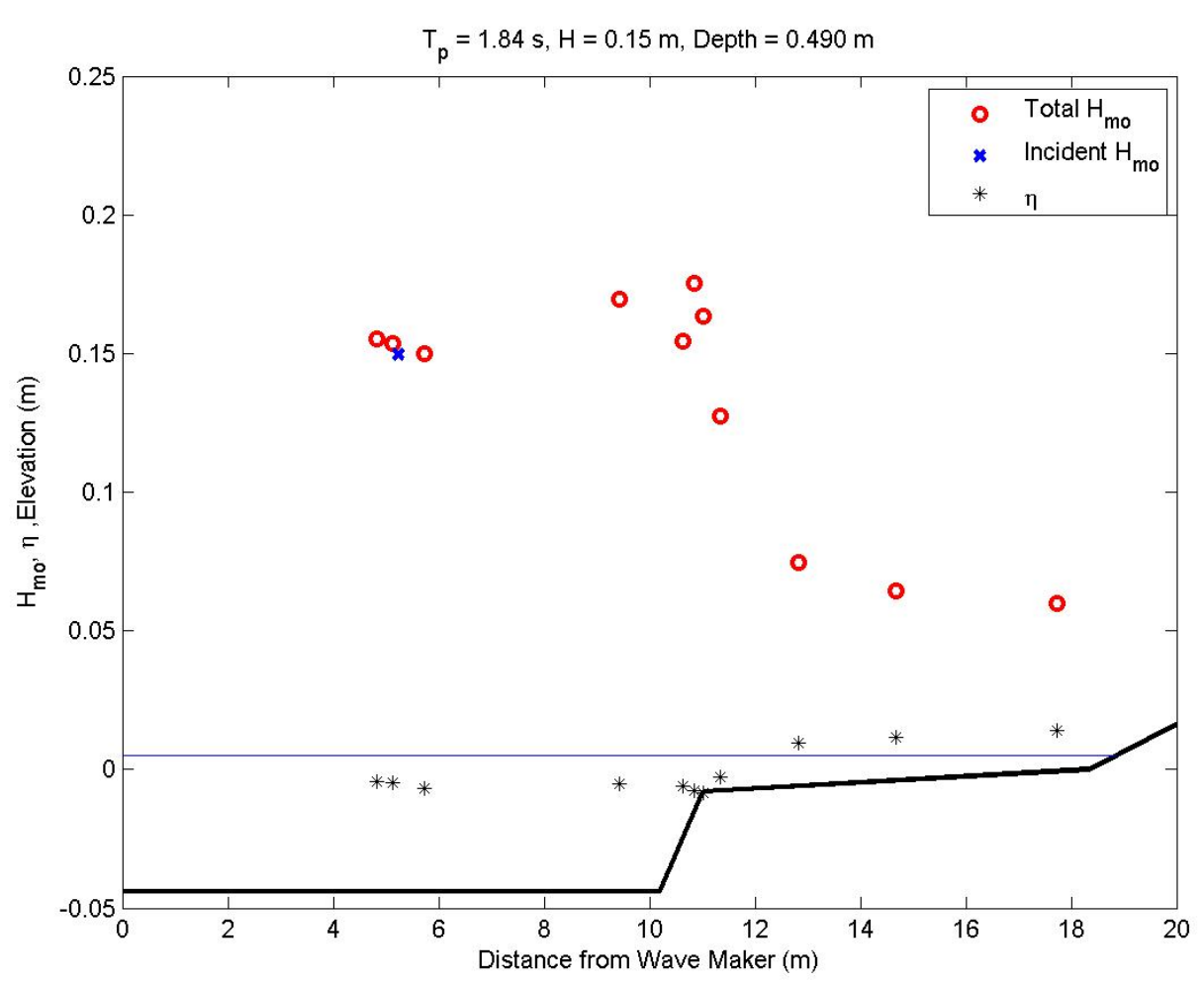

Figure D36. Wave transformation for 1.8-sec, $0.15 \mathrm{~m}$ waves at high water with a 1:25 reef slope.

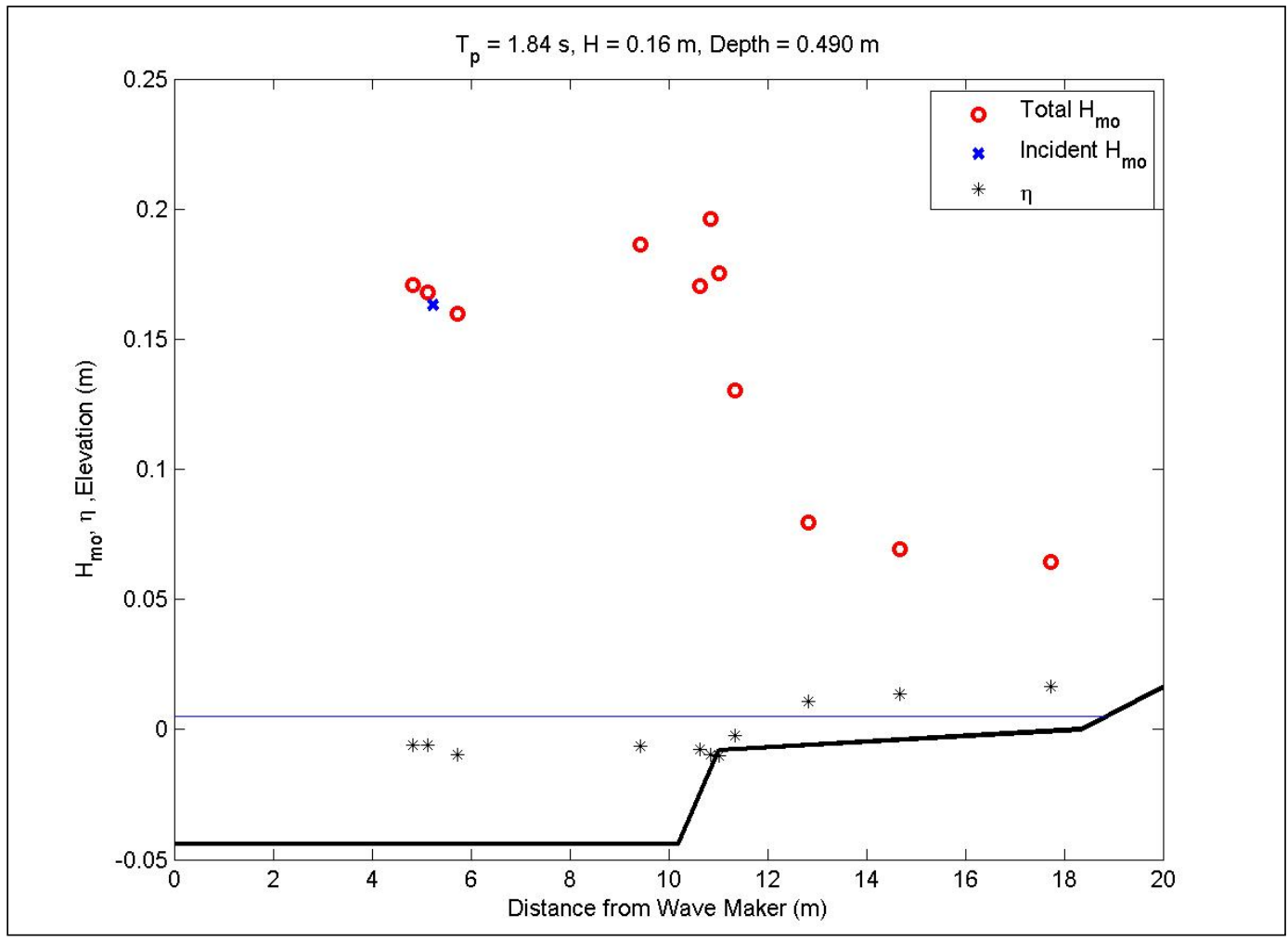

Figure D37 Wave transformation for 1.8-sec, $0.16 \mathrm{~m}$ waves at high water with a 1:25 reef slope. 


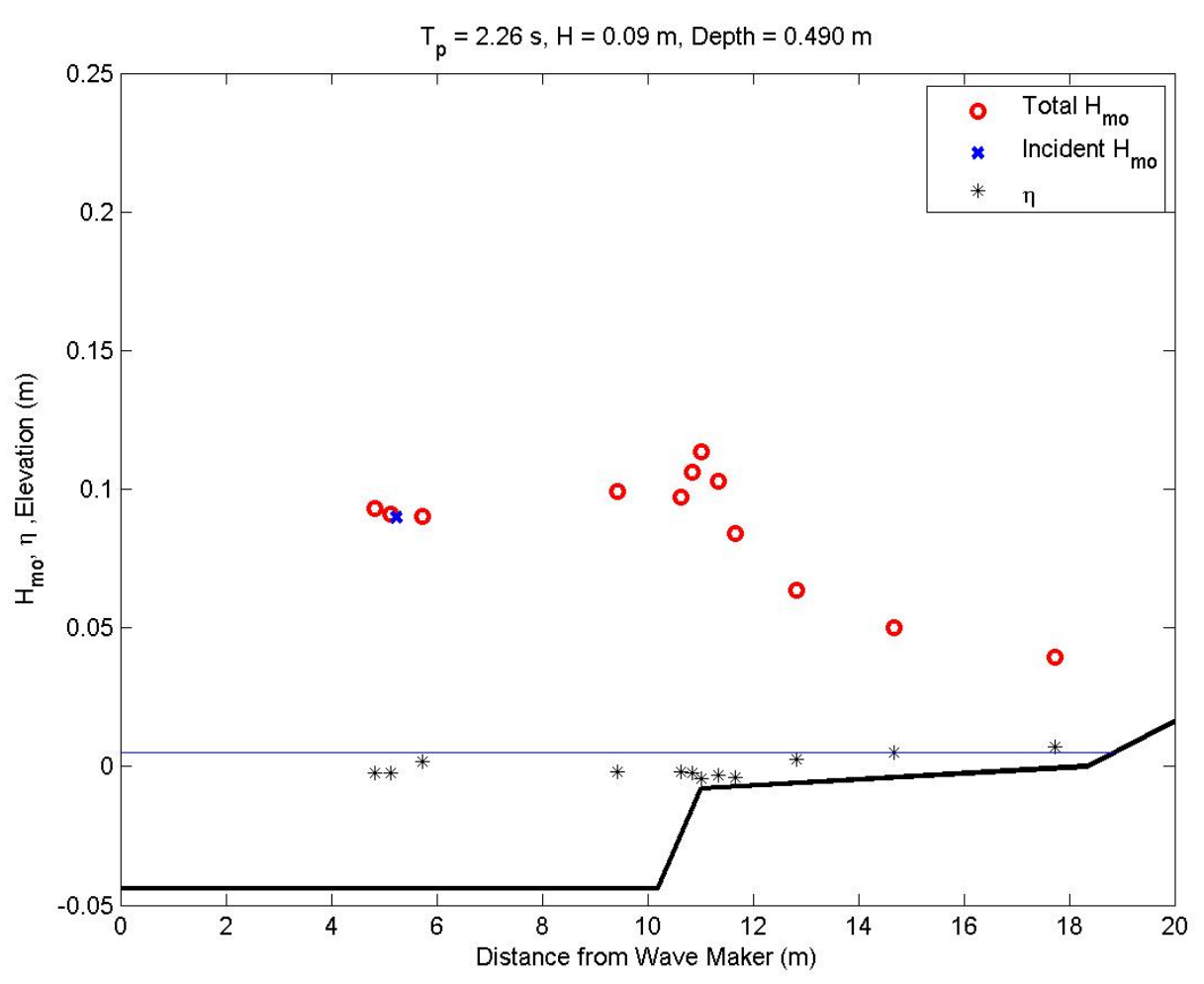

Figure D38. Wave transformation for 2.3-sec, $0.09 \mathrm{~m}$ waves at high water with a 1:25 reef slope.

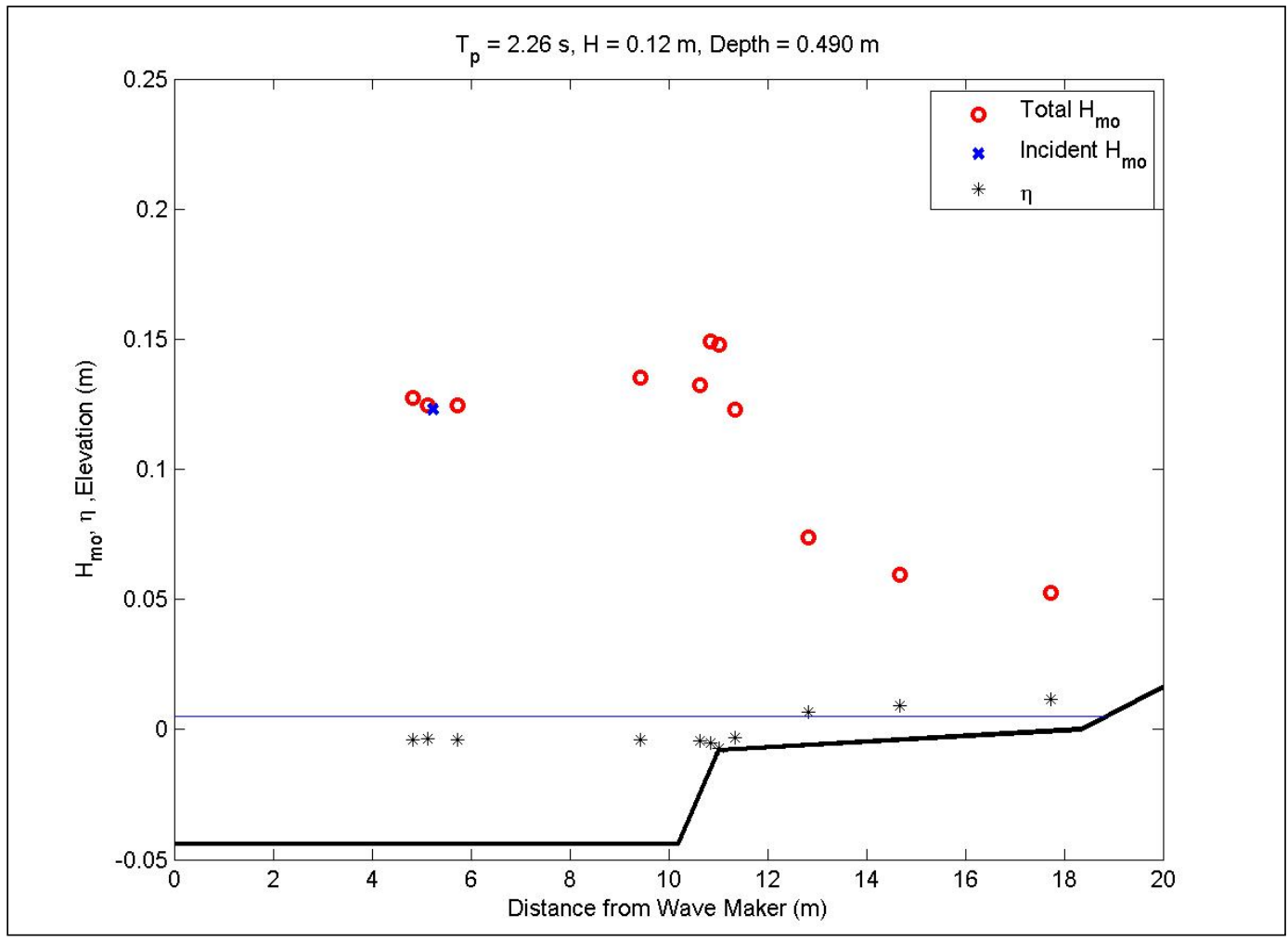

Figure D39. Wave transformation for 2.3-sec, $0.12 \mathrm{~m}$ waves at high water with a 1:25 reef slope. 


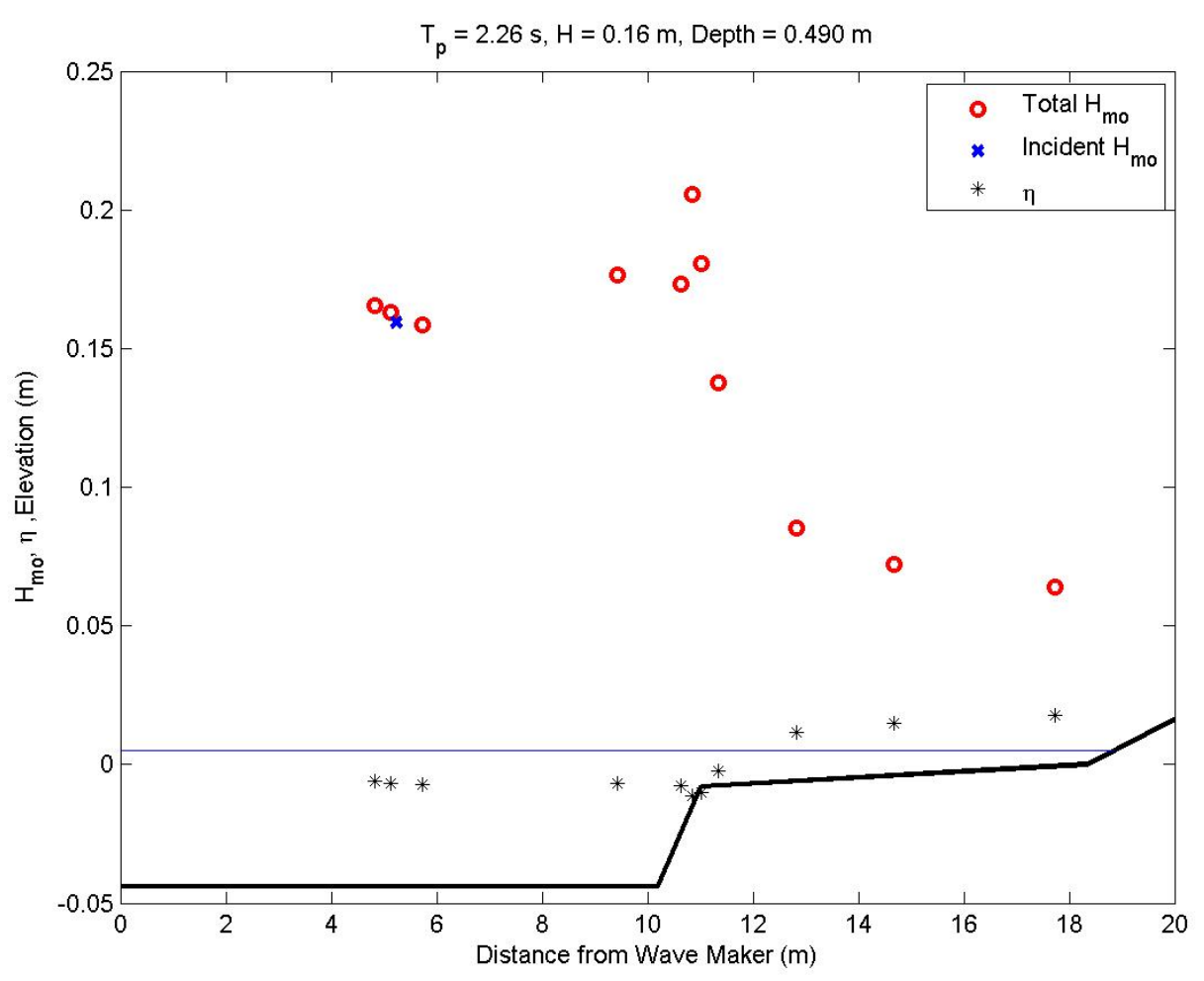

Figure D40. Wave transformation for 2.3-sec, $0.16 \mathrm{~m}$ waves at high water with a 1:25 reef slope.

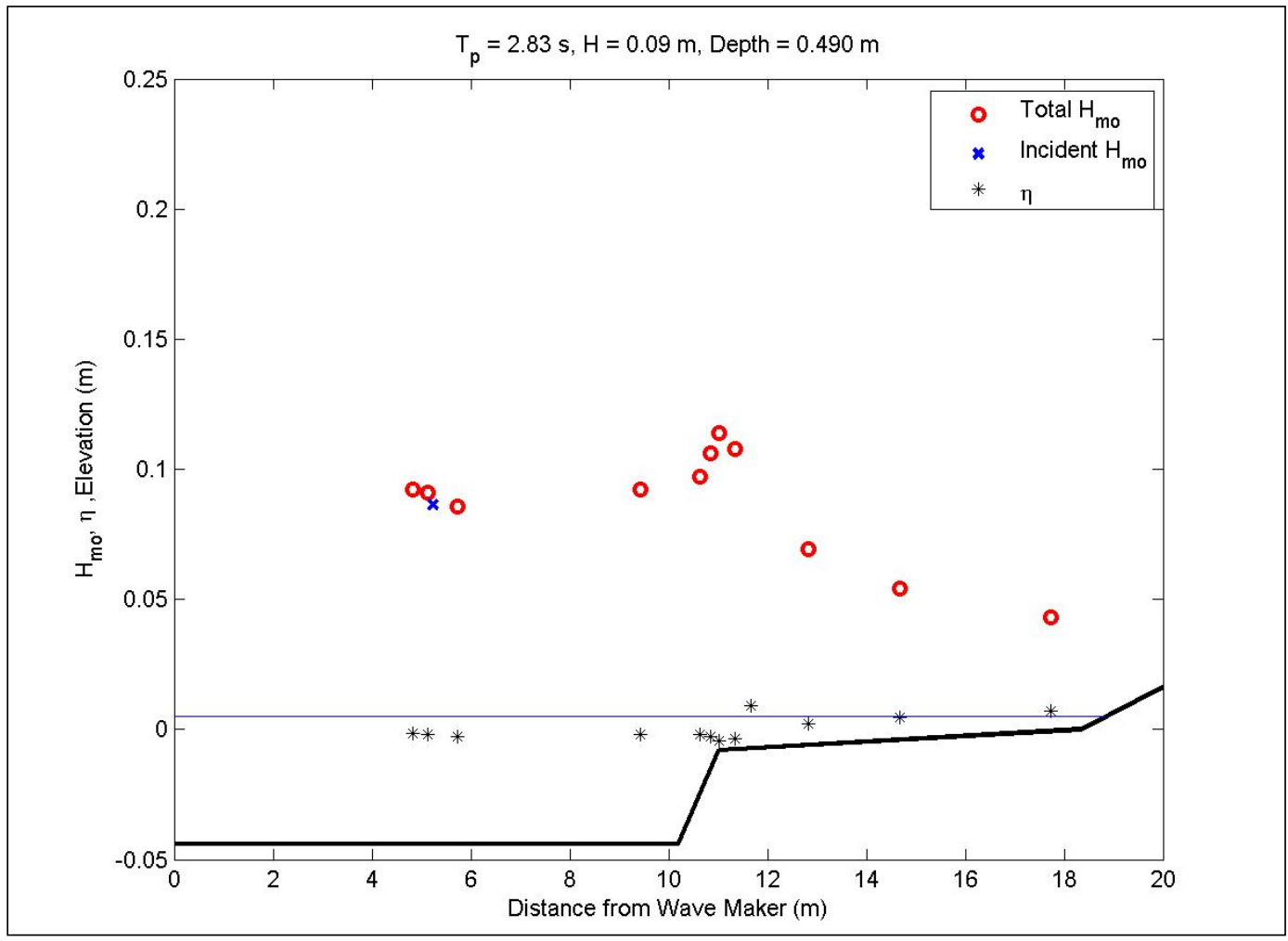

Figure D41. Wave transformation for 2.8-sec, $0.09 \mathrm{~m}$ waves at high water with a 1:25 reef slope. 


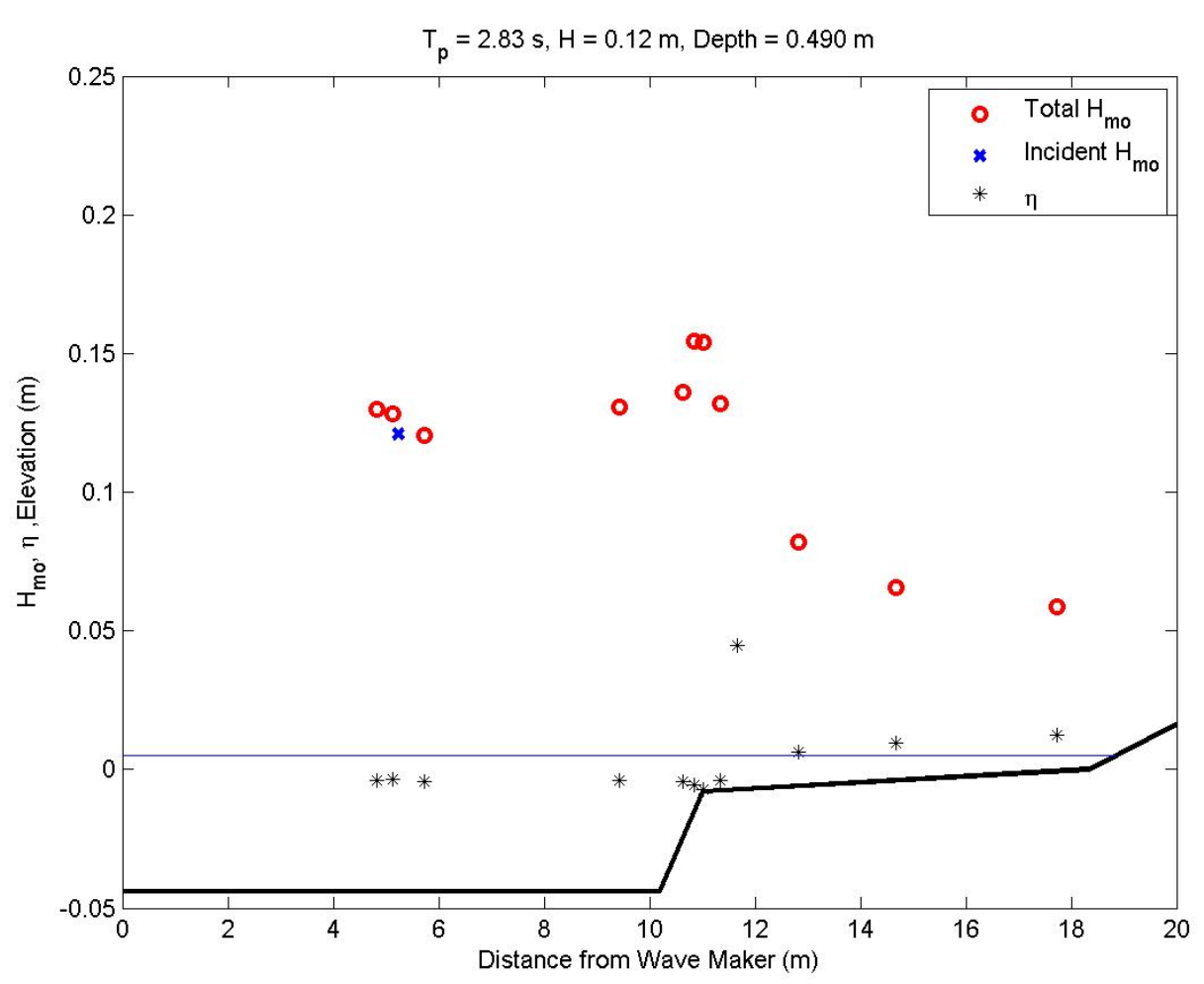

Figure D42. Wave transformation for 2.8-sec, $0.12 \mathrm{~m}$ waves at high water with a 1:25 reef slope.

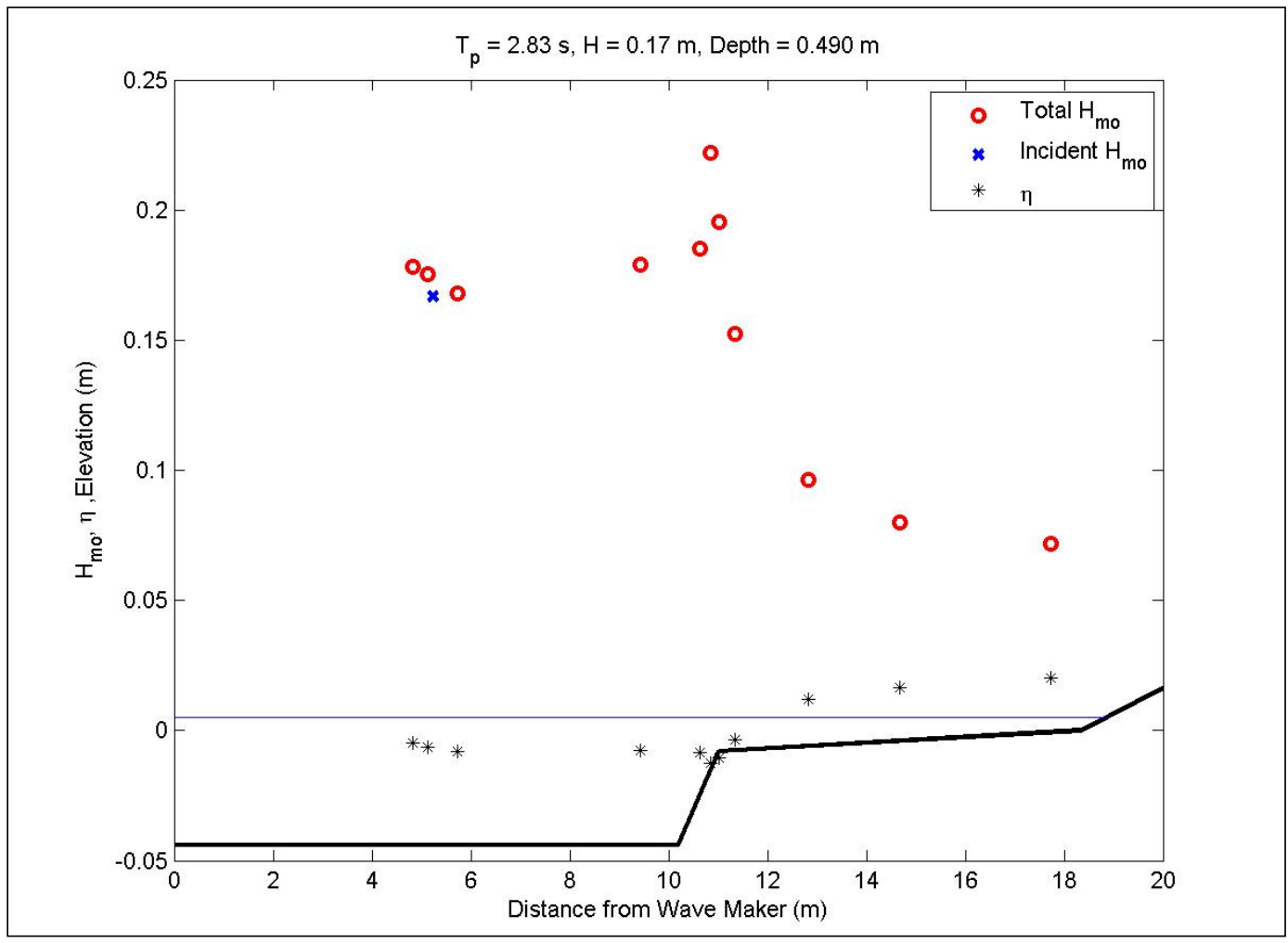

Figure D43. Wave transformation for $2.8-\mathrm{sec}, 0.17 \mathrm{~m}$ waves at high water with a 1:25 reef slope. 


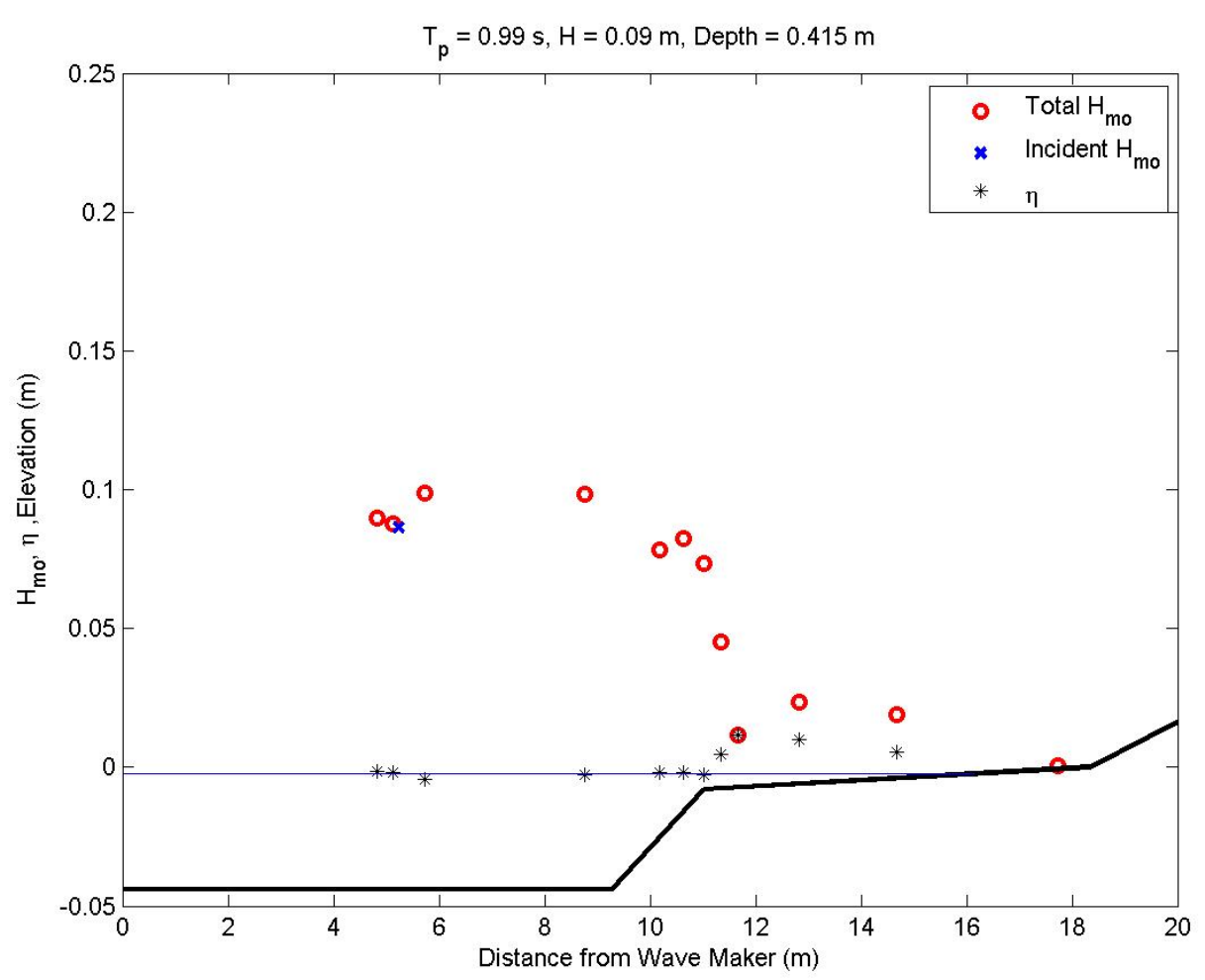

Figure D44. Wave transformation for 1-sec, 0.09-m waves at low water with a 1:5 reef slope.

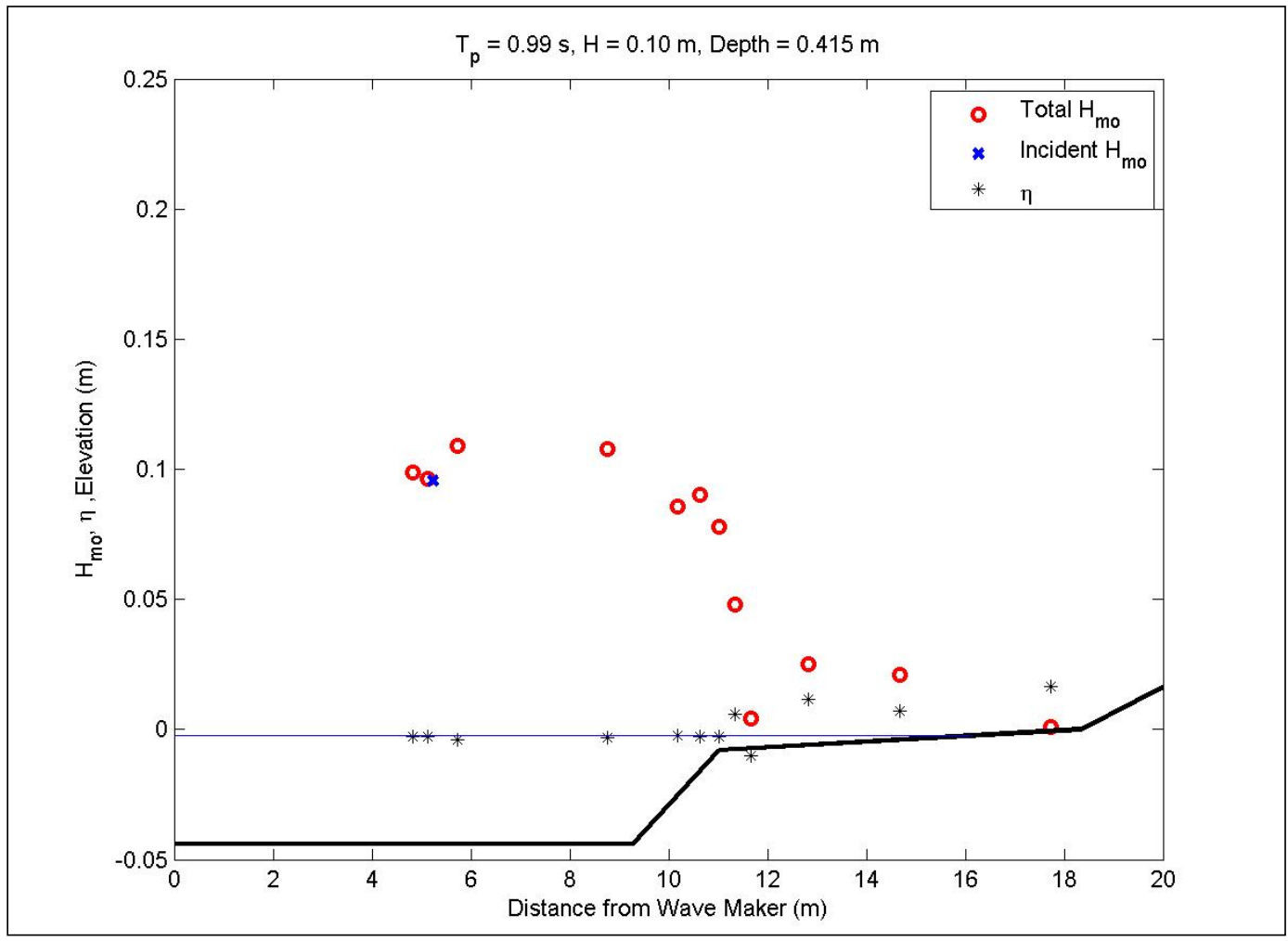

Figure D45. Wave transformation for 1-sec, 0.1-m waves at low water with a 1:5 reef slope. 


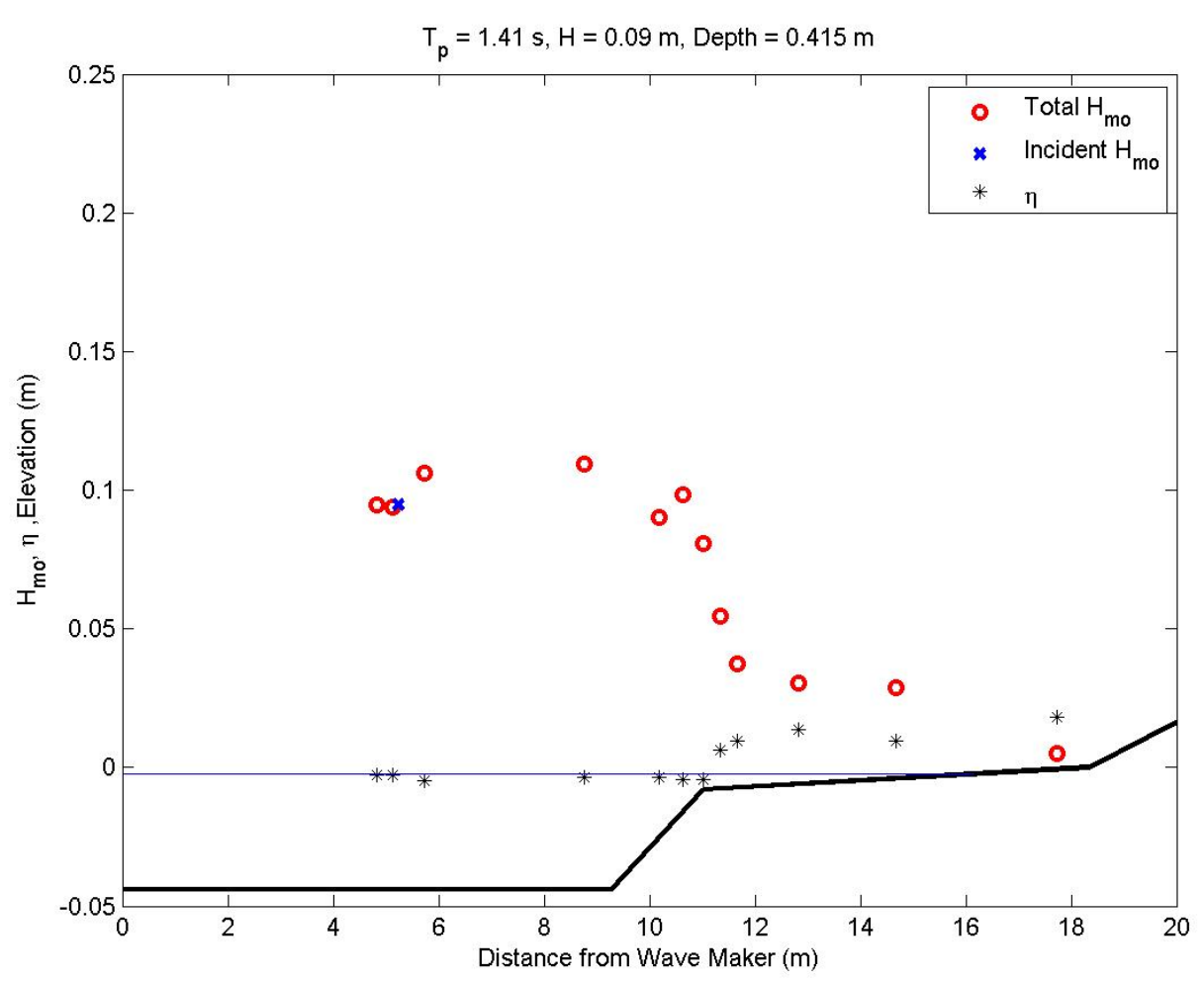

Figure D46. Wave transformation for 1.4-sec, 0.09-m waves at low water with a 1:5 reef slope.

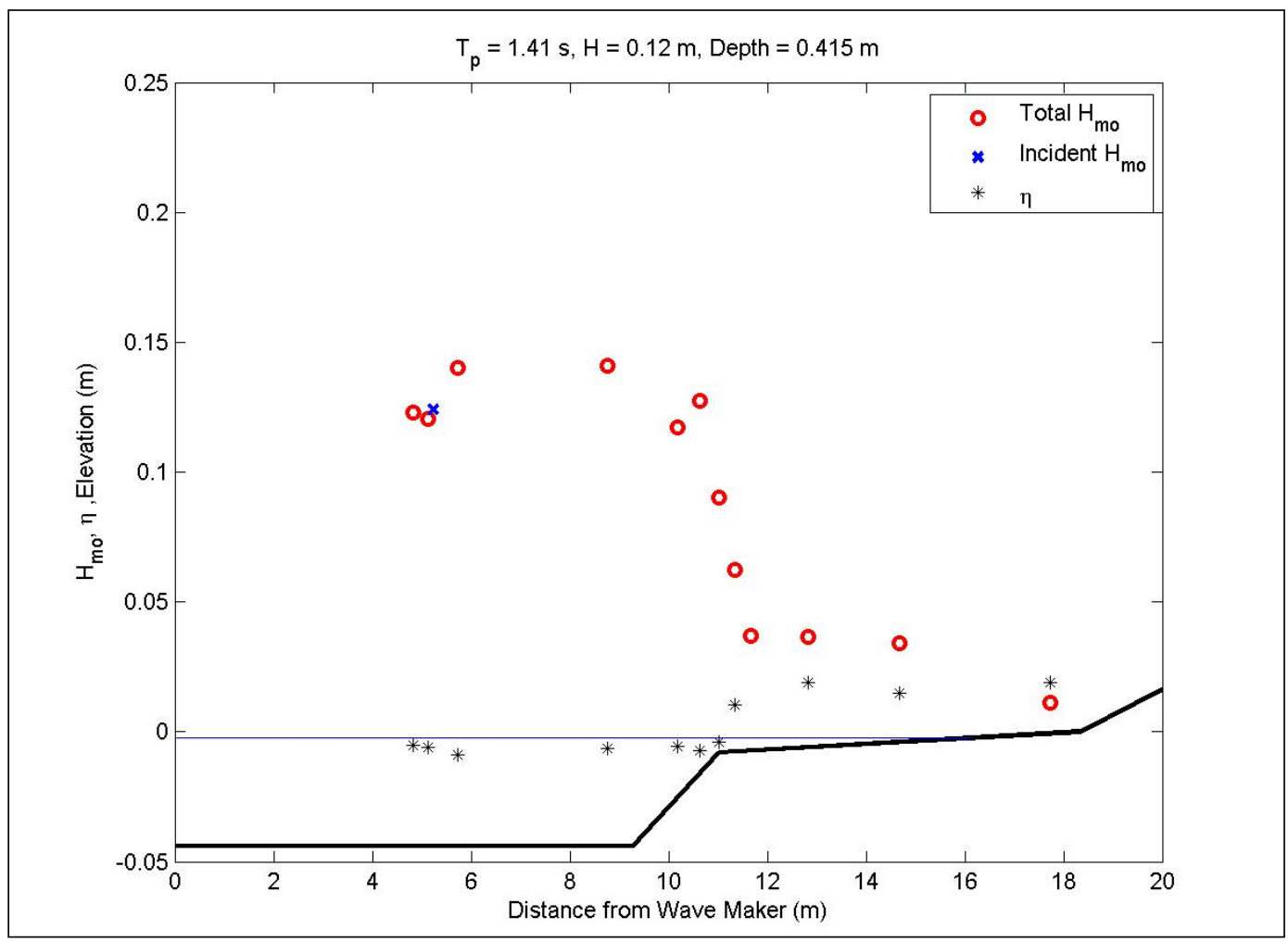

Figure D47. Wave transformation for 1.4-sec, 0.12-m waves at low water with a 1:5 reef slope. 


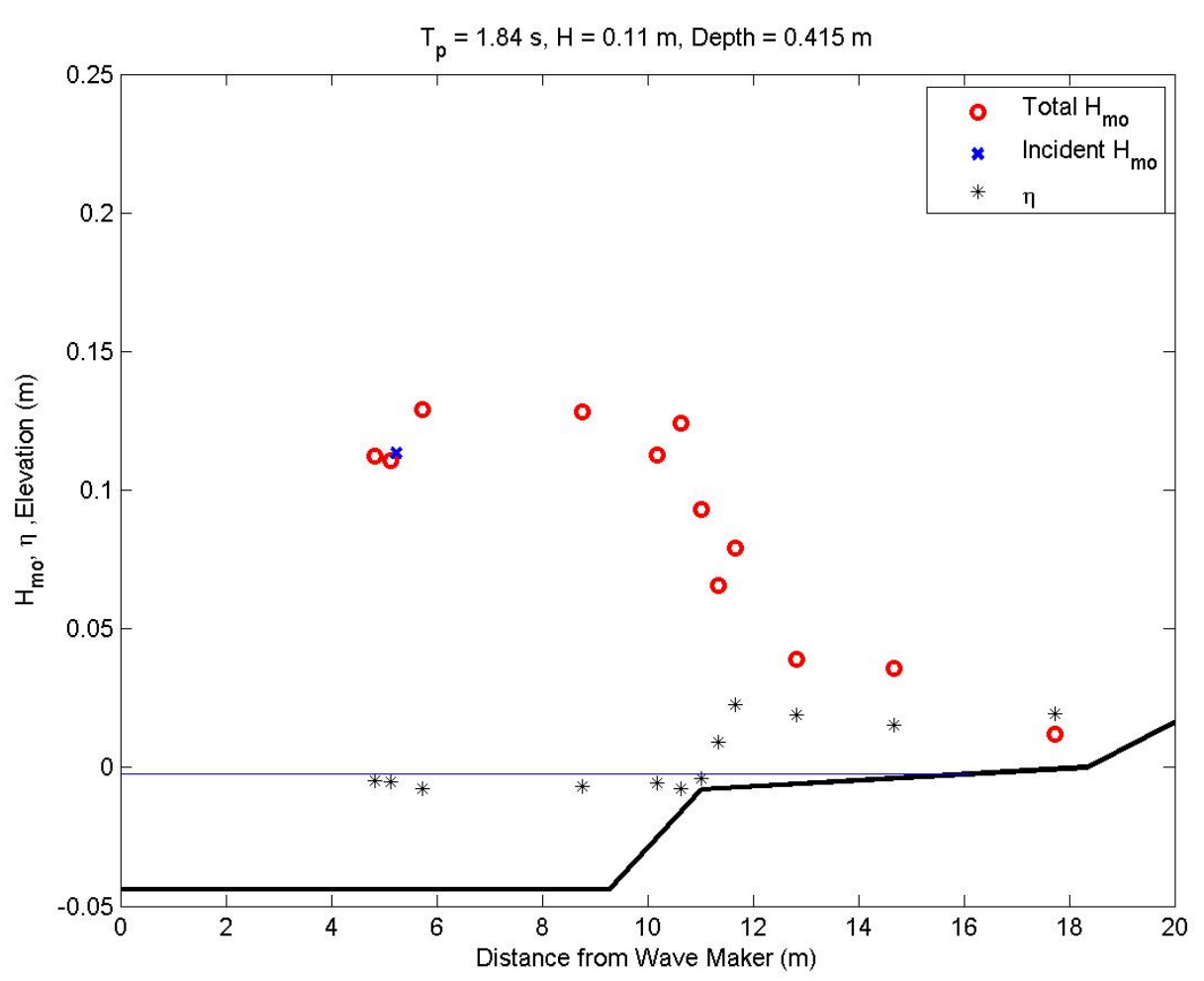

Figure D48. Wave transformation for 1.8-sec, $0.11-\mathrm{m}$ waves at low water with a 1:5 reef slope.

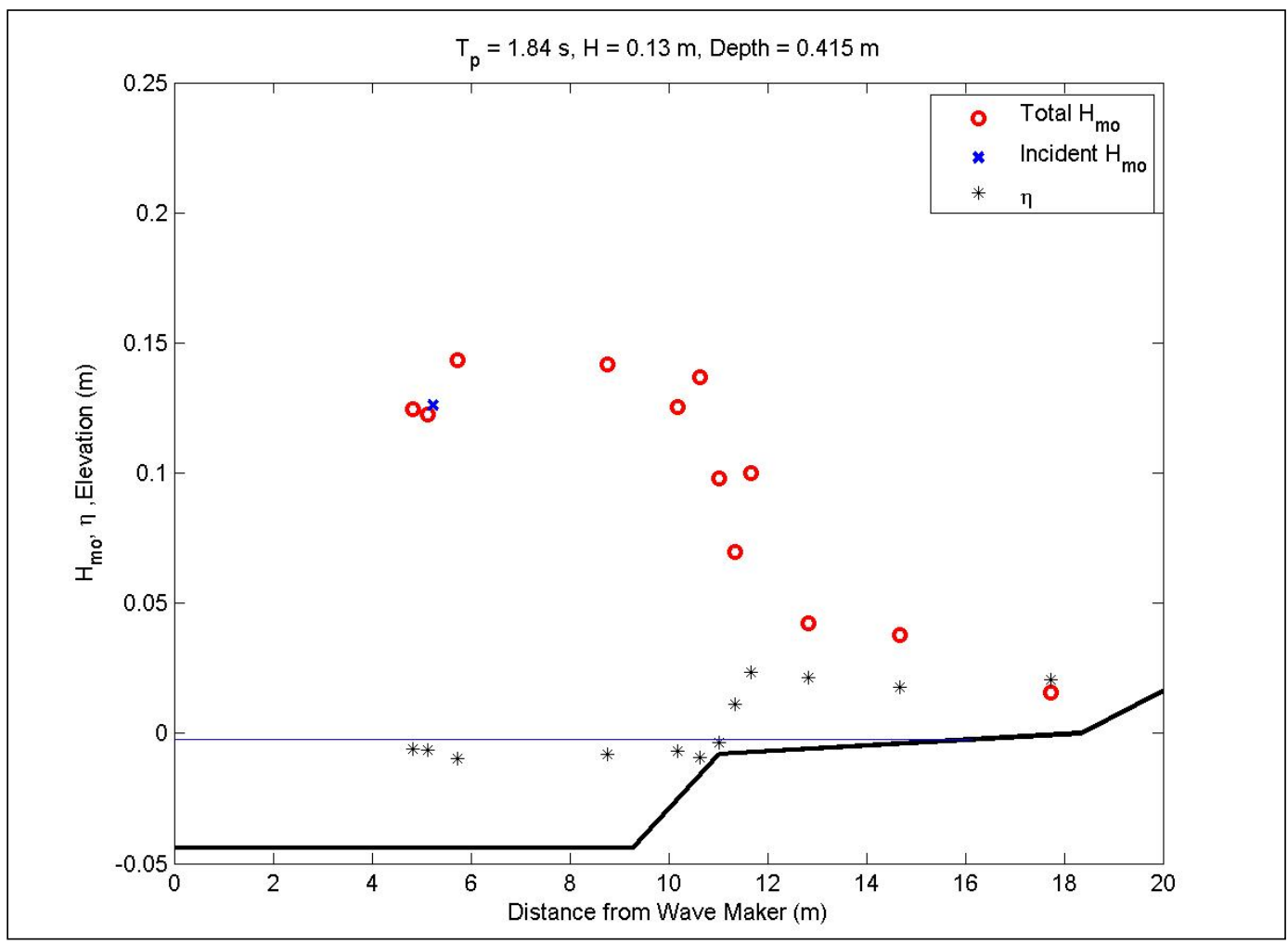

Figure D49. Wave transformation for 1.8-sec, 0.13-m waves at low water with a 1:5 reef slope. 


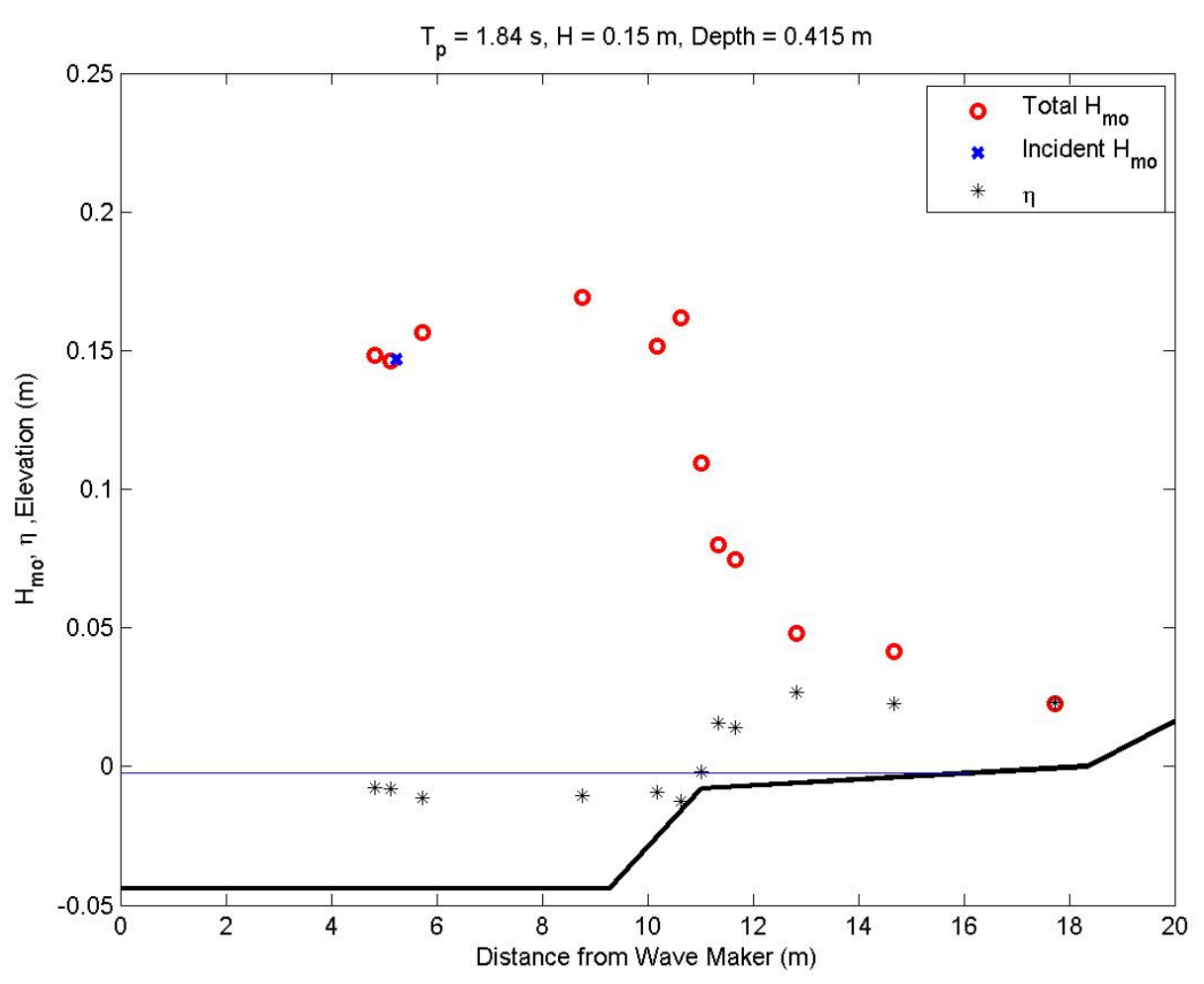

Figure D50. Wave transformation for 1.8-sec, 0.15-m waves at low water with a 1:5 reef slope.

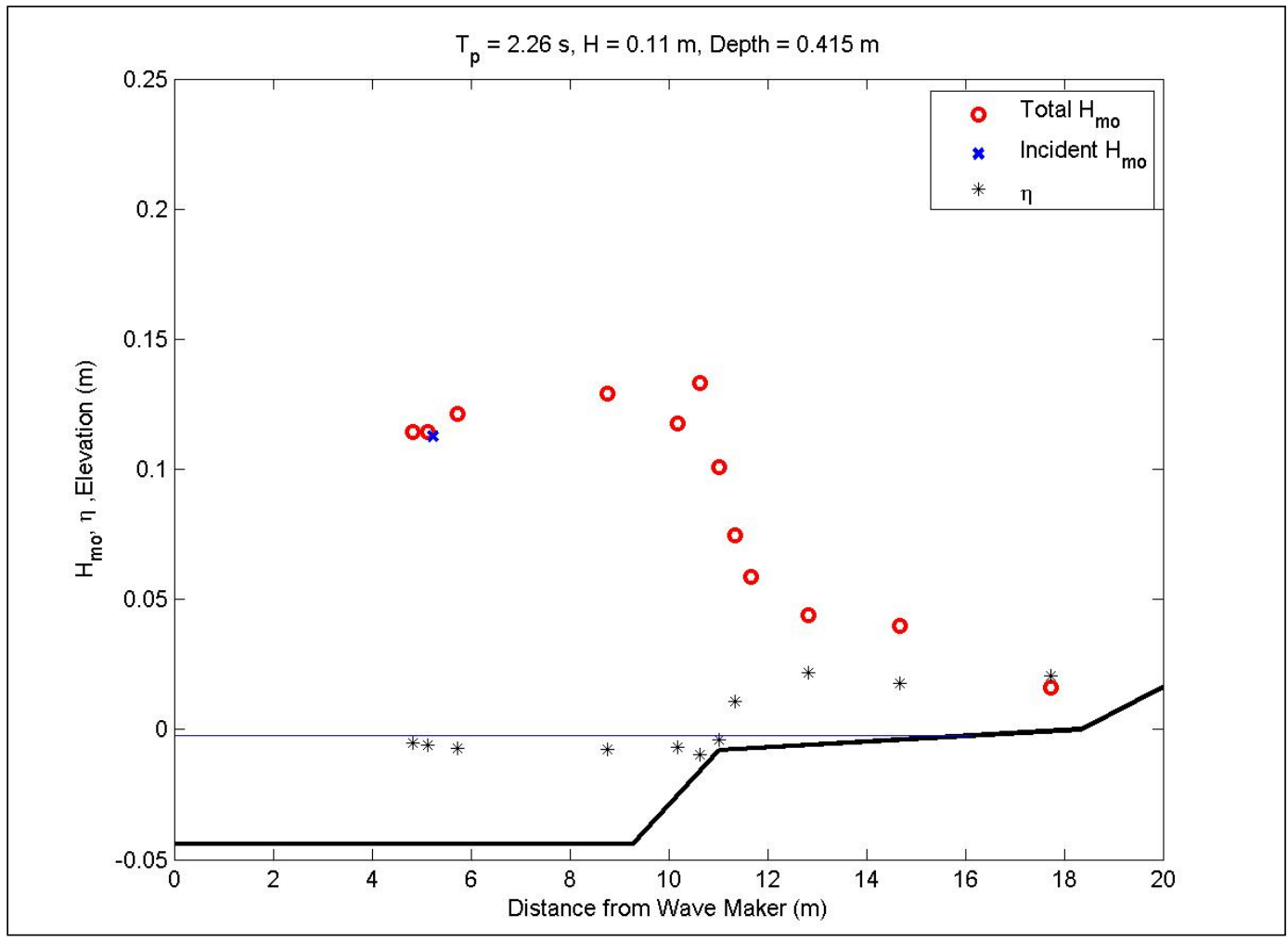

Figure D51. Wave transformation for 2.3-sec, 0.11-m waves at low water with a 1:5 reef slope. 


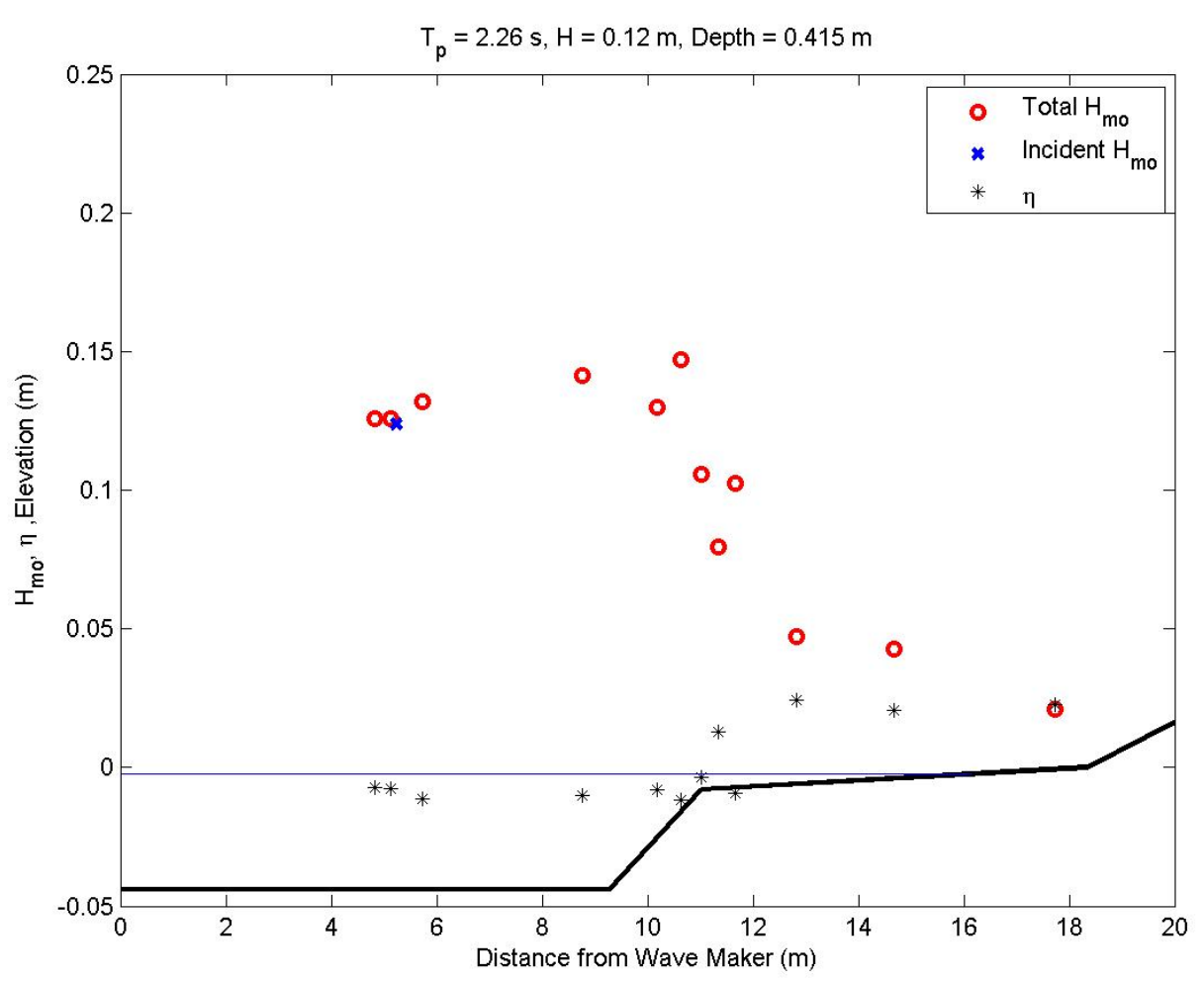

Figure D52. Wave transformation for 2.3-sec, 0.12-m waves at low water with a 1:5 reef slope.

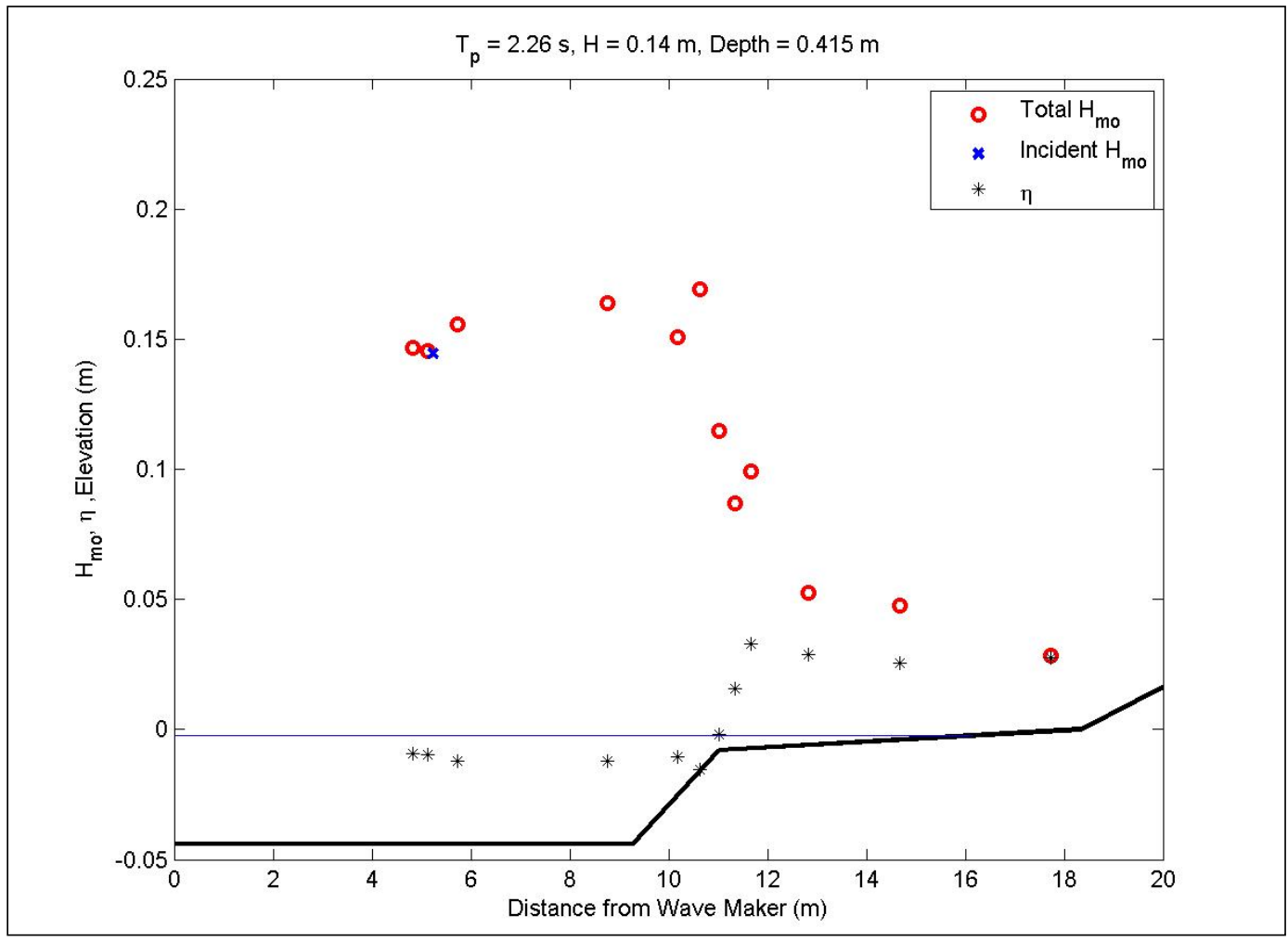

Figure D53. Wave transformation for 2.3-sec, 0.14-m waves at low water with a 1:5 reef slope. 


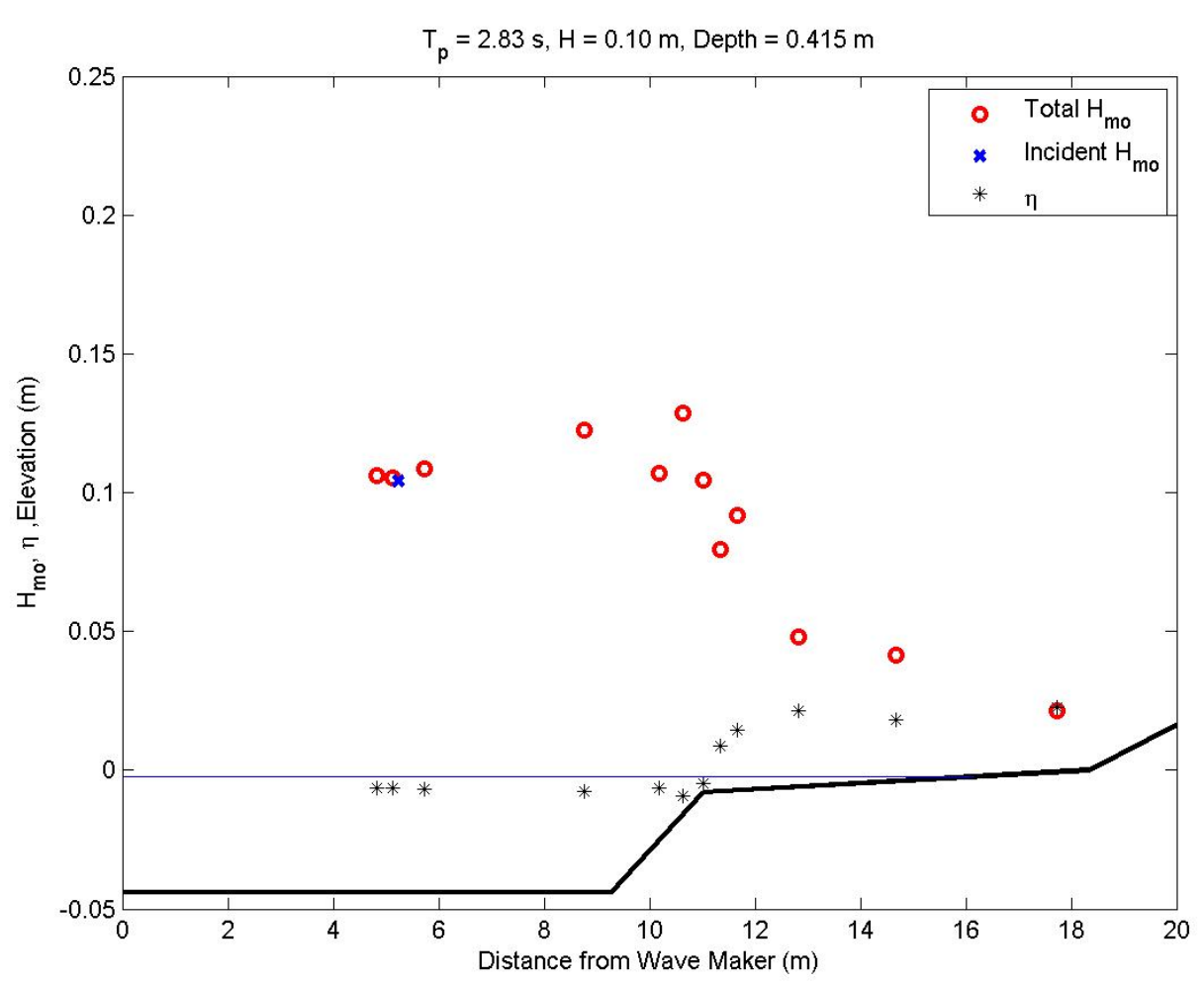

Figure D54. Wave transformation for 2.8-sec, 0.10-m waves at low water with a 1:5 reef slope.

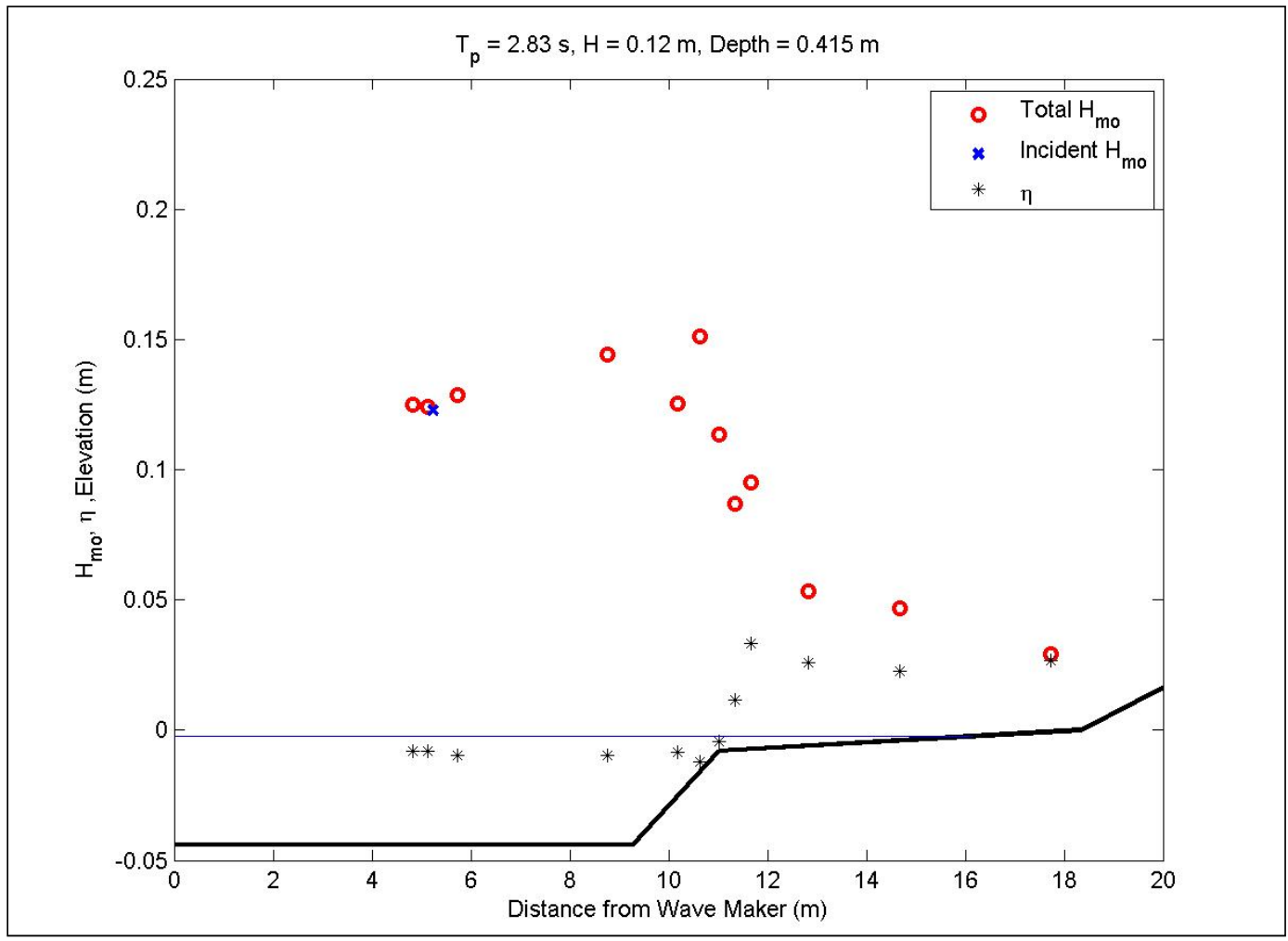

Figure D55. Wave transformation for 2.83-sec, 0.12-m waves at low water with a 1:5 reef slope. 


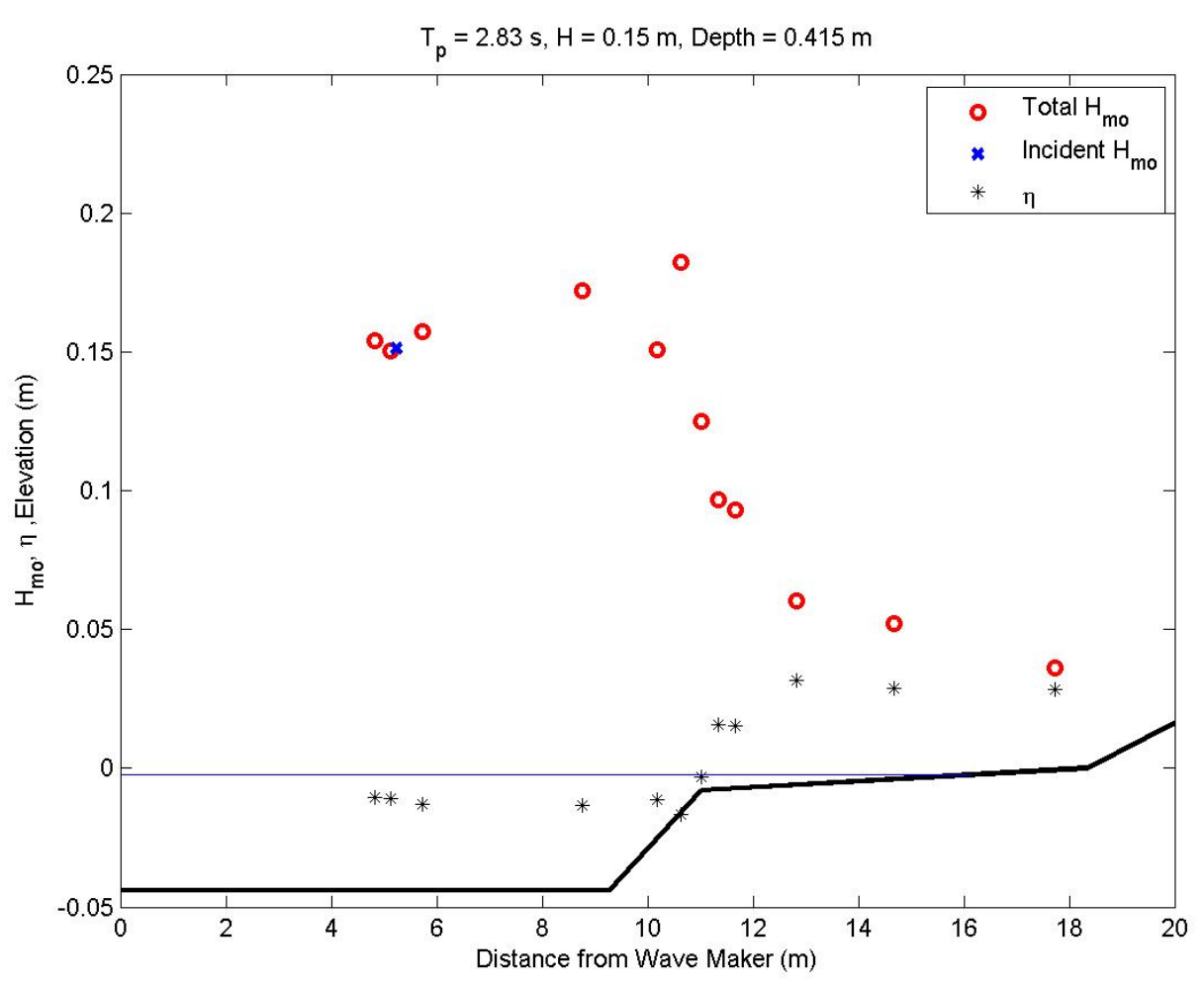

Figure D56. Wave transformation for 2.83-sec, 0.15-m waves at low water with a 1:5 reef slope.

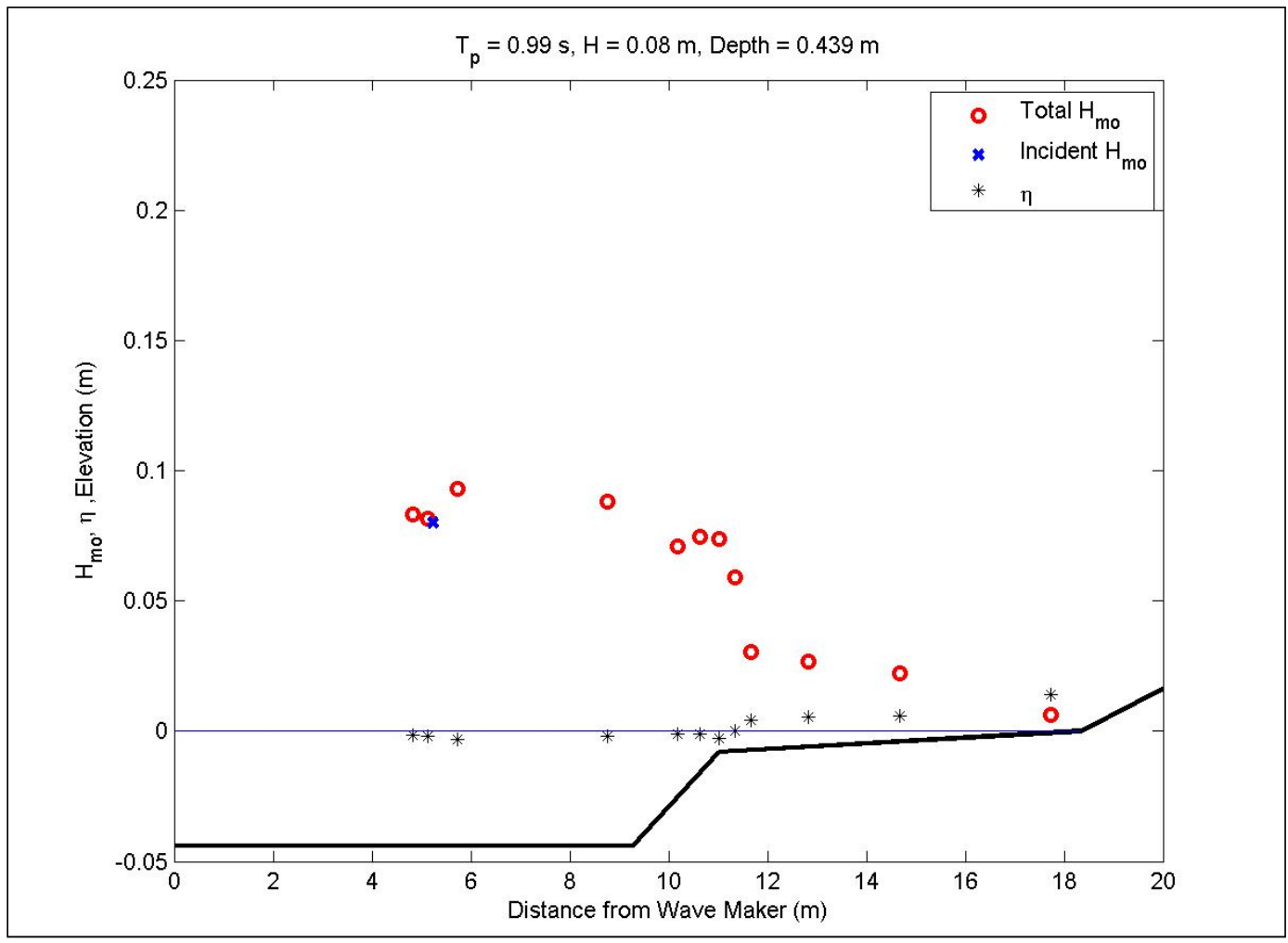

Figure D57. Wave transformation for 1-sec, 0.08-m waves at mid water with a 1:5 reef slope. 


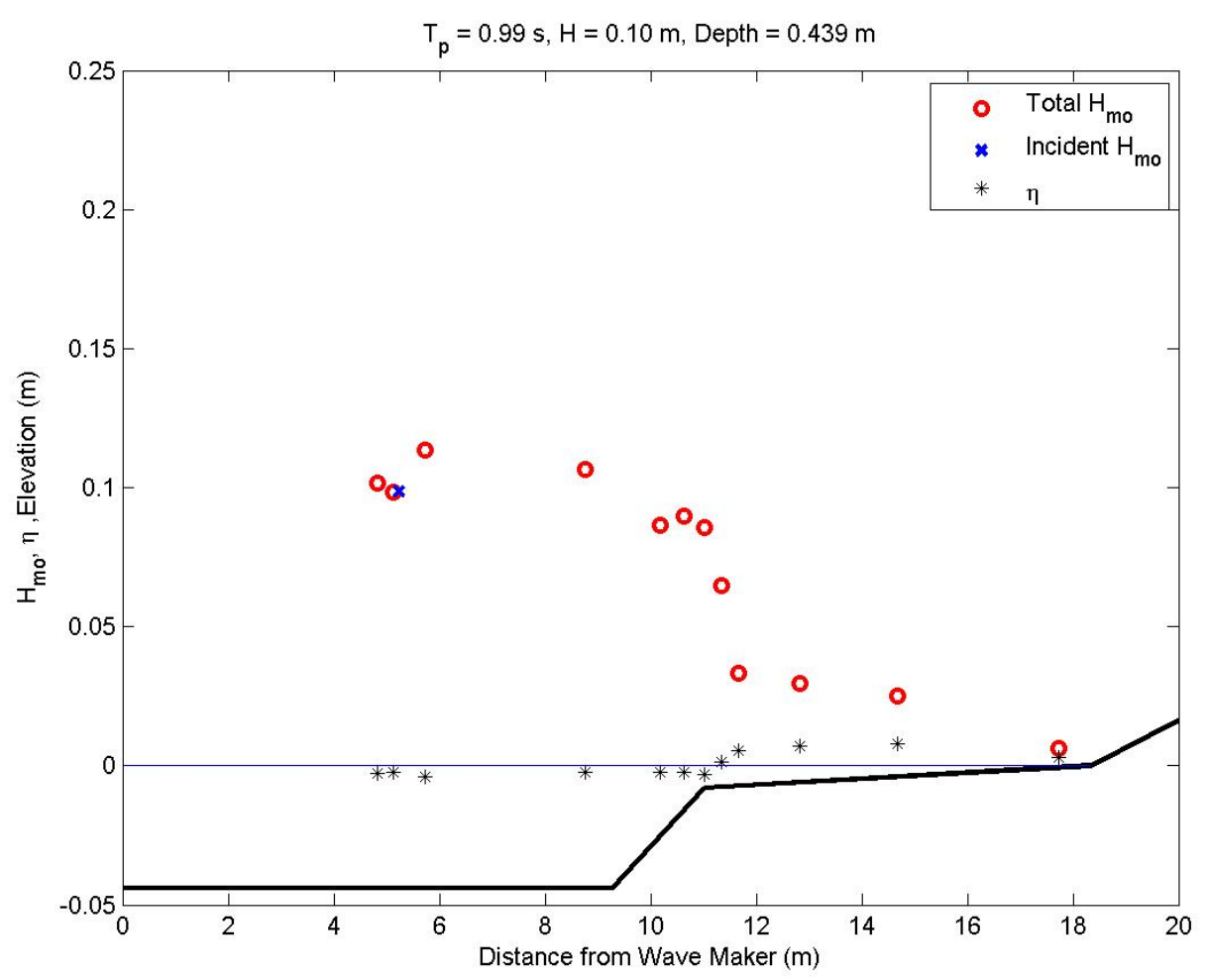

Figure D58. Wave transformation for 1-sec, 0.10-m waves at mid water with a 1:5 reef slope.

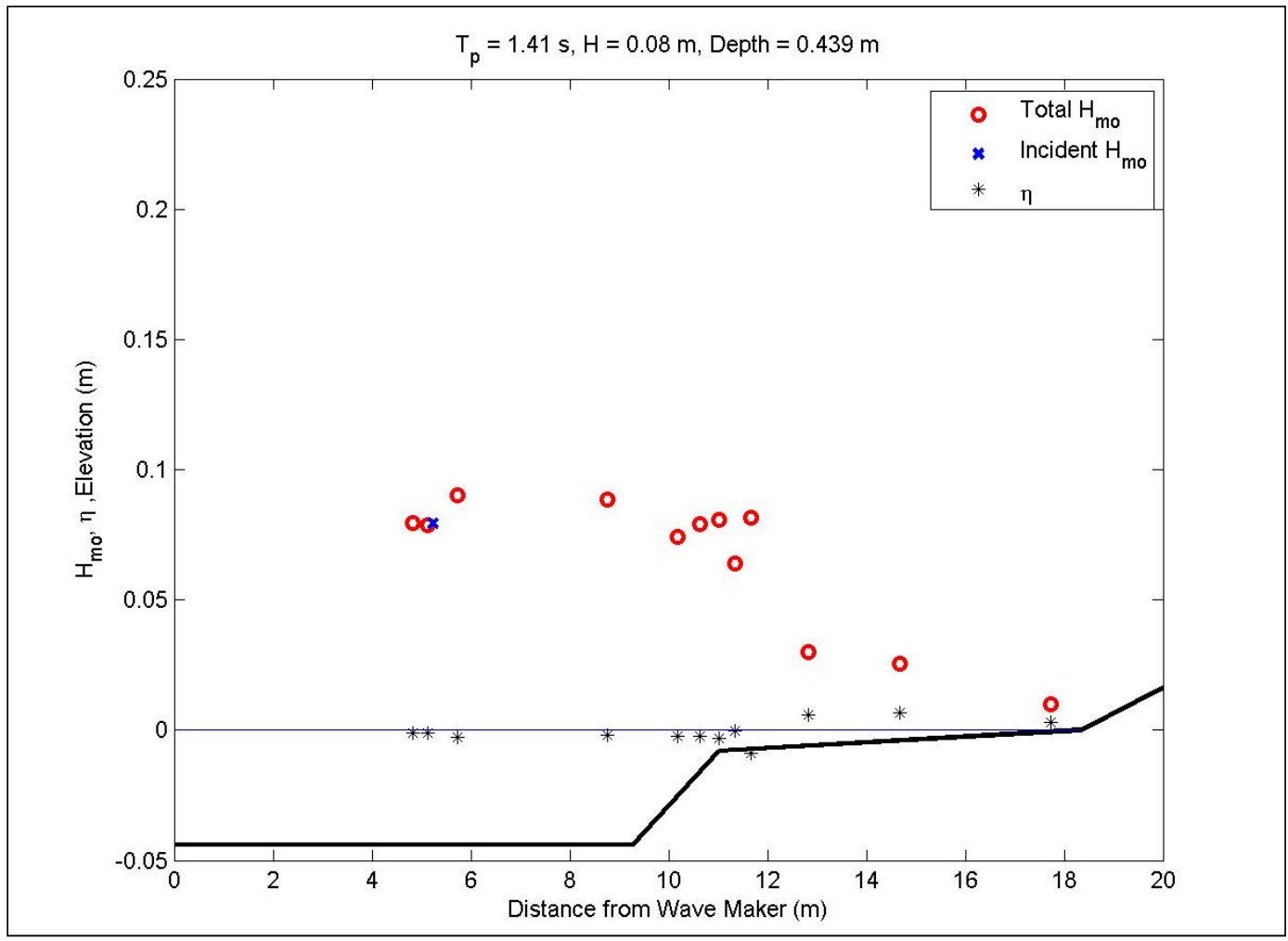

Figure D59. Wave transformation for 1.4-sec, 0.08-m waves at mid water with a 1:5 reef slope. 


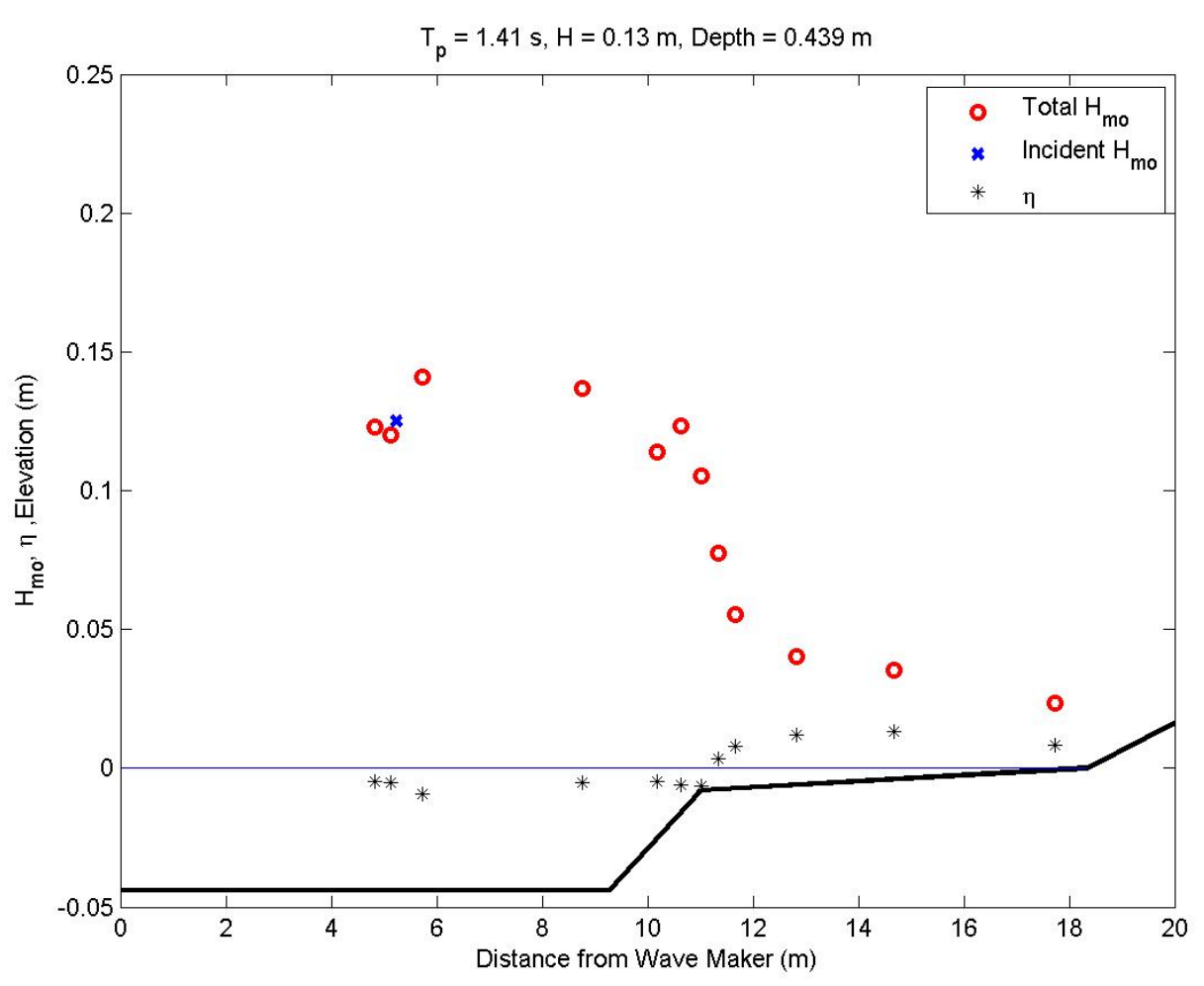

Figure D60. Wave transformation for 1.4-sec, 0.13-m waves at mid water with a 1:5 reef slope.

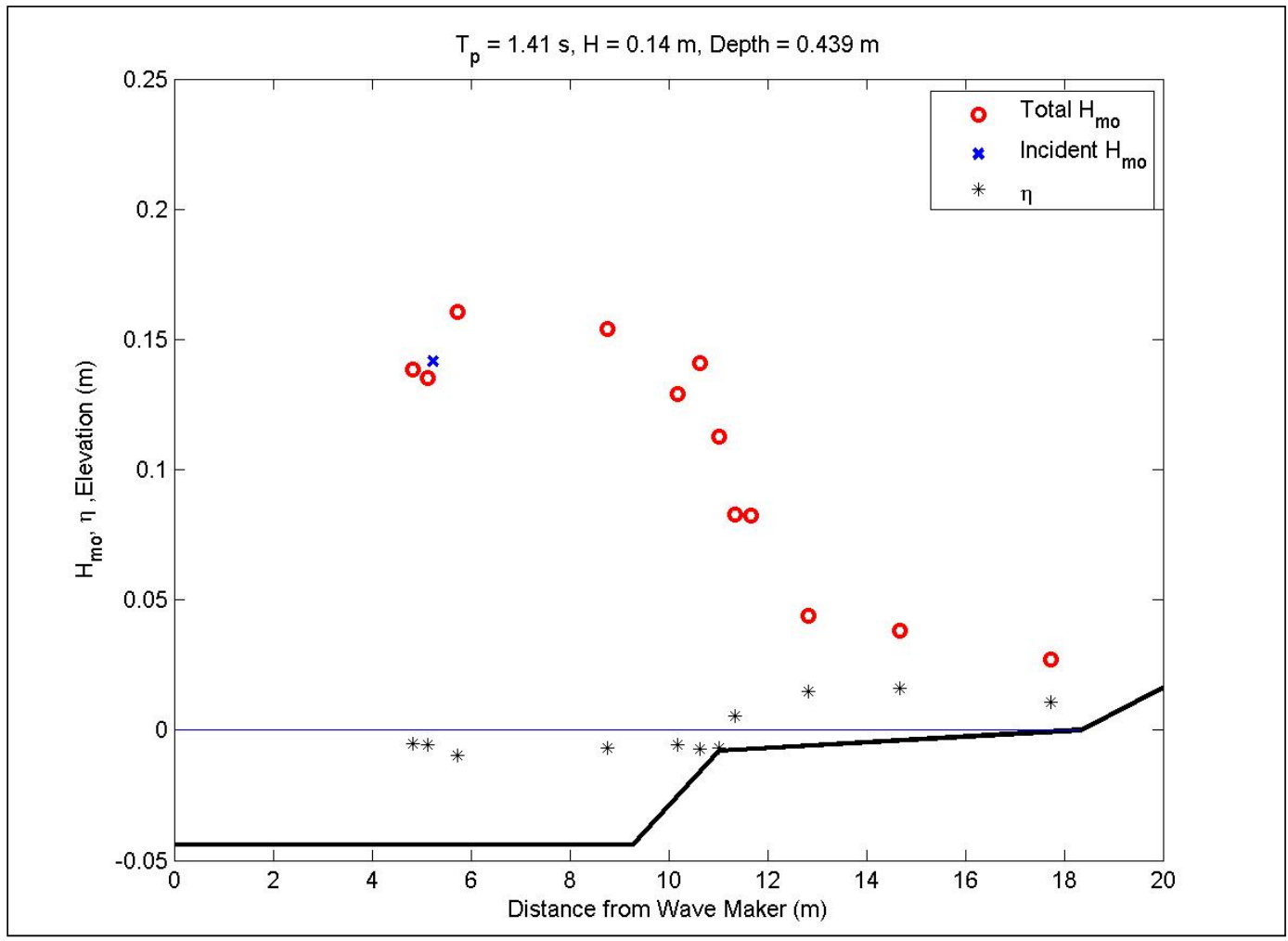

Figure D61. Wave transformation for 1.4-sec, 0.14-m waves at mid water with a 1:5 reef slope. 


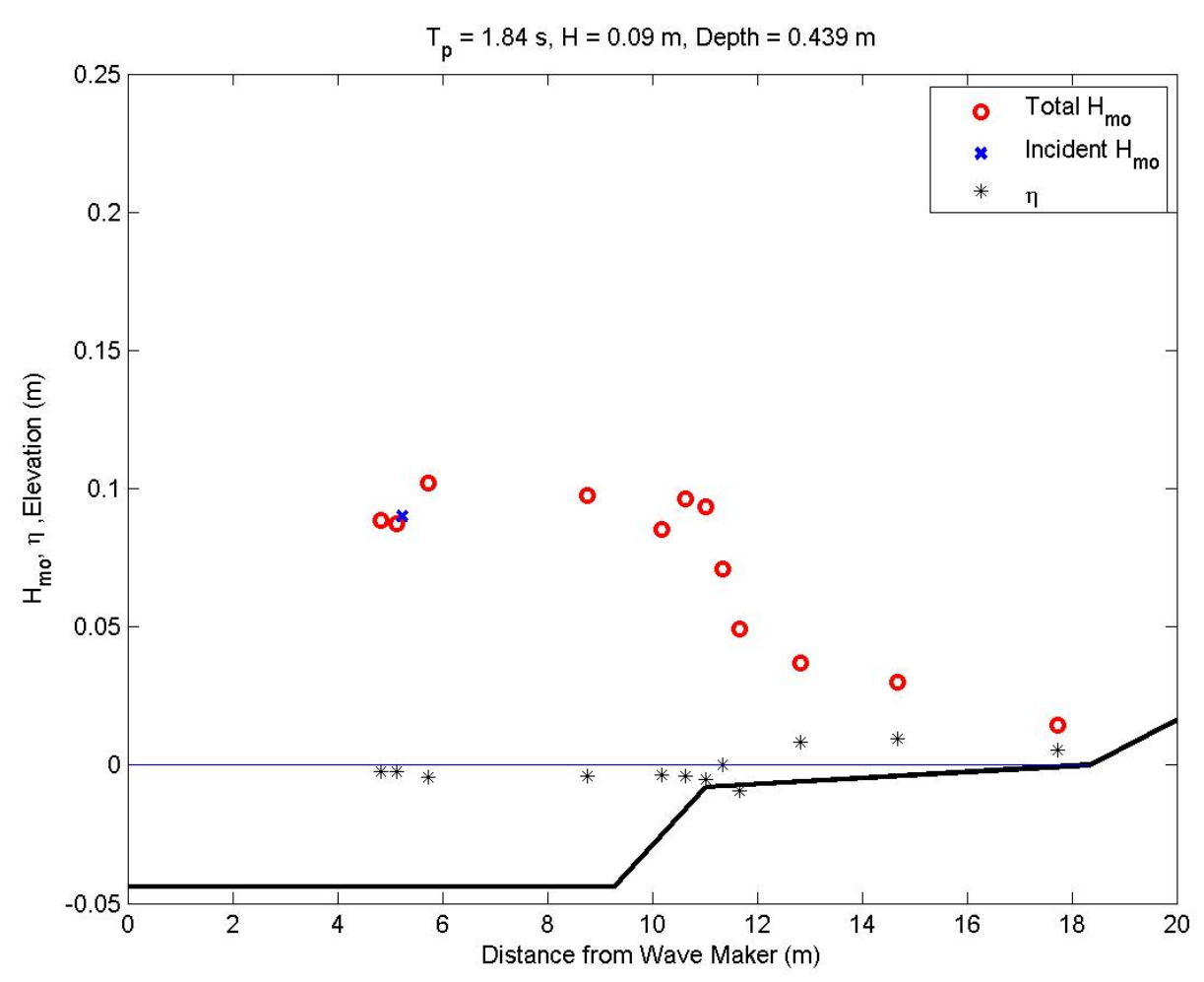

Figure D62. Wave transformation for 1.8-sec, 0.09-m waves at mid water with a 1:5 reef slope.

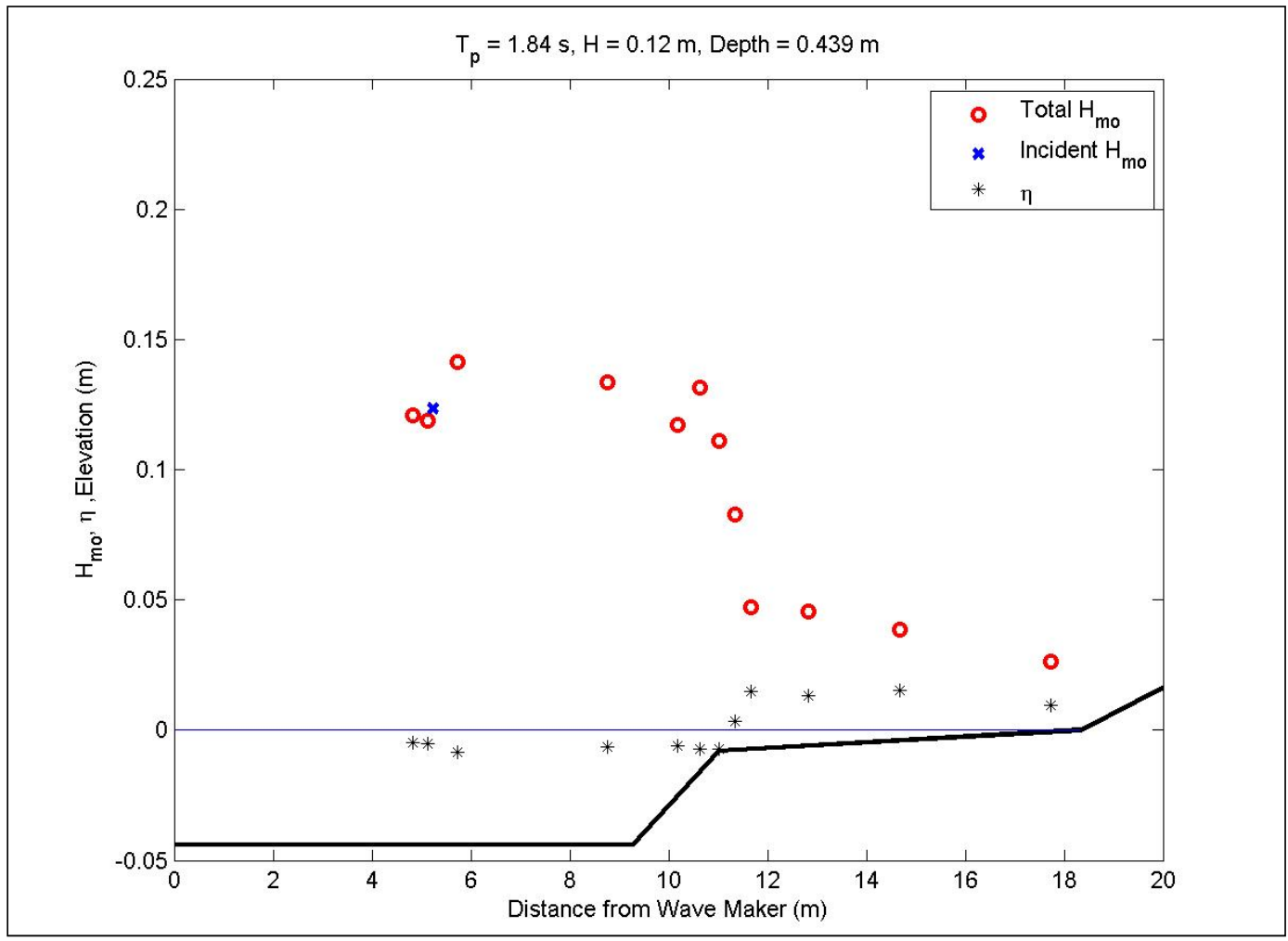

Figure D63. Wave transformation for 1.8-sec, 0.12-m waves at mid water with a 1:5 reef slope. 


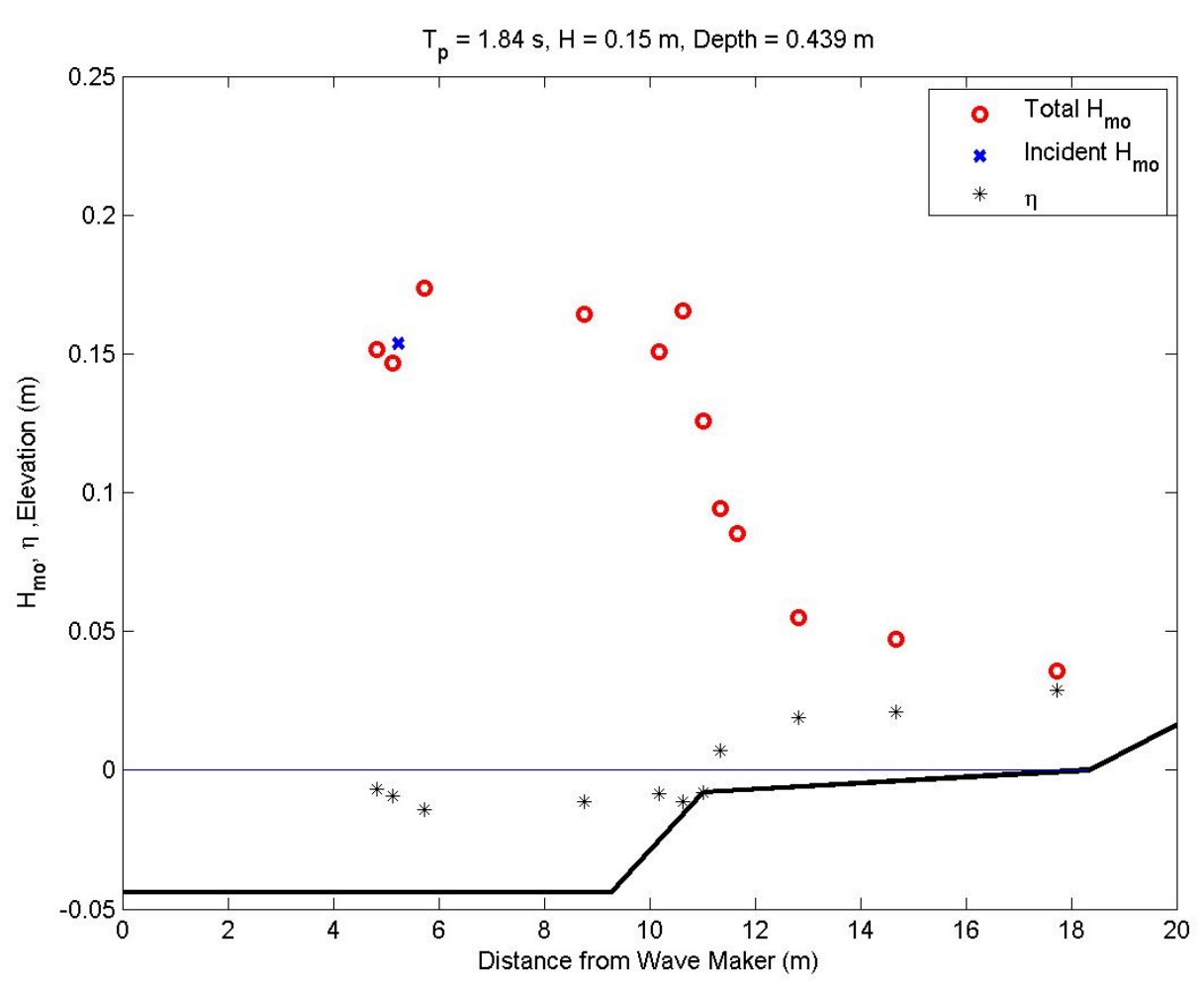

Figure D64. Wave transformation for $1.8-\mathrm{sec}, 0.15-\mathrm{m}$ waves at mid water with a 1:5 reef slope.

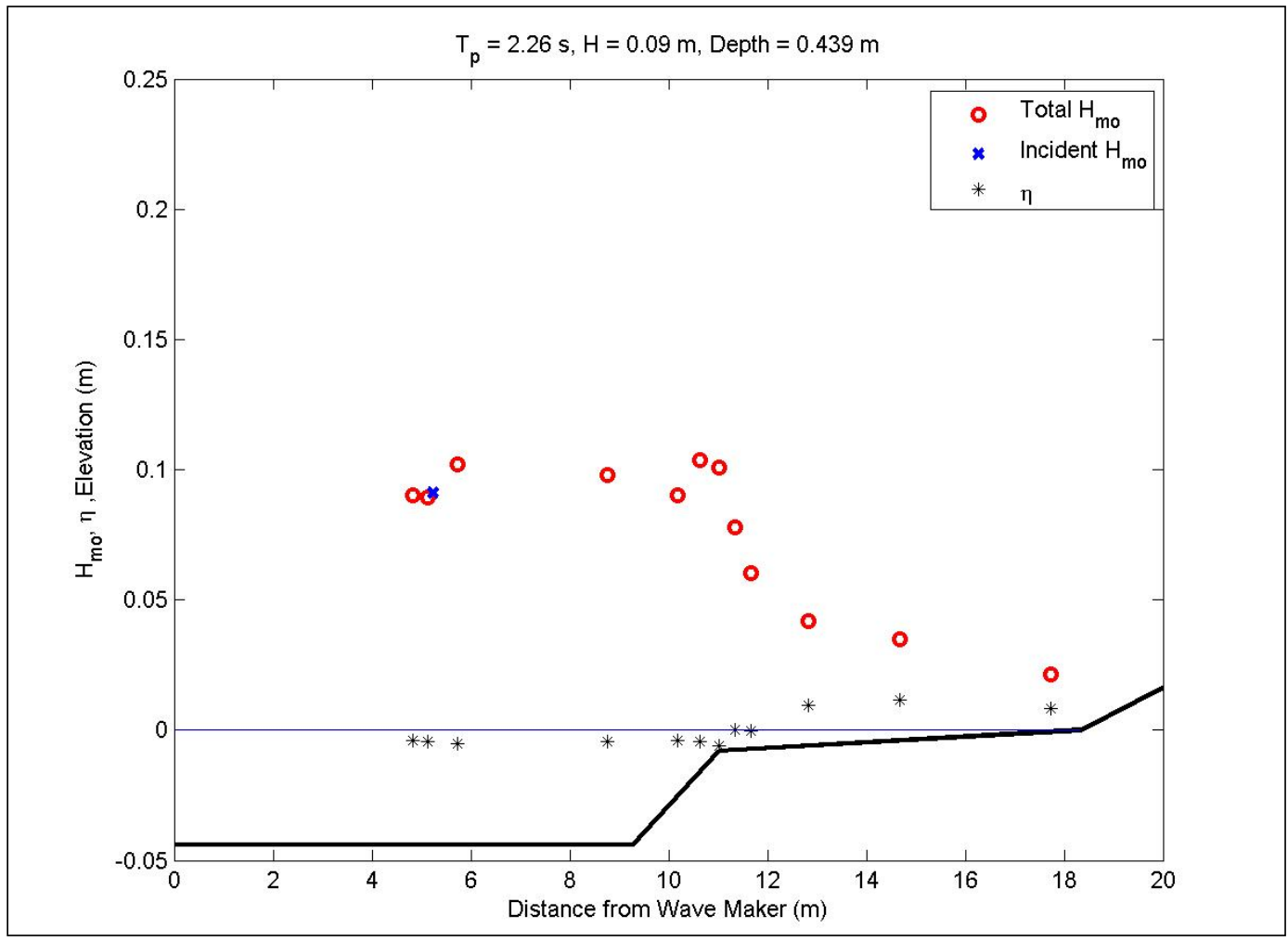

Figure D65. Wave transformation for 2.3-sec, 0.09-m waves at mid water with a 1:5 reef slope. 


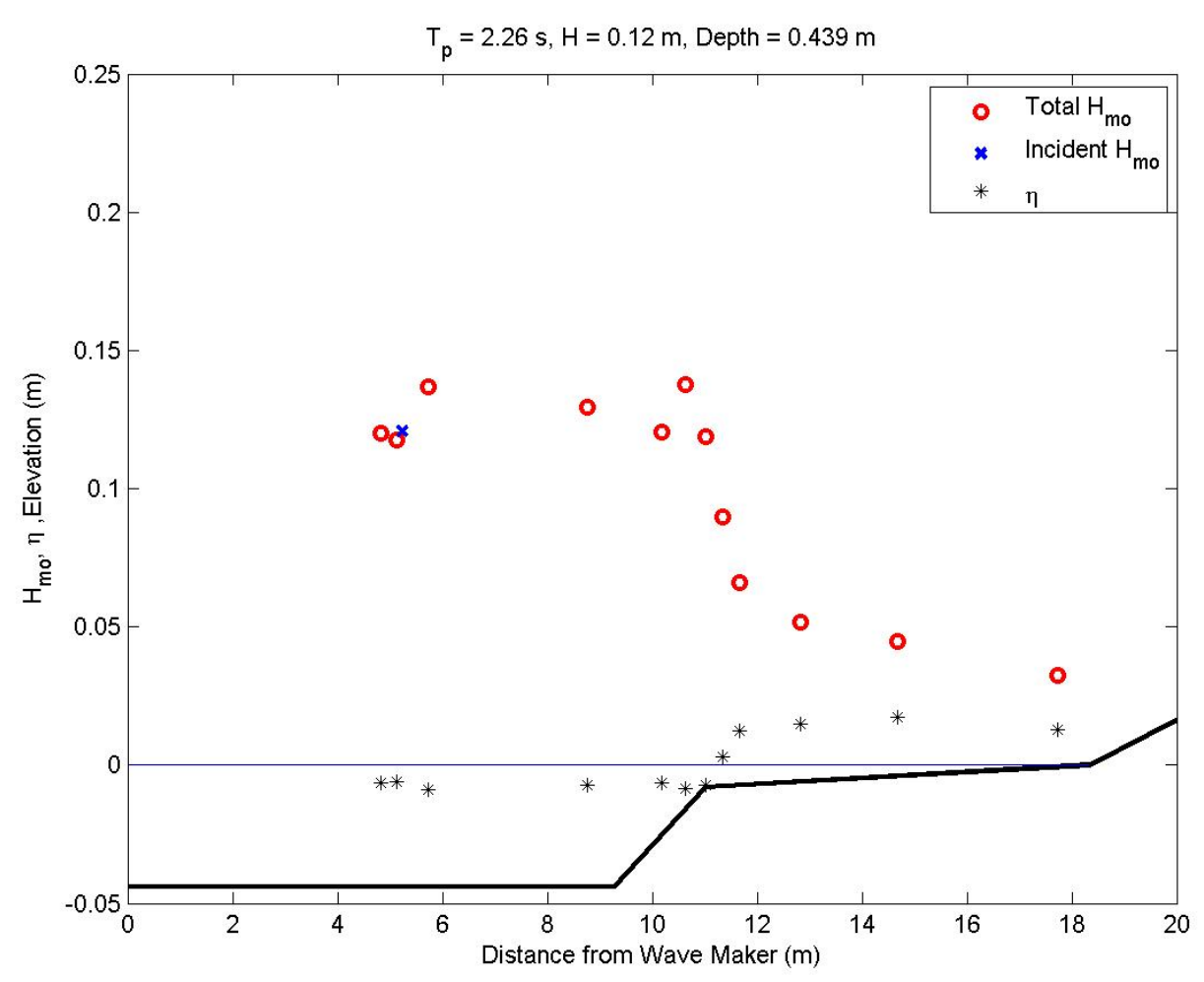

Figure D66. Wave transformation for 2.3-sec, 0.12-m waves at mid water with a 1:5 reef slope.

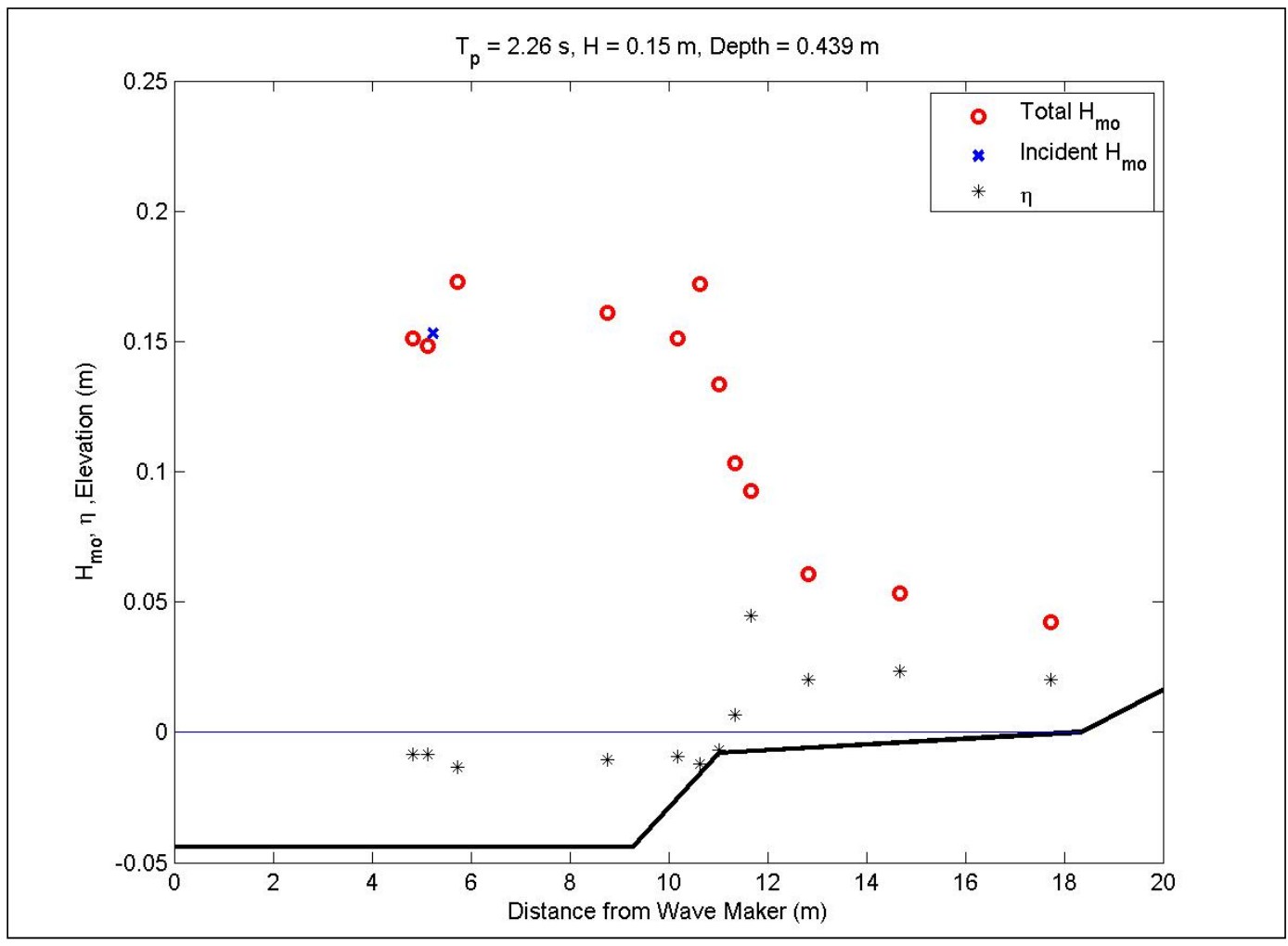

Figure D67. Wave transformation for 2.3-sec, 0.15-m waves at mid water with a 1:5 reef slope. 


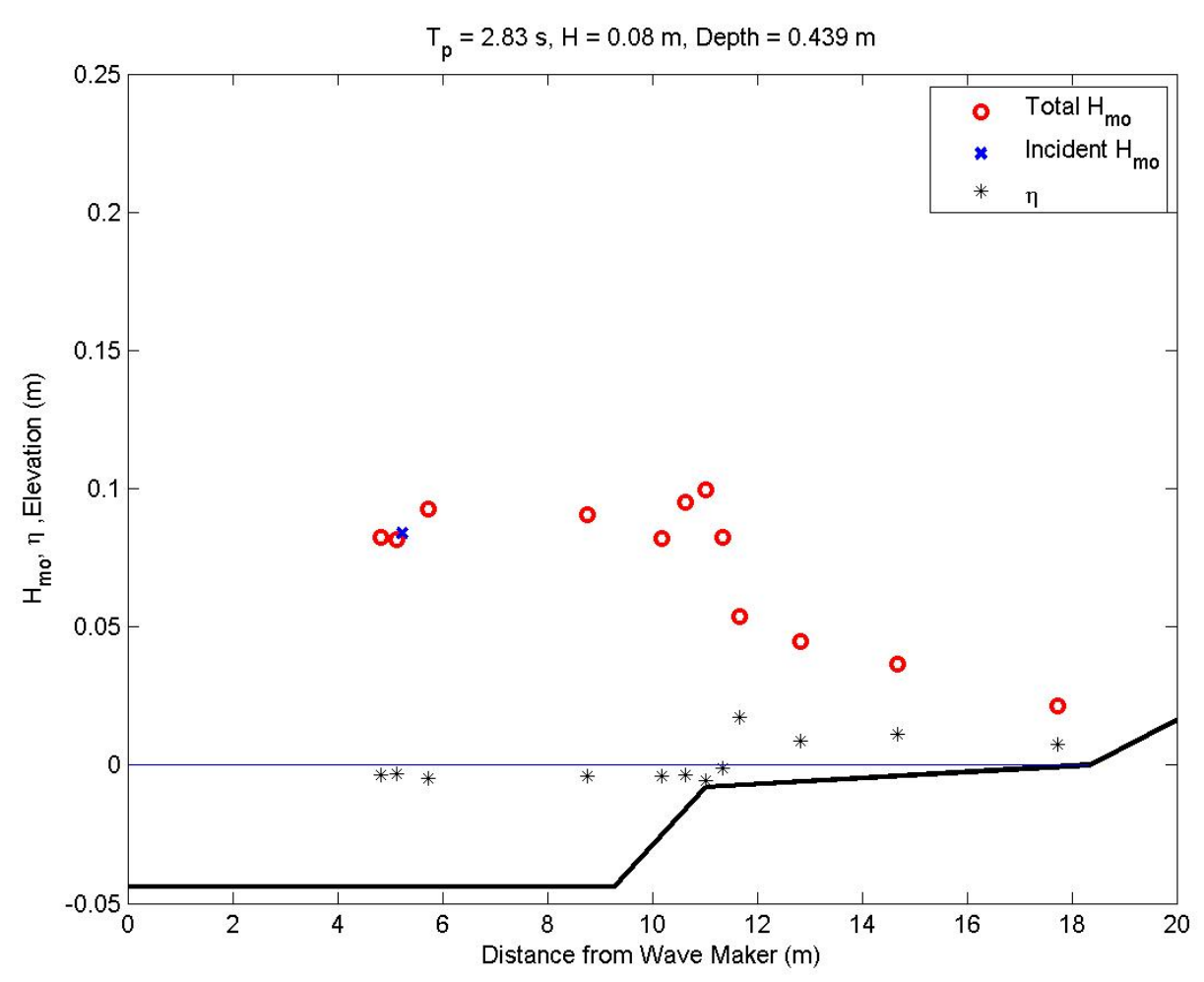

Figure D68. Wave transformation for 2.8-sec, 0.08-m waves at mid water with a 1:5 reef slope.

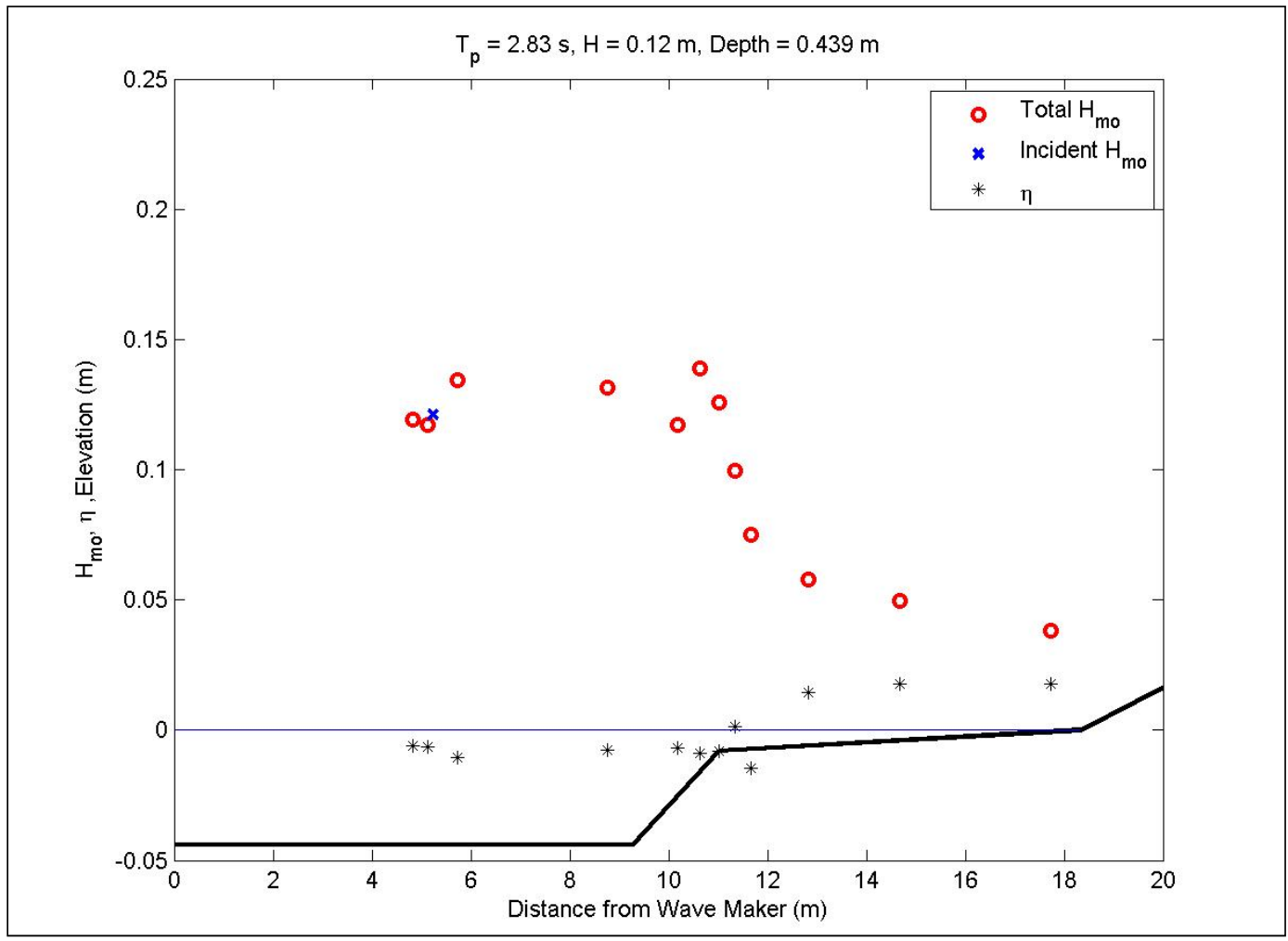

Figure D69. Wave transformation for 2.8-sec, 0.12-m waves at mid water with a 1:5 reef slope. 


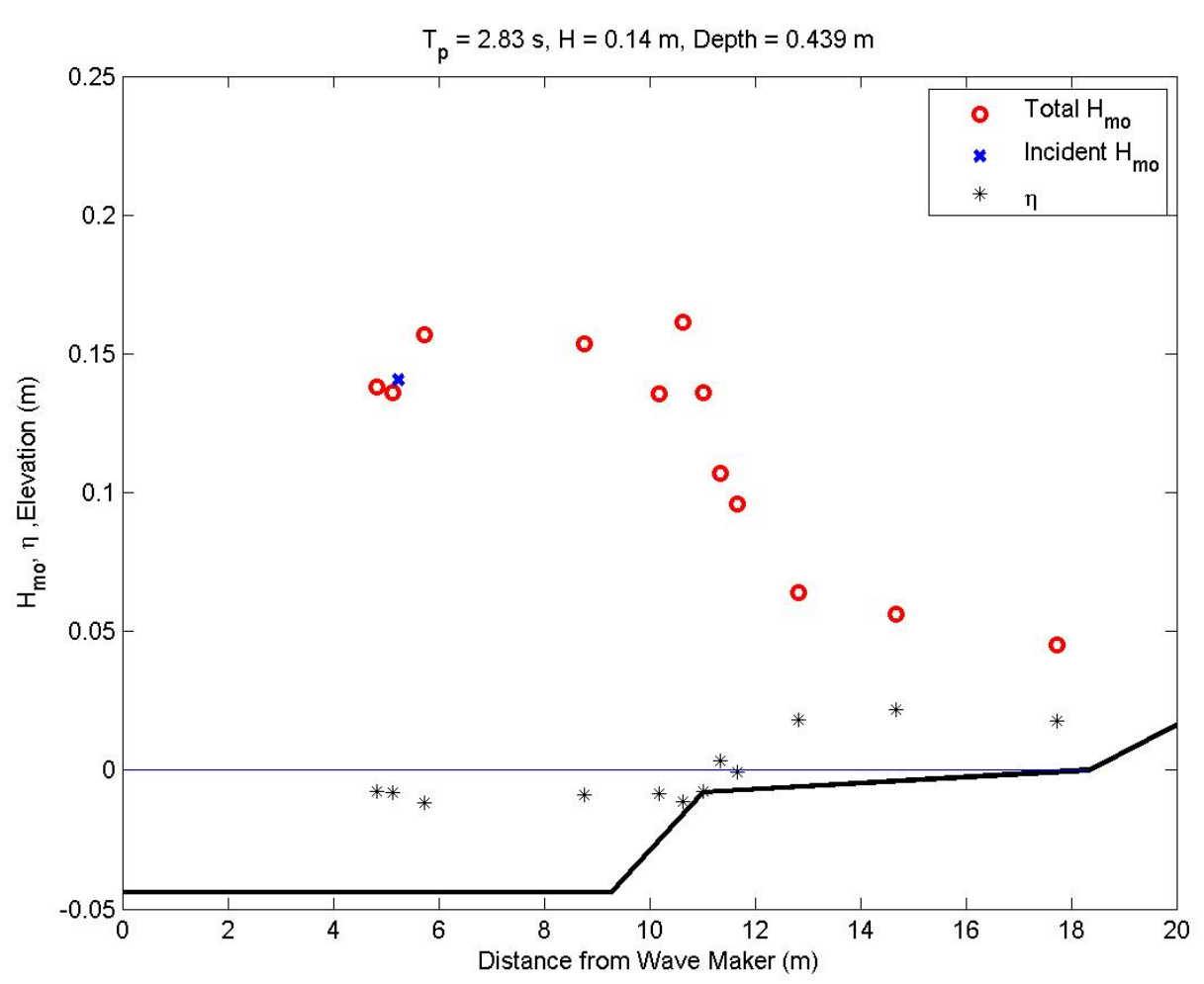

Figure D70. Wave transformation for 2.8-sec, 0.14-m waves at mid water with a 1:5 reef slope.

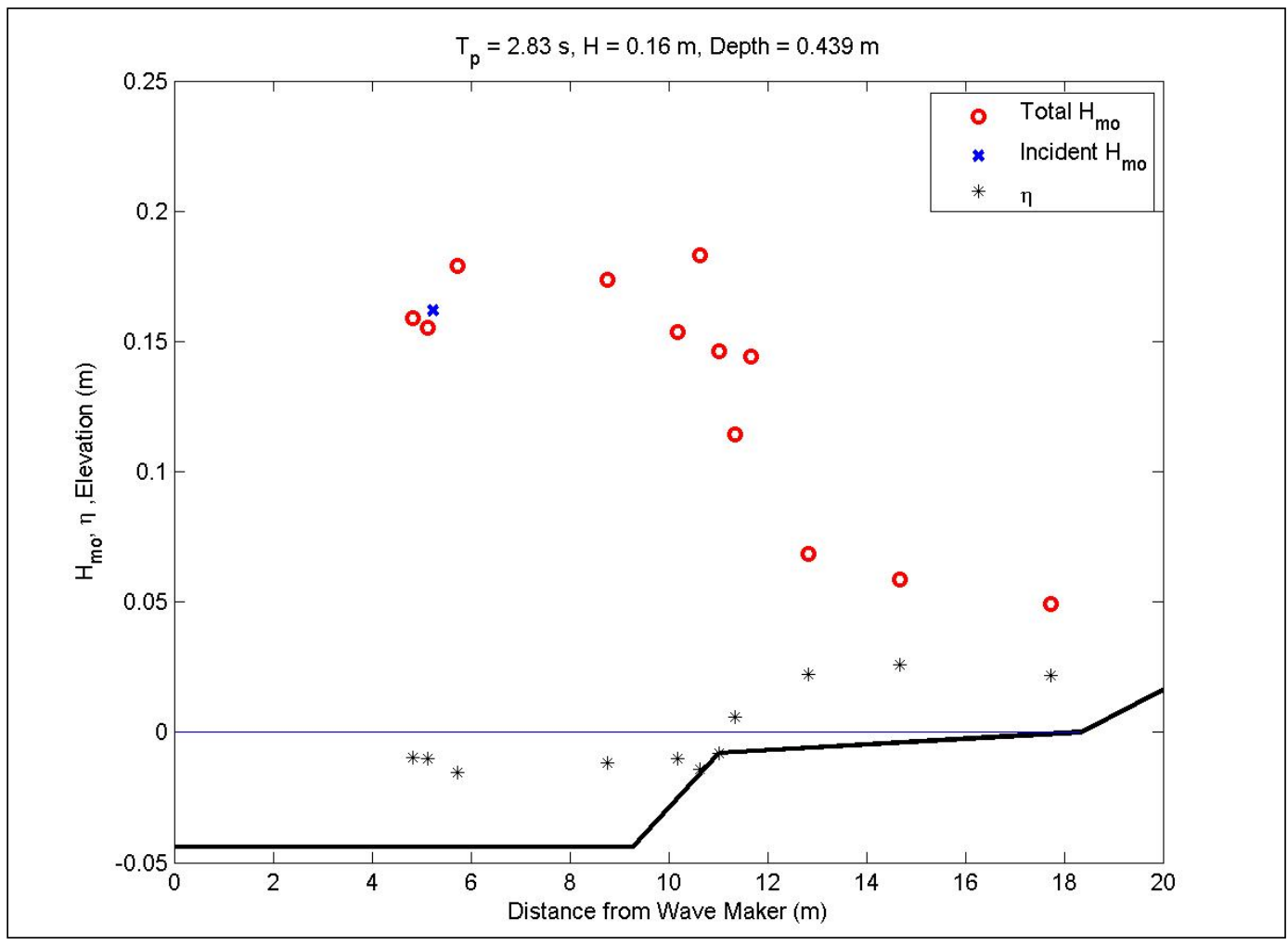

Figure D71. Wave transformation for 2.8-sec, 0.16-m waves at mid water with a 1:5 reef slope. 


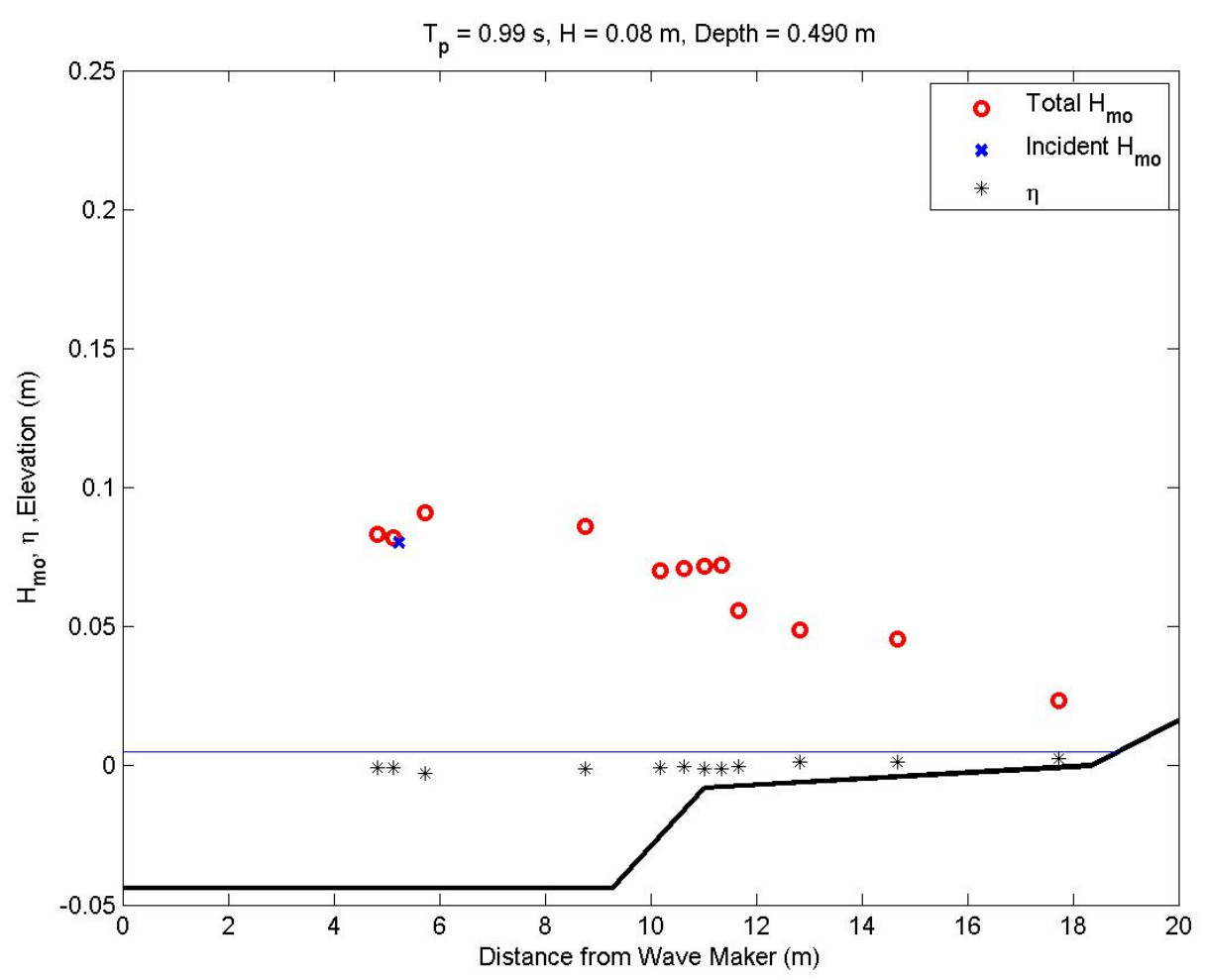

Figure D72. Wave transformation for 1-sec, 0.08-m waves at high water with a 1:5 reef slope.

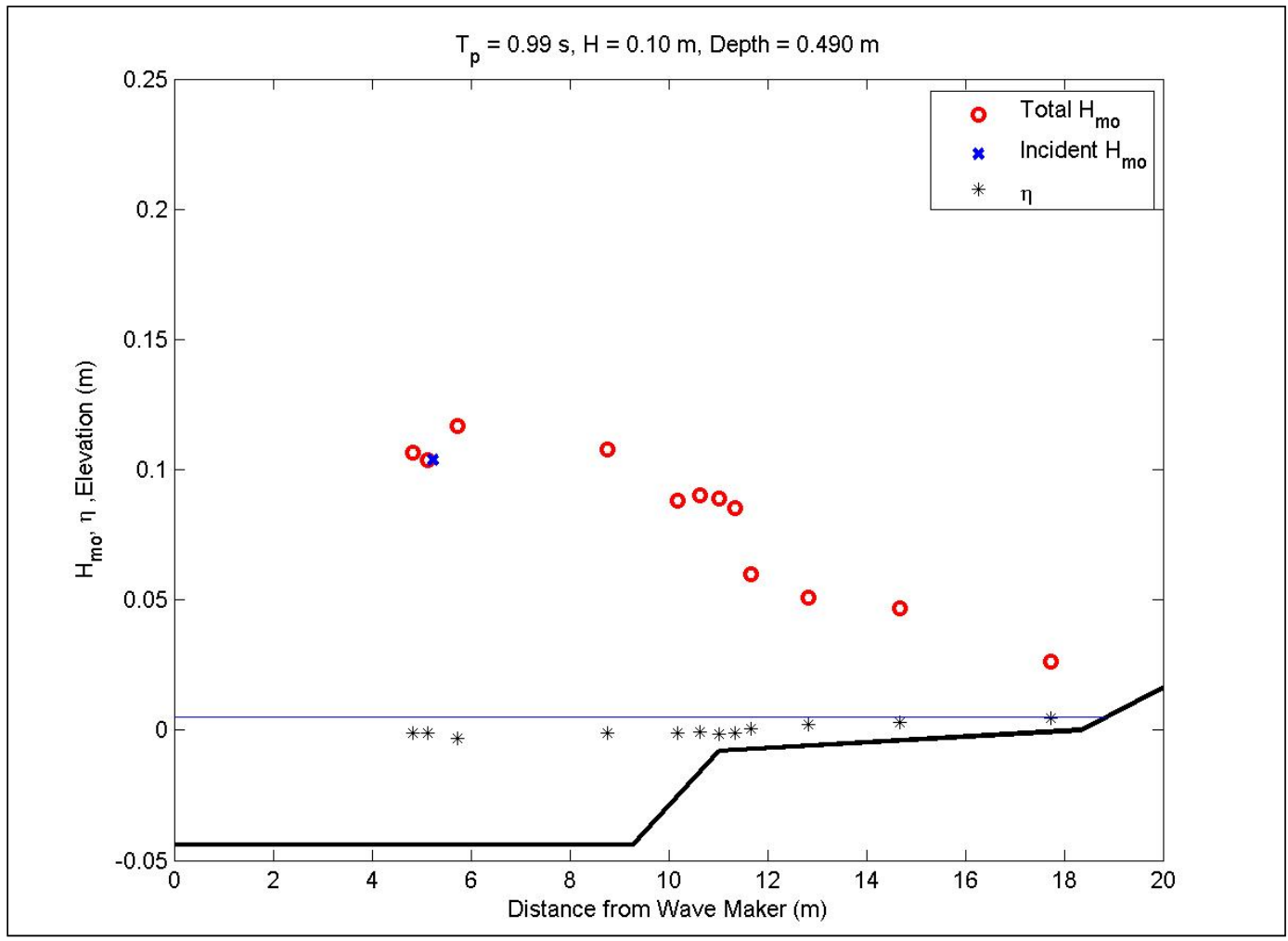

Figure D73. Wave transformation for 1-sec, 0.1-m waves at high water with a 1:5 reef slope. 


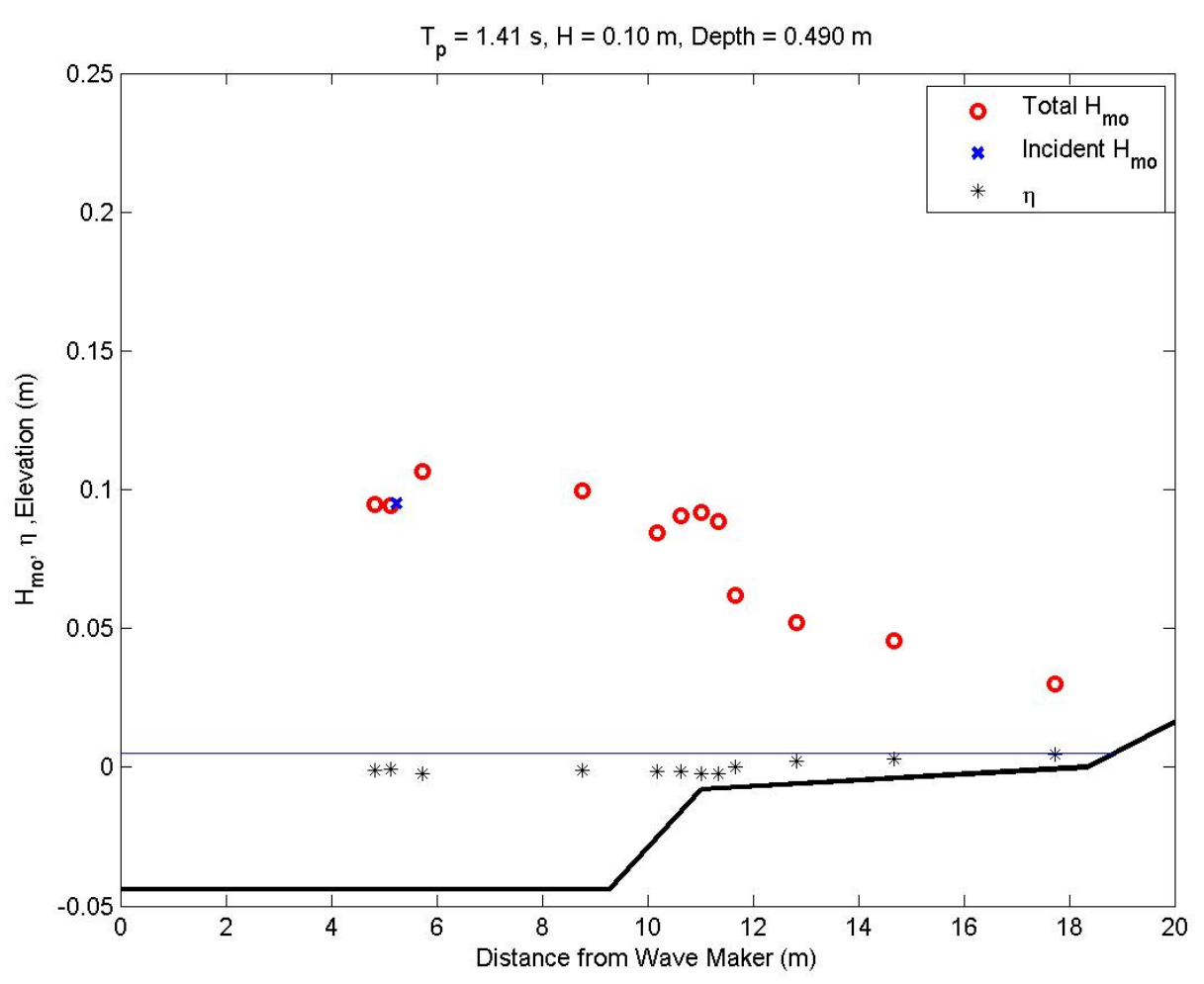

Figure D74. Wave transformation for 1.4-sec, 0.1-m waves at high water with a 1:5 reef slope.

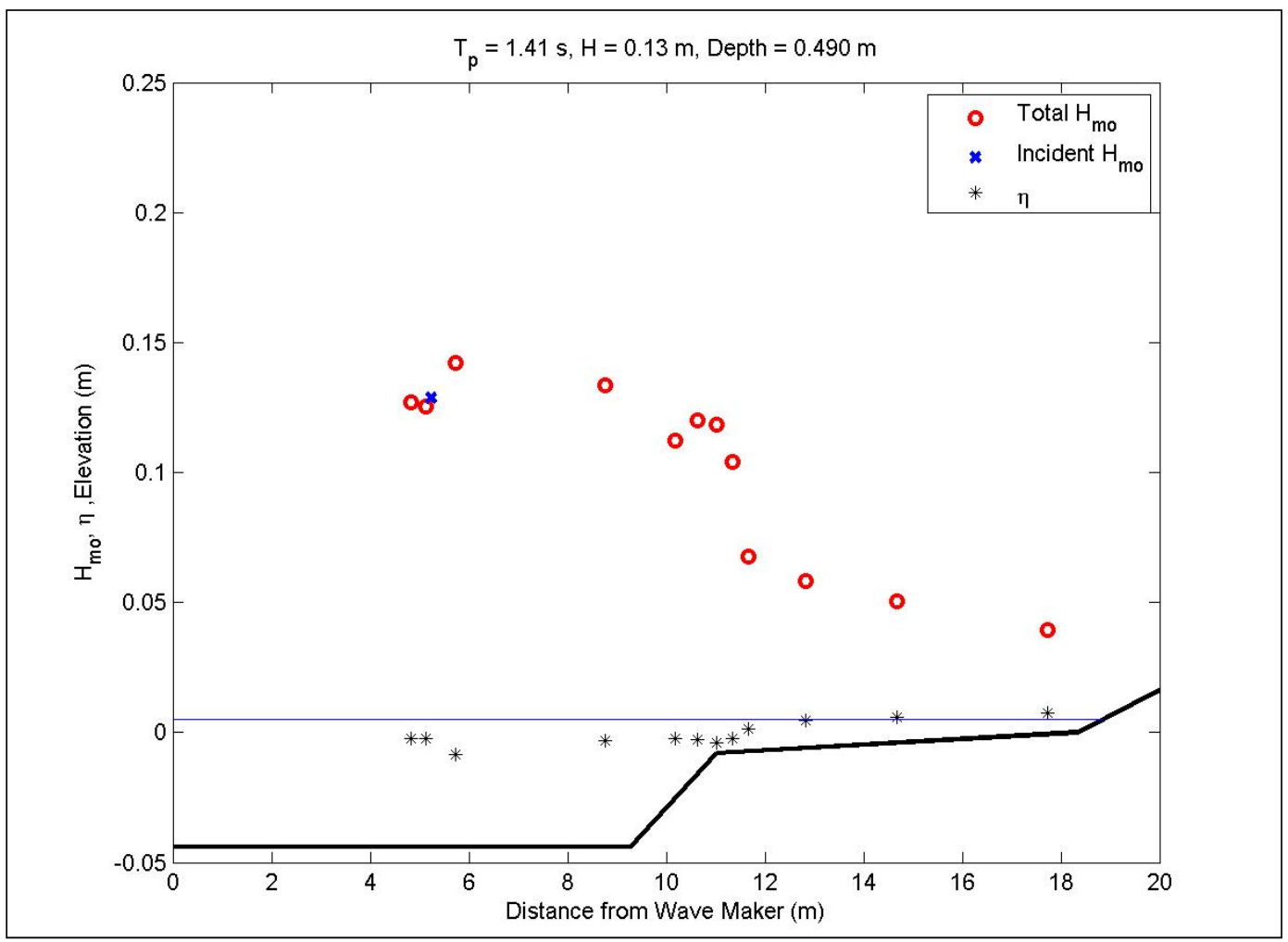

Figure D75. Wave transformation for 1.4-sec, 0.13-m waves at high water with a 1:5 reef slope. 


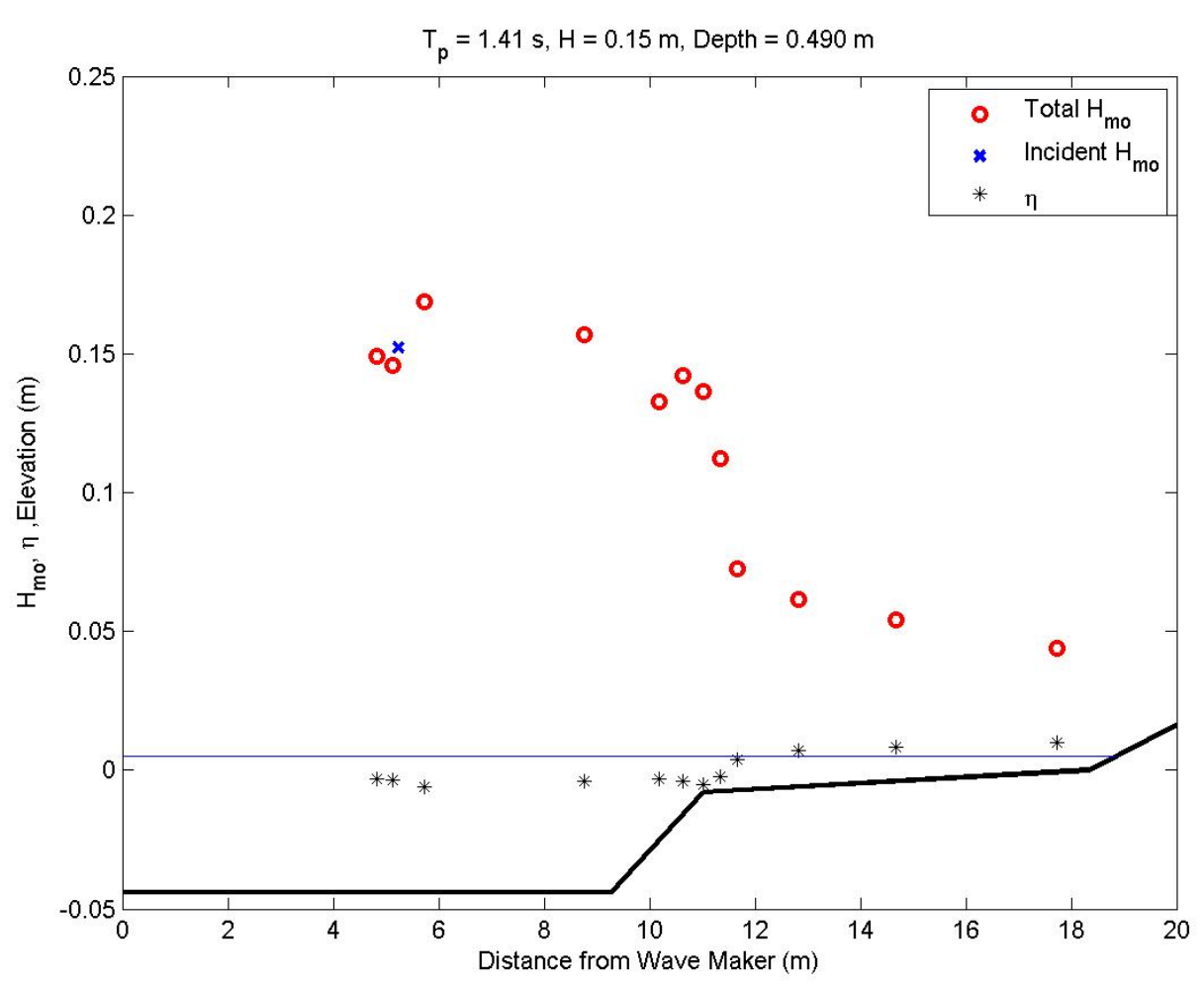

Figure D76. Wave transformation for 1.4-sec, 0.15-m waves at high water with a 1:5 reef slope.

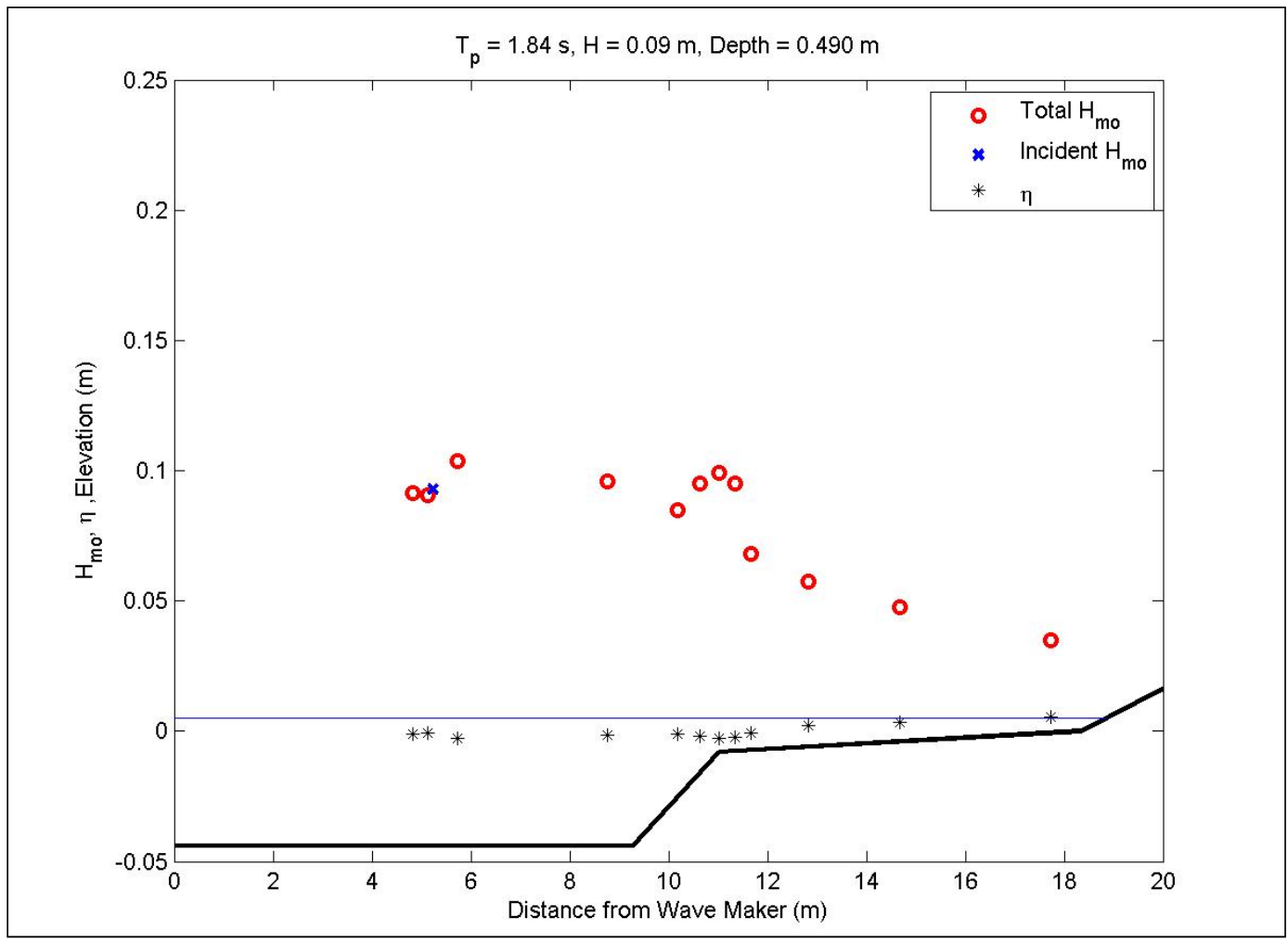

Figure D77. Wave transformation for 1.8-sec, 0.09-m waves at high water with a 1:5 reef slope. 


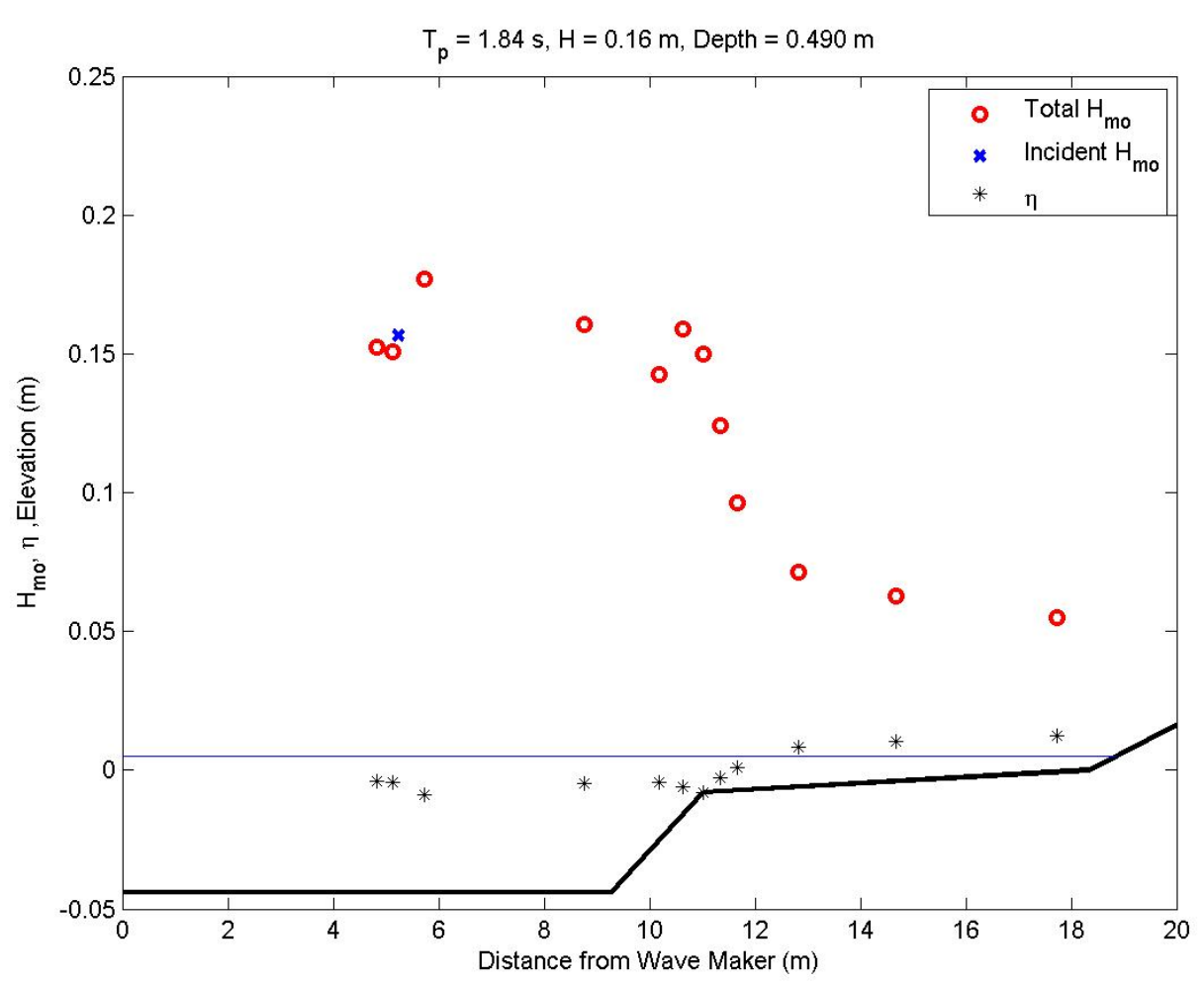

Figure D78. Wave transformation for $1.8-\mathrm{sec}, 0.16-\mathrm{m}$ waves at high water with a 1:5 reef slope.

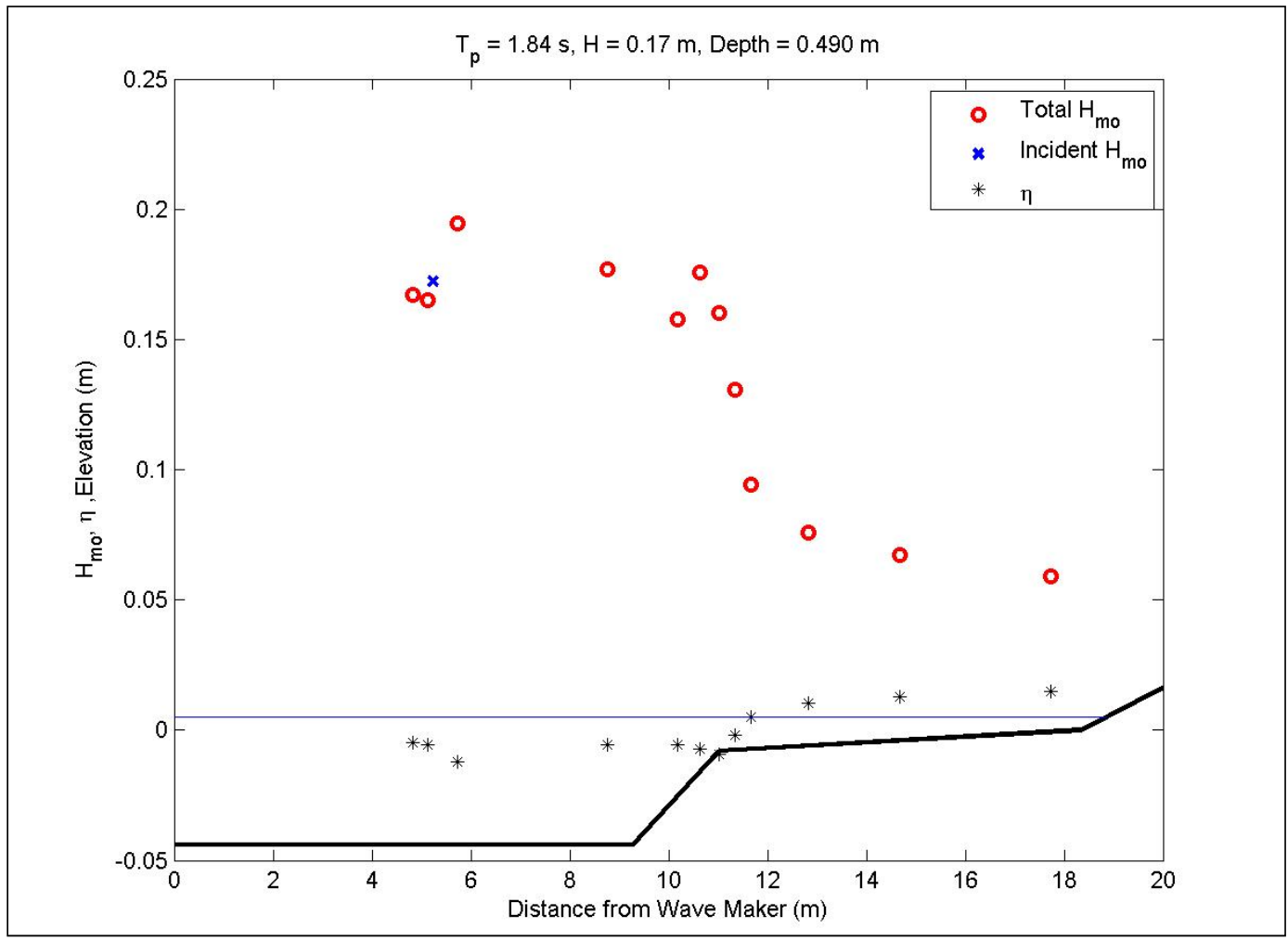

Figure D79. Wave transformation for 1.8-sec, 0.17-m waves at high water with a 1:5 reef slope. 


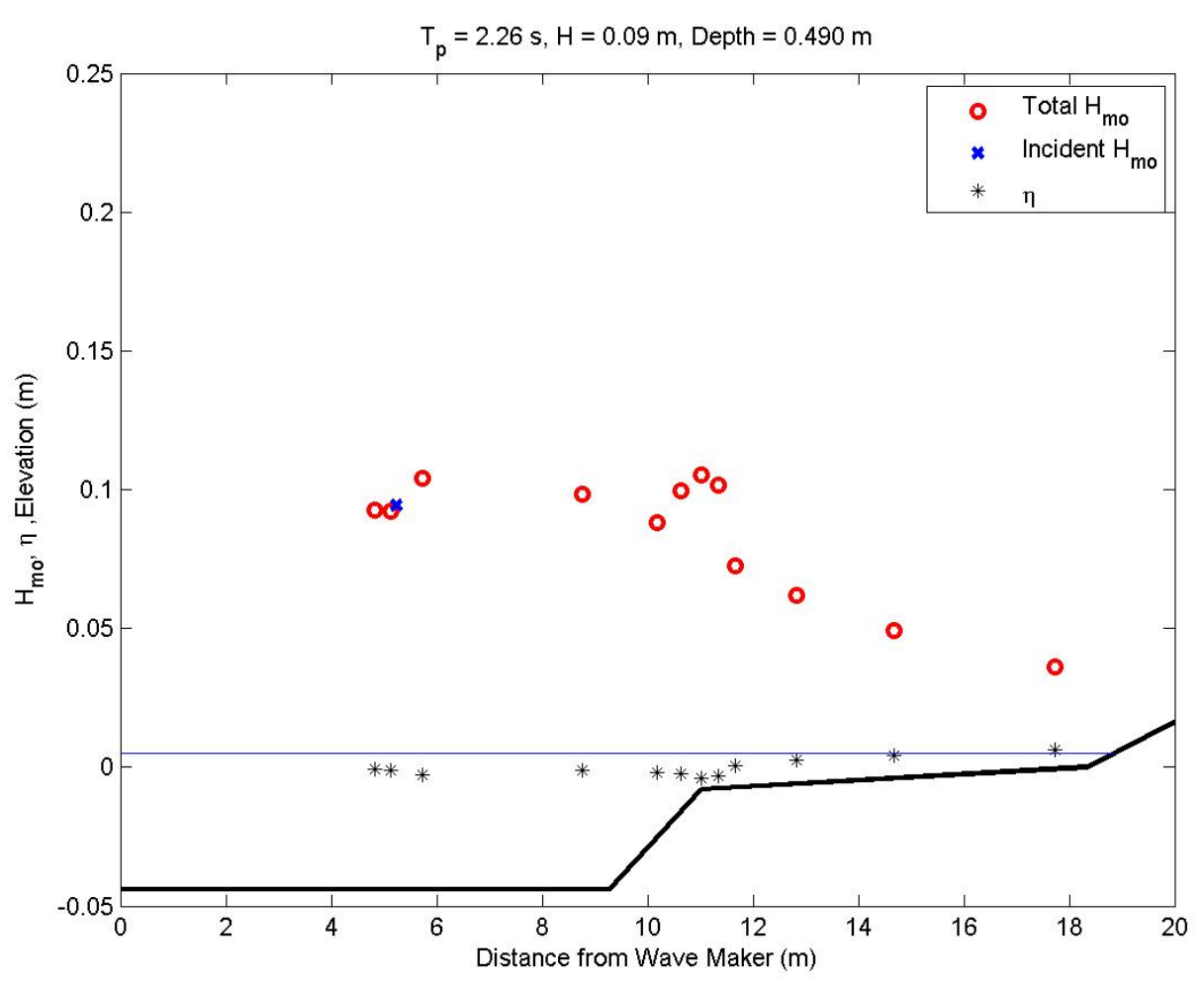

Figure D80. Wave transformation for 2.3-sec, 0.09-m waves at high water with a 1:5 reef slope.

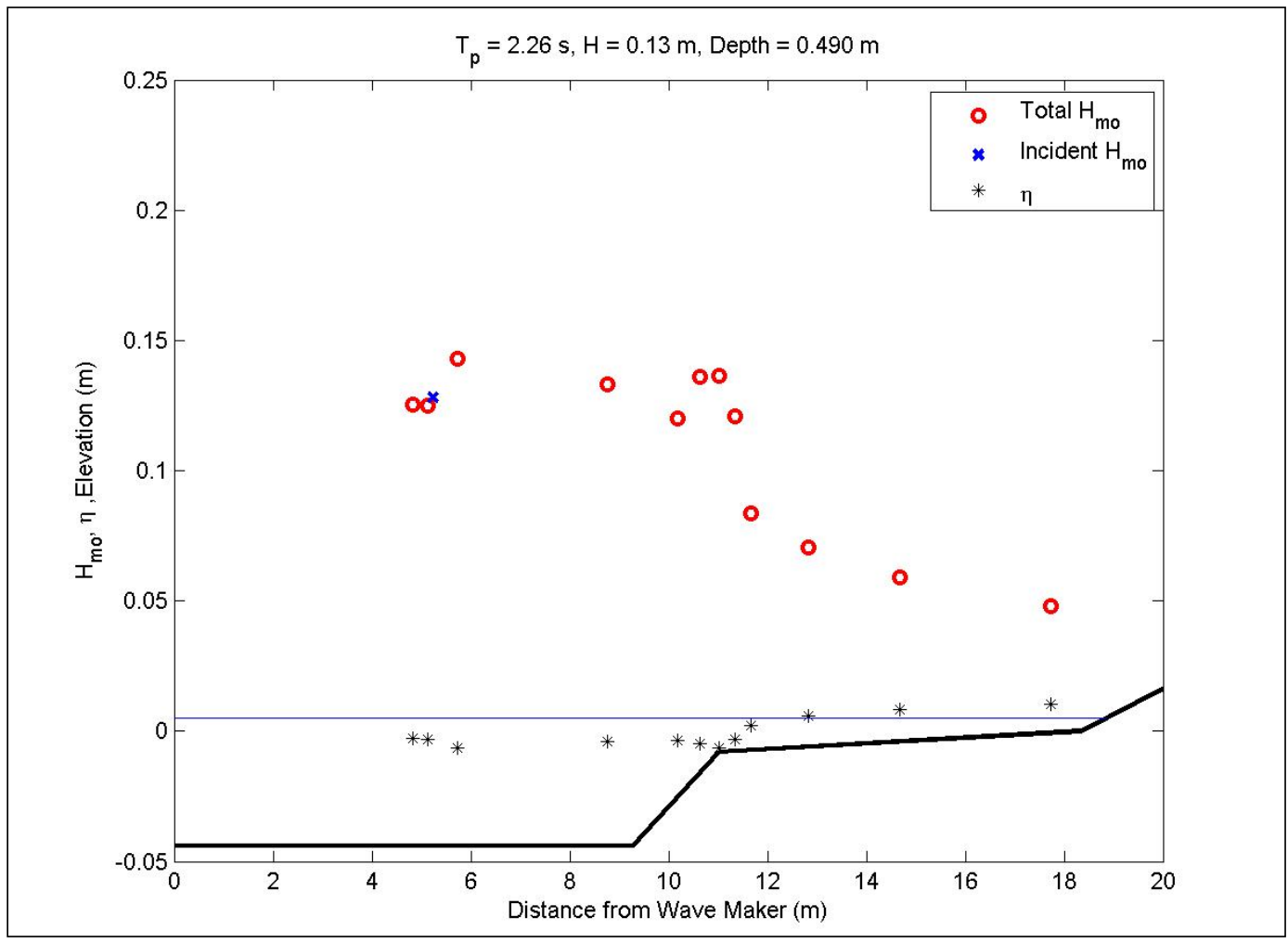

Figure D81. Wave transformation for 2.3-sec, 0.13-m waves at high water with a 1:5 reef slope. 


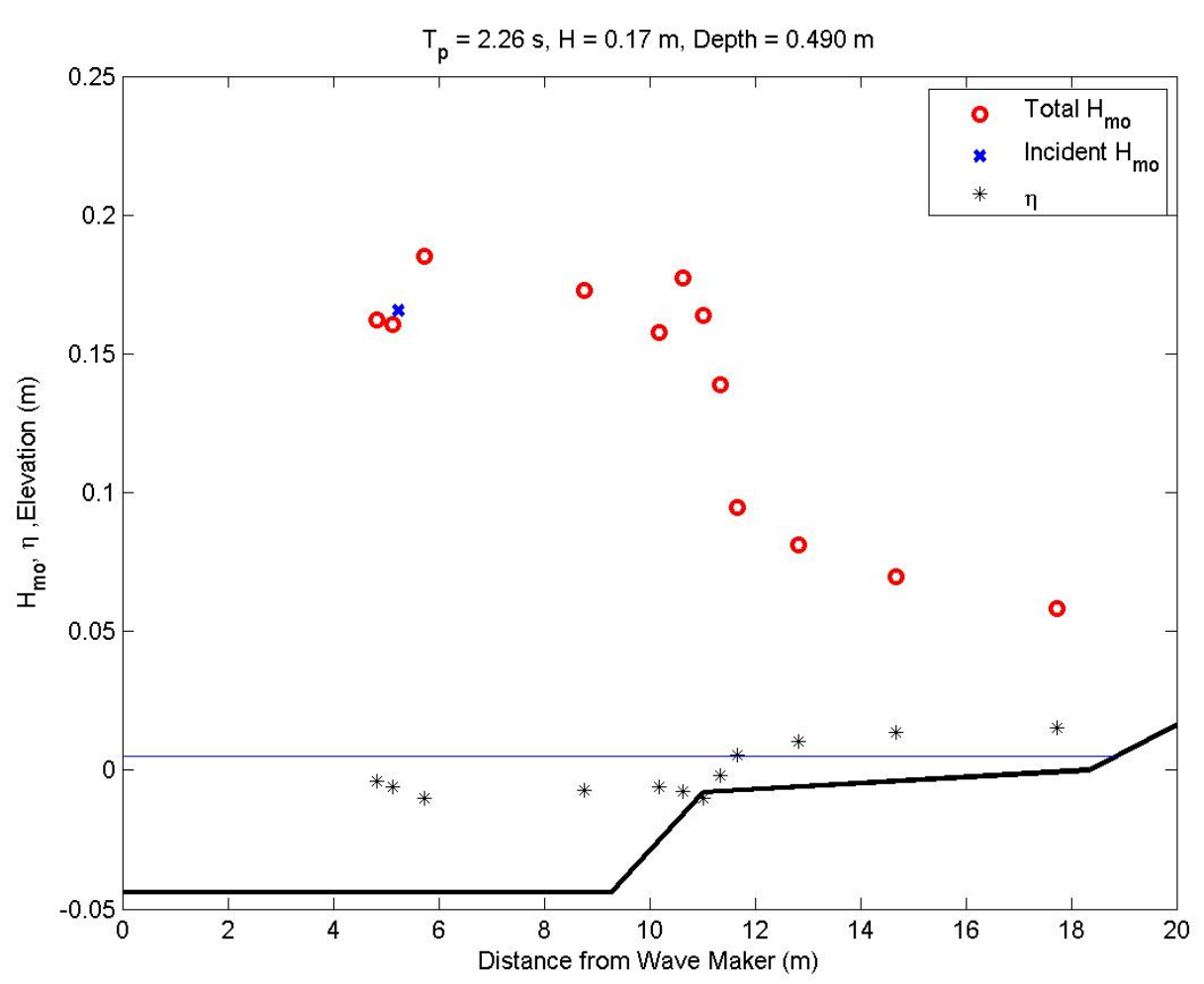

Figure D82. Wave transformation for 2.3-sec, 0.17-m waves at high water with a 1:5 reef slope.

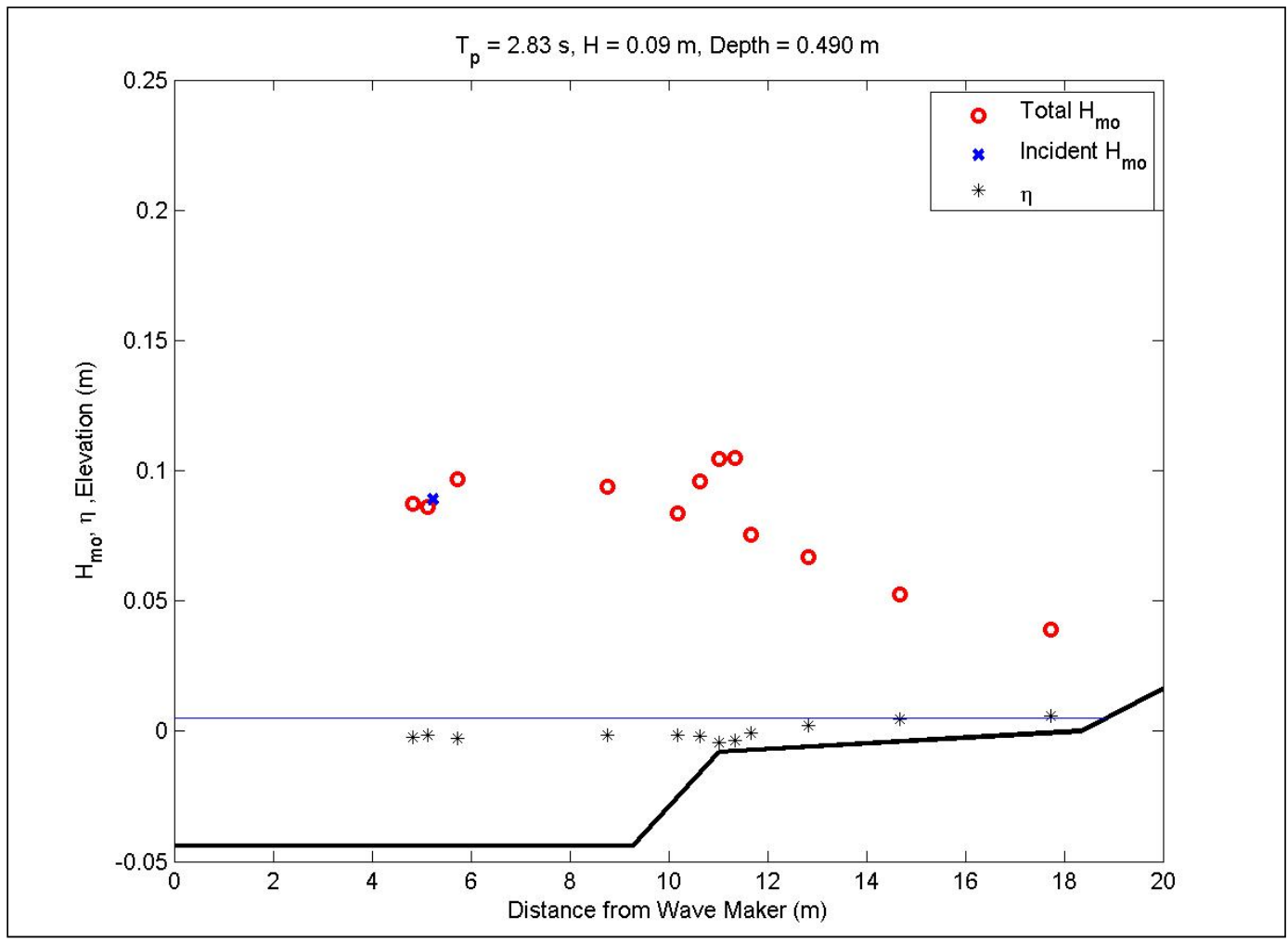

Figure D83. Wave transformation for 2.8-sec, 0.09-m waves at high water with a 1:5 reef slope. 


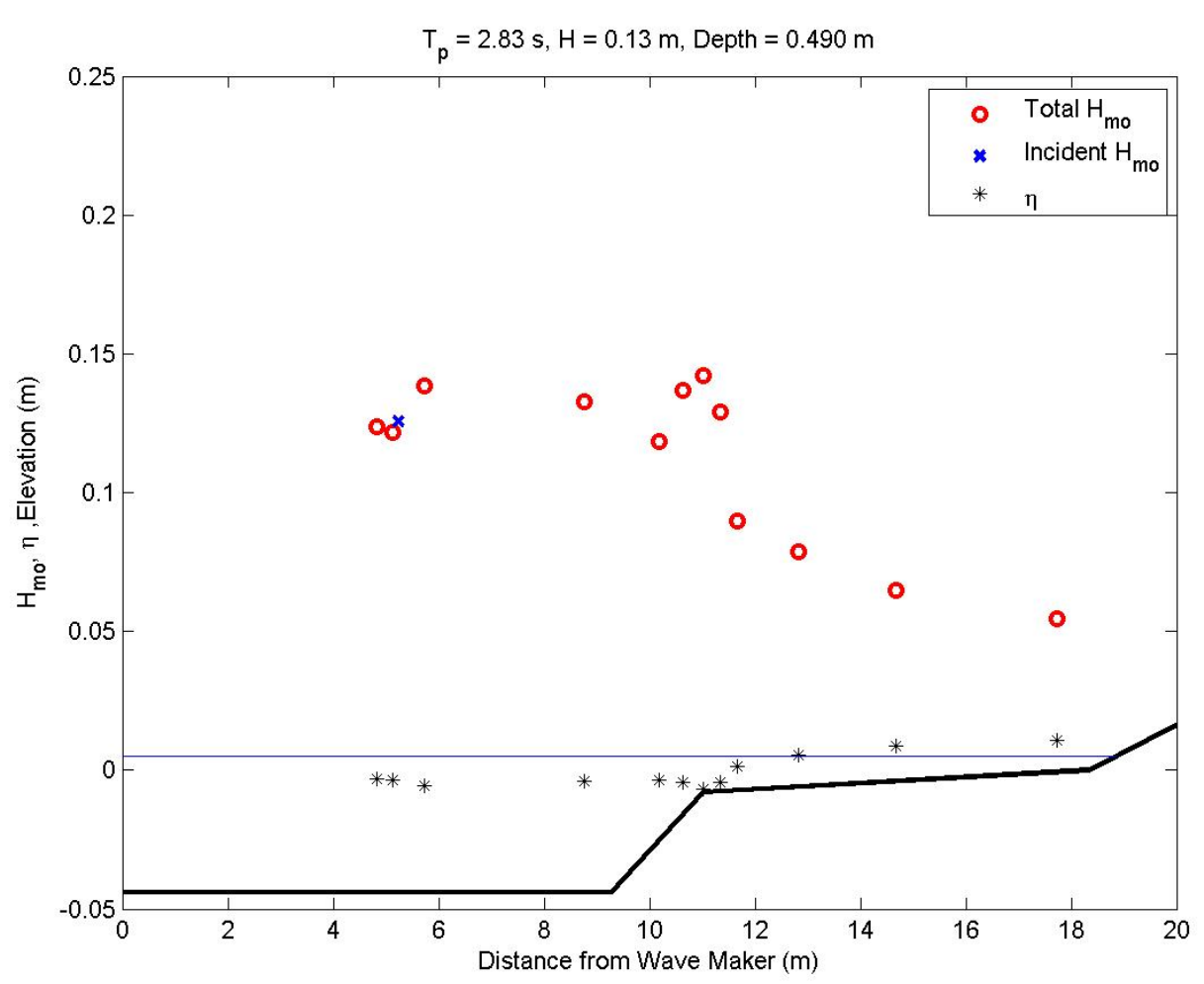

Figure D84. Wave transformation for 2.8-sec, 0.13-m waves at high water with a 1:5 reef slope.

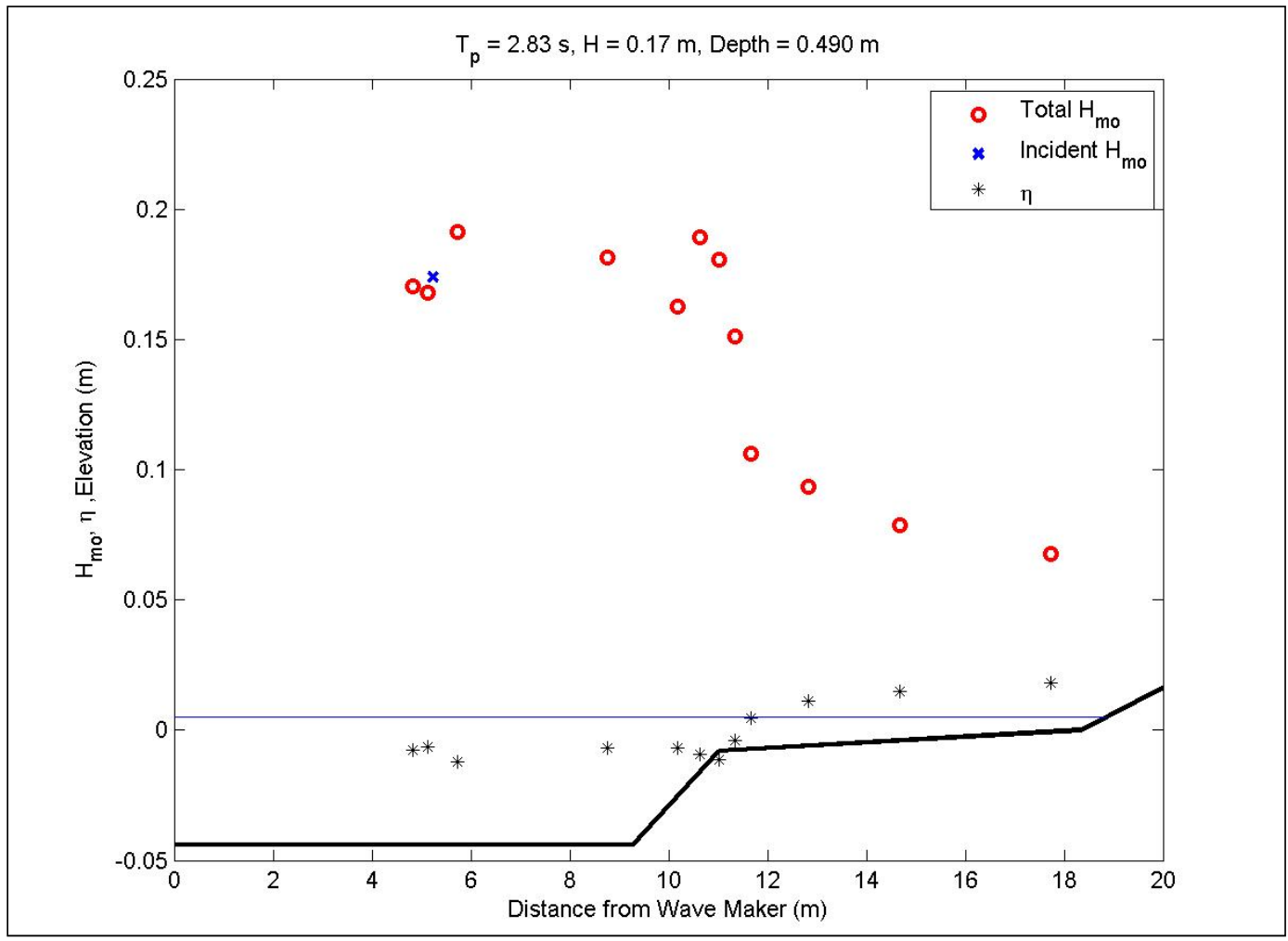

Figure D85. Wave transformation for 2.8-sec, 0.17-m waves at high water with a 1:5 reef slope. 


\section{Appendix E: Wave transformation figures from the two-dimensional experiments}

The figures presented in Appendix E show zero-moment wave heights and mean water levels as a function of distance in the 2-D wave tank. Each figure includes wave height and mean water level measured on the smooth and roughened bathymetry for similar wave conditions. The model bottom also is presented as a reference, but is scaled by a factor of 10 .

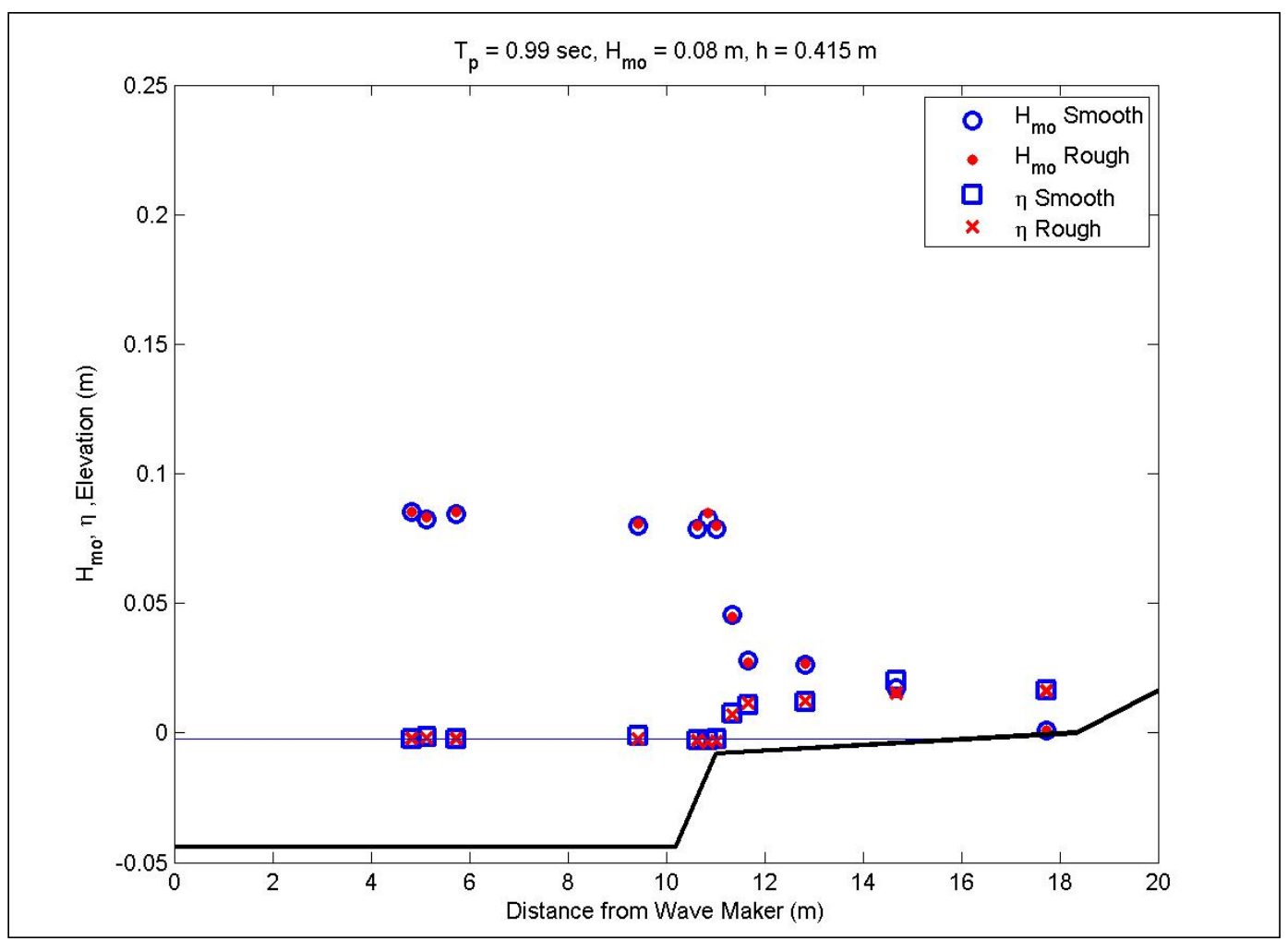

Figure E1. Wave transformation for 1-sec, 0.08-m waves at low water with a 1:25 reef slope. 


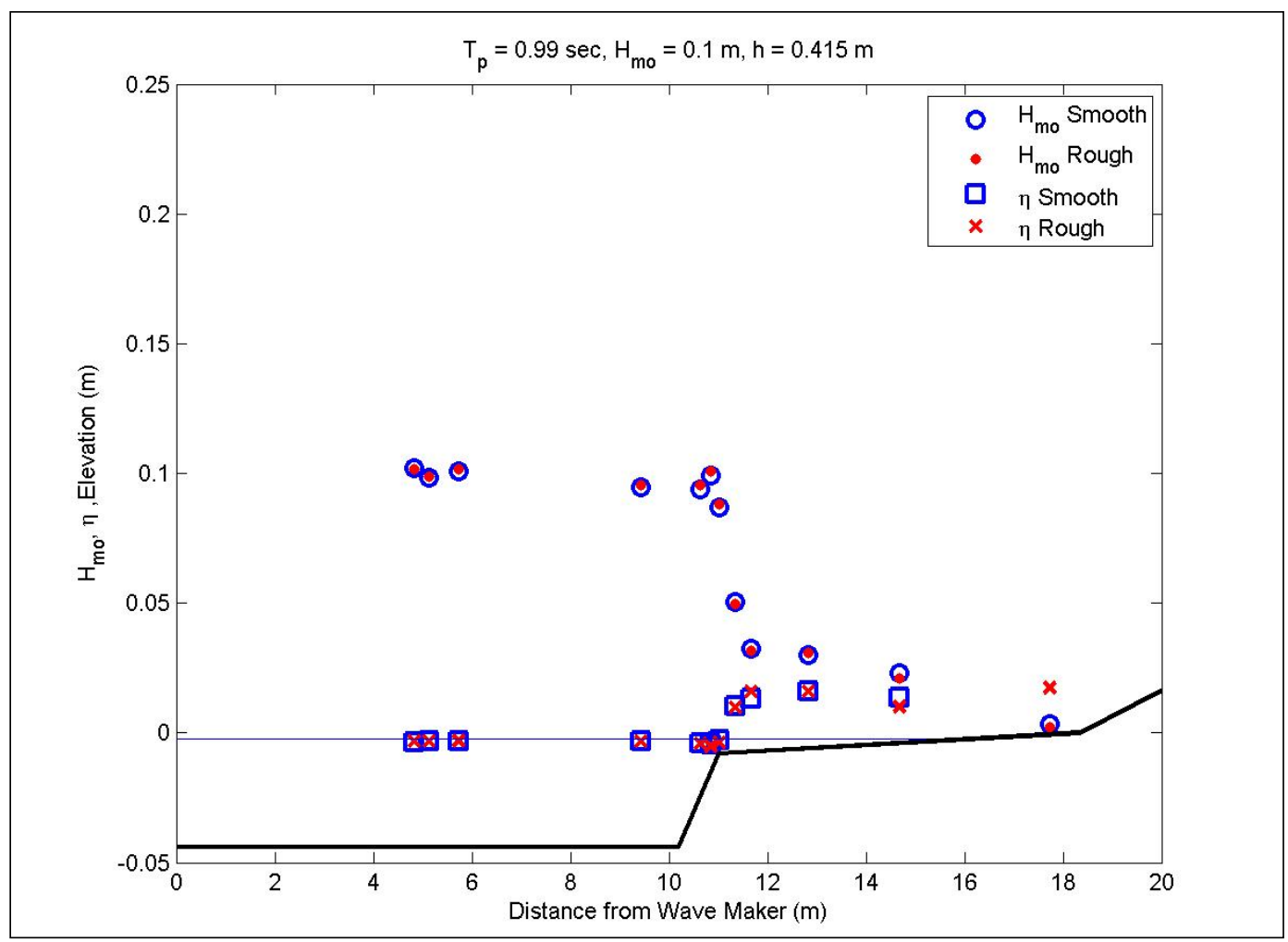

Figure E2. Wave transformation for 1-sec, 0.1-m waves at low water with a 1:25 reef slope.

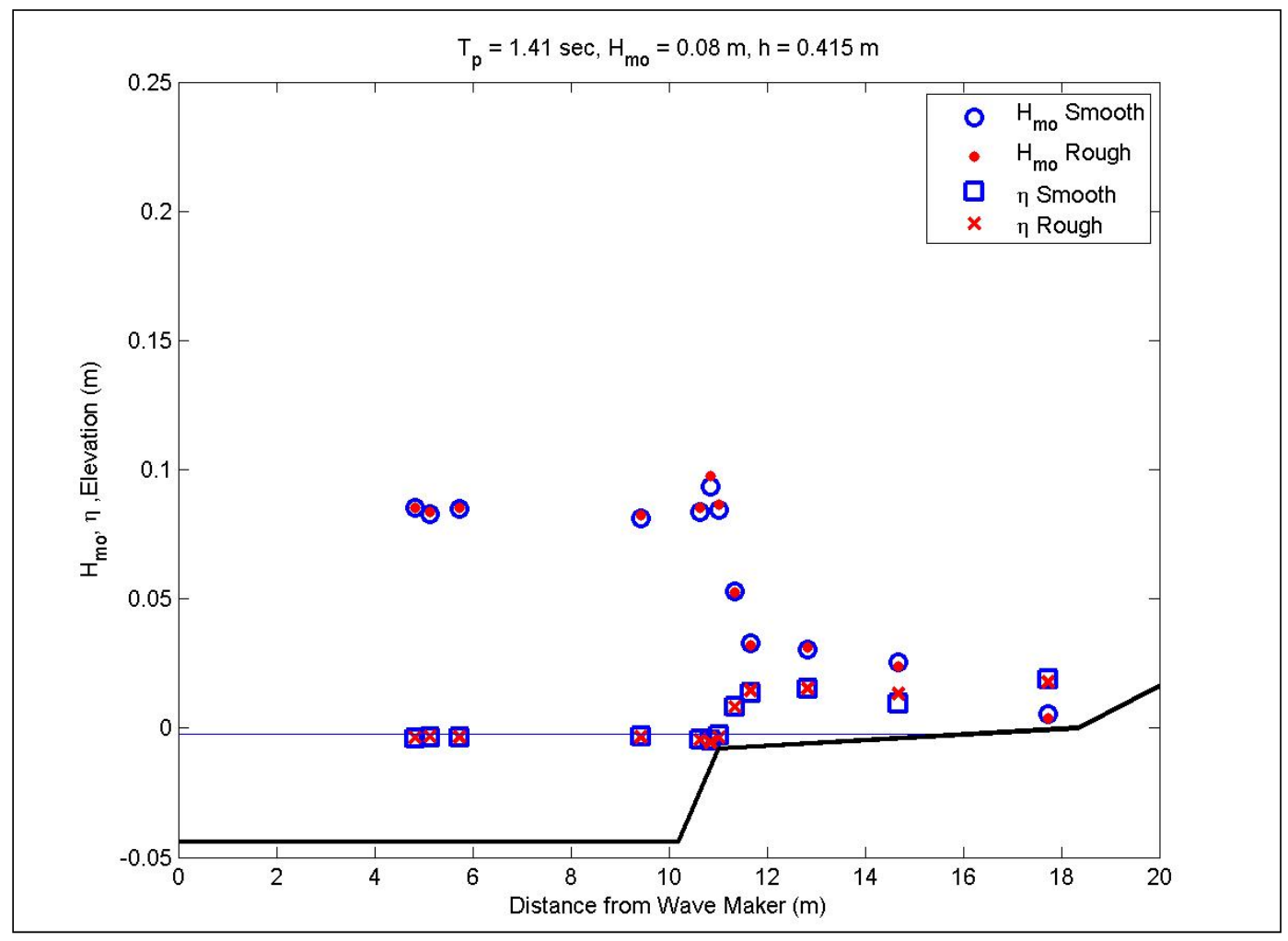

Figure E3. Wave transformation for 1.4-sec, 0.08-m waves at low water with a 1:25 reef slope. 


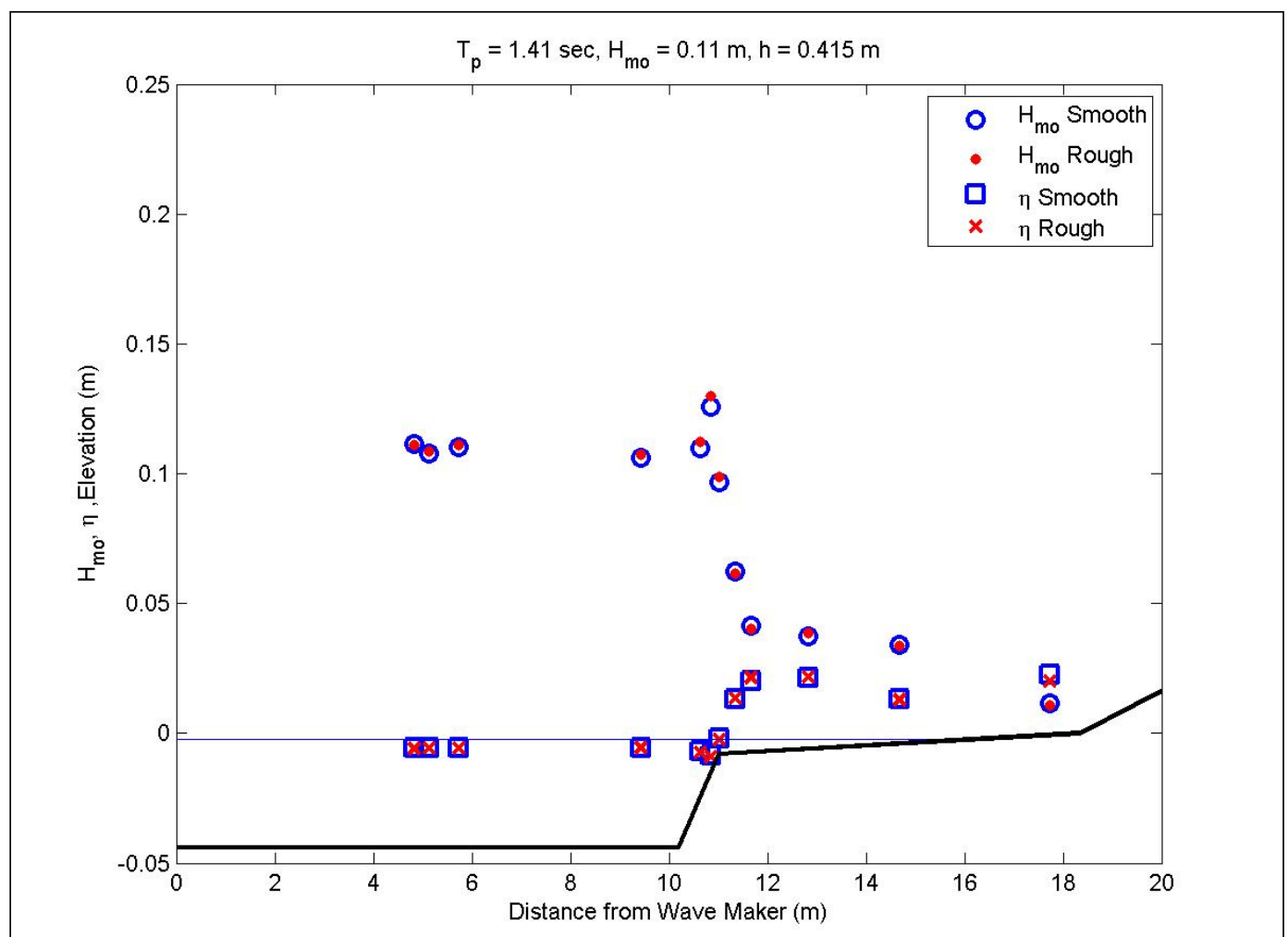

Figure E4. Wave transformation for 1.4-sec, 0.11-m waves at low water with a 1:25 reef slope.

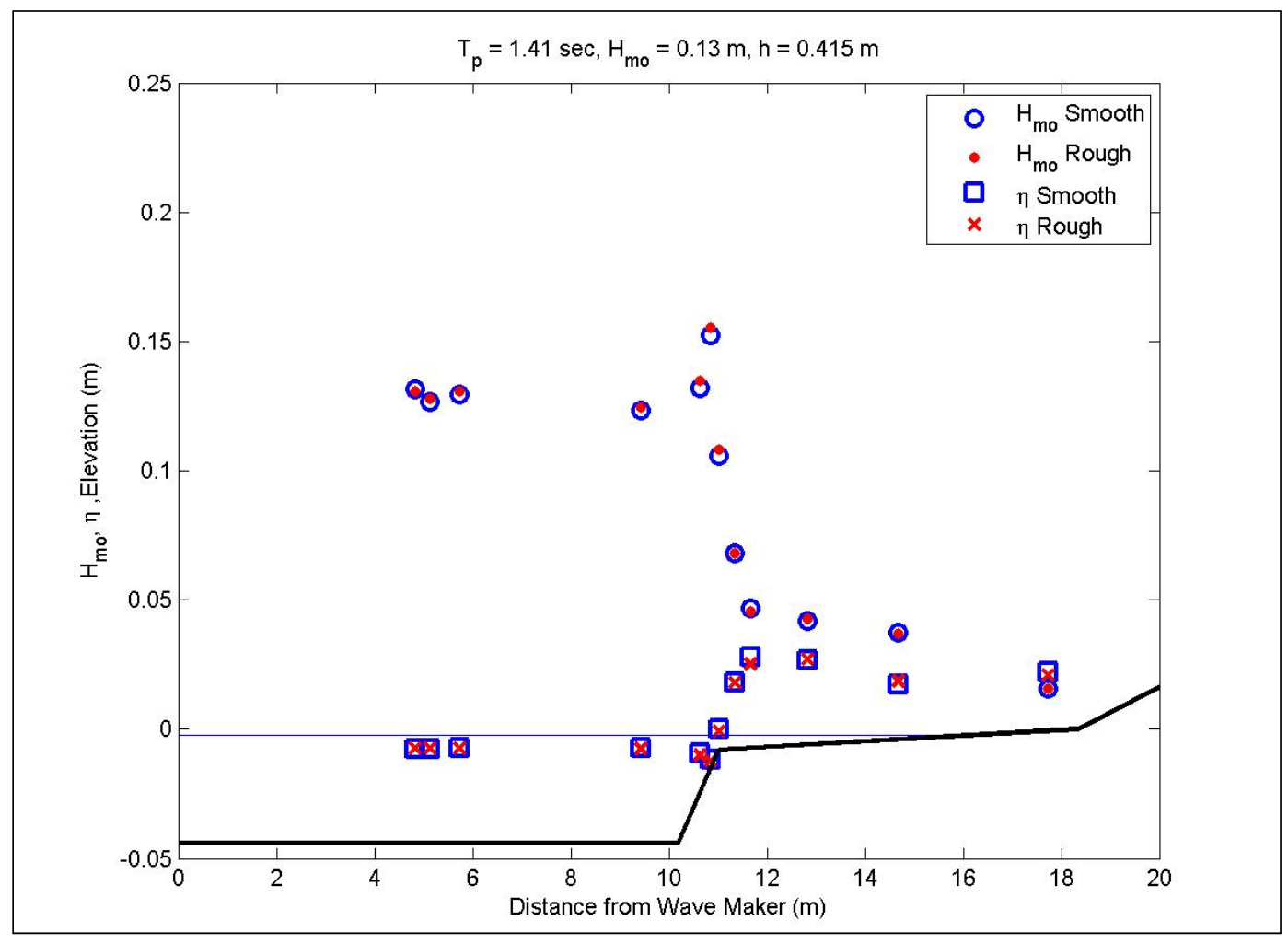

Figure E5. Wave transformation for 1.4-sec, 0.13-m waves at low water with a 1:25 reef slope. 


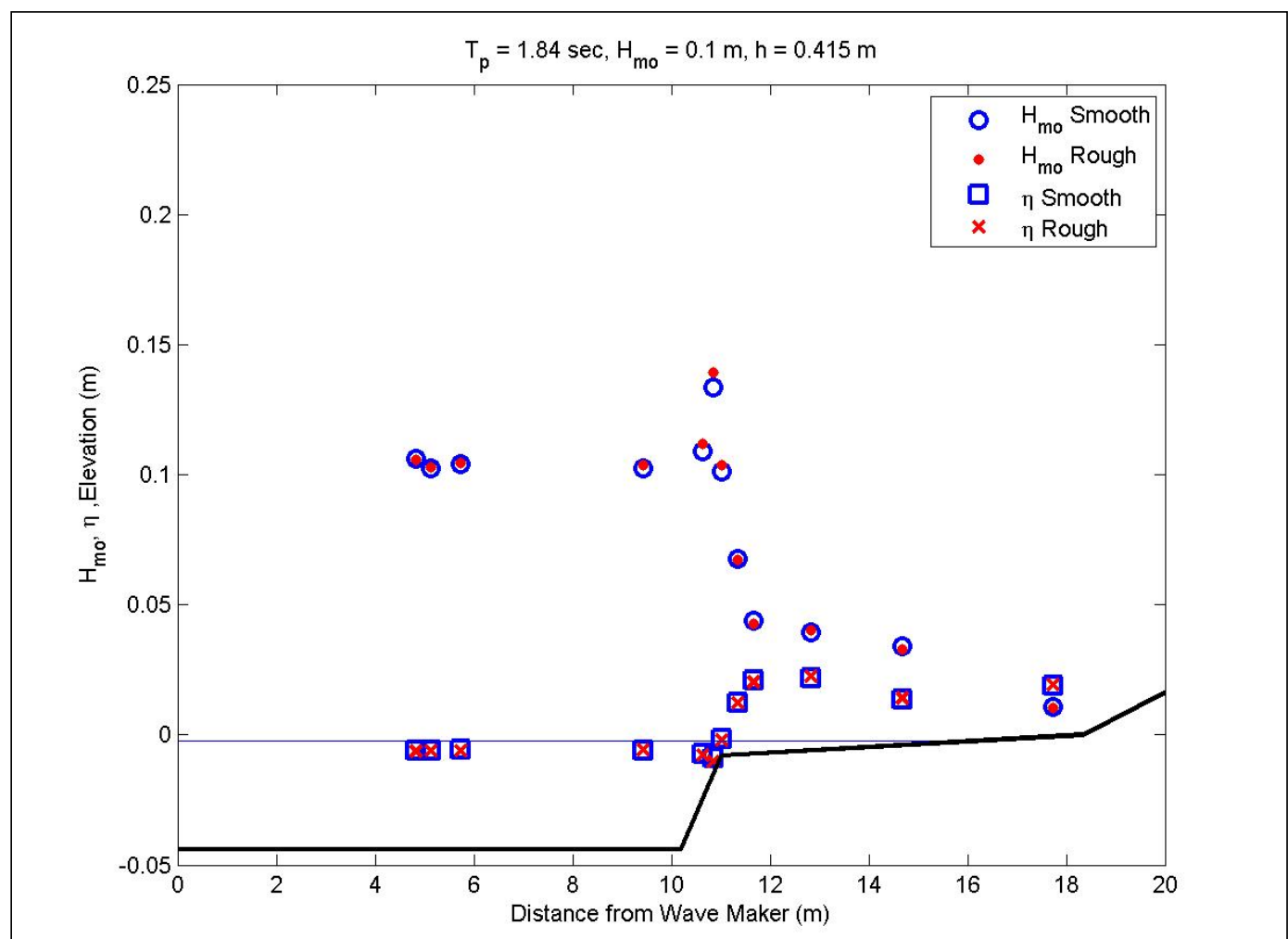

Figure E6. Wave transformation for 1.8-sec, 0.1-m waves at low water with a 1:25 reef slope.

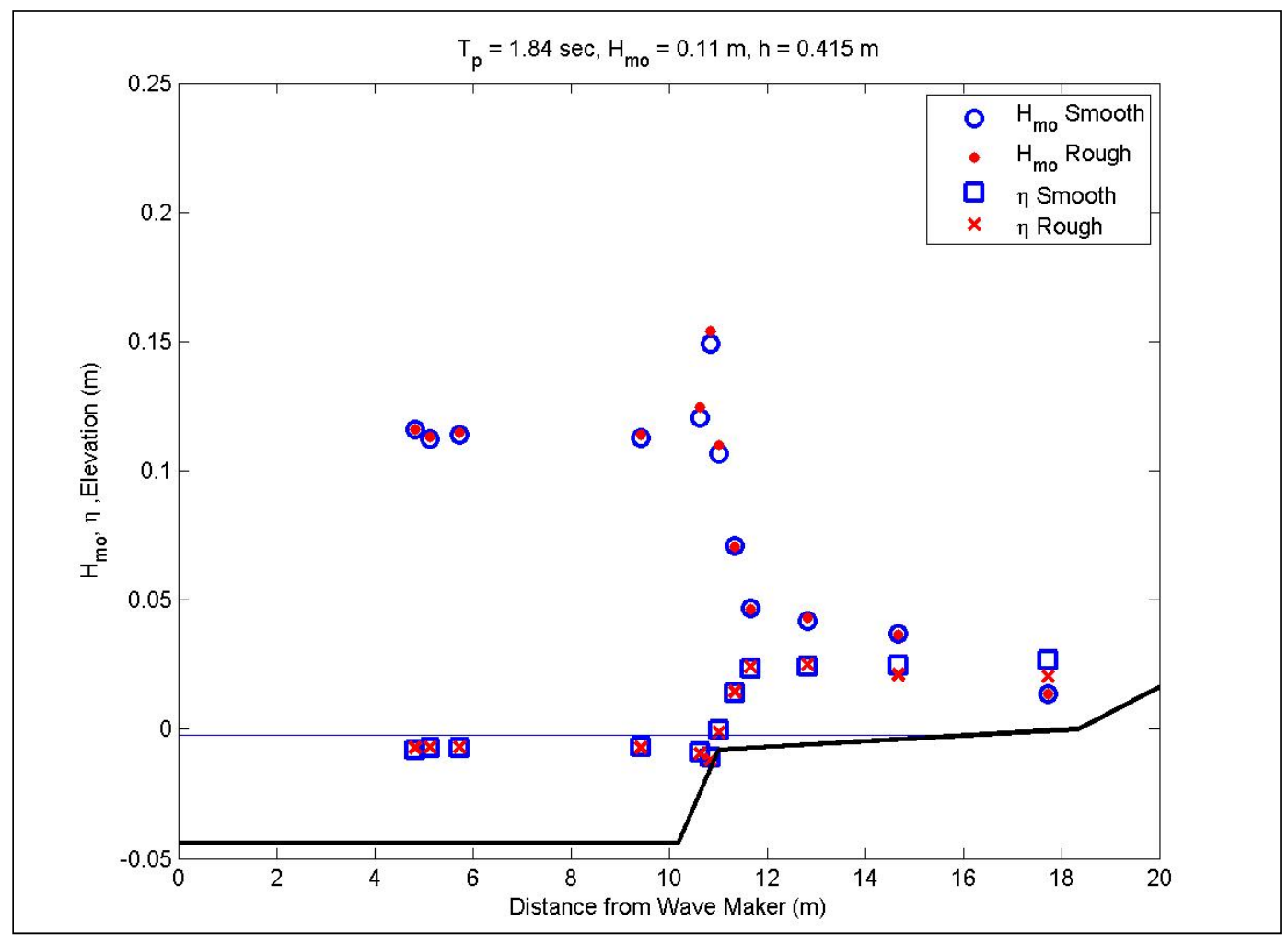

Figure E7. Wave transformation for 1.8-sec, 0.11-m waves at low water with a 1:25 reef slope. 


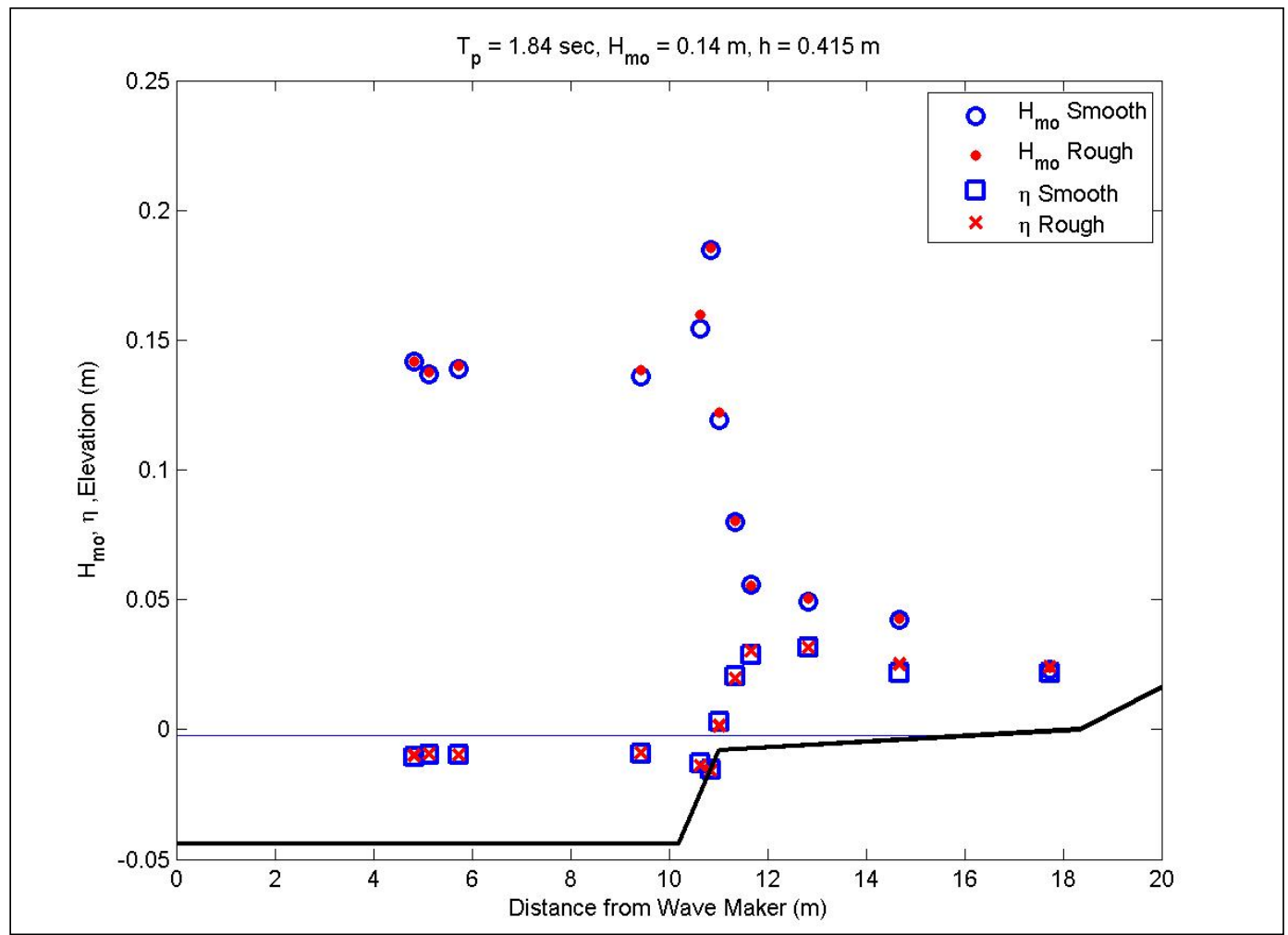

Figure E8. Wave transformation for 1.8-sec, 0.14-m waves at low water with a 1:25 reef slope.

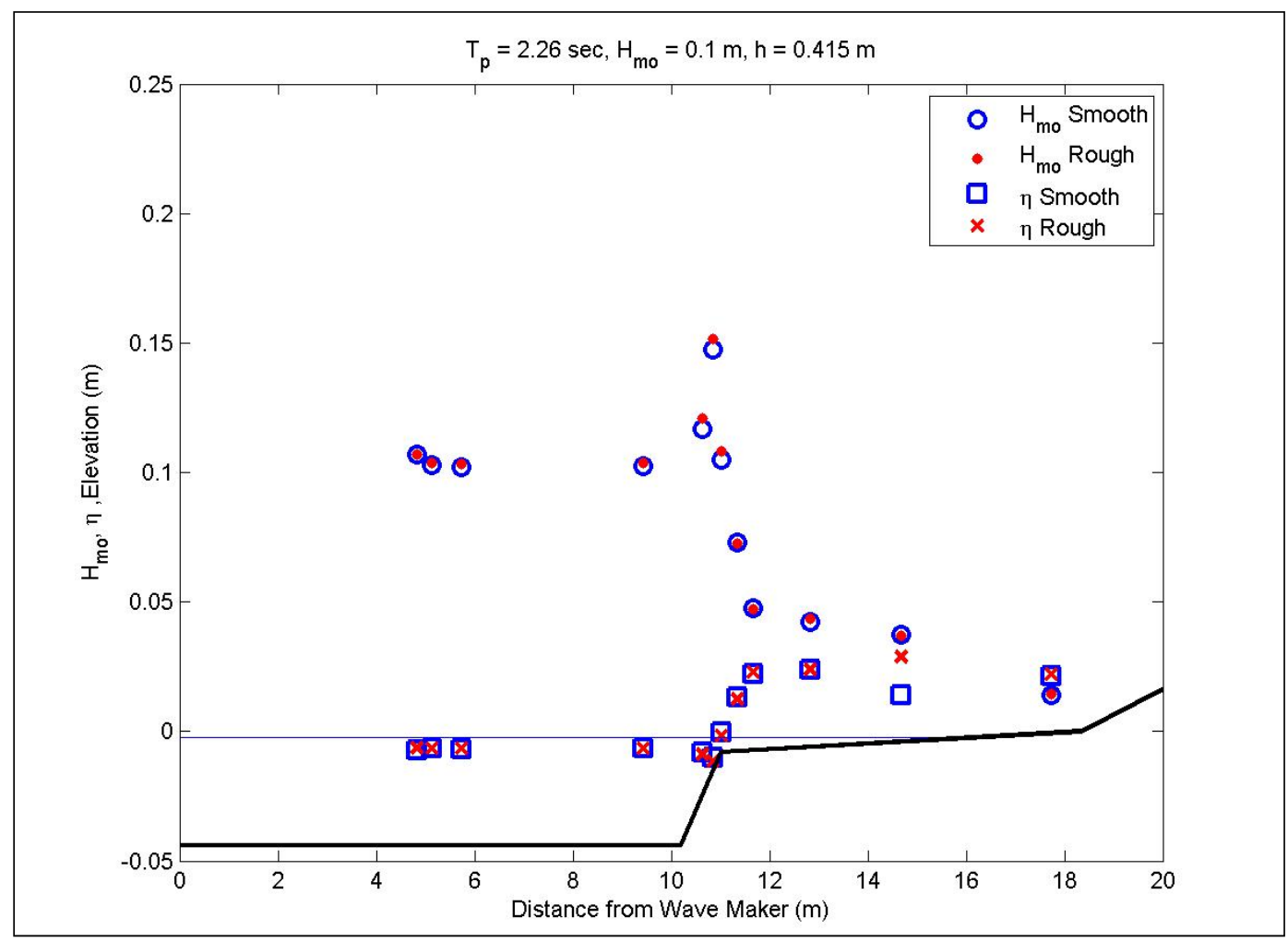

Figure E9. Wave transformation for 2.3-sec, 0.1-m waves at low water with a 1:25 reef slope. 


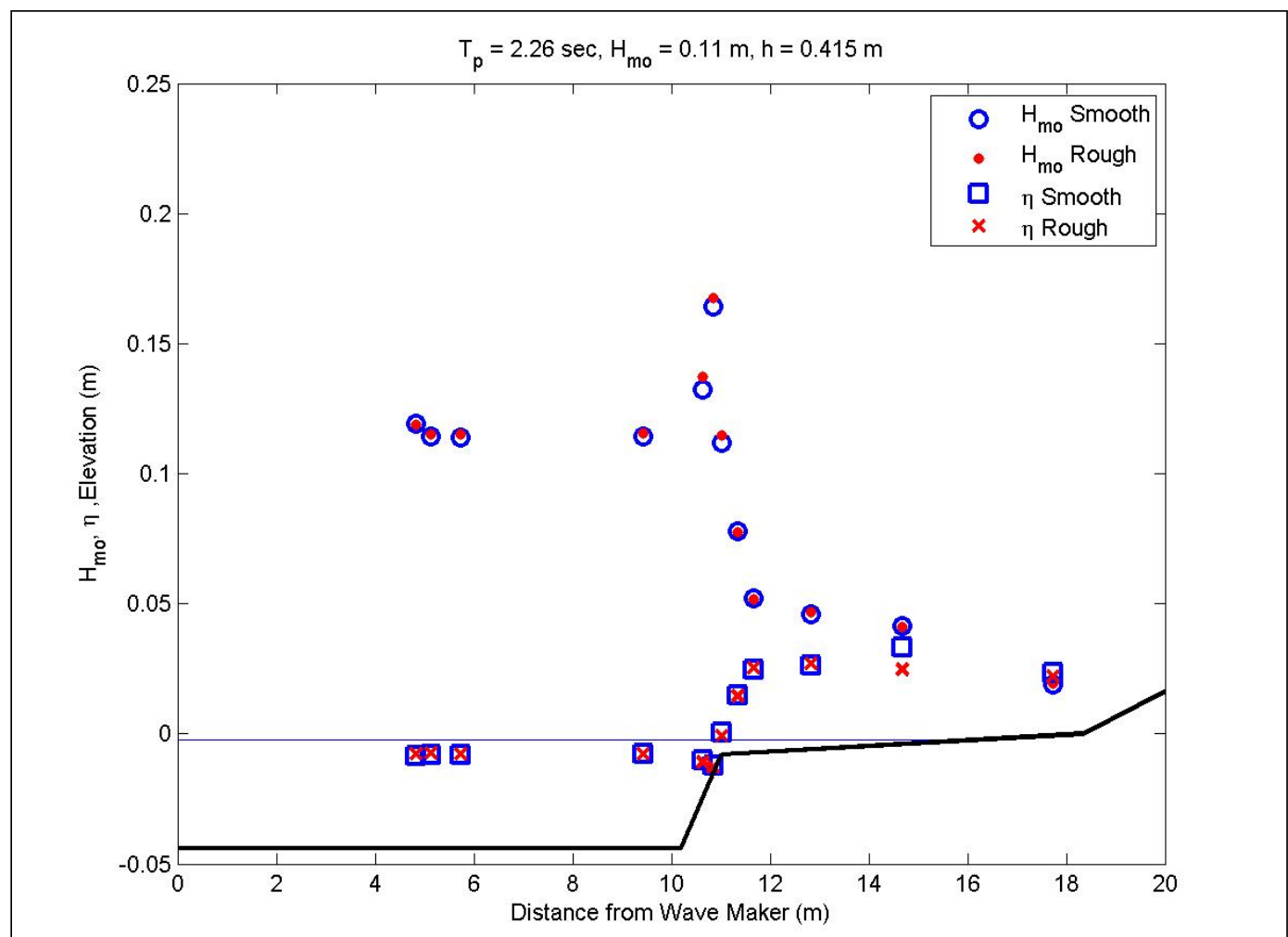

Figure E10. Wave transformation for 2.3-sec, 0.11-m waves at low water with a 1:25 reef slope.

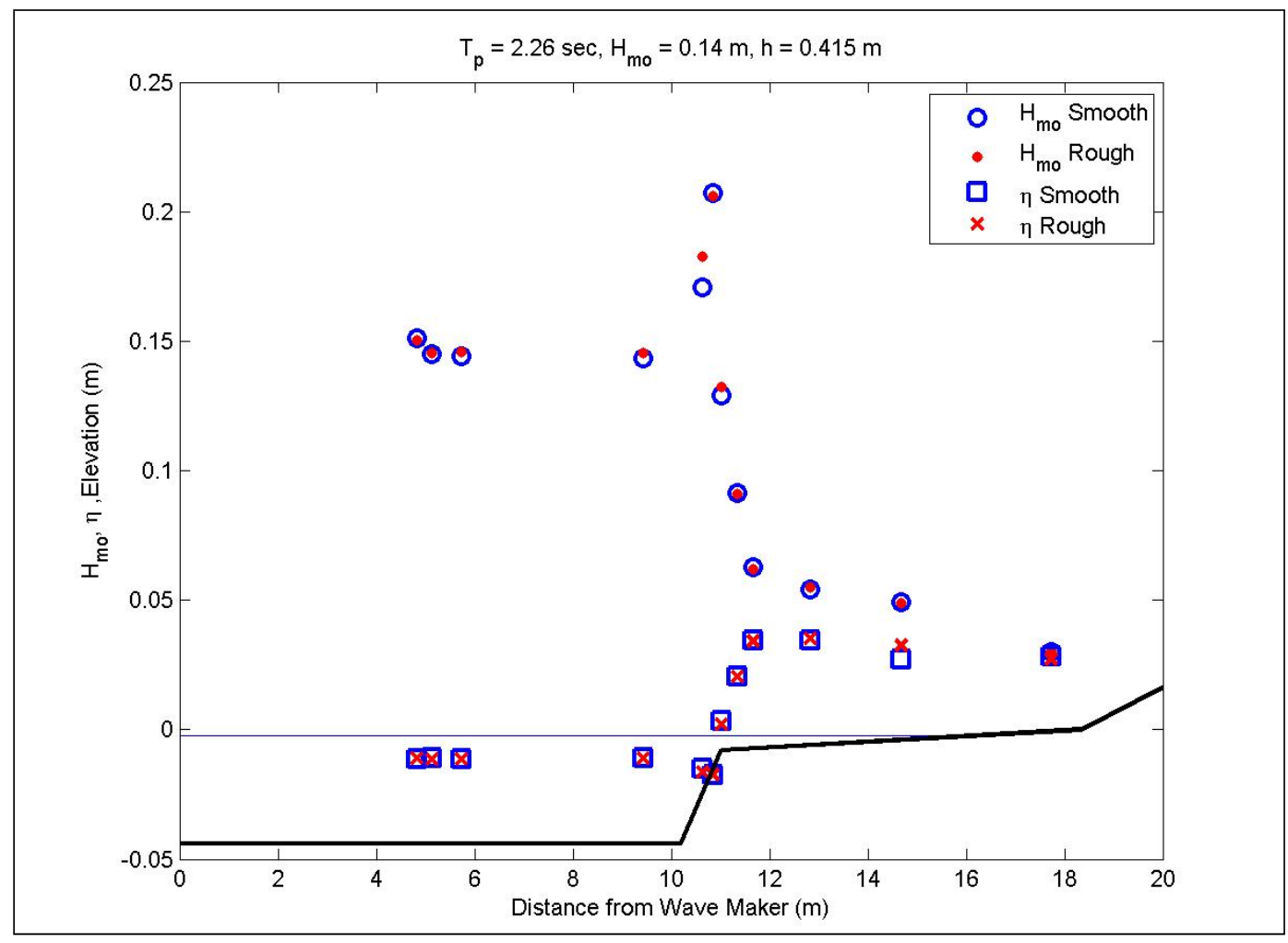

Figure E11. Wave transformation for 2.3-sec, 0.14-m waves at low water with a 1:25 reef slope. 


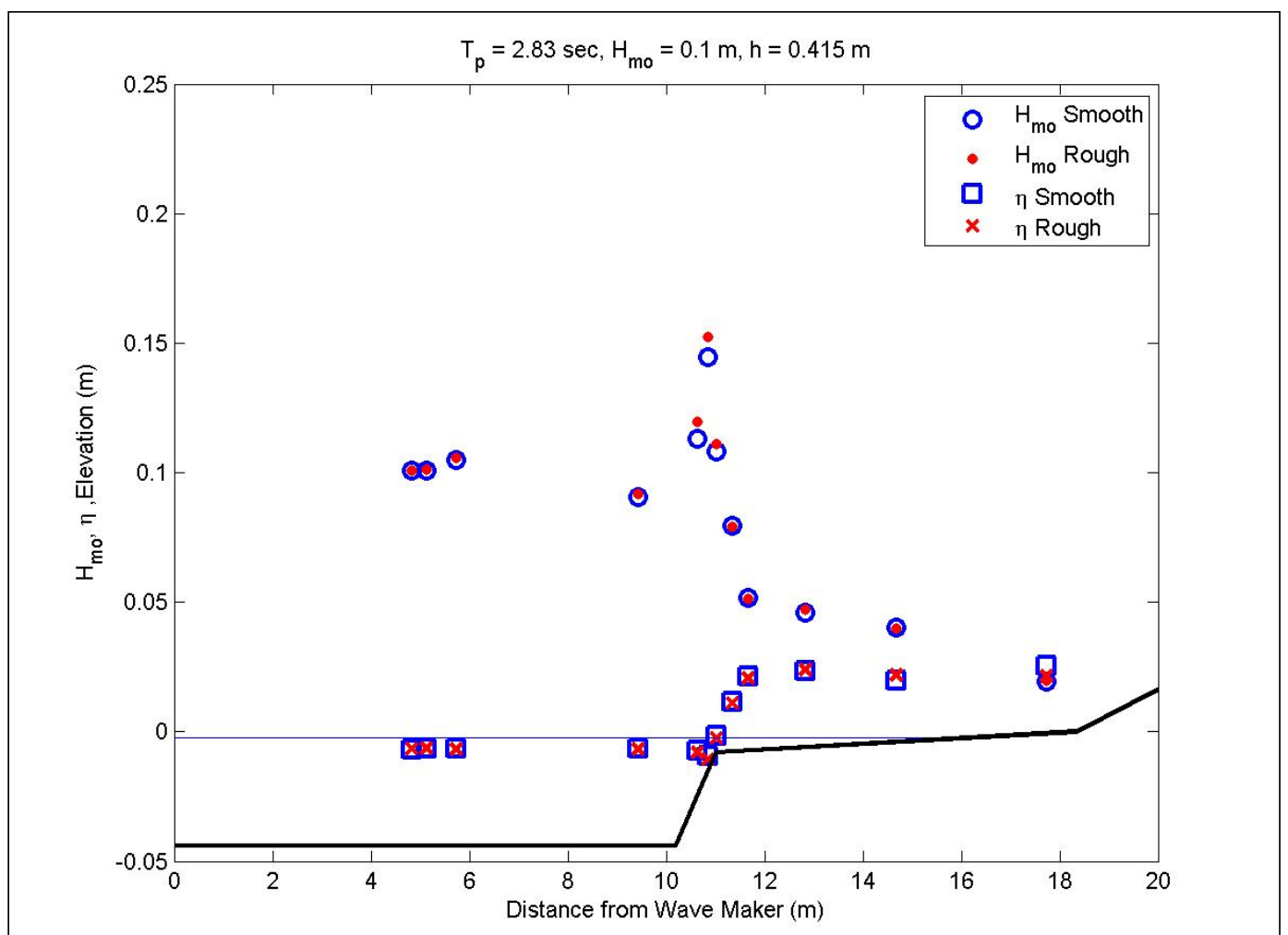

Figure E12. Wave transformation for 2.8-sec, 0.1-m waves at low water with a 1:25 reef slope.

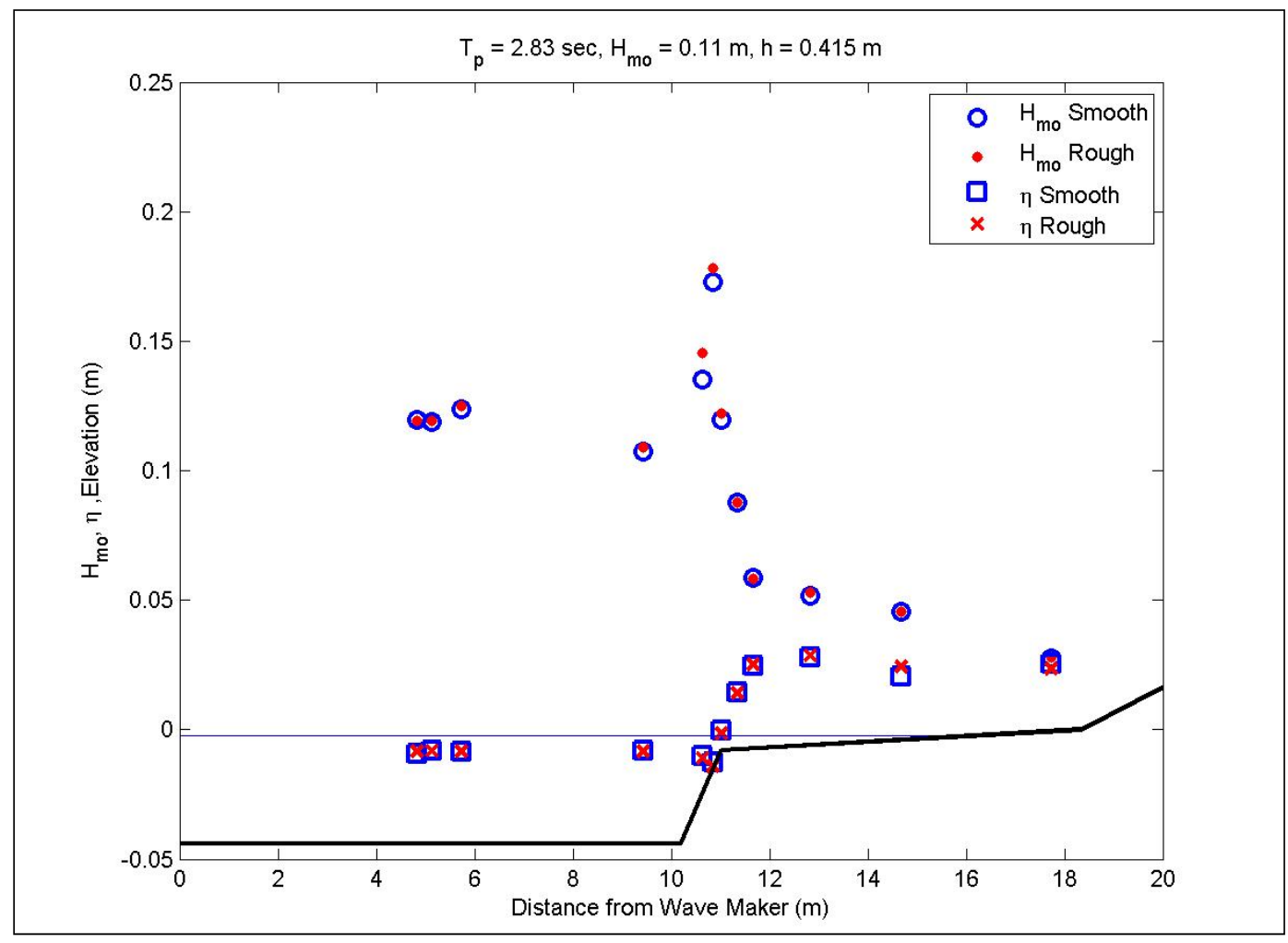

Figure E13. Wave transformation for 2.8-sec, 0.11-m waves at low water with a 1:25 reef slope. 


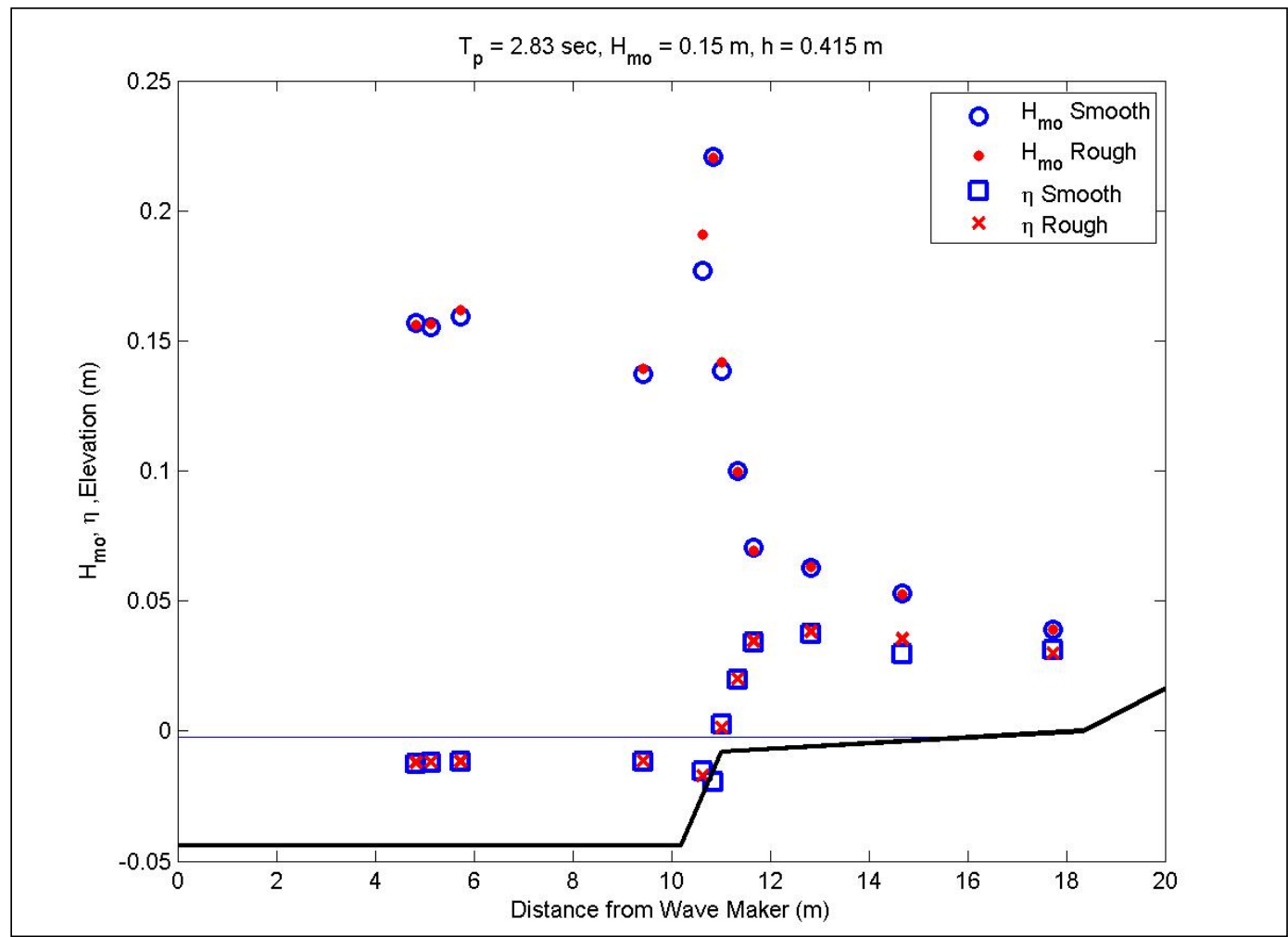

Figure E14. Wave transformation for 2.8-sec, 0.15-m waves at low water with a 1:25 reef slope.

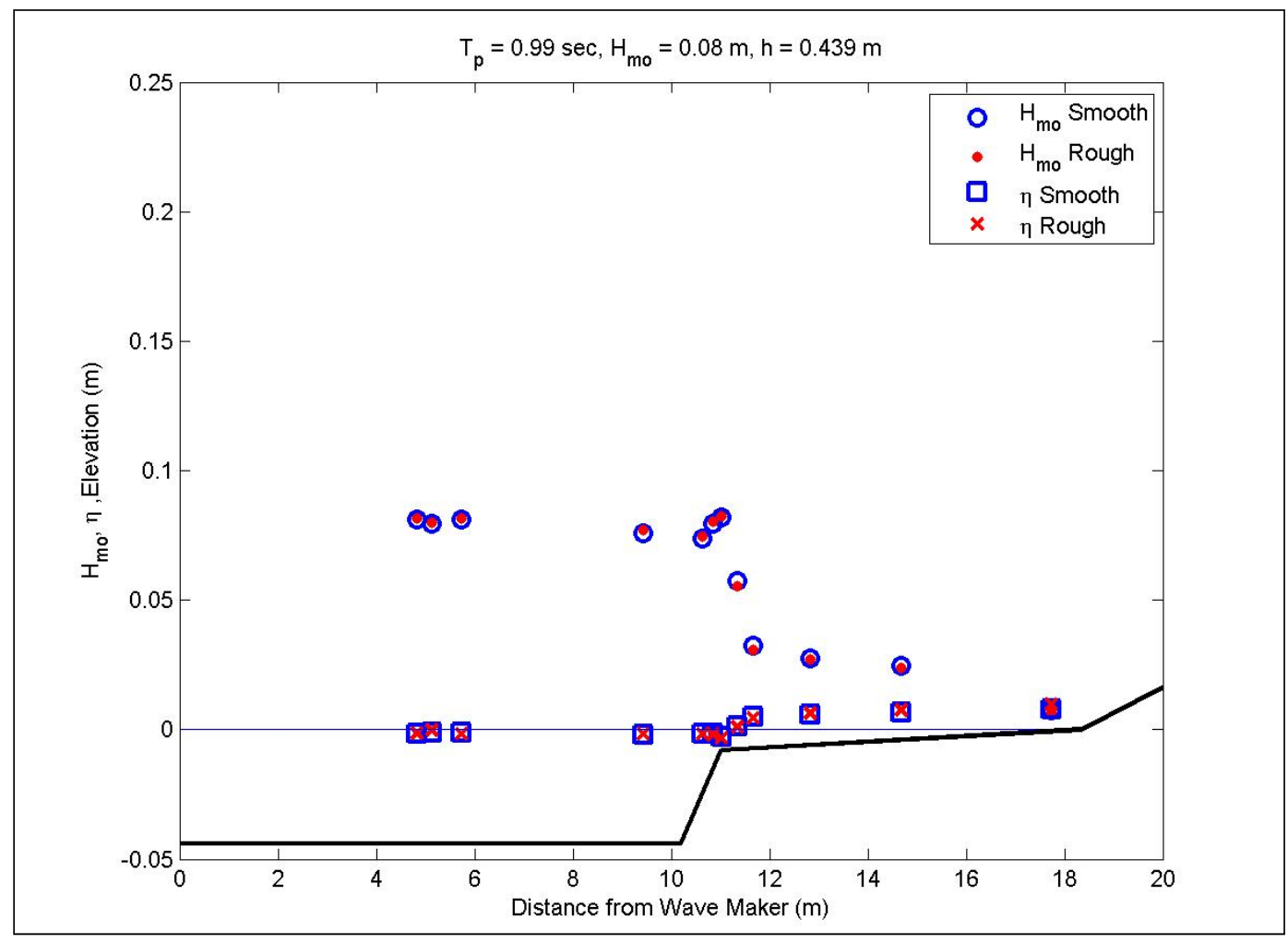

Figure E15. Wave transformation for 1-sec, 0.08-m waves at mid water with a 1:25 reef slope. 


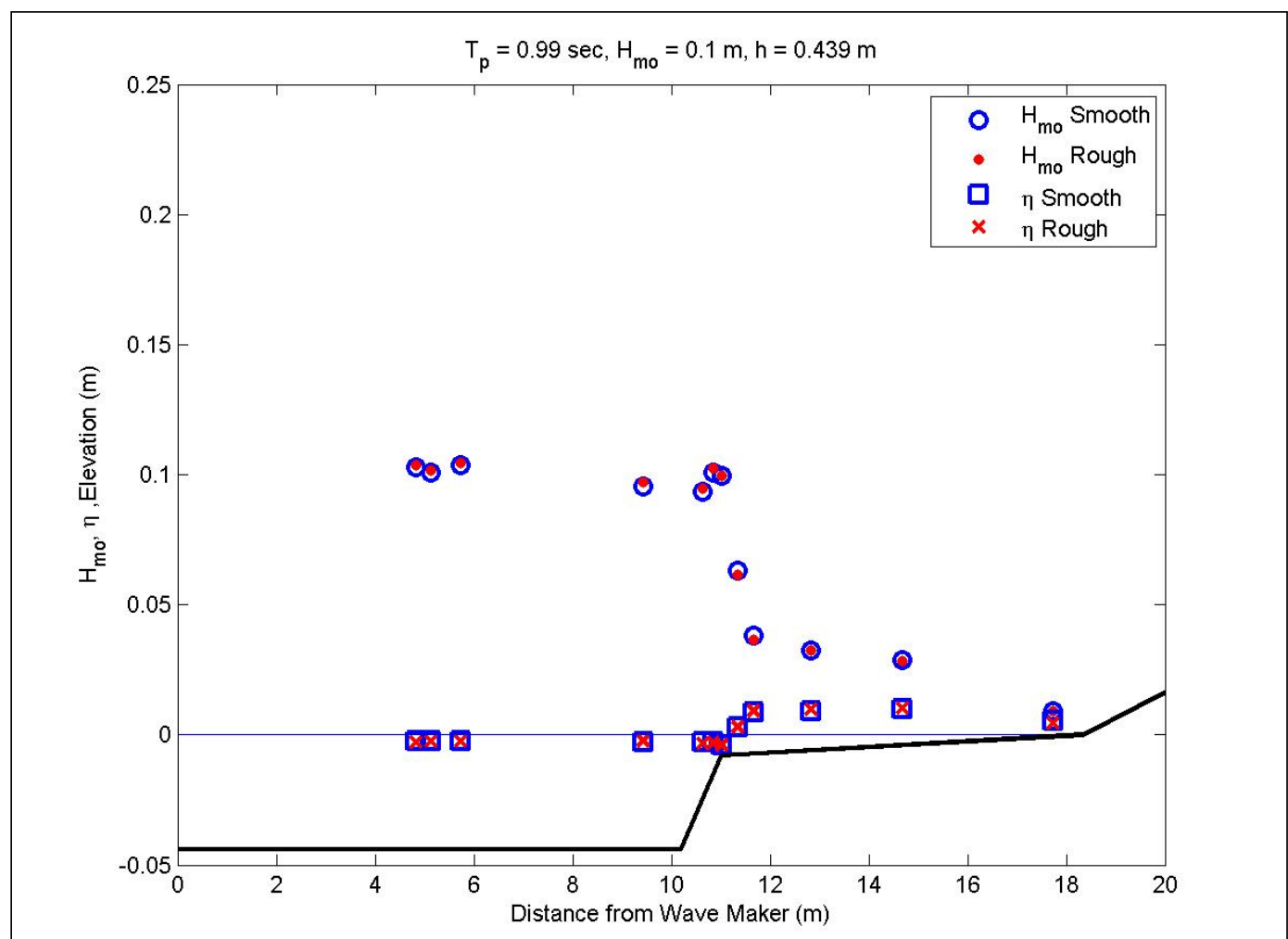

Figure E16. Wave transformation for 1-sec, 0.1-m waves at mid water with a 1:25 reef slope.

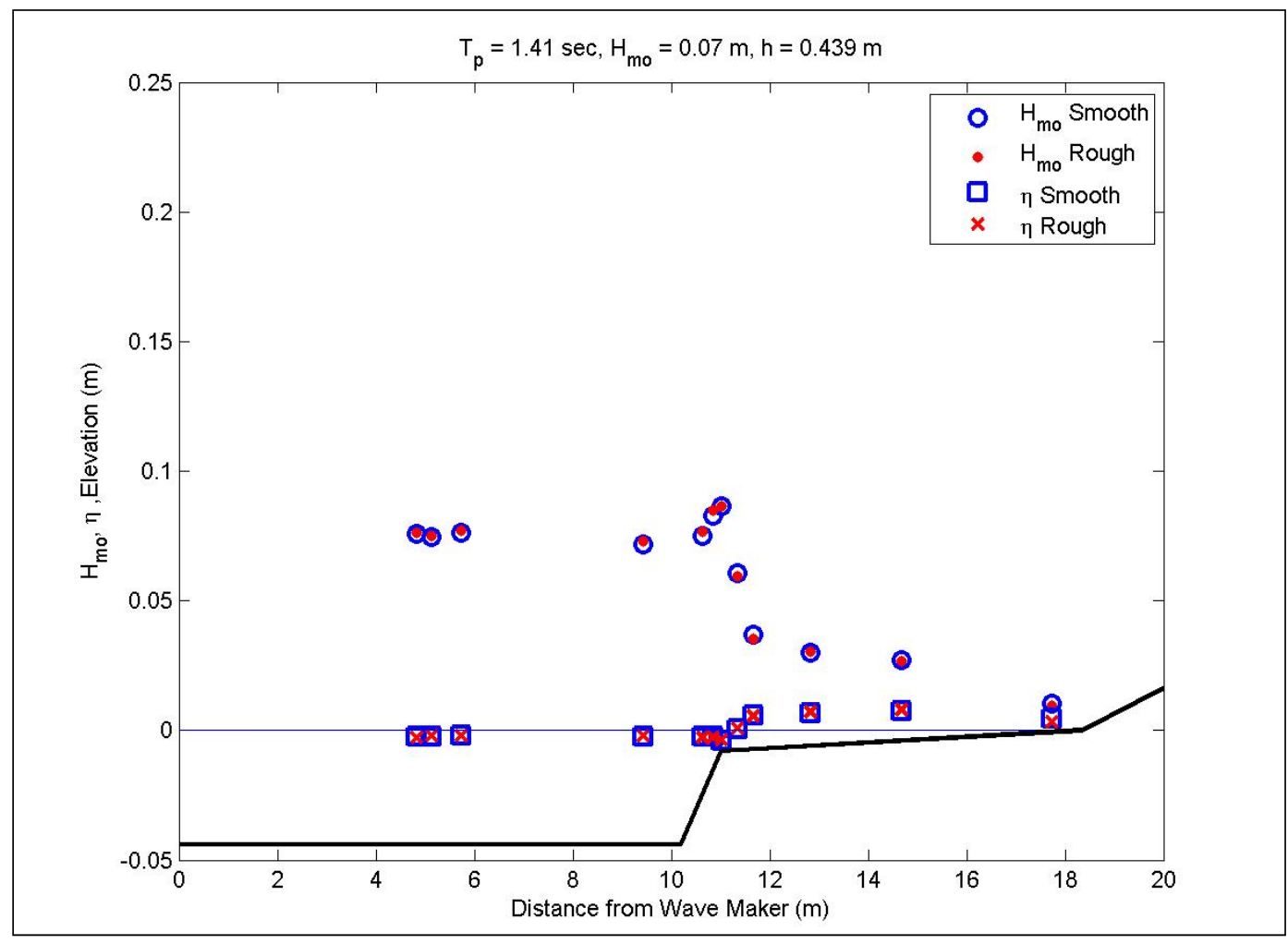

Figure E17. Wave transformation for 1.4-sec, 0.07-m waves at mid water with a 1:25 reef slope. 


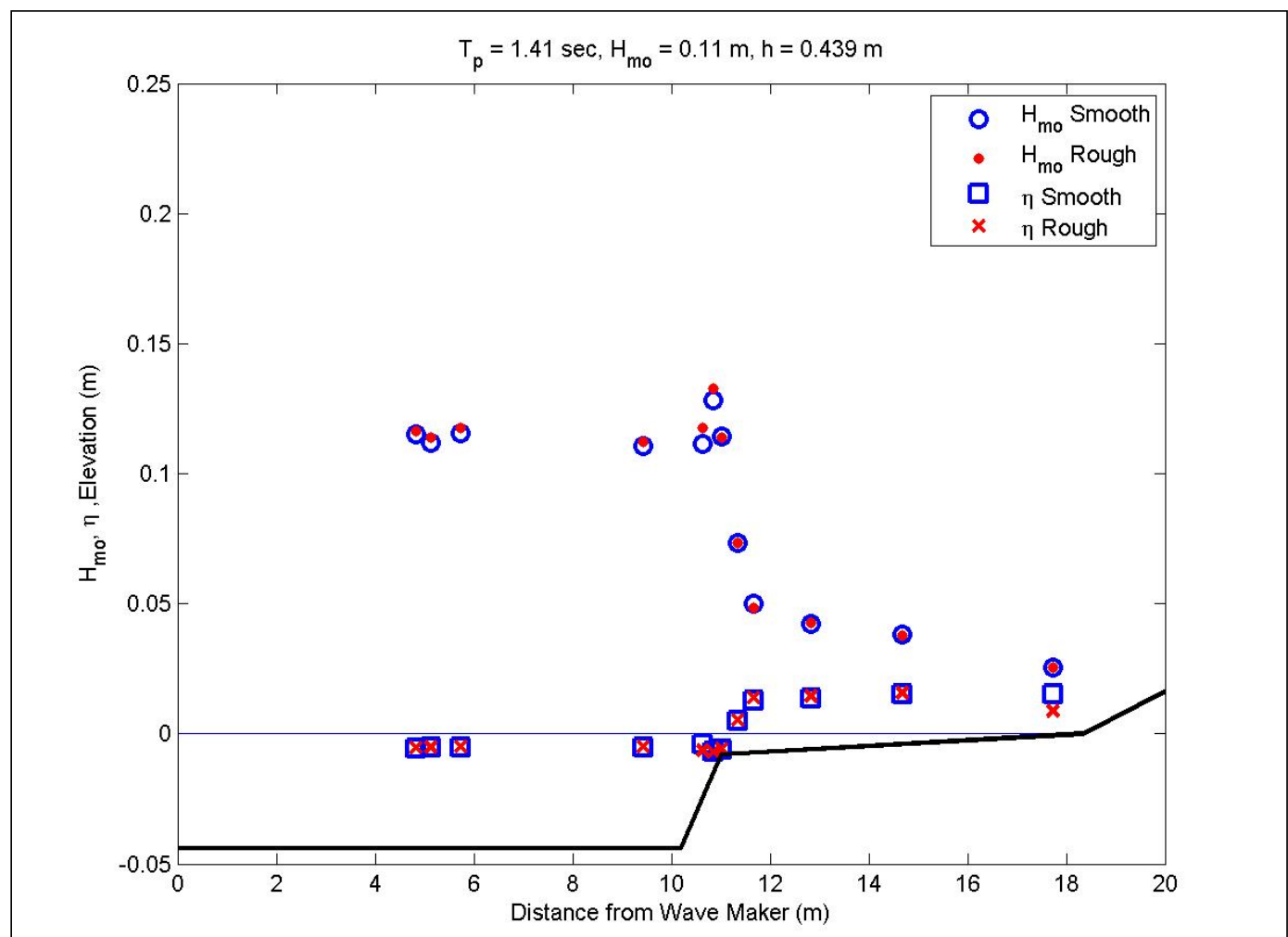

Figure E18. Wave transformation for 1.4-sec, 0.11-m waves at mid water with a 1:25 reef slope.

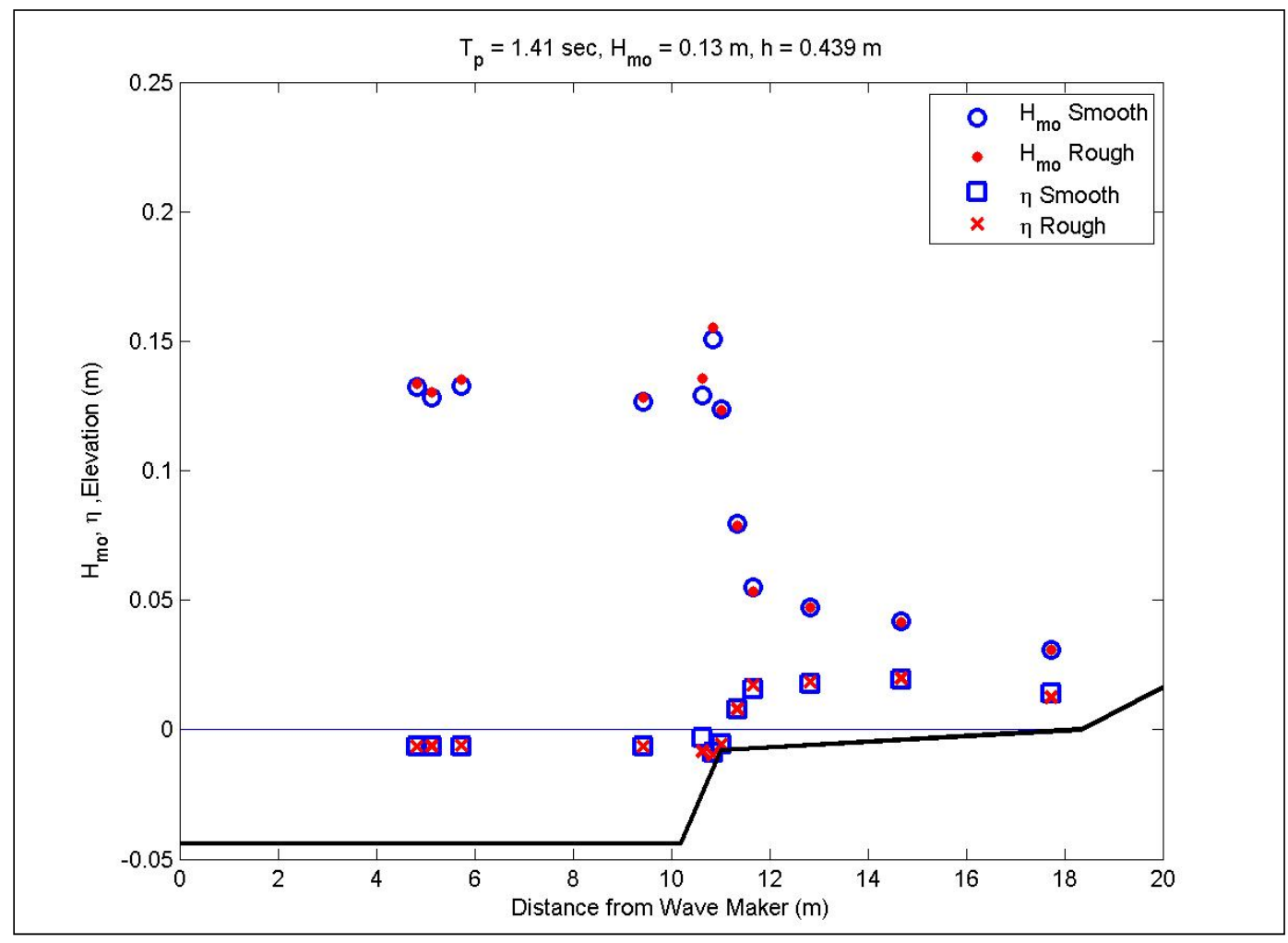

Figure E19. Wave transformation for 1.4-sec, 0.13-m waves at mid water with a 1:25 reef slope. 


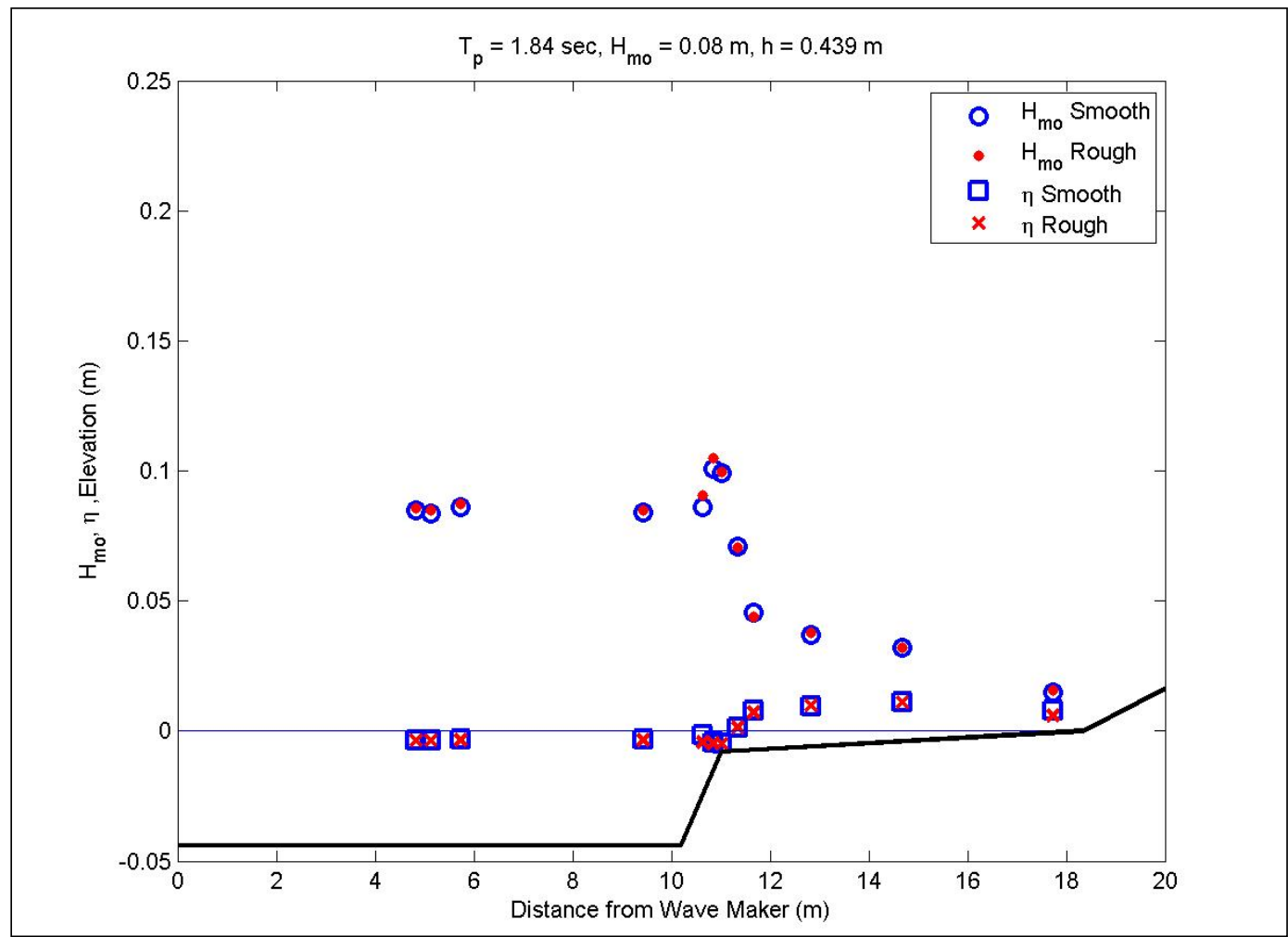

Figure E20. Wave transformation for 1.8-sec, 0.08-m waves at mid water with a 1:25 reef slope.

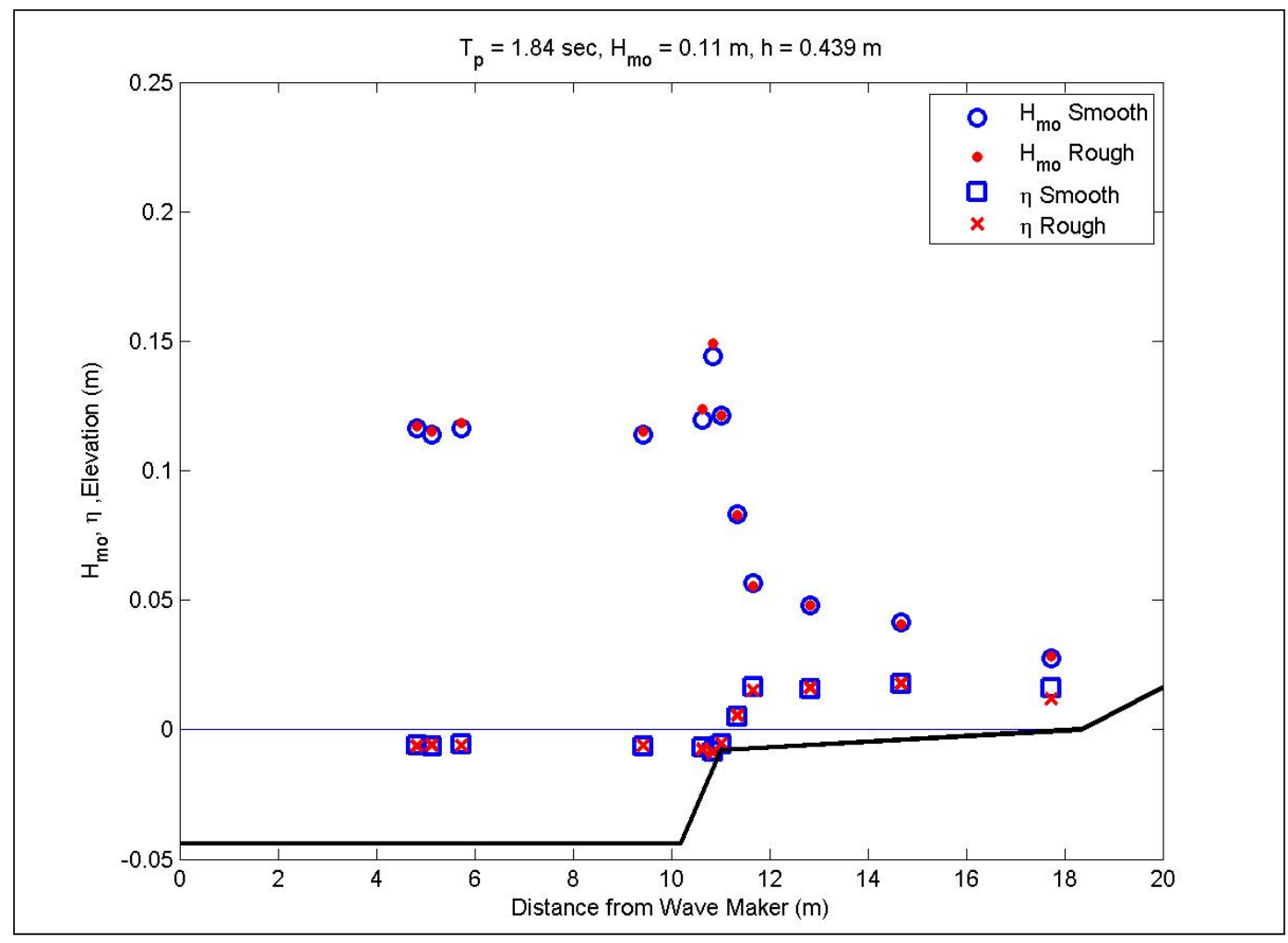

Figure E21. Wave transformation for 1.8-sec, 0.11-m waves at mid water with a 1:25 reef slope. 


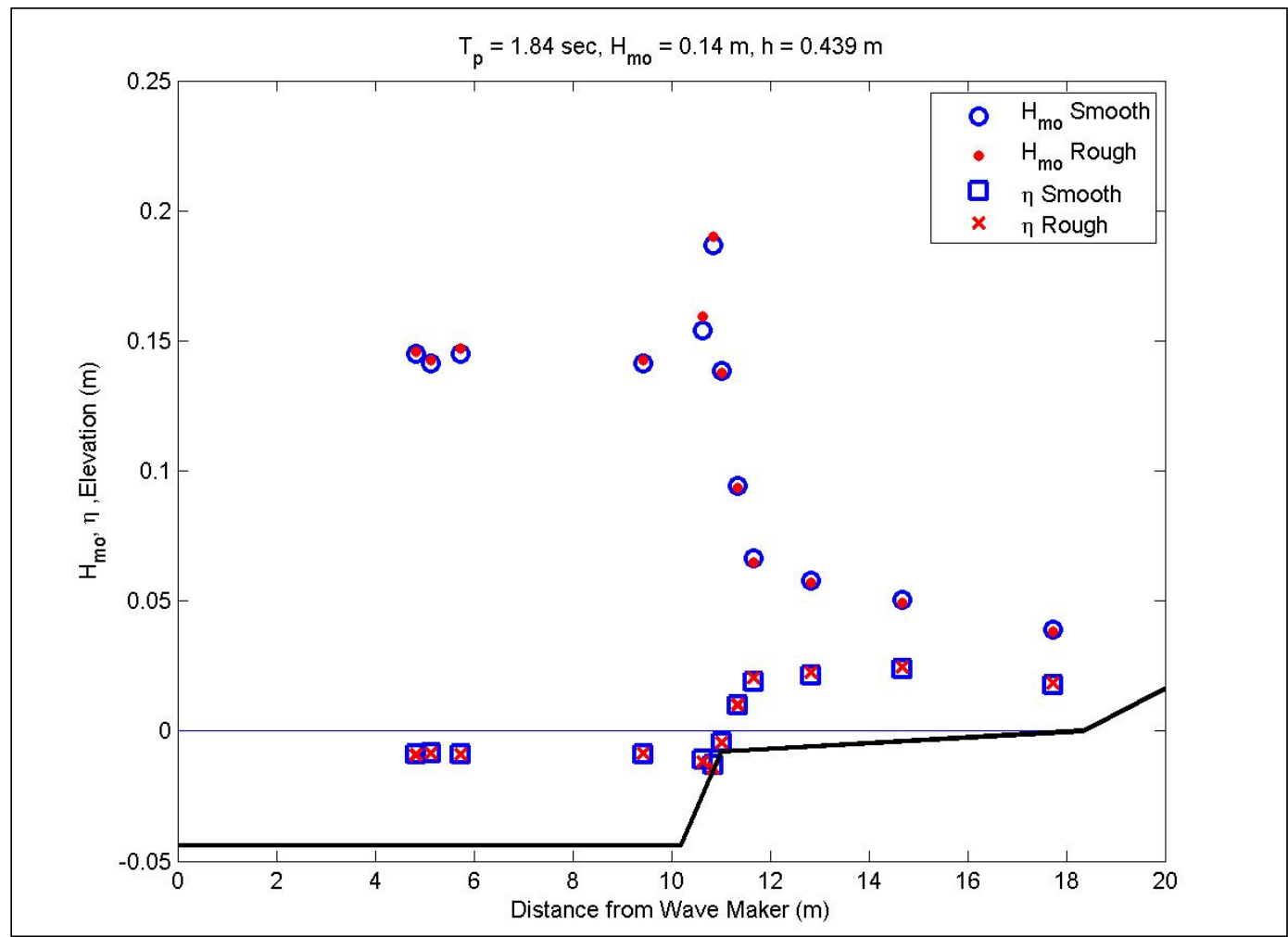

Figure E22. Wave transformation for 1.8-sec, 0.14-m waves at mid water with a 1:25 reef slope.

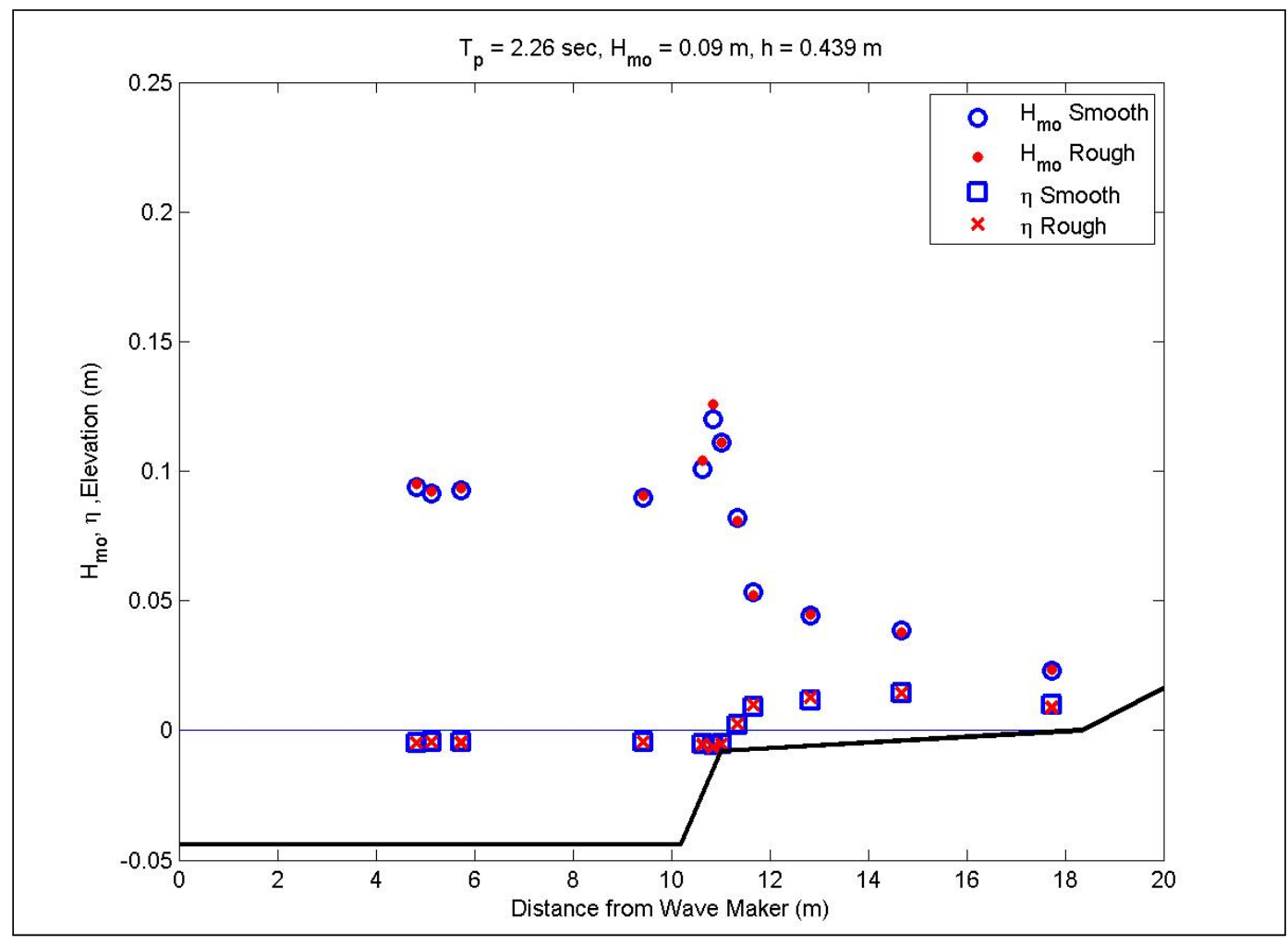

Figure E23. Wave transformation for 2.3-sec, 0.09-m waves at mid water with a 1:25 reef slope. 


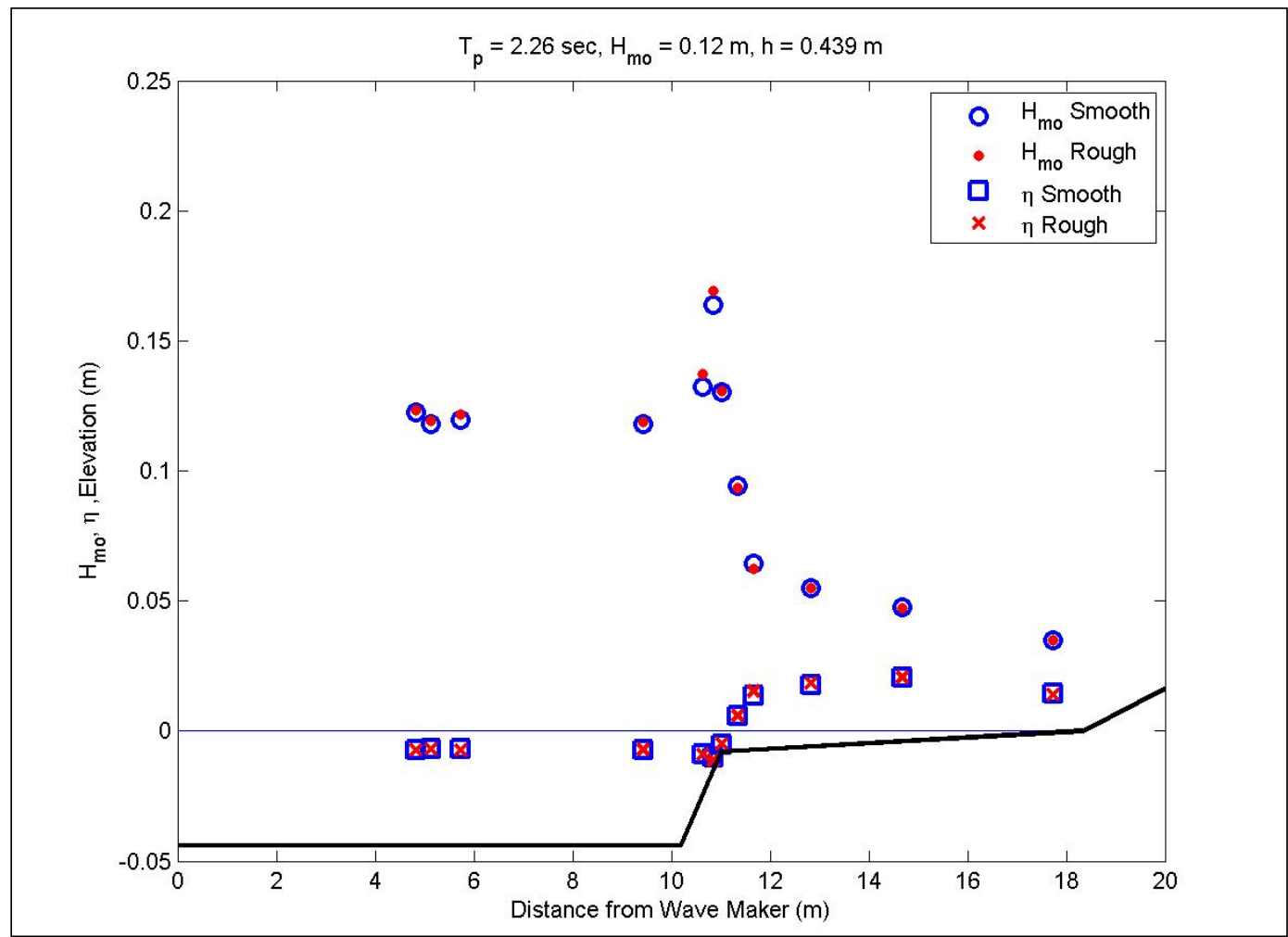

Figure E24. Wave transformation for 2.3-sec, 0.12-m waves at mid water with a 1:25 reef slope.

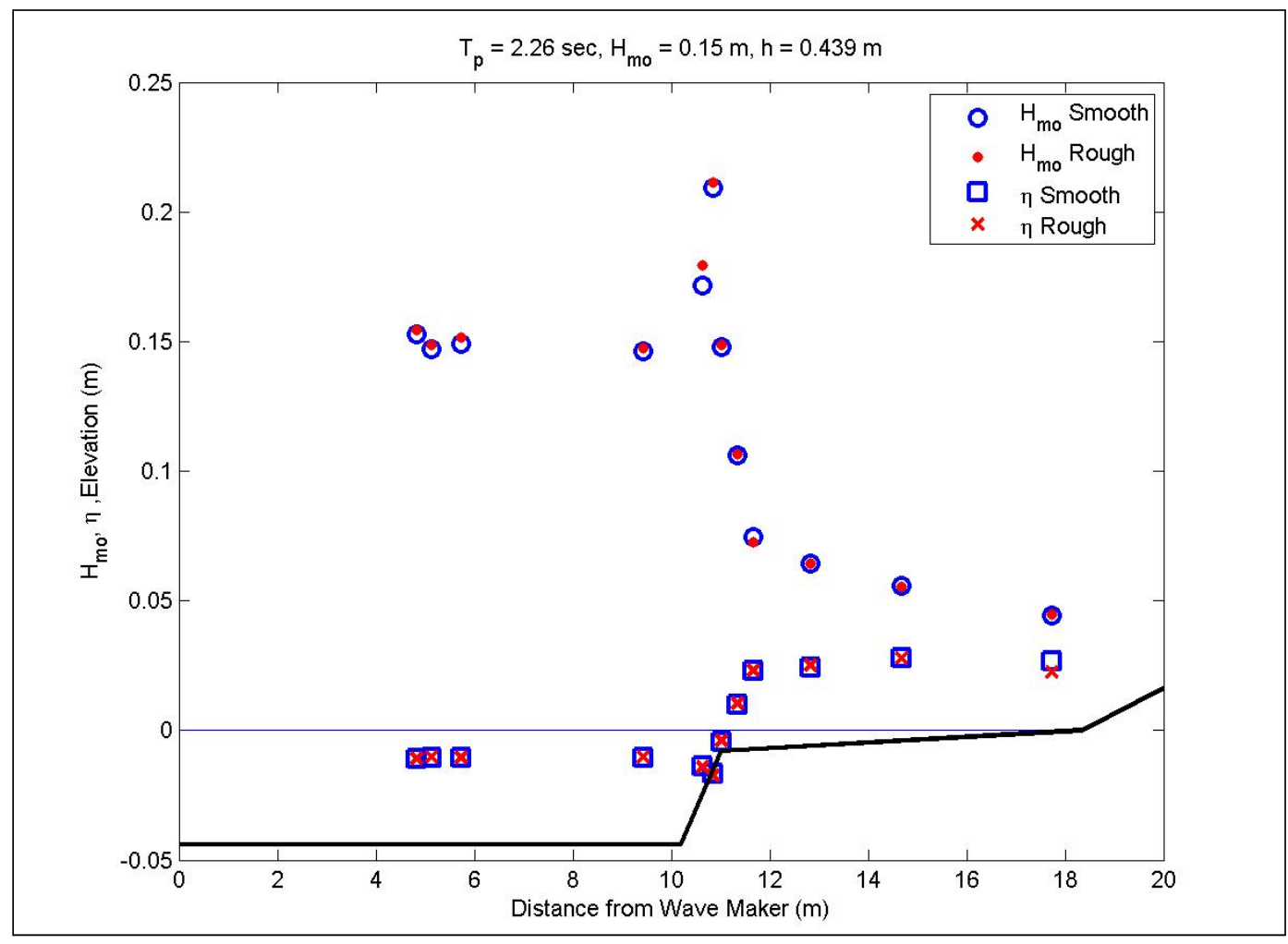

Figure E25. Wave transformation for 2.3-sec, 0.15-m waves at mid water with a 1:25 reef slope. 


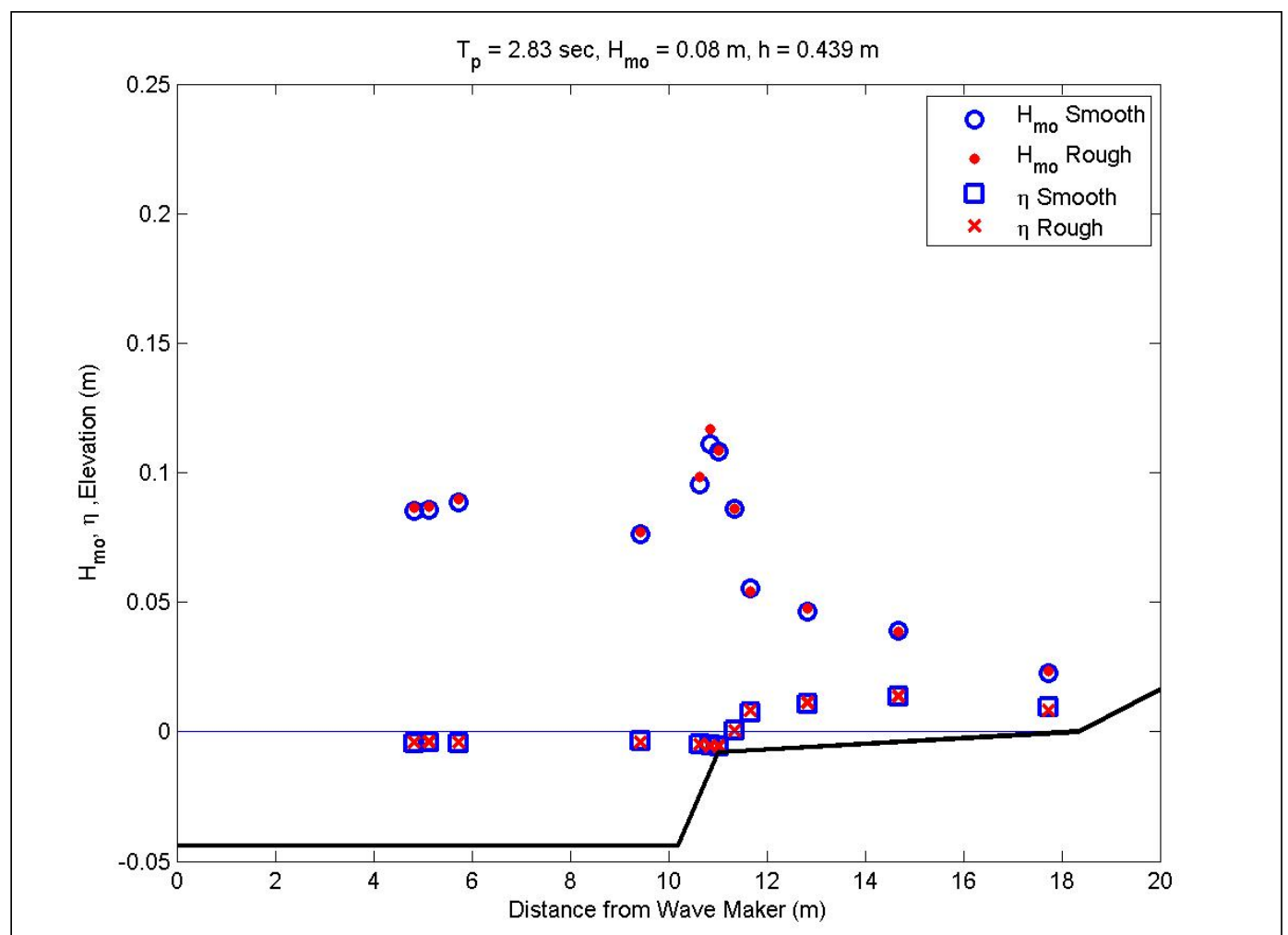

Figure E26 Wave transformation for 2.8-sec, 0.08-m waves at mid water with a 1:25 reef slope.

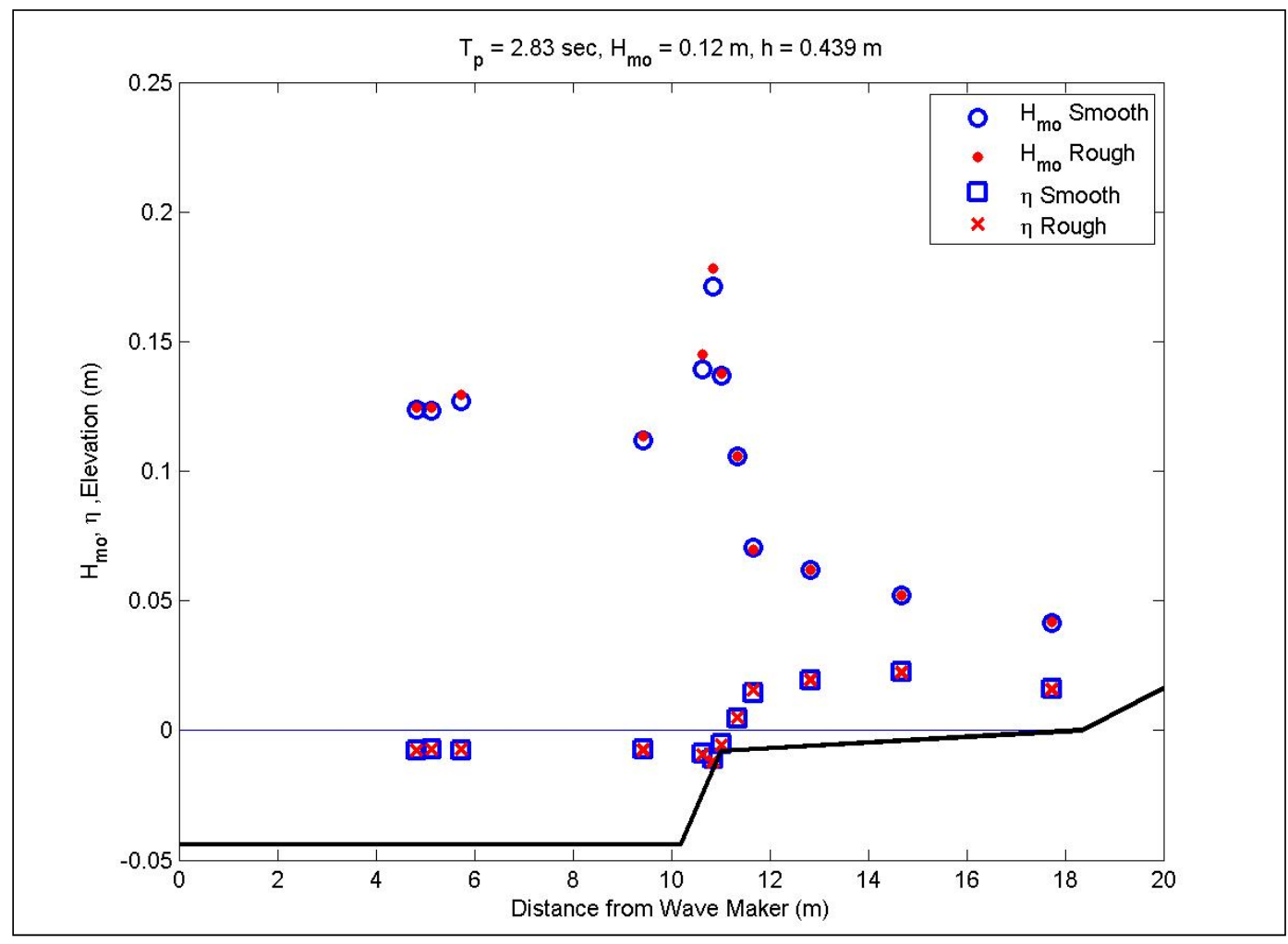

Figure E27. Wave transformation for 2.8-sec, 0.12-m waves at mid water with a 1:25 reef slope. 


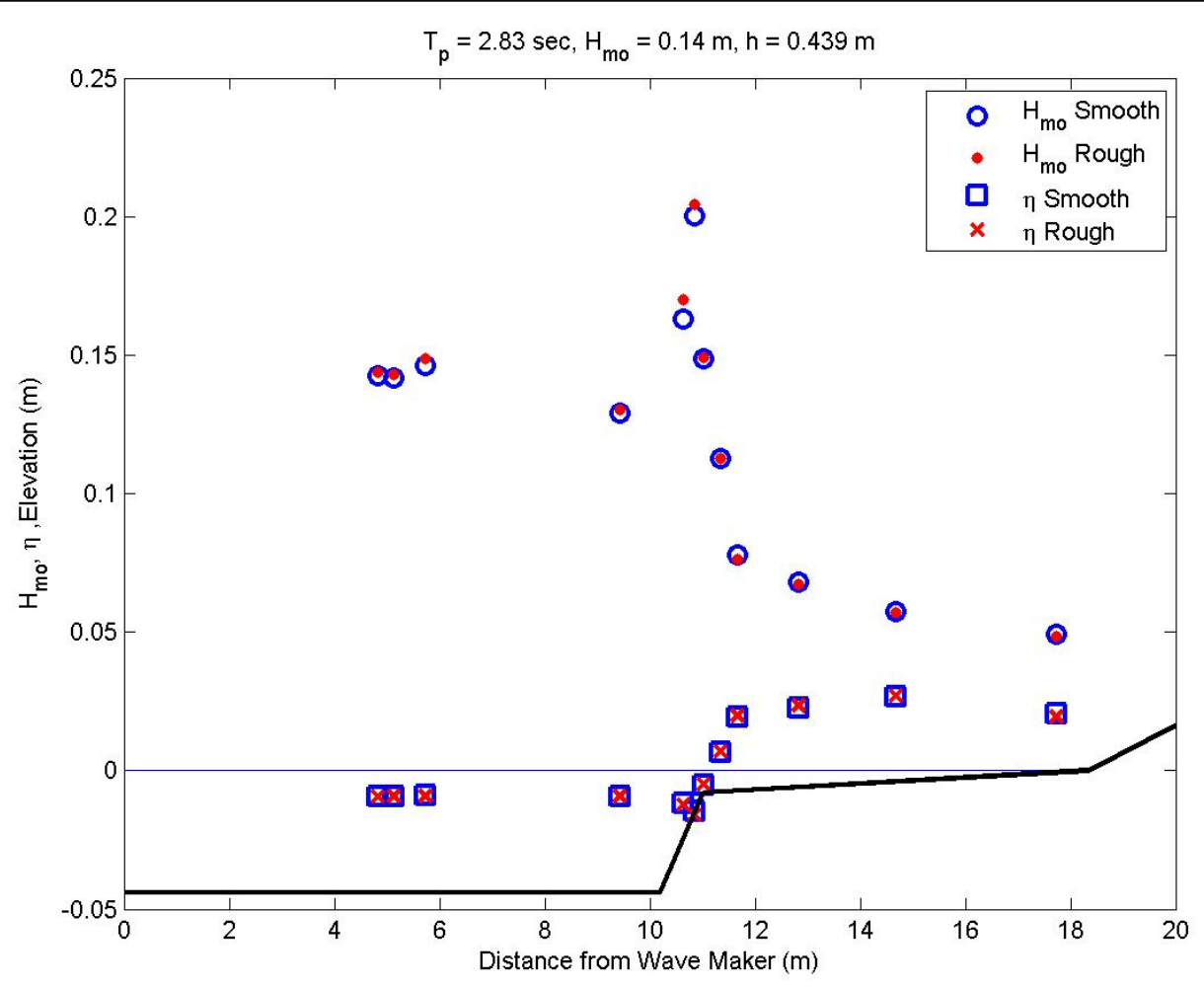

Figure E28. Wave transformation for 2.8-sec, 0.14-m waves at mid water with a 1:25 reef slope.

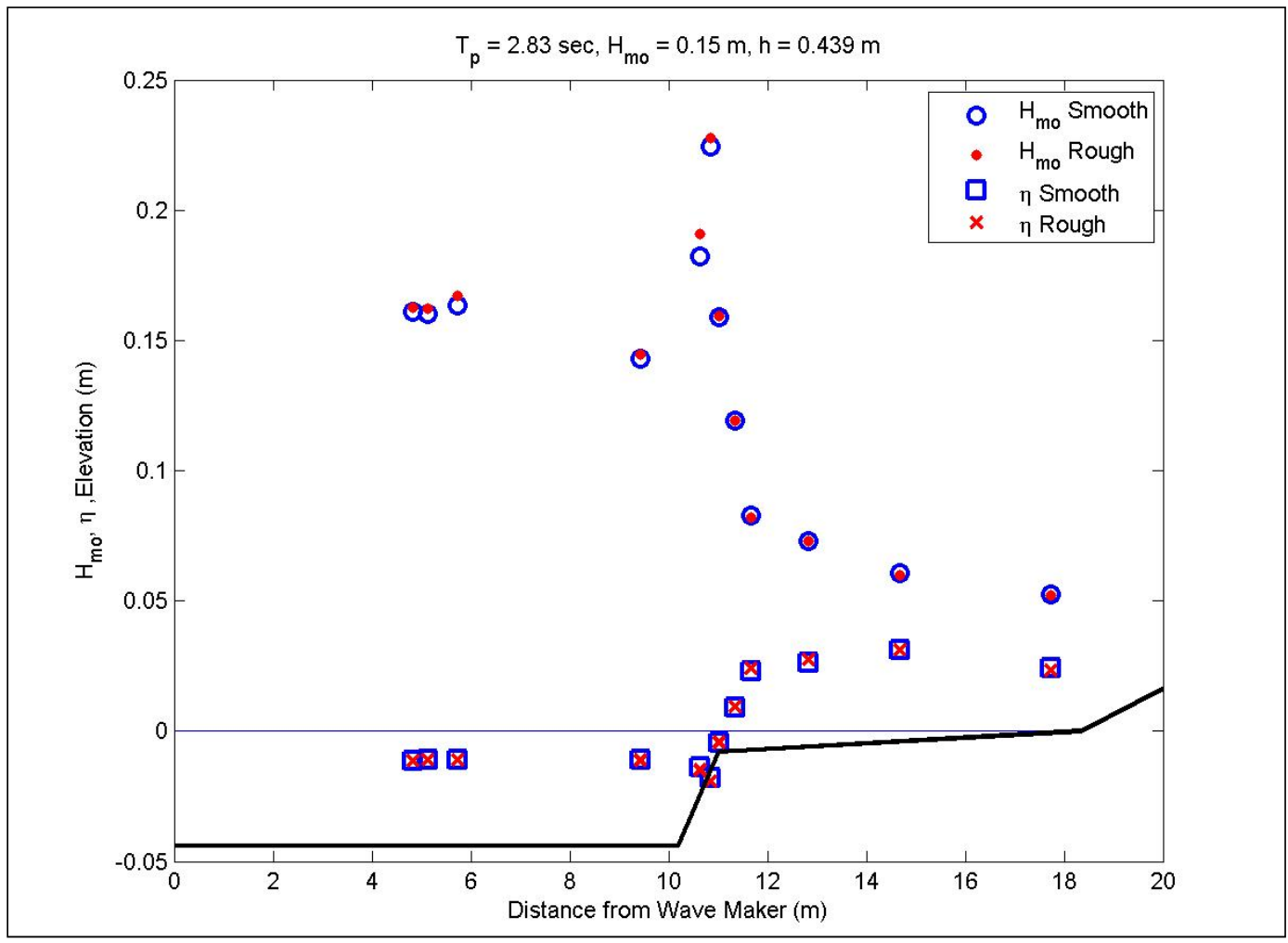

Figure E29. Wave transformation for 2.8-sec, 0.15-m waves at mid water with a 1:25 reef slope. 


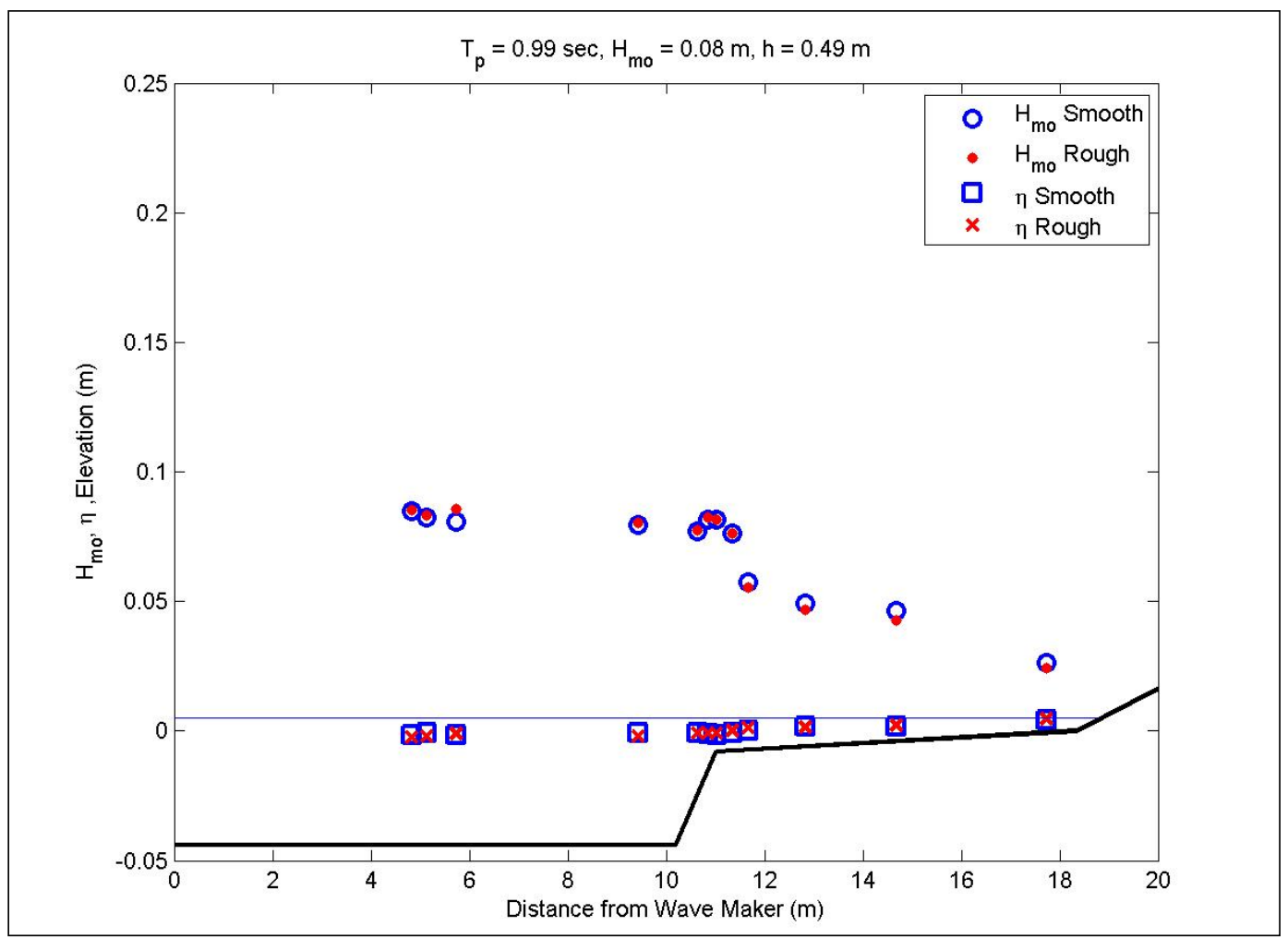

Figure E30. Wave transformation for 1-sec, 0.08-m waves at high water with a 1:25 reef slope.

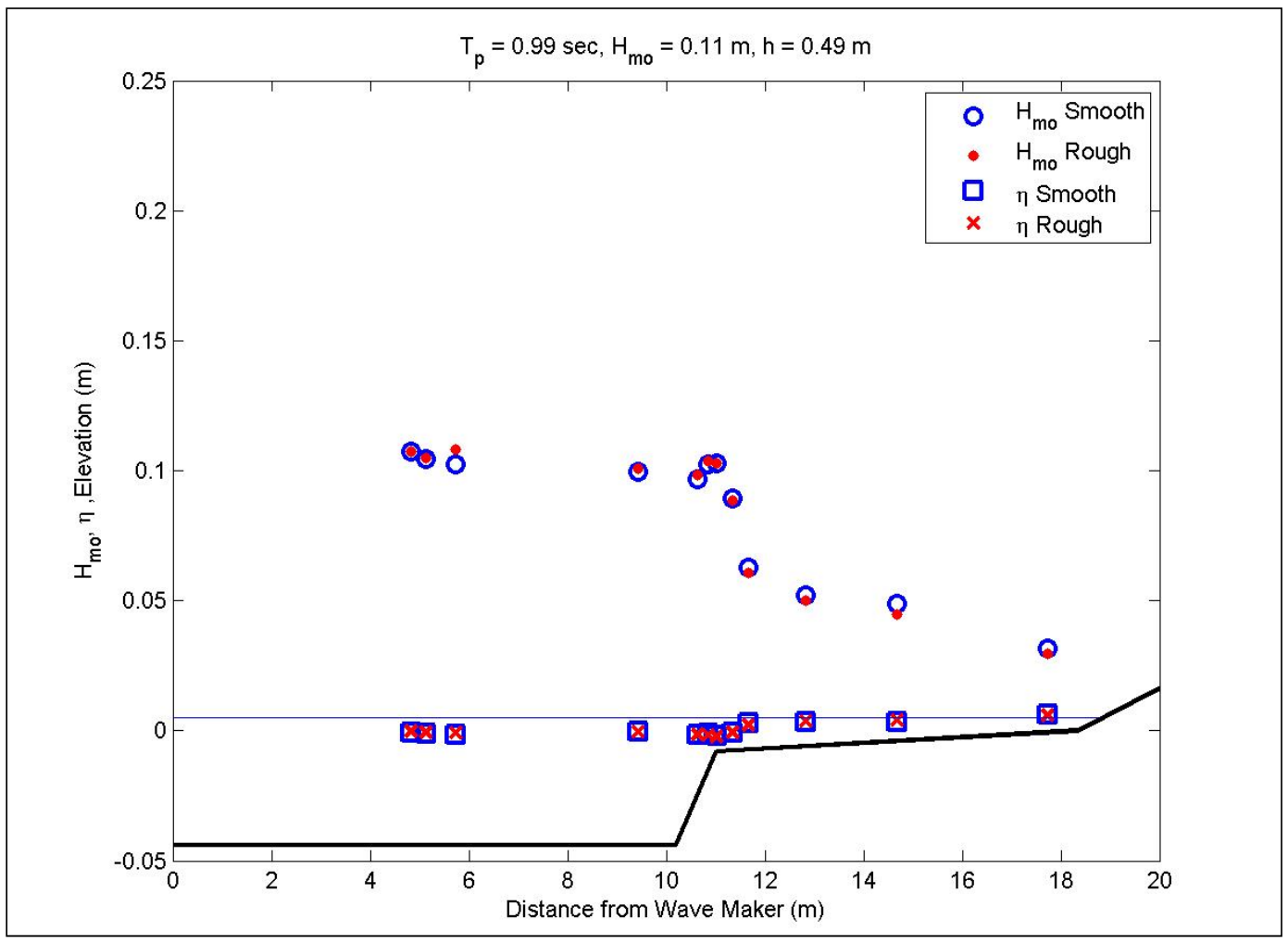

Figure E31. Wave transformation for 1-sec, 0.11-m waves at high water with a 1:25 reef slope. 


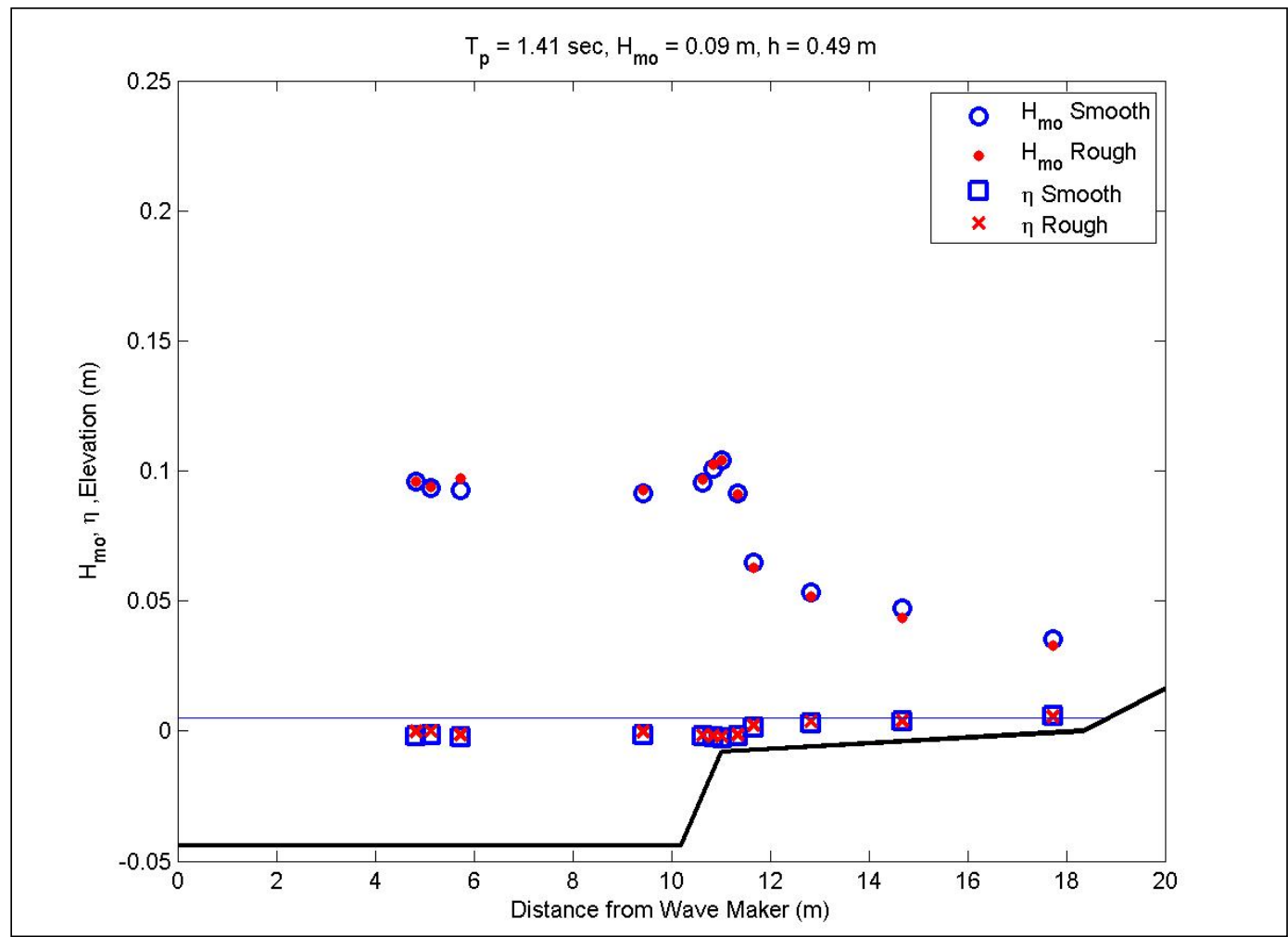

Figure E32. Wave transformation for 1.4-sec, 0.09-m waves at high water with a 1:25 reef slope.

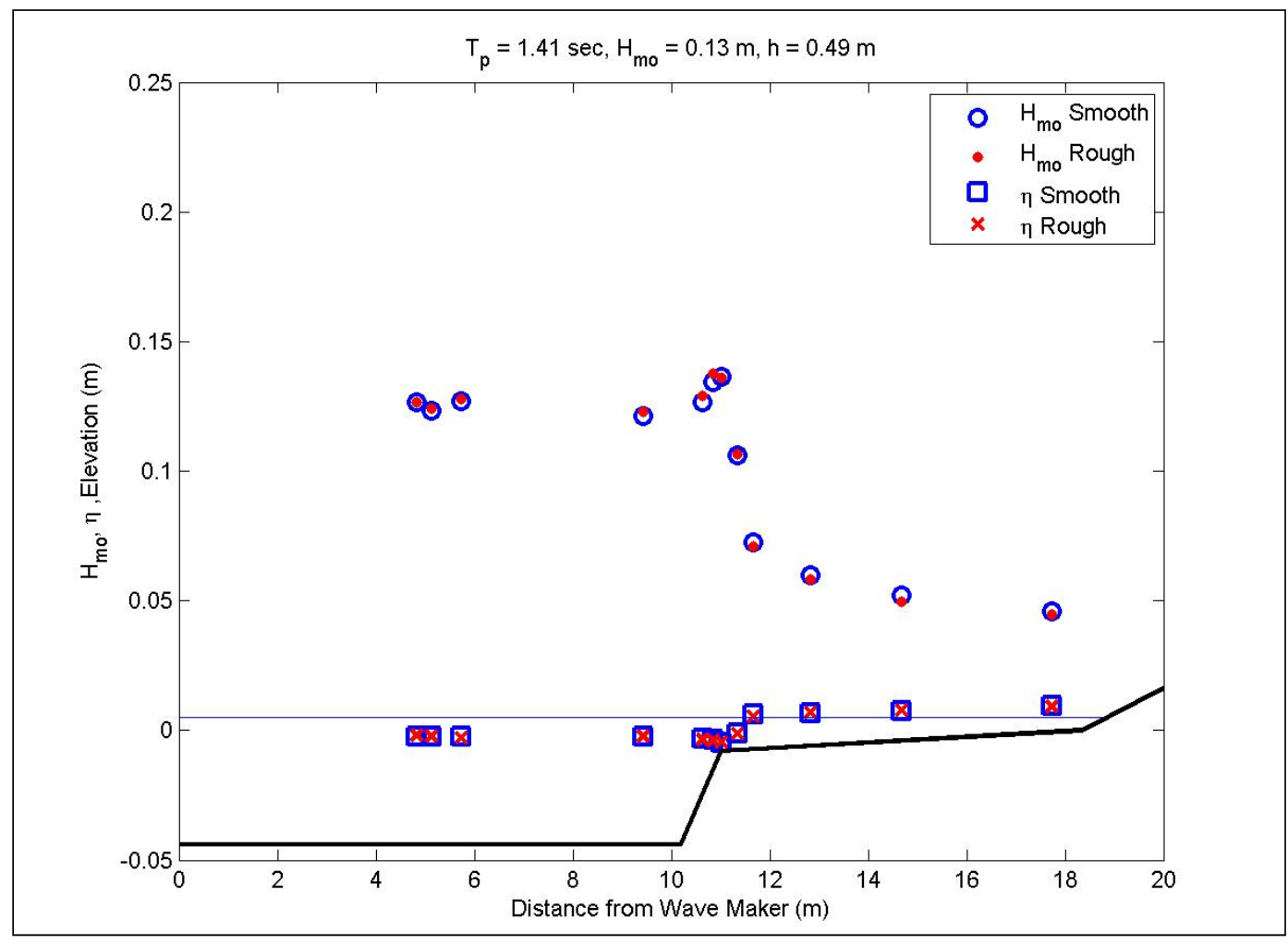

Figure E33. Wave transformation for 1.4-sec, 0.13-m waves at high water with a 1:25 reef slope. 


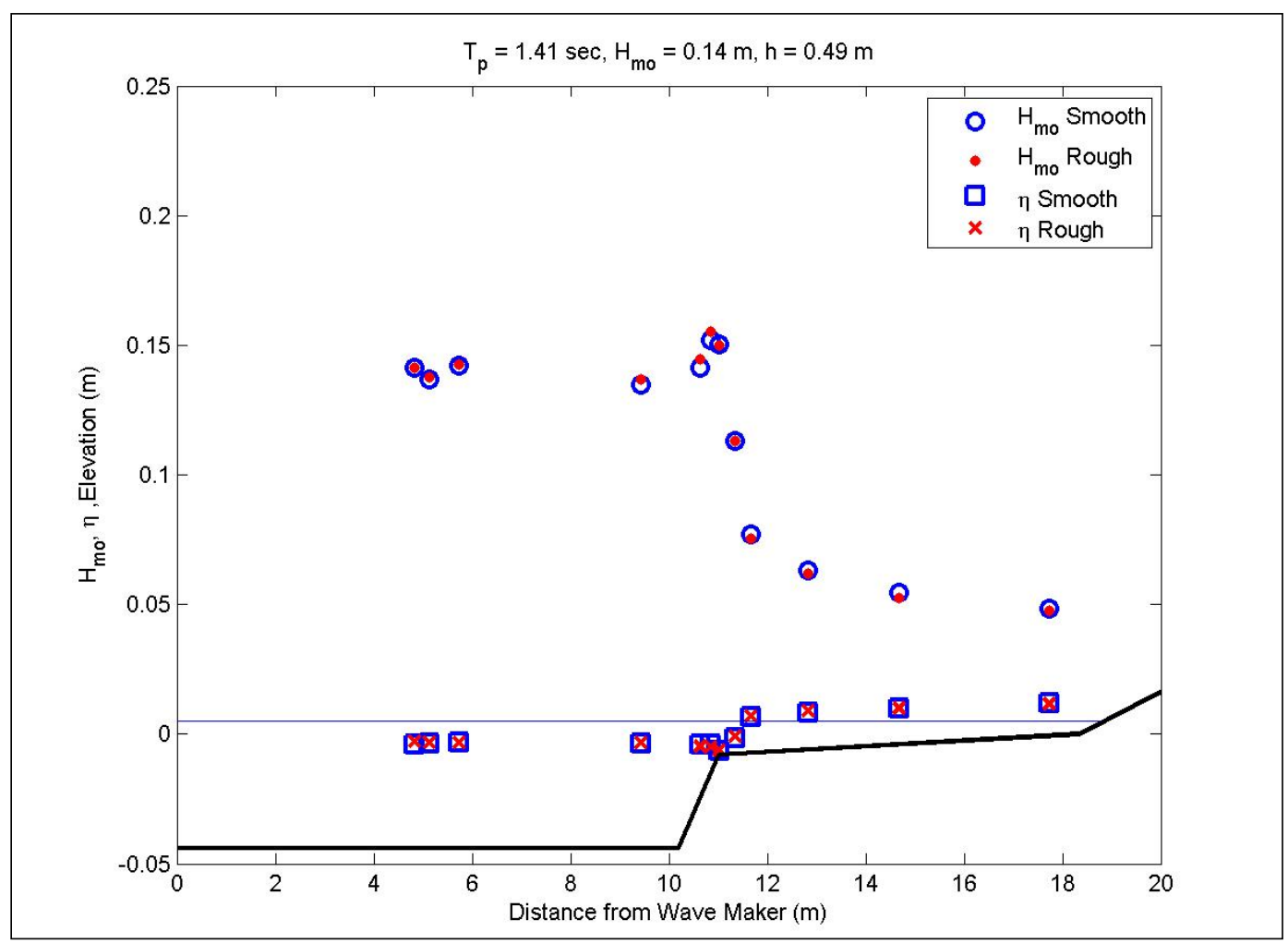

Figure E34. Wave transformation for 1.4-sec, 0.14-m waves at high water with a 1:25 reef slope.

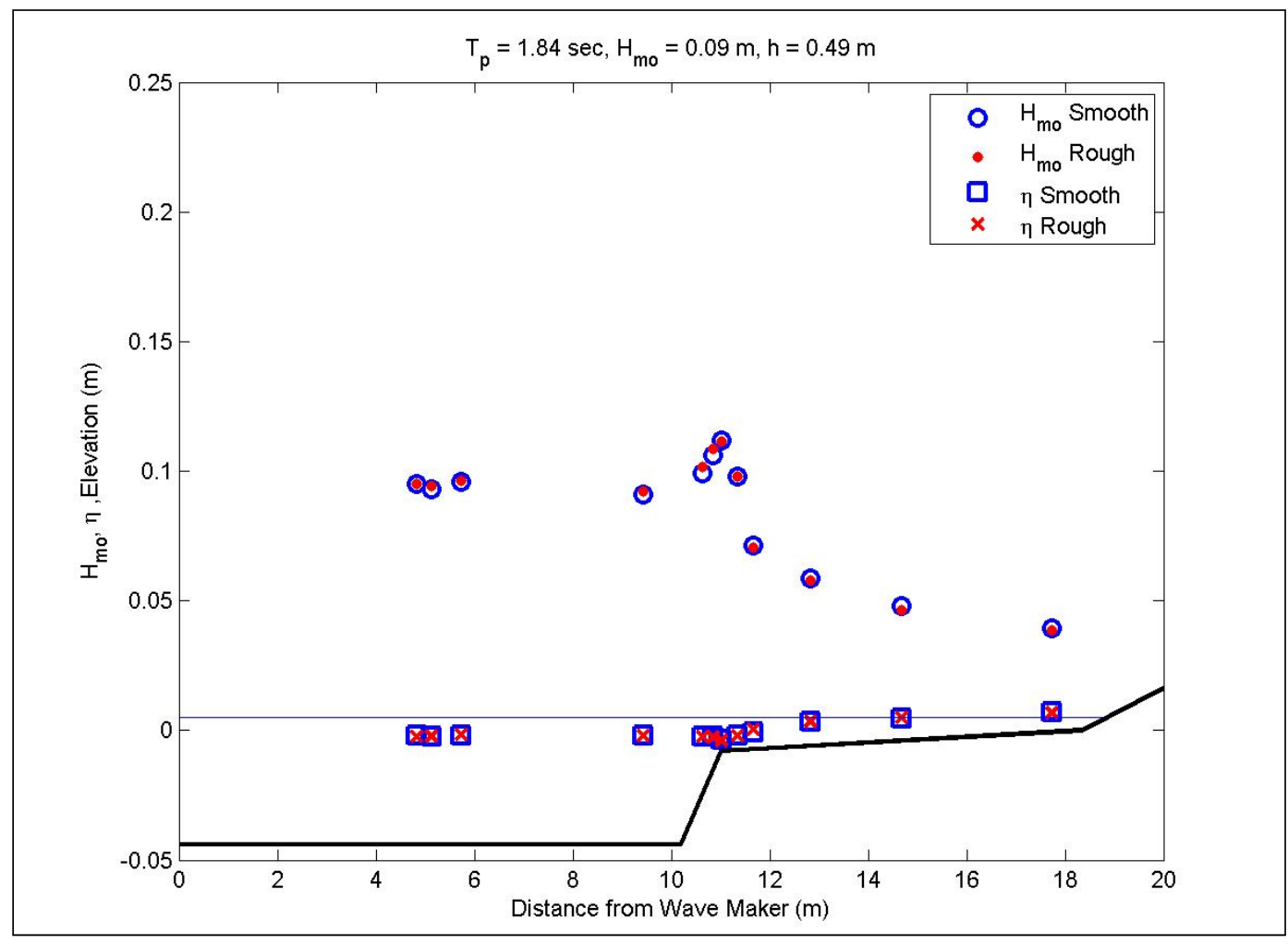

Figure E35 Wave transformation for 1.8-sec, 0.09-m waves at high water with a 1:25 reef slope. 


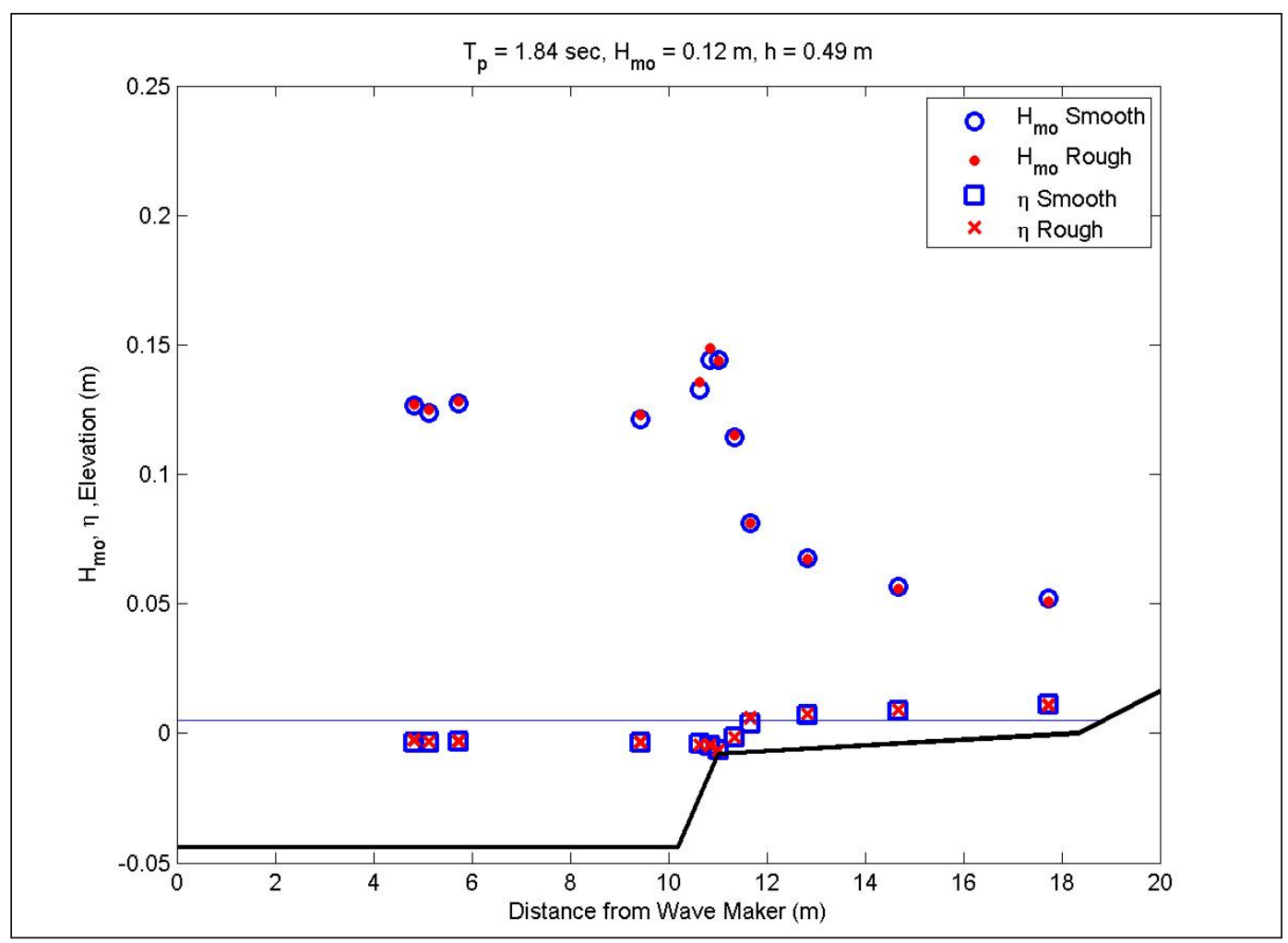

Figure E36. Wave transformation for 1.8-sec, 0.12-m waves at high water with a 1:25 reef slope.

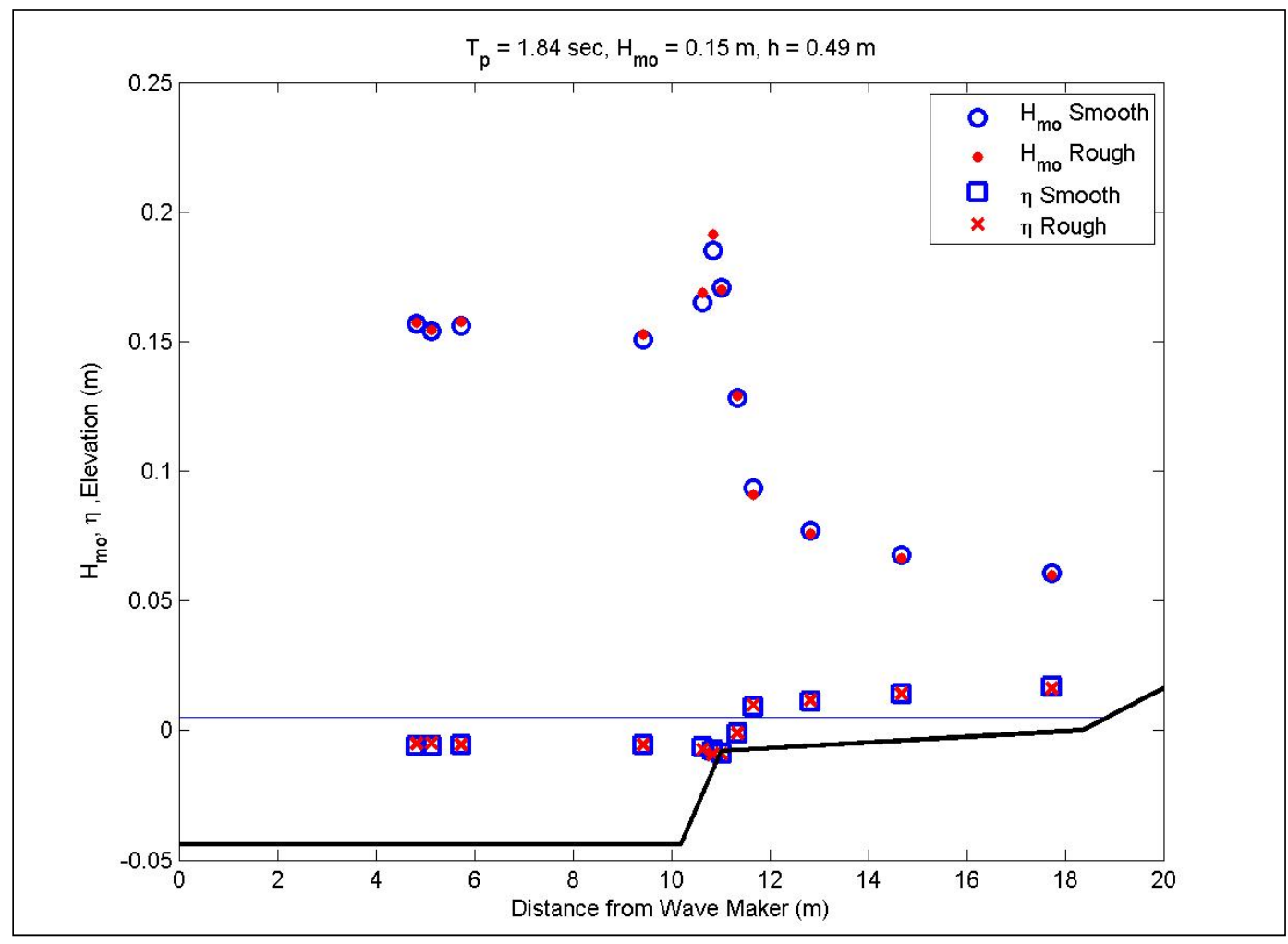

Figure E37. Wave transformation for 1.8-sec, 0.15-m waves at high water with a 1:25 reef slope. 


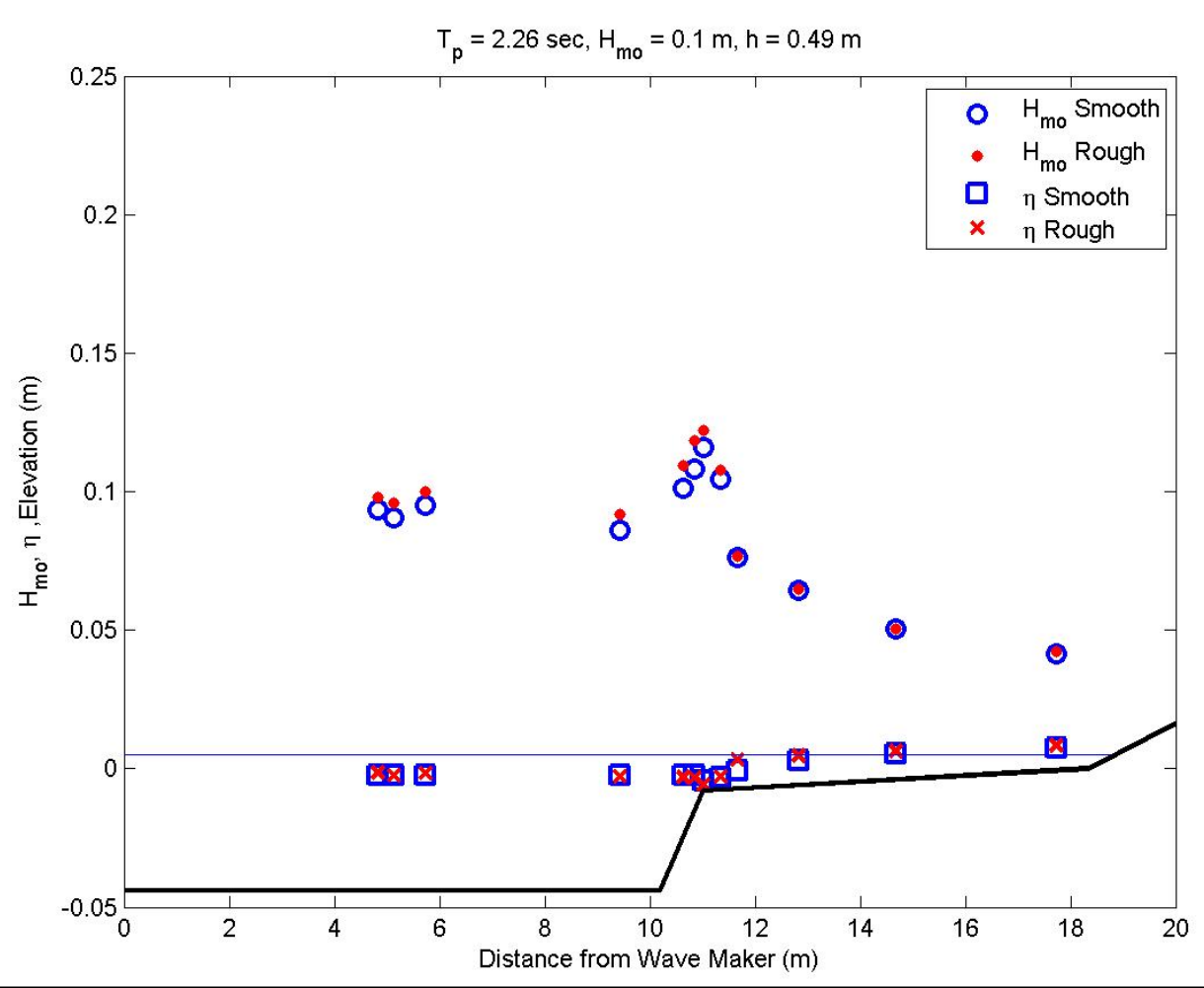

Figure E38. Wave transformation for 2.3-sec, 0.1-m waves at high water with a 1:25 reef slope.

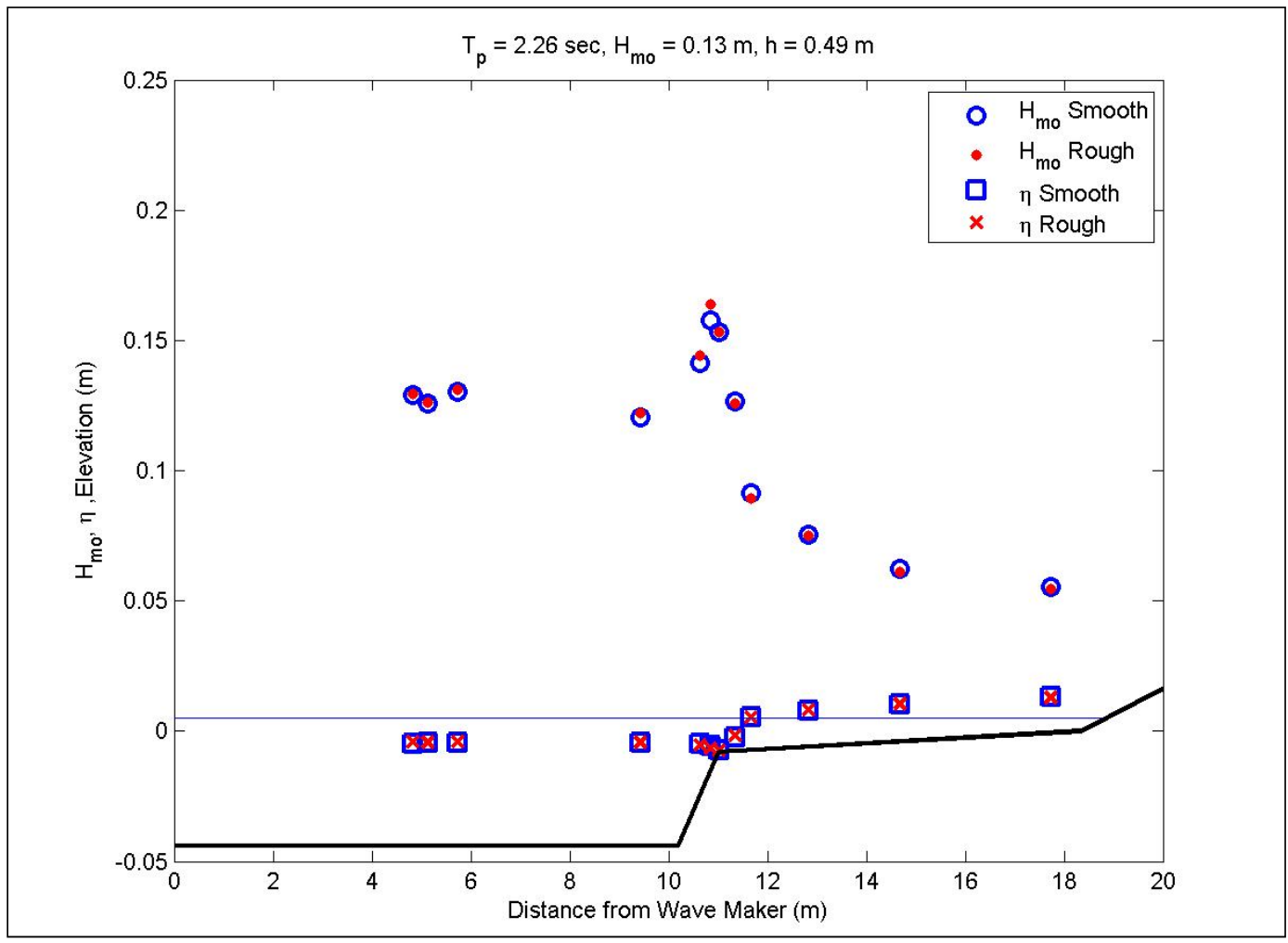

Figure E39. Wave transformation for 2.3-sec, 0.13-m waves at high water with a 1:25 reef slope. 


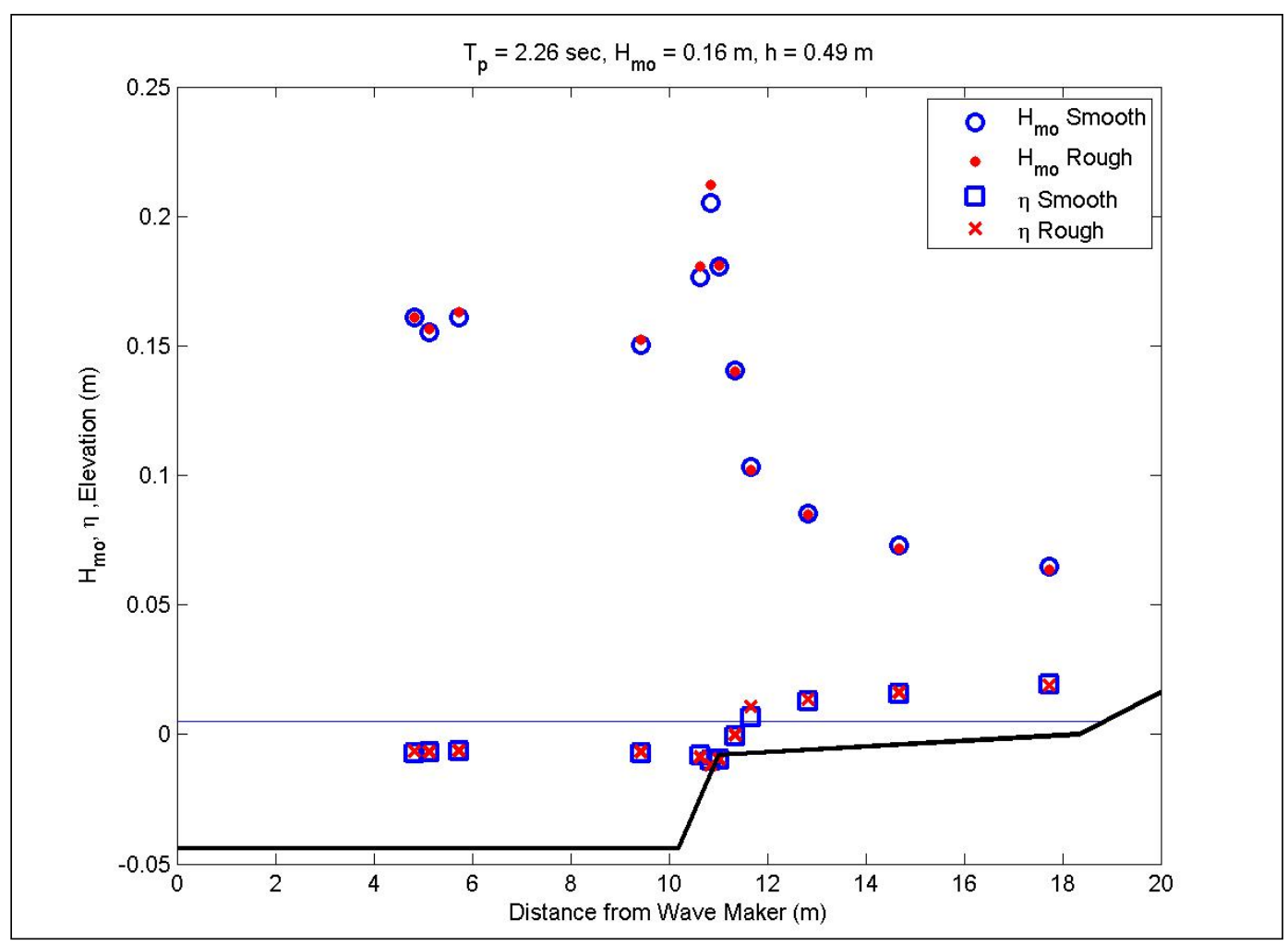

Figure E40. Wave transformation for 2.3-sec, 0.16-m waves at high water with a 1:25 reef slope.

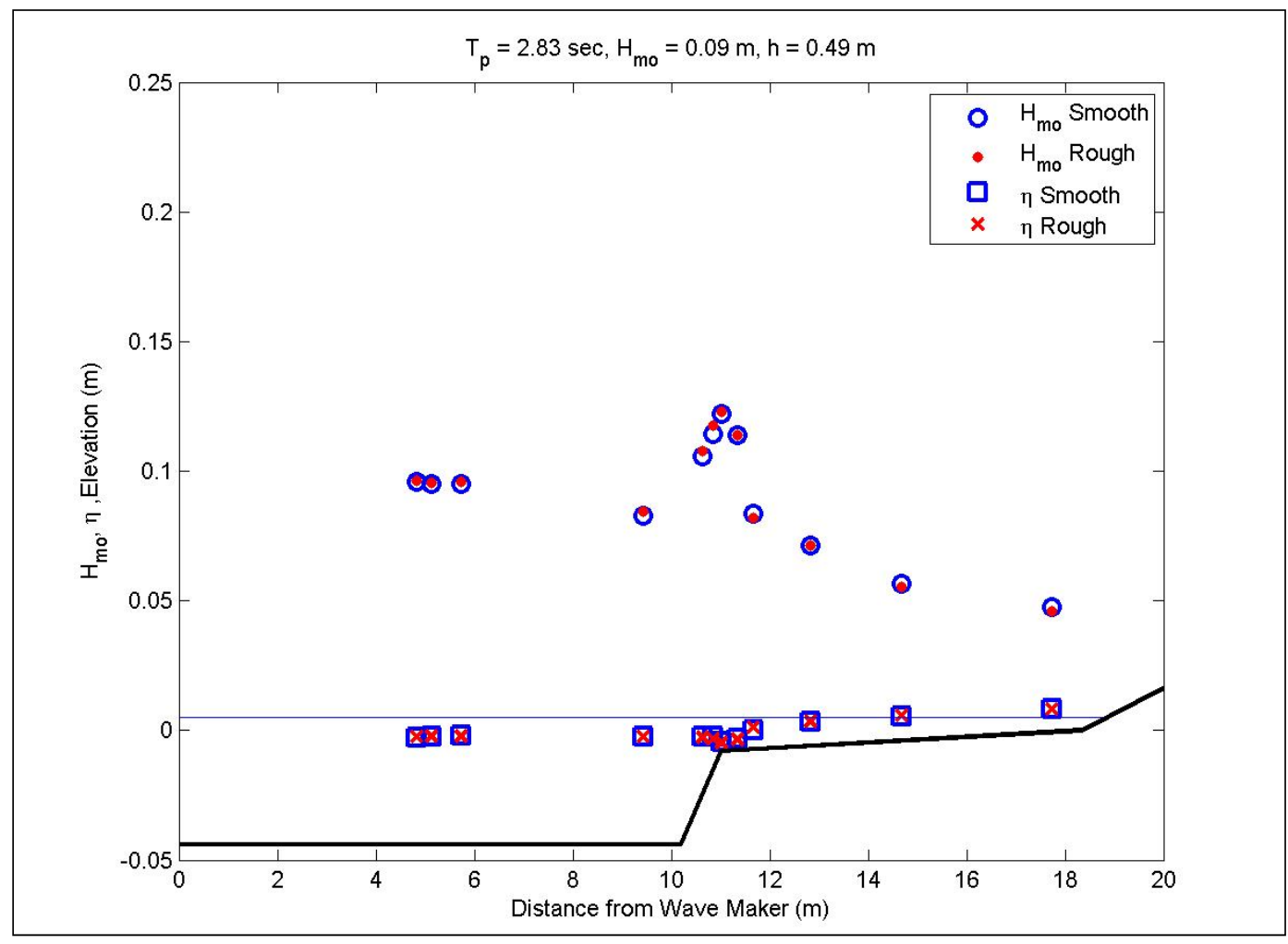

Figure E41. Wave transformation for 2.8-sec, 0.09-m waves at high water with a 1:25 reef slope. 


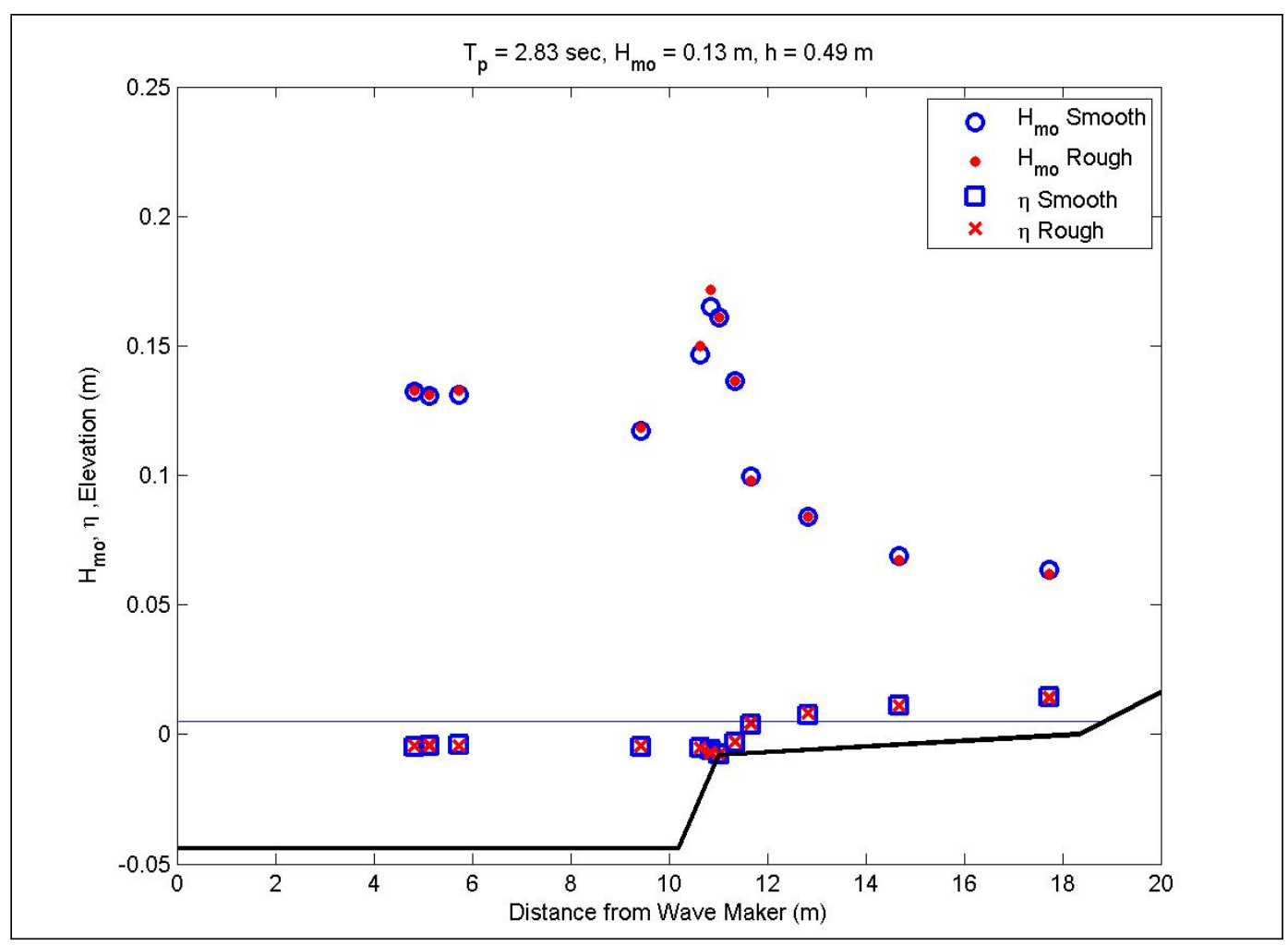

Figure E42. Wave transformation for 2.8-sec, 0.13-m waves at high water with a 1:25 reef slope.

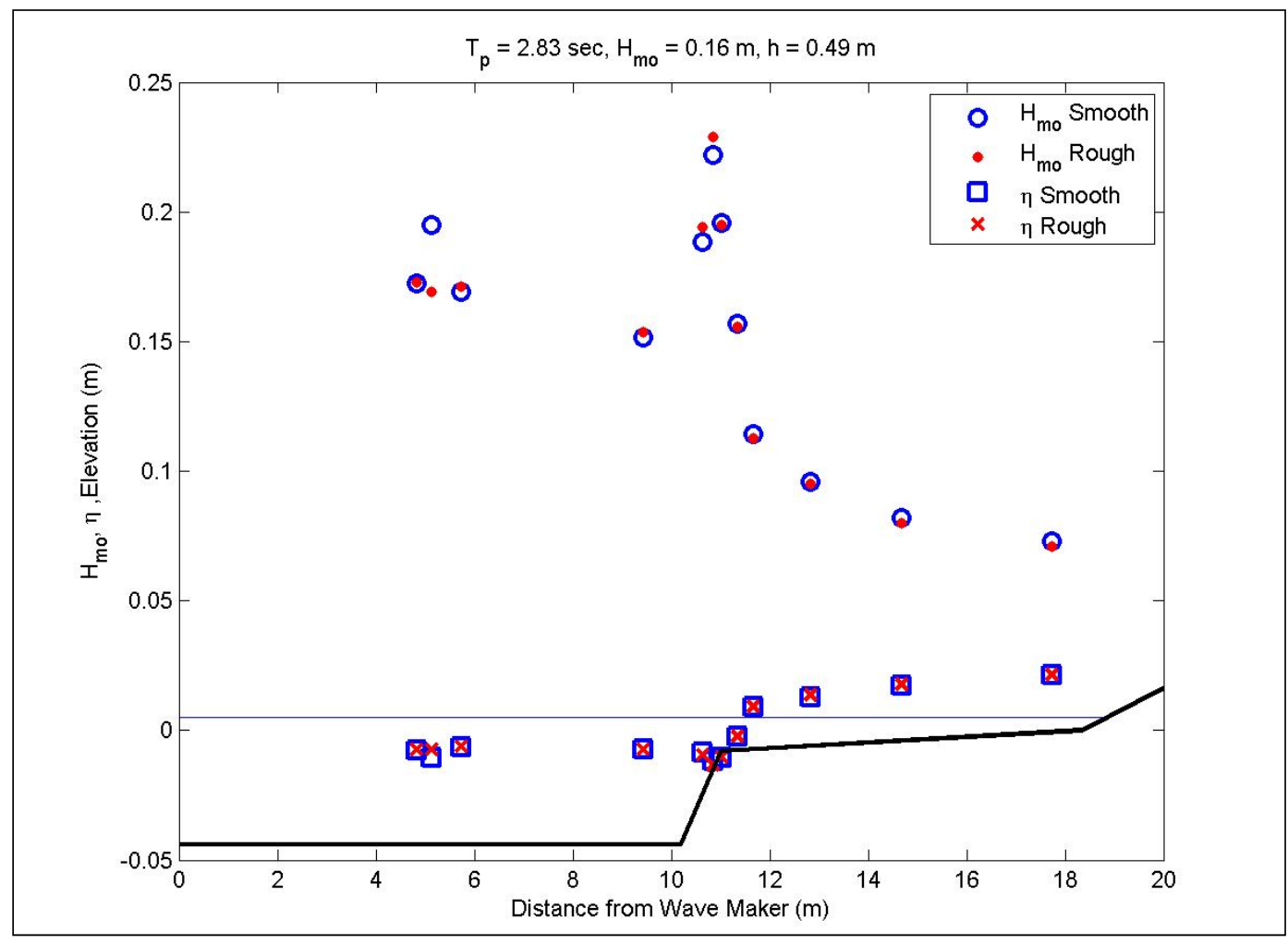

Figure E43. Wave transformation for 2.8-sec, 0.16-m waves at high water with a 1:25 reef slope. 


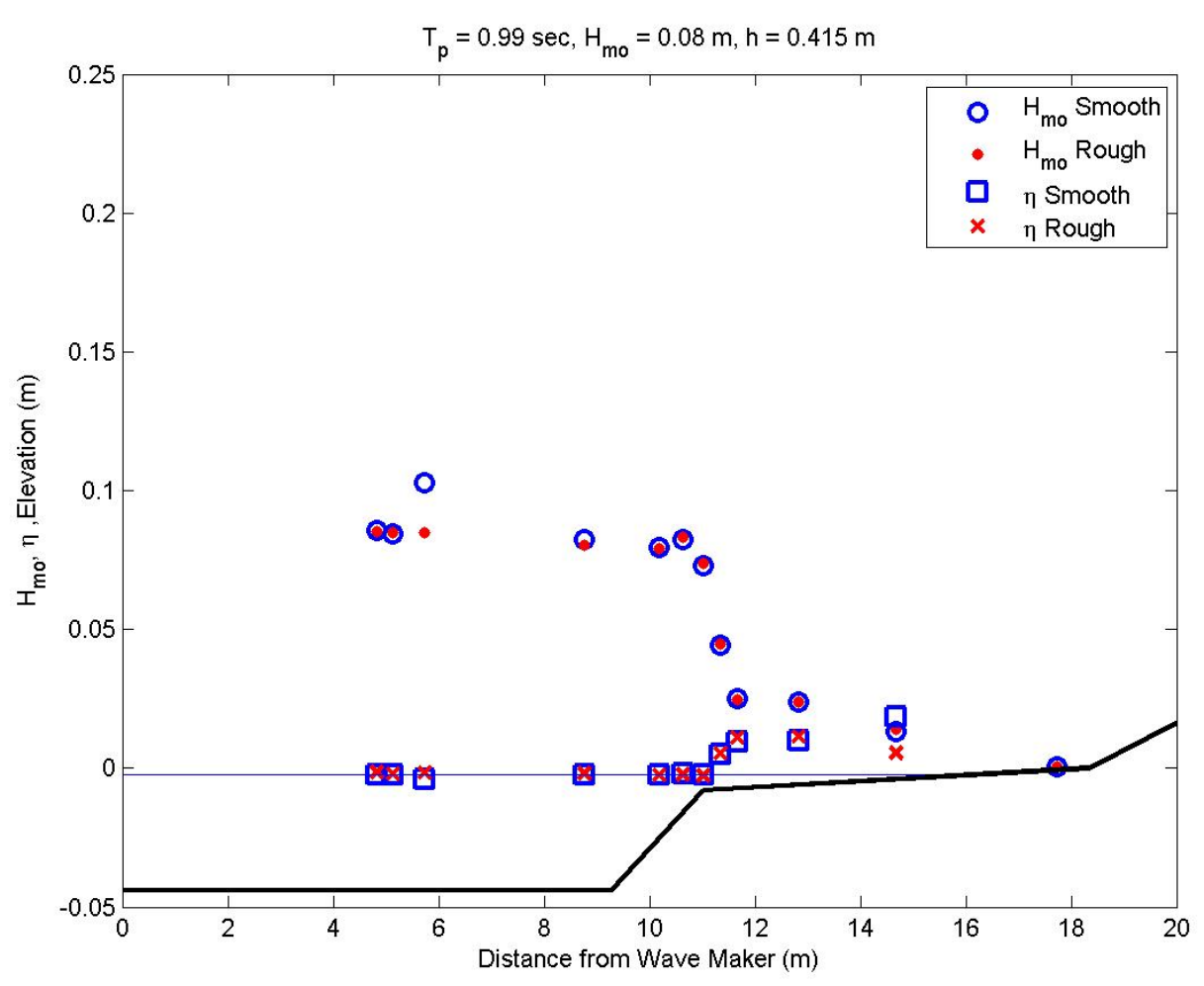

Figure E44. Wave transformation for 1-sec, 0.08-m waves at low water with a 1:5 reef slope.

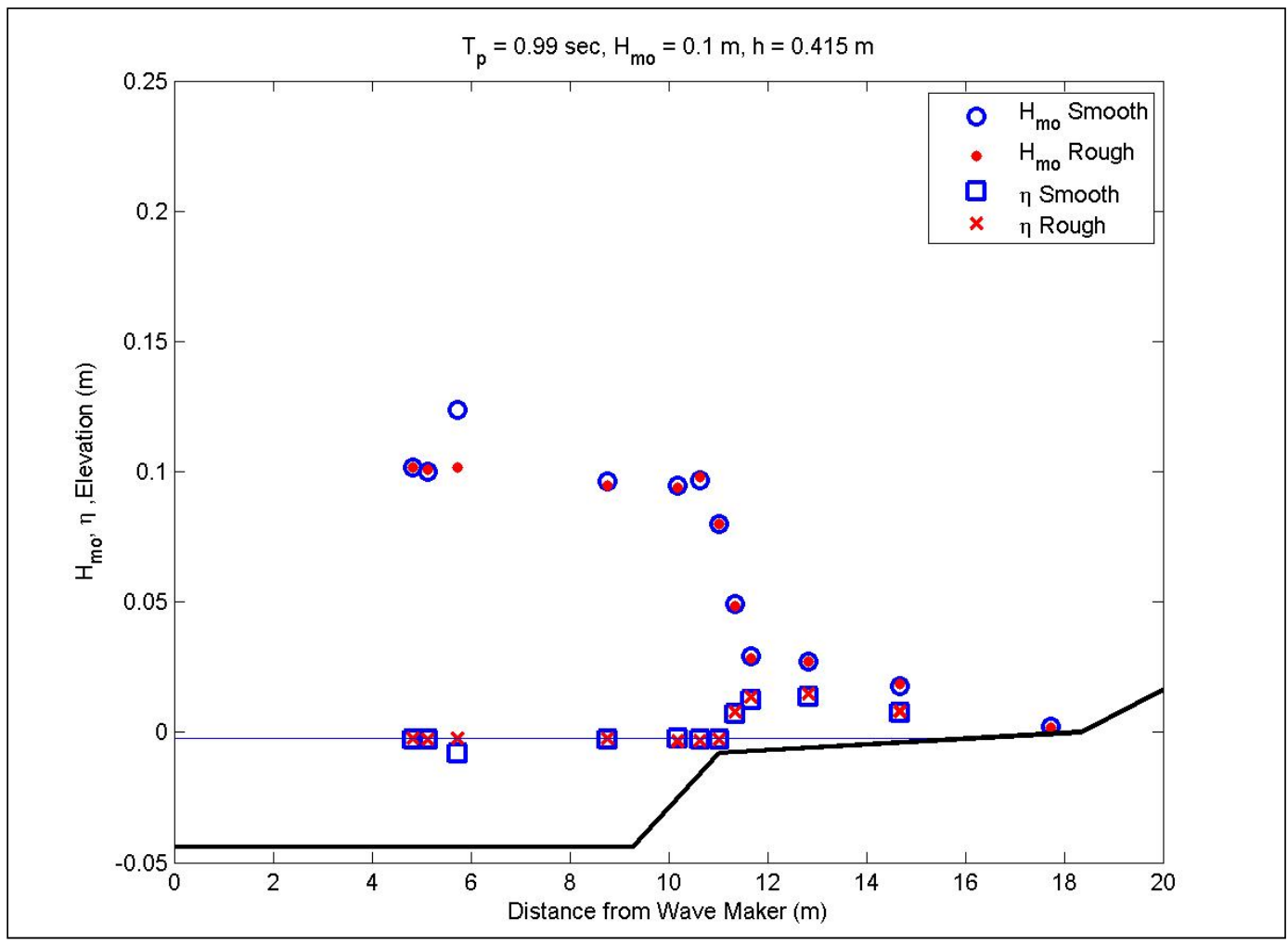

Figure E45. Wave transformation for 1-sec, 0.1-m waves at low water with a 1:5 reef slope. 


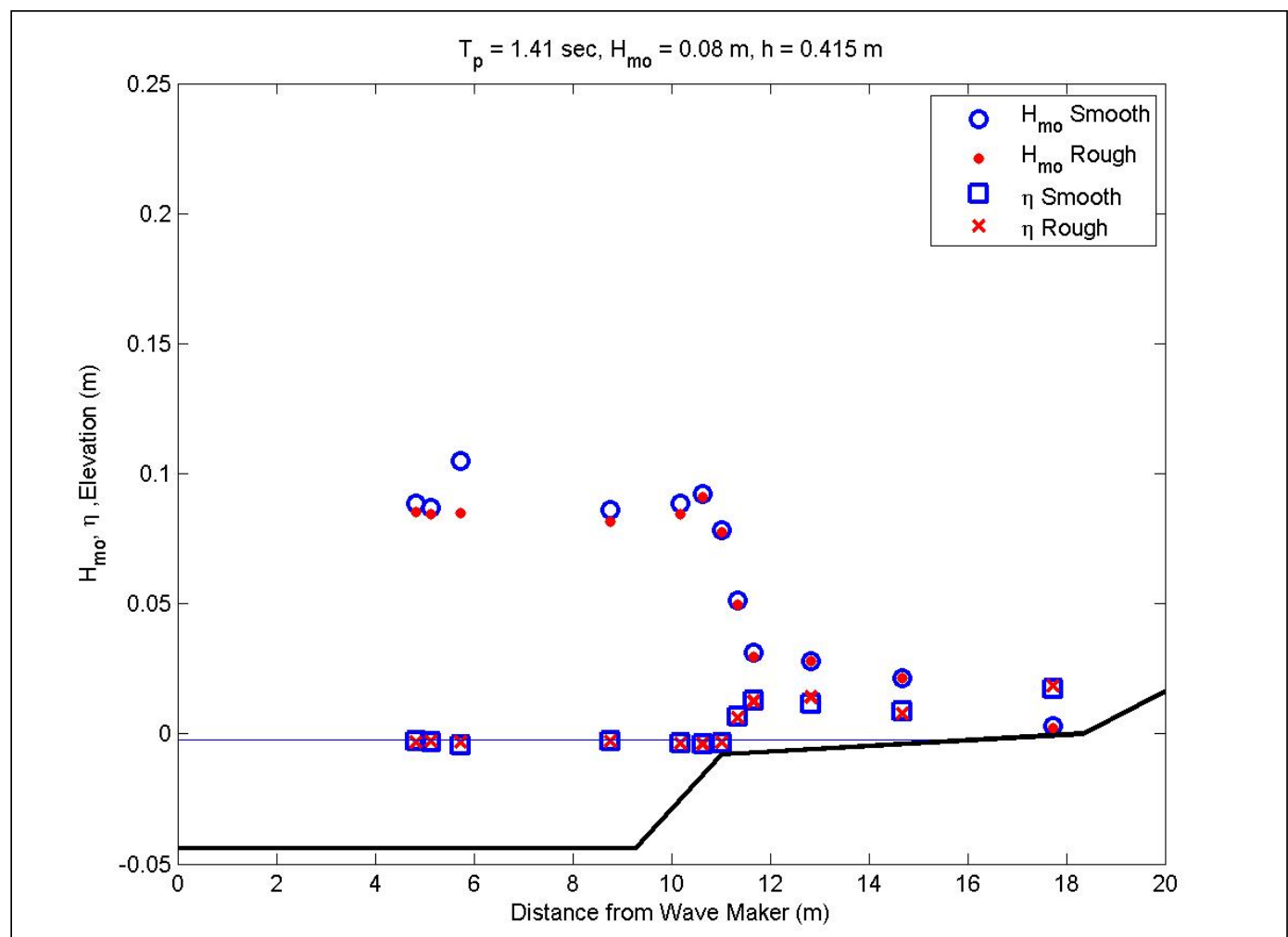

Figure E46. Wave transformation for $1.4-\mathrm{sec}, 0.08-\mathrm{m}$ waves at low water with a 1:5 reef slope.

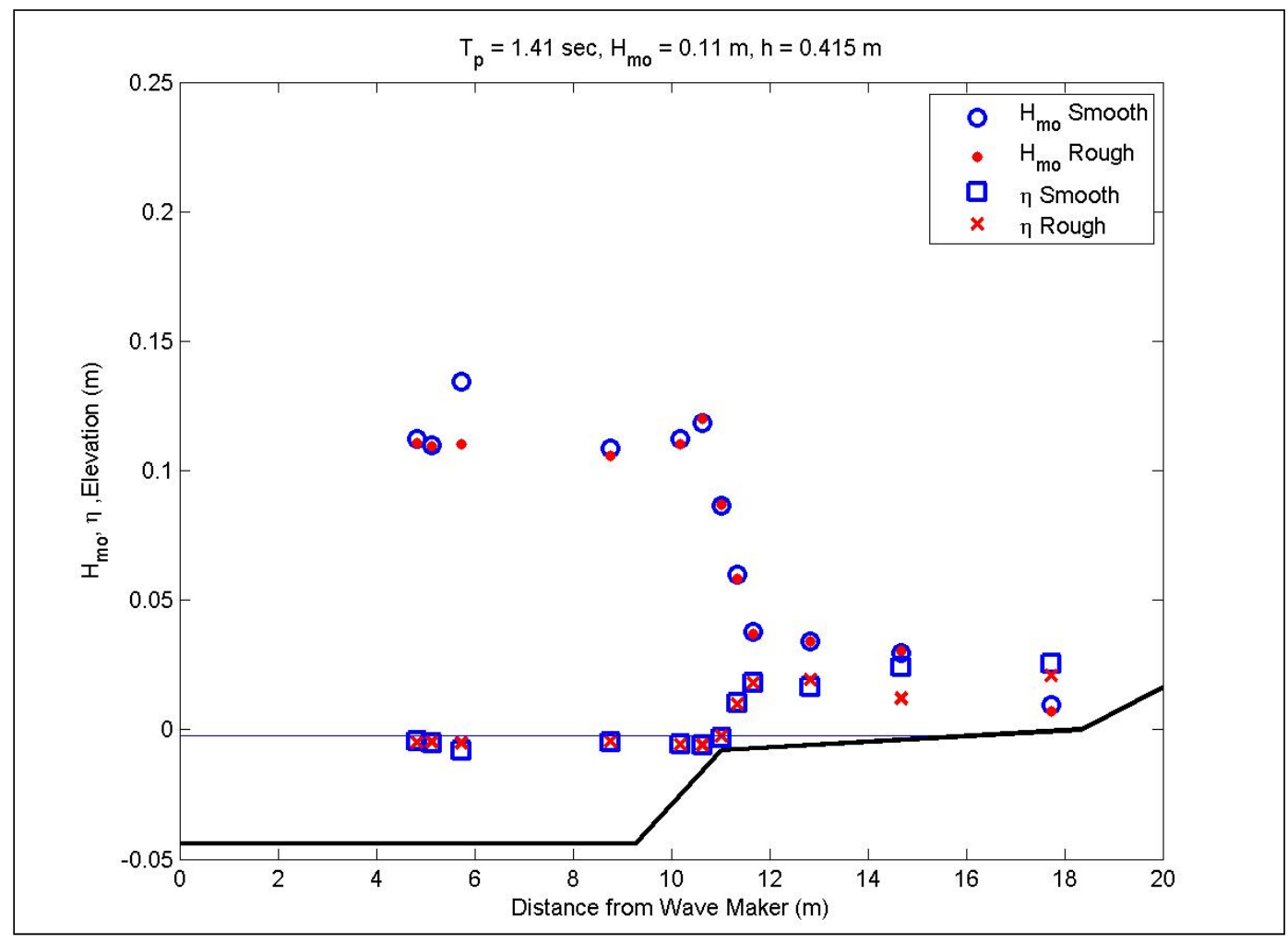

Figure E47. Wave transformation for 1.4-sec, 0.11-m waves at low water with a 1:5 reef slope. 


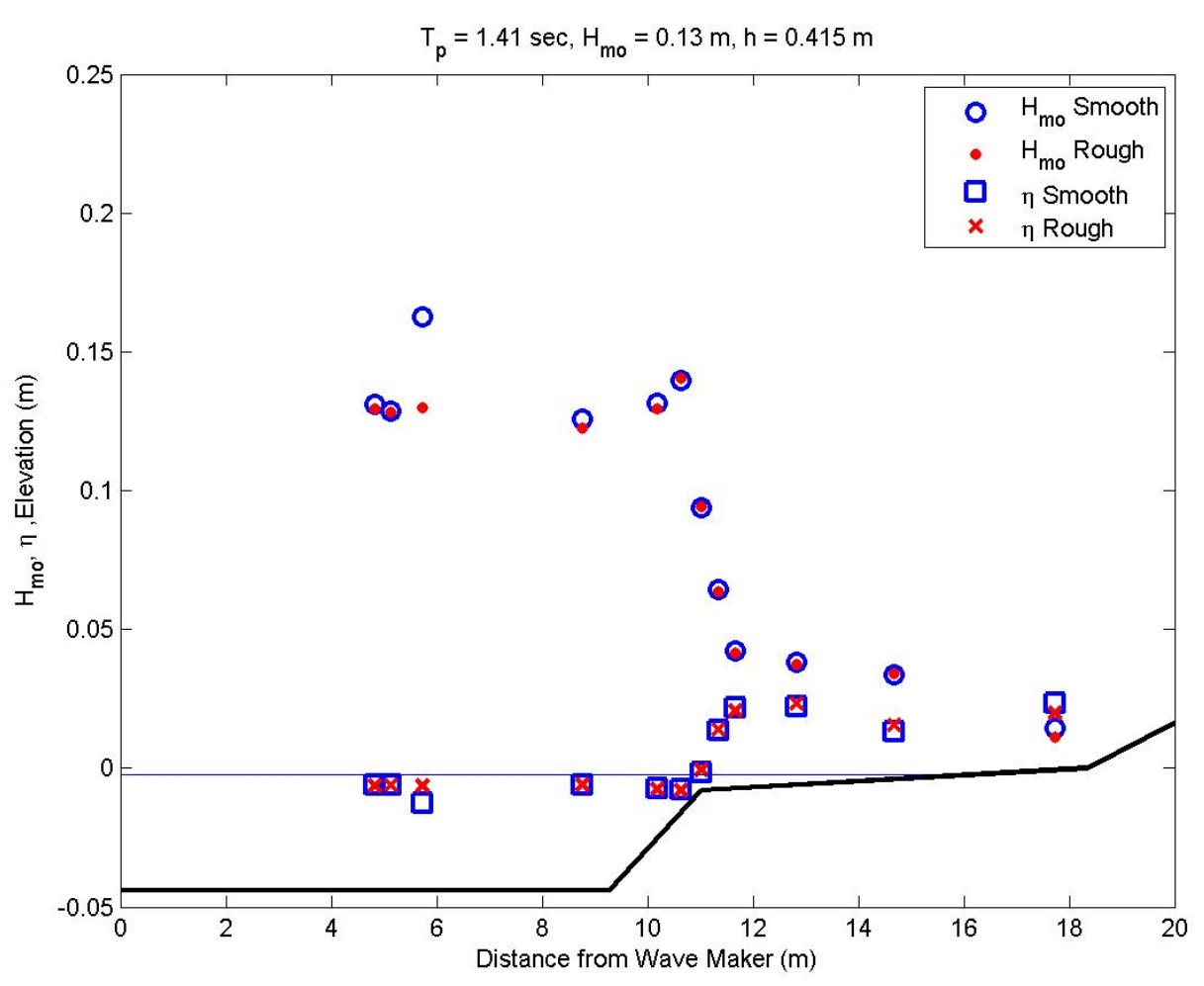

Figure E48. Wave transformation for 1.4-sec, 0.13-m waves at low water with a 1:5 reef slope.

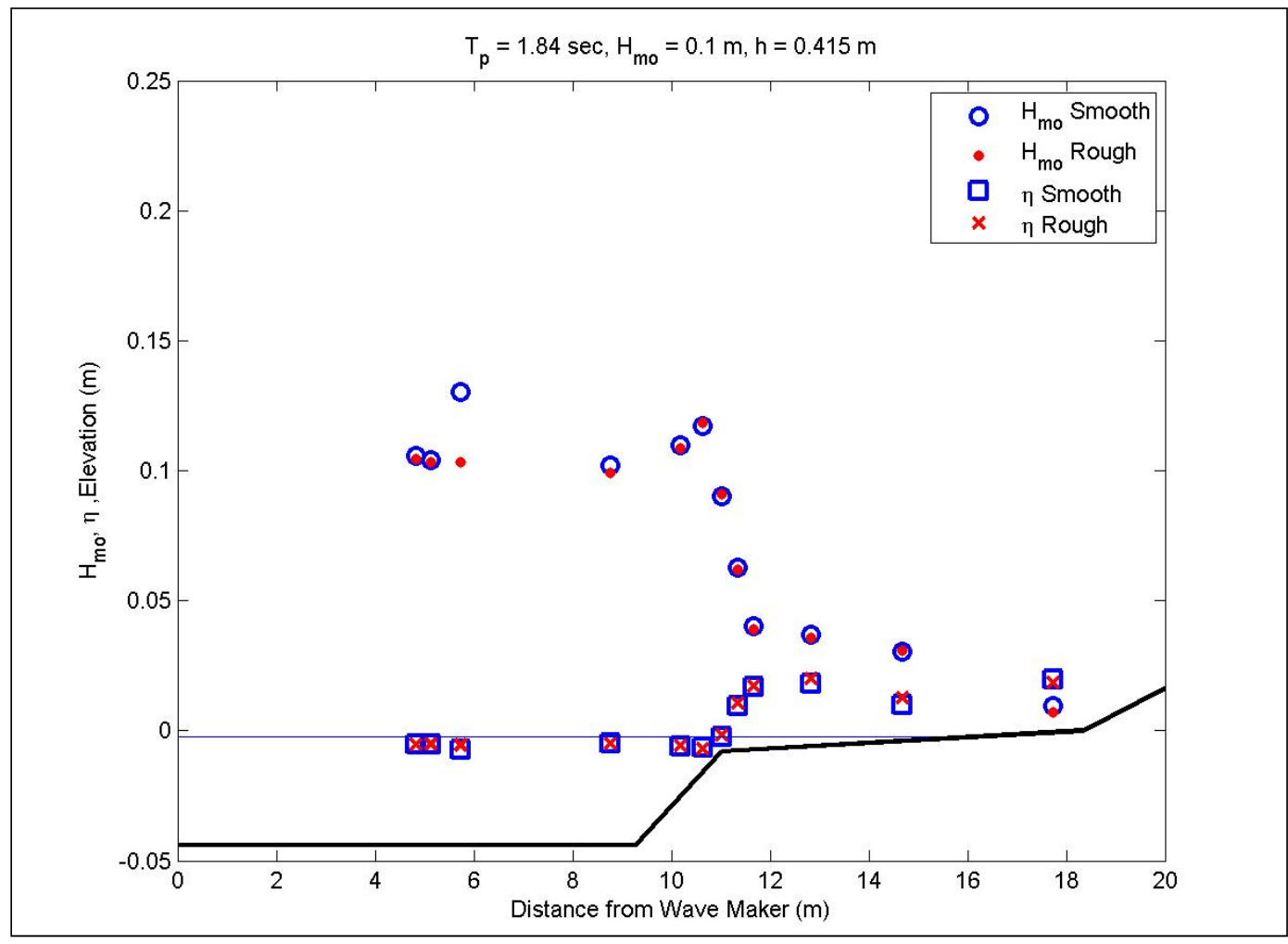

Figure E49. Wave transformation for 1.8-sec, 0.1-m waves at low water with a 1:5 reef slope. 


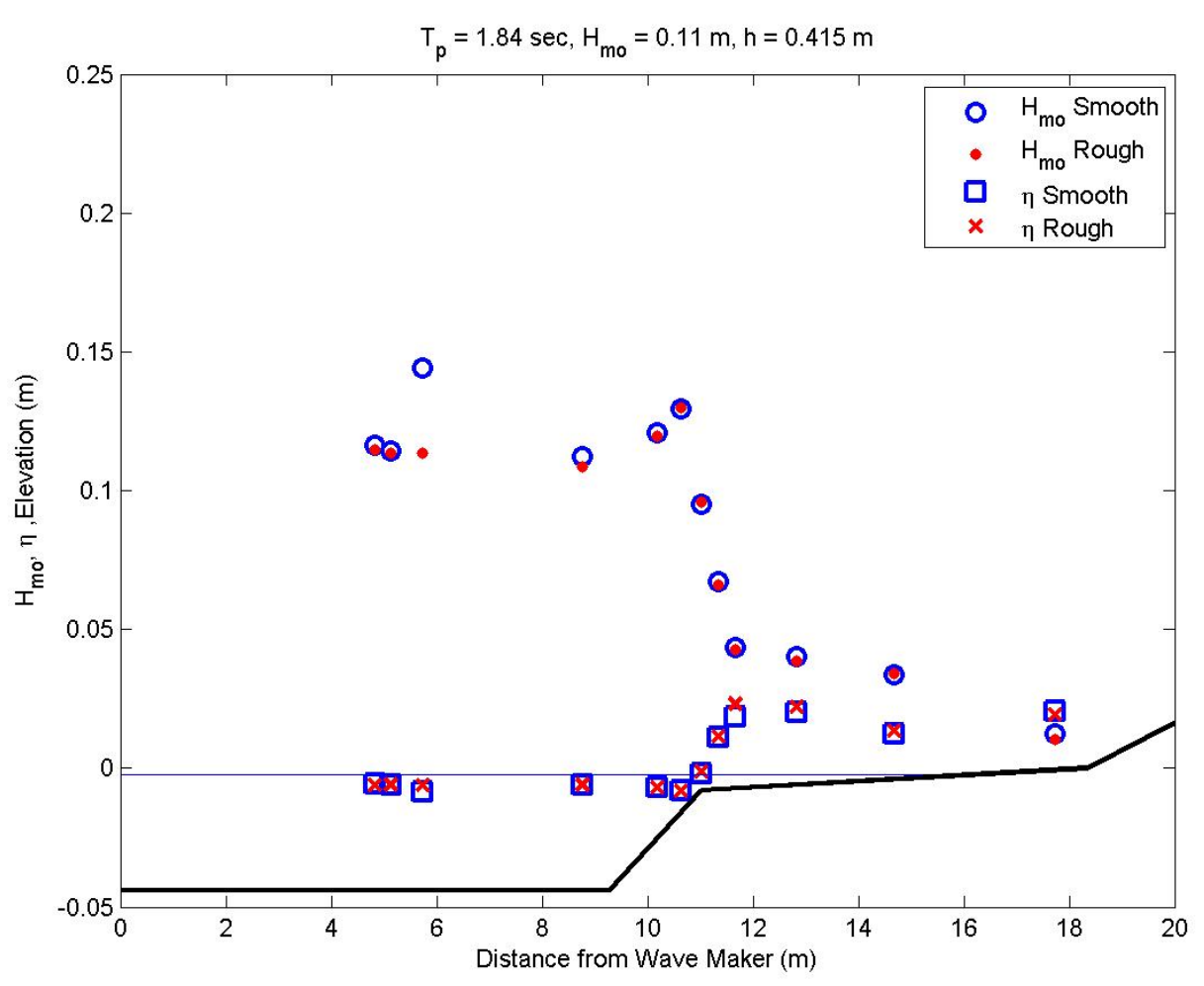

Figure E50. Wave transformation for 1.8-sec, 0.11-m waves at low water with a 1:5 reef slope.

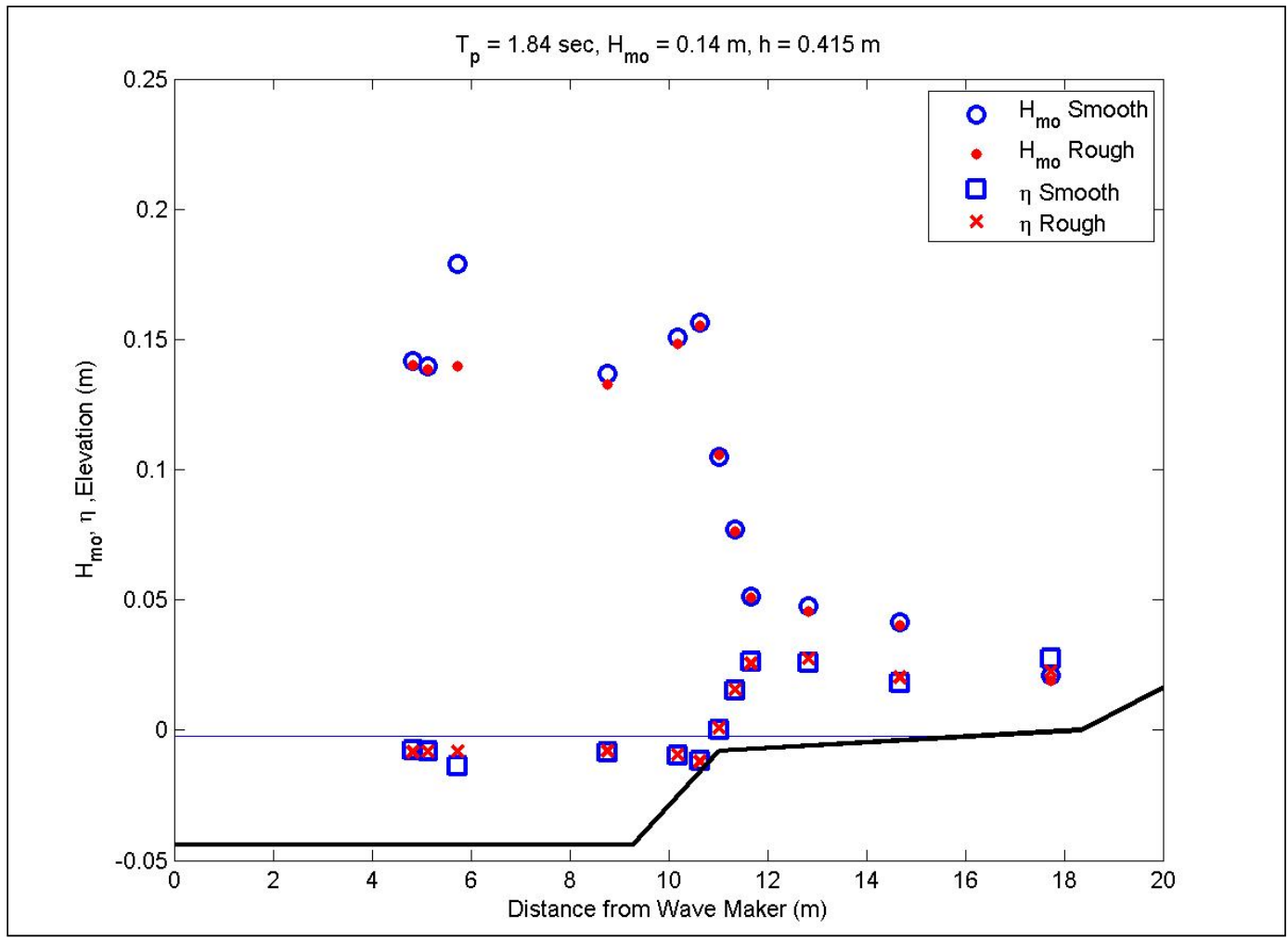

Figure E51. Wave transformation for 1.8-sec, 0.14-m waves at low water with a 1:5 reef slope. 


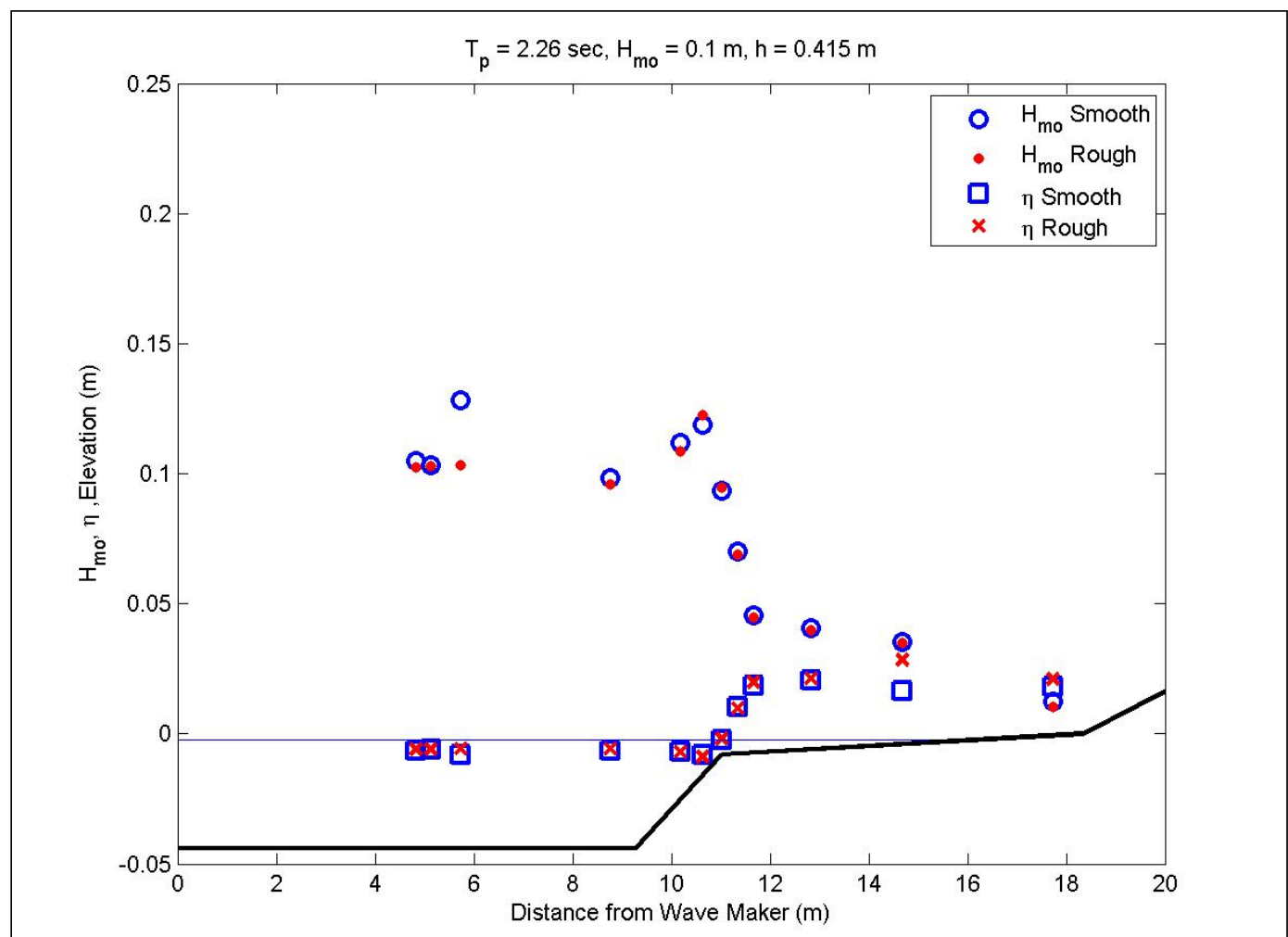

Figure E52. Wave transformation for 2.3-sec, 0.1-m waves at low water with a 1:5 reef slope.

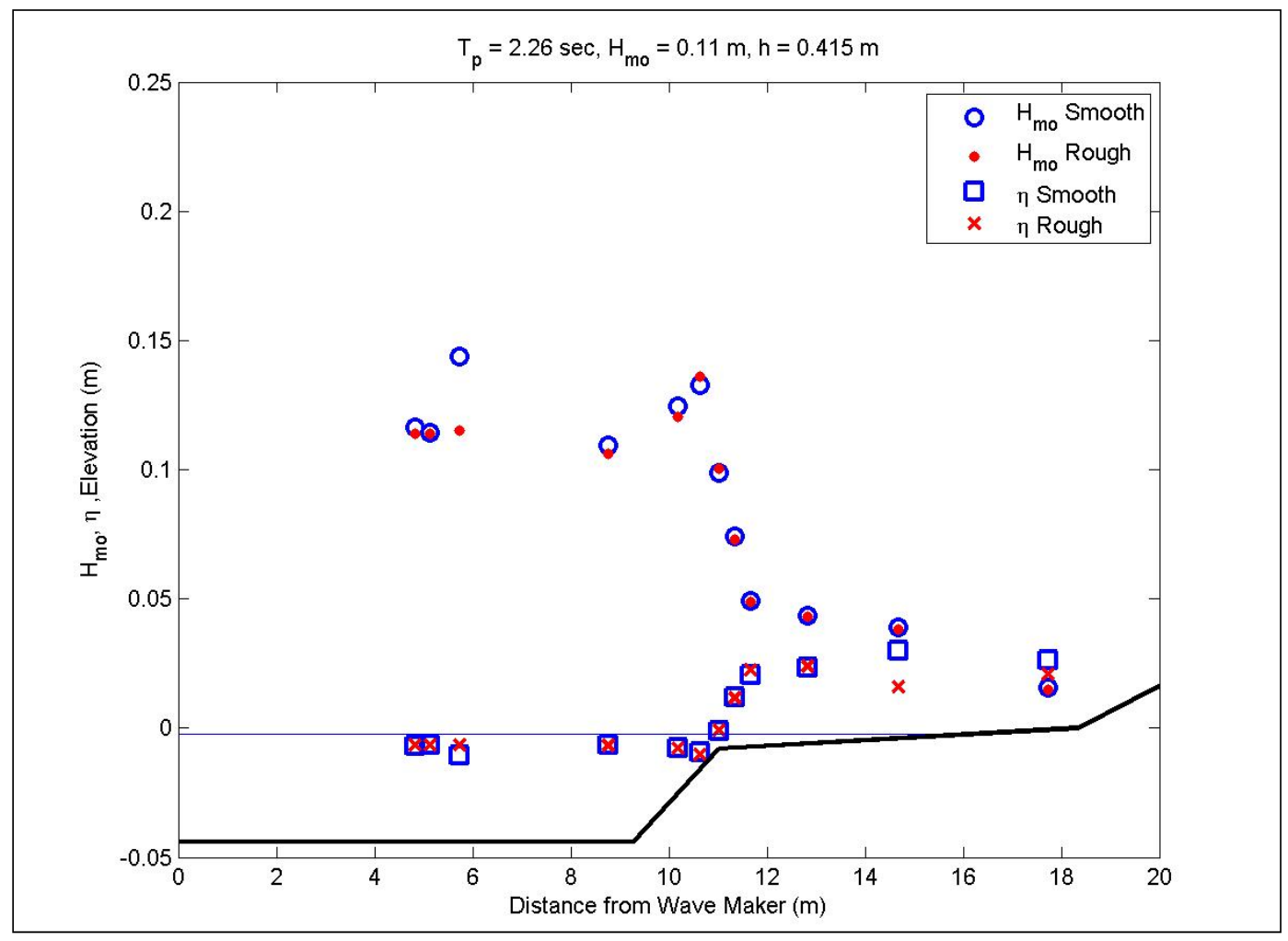

Figure E53. Wave transformation for 2.3-sec, 0.11-m waves at low water with a 1:5 reef slope. 


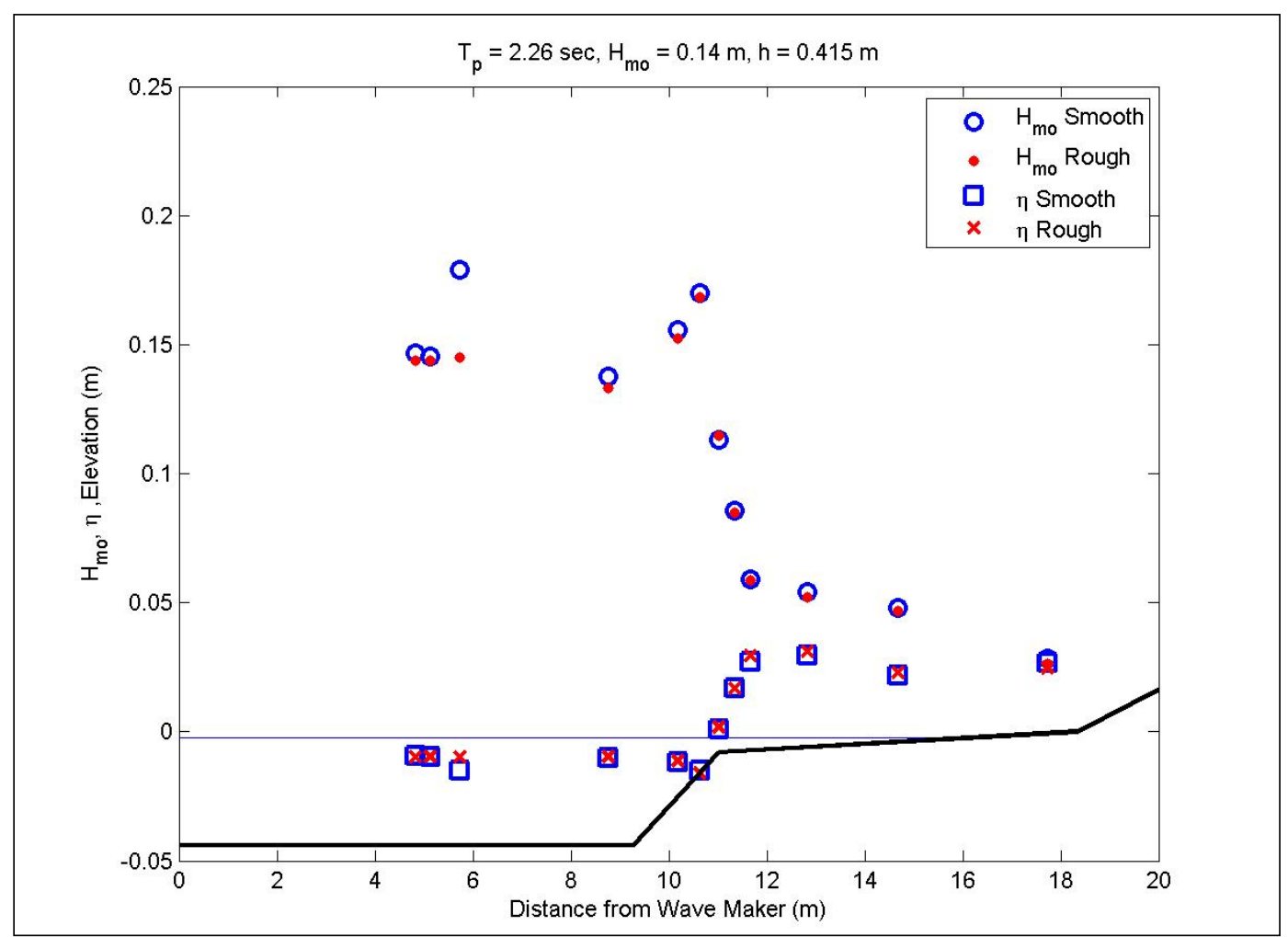

Figure E54. Wave transformation for 2.3-sec, 0.14-m waves at low water with a 1:5 reef slope.

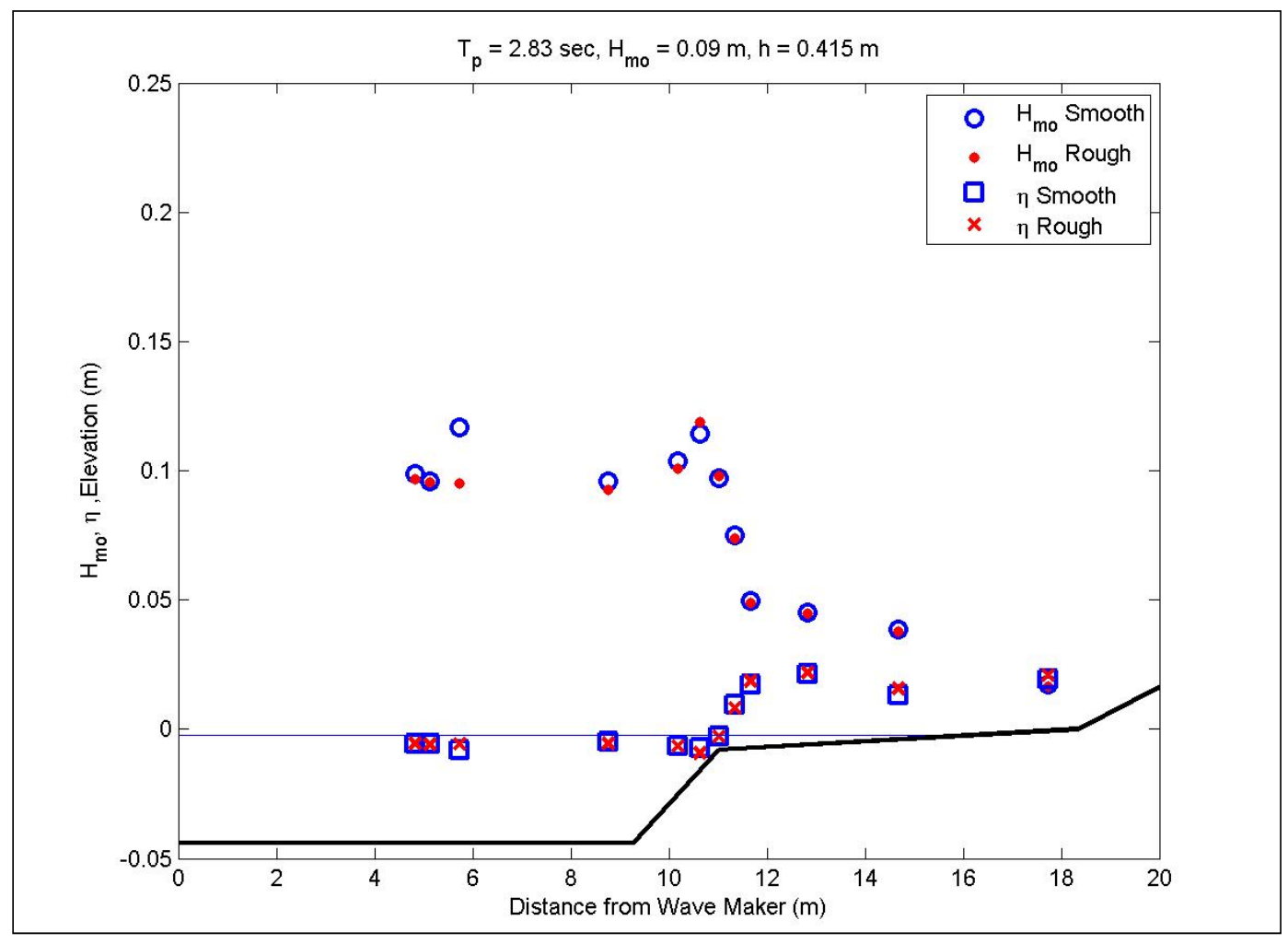

Figure E55. Wave transformation for 2.8-sec, 0.09-m waves at low water with a 1:5 reef slope. 


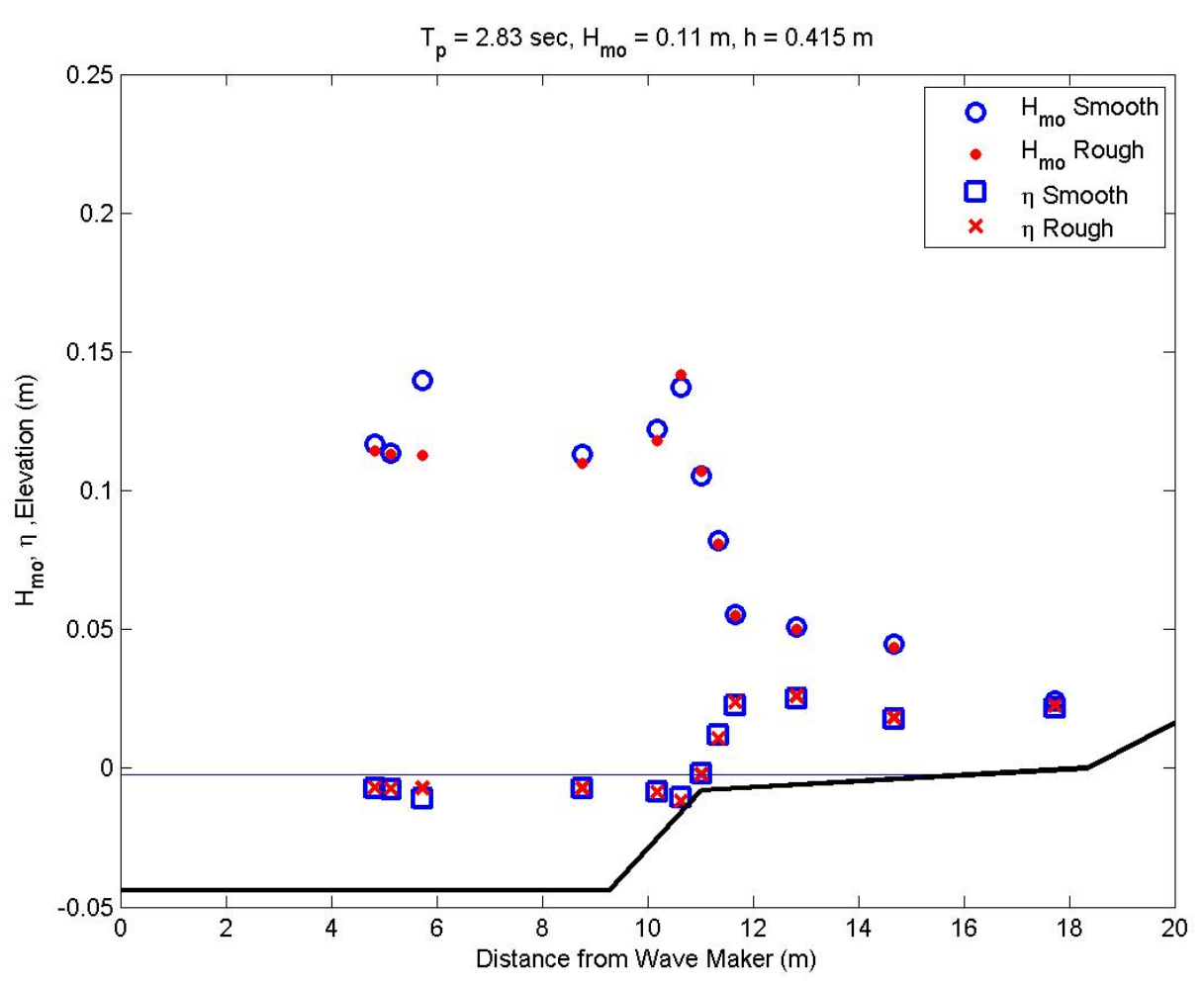

Figure E56. Wave transformation for 2.8-sec, 0.11-m waves at low water with a 1:5 reef slope.

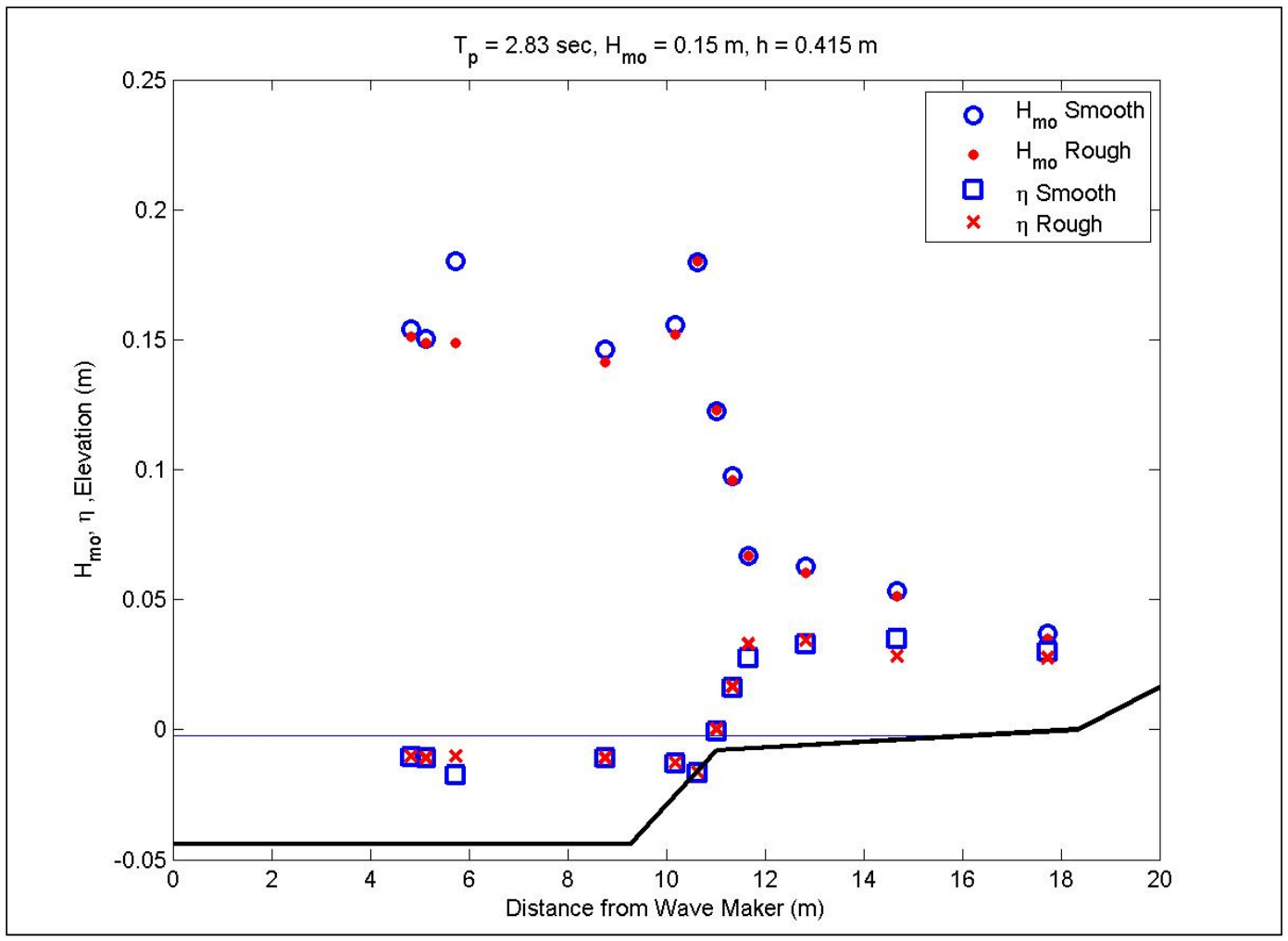

Figure E57. Wave transformation for 2.8-sec, 0.15-m waves at low water with a 1:5 reef slope. 


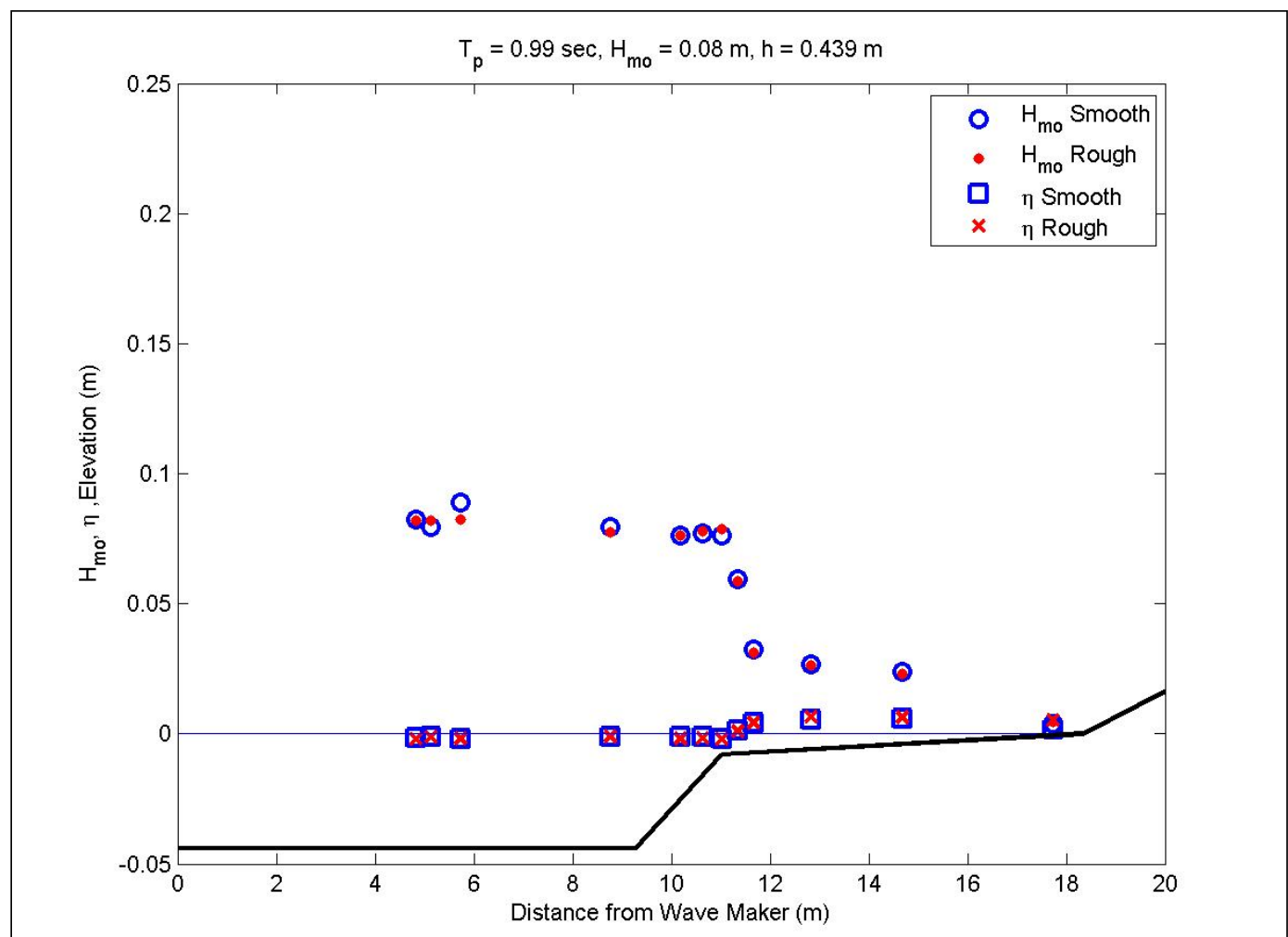

Figure E58. Wave transformation for 1-sec, 0.08-m waves at mid water with a 1:5 reef slope.

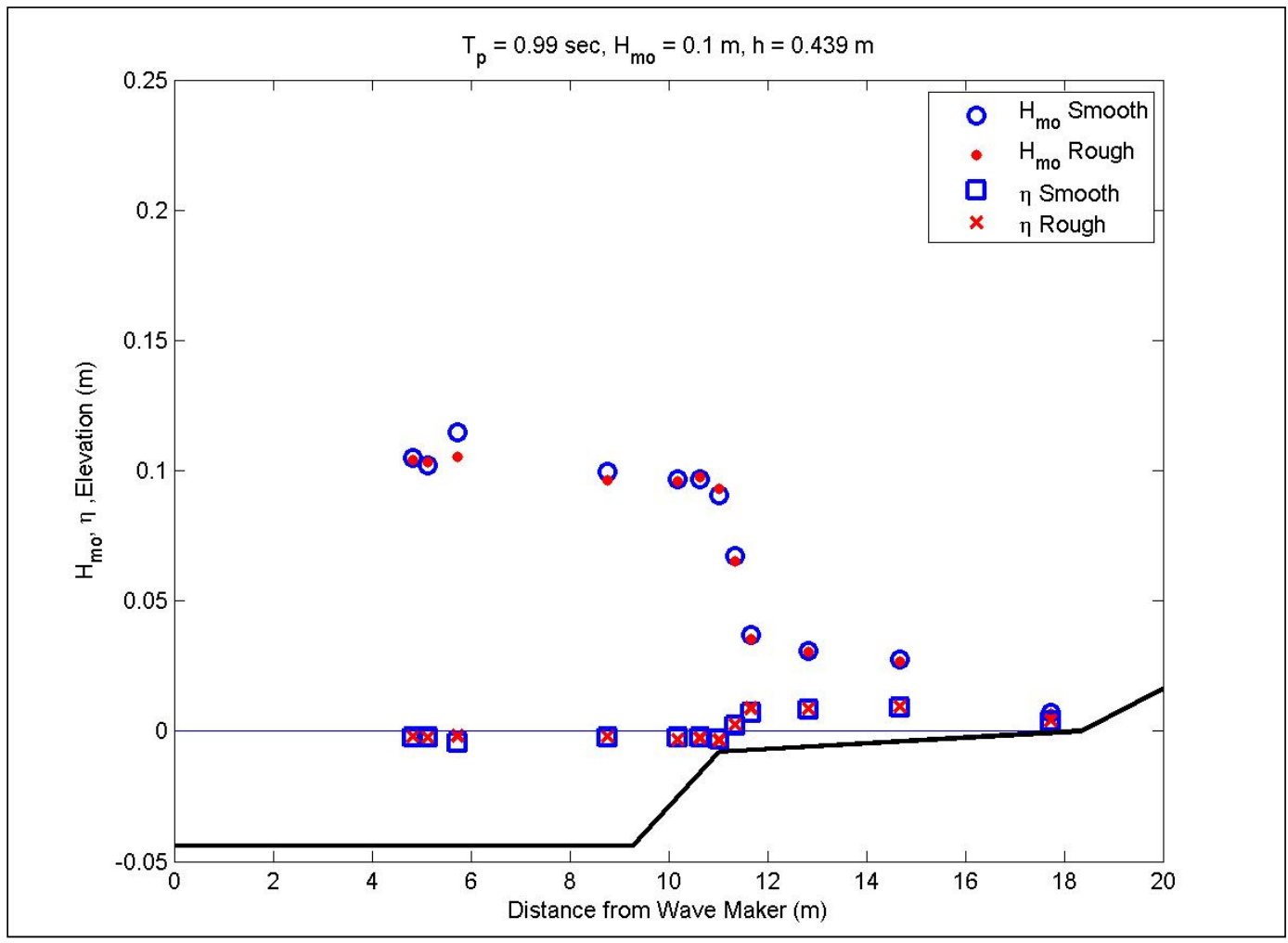

Figure E59. Wave transformation for 1-sec, 0.1-m waves at mid water with a 1:5 reef slope. 


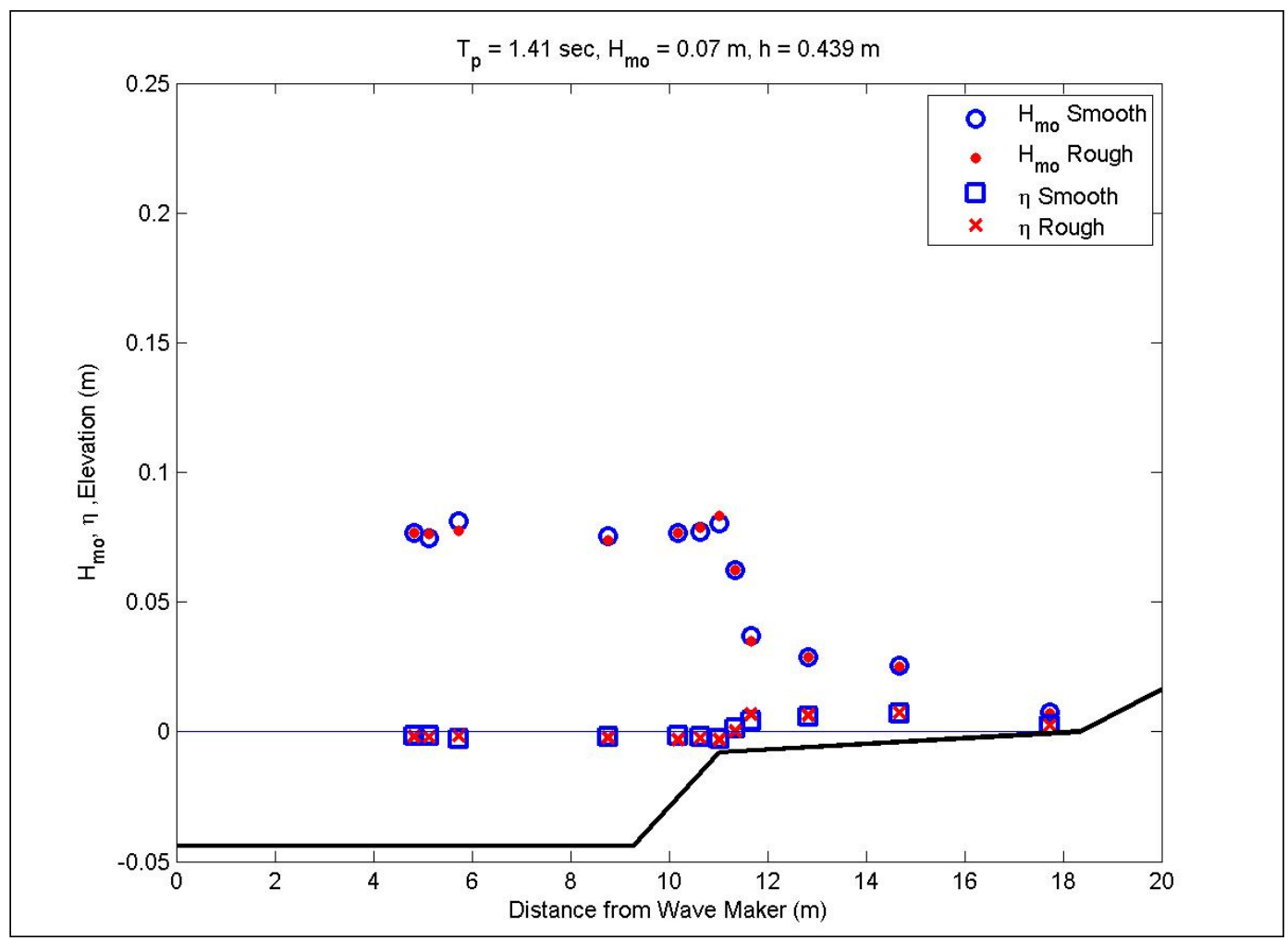

Figure E60. Wave transformation for 1.4-sec, 0.07-m waves at mid water with a 1:5 reef slope.

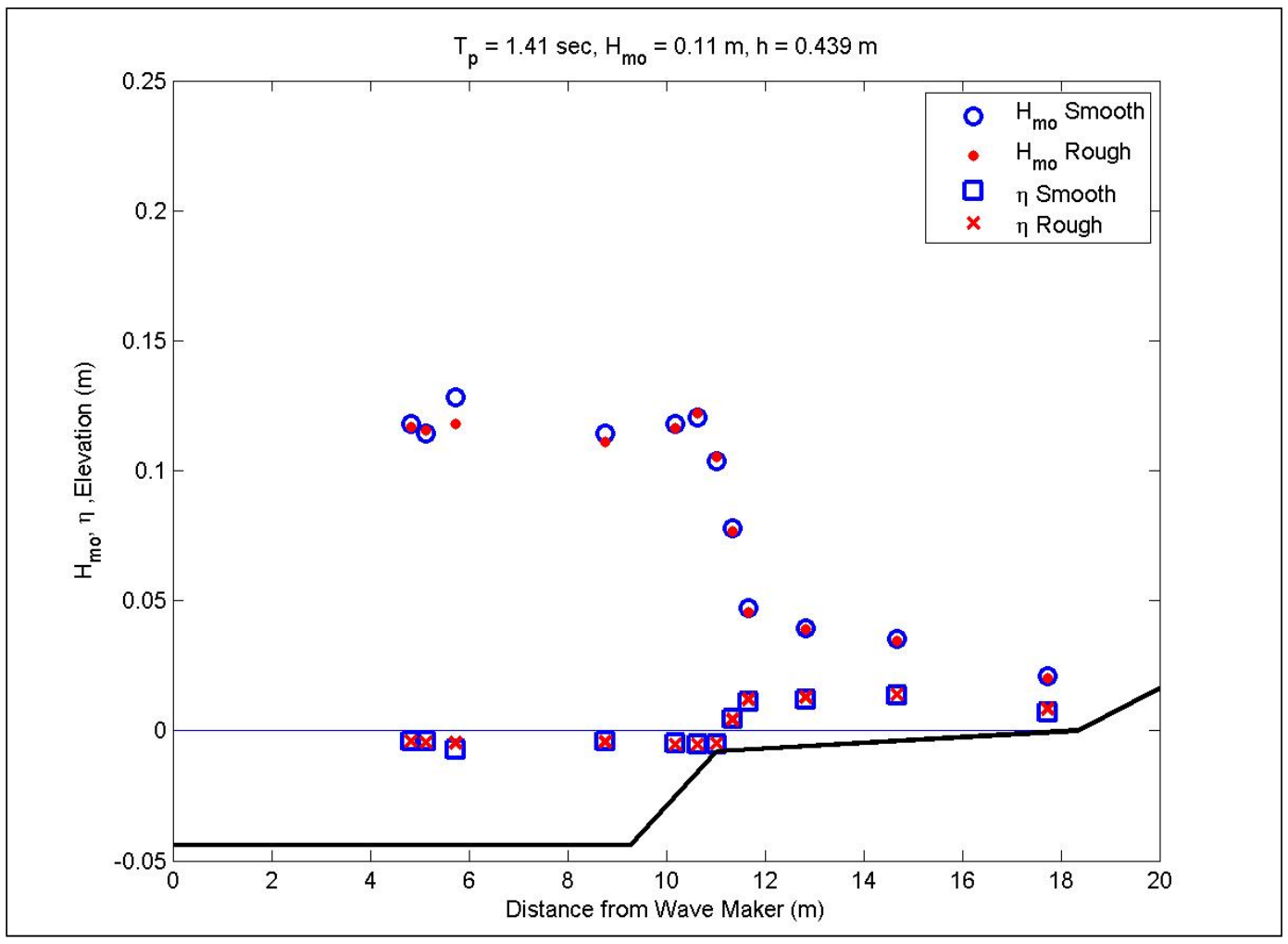

Figure E61. Wave transformation for 1.4-sec, 0.11-m waves at mid water with a 1:5 reef slope. 


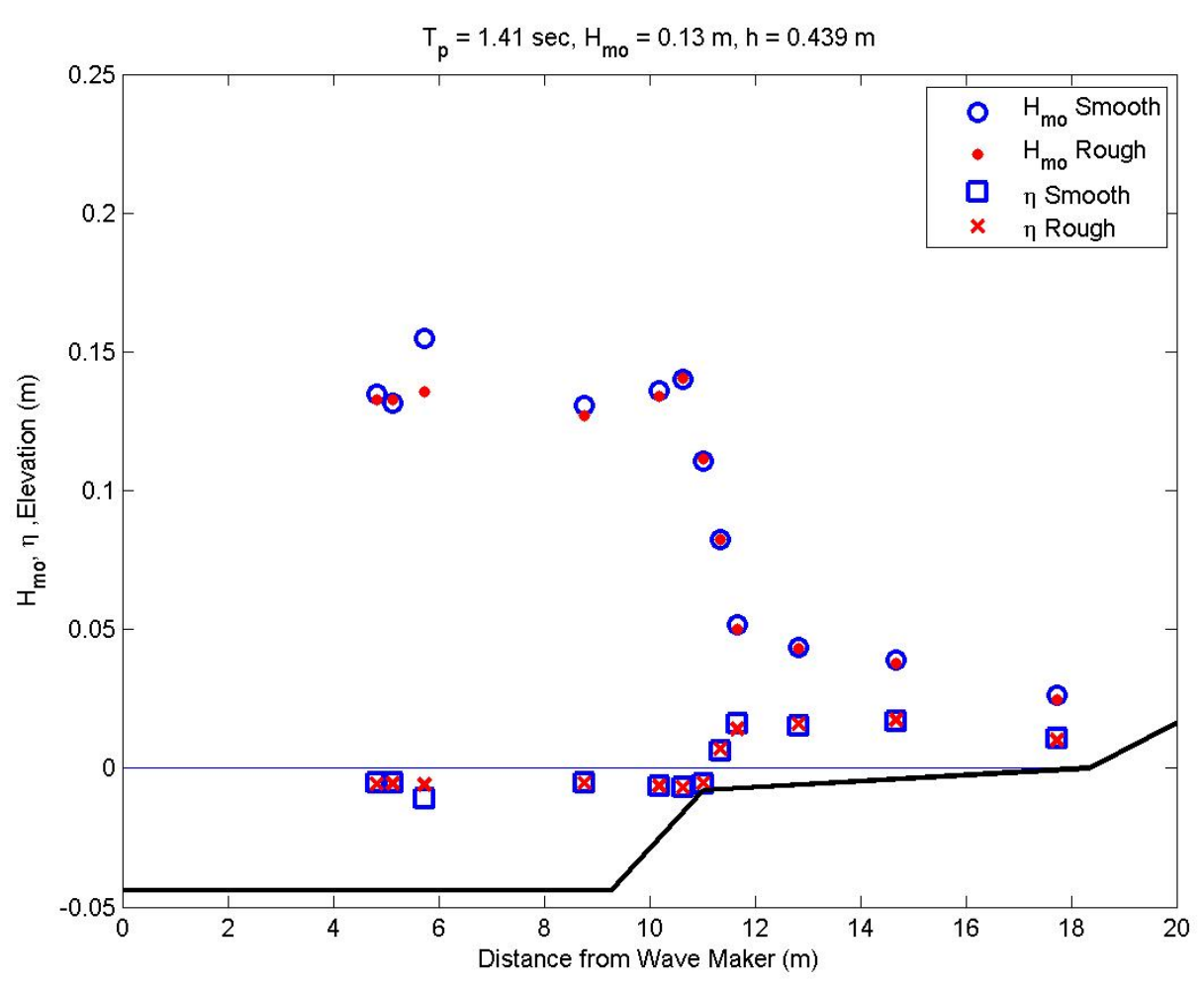

Figure E62. Wave transformation for 1.4-sec, 0.13-m waves at mid water with a 1:5 reef slope.

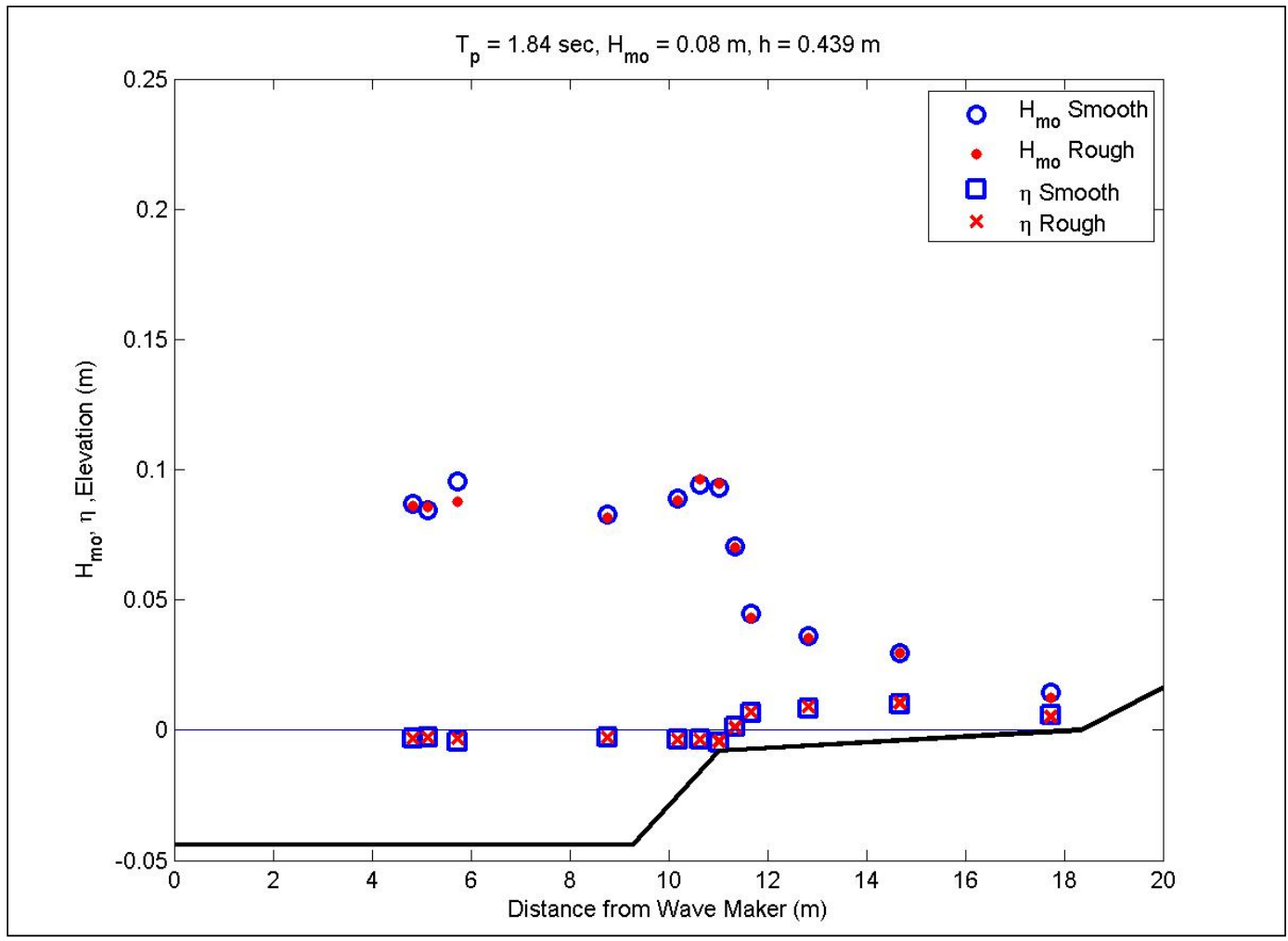

Figure E63. Wave transformation for 1.8-sec, 0.08-m waves at mid water with a 1:5 reef slope. 


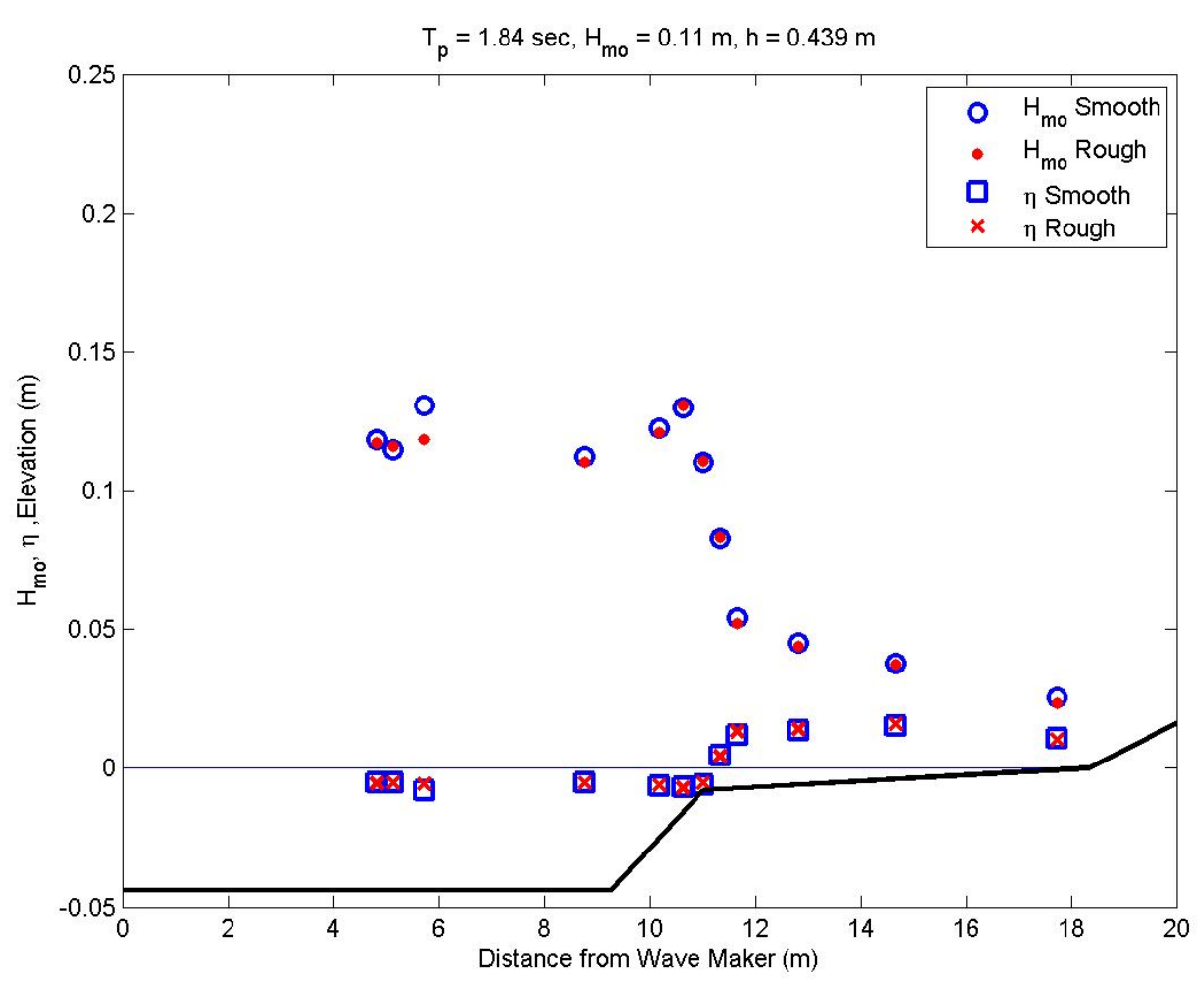

Figure E64. Wave transformation for 1.8-sec, 0.11-m waves at mid water with a 1:5 reef slope.

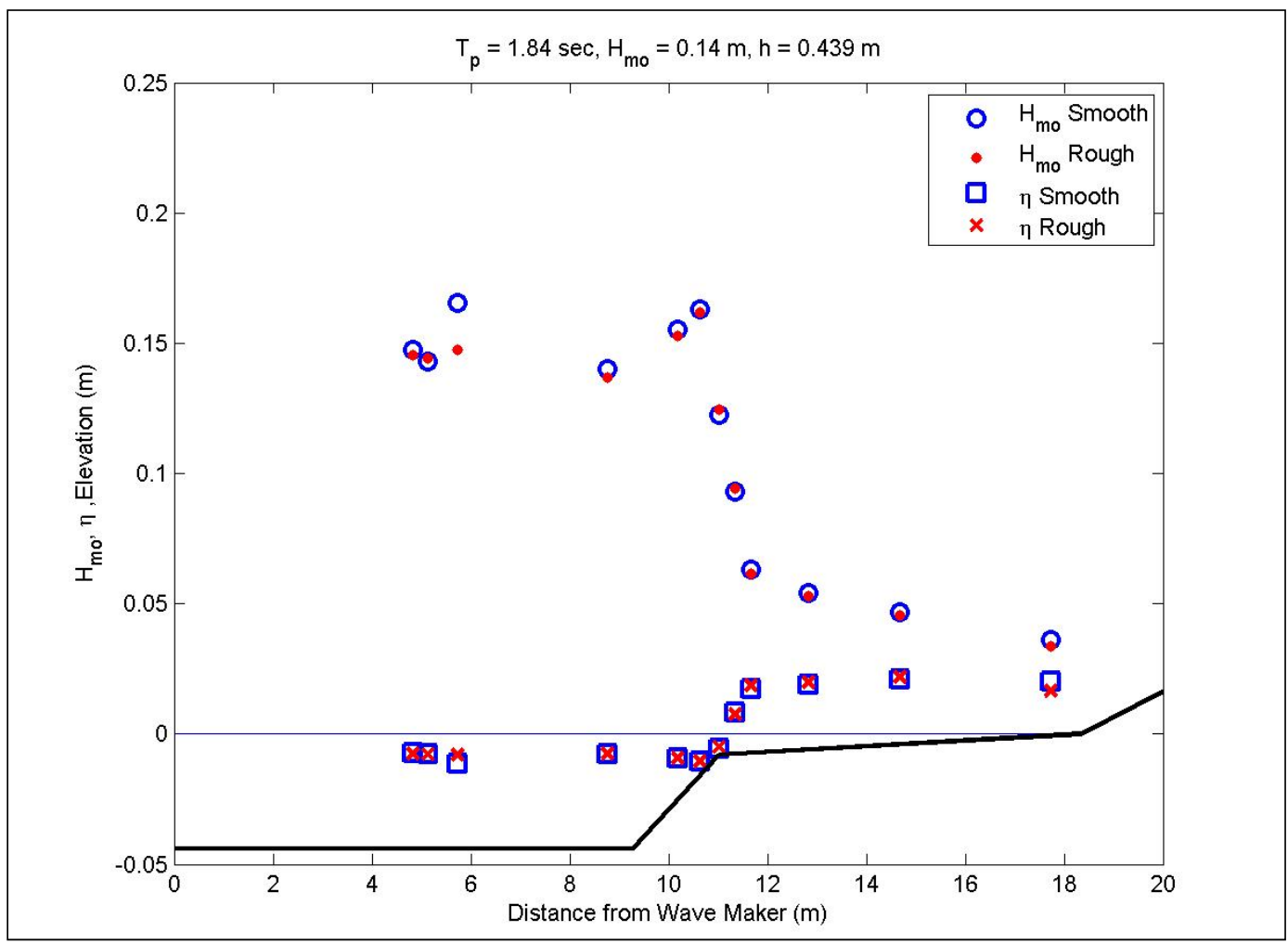

Figure E65. Wave transformation for 1.8-sec, 0.14-m waves at mid water with a 1:5 reef slope. 


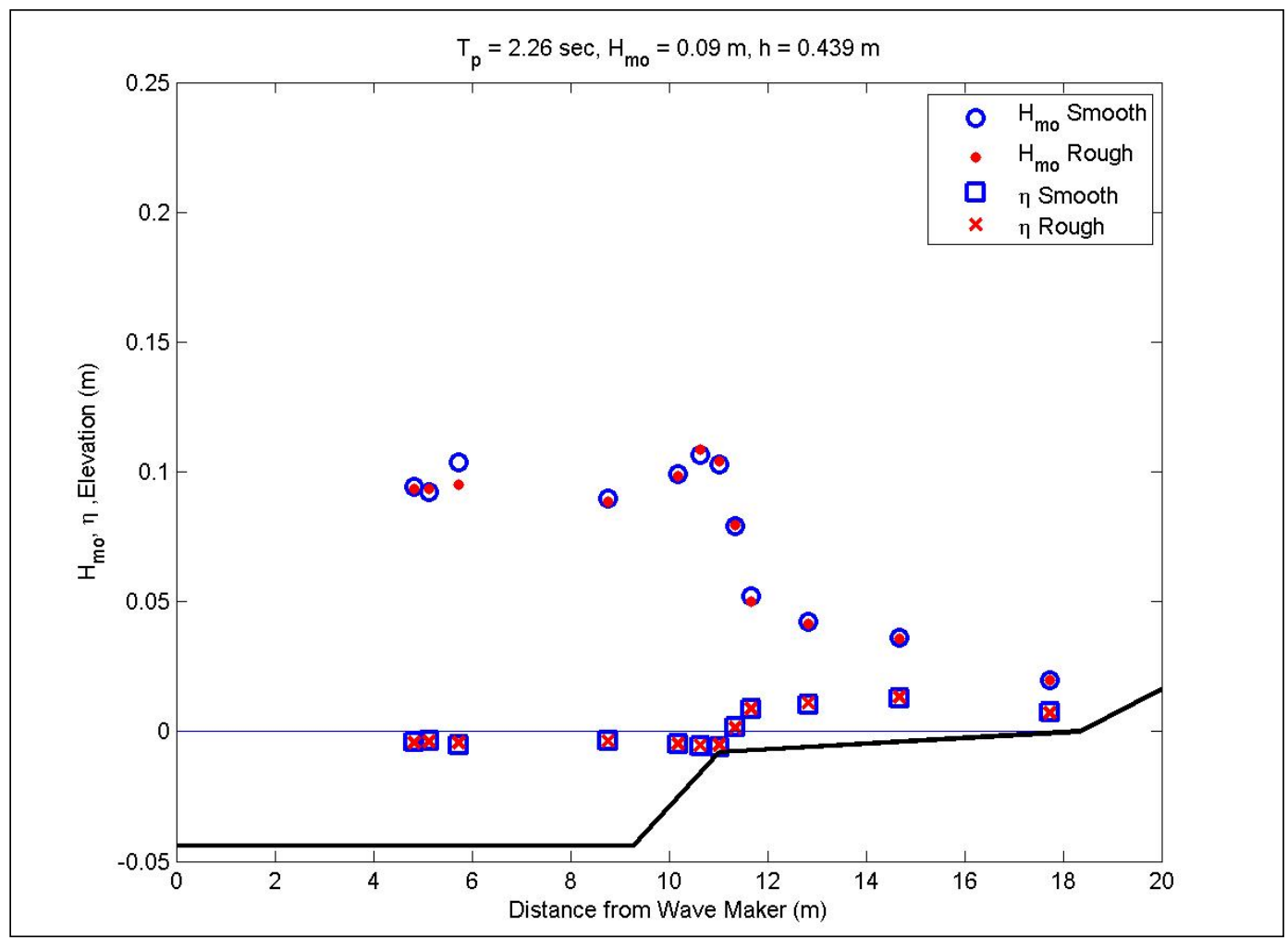

Figure E66. Wave transformation for 2.3-sec, 0.09-m waves at mid water with a 1:5 reef slope.

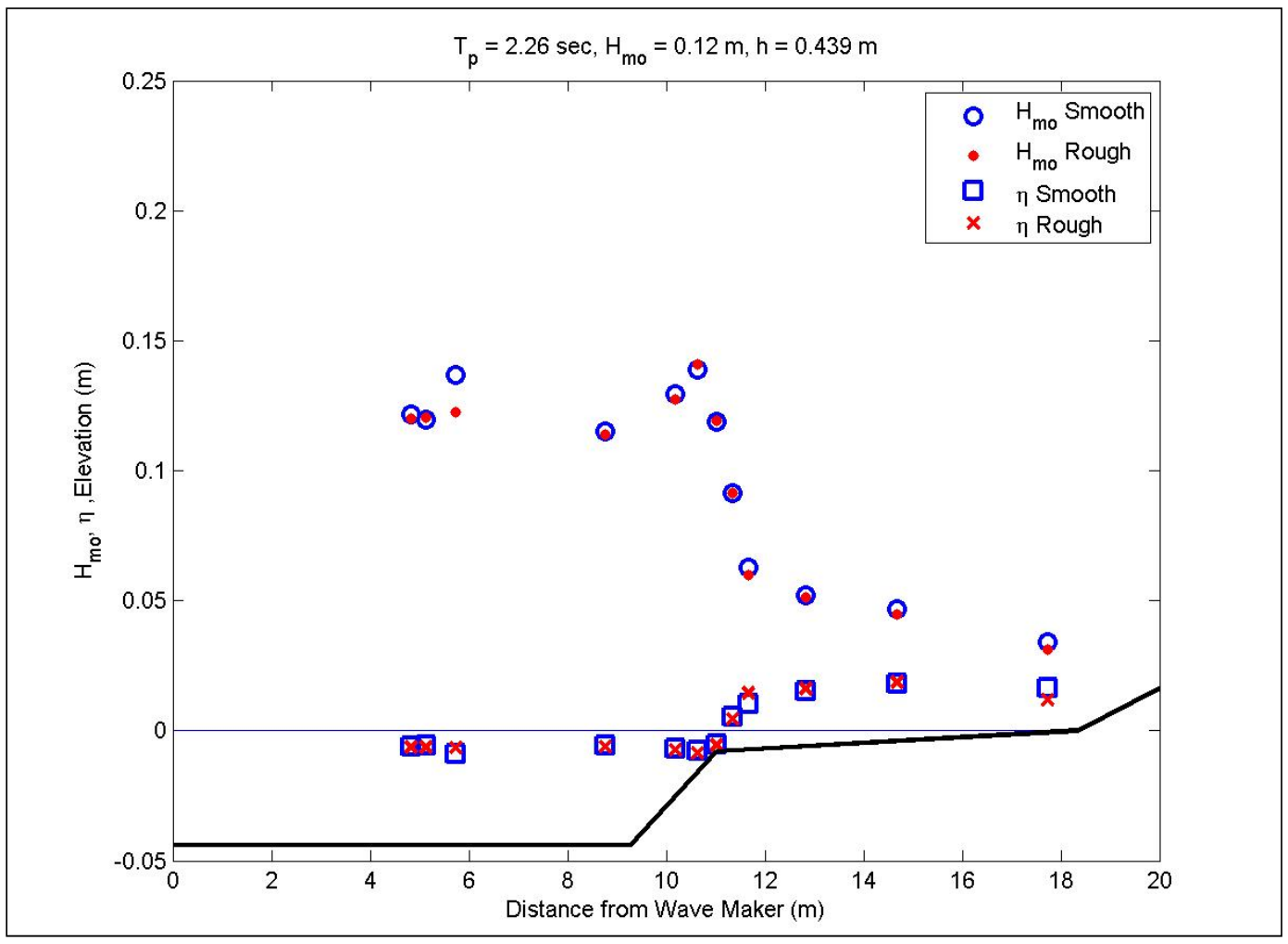

Figure E67. Wave transformation for 2.3-sec, 0.12-m waves at mid water with a 1:5 reef slope. 


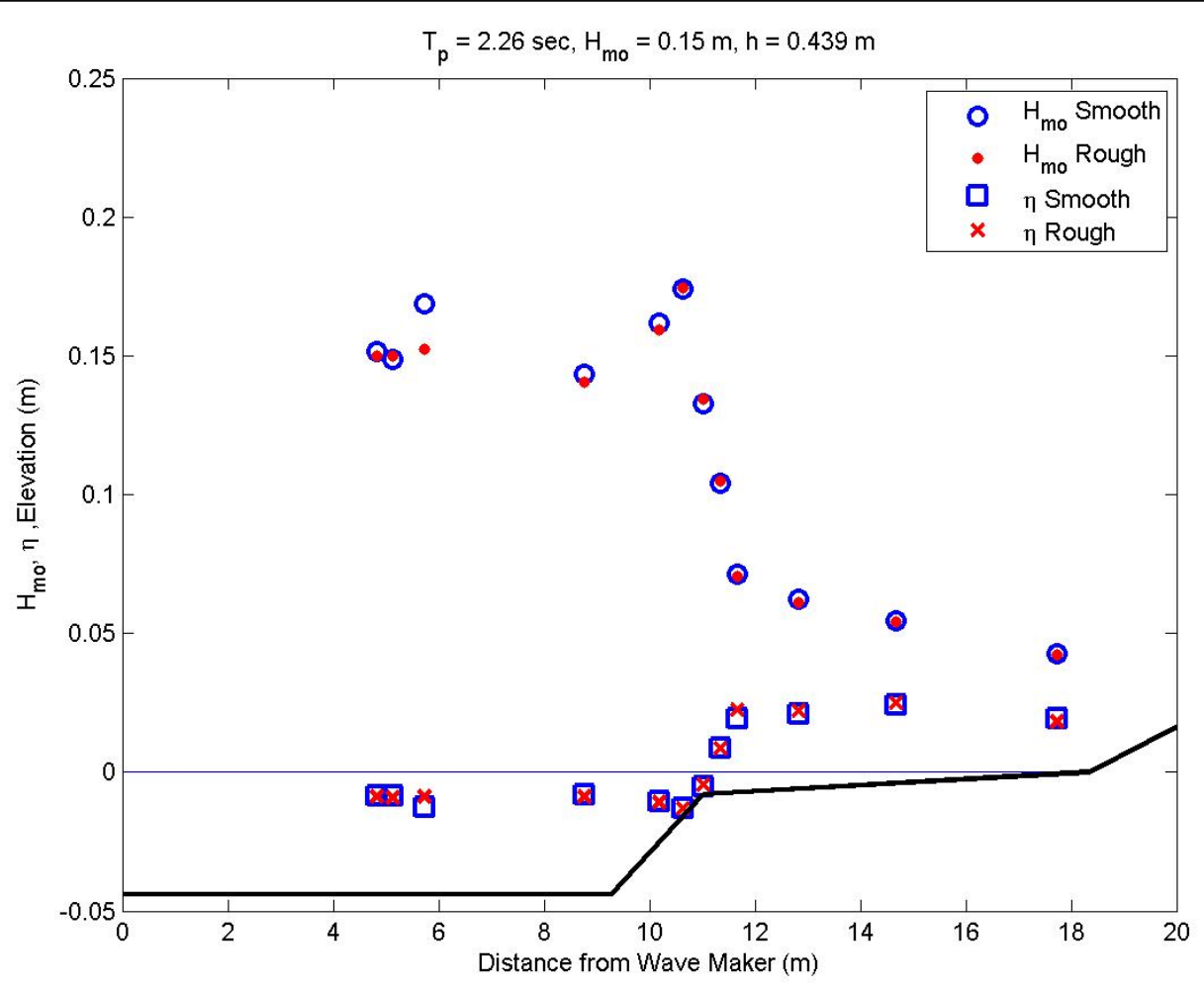

Figure E68. Wave transformation for 2.3-sec, 0.15-m waves at mid water with a 1:5 reef slope.

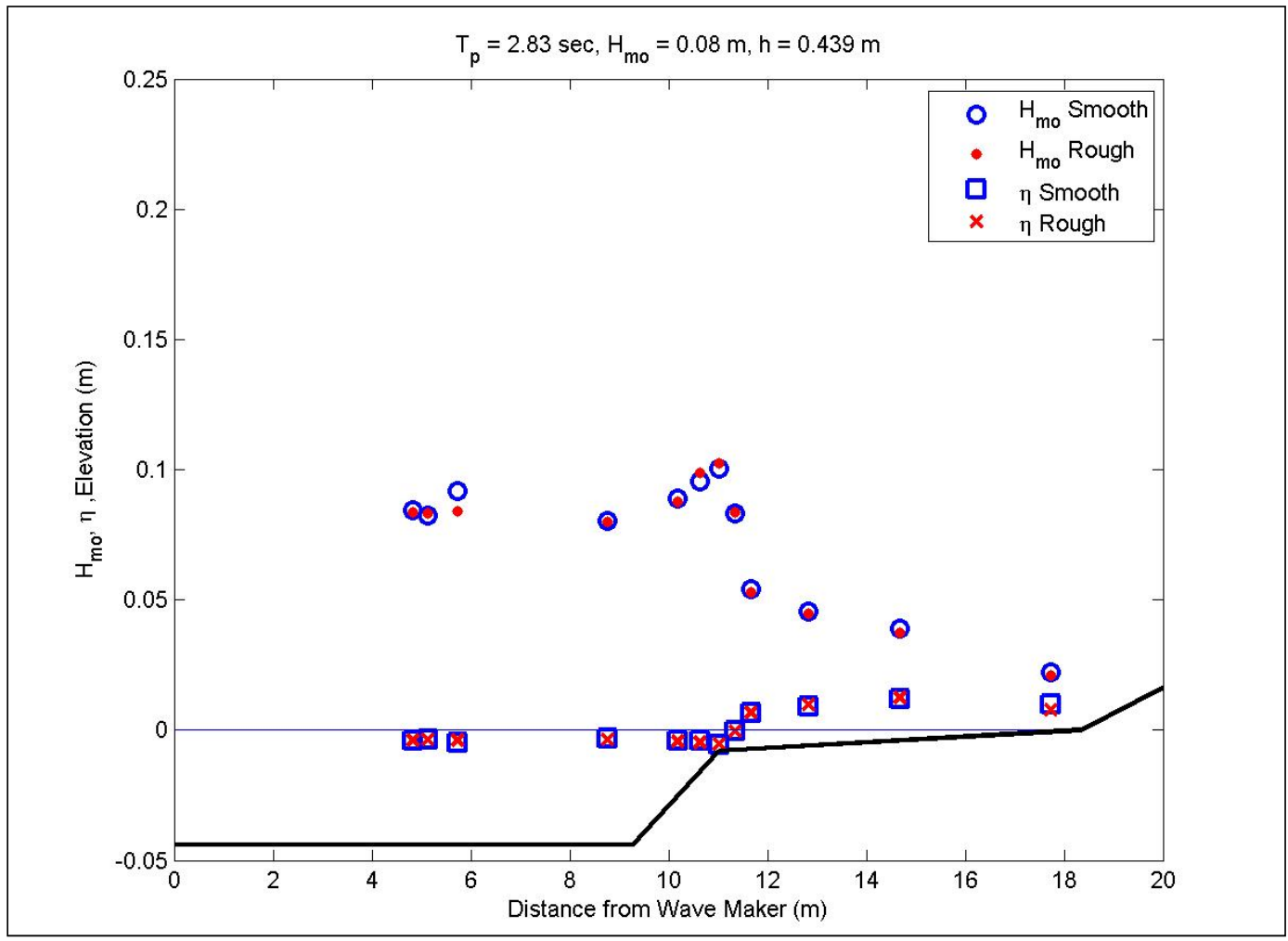

Figure E69. Wave transformation for 2.8-sec, 0.08-m waves at mid water with a 1:5 reef slope. 


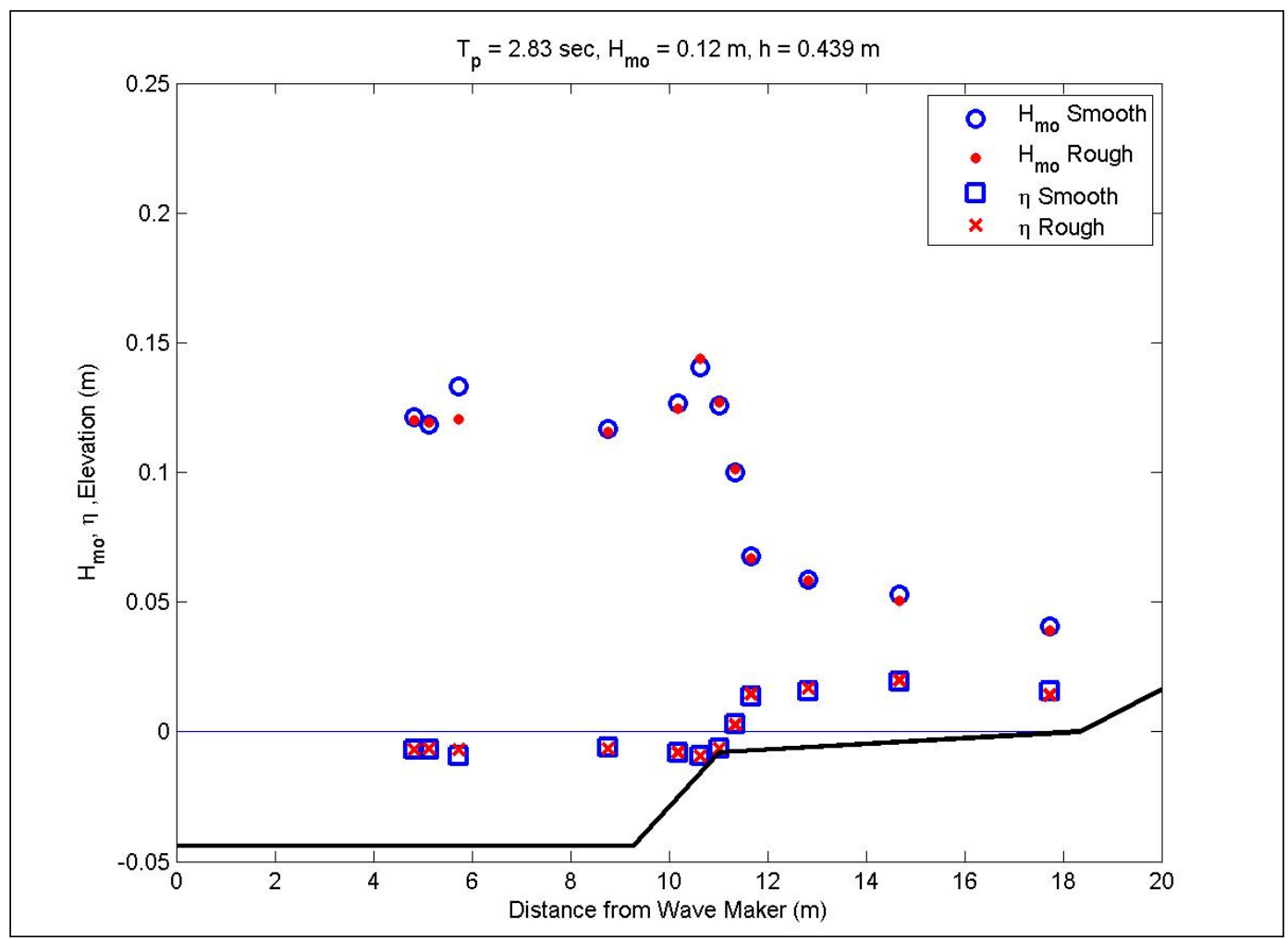

Figure E70. Wave transformation for 2.8-sec, 0.12-m waves at mid water with a 1:5 reef slope.

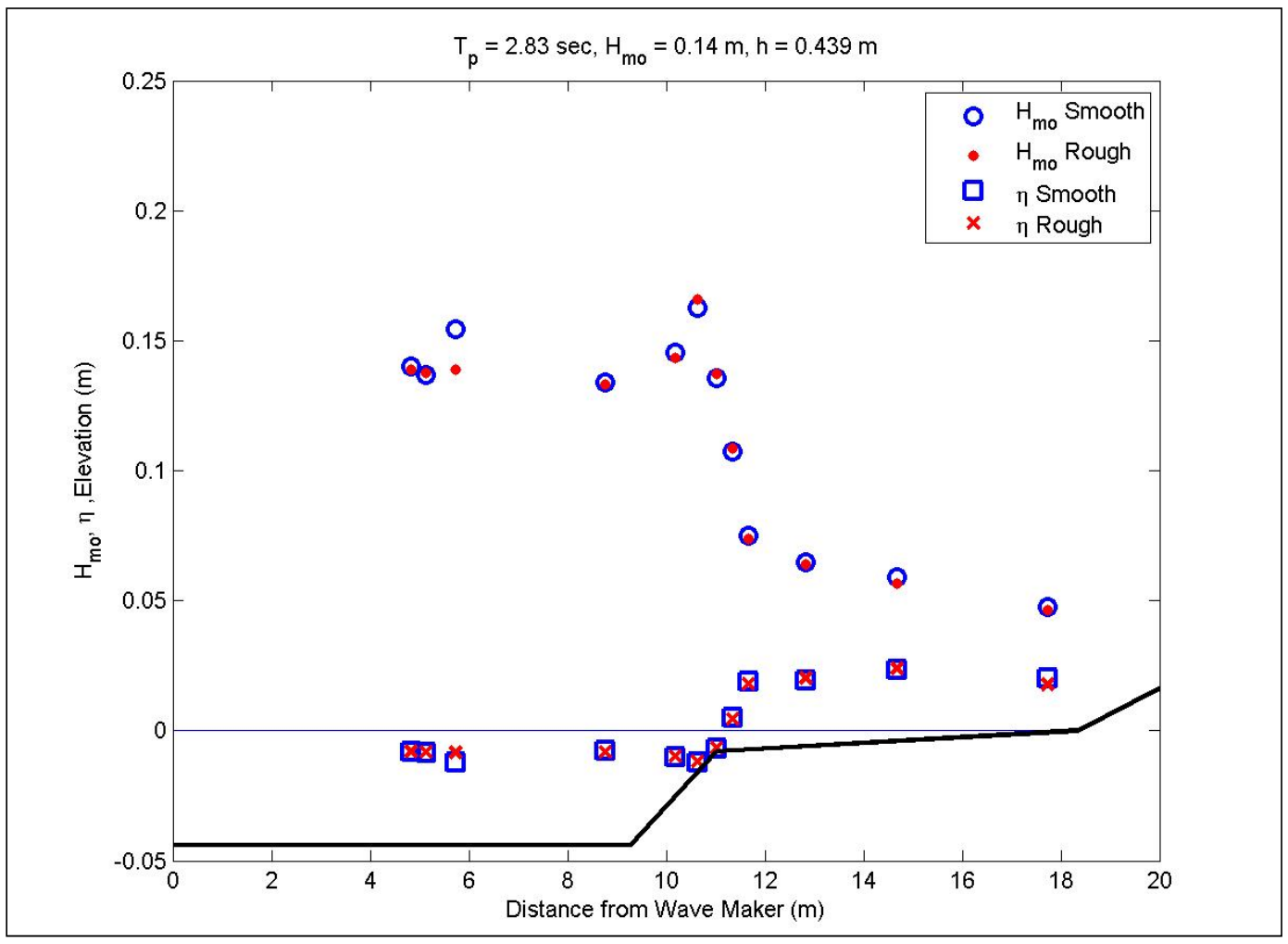

Figure E71. Wave transformation for 2.8-sec, 0.14-m waves at mid water with a 1:5 reef slope. 


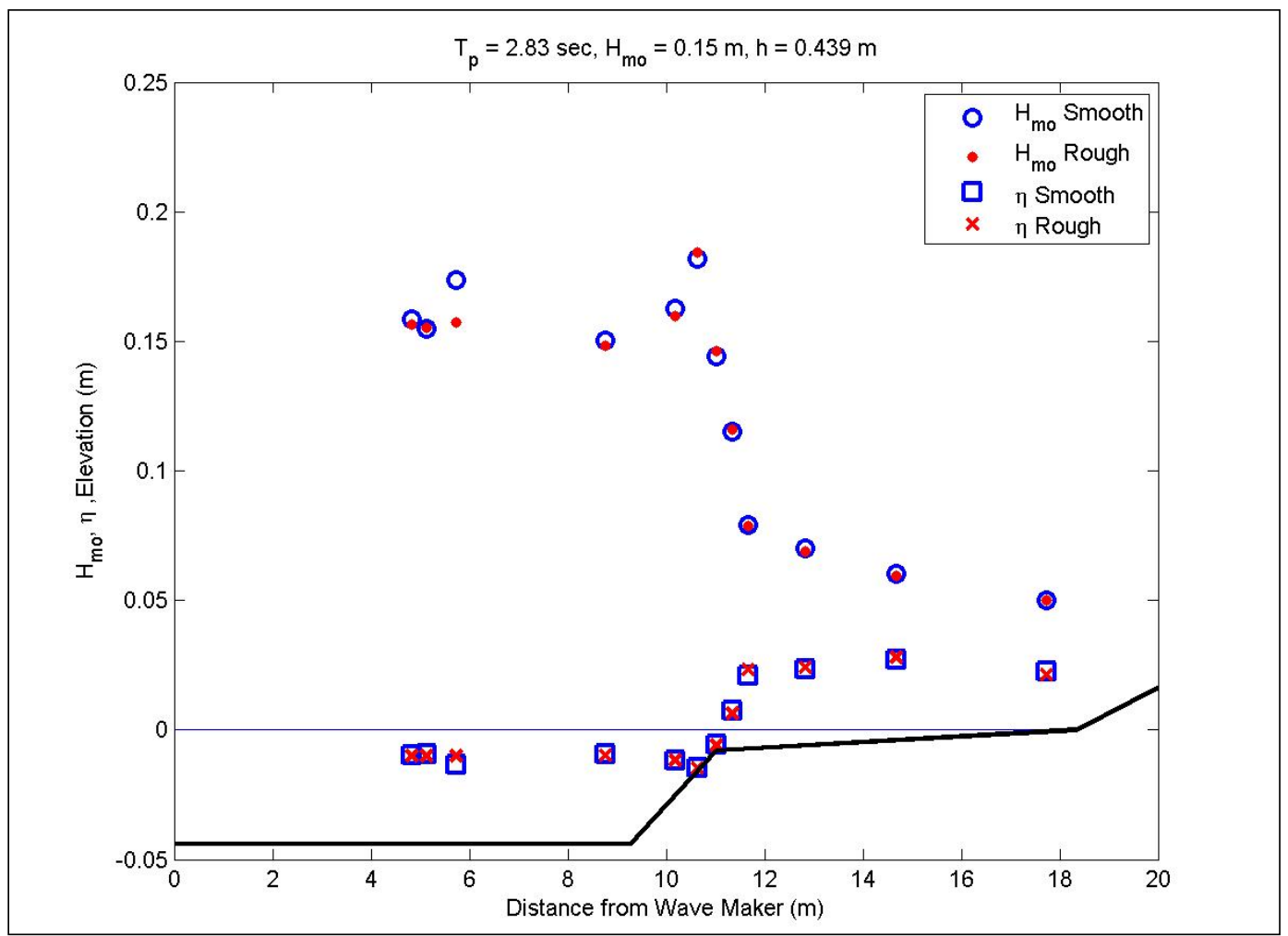

Figure E72. Wave transformation for 2.8-sec, 0.15-m waves at mid water with a 1:5 reef slope.

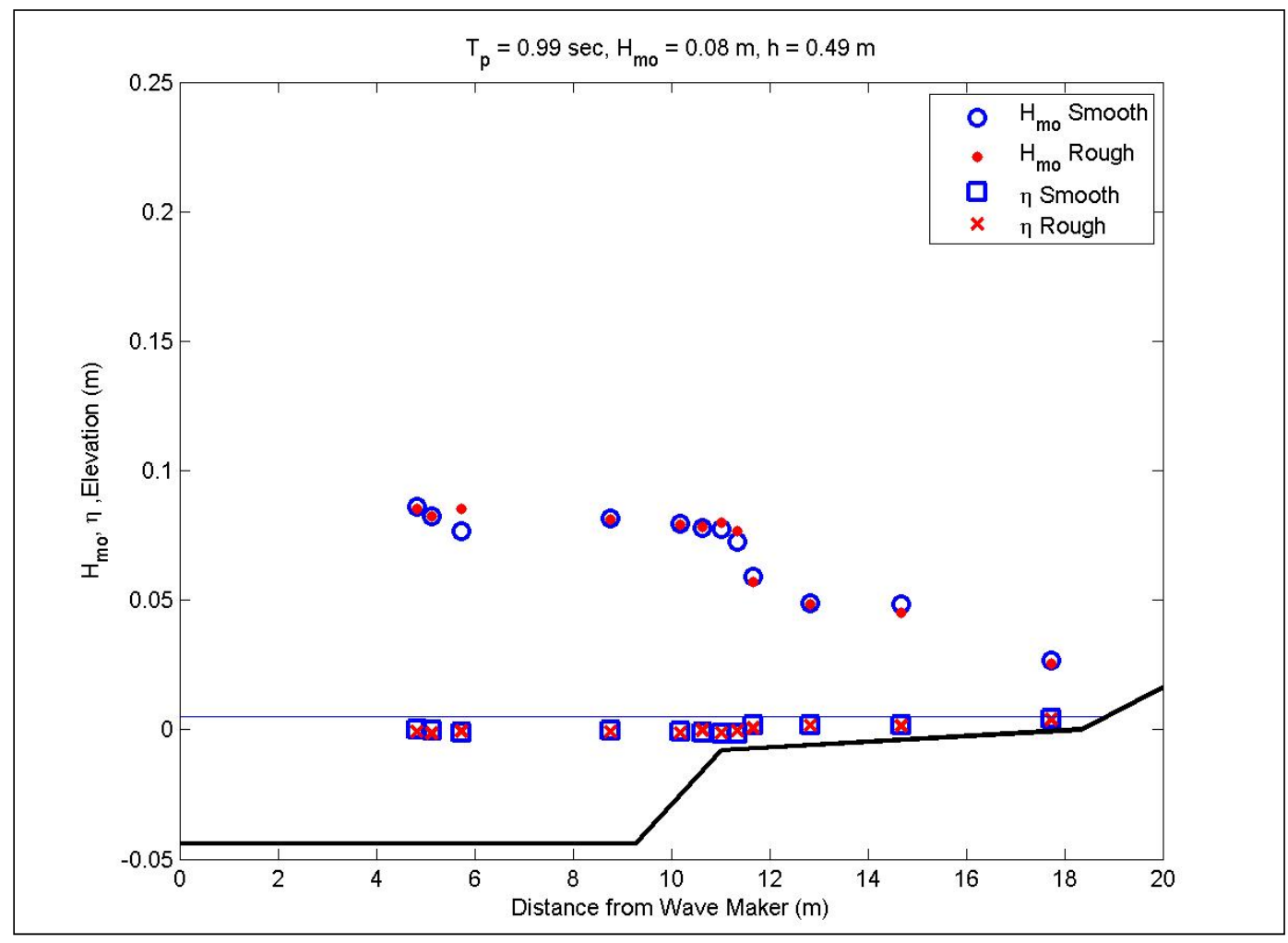

Figure E73. Wave transformation for 1-sec, 0.08-m waves at high water with a 1:5 reef slope. 


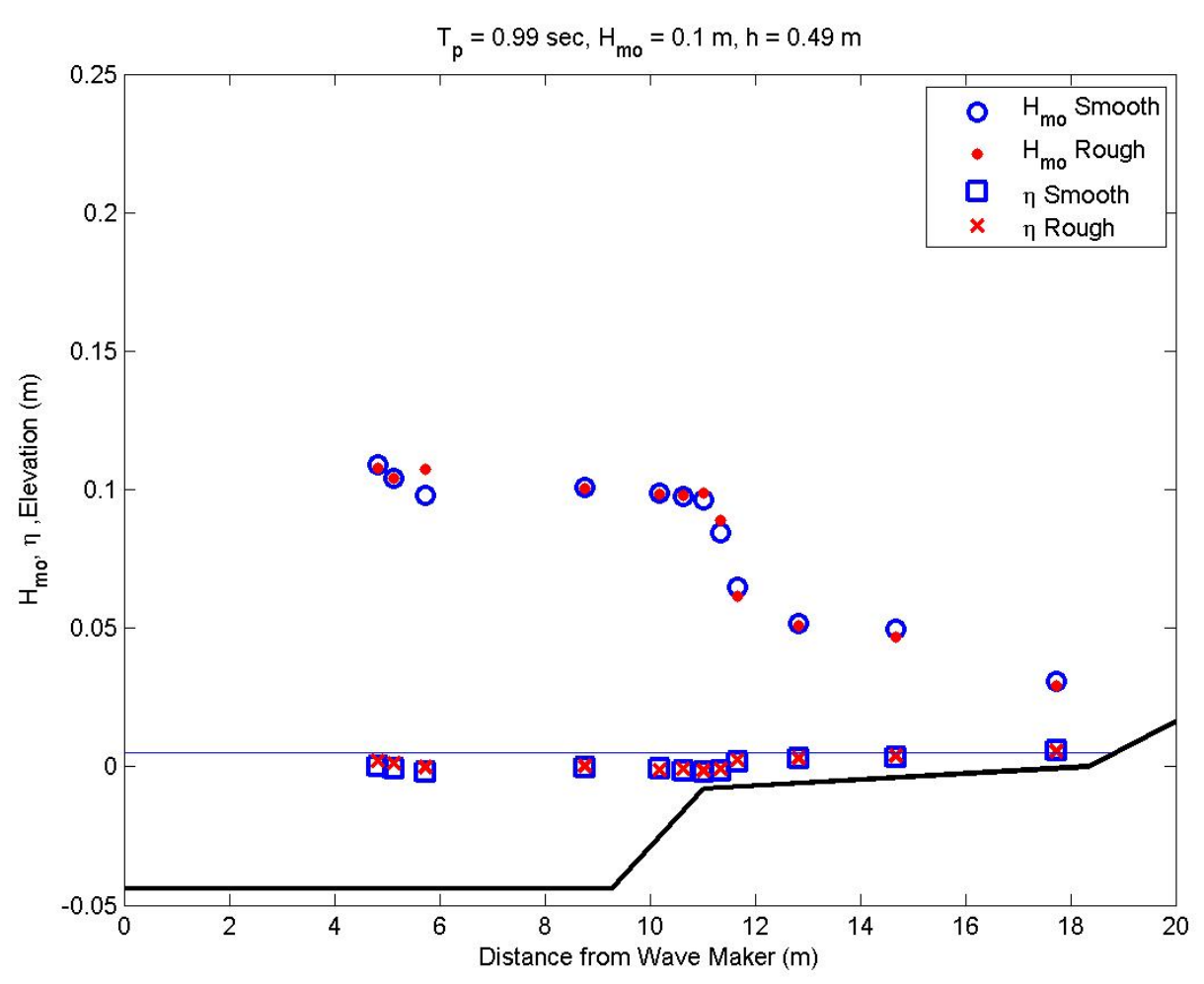

Figure E74. Wave transformation for 1-sec, 0.1-m waves at high water with a 1:5 reef slope.

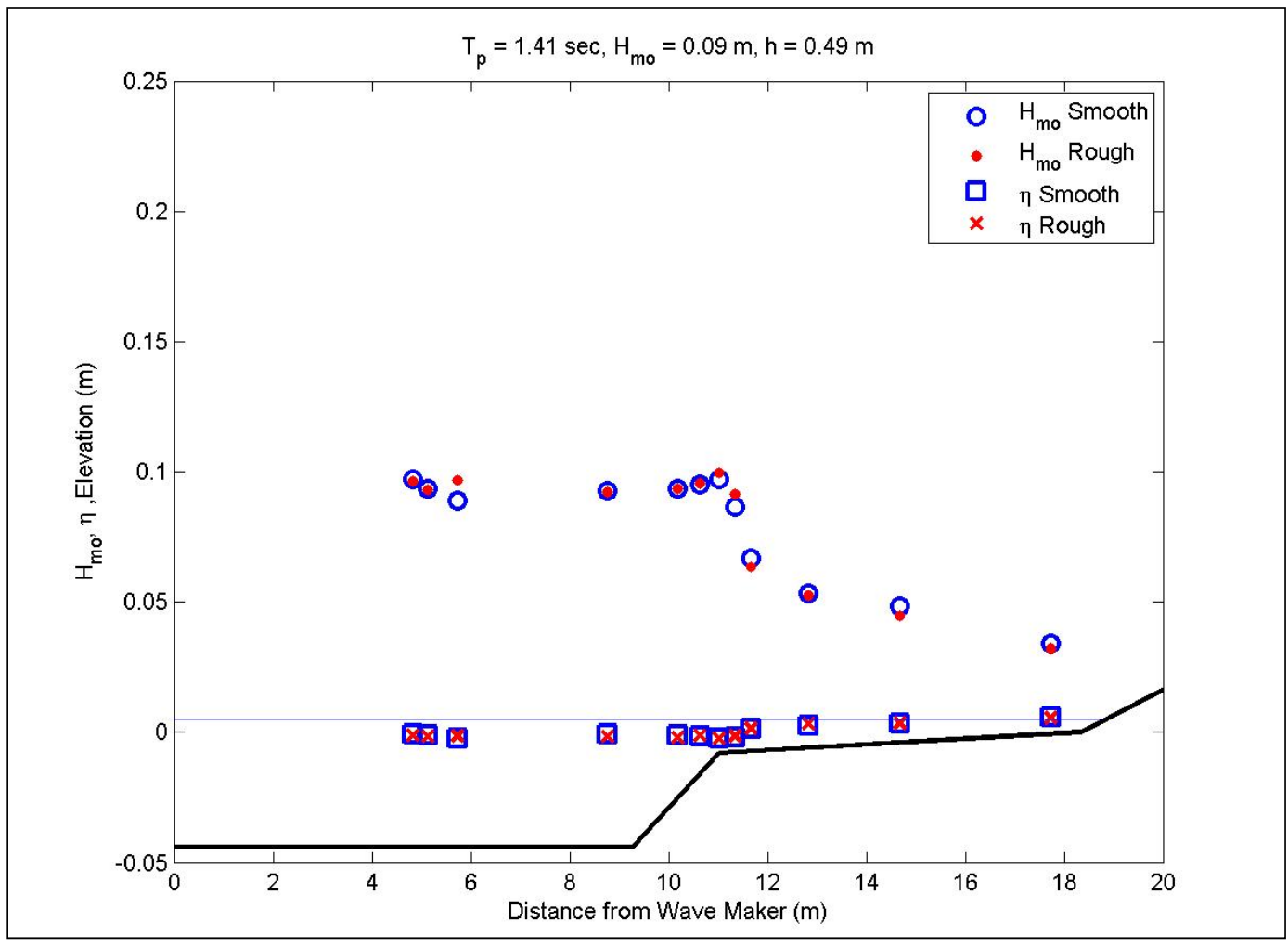

Figure E75. Wave transformation for 1.4-sec, 0.09-m waves at high water with a 1:5 reef slope. 


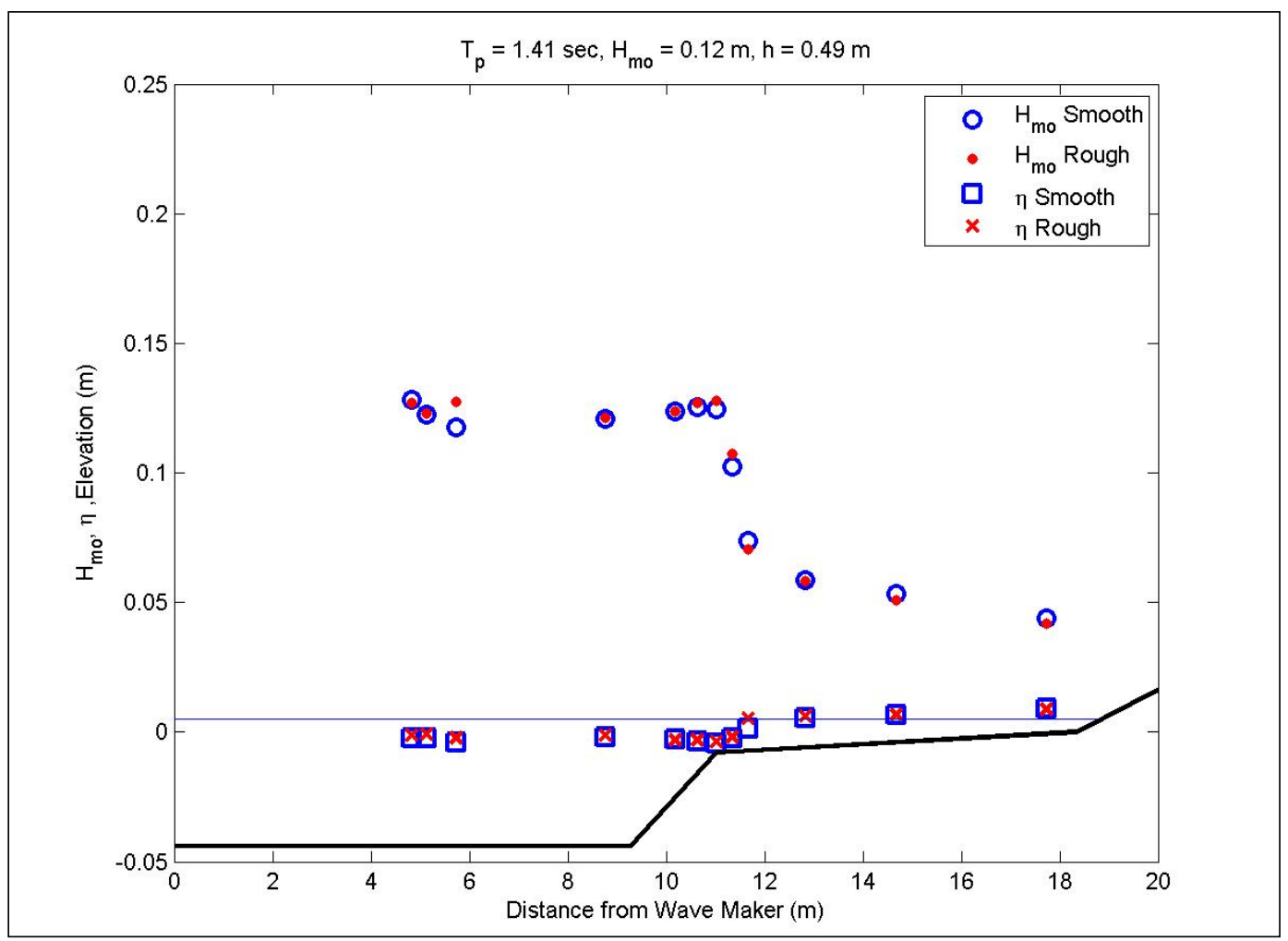

Figure E76. Wave transformation for 1.4-sec, 0.12-m waves at high water with a 1:5 reef slope.

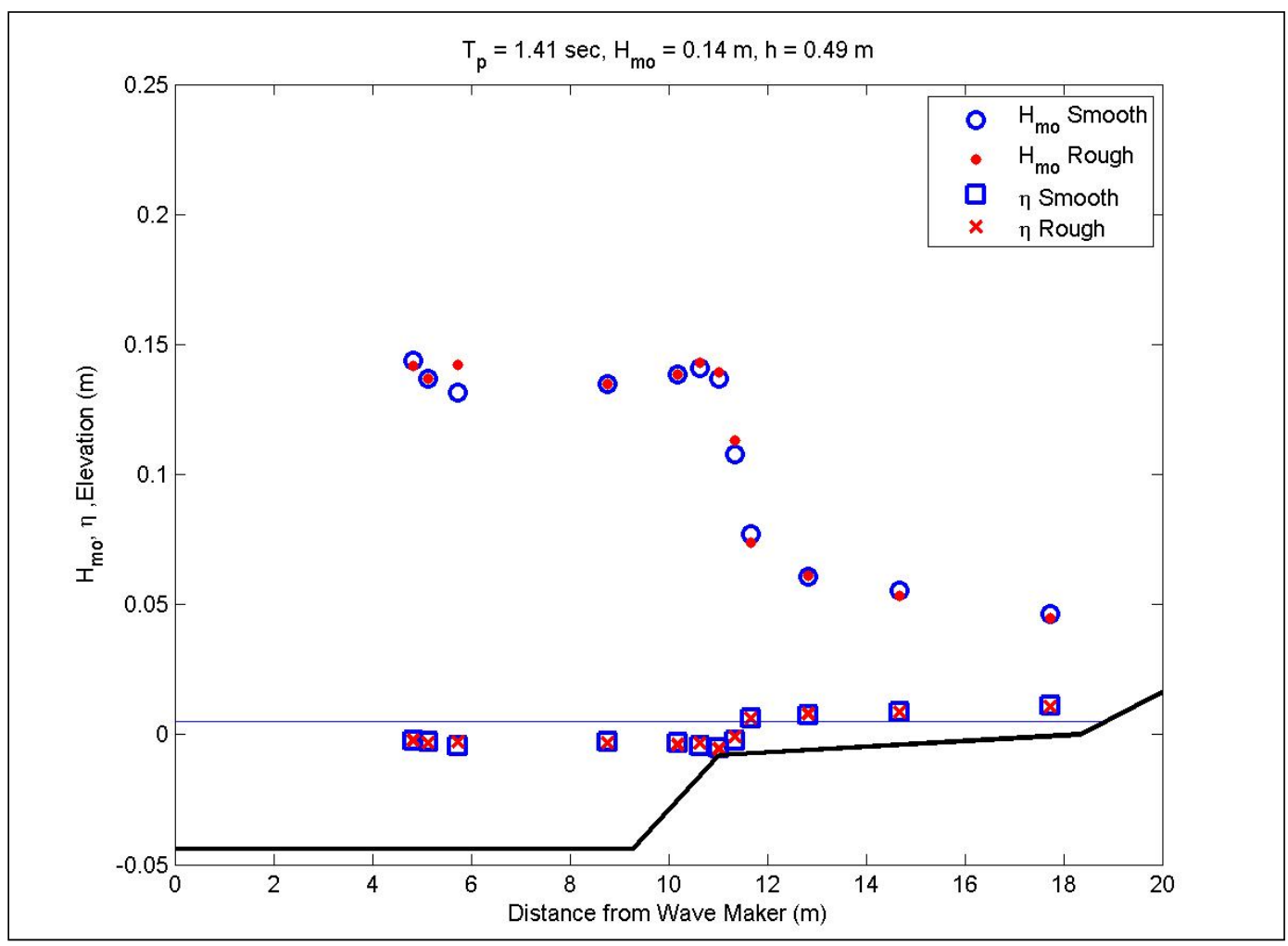

Figure E77. Wave transformation for 1.4-sec, 0.14-m waves at high water with a 1:5 reef slope. 


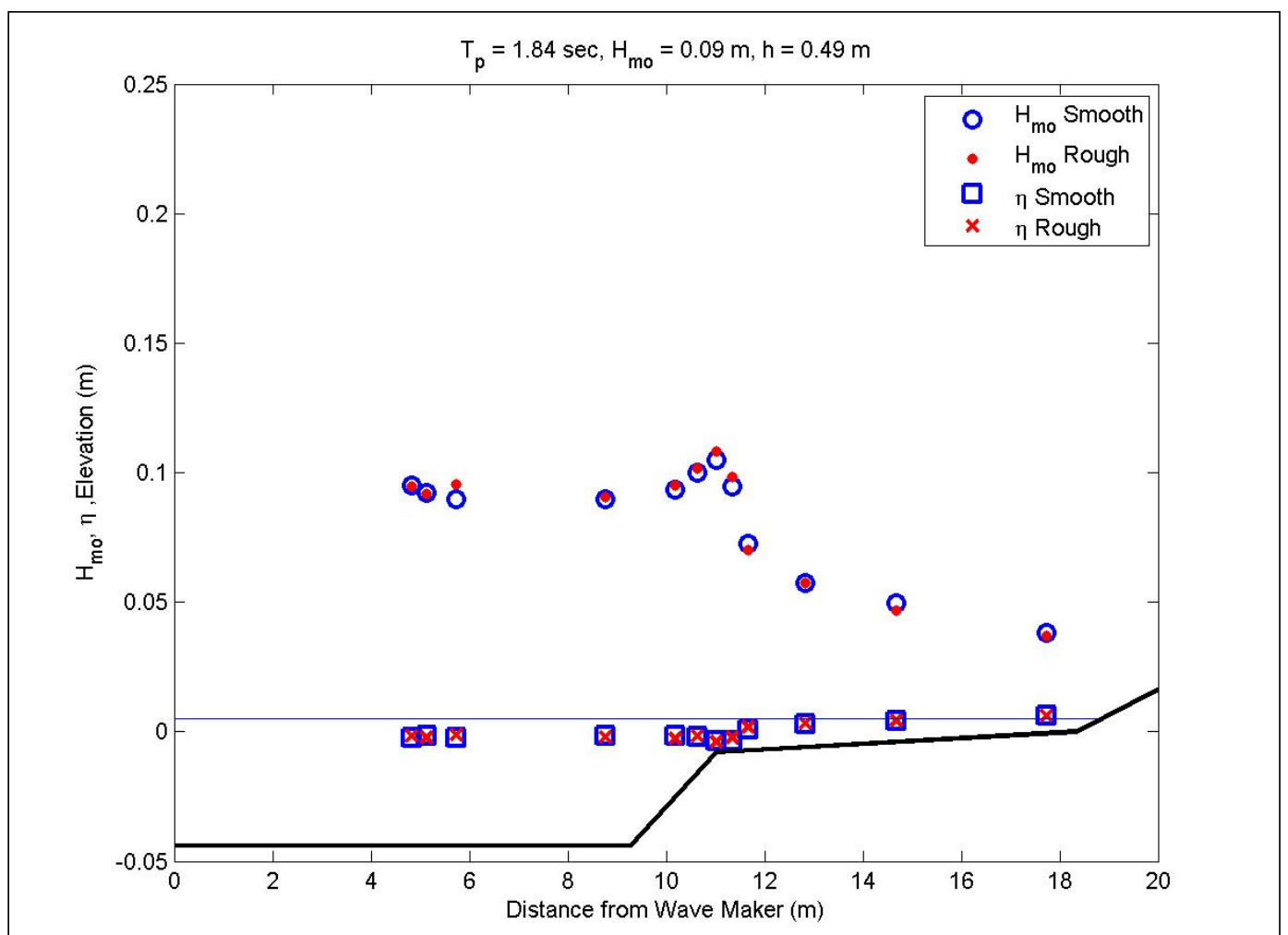

Figure E78. Wave transformation for 1.8-sec, 0.09-m waves at high water with a 1:5 reef slope.

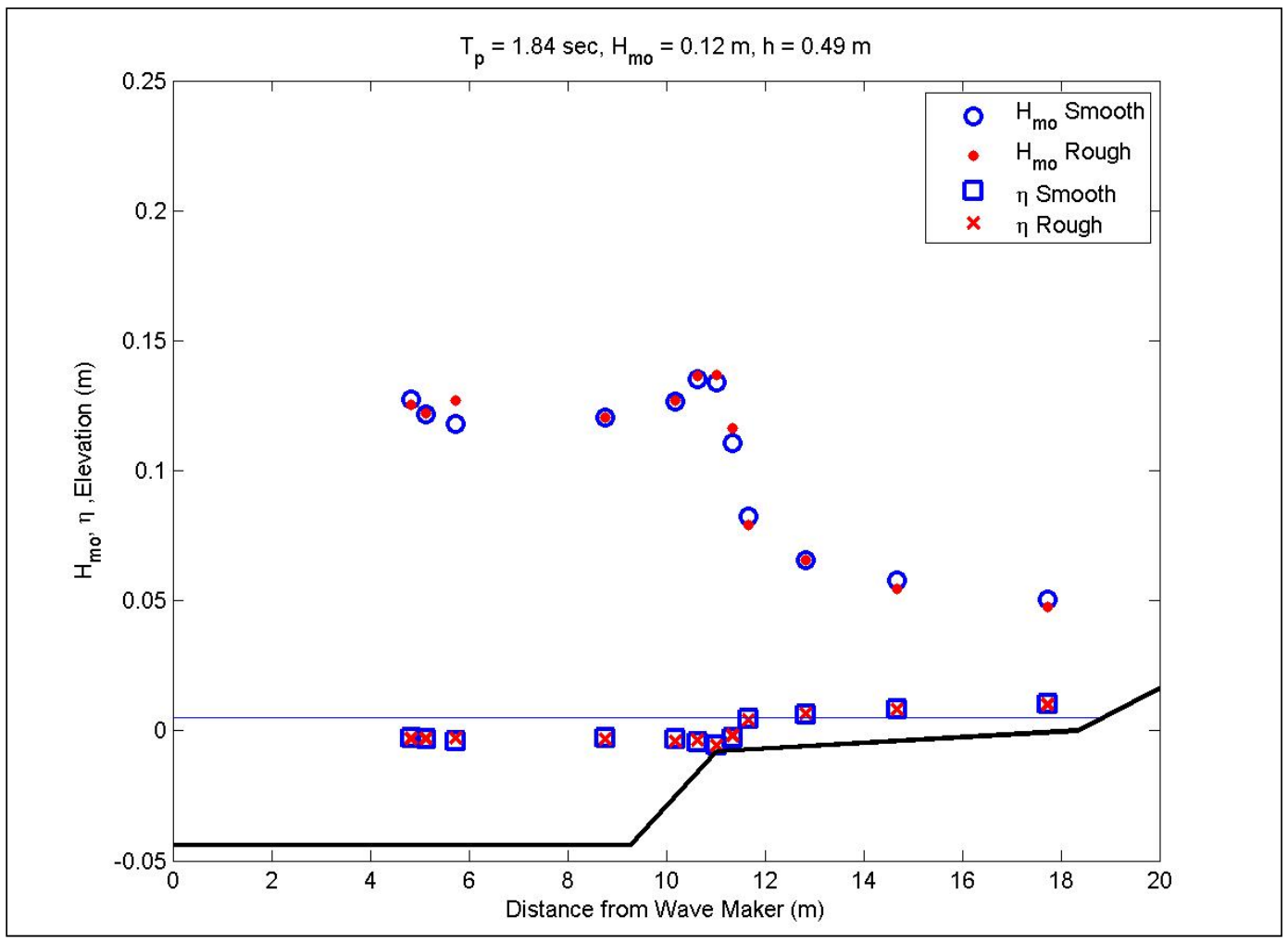

Figure E79. Wave transformation for 1.8-sec, 0.12-m waves at high water with a 1:5 reef slope. 


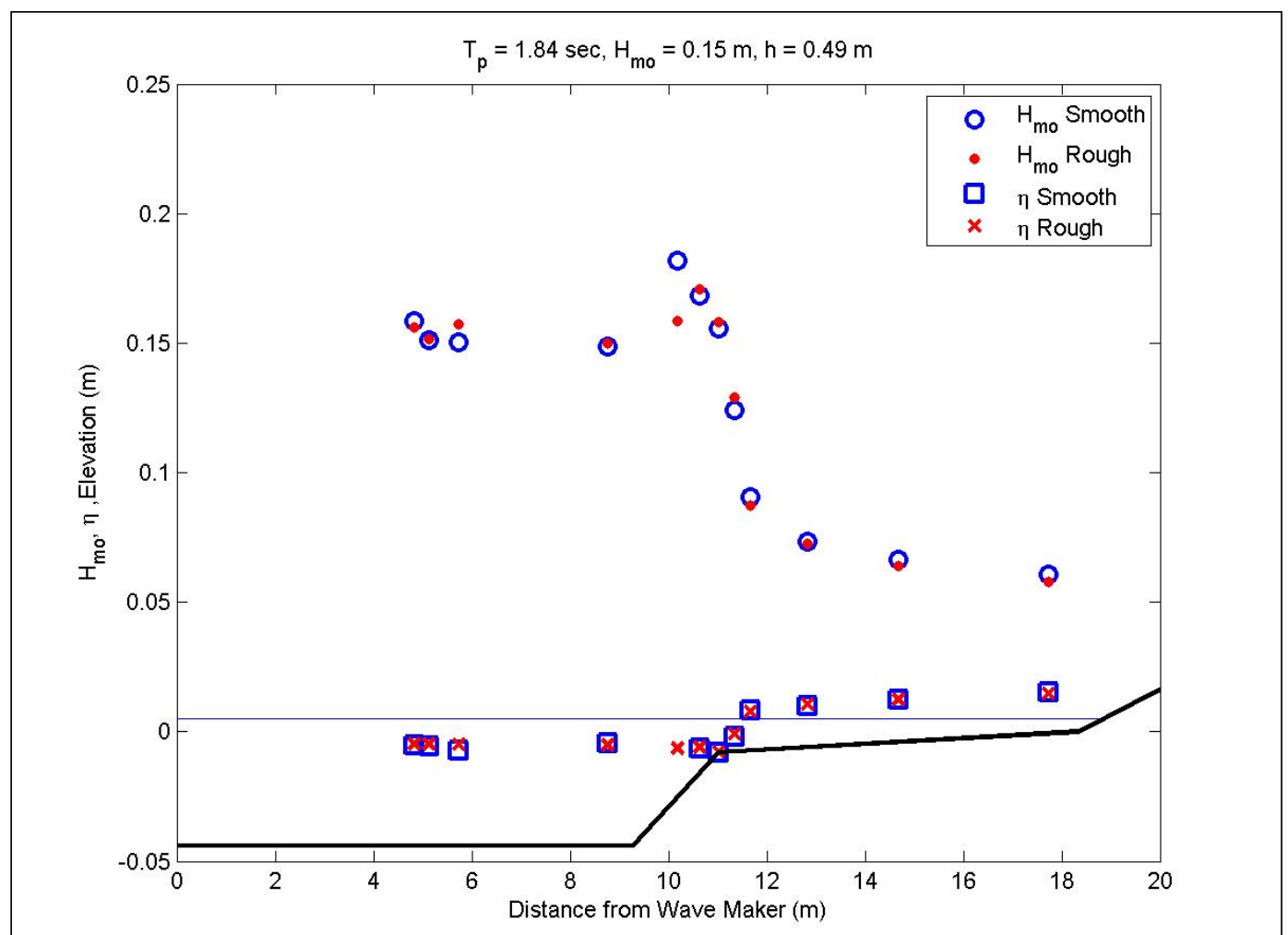

Figure E80. Wave transformation for 1.8-sec, 0.15-m waves at high water with a 1:5 reef slope.

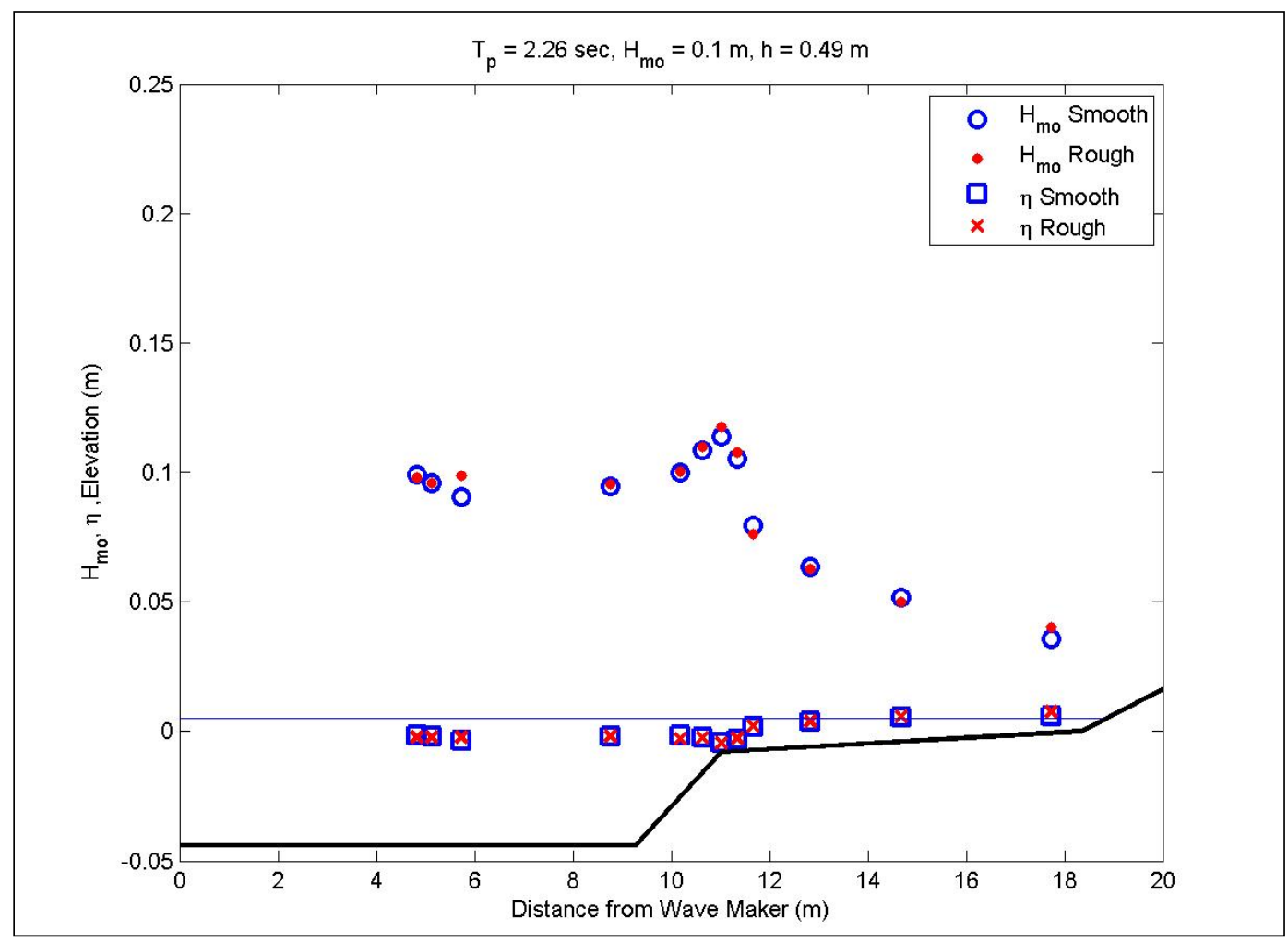

Figure E81. Wave transformation for 2.3-sec, 0.1-m waves at high water with a 1:5 reef slope. 


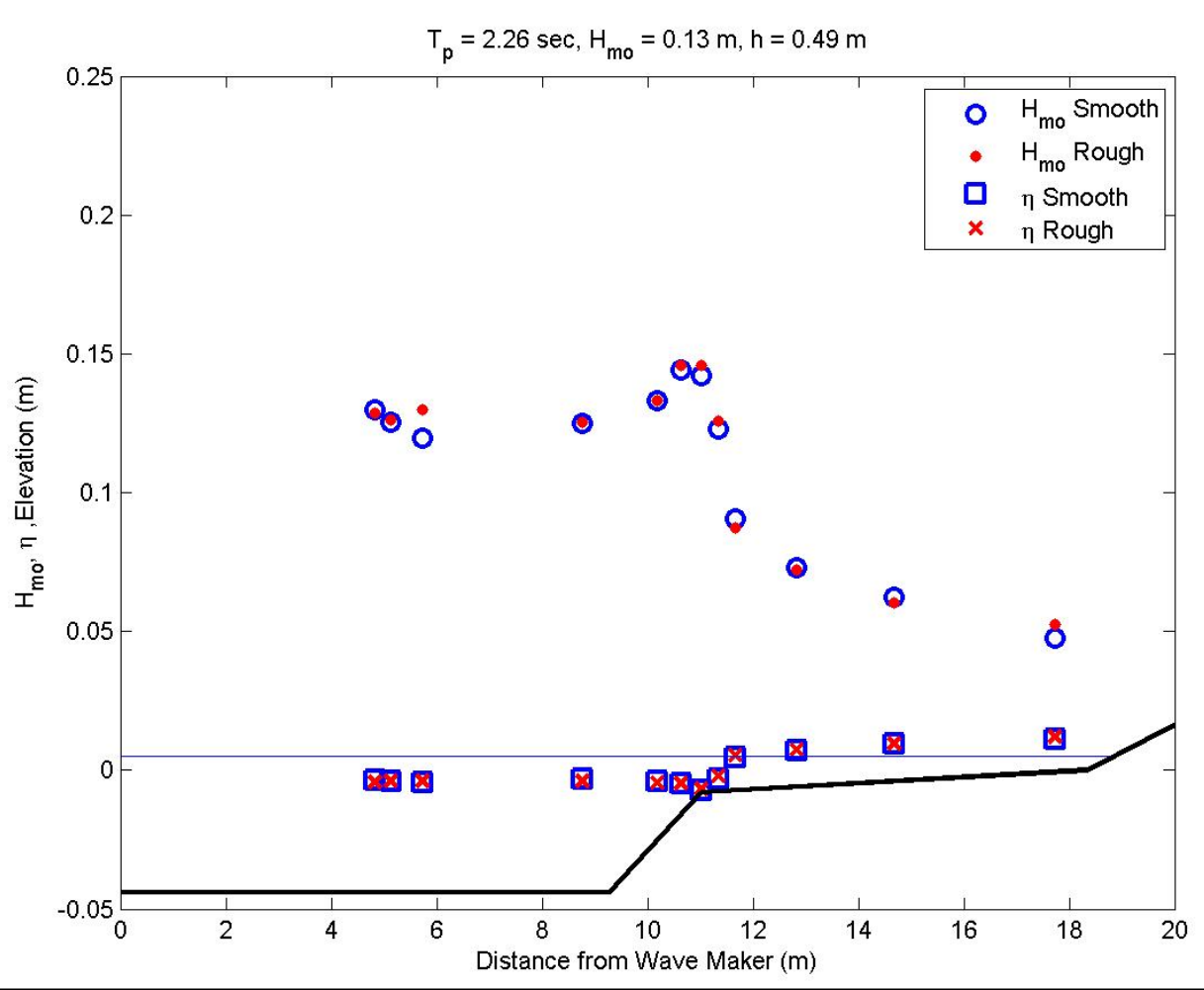

Figure E82. Wave transformation for 2.3-sec, 0.13-m waves at high water with a 1:5 reef slope.

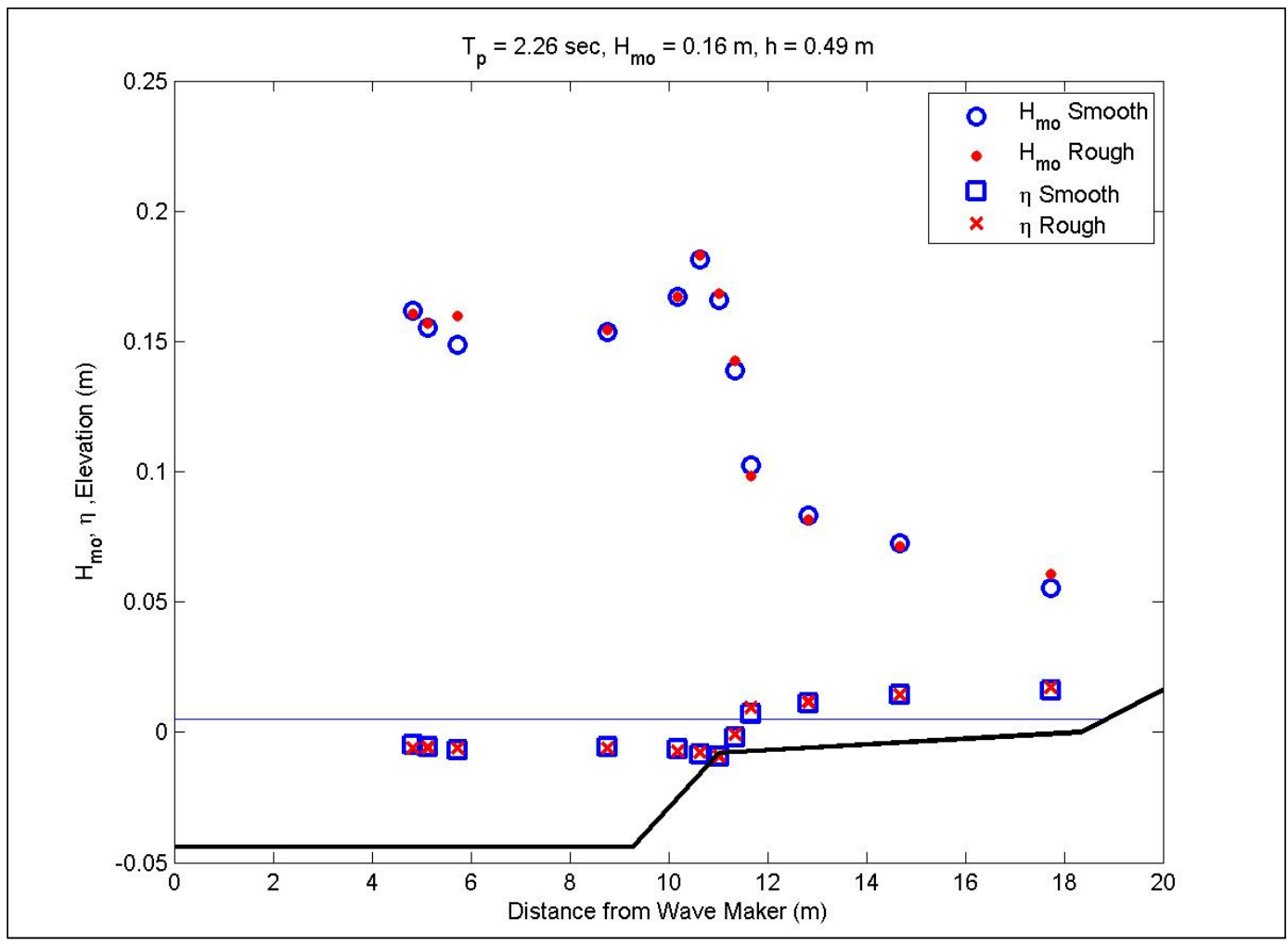

Figure E83. Wave transformation for 2.3-sec, 0.16-m waves at high water with a 1:5 reef slope. 


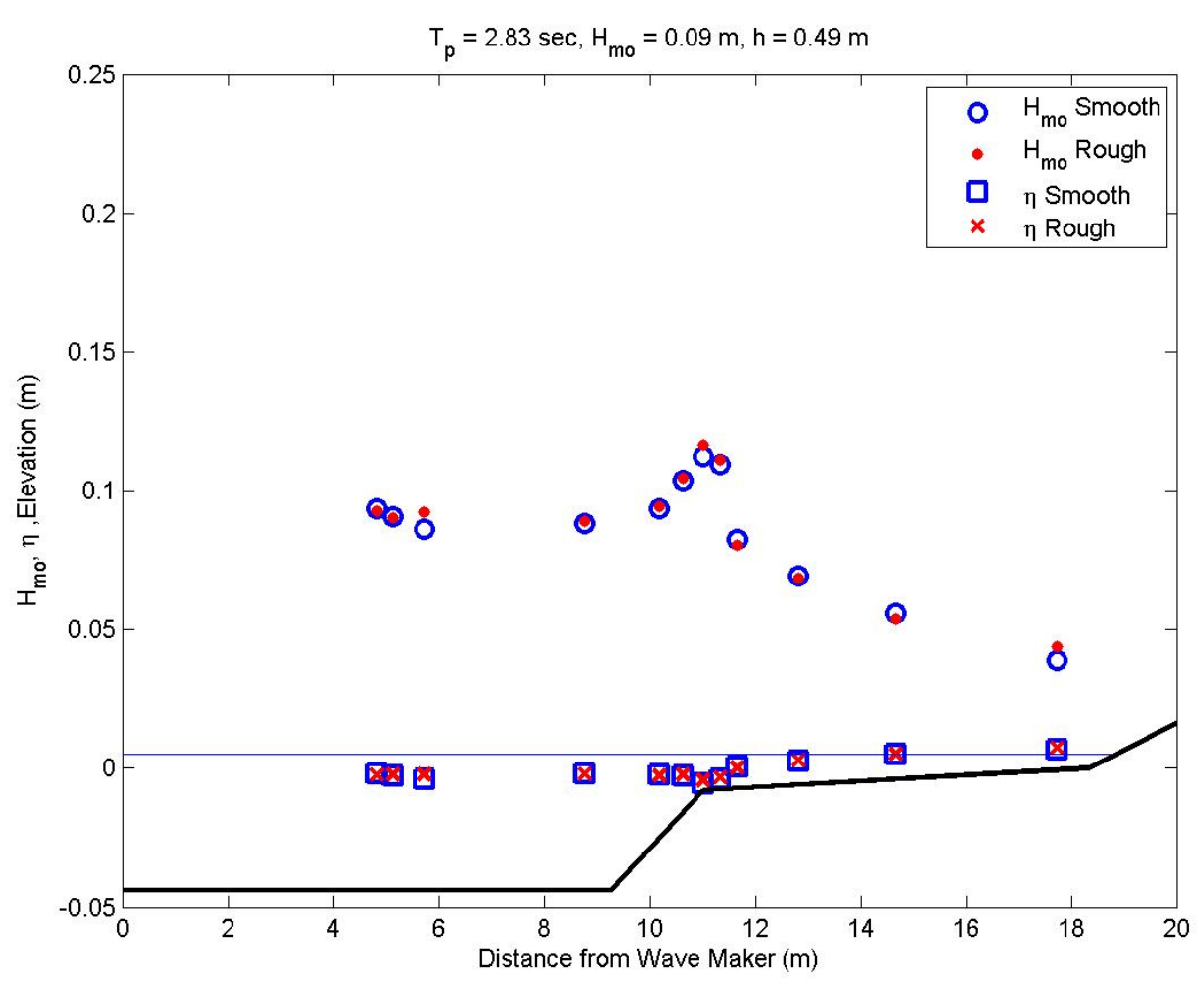

Figure E84. Wave transformation for 2.8-sec, 0.09-m waves at high water with a 1:5 reef slope.

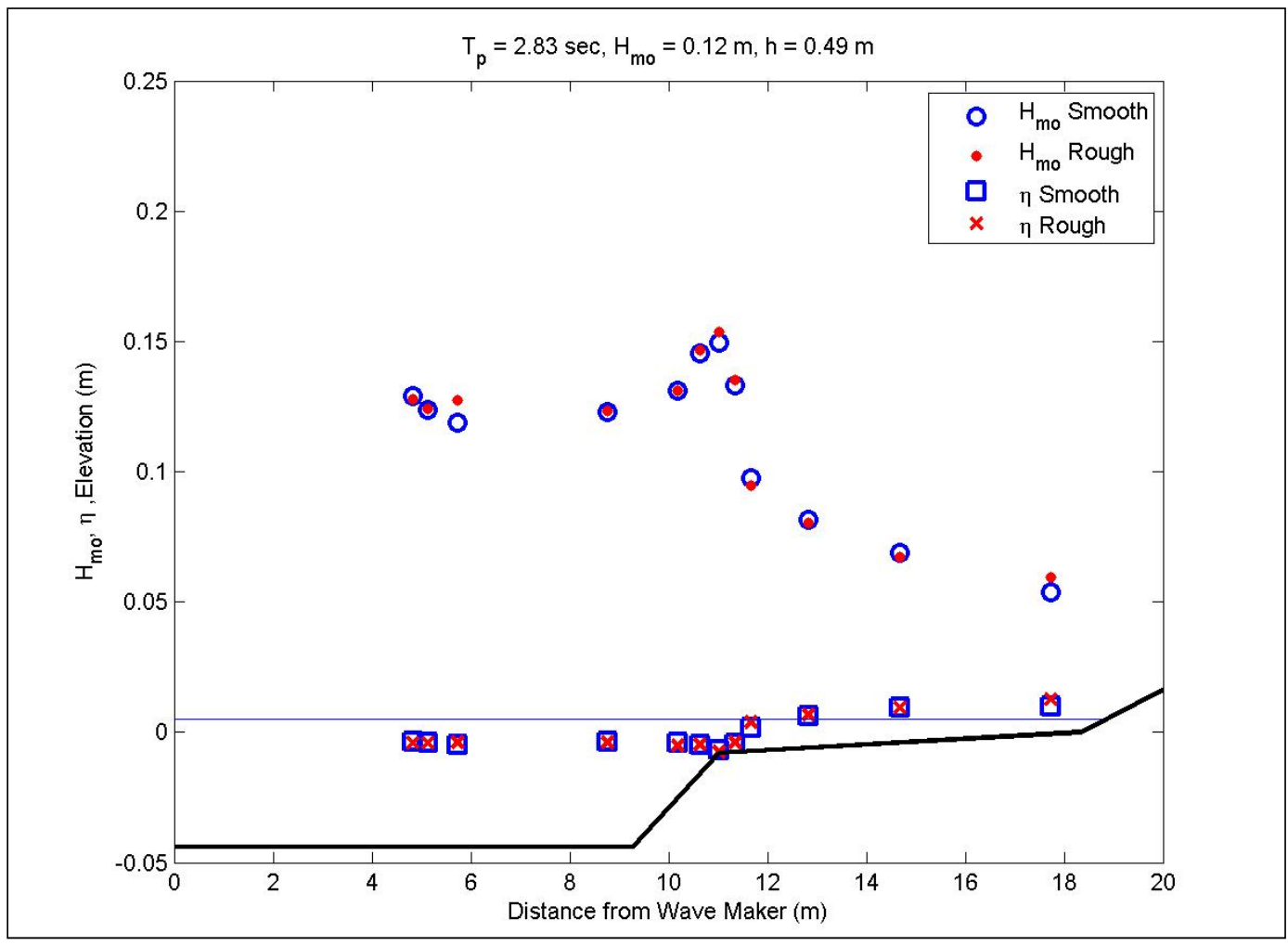

Figure E85. Wave transformation for 2.8-sec, 0.12-m waves at high water with a 1:5 reef slope. 


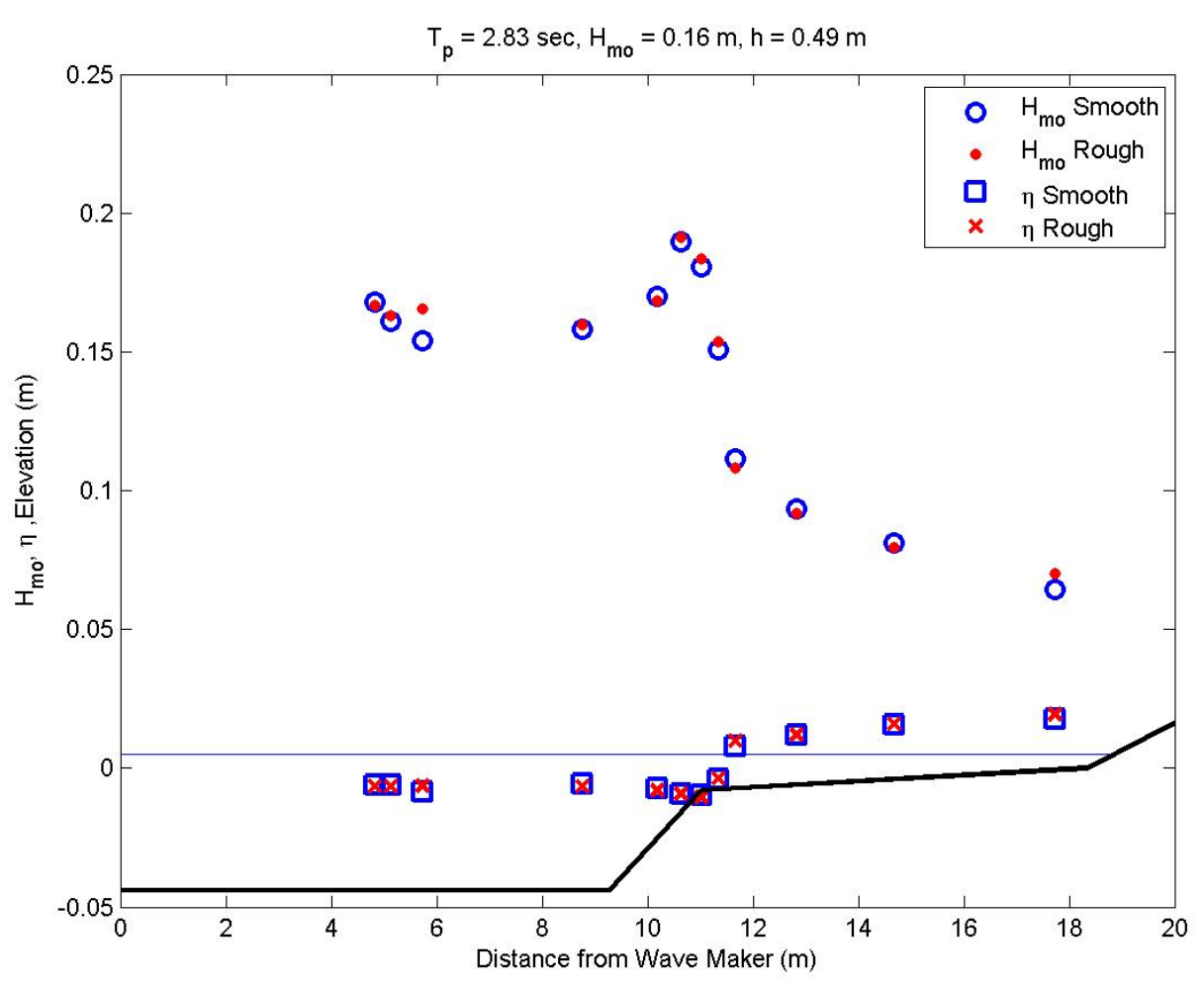

Figure E86. Wave transformation for 2.8-sec, 0.16-m waves at high water with a 1:5 reef slope. 


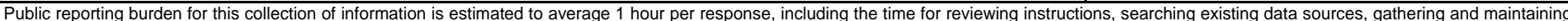

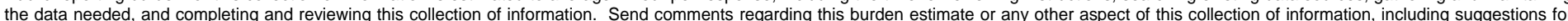

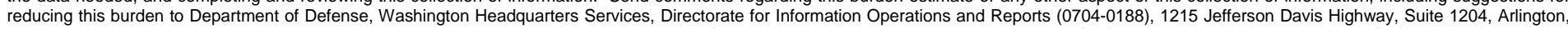

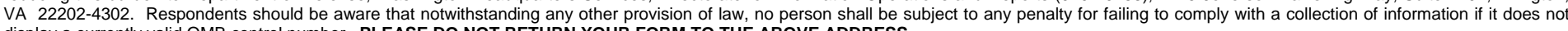
display a currently valid OMB control number. PLEASE DO NOT RETURN YOUR FORM TO THE ABOVE ADDRESS.

\begin{tabular}{l|c}
$\begin{array}{l}\text { 1. REPORT DATE }(D D-M M-Y Y Y Y) \\
\text { September } 2012\end{array}$ & $\begin{array}{c}\text { 2. REPORT TYPE } \\
\text { Final report }\end{array}$ \\
\hline
\end{tabular}

\section{TITLE AND SUBTITLE}

Two- and Three-Dimensional Laboratory Studies of Wave Breaking, Dissipation, Setup, and Runup on Reefs

3. DATES COVERED (From - To)

5a. CONTRACT NUMBER

5b. GRANT NUMBER

5c. PROGRAM ELEMENT NUMBER

\section{AUTHOR(S)}

5d. PROJECT NUMBER

Ernest R. Smith, Tyler J. Hesser, and Jane McKee Smith

5e. TASK NUMBER

5f. WORK UNIT NUMBER

\section{PERFORMING ORGANIZATION NAME(S) AND ADDRESS(ES)}

8. PERFORMING ORGANIZATION REPORT NUMBER

U.S. Army Engineer Research and Development Center

Coastal and Hydraulics Laboratory

ERDC/CHL TR-12-21

3909 Halls Ferry Road

Vicksburg, MS 39180-6199

9. SPONSORING I MONITORING AGENCY NAME(S) AND ADDRESS(ES)

10. SPONSOR/MONITOR'S ACRONYM(S)

U.S. Army Corps of Engineers

441 G. Street, NW

Washington, DC 20314-1000

11. SPONSOR/MONITOR'S REPORT

NUMBER(S)

\section{DISTRIBUTION I AVAILABILITY STATEMENT}

Approved for public release; distribution is unlimited

\section{SUPPLEMENTARY NOTES}

\section{ABSTRACT}

Physical model studies were performed at a 1-to-50 model to prototype scale in a three-dimensional (3-D) basin and a two-dimensional (2-D) flume to measure wave transformation, setup, and runup on a generalized reef to improve understanding of the processes. Incident wave and water level conditions encompassed a range of combinations representative of the geographical locations where reefs are present.

Results from the 3-D experiment indicated higher frequency wave energy dissipated across the reef, whereas low frequency wave energy remained nearly constant. Three-dimensional patterns were observed on the reef, which was attributed to non-symmetrical bathymetry, wave-wave interactions, wave refraction, and reflection. Additional tests with an angled channel cut into the reef showed net offshore flow in the channel and near the reef centerline, where a net alongshore velocity also was measured. Wave heights were similar to the nonchannel tests, but longer-period waves with higher incident heights generally showed greater heights in the shoaling region.

A cross-section of the 3-D reef was duplicated and installed in a wave tank. The 2-D experiments were performed for similar wave and water level conditions as in the three-dimensional tests. Reef slopes of 1:2.5 and 1:5 were examined. Transformation results were similar to the 3-D tests except that a strong return flow was observed which increased breaker height. Reef roughness was varied for both the 2-D and 3-D tests, but had little effect on the waves, indicating that a rougher surface would be necessary to reduce wave height

\begin{tabular}{lll}
\hline 15. SUBJECT TERMS & (SWIMS) & Wave breaking \\
Fringing reefs & Wave transformation & Wave reflection
\end{tabular}

Physical modeling

Wave Setup

Surge and Wave Island Modeling Studies Wave runup

16. SECURITY CLASSIFICATION OF:

a. REPORT

UNCLASSIFIED

\section{b. ABSTRACT} UNCLASSIFIED c. THIS PAGE UNCLASSIFIED

\begin{tabular}{|c|c|}
\hline $\begin{array}{l}\text { 17. LIMITATION } \\
\text { OF ABSTRACT }\end{array}$ & $\begin{array}{c}\text { 18. NUMBER } \\
\text { OF PAGES }\end{array}$ \\
UNCLASSIFIED & 274 \\
\hline
\end{tabular}

19a. NAME OF RESPONSIBLE PERSON

19b. TELEPHONE NUMBER (include area code) 